LA.8251-MS

MASTER

Informal Report

UC-34a

issued: July 1980

\title{
An Atlas of Uranium Emission Intensities in \\ A Hollow Cathode Discharge
}

Byron A. Palmer

Richard A. Keller

Rolf Engleman, Jr.
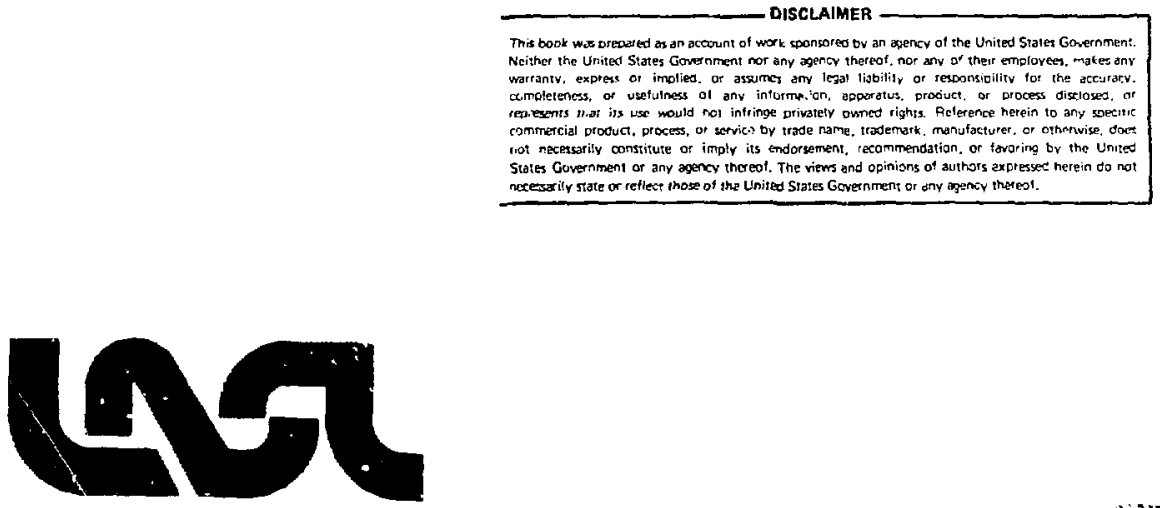
AN ATLAS OF URANIUM EMISSION INTENSITIES

IN A HOLLOW CATHODE DISCHARGE

by

Byron A. Palmer, Richard A. Keller, and Rolf Engleman, Jr.

\section{ABSTRACT}

The uranium emission spectrum from a hollow cathode discharge is displayed from 11000 to $26000 \mathrm{~cm}^{-1}$. This atlas lists 4928 spectral lines of uranium; 3949 are classified to the neutral spectrum and 431 are classified to the singly Ionized spectrum. Listed wavenumbers are accurate to $\pm 0.003 \mathrm{~cm}^{-1}$ aud the 1 isted relative intensities to $\pm 8 \%$. The richness of the spectrum makes this atlas useful for wavenumber caltbration of lasers, spectrographs, and monochromators to an accuracy of 1 part in $10^{7}$. This at las is also useful as a guide to the uranium spectrum, and relative oscillator strengths ( $g f$ values) can be calculated from the intensities to a preciston of $\pm 20 \%$. 


\section{INTRODUCTION}

This atlas, a series of graphs depicting the spsctrum of uranium and accompanied by tables of waverumbers, relative intensities, and assignments, provides an excellent source of information on the spectrum of uranium. Because of its wavenumber accuracy, this atlas is useful for calibration of spectrographs, monochromators, and tunable lasers (see the Appendix for a description of laser wavenumber calibration using the optogalvanic effect ${ }^{1}$ ). The relative intensities of the spectral lines measured are accurate to $\pm 8 \%$, a result of the spectroneter used, and are useful in mapping the spectrum and for the determination of relative oscillator strengths ( $g f$ values).

The spectral lines from our lamp, a commercially ayailable hollow cathode discharge tube (HCDT), are sharp and are reproducible. The sharpness of the lines and the excellent wavenumber accuracy of the Fourter Transform Spectrometer (FTS) at Kitt Peak National Observatory (KPNO) results in a set of wavenumber standards superior to any other list of wavenumber standards with a comparable number of lines. The uranium emission spectrum has numerous very sharp IInes that are widely distributed throughout lia ir, visible, and uv spectral regions with the result that there is a selection of strong lines in any given region of the spectrum.

This report provides one of the best sources of information on the spectrum of uranium. The tables not only list the wavenumbers of the strong lines, but also the stage of ionization, erergy levels and $\mathrm{J}$ values for most of these transitions. This detailed knowledge is useful to anyone working with uranium in plasmas, furnaces, or in isotope enrichment.

\section{SOURCE AND SPECTROMETER}

The spectrum of uranium was obtained from a commercial hollow cathode discharge tube* (HCDT) manufactured by the Westingho'sse Electric Corporation. The HCDT was filled with a carrier gas of neon, although argon may also be

\footnotetext{
* Westinghouse Industrlal and Government Tube Division, Westinghouse Circle, Horseheads, NY, 14854. Lamp order number WL-36077. There are other sources of this type of lamp, which is commonly used for atomic absorption measurenents.
} 
used. We chose to use a commercially available HCDT because it is a readily avallable, reproducible source. The spectral lines emitted by tr. HCDT are sharp, display no hyperfine structure, and have small shifts (less than our precision) due to pressure and electric field effects. Because uranium is a heavy element and the Doppler broadening for a given temperature vartes inversely as the square root of the atomic weight, the spectral lines are narrow. The lines are not shifted or broadened by pressure effects because the gas pressure is low (about 5 torr). The cathode was constructed from uranium.whose composition was about $99.75 \%{ }^{238} \mathrm{U}$ and $0.25 \%{ }^{235} \mathrm{U}$; therefore, there is no hyperfine structure to broaden or split the lines (even-even isotopes have no hyperfine structure). The stark shifts caused by the electric field are small since the elcctric field inside the cathode discharge region is on the order of a few tens of volts.

The HCIJT is rated at 25-mA maximum current, but to obtain more sputtering and thereby increase the signal, the lamp was run at $75 \mathrm{~mA}$. This required cooling the lamp envelope by circulating water through a jacket surrounding the lamp. From the results of several tests, we have concluded that the ratio of the intensities of the lines is a constant for moderate variations in the lamp current. 1 The spectrum that would be measured with 25-mA current would be very similar to that which we obtalned at the higher current. The lifetime of the tube is rated in terms of amp-hours, so running at $75 \mathrm{~mA}$ should shorten the life of the tube by about a factor of three, which may be an important constderatien for some users. For further details on line intensities, see Sec. $x$.

We took the spectrum on the FTS at KPNO. This spectrometer was designed by Brault $^{2}$ for use with the McM … solar telescope. The instrument has a total path difference of $\sim 1 \mathrm{~m}$ and is capable of a resolution approaching one million. The data are collected as an interferogram and must be Fourier-transformed to obtain the spectrum. To reduce the noise level of the data, six to eight scans of the interferogram were added potnt to point to form one final interferogram (time for one scan is about $7 \mathrm{~min}$ ). To reduce noise further, optical band-pass filters were used to limit the spectral region viewed in each interferogram. In order to cover the region displayed in this atlas, we used three separate final interferograms. Table I lists the parameters for the three final interferograms. 
TABLE I

PARAMETERS OF THE INTERFEROGRAMS ${ }^{a}$

\section{Section 1}

$$
\begin{aligned}
& \text { Start }\left(\mathrm{cm}^{-1}\right) \\
& \text { End }\left(\mathrm{cm}^{-1}\right) \\
& \text { FSR }\left(\mathrm{cm}^{-1}\right) \\
& \text { MP }(\mathrm{cm}) \\
& \operatorname{Res}(\mathrm{mK})^{b} \\
& \text { NP } \\
& \text { ZP } \\
& \text { AC }
\end{aligned}
$$

11000

18000

$22 \quad 219$

23.55

50

$1046 \quad 394$

209280

$5.6236 \times 10^{-7}$
Section 2

18000

22060

$28 \quad 920$

18.05

65

1044194

208840

$6.1907 \times 10^{-7}$
Section 3

$22 \quad 060$

25980

41239

16.72

75

1044546

208910

$5.7743 \times 10^{-7}$

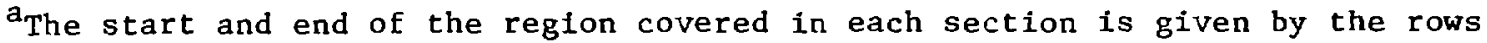
labeled start and End. The free spectral range (FSR) is the maximum wavenumber that can be observed without overlap. The maximum path difference is given in the row labeled MP, and the resolution is in the row labeled Res. The total number of points in the interferogram is listed in the row labeled $\mathrm{NP}$, and the number of points taken before zero path difference is listed in the row labeled $Z P$. The aperture correction fartor minus one is listed in the row labeled $\mathrm{AC}$.

$b_{\text {One }} \mathrm{mK}$ is $0.001 \mathrm{~cm}^{-1}$.
}

Short low-resolution interferograms of an incandescent standard lamp * were interspersed with the uranfum interferogrami. This allowed us to calfbrate the Intensity response of the system composed of the detector, filter, and instrument. The interferograms were subsequently transformed at KPNO and the transforms brought to the Los Alamos Scientific Laboratory (LASL) on magnetic tape for data reduction.

Optrontc Laboratories, Inc.: 7676 Fenton Street, Silver Springs, MD, 20910. Ribbon filament lamp, Model 550 designated IR-15 operated at the rated current of 15 amp dc. This lamp is callbrated by the manufacturer against NBS standard lamps. 


\section{INTENSITY CORRECTION}

Using the transformed interferogram of the standard lamp, and a table of intensity vs wavelength supplied by the manufacturer, we calculated a multiplicative correction curve for each section of the atlas, as shown in Fig. 1. It was convenient to fit a functional form to the tabulated data; for this purpose a modified Planck's blackbody equation was used. We introduced a $\lambda^{3}$ factor to convert from ergs/nm to photons $/ \mathrm{cm}^{-1}$ :

$$
R=\frac{\lambda^{3}}{\left(\lambda^{5}\left(e^{5.0 / \lambda}-1\right)\right)^{1.15}}
$$

where $R$ is the relative number of photons $/ \mathrm{cm}^{-1}$ and $\lambda$ is in $\mu \mathrm{m}$. This equation fits all the points in the tabulated data to better than $1 \%$. We chose an arbitrary scale factor for the $f_{1} t$ section such that the strongest line was about 1000; this determined the scale factors for the other two sections. The intensities of the first and second sections were matched in a region of $1000 \mathrm{~cm}^{-1}$ centered on $18000 \mathrm{~cm}^{-1}$. The variation in intensity between the two sections in this overlap region was observed to be less than $4 \%(1 \sigma)$. We matched the third section to the second section for $1000 \mathrm{~cm}^{-1}$ centered at $22000 \mathrm{~cm}^{-1}$, and again the variation in intensity between the two sections was $4 \%(1 \sigma)$ over the region at the overlap. Since the intensities between the sections agreed to $4 \%$, we claim a precision of better than $8 \%(2 \sigma)$ in our intensities.

The relative intensities listed were calculated from the intensitycorrected spectrum by fitting a second-degree polynomial to the top portion of the line, and using the intensity at the maximum value of this polynomial. All intensities were determined from the smoothed data (see discussion in Sec. IV).

IV. INTERPOLATION AND SMOOTHING

The transformed interferograms have several disadvantages that make them difficult to use. The minimum number of points of the digitized interferogram were transformed, which results in having only 3 or 4 points 
per half-width on uranium lines. Neon lines are usually two to three times broader and have $8-12$ points per half-width. To determine uranium line positions and intensities correctly, new points must be found by interpolation. Using the theory of Fourier transforms and the sampling theorem, 3,4 we decided to interpolate with an apodized sinc function* with 3 points between primary points and spanning 25 primary points. We tested the adequacy of this interpolation by varying the number of points interpolated between primary points, and the number of points used in the interpolation. Increasing these numbers gave nearly the same results as with 3 and 25 , but decreasing them affected the accuracy, espectally on the weak lines near the noise level.

Due to limitaitons of the FTS apparatus, mainly disk space to hold the data, our Interferograms do not provide full resolution for our light source. Full resolution means taking enough data points so that the interferogram, at the maximum path difference, has reached a steady state and no further information can be obtained. Stopping short of this poinc has two major effects-wider lines (decreased resolution), and the introduction of "ringing" due to the Gibbs phenomenon.3,4 Ringing is only evident on the uranium lines since the neon lines are wider and are fuily resolved. The ringing of strong lines tends to mask weak lines by as much as several wavenumbers on either side, affecting the weak line positions and intensities. To decrease the ringing effect we smoothed the data by convoluting the transform with a Gaussian function. The smoothing has the effect of increasing the line widths (degrading the resolution), but allows the location of the weak lines in the spectrum. In some cases of close lines where the smoothing blended the two lines, we measured the wavenumbers of the lines without smoothing. The effective resolution (full width at half maxinum) with smoothing was $50 \mathrm{mK}$ in the ftrst section, $65^{\circ} \mathrm{mK}$ in the second section, and $75 \mathrm{mK}$ in the third section of the atlas. Without smoothing the resolution improves by a factor of 25 to $35 \%$.

$\star \operatorname{sinc}(x)=\sin (x) / x$. 


\section{WAVENUMBER MEASUREMENT}

We determined the positions of the spectral lines by searching for the zero crossing of the first derivative of the spectrum. The first derivative is calculated using the method of convoluting numbers.5,6 In essence, this technique performs a least squares fit (LSF) to the data and calculates the derivatives from the LSF function at the center of the region of fit. We chose a quadratic polynomial calculated for seven points centered on the point of interest. The line position was determined by a linear interpolation of the two derivatives on either side of the zero crossing. To ensure that only peaks were measured, we calculated the second derivative and rejected those crossings with a positive second derivative. As a final test of the measurement, lines with a very small half-width were also eliminated, thus removing the ringing of the strong lines from the preliminary line list.

The measured wavenumber was adjusted for the index of refraction and the effect of a finite entrance aperture. We used the formula given by Edlén ${ }^{7}$ to calculate the index of refraction, which was then corrected for the temperature and pressure using the procedure given in the Table of Wavenumbers. ${ }^{8}$ The effect of the finite aperture is to lengthen the path the sampled light travels with respect to the reference path. The aperture correction is a constant multiplicative factor applied to all wavenumbers. This correction can be expressed as

$$
\sigma^{*}=k \sigma=\sigma\left(1+\frac{\Omega}{4 \pi}\right),
$$

where $\sigma^{\prime}$ is the corrected wavenumber, $K$ is the aperture correction, $\sigma$ is the measured wavenumber, and $\Omega$ is the solld angle subtended by the aperture. The solid angle is defined as

$$
\Omega=\frac{\pi h^{2}}{4 F^{2}},
$$

where $h$ is the diameter of the aperture and $F$ is the focal length of the 
collimating optics. For the first section, this factor is 1.00000056236 , which yields a maximum change of $10 \mathrm{mK}$.

We chose the first section of the atlas to be the wavenumber reference for the other two sections because it had the fewest wavenumber problems--a less dense spectrum and the best phase correction (which will be discussed in Sec. VI) of the three sections. To match the wavenumbers in the overlap regions, an additional factor was applied to the second and third sections. This changed the wavenumbers by a maximum of $1.3 \mathrm{mK}$ in the second section and a maximum of $0.4 \mathrm{mK}$ in the third section.

\section{WAVENUMBER ACCURACY AND PRECISION}

The wavenumbers reported in this atlas are based on the vacuum wavelength of the reference He-Ne laser. This 1 laser ${ }^{9}$ has an accuracy of $\sim 1$ $\mathrm{mK}$ and a stability better than $0.1 \mathrm{mK}$. Additional sources of systematic errors can be introduced by the measurement system. The most important of these systematic errors are due to alignment and phase correction.

Improper alignment of the source can cause wavenumber errors as large as $5 \mathrm{mK}$. However, with reasonable care, these errors are reduced to less than 2 $\mathrm{mK}$. This fact is documented by the reproducibility of wavenumber measurements over many different realignments.

The other major source of systematic errors is in the determination of the phase correction. Phase errors are caused by wavelength-dependent phase shifts upon reflection at the mirrors, and the inability to locate zero path difference ( $Z P D)$. The theory of Fourier transforms ${ }^{4}$ requires there to be a point where the path difference for all wavelengths is equal to zero, ZPD. The phase correction adjusts the interferogram to satisfy this condition by taking a small number of points around the ZPD point, transforming them, and calculating the phase from their transform. The phase thus determined is then used to correct the interferogram. Because only a few (not more than 1024) points are used, the strong features are "smeared" out over the rest of the spectrum. As opposed to a continuous spectrum where phase corrections can be calculated preclsely, the phase correction of a line spectrum is unduly influenced by the strong lines, and the phase distant from strong lines may be in error. Our study of the transform indicates that in some regions the phase problem may cause errors of several mk. In fact, we believe that the disagreement of $1.3 \mathrm{mK}$ for wavenumbers of lines in the 
overlap ragion between the first and second sections is caused by improper phase corrections in section two.

To determine the magnitude of the systematic error, we measured the wavenumbers of a group of thorium lines. The inteferogram we used for these measurements was taken on the FTS shortly before the uranium interferograms were collected. The thorium light source was a HCDT operated in a manner similar to the uranium lamps, and the data were processed using the same procedures. These measurements were over a limfted spectral range of 100 $\mathrm{cm}^{-1}$ and the deviation from the reported wavenumbers ${ }^{10}$ for this secondary standard is $0.64 \pm 1.2 \mathrm{mK}$. It is not clear whether this $0.64 \mathrm{mK}$ is indicative of a systematic error in our measurements, in the thorium measurements, or in both. In summary, we believe that systematic errors in this atlas are less than $3 \mathrm{mK}$.

Random errors in measurements result in statistical deviations of the ine positions. The random errors in our measurements have several causes:

- line position refinement problems,

- weak lines where signal to noise is low, and

- blended lines.

Using several procedures, we determined the magnitude of the random errors to be less than $1 \mathrm{mK}$ for most lines.

The size of the random errors is very dependent on the signal-to-noise ratio $(\mathrm{S} / \mathrm{N})$ of the spectrum. Every line adds noise to all regions of the spectrum; thus, by limiting the spectral range, a better $S / N$ can be achieved--this is why we separated the atlas into three sections. Therefore, the number of lines and the relative intensity of these lines partially determines the precision. Since uranium has many lines, with few strong lines, the $S / N$ for most lines listed in this atlas is at least 100 . Our experience shows that the peak positions of lines with this $\mathrm{S} / \mathrm{N}$ can be determined to within $1 \%$ of the line width, which in this spectrum would be less than $1 \mathrm{mK}$. Uranium has so many lines that blending becomes a problem and shifts caused by blending may approach $15 \mathrm{mK}$. Blending becomes increasingly severe toward the blue end of the spectrum.

Two metirods of determining the precision of the measurements are avallable to is. The first method is to determine the differences between the wavenumbers measured for the same lines in the overlap regions. In the first overlap region, the root mean square (RMS) error is $0.62 \mathrm{mK}$, and in the xii 
secona overlap region it is $0.58 \mathrm{mK}$, which leads us to believe that the precision is better than $1.2 \mathrm{mK}(2 \sigma)$ over the entire spectrum. The second method is to examine the self-consistency of the energy levels derived from the wavenumbers. We have data in the atlas for 3949 transitions to 245 odd-parity levels and 9 is even-parity levels in the neutral. spectrum of uranium. We rejected 64 lines that had deviations from the calculated wavenumbers greater than three times the estimated uncertainty and whose devtations were greater than $3 \mathrm{mK}$ (these lines are listed in Table $I I$ and marked in the atlas with a ?). Levels determined uniquely by one transition are irrelevant to the statistics of the calculation. Those lines that originate or terminate on such levels were discarded (a. total of 633 1ines). We carried out a least squares fit to the encrgy levels 11,12 with the remaining 3362 transitions connecting 129 odd levels and 526 even levels. The lines for the level calculation were weighted by the reciprocal of the square of their estimated uncertainty. The uncertainty, $\delta$, of the lines was determined by first using unit weights in the level calculation. Next we calculated the RMS devfation for small ranges in intensities. Finaily the RMS deviations were $f i t$ to an equation of the form $a+b / I$, where $I$ is the intensity of the transition as listed in the atlas. This process yielded

$$
\delta=0.5458+\frac{0.8678}{I},
$$

where the $\delta$ is in $\mathrm{mK}$. The lines thus weighted were used in a second level calculation. We verified that the weighting scheme used was consistent by comparing the RMS deviation computed for ranges of intensities with that predicted by Eq. (4). The actual RMS values and $\delta$ agreed to within $0.1 \mathrm{mK}$ over most of the range of intensities. This comparison indicated that the higher intenstties are slightly more accurate than predicted, and that the weaker lines are slightly less accurate. Therefore, we feel that Eq. (4) gives a reasonable estimate of the uncertainty in a given measurement. It does not, however, take into account the blending or misidentification of lines, although the weaker lines will tend to be more blended than stronger ones. 
TABLE II

TRANSITIONS WITH LARGE DEVIATIONS ${ }^{a}$

\begin{tabular}{|c|c|c|c|c|c|}
\hline Wavenumber & Even & odd & Int & Dev & Comment \\
\hline 11060.2463 & 2894310 & 1788209 & $2 \cdot n_{2}$ & 5.98 & wide \\
\hline 11220.8621 & 2262403 & 1140304 & $1 \cdot 30$ & 9.09 & \\
\hline 11476.7091 & 2246406 & 1098706 & 1.93 & $-4 \cdot 32$ & blend \\
\hline 11766.4809 & 2275406 & 1098706 & 1.85 & -8.34 & blend \\
\hline 11914.3539 & 2979708 & 788209 & 4.47 & -15.71 & wide \\
\hline 12082.1323 & 2371506 & 1163305 & 0.92 & -5.77 & \\
\hline 12249.2113 & 2612504 & 1387605 & 1.31 & 9.73 & \\
\hline 12573.1125 & 2278605 & 1020804 & 0.85 & 4.44 & wide \\
\hline 12770.5277 & 2565504 & 1288404 & 0.98 & -6.66 & \\
\hline 12813.2577 & $19 i 9204$ & 3200505 & 0.81 & $-11 \cdot 38$ & \\
\hline 12899.5489 & 2744305 & 1454306 & 1.30 & -5.92 & \\
\hline $12963.3 ; 54$ & 2632405 & 1336106 & 0.76 & 7.20 & wide \\
\hline 13298.3122 & 2411803 & 1081903 & 0.80 & 14.27 & blend \\
\hline 14041.1721 & 1746804 & 3150905 & 1.08 & 14.29 & wide \\
\hline 14276.3612 & 2240903 & 813304 & 2.03 & -6.56 & blend \\
\hline 14399.2345 & 2867303 & 1427404 & 0.99 & 8.83 & wide \\
\hline 14594.3335 & 2599703 & 1140304 & 1.03 & -9.08 & wide \\
\hline 14934.1037 & 2203804 & 710303 & 2.81 & 9.31 & \\
\hline 15242.1616 & 2337504 & 813304 & 1.40 & 7.50 & \\
\hline 15322.7291 & 2318604 & 786405 & 1.64 & 4.93 & \\
\hline $1577: \cdot 3058$ & 2675807 & 1098706 & 1.75 & -6.53 & hlend \\
\hline 16047.8600 & 2703507 & 1098706 & 3.82 & 4.62 & \\
\hline i 6441.7849 & 2456007 & 811807 & 9.17 & -6.94 & wide \\
\hline 16600.6587 & 2392608 & 732607 & 1.25 & 5.50 & \\
\hline 16637.5052 & 2689206 & 1025405 & 4.03 & -4.54 & wide \\
\hline 16807.2364 & 2494004 & 813304 & i. 79 & 9.17 & blend \\
\hline 16860.6745 & 2066106 & 380007 & 15.8 & 8.72 & \\
\hline 17532.0427 & 3140805 & 1387605 & 1.23 & 9.69 & \\
\hline 17771.5025 & 2811807 & 1034708 & 1.17 & -5.57 & wide \\
\hline 17933.4053 & 2606605 & 813304 & 2.16 & 5.24 & blend \\
\hline 18113.7209 & 2979007 & 1167707 & 1.58 & $-21 \cdot 24$ & \\
\hline 18293.5823 & 3026205 & 1196805 & 0.82 & 8.12 & wiae \\
\hline 18307.9980 & 2859607 & 1028806 & 1.04 & -5.87 & wide \\
\hline 18322.9177 & 3026604 & 1194303 & 2.09 & 6.57 & blend \\
\hline
\end{tabular}

\footnotetext{
${ }^{a}$ This table lists those lines that were excluded from the level calculation. The level designators are a comblned level value and two digits representing the $\mathrm{J}$ value--designation 2894310 is the level at $28943 \mathrm{~cm}^{-1}$ with a $\mathrm{J}$ value of 10. The intensity is listed in INT column with the deviation in mK given in the colurn under Dev. The comment column lists the reason for the large devlation if known-a known blend of two or more lines or an unknown blend which we label as wide. The rest of the lines listed may be misassignments, blends that we cannot detect, or have other problems.
} 
Wavenumber

18458.5662

18590.0960

18702. 7095

18800.7717

19595.5862

19858.3377

19888.9236

20081.2970

20525.5554

20672.8324

20722.7480

20881.4581

21073.2657

21236.4897

22029.4154

$22141 \cdot 1476$

22464.6421

22785.2191

22865.3817

23030.7407

23818.8309

24387.5212

24765.5468

24960.8545

25156.7195

25200.2792

25512.6346

25612.4529

25705.5752

25949.4043
TABLE II(cont)

\begin{tabular}{|c|c|c|c|c|}
\hline Even & odd & Int & Dev & Compent \\
\hline 2422005 & 576205 & 2.27 & 3.20 & \\
\hline 3004706 & 1145706 & 1.41 & -7.28 & wide \\
\hline 2656600 & 786405 & 3.11 & 6.27 & \\
\hline 3058903 & 1178803 & 1.01 & 8.82 & \\
\hline 2346403 & 386803 & 3.26 & -4.53 & \\
\hline 2696204 & 710303 & 1.04 & 6.97 & \\
\hline 2775304 & 786405 & 2.71 & $7 \cdot 36$ & \\
\hline 1346305 & 3354404 & 2.27 & 8.08 & \\
\hline 2628706 & 576205 & 2.51 & -4.11 & \\
\hline 1203504 & 3270804 & 1.15 & 8.39 & blerd \\
\hline 2697107 & 624906 & 7.47 & 5.71 & \\
\hline 2788607 & 700506 & 2.95 & 4.22 & \\
\hline 2534806 & 427506 & 3.28 & 4.58 & \\
\hline 2510404 & 386803 & 1.64 & 6.58 & wide \\
\hline 2630505 & 427506 & 3.31 & -7.09 & wide \\
\hline 1005105 & 3219204 & 2.75 & 13.75 & \\
\hline 2979007 & 73ลล007 & 1.66 & $-21 \cdot 50$ & \\
\hline 2979007 & $700 \leq 06$ & 4.88 & -12.89 & \\
\hline 3019106 & 732607 & 4.52 & 6.97 & wide \\
\hline 3470707 & 1167707 & 1.77 & -7.39 & \\
\hline 2981003 & 599104 & 2.85 & -3.60 & \\
\hline 2884005 & 445304 & 3.81 & -5.17 & \\
\hline 2856607 & 380007 & 5.13 & 8.73 & \\
\hline 2923607 & 427506 & 15.0 & 7.22 & blend \\
\hline 2961004 & 445304 & 6.00 & -6.28 & wide \\
\hline 2947507 & 427506 & 2.43 & 7.35 & \\
\hline 2931306 & 380007 & 2.55 & 5.94 & wide \\
\hline 2941308 & 380007 & 3.11 & 6.06 & blend \\
\hline 3303108 & 732607 & 15.54 & 5.45 & blend \\
\hline 3359509 & 764508 & 4.05 & 4.84 & \\
\hline
\end{tabular}

TABLE III

LEVEL CALCULATION RESULTS

\begin{tabular}{|c|c|c|c|}
\hline Intensity & Unc. $(\mathrm{mK})$ & Number & $\underline{\mathrm{RMS}}(\mathrm{mK})$ \\
\hline$>100$ & 0.55 & 52 & 0.54 \\
\hline $50-100$ & 0.56 & 66 & 0.41 \\
\hline $20-50$ & 0.58 & 168 & 0.46 \\
\hline $10-20$ & 0.61 & 293 & 0.58 \\
\hline $5-10$ & 0.67 & 433 & 0.65 \\
\hline $4-5$ & 0.74 & 183 & 0.69 \\
\hline $3-4$ & 0.80 & 332 & 0.88 \\
\hline $2-3$ & 0.91 & 500 & 0.98 \\
\hline $1-2$ & 1.20 & 981 & 1.31 \\
\hline$<1$ & $1 \cdot 70$ & 243 & 1.60 \\
\hline rejected & & 64 & 8.51 \\
\hline
\end{tabular}




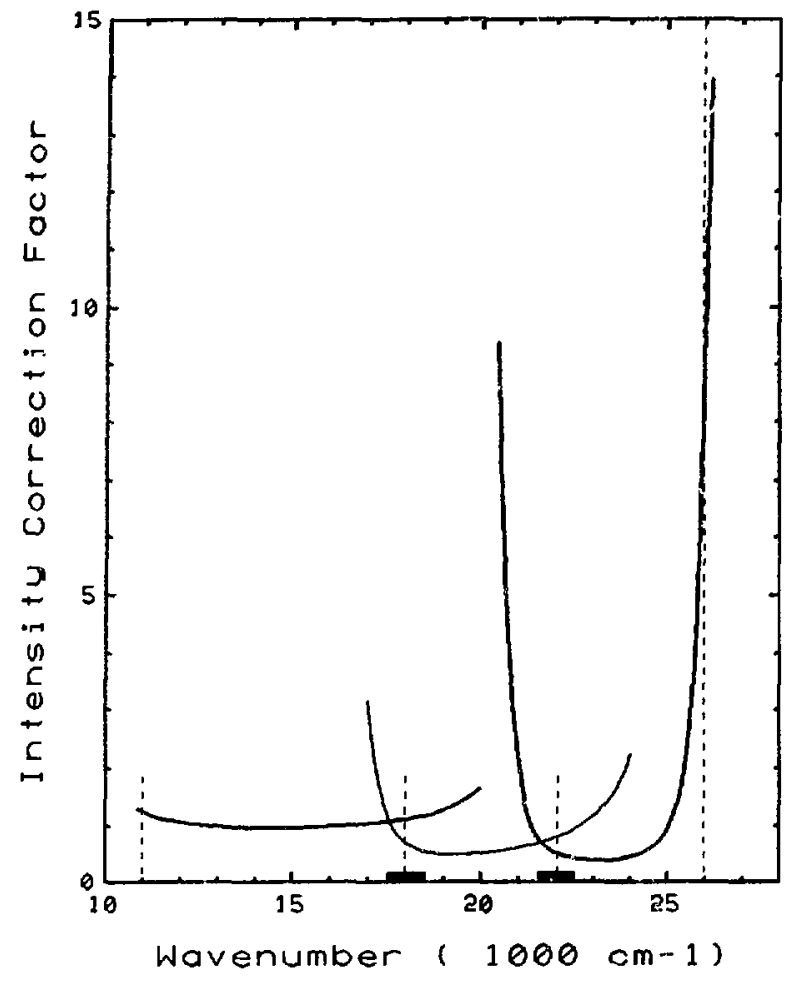

Fig. 1

Intensity correction curves. This factor multiplies the intevsities determined from the transforms. There is a curve for earl: section of the atlas. The dashed lines indicate the break from one section to the next. The bars near the bottom indicate the overlap regions.

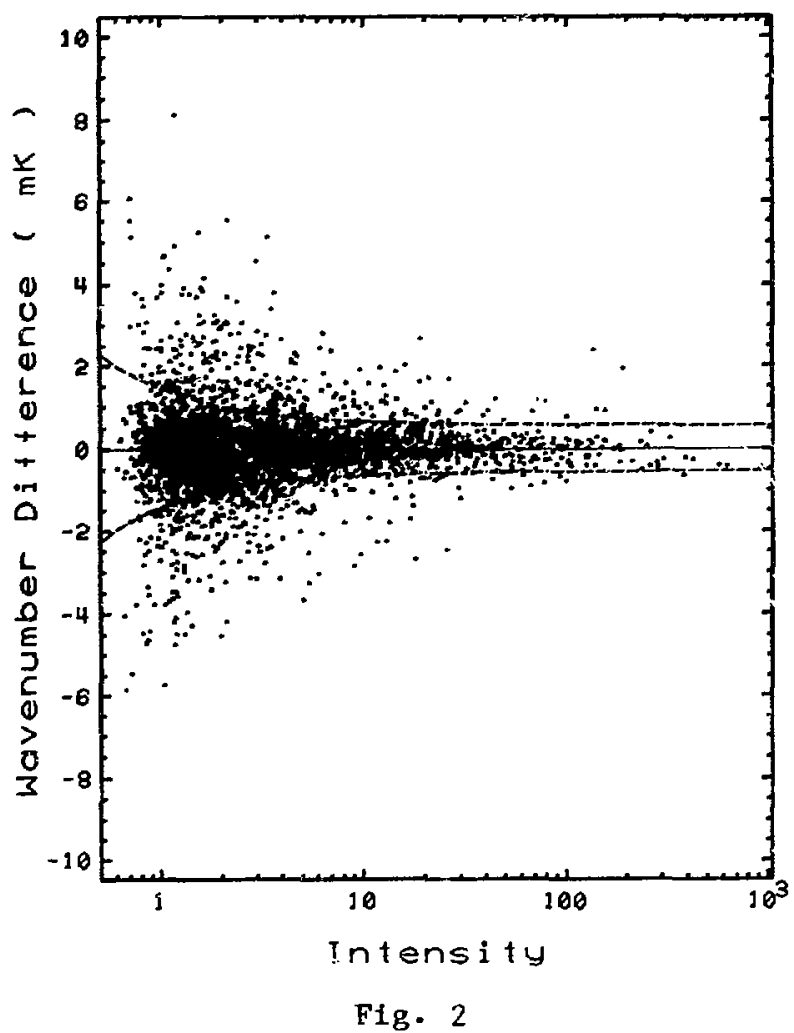

Wavenumber difference vs intensity as determined in the level calculation. The dashed lines indicate the uncertainty used for that intensity in Eitc level calculatinn.

The resultant standard deviation in the energy levels calculated was $0.58 \mathrm{mK}$, and the lines fit to within $0.53 \mathrm{mK}(1 \sigma)$ for the 628 lines with an intensity greater than 10. Table III lists the statistics of this fit and Fig. 2 is a plot of wavenumber devlation vs intensity that illustrates the inverse relationship between intensity and error.

The two sources of wavenumber measurements on uranium with which we can compare our measurenents are the compilation 13 in LASL report LA-4501 and a list of 523 lines proposed for use as secondary standards by Gerstenkorn, Luc, Perrin, and Chauville. 14 
The LASL list was based on more than 8000 lines measured on a Fabry-Perot interferometer by Steinhaus. 15 He augmented this list with measurements nade on a 5-m Czerny-Turner spectrograph. The Fabry-Perot measurements were compared with thorium standards 10 and it was necessary to adjust the Fabry-Perot data by as much as $40 \mathrm{mK}$. These thorium-besed uranium wavenumbers were then used as the standards for the 5-m spectrograph determinations. Because of the sparsity of lines in the thorium spectrum ised for standards, the sizable adjustment of the Fabry-Perot measurements, and the taherent difficulties of measurements made on photographic plates taken on grating spectrographs, there are small systematic errors in the reported wavenumbers. Figure 3 shows a comparison between our measurements of 4847 lines and that of the LASL line list. The average difference between the two lists is $-0.6 \mathrm{mK}$ with a RMS error of $1.8 \mathrm{mK}$.

The Gerstenkorn, et al. 1ist of uranium wavenumber standards was compiled from data taken on an FTS in France, and should be more accurate than the JASL line list. In comparing our data with this list we find a systamatic error in one or the other, or both. This systematic deviation is shown in Fig. 4. A total of 514 lines were matched between the two lists, and the average difference is $-0.4 \mathrm{mK}$ with a RMS error of $1.2 \mathrm{mk}$. The agreement of the lists is within our estimated accuracy of $3 \mathrm{mK}$.

VII. CLASSIFICATION

The level classification for the 1 ines listed in this atlas was obtalned from the LASL line list. 13 Those lines that differed from the LASL line Iist by more than $5 \mathrm{mK}$ were reviewed and adjusted as needed for blending in our spectrum and for misidentifications in the LASL line 1ist. One region of the spectrum, from 11000 to $12000 \mathrm{~cm}^{-1}$, revealed more Iines in our 1 ist than in the LASL line 1ist. Otherwise, all the lines listed in the tables matched known uranium 1ines. The neon lines were removed by comparing the lines with those listed in striganov and sventitskii, 16 and by noting that neon half-widths were approximately twice that of an unblended uranium line. 


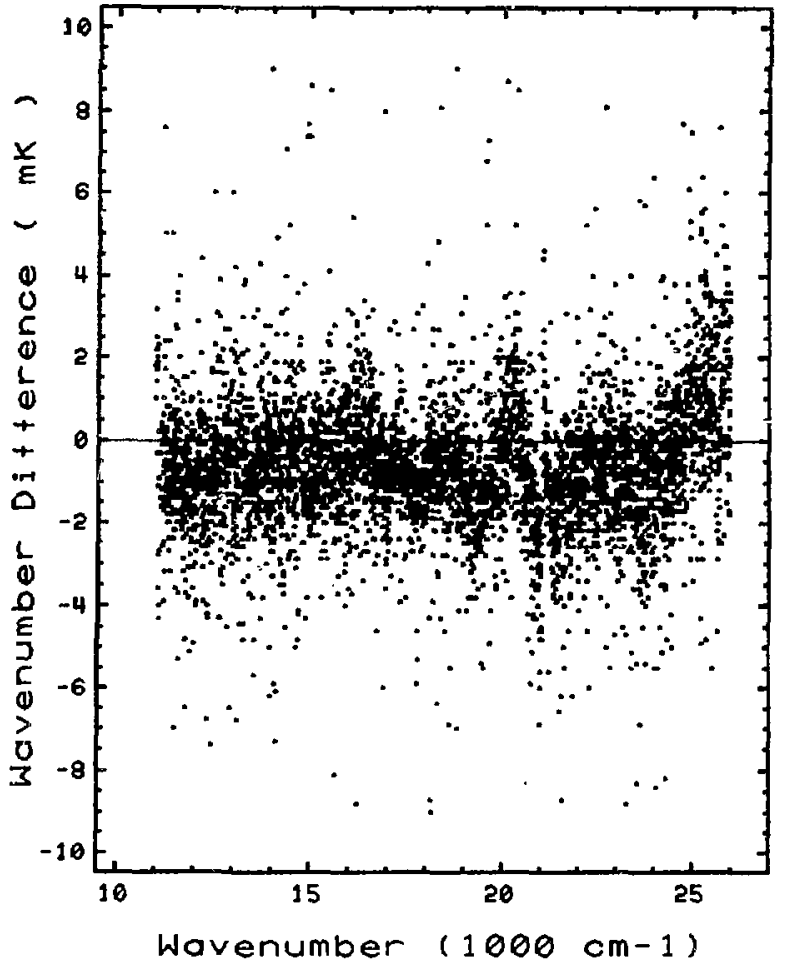

Fig. 3

Wavenumber difference between the LASL 1 ist and this atlas. The average difference between the two lists is $-0.6 \mathrm{mK}$, and the RMS difference is $1.8 \mathrm{mK}$. Note the systematic variation toward the blue end of the atlas.

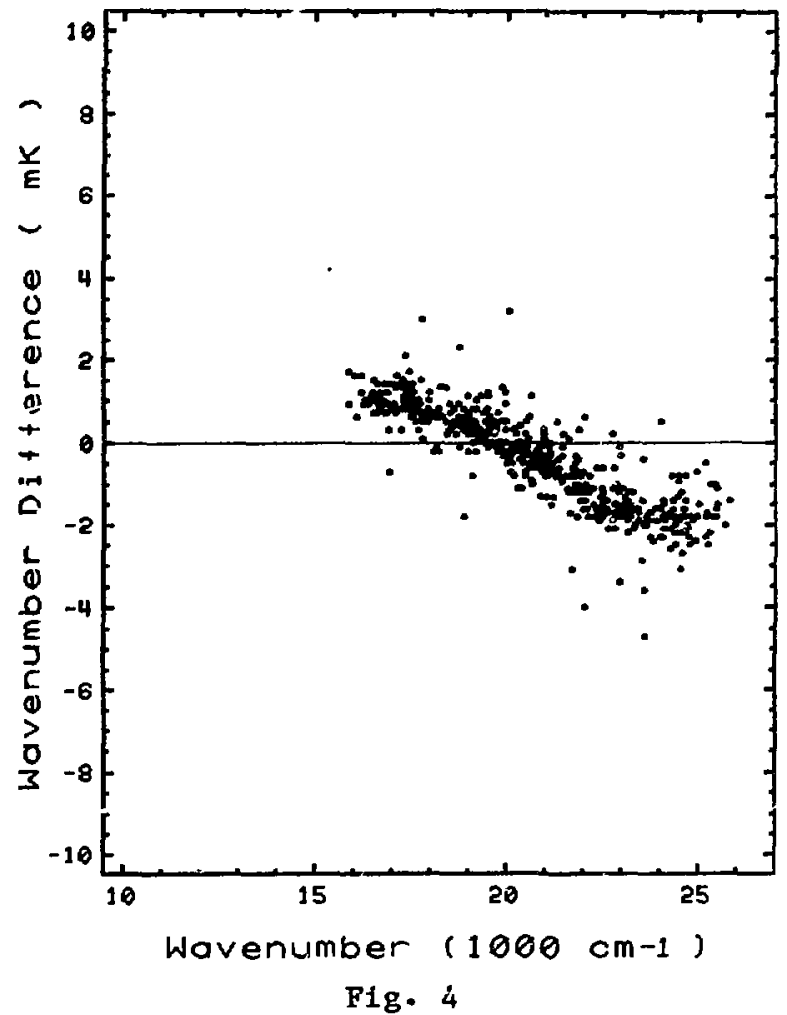

Wavenumber difference between the list of Gerstenkorn et al. and this atlas. The average difference between the lists is $-0.4 \mathrm{mK}$, and the RMS difference is $1.2 \mathrm{mK}$. If the systematic offset is removed, the RMS difference would decrēase to $\sim 0.5 \mathrm{mK}$.

\section{URANIUM SPECTRAL LINE TABLES}

The alr wavelength of the measured uranium lines is listed in the LAMBDA(A) column in units of angstroms. The vacuum wavenumber is tabulated in the SIGMA(CM-1) column and is given in $\mathrm{cm}^{-1}$. The INT column gives the relative intensity (photons $/ \mathrm{cm}^{-1}$ ) reported to three digits. The ION column gives the stage of ionization, I for neutral uranium, and II for singly Ionized uranium. A blank line in this column is at present an unclassified line, and $a$ UB indicates that this line is a blend of two or more uranium 11nes. There are a few cases where a uranium line was close to a neon line, 
and where the neon line was comparable in intensity to that of the uranium line, we listed it as NE+UB. The next four columns provide the levs1 designation of the spectral 1ine. The first wo give the truncated energy level value for the even level involved in the transition, and the associated . value. The next two columns provide the same information for the odd level. More detailed information on the levels of neutral uranium can be found in a paper by Blaise and Radziemski. 17

To keep the pages matched to the spectral plots, and to maximize the ease of use, weak lines were discarded from our list unt 1147 or fewer lines remained per page. The weakest line listed has a relative intensity greater than 0.7 and the strongest line has a relative intensity 912. Those lines which are evident on the plots and are not listed may usually be found in the LASL line list, 13 but with reduced precision.

\section{SPECTRAL DISPLAYS}

The graphs in this atlas display the spectrum of uranium $\neq 1$ th neon. There are two panels on each page; each panel covers $70 \mathrm{~cm}^{-1}$ of the spectrum. The bottom scale on the panel is the vacuum wavenumber in $\mathrm{cm}^{-1}$ and the top scale is the air wavelength in angstroms. The vertical scale is the relative intensity of the spectrum (photons $/ \mathrm{cm}^{-1}$ ). Five vertical scales are used for display, ranging from $0-5$ to a maximum of $0-100$. Although this change in scales might be confusing, we feel that this was the best way to display the spectrum, showing the weak lines as well as giving an indication of the strong lines. On any given panel there may be up to five uranium lines off-scale--intensities greater than the maximum that can be displayed. When a line goes off-scale its intensity is labeled. Neon lines are indicated in the plots with $\mathrm{NE}$ at the base of the line. If a neon line is off-scale, its intensity is also labeled. A linear plotting scale was chosen since most users wil be recording the spectrum using linear devices.

\section{RELATIVE OSCILLATOR S'TRENGTHS IN NEUTRAL URANIUM}

Oscillator strengths (gf values) may be calculated from the intensity of the transitions if certain parameters of the discharge are known. One must first assume that the atoms are in local thermodynamic equilibrium (LTE) amoung the electronic energy levels, which is the same as saying that 
collisions are the primary excitation and de-excitation process. If the discharge is in LTE, then Boltzmann's distribution holda. This is expressed as

$$
N_{u}=N_{0} \frac{g_{u}}{g_{0}} e^{-E_{u} / k T}
$$

where $\mathrm{N}_{\mathrm{u}}\left(\mathrm{N}_{0}\right)$ is the number of atoms in the upper (ground) level, $g_{u}\left(g_{0}\right)$ is the statistical weight of the upper (ground) level, $E_{u}$ is the energy of the upper level, $k$ is Boltzmann's constant, and $T$ is the excitation temperature of the discharge.

Standard equations ${ }^{18}$ relate the emission intensity to the gf value and the upper energy level $\left(E_{u}\right)$. The expression for the intensity is

$$
I \propto g f \sigma^{2} e^{-E_{u} / k T} \text { or } \frac{I \lambda^{2}}{g f} \propto e^{-E_{u} / k T}
$$

Note that the exponent of $\lambda$ is 2 , a result of our choice of units for the intensity (relative number of photons/s). Equation (6) shows that the temperature of the plasma and a proportionality constant are needed in order to calculate relative osclllator strengths. Our data are the relative intensities of the spectral lines. To determine the temperature and the proportionality constant, previous measurements of oscillator strengths are needed.

The three sets of measurements of oscillator strengths in uranium are those of Corliss, 19 klose and Voigt, 20 and Bleniewski. 21 only the first two sets of measurements overlap the range of this atlas. Corliss' 1 ist is the most extensive but has the least precision; he estimated uncertainties of greater than 35\%. Klose and Voigt list only 28 lines that were usable--two lines were not used because of blending problems. They estimated theli measurements were accurate to $\pm 20 \%$.

To determine the temperature and the proportionality constant, we did a least squares $f$ it (LSF) of $\log \left(I \lambda^{2} / g f\right)$ to the upper energy level, using both the data from Corliss and those from Klose and Voigt. The fact that we 
obtain a straight line indicates the discharge is in LTE. With the Corliss data, we obtalned an excitation temperature of $4100 \pm 80 \mathrm{~K}$ with an average of $\pm 50 \%$ error in the calculated gf values. Klose and Volgt's data gave a temperature of $4660 \pm 230 \mathrm{~K}$, but with a much closer fit to a straight line (average error of $\pm 20 \%$ in calculated gf values). Figures 5 and 6 show the least square fit for the Corliss data, and for the Klose and volgt measurements. For the lower energy levels, the LSF to Corllss' data predicts smaller gf values than the LSF to Klose and Voigt's data by $14 \%$, and for the higher levels, the LSF of Corliss data is $30 \%$ larger. We chose the LSF to

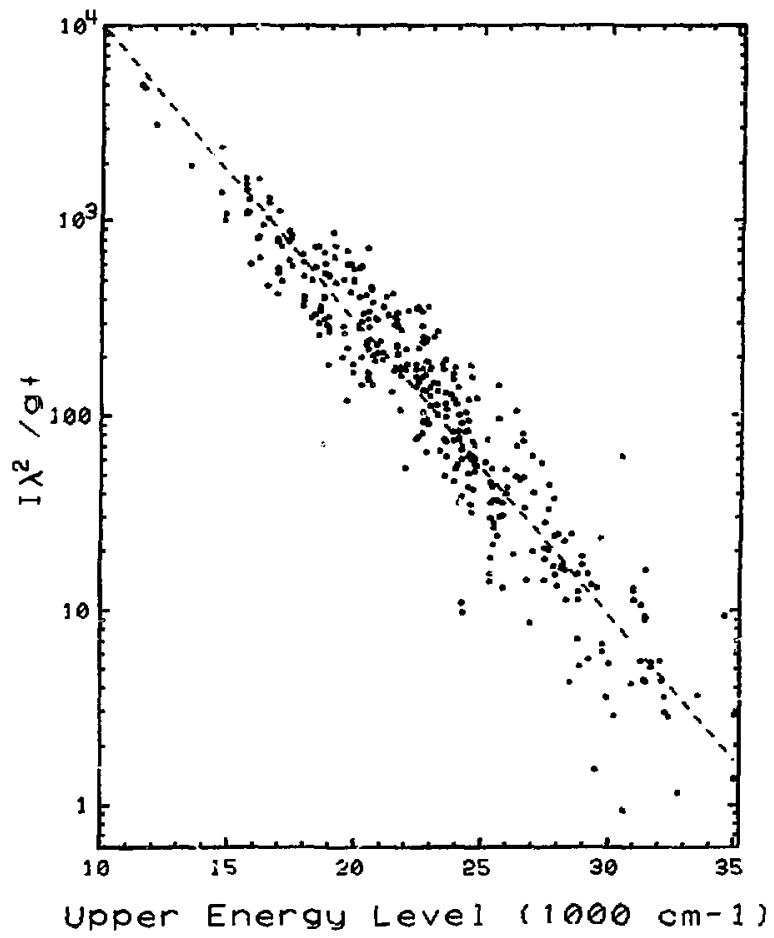

Fig. 5

$I \lambda^{2} / g f$ vs energy of emitting level. The gf values used in this figure are from Corliss. Intensities are Erom this atlas. From these data we determined the temperature to be 4100 $\pm 80 \mathrm{~K}$.

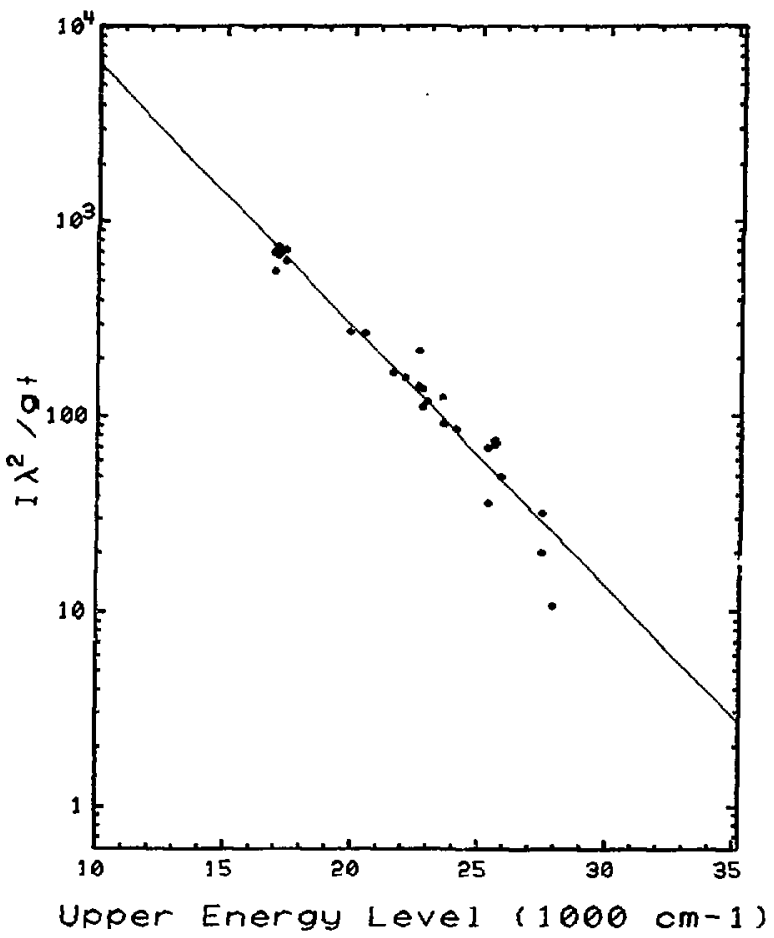

Fig. 6

$I \lambda^{2} / g f$ vs energy of emitting level. The gf values used in this figure are from $x$ lose and Voigt. Intensities are from this atlas. From these data we determined the temperature to be $4660 \pm 230 \mathrm{k}$. 
Corliss' data because there were more measurements and the temperature error was smaller. The equation we calculated from the Corliss data for the gf values is

$$
g f=\frac{3.15 \times 10^{-14} \mathrm{I} \lambda^{2}}{e^{-3.5 \times 10^{-4} E_{u}}}
$$

where $I$ is the intensity 1isted $1 n$ the atlas, $\lambda$ is in angstroms, and $E_{u}$ is in $\mathrm{cm}^{-1}$. The error introduced by the uncertainty in the temperature, derived from Corliss' data, is small (2 to $4 \%$ change in gf) in comparison to the other sources of errors. The errors we see may be due to problems in the gf values used, or in small deviations from LTE. Because of the accuracy of our fit to the data of Klose and Volgt, the relative gf values calculated from Eq. (7) should be accurate to $\pm 20 \%$. Absolute gf values may have a larger error, and we awalt accurate $g f$ value measurements in the range of our atlas.

ACKNOWL EDGMENTS

We thank Kitt Peak National Observatory for allowing us to use their facilities to obtain the spectra, and are extremely grateful to Jim Brault for the operation of the FTS instrumenc. We thank Larry Testerman for his assistance in performing the Fourier transforms. We also thank John Kline and Dave Steinhaus for reviewing the manuscript.

\section{REFERENCES}

1. R. A. Keller, R. Engleman, Jr., and B. A. Palmer, "Atlas for optogalvanic wavelength calibration," Appl. Opt. 19, 836-837 (1980).

2. J. W. Brault, "Rapid Scan High Resolution Fourler Spectrometer for the Visible," J. Opt. Soc. Am. 66, 1081 (1976).

3. R. J. Bel1, Introductory Fourier Transform Spectroscopy (Academic Press, New York, 1972). 
4. R. N. Bracewe11, The Fourter Trangform and Its Applirations, Second Edition (McGraw H111 Book Co., New York, 1978), Chaps. 2,10, and 18 .

5. J. K. Sterrett, "Manual for Moving Polynomial ARC Smoothing," Ballistic Research Laboratory, report RP 840, no 52. (1952).

b. A. Savitzky and M. J. E. Golay, "Smoothing and Differentiation of Data by Simplified Least Squares Procedures," Anal. Chem 30, 1627-1639 (1964).

7. B. Edlén, "The Refractive Index of Alr," Metrologia 2, 71-80 (1956).

8. C. D. Coleman, W. R. Bozman, and W. F. Meggers, Table of Wavenumbers, NBS monograph 3 (1960).

9. Zeeman stablilized He-Ne laser, Hewlett Packard Model 5526A. The specifled wavelength accuracy is 5 parts in $10^{7}$. Measurements on 15 lasers by NBS in the period 1972-1977 displayed a maximum wavelength deviation of 1.2 parts in $10^{7}$. Private coimunication, Kent Truscott, Laser Interferometer Division, Hewlett Packard Corp. (1980).

10. A. Giacchetti, R. W. Stanley, and R. Zalubus, "Proposed Secondary Standard Wavelengths in the Spectrum of Thorium," J. Opt. Soc. Am. 69 , 474-489 (1970).

11. L. J. Radziemski, Jr., K. J. Fisher, and D. W. Steinhaus, "Calculation of Atomic-Energy-Level Values," Los Alamos Scientific Laboratory report LA-4402 (1970).

12. L. J. Radziemski, Jr., K. J. Fisher, D. W. Steinhaus, and A. S. Goldman, "Calculation of Atomic Energy Level Values," Comp. Phys. Comm. 3, 9-18 (1972). 
13. D. W. Stelnhaus, L. J. Radzlemsk1, R. D. Cowan, J. Blaise, G. Guelachvil1, Z. B. Osman, and J. Verges, "Present Status of the Analysis of the First and Second Spectra of Urantum (U I and $U$ II) as Derived frow Measurements of Optical Spectra," Los Alamos Scientific Laboratory report LA-4501 (1971).

14. S. Gerstenkorn, P. Luc, A. Perrin, and J. Chauville, "Sur la prècision des nombres d'ondes mesurés par spectroscople de Fourler dans le visible, Etalons secondatres de nombres d'ondes," Astron. Astrophys. $\underline{58}$, 255-266 (1977).

15. D. W. Steinhaus, "Rap1d Precision Wave Number Measurements from Fabry-Perot Interferograms," J. Opt. Soc. Am. 50, 672-675 (1960).

16. A. R. Striganov and N. S. Sventitski1, Tables of Spectral Lines of Neutral and Ionized Atoms (IFI/Plenum Press, 1968), pp. 199-222.

17. J. Blaise, and L. J. Radziemski, Jr., "Energy levels of neutral atomic uranium (UI)," J. Opt. Soc. Am. 66, 644-659 (1976).

18. A. P. Thorne, Spectrophysics (William clowes \& Sons Ltd., London, England, 1974).

19. C. H. Corliss, "Line Strengths and Lifetimes of Levels in Neutral Uranium," J. Res. Nat. Bur. Stds. ㅁA, 1-7 (1977).

20. J. Z. Klose and P. A. Volgt, "Mean lives and gf values in U I," Phys. Rev. A 16, 2032-2037 (1977).

21. T. M. Bieniewsk1, "Absolute osc1llator strengths for neutral atomic uranium," J. Opt. Soc. Am. 68, 1173-1181 (1978). 
AN ATLAS OF URANIUM EMISSION INTENSITIES

IN A HOLLOW CATHODE DISCHARGE 


管

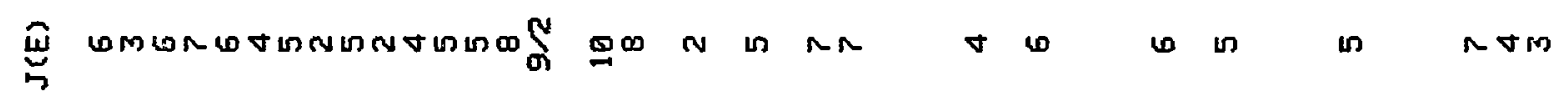

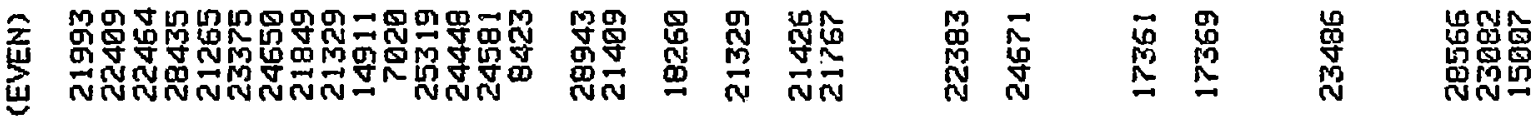

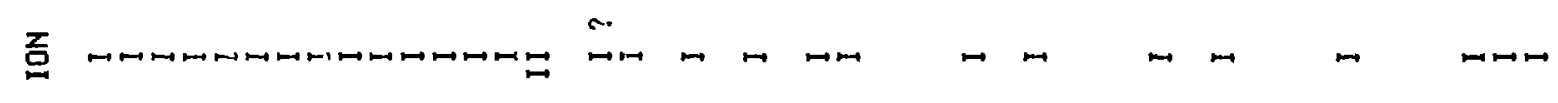

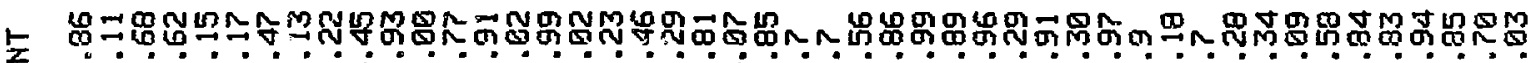
I

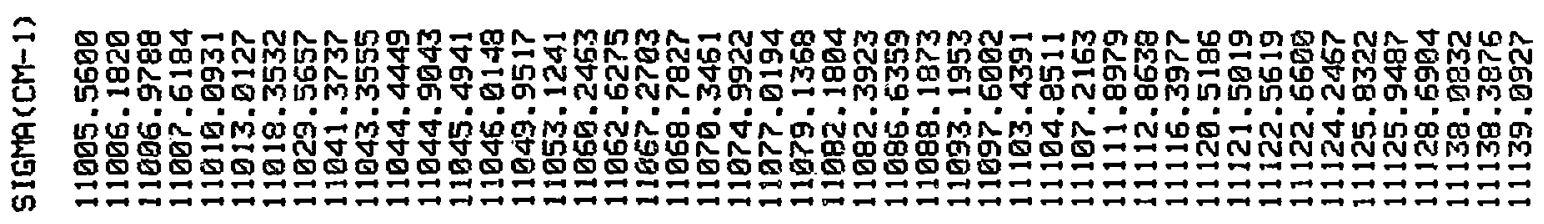

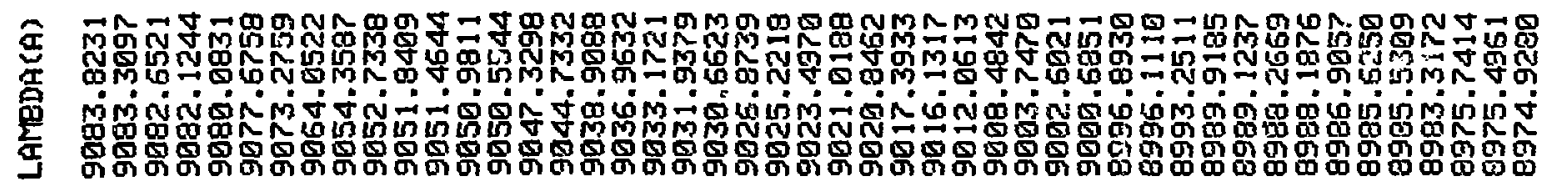



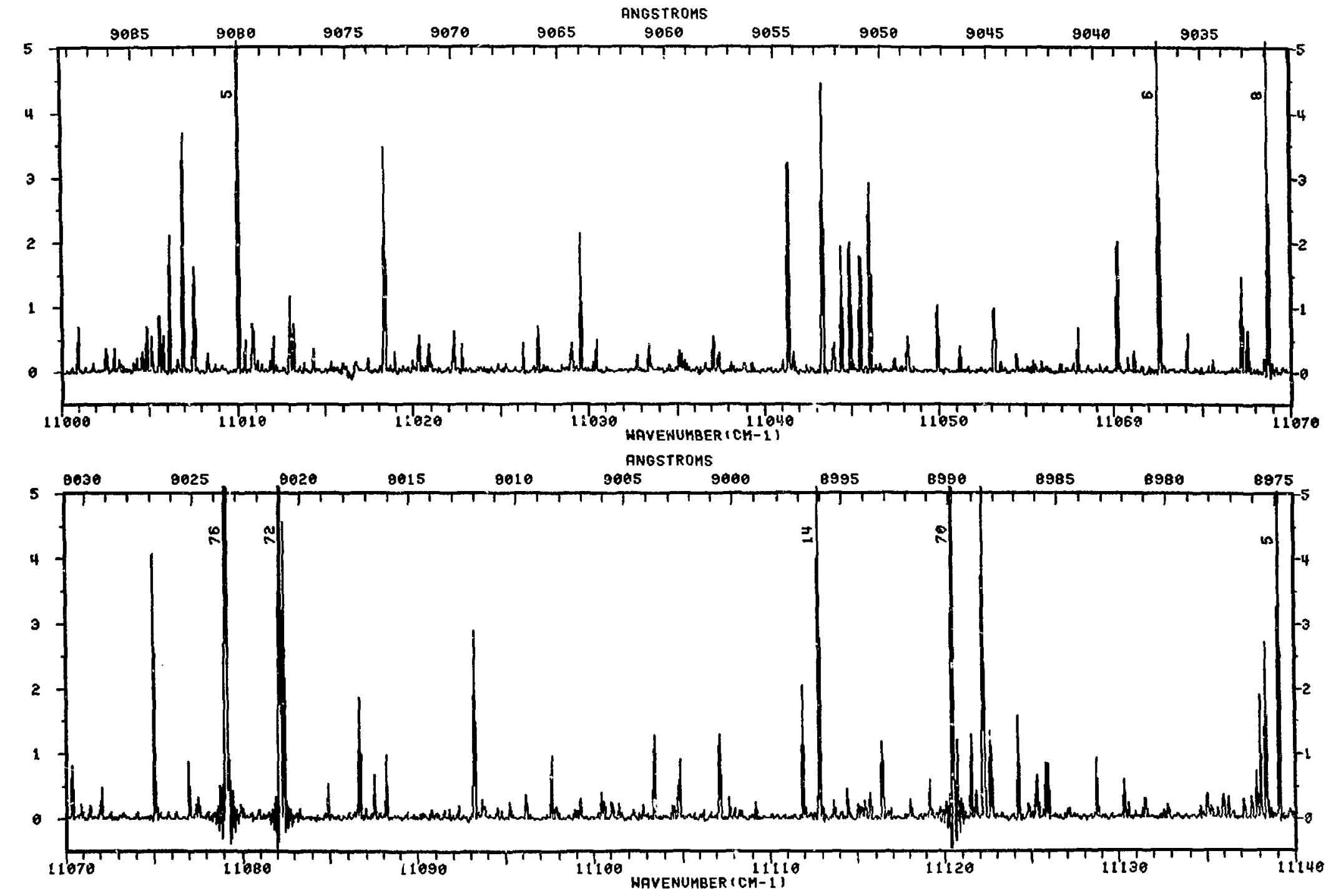


\begin{tabular}{|c|c|c|c|c|c|c|c|c|}
\hline$\hat{\theta}$ & $\frac{1}{2}$ & $m \nabla n$ & $m \nabla$ & $\ln n$ & $\boldsymbol{N}$ & $\omega M \Omega$ & $\nabla \nabla D \Omega N \Omega \Lambda \rightarrow M \nabla m$ & オんம \\
\hline 忩 & $\begin{array}{l}n \\
\text { no } \\
\text { n }\end{array}$ & 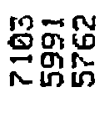 & 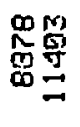 & $\overrightarrow{0} \tilde{0}$ & $\begin{array}{c}\stackrel{0}{10} \\
\mathbf{m} \\
\mathbf{m}\end{array}$ & 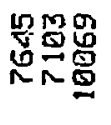 & 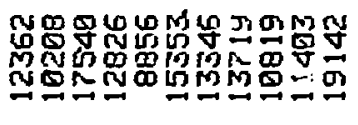 & 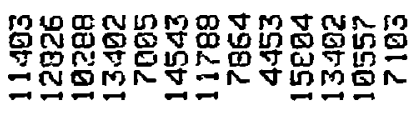 \\
\hline
\end{tabular}

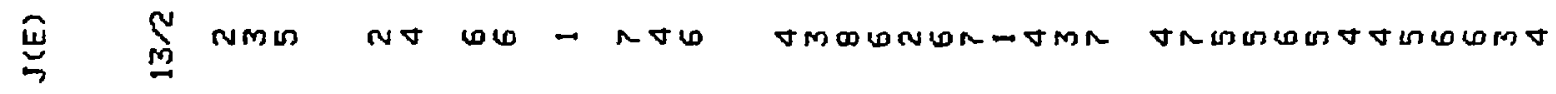

\begin{tabular}{|c|c|c|c|c|c|c|c|}
\hline$\stackrel{N}{N}$ & 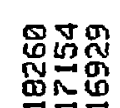 & 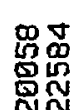 & W & ڤัง & 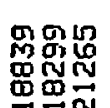 & 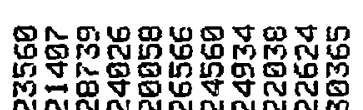 & 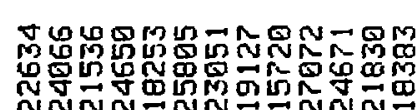 \\
\hline
\end{tabular}

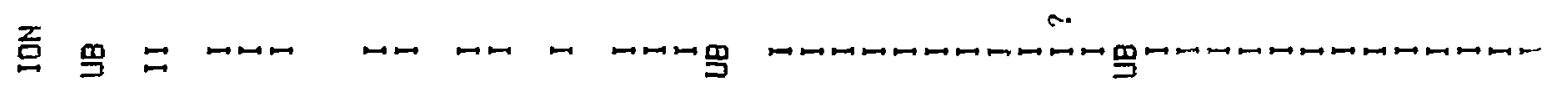

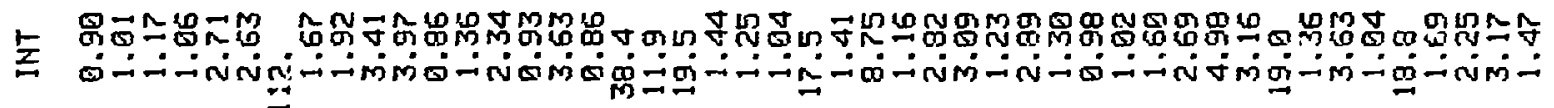

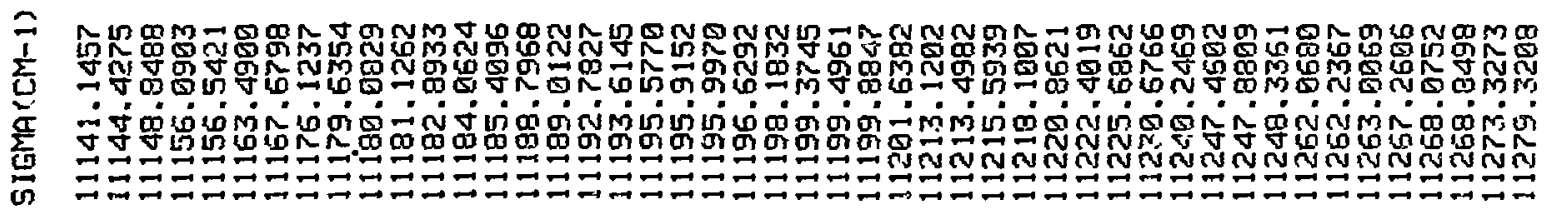

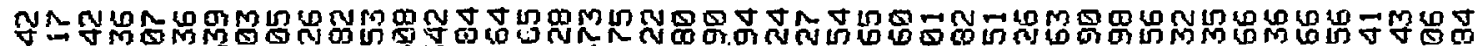

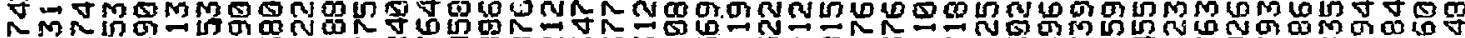

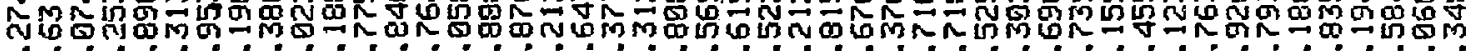
M⿻日禸 N

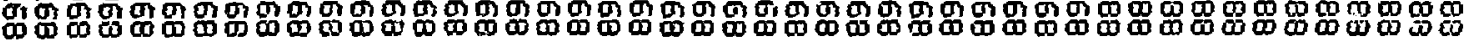



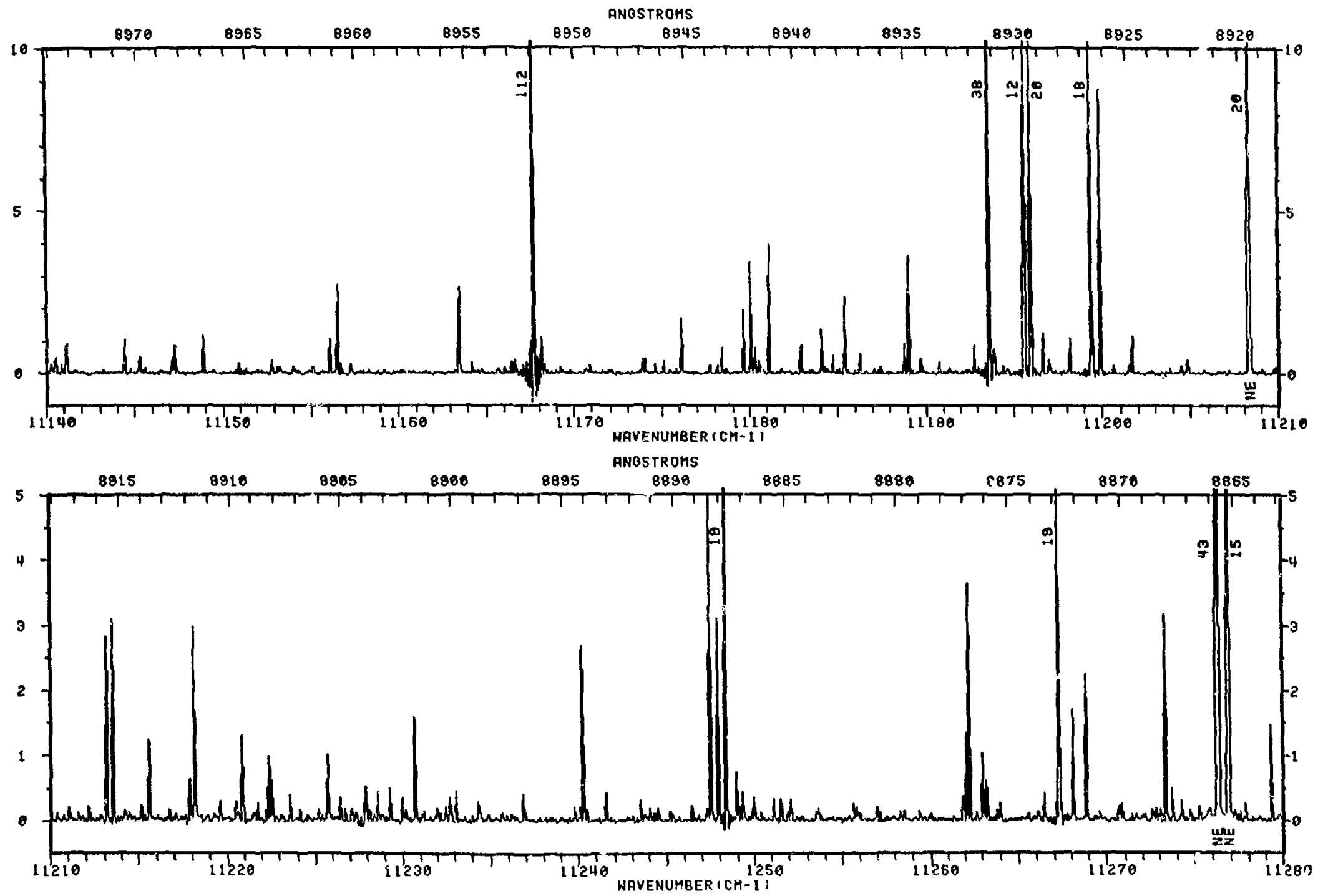
马 " जU

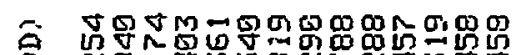

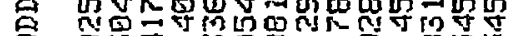

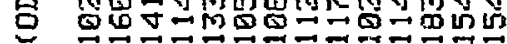

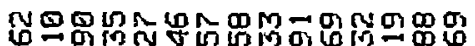

舟

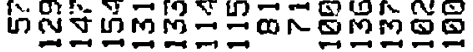

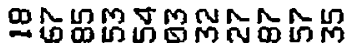

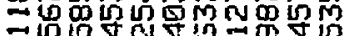

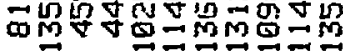

mQ

ถึกตำ

息至

कै

bUNR

$\operatorname{con} \int_{m}^{N} \sin \theta \cos \cos$

$\cos \theta$

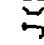

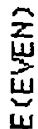

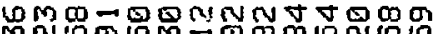

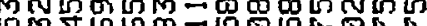

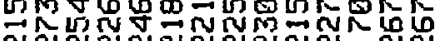

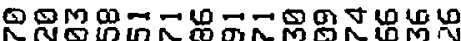

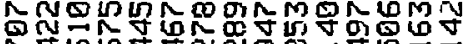

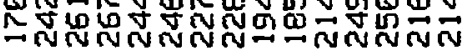

MN.

OM⿻mM⿻コ一𠃌

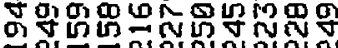

$\cos 20$

nMN

भing NVNWMNNNANN NN

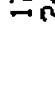

$\rightarrow$ WUNWNNWN $N N=\vec{N}$

z

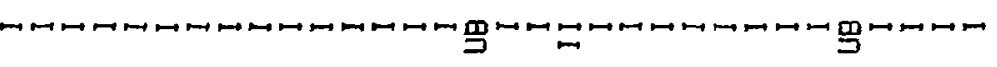

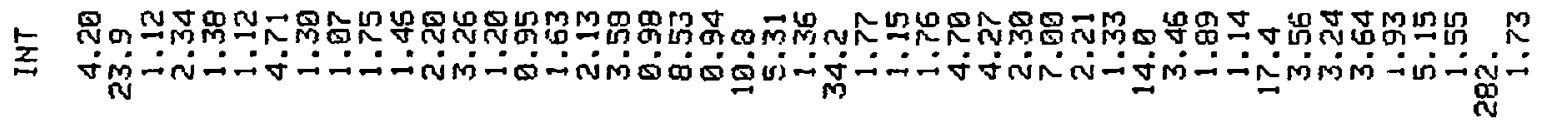

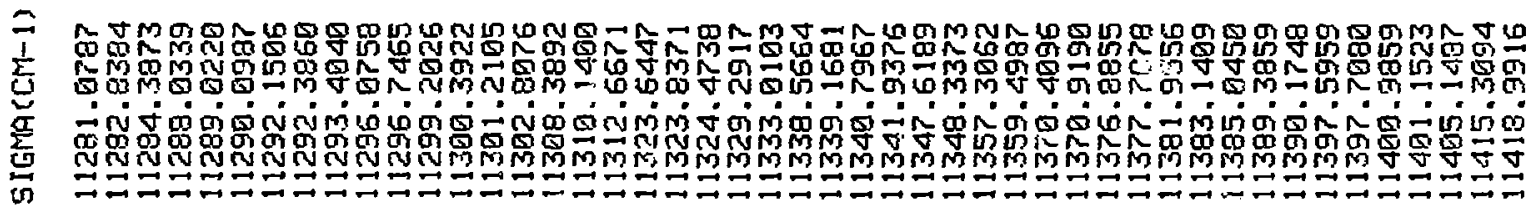

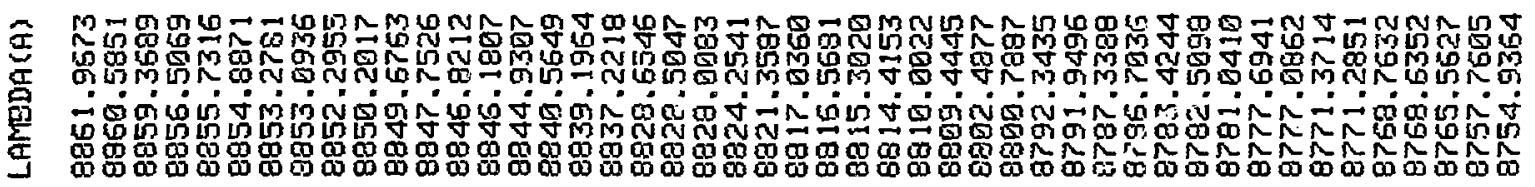




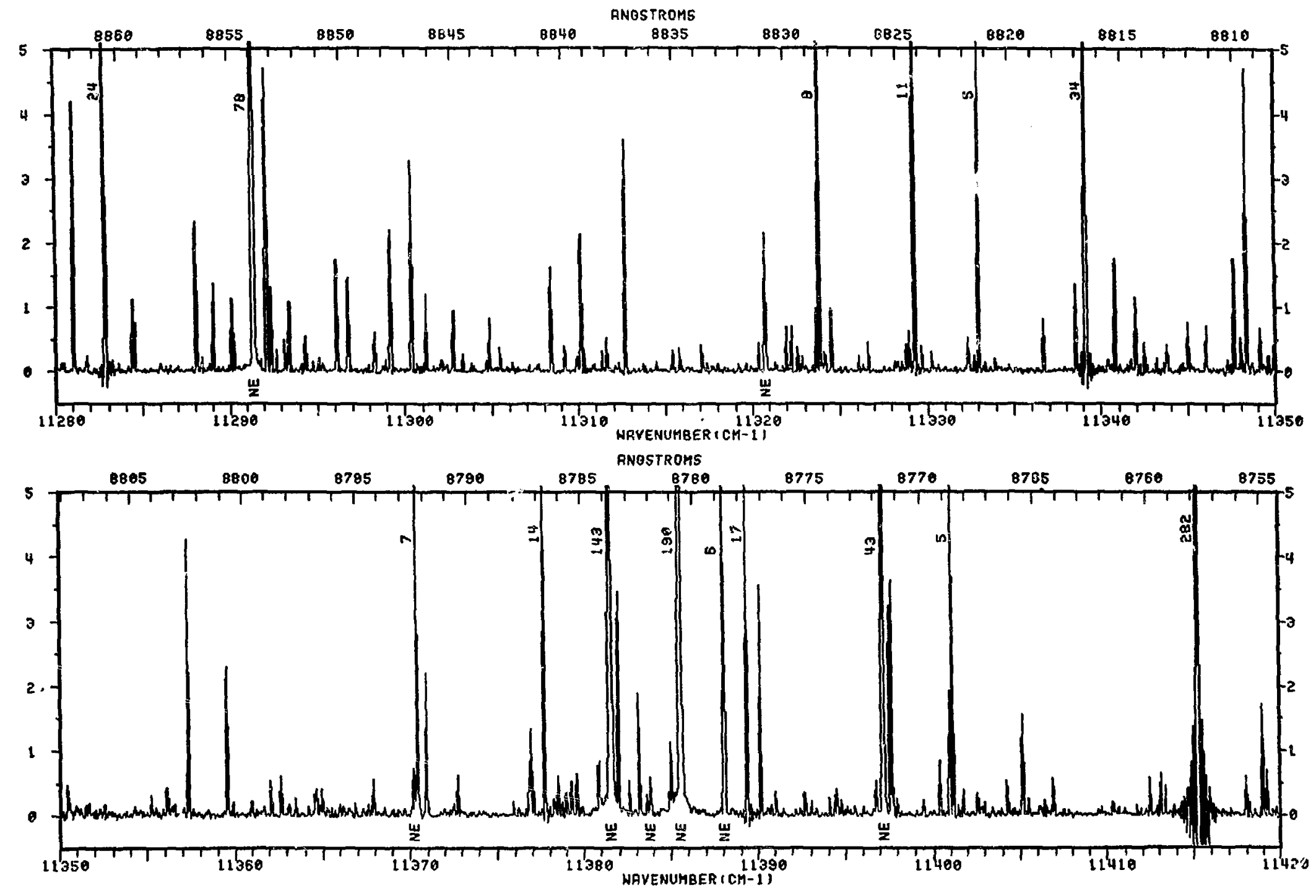

7 


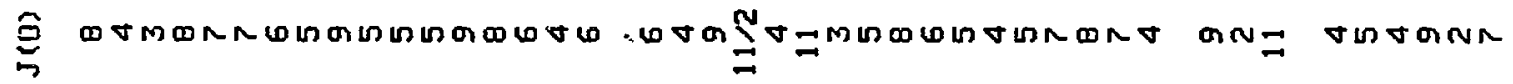

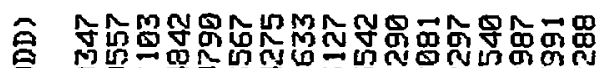

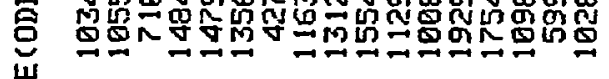

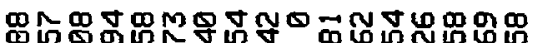

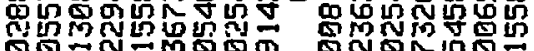

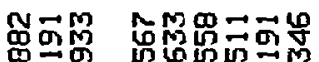

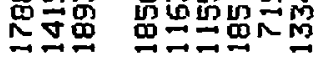

出

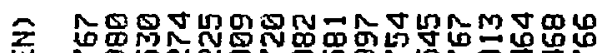

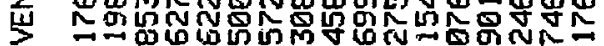

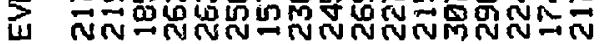

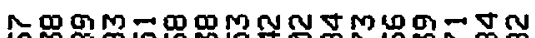

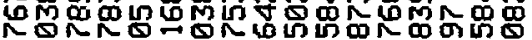

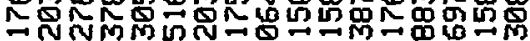

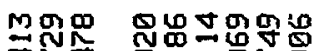

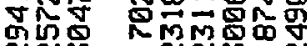
NWNOMNAM

$\sim$

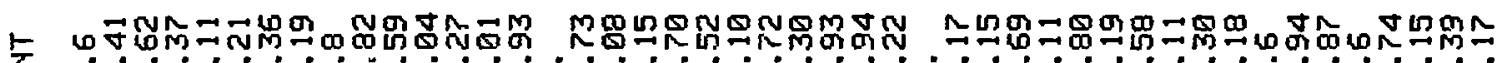

I $\quad$ J

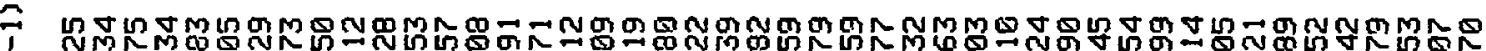

I NNNM⿻日禸 प ك

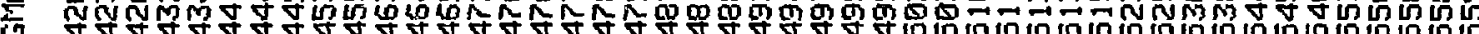
9 \&

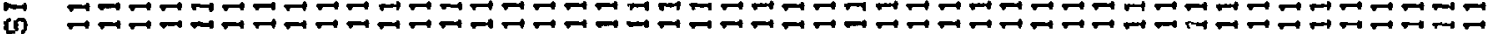

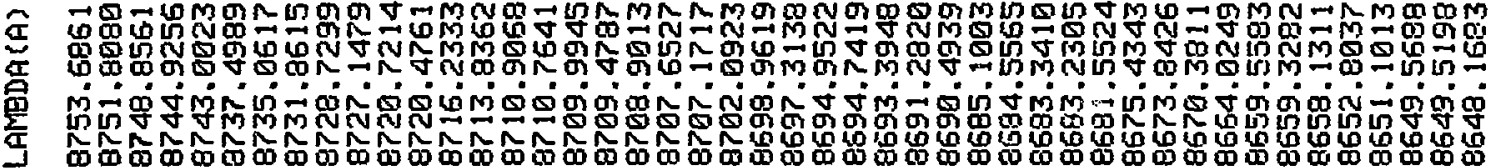




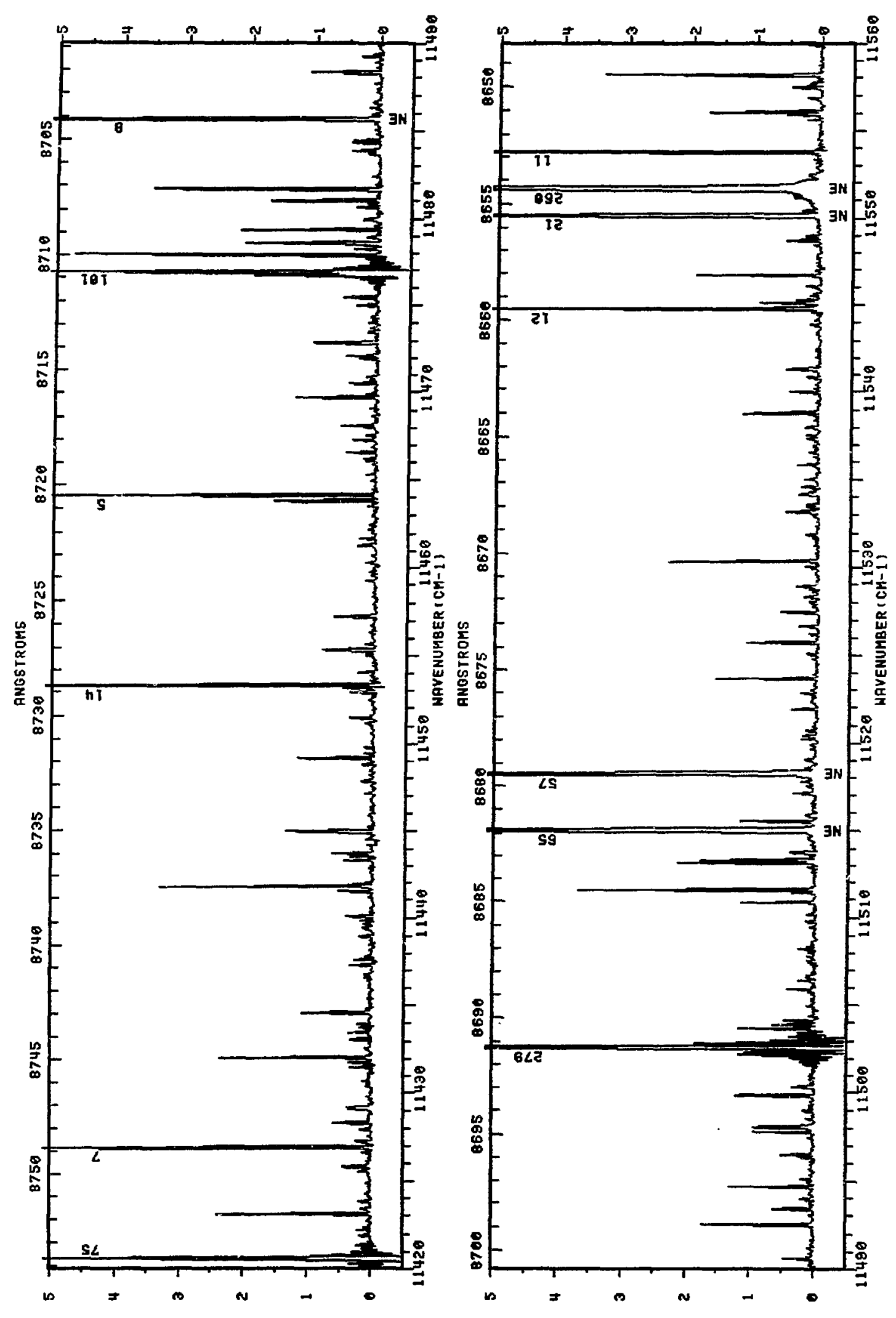




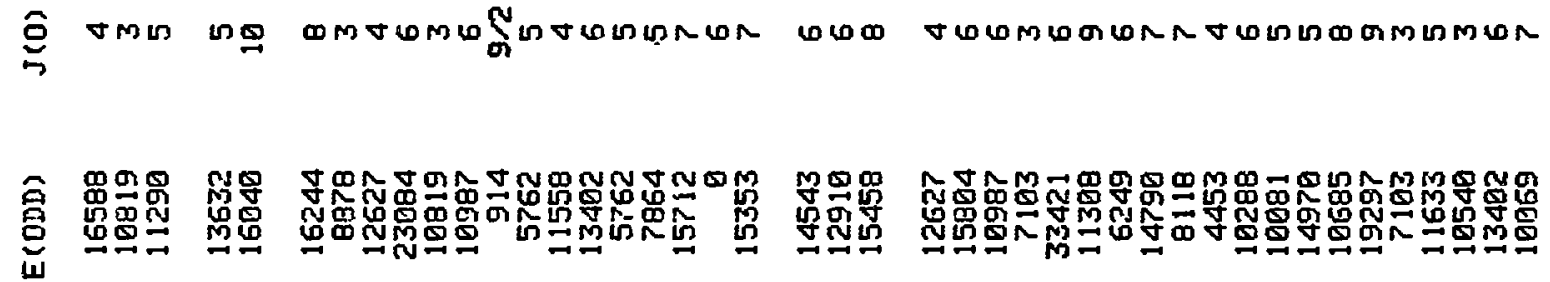

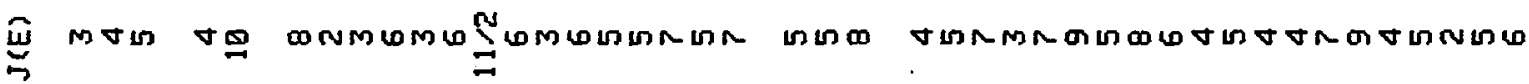

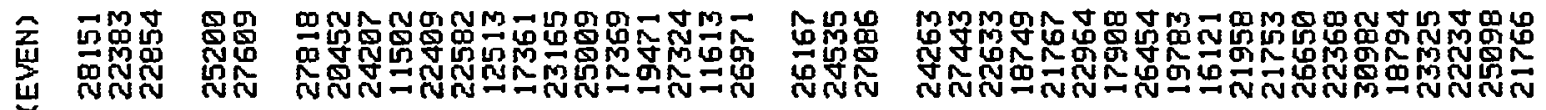

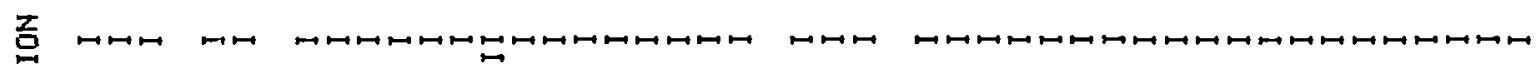

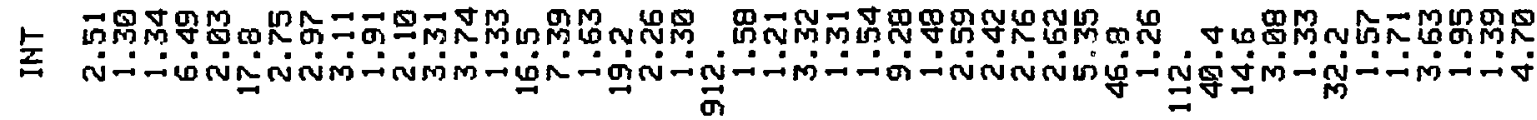

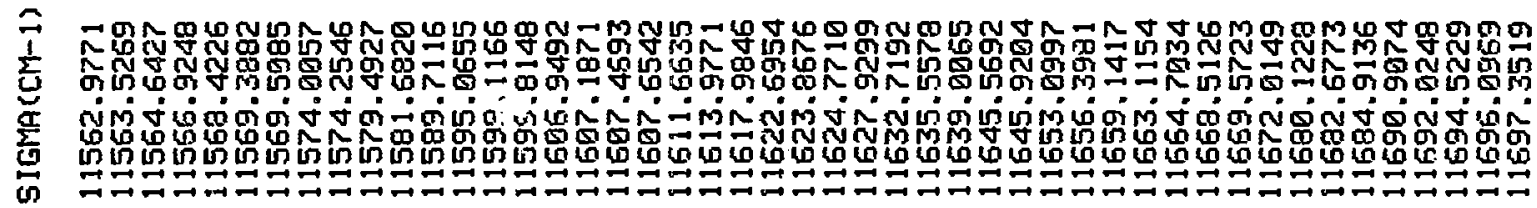

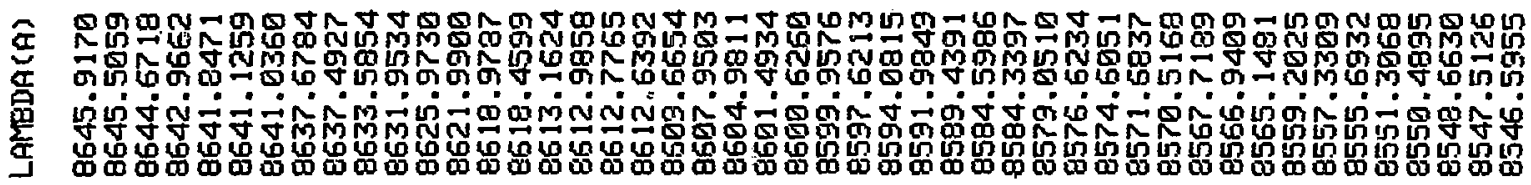



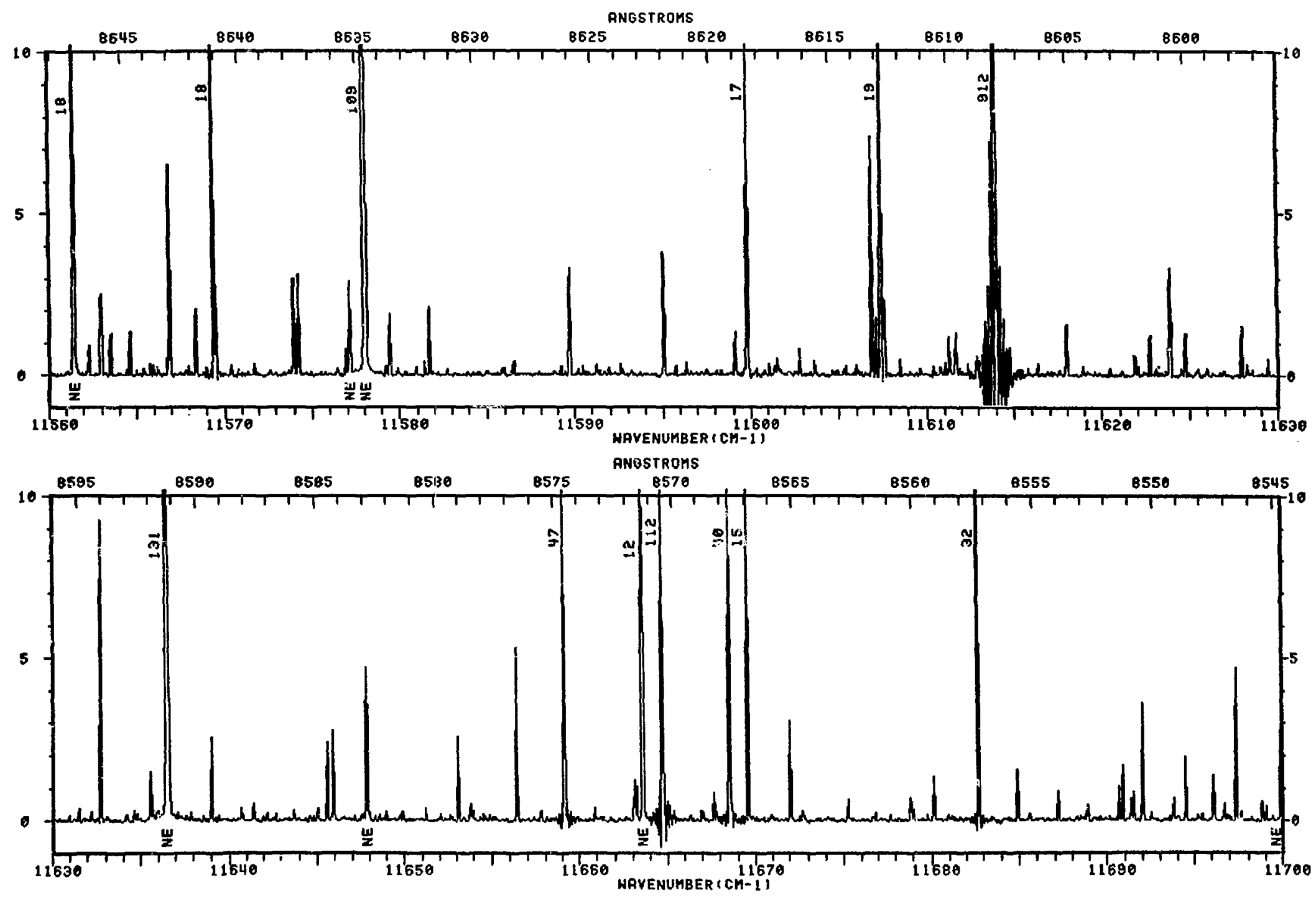


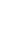

$$
\text { (1) }
$$

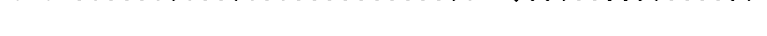

岂

$\infty$ ช

$\omega \rightarrow$

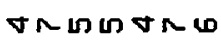

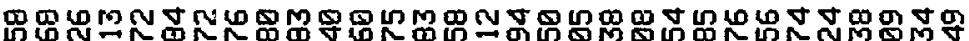

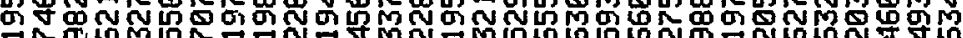

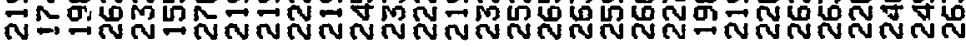

罚界男 $\rightarrow$ 出

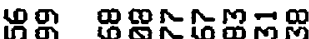

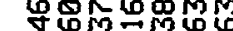

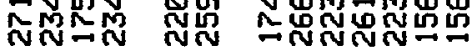

要

a.

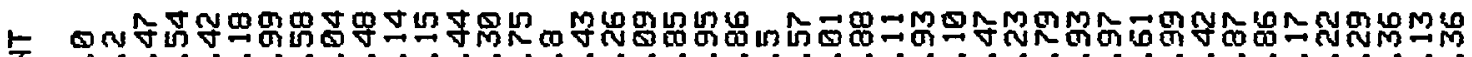

Z

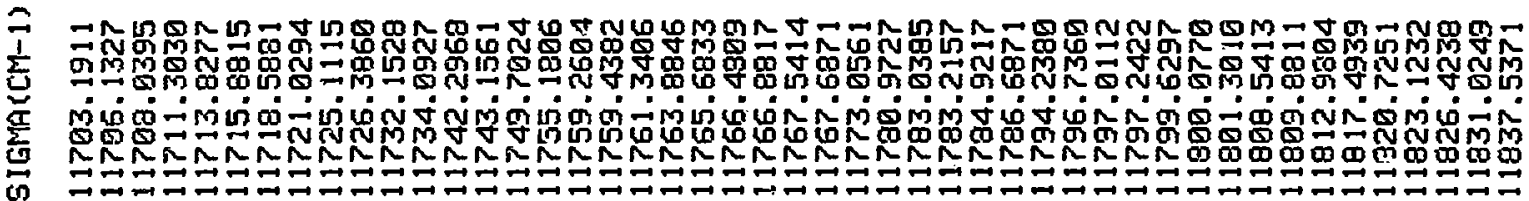

东

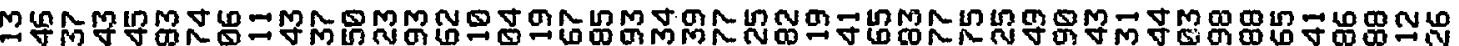
móñ moर

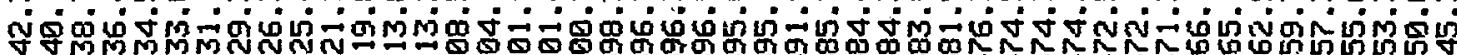

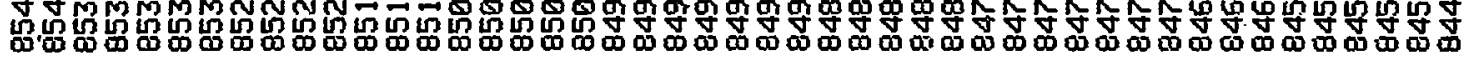



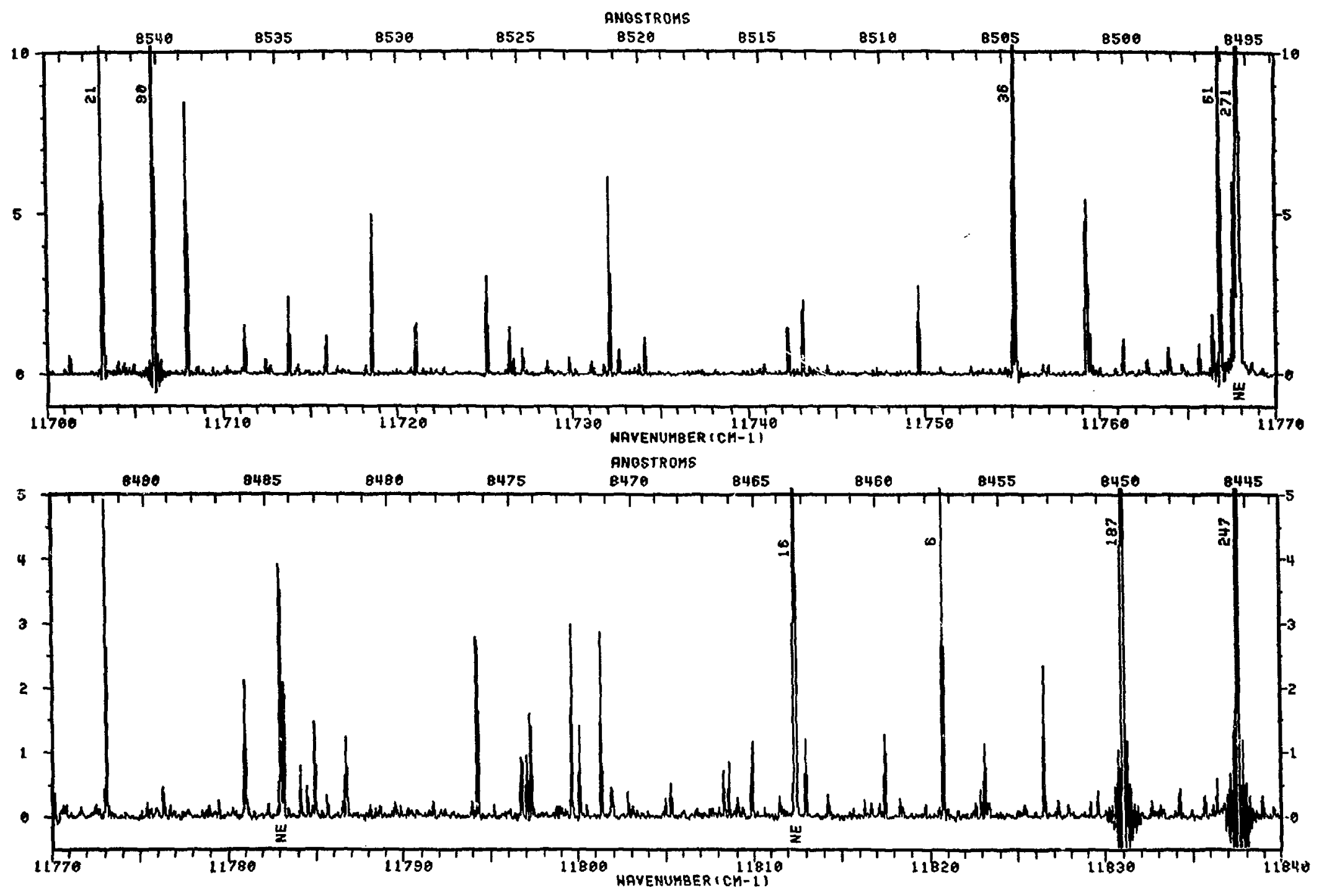

13 
S

nM $\backsim \nabla$

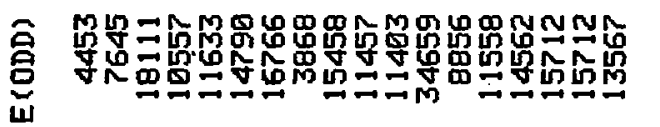

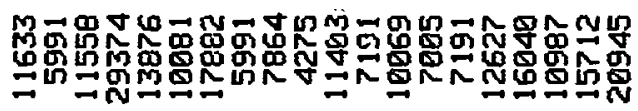

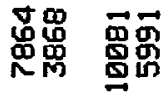

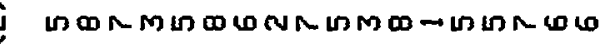

UR

$\omega M$

$10 \mathrm{~m}$

岕

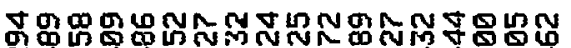

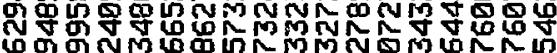

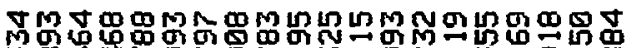

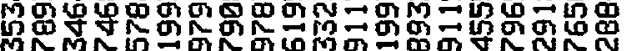

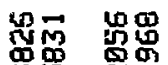
ㅁํำN

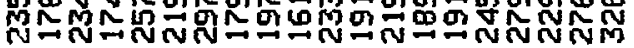

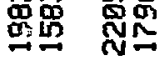

즘

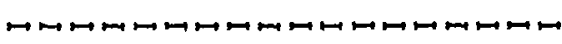

c.

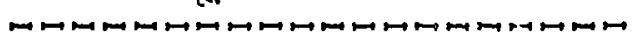

$\mapsto m \quad \mapsto$

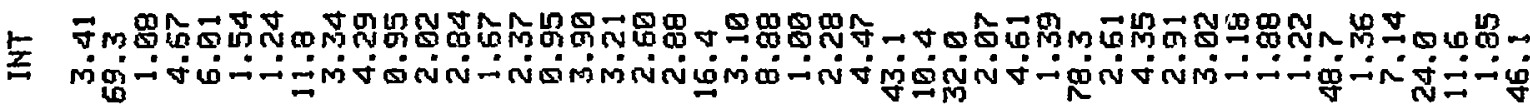

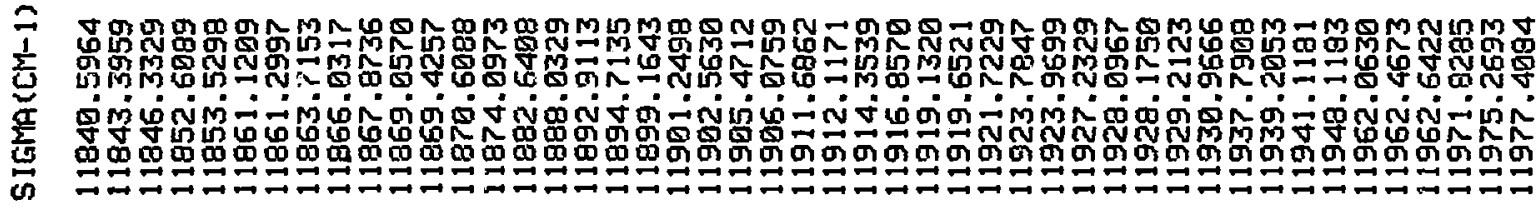

10008-MM-7

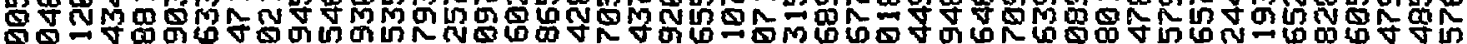

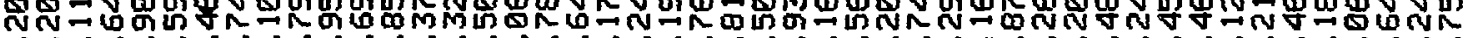
m

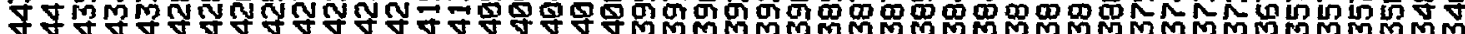

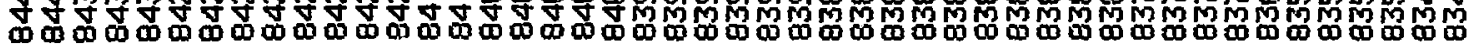




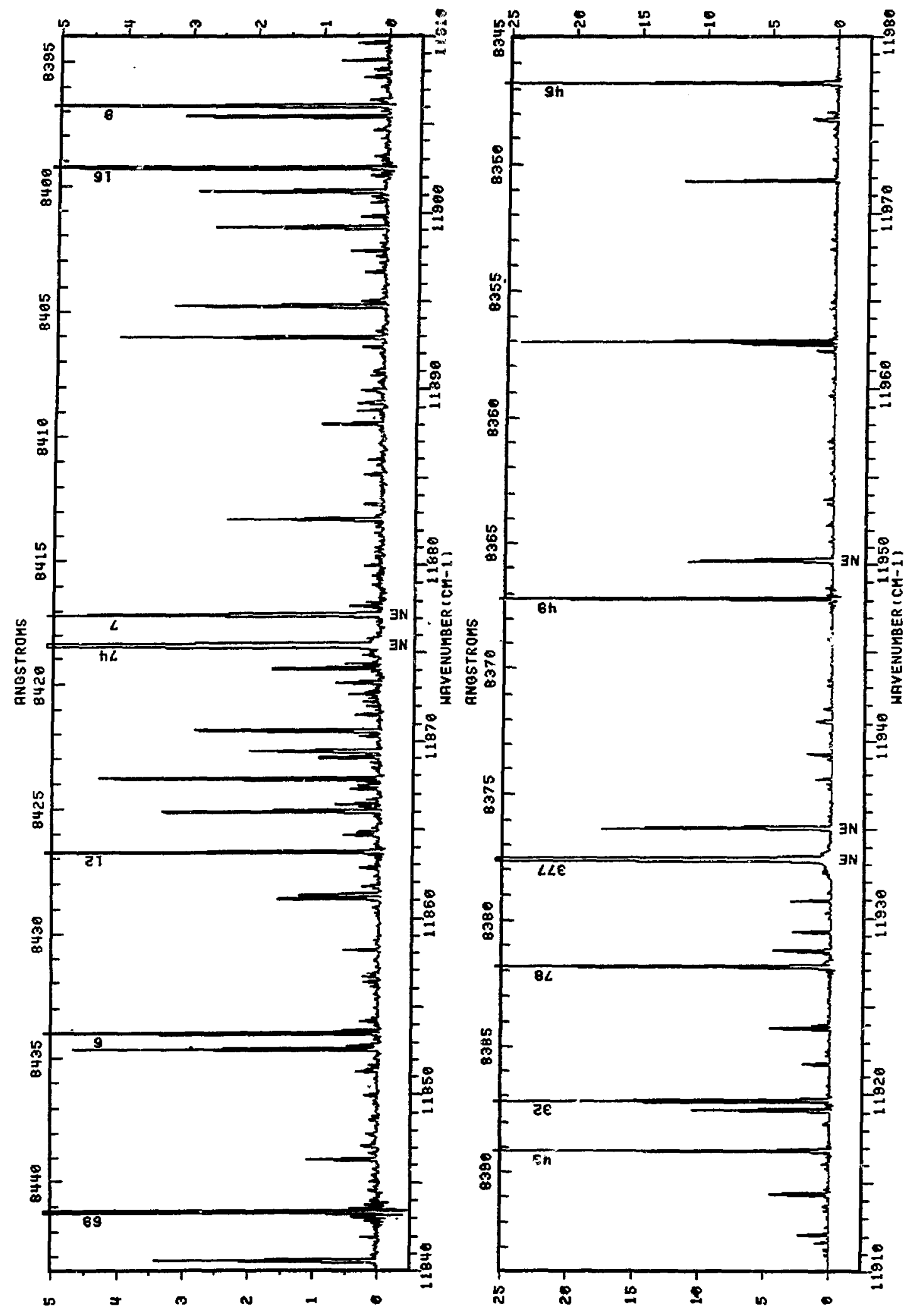


s̆

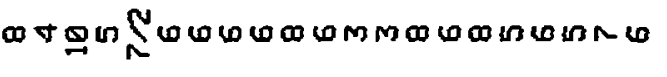

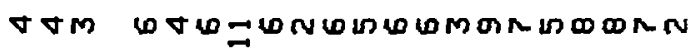

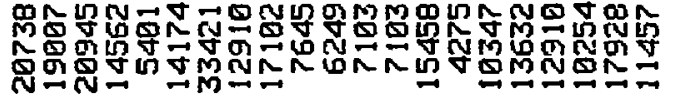

过思 舟足 $M \underset{N}{M}$

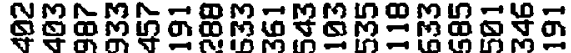

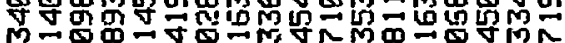

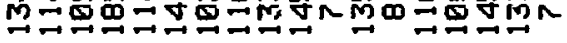

జே

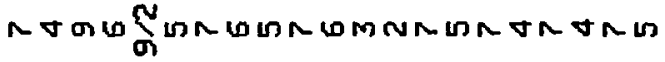

站

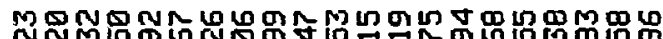

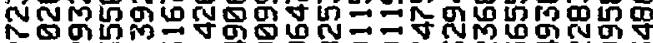

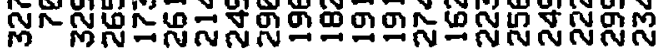

㝵昌盇 Nim
MMV WMNgUNA

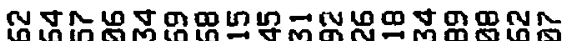

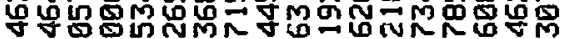

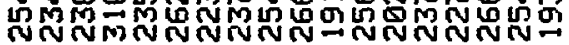

z

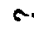

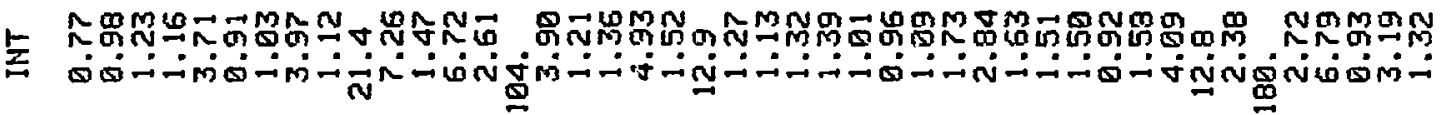

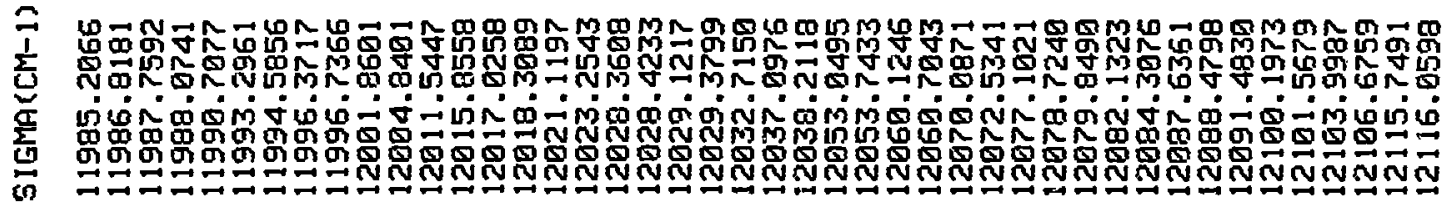

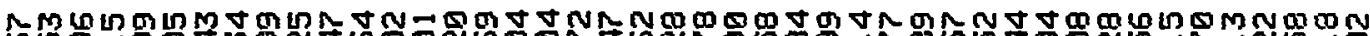

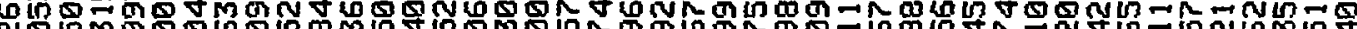

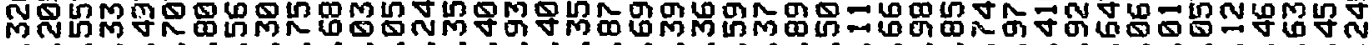
-

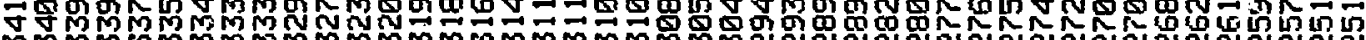

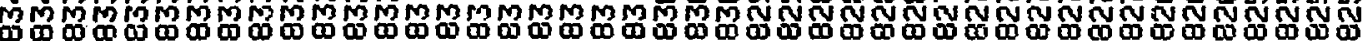



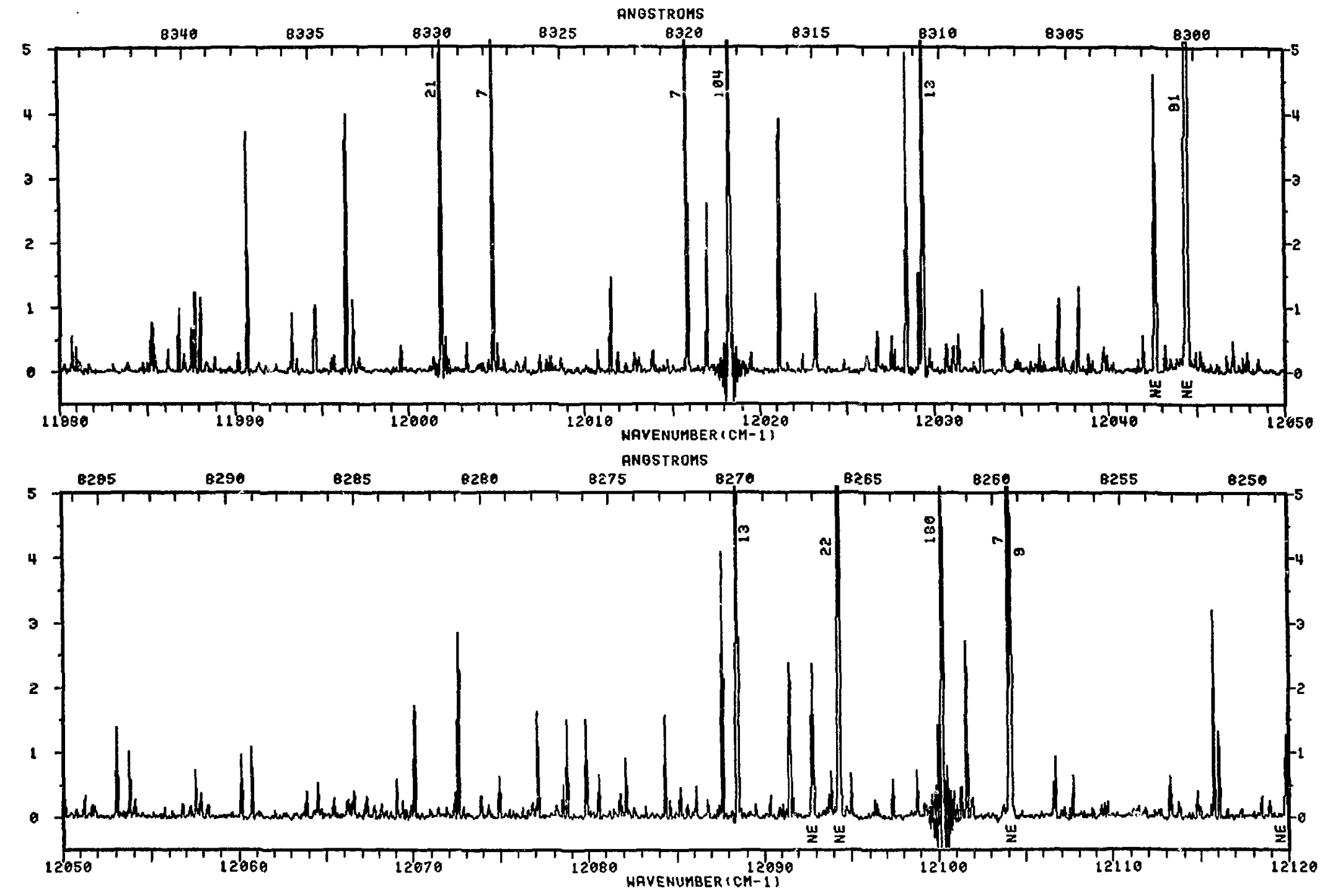


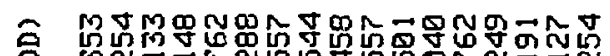

क m

ш

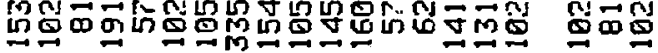

$\stackrel{2}{3}$

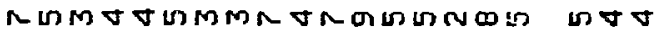

端

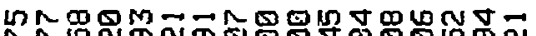

ร

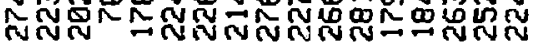

$\sim ⿻ 上$

m䍜

N胥

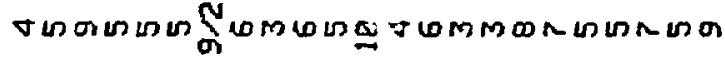

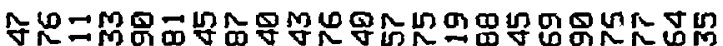

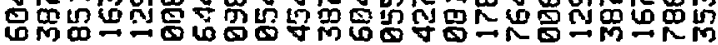

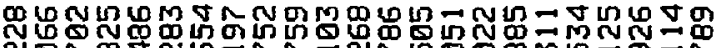

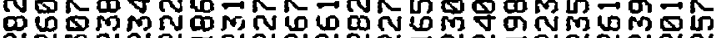

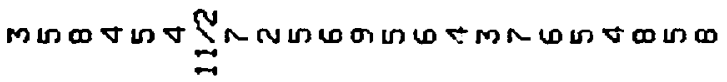

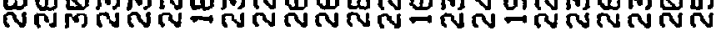

吉

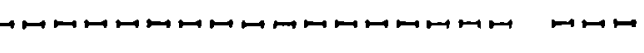

号

我

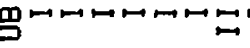

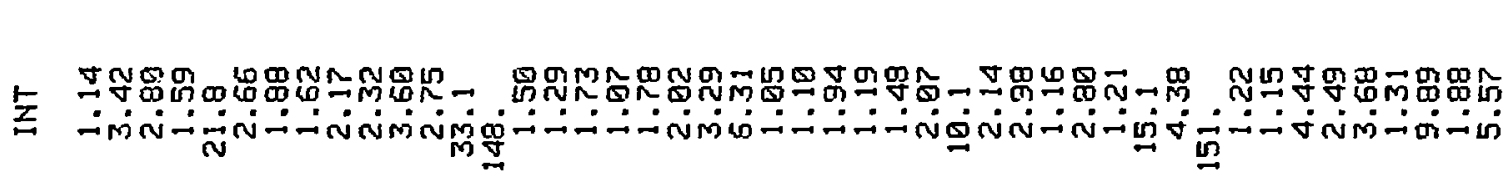

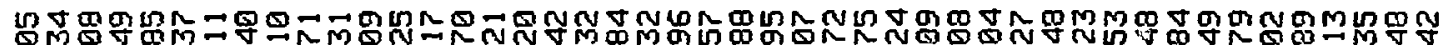

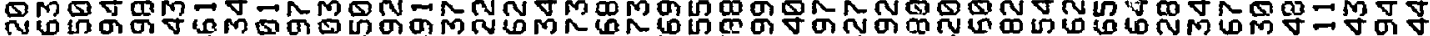

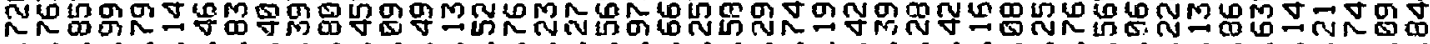

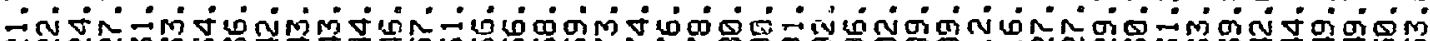

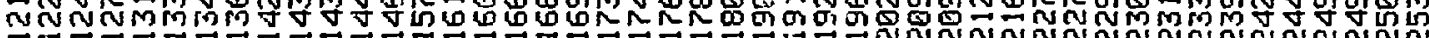

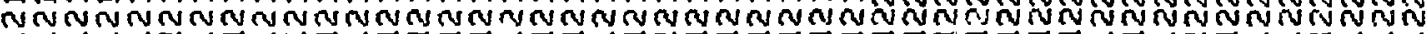

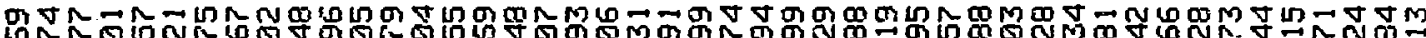

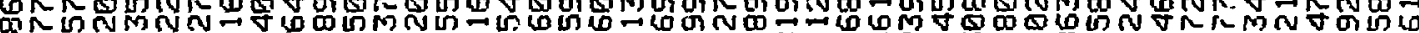
M -

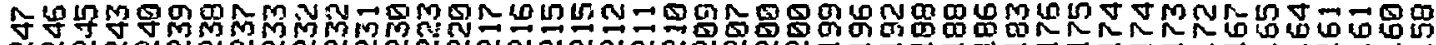

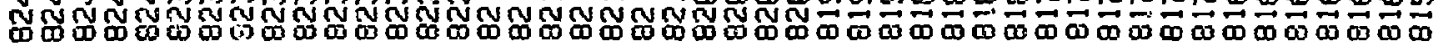



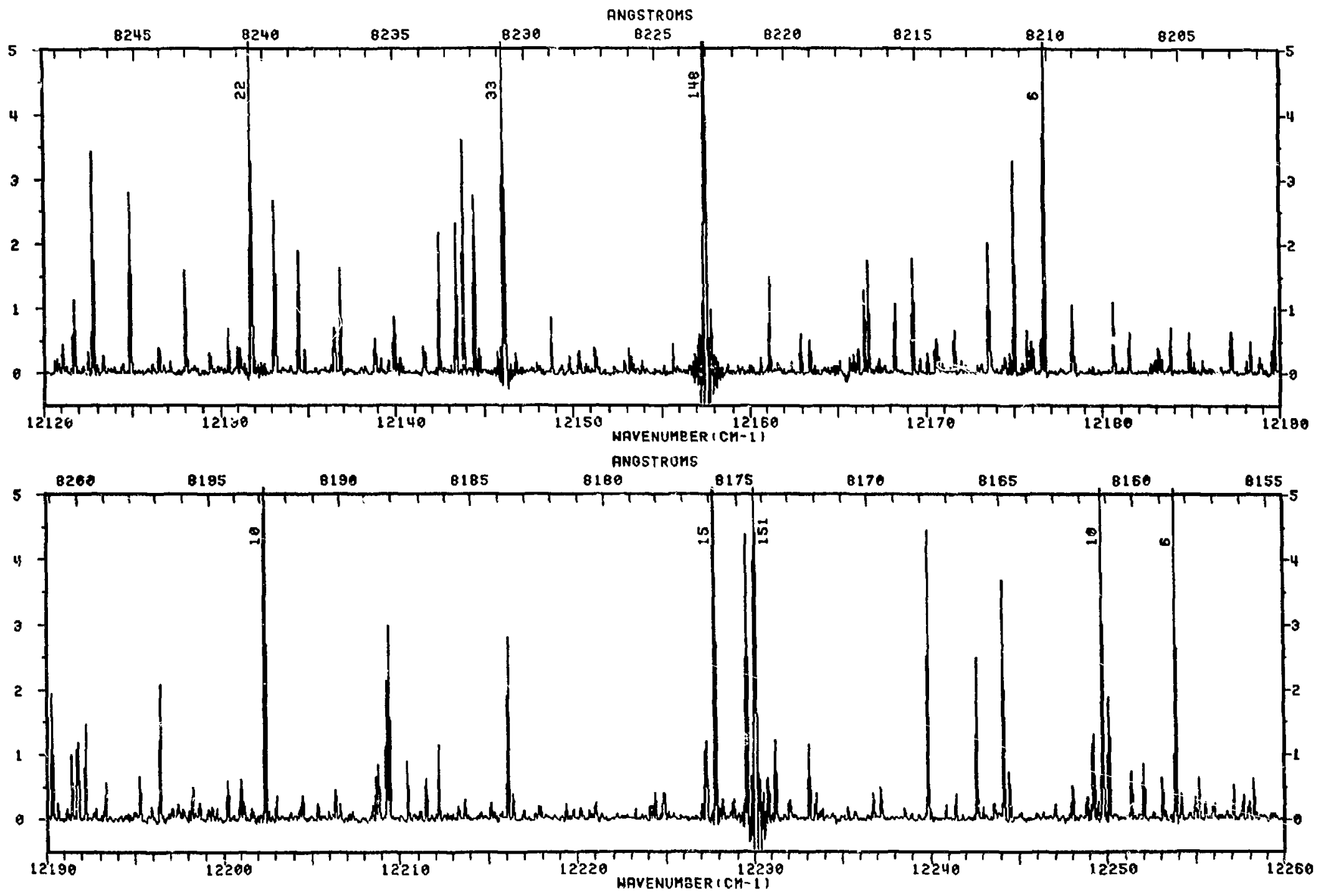

19 
g

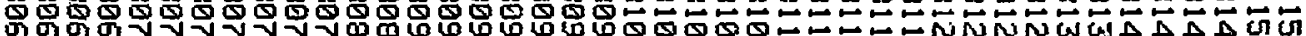

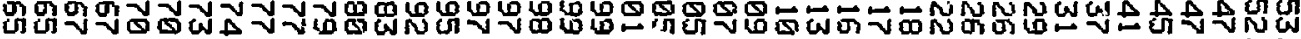
"

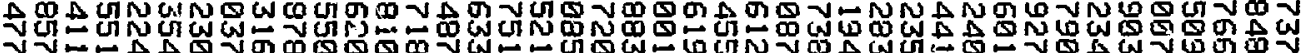

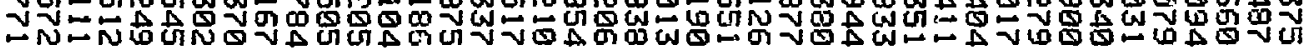

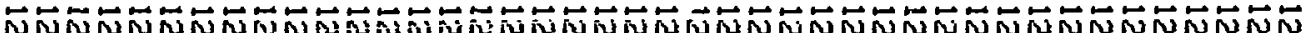

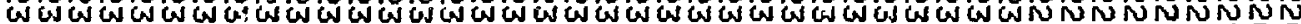
w出

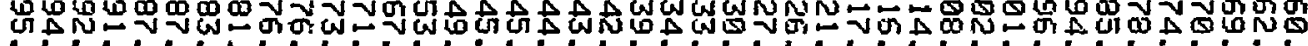

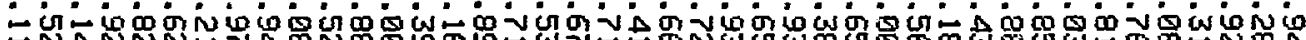

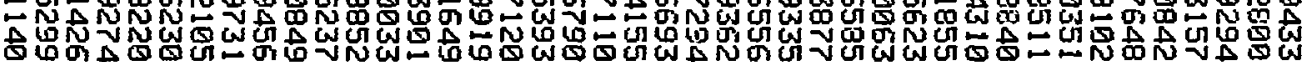

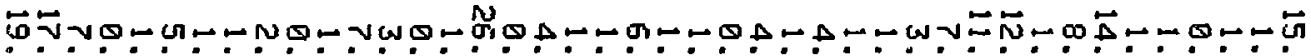
ivion on

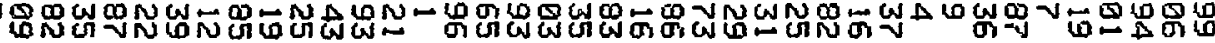

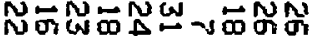

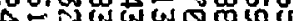

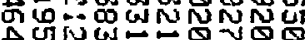

N 䁬盅 ANNNANRN

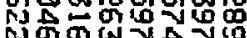

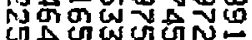

\%Nํำ

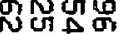
學步

UN-NNNNNNNGN GW कल心

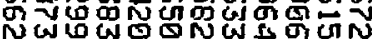

N

\%

O) VGนA $D$ G

D Rnos

GWA

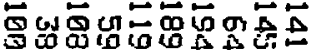

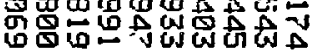

田怘 界:

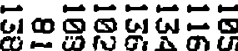

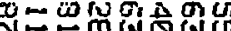

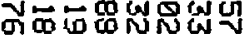

茞灾

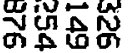

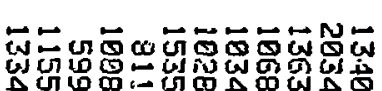

灾 罗

- vNQ

$\infty \omega$
ต $\cos \theta \cos \Delta$

GกN

$\Delta \Delta$ แ

10 


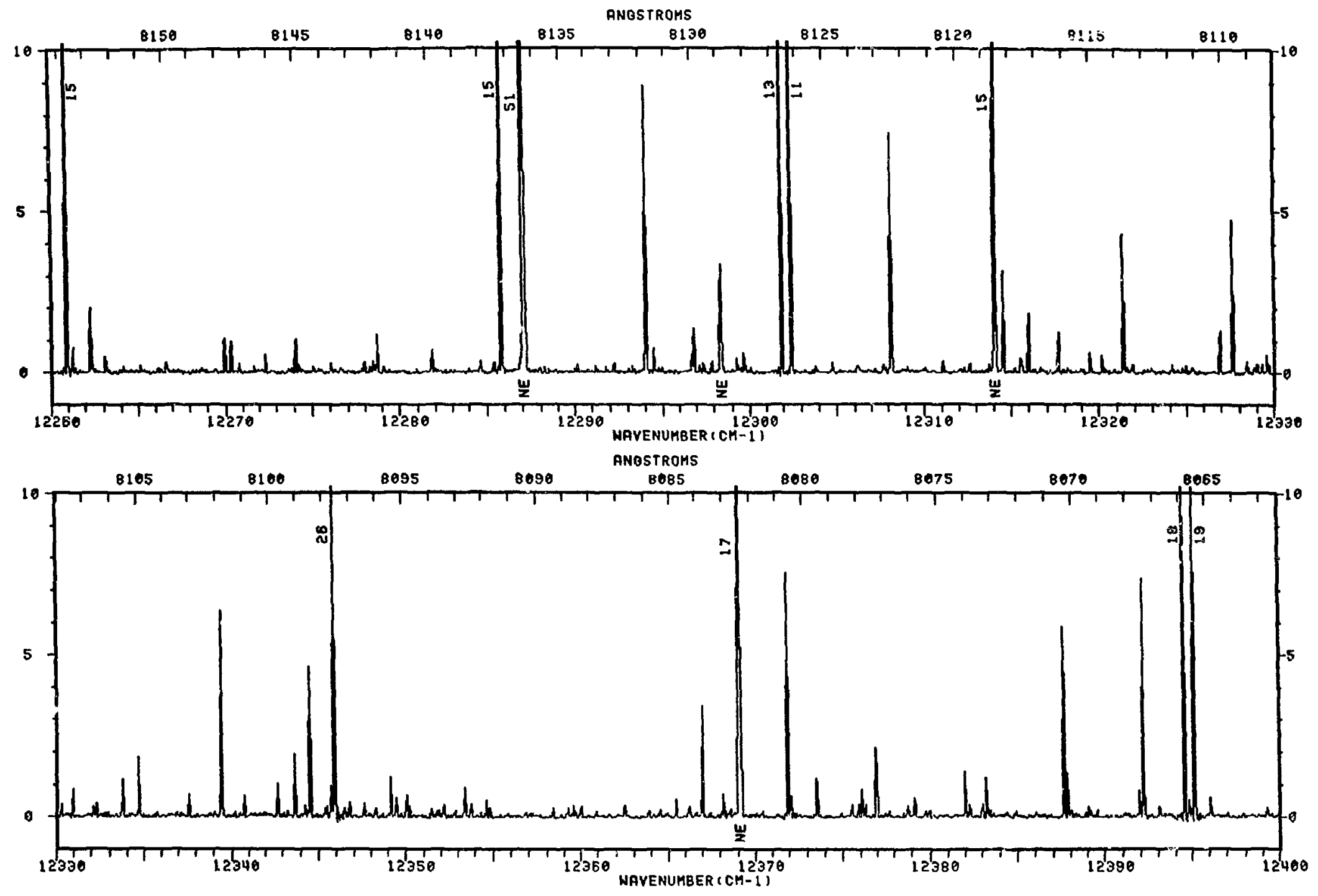




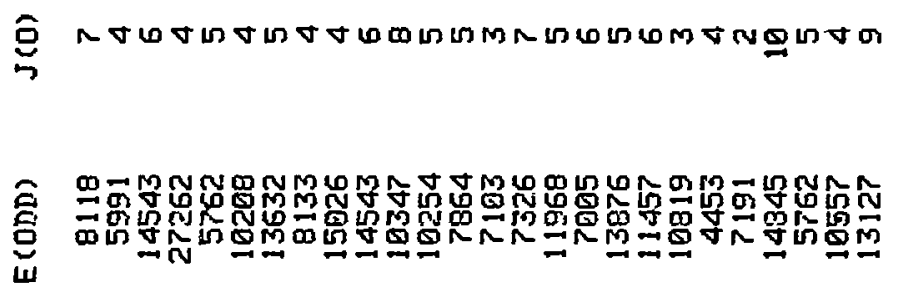

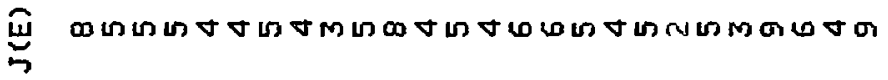

$\underset{w}{\frac{2}{4}}$

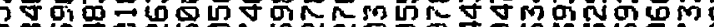

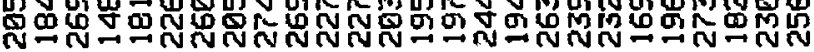

Nun w on

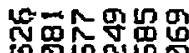

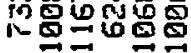

$\operatorname{mos} \sin \alpha \sin \alpha \sin$

DQNOMN Non

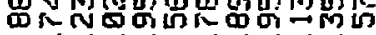

corrore MRMuv

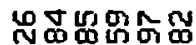

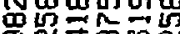

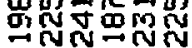

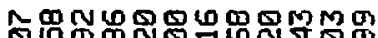

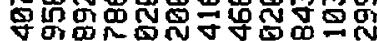

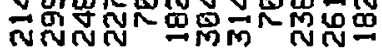

늠

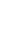

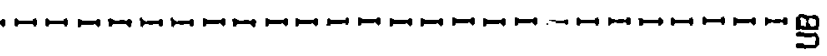

卡

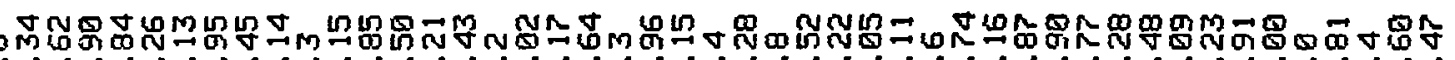

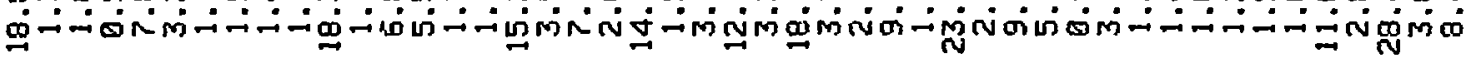

空

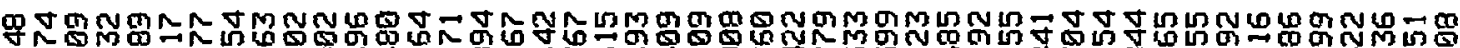

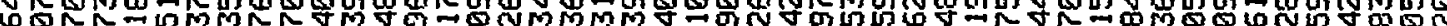

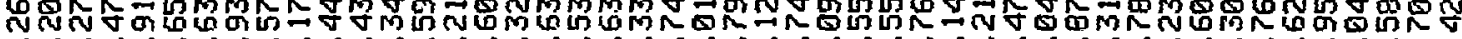

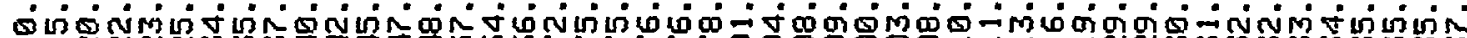

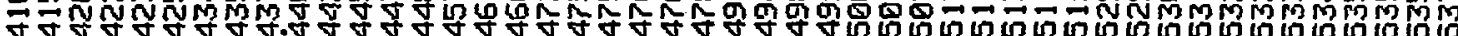
NलMN⿴囗十心

喾

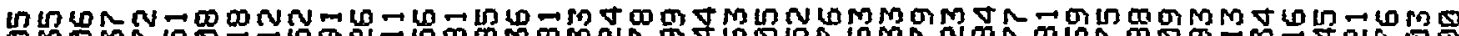
МMSMN

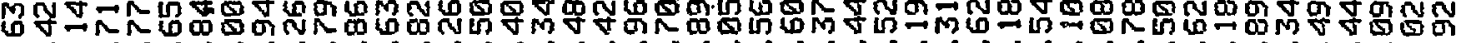
ต

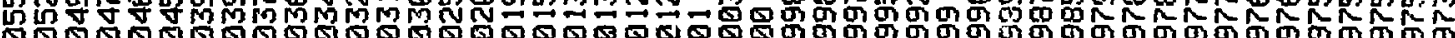

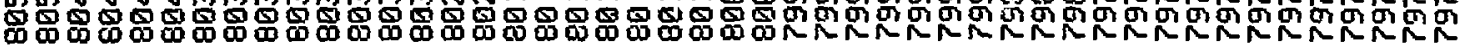



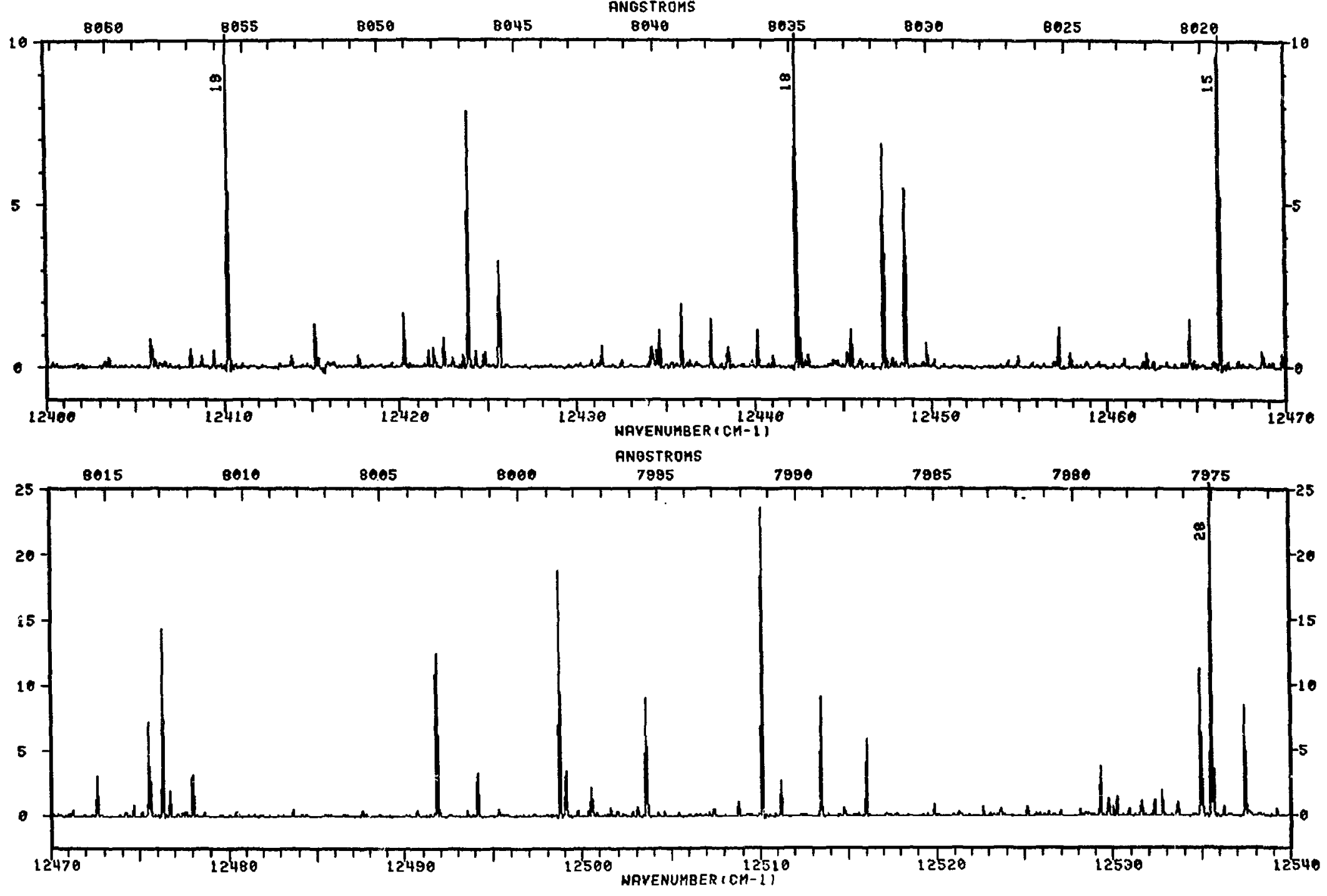
6

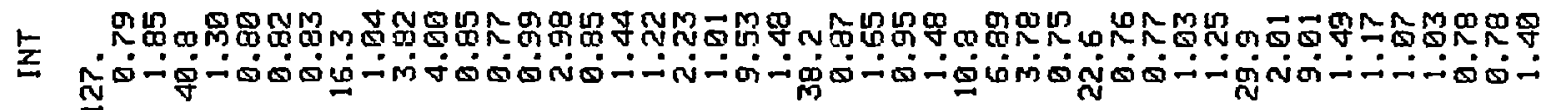

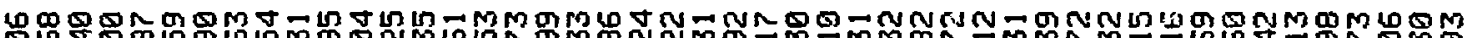
SW

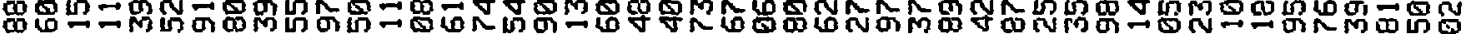

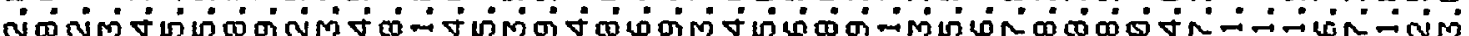

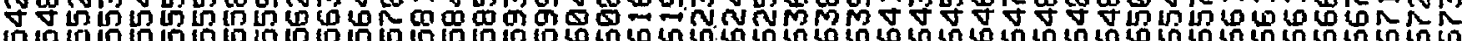
ต

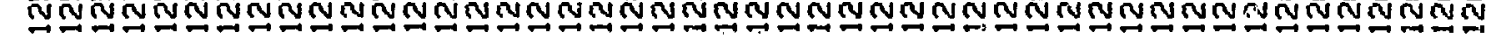

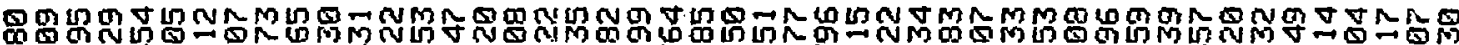

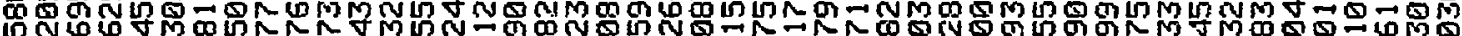

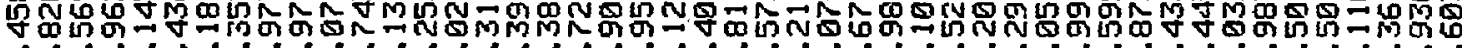
心

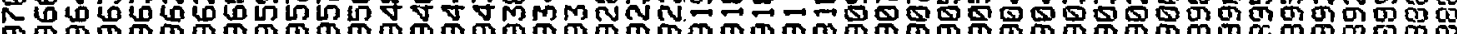

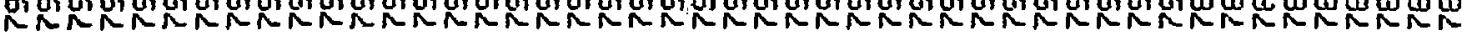



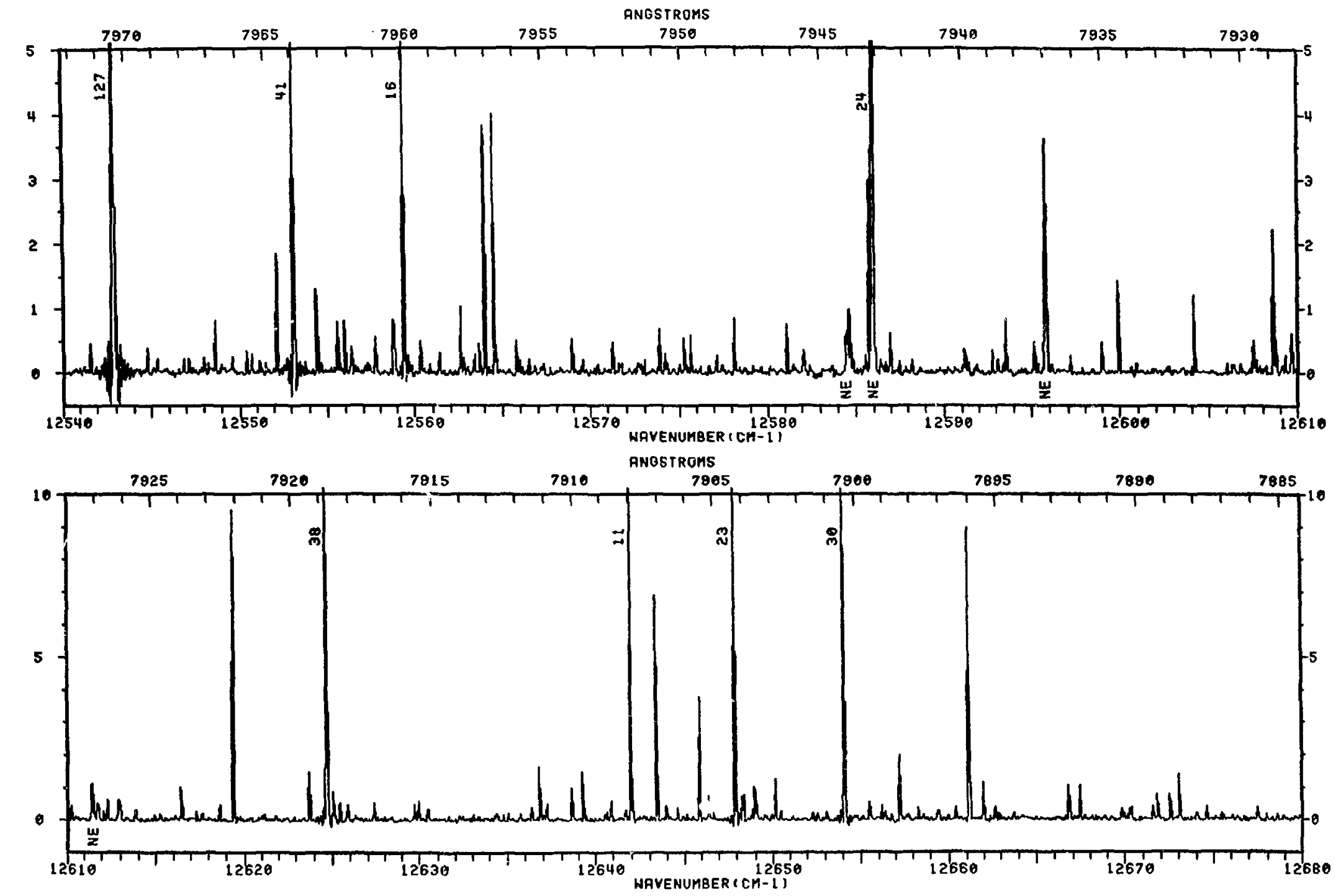


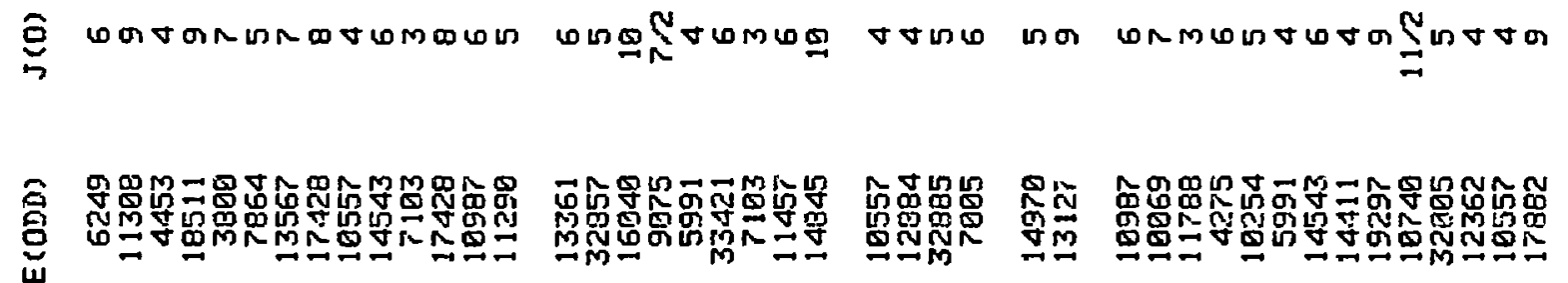

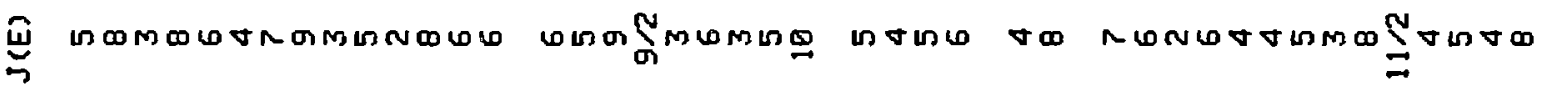
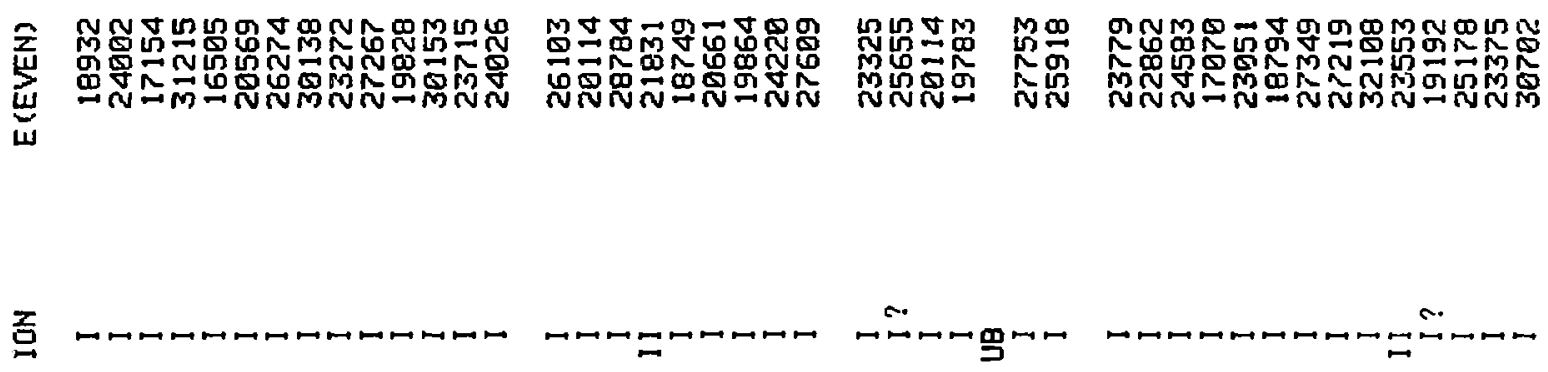

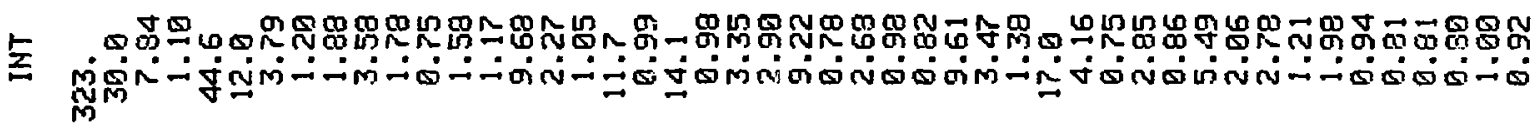

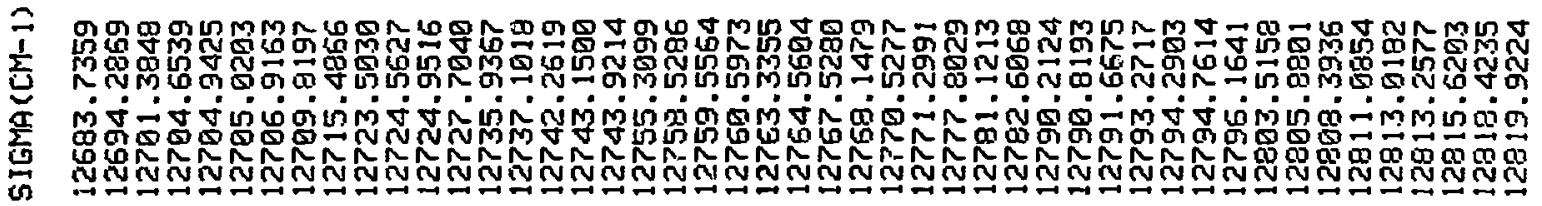

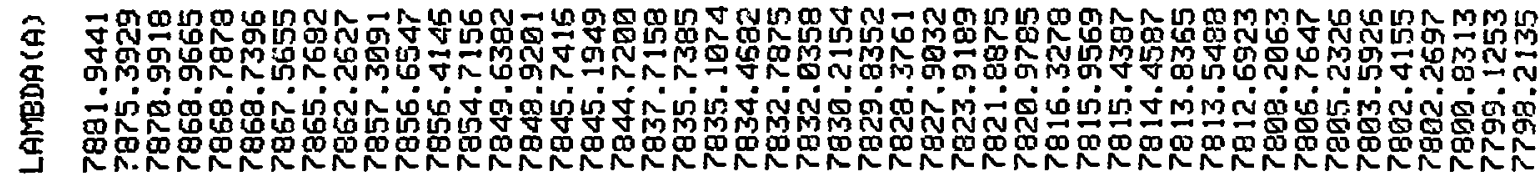



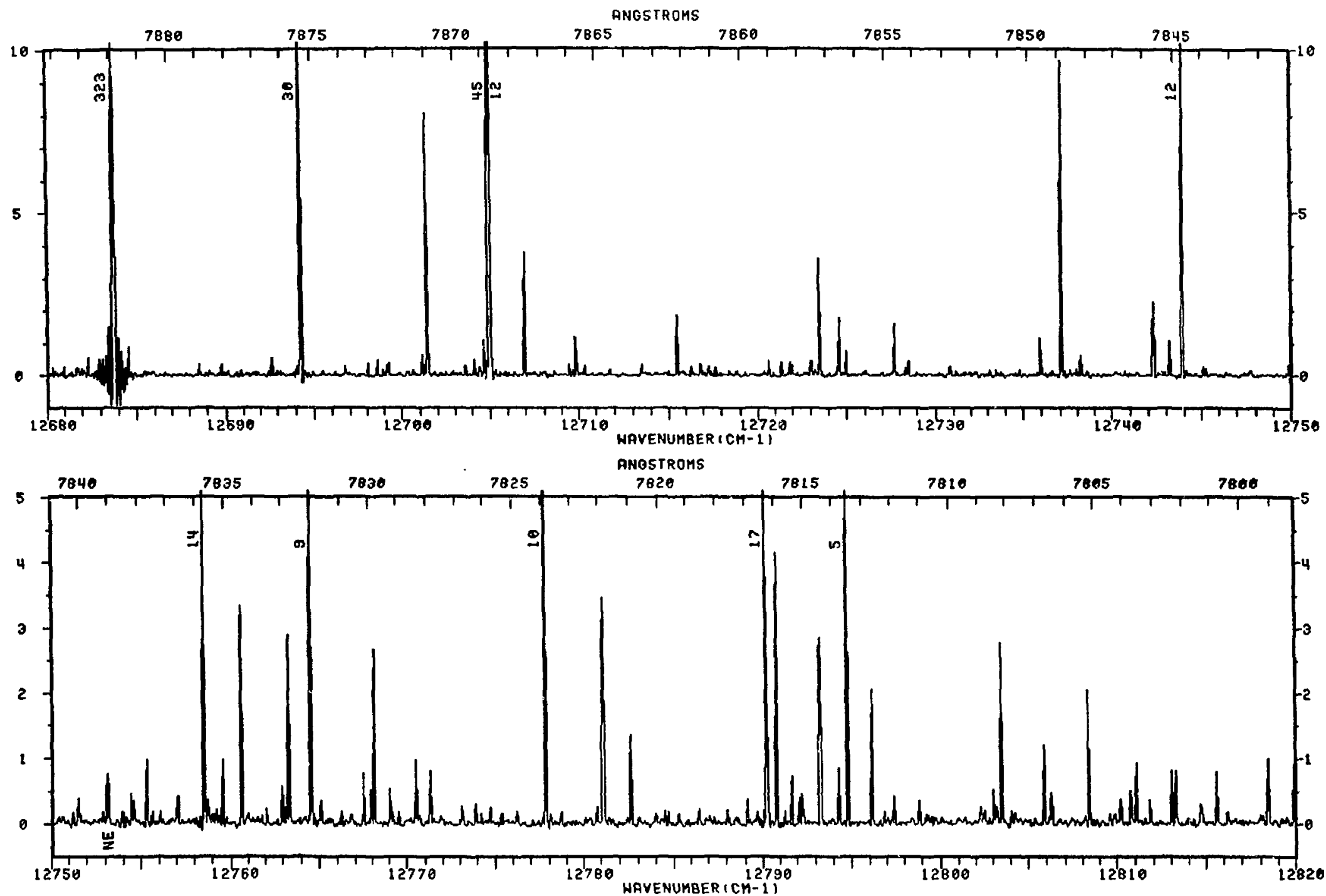


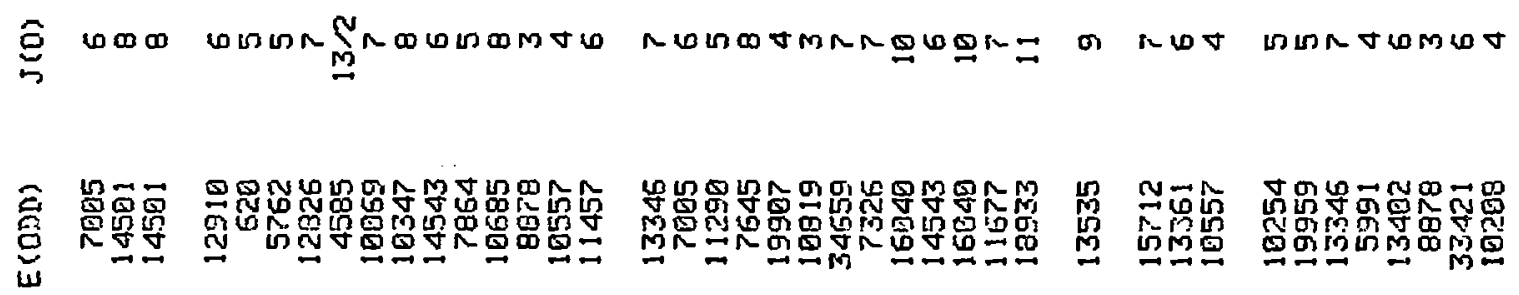

تै

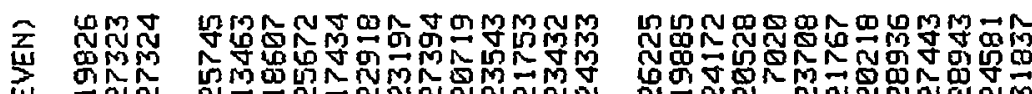

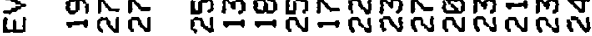

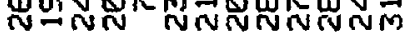
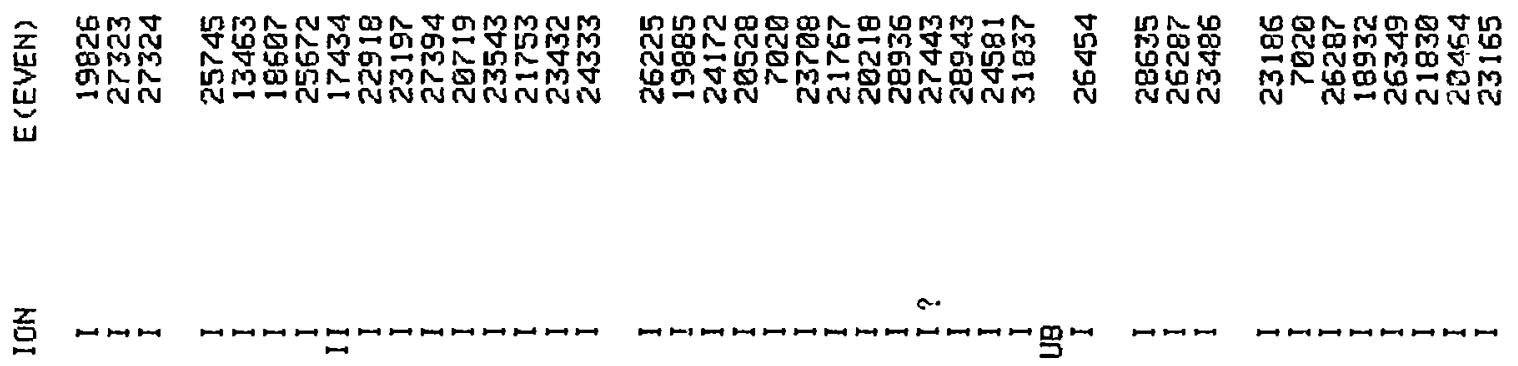

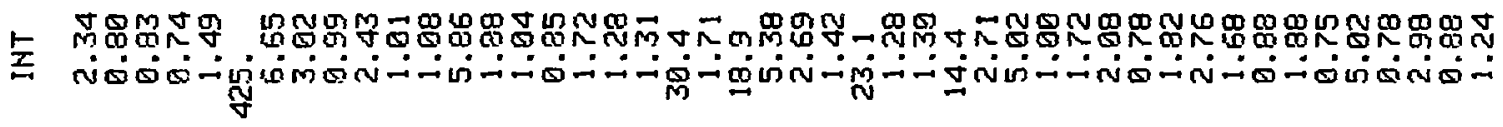
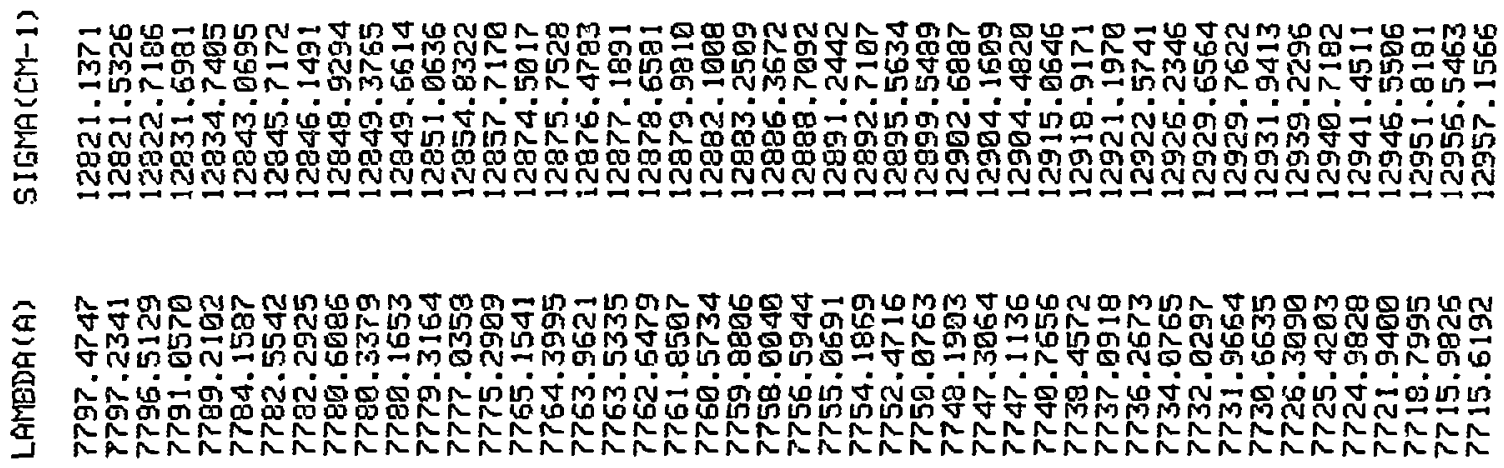

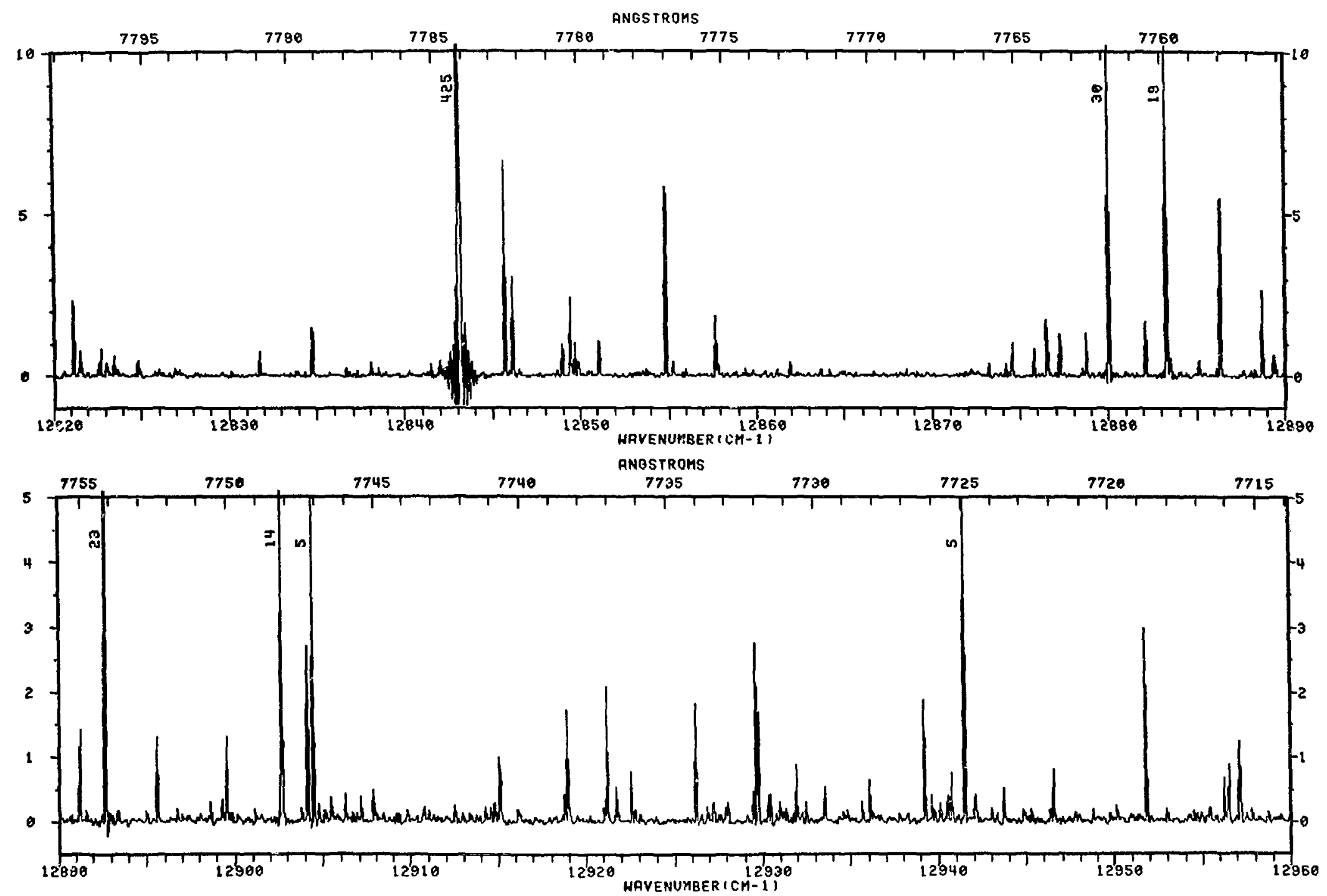
官

மตแM

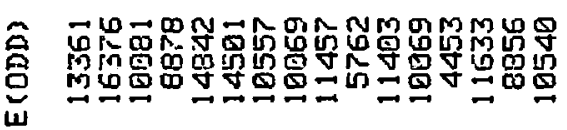

$\cos$

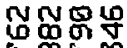
is m

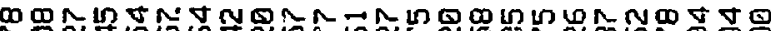
NN

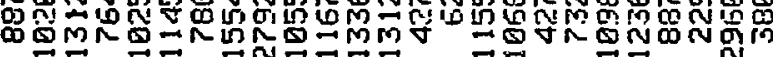

$\underset{\omega}{\omega}$

$\sum_{\text {Lu }}^{\sum_{\text {Lut }}^{2}}$ $\ln \sin \theta \cos \sin \sin \theta \sin \theta$

Ding

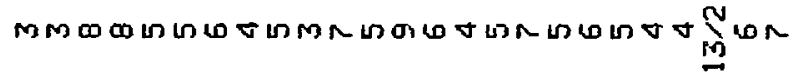

츠ำ riom

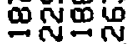

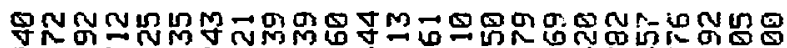

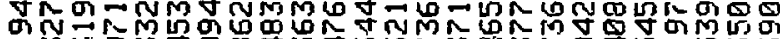

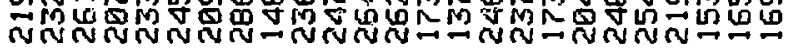

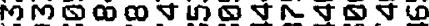

늠

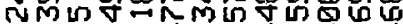

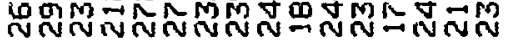

r.

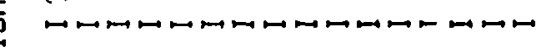

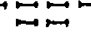

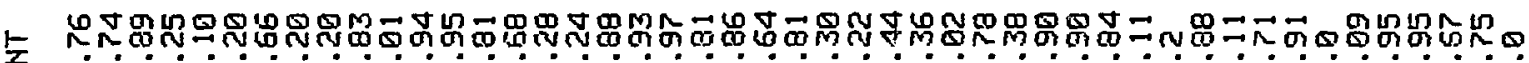

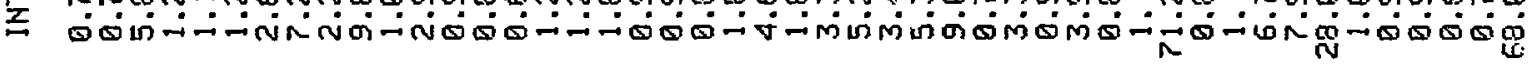

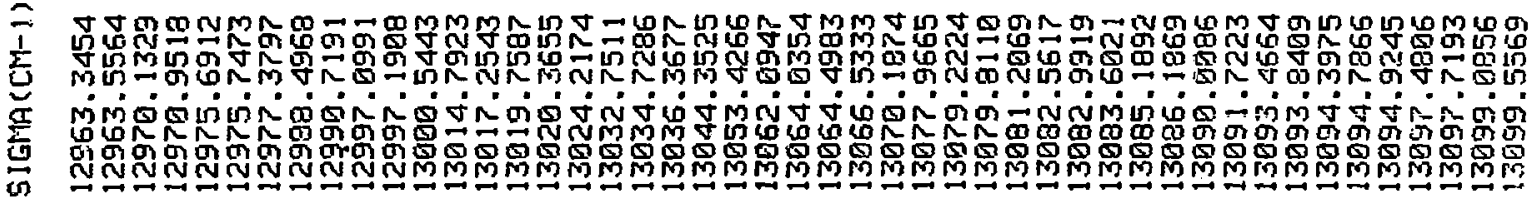

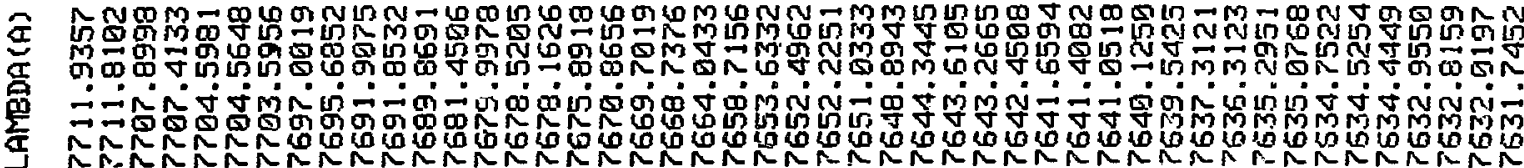



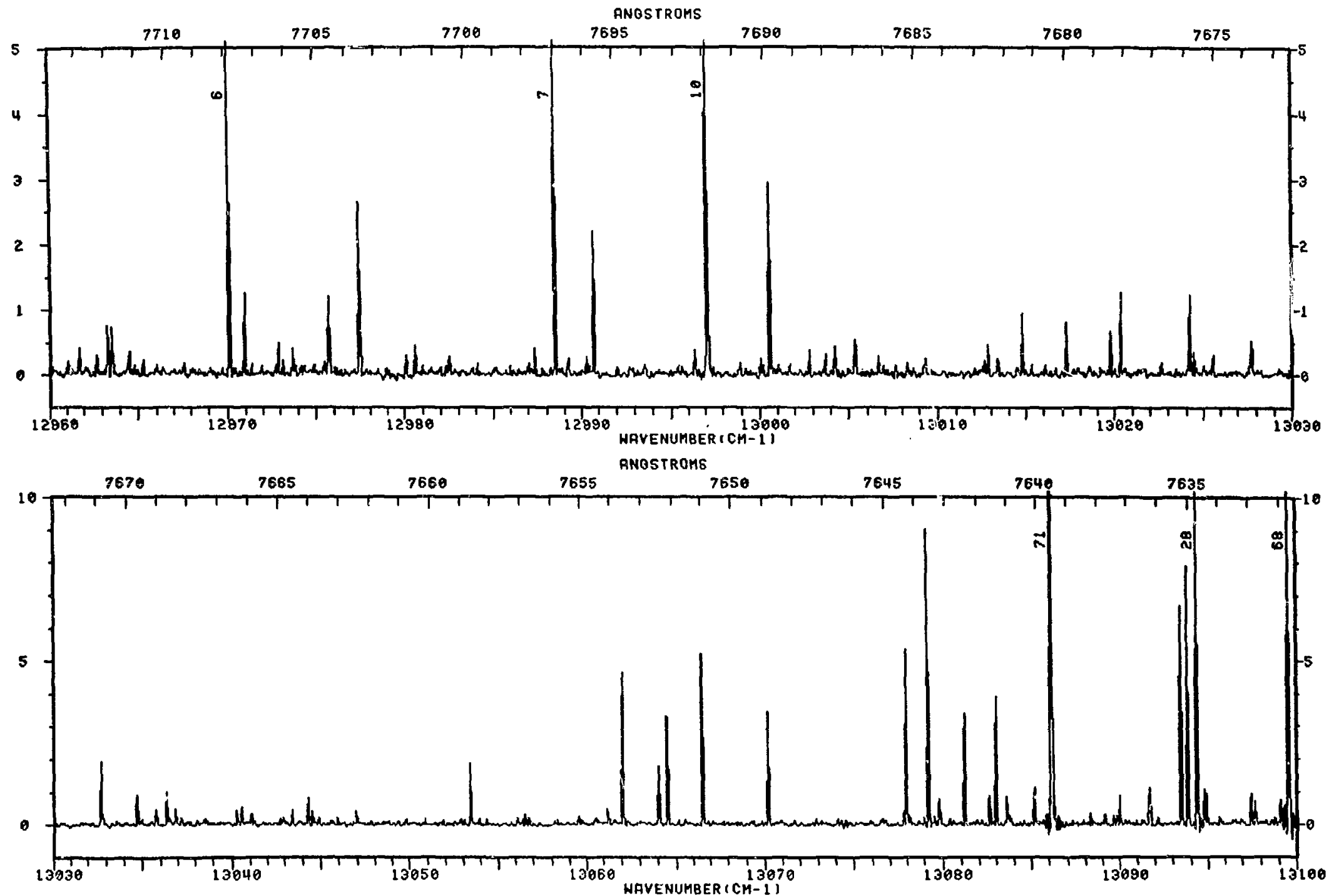
D

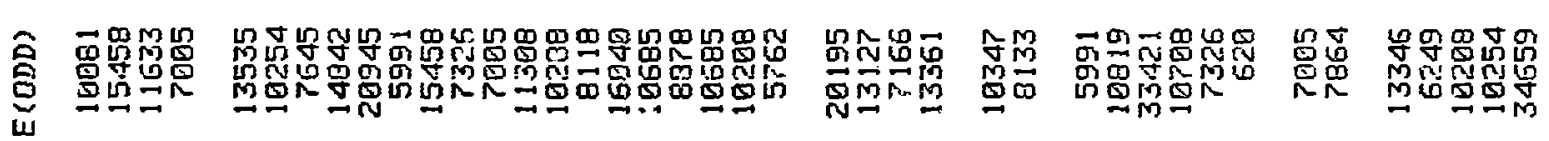

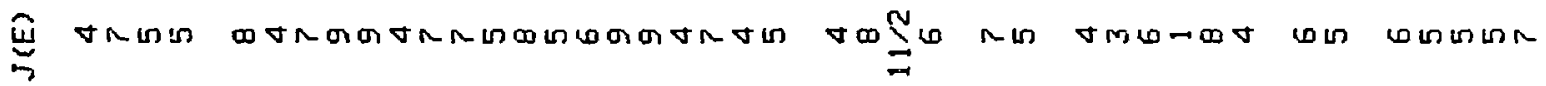

空

产 品要

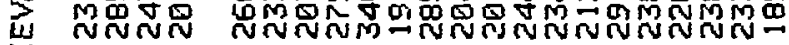

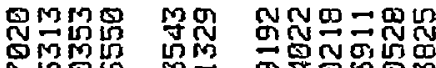

品品

nim

जि कण ज尚m

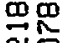

त禺

Q

品等罸变 onm $w$

疋

$\mapsto \mapsto \mapsto ロ$
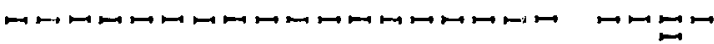

$\rightarrow$ N

No

NMNON

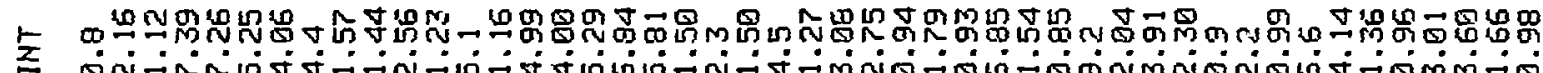

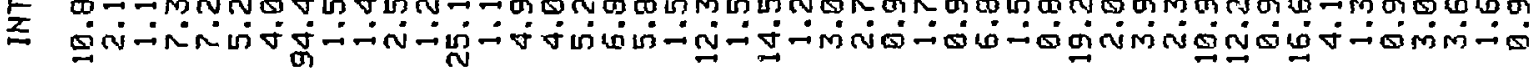

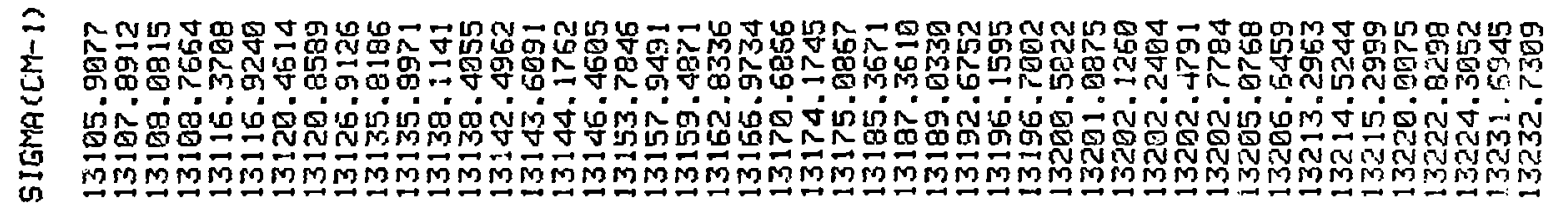

6

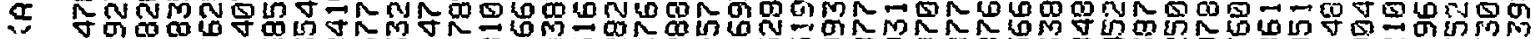

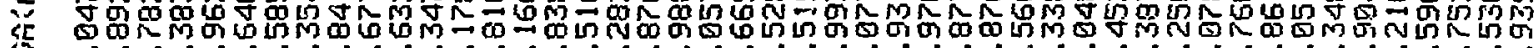
कि NलMNA- -

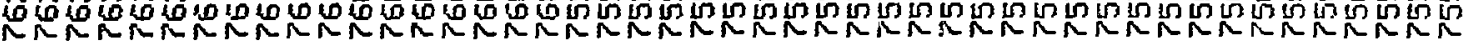



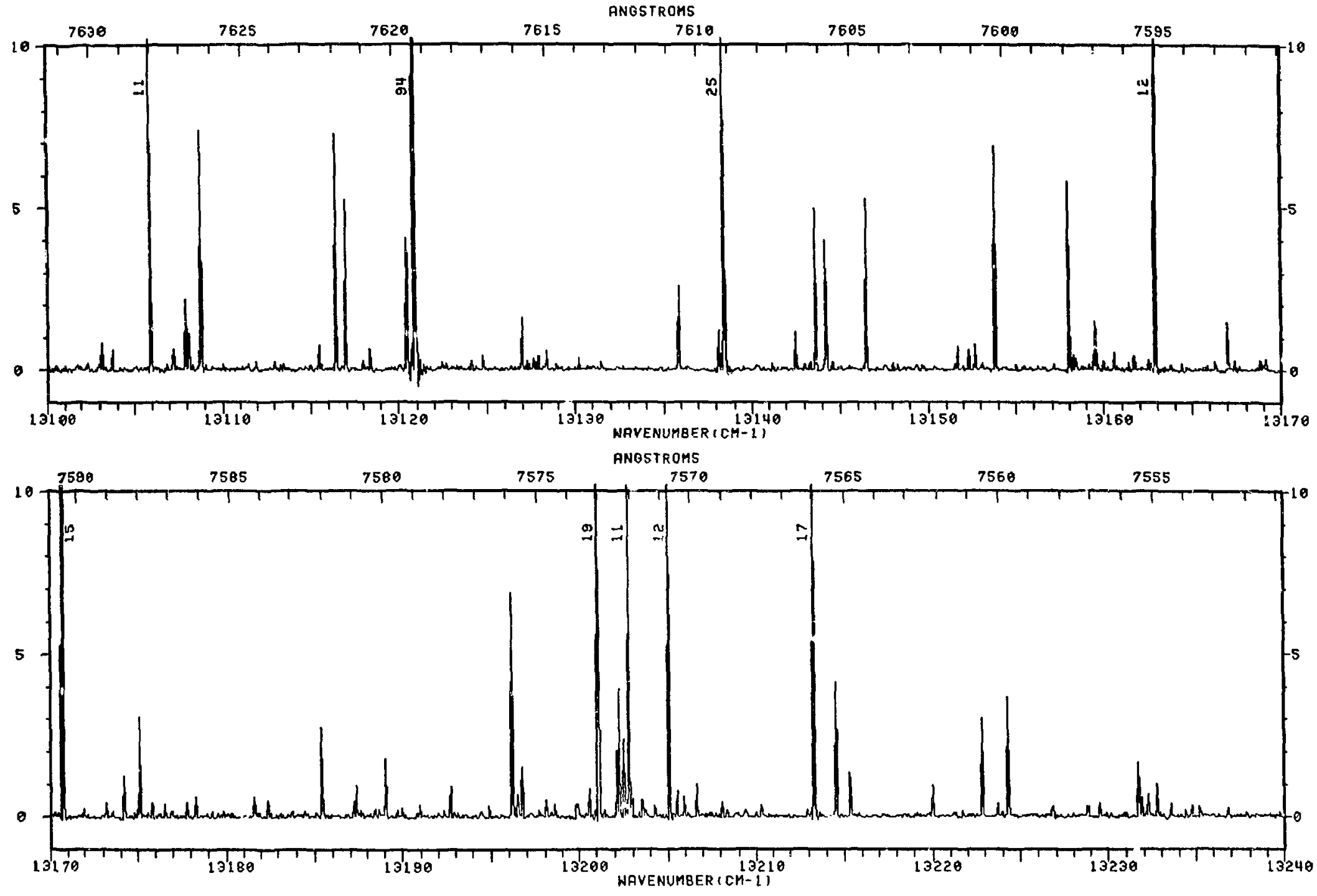

ค
r ton menseng
MUナmg

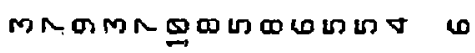
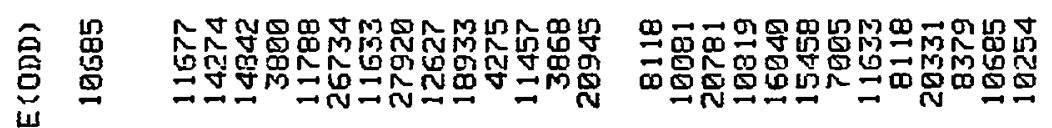

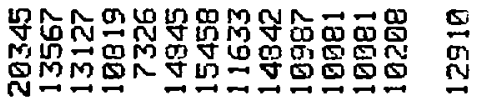

$\stackrel{\ddot{\sim} \infty}{\sim}$

$\cos$ menus

m

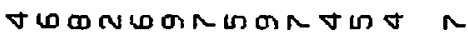

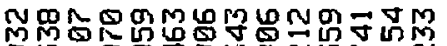

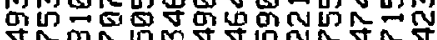

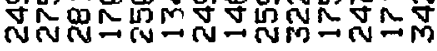

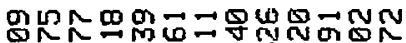

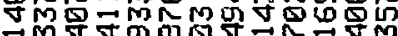

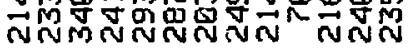

s.

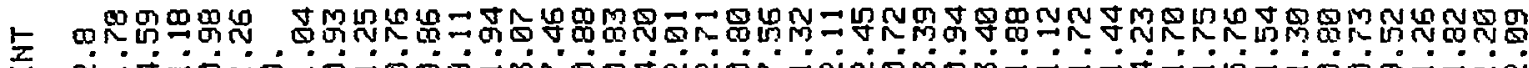

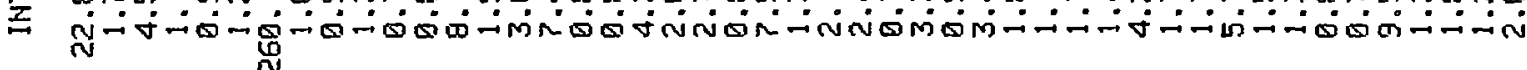

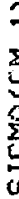

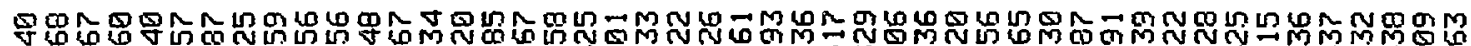

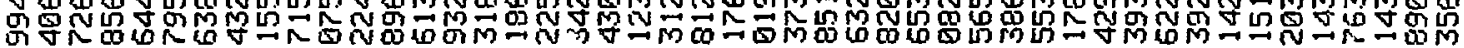
- -

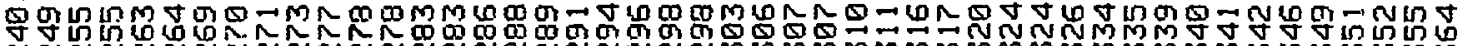

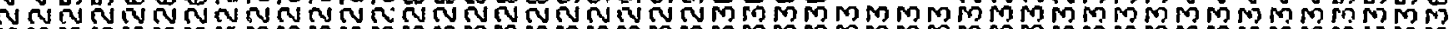

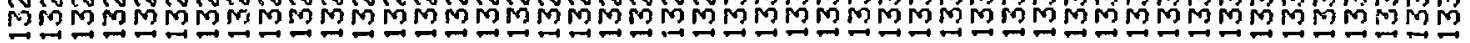

MNON⿴囗十丁 SN⿴囗十心 구유.

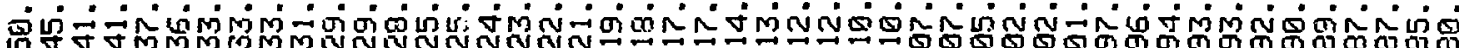

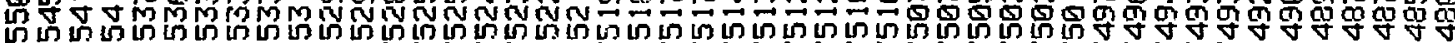

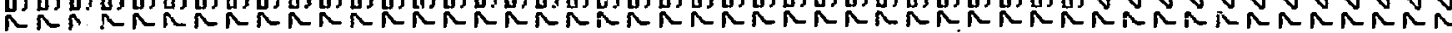



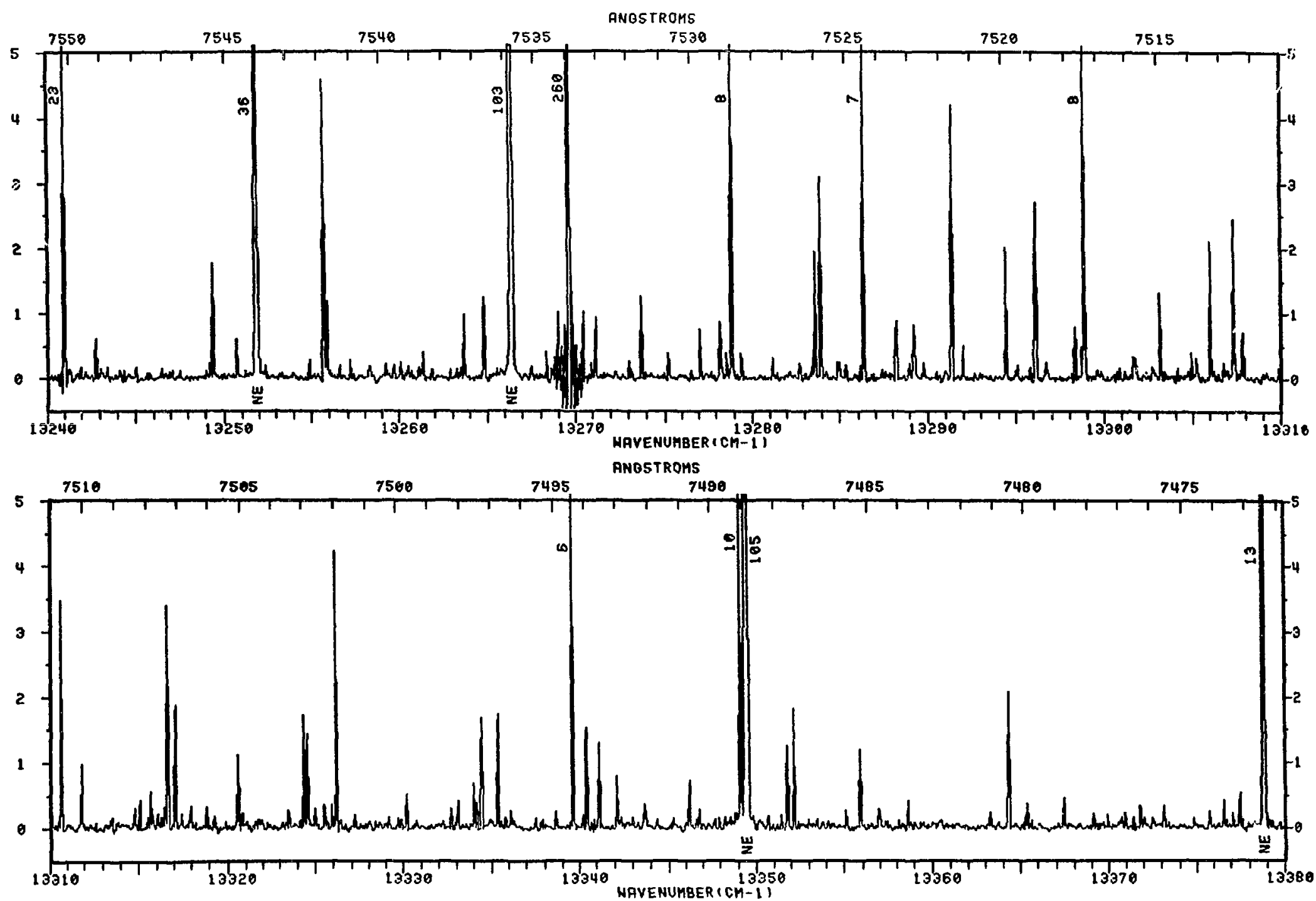


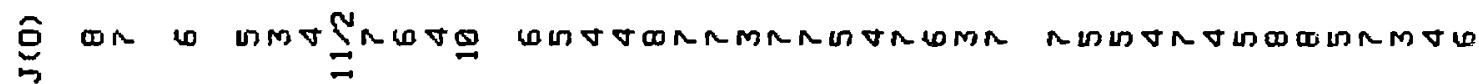

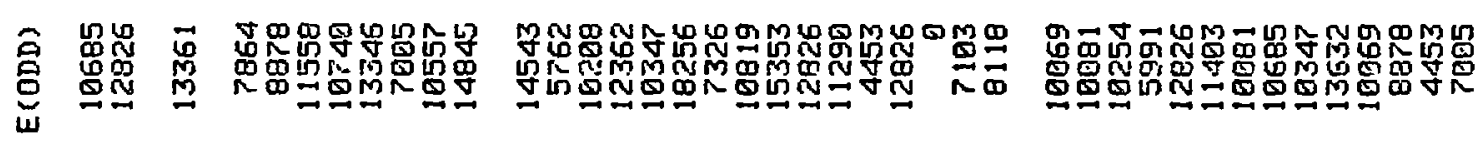

कु

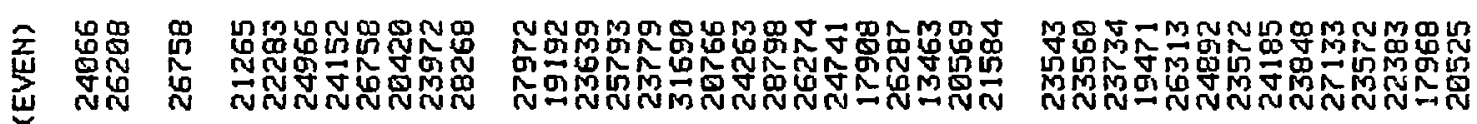

总 $-\cdots+\cdots$

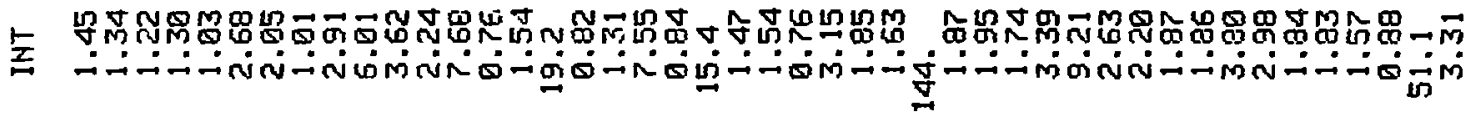
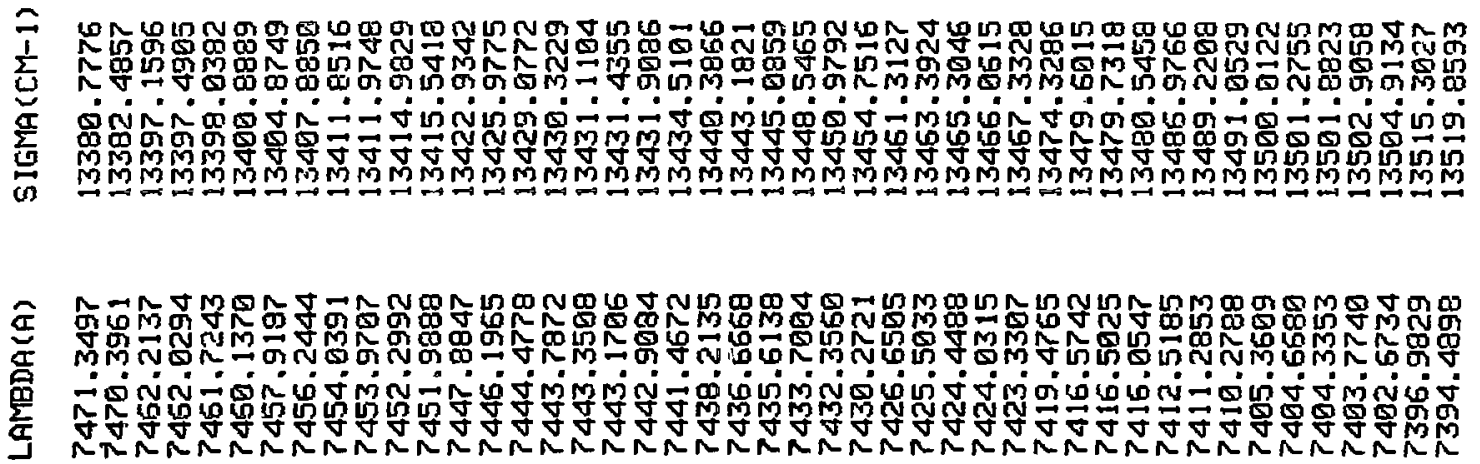

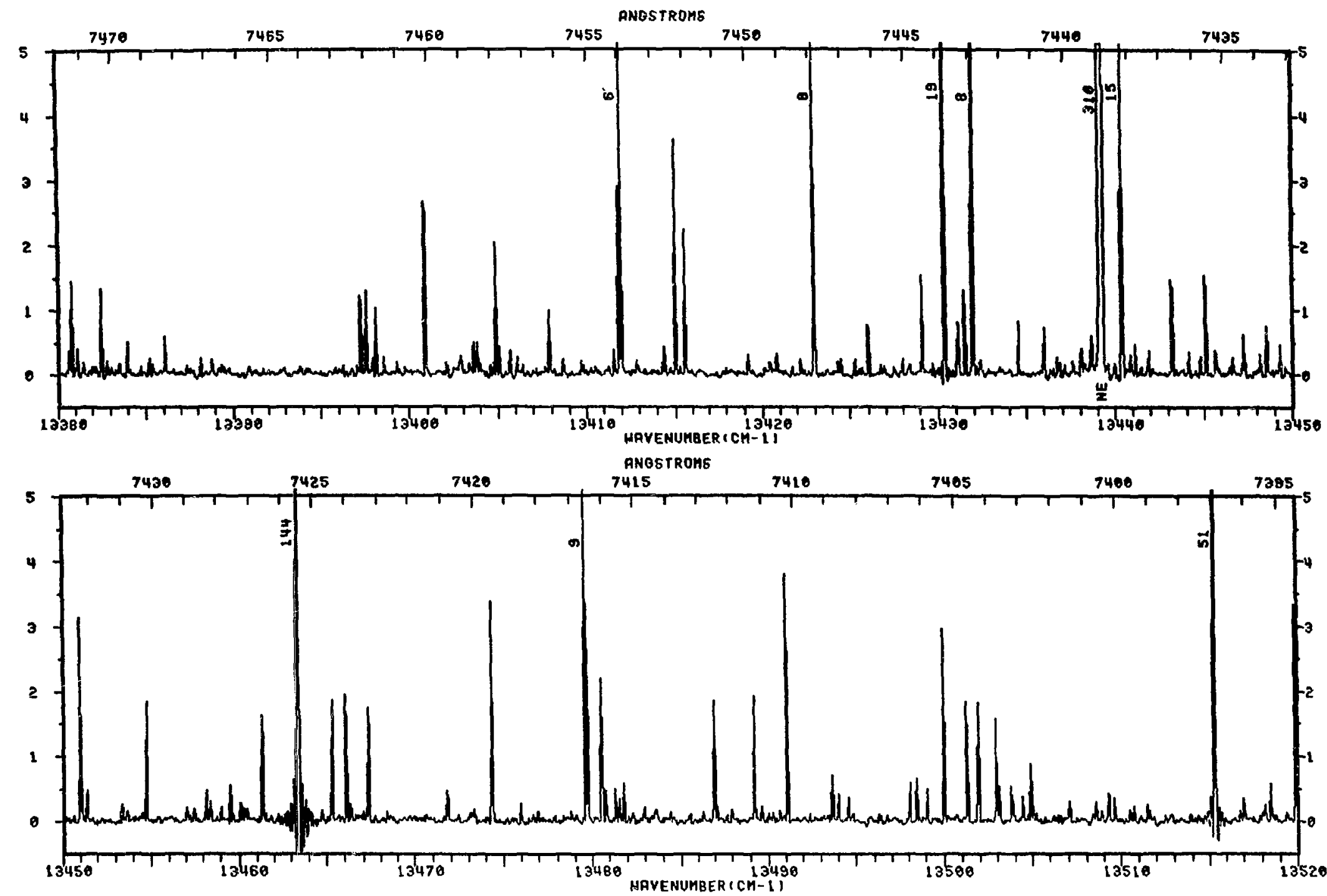


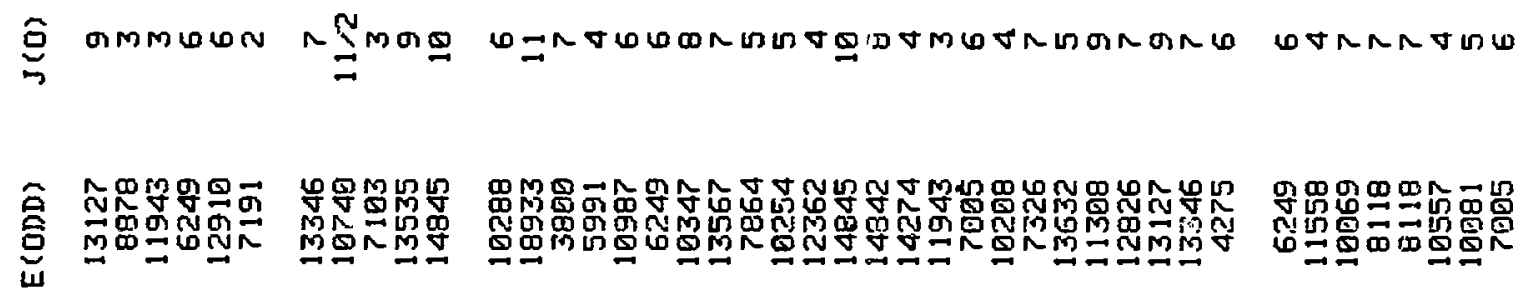

过

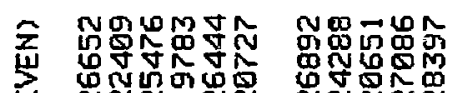

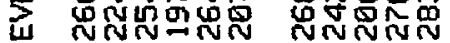

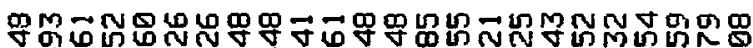

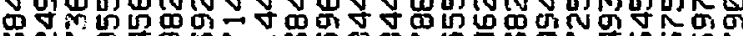

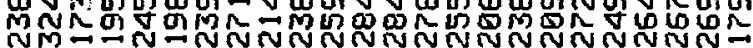

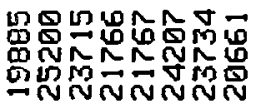

亮 ロロロロロロ ロロッロー

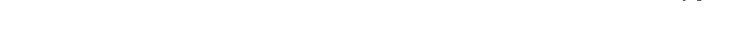

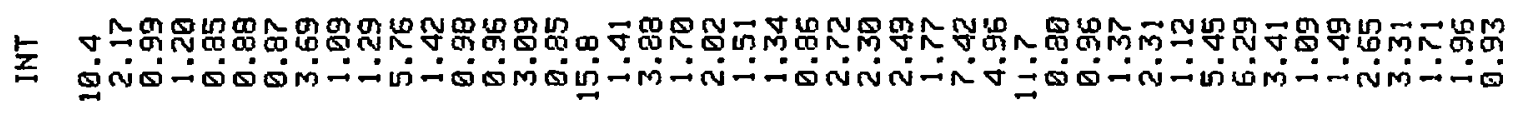
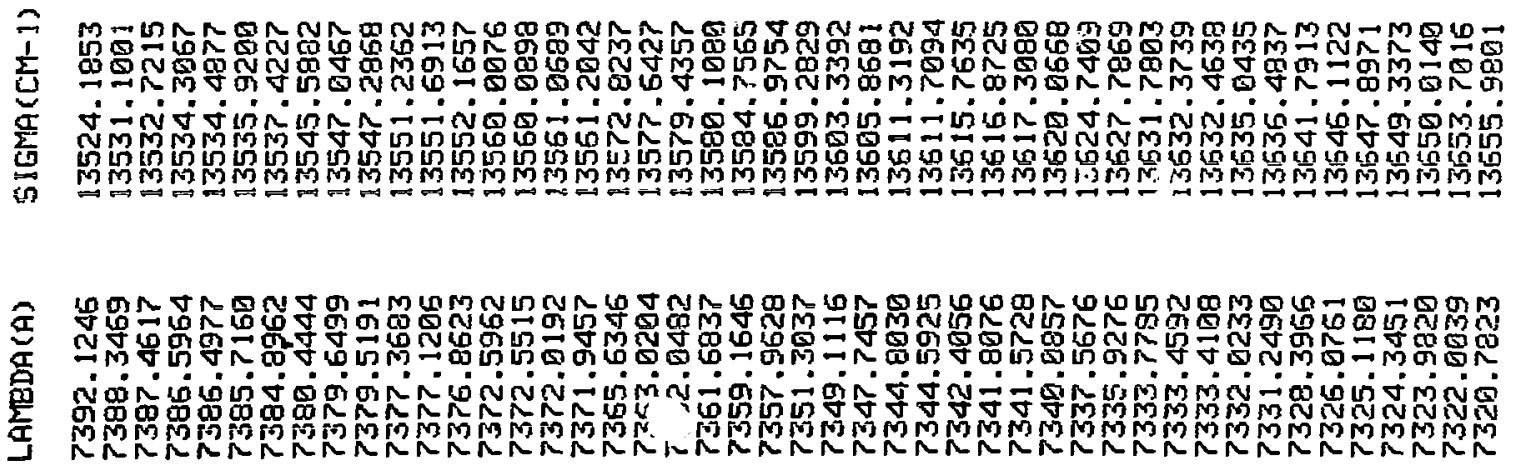

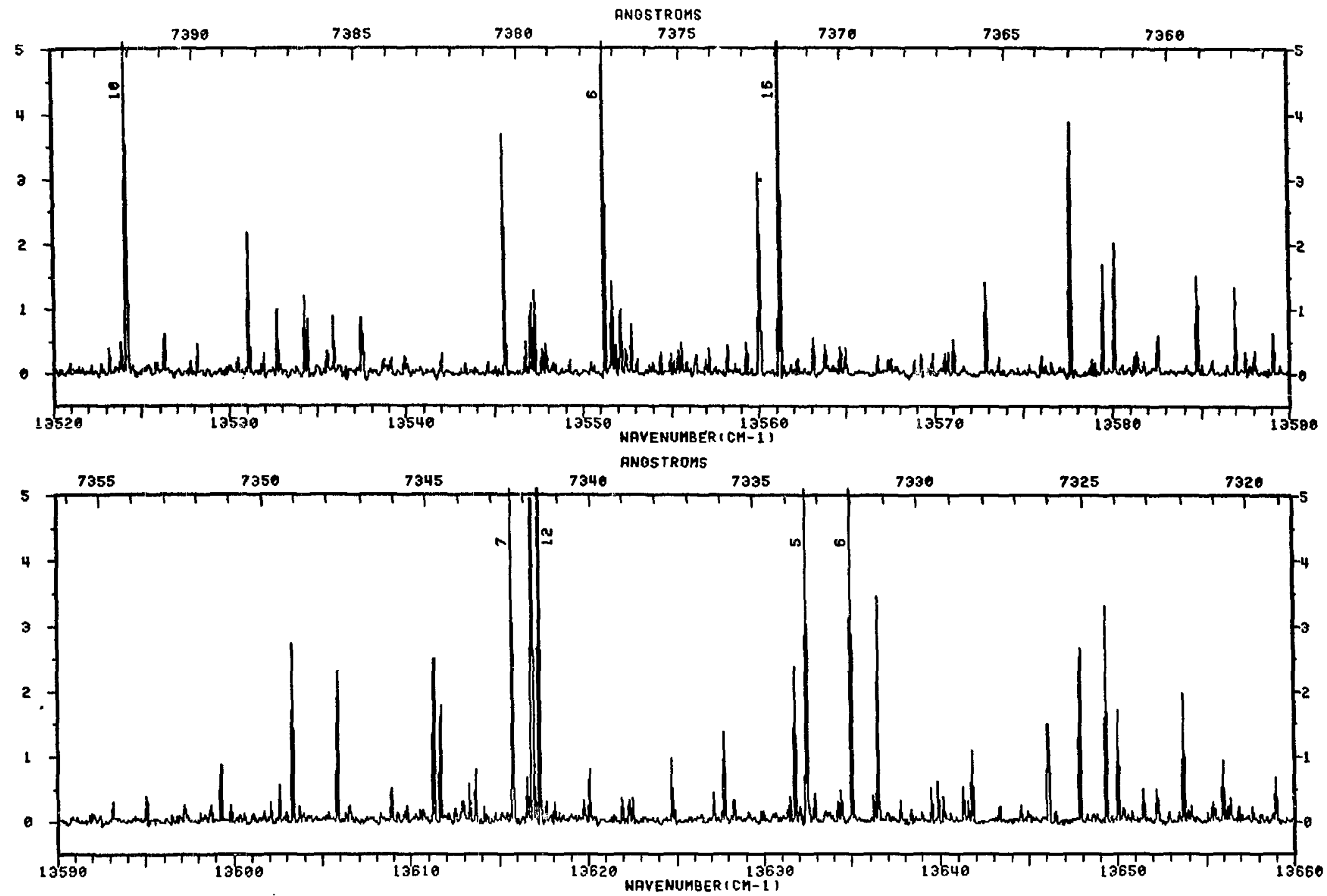


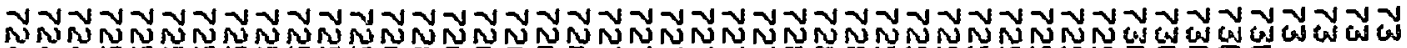

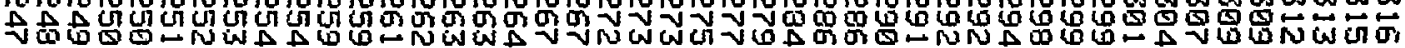

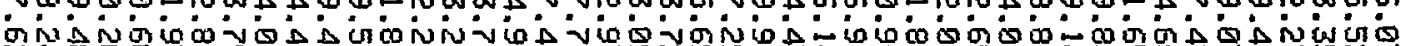

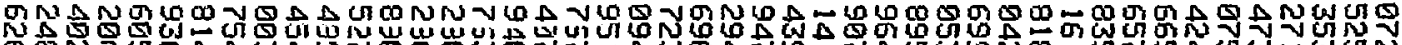

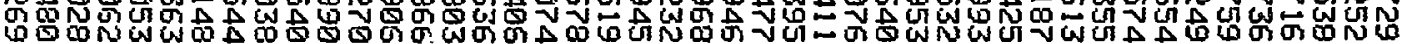

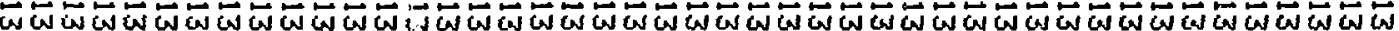

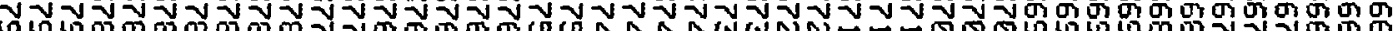

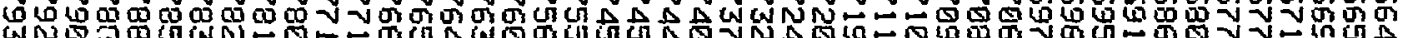

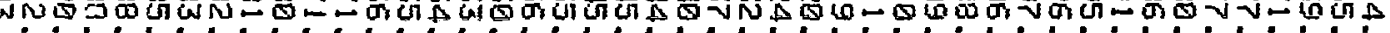
og

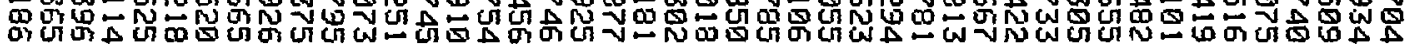

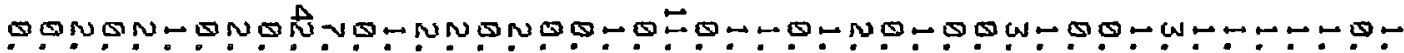

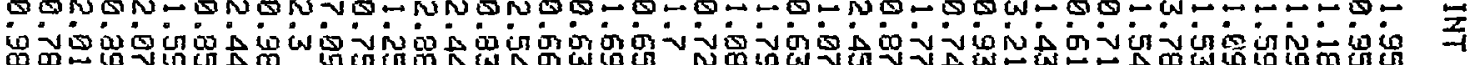

NANNNNNNNRNMNNNNNGNNNNN: T

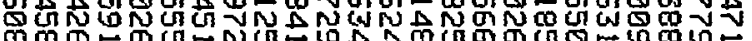

NNNANMNAGNNMN

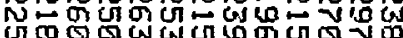

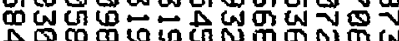



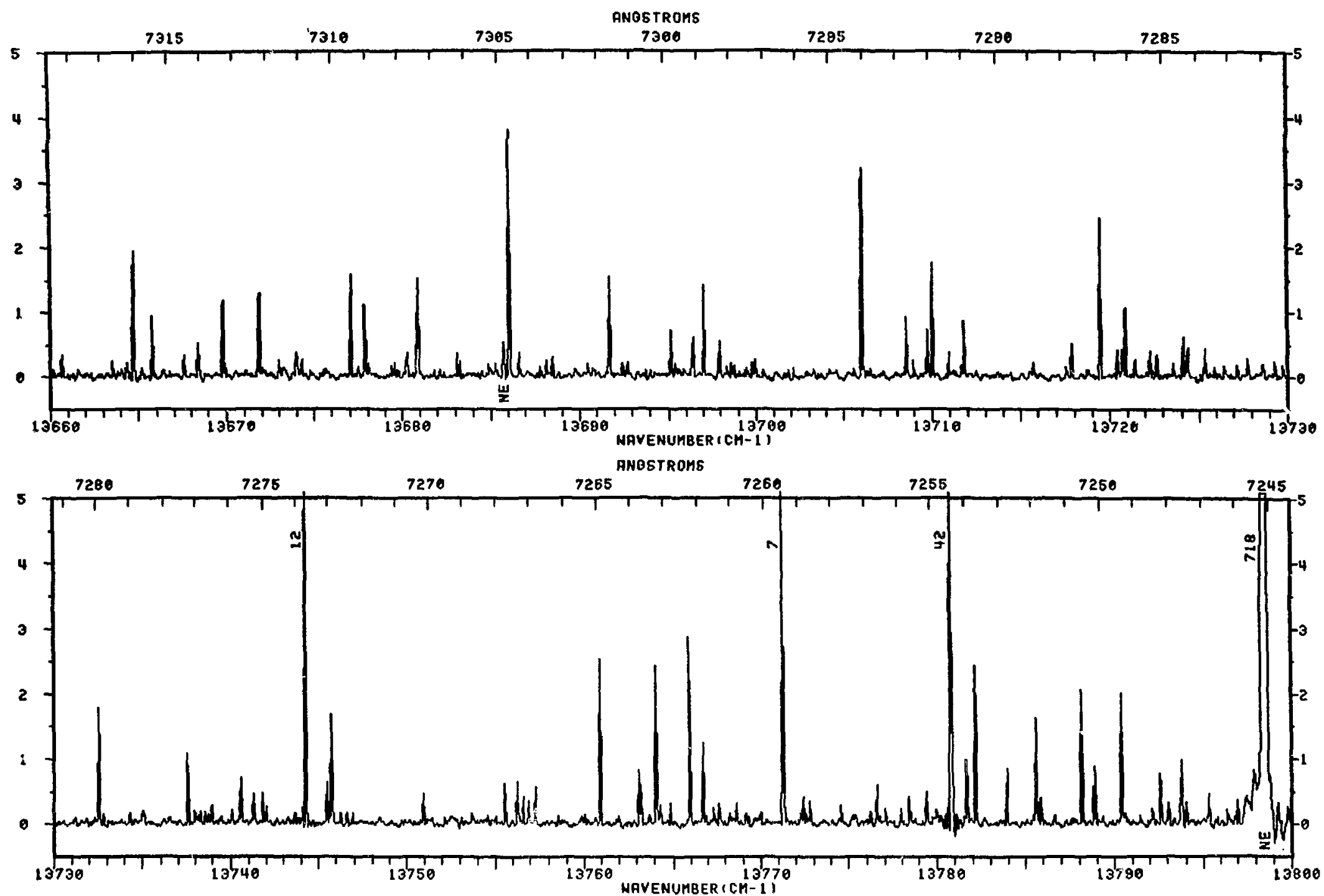


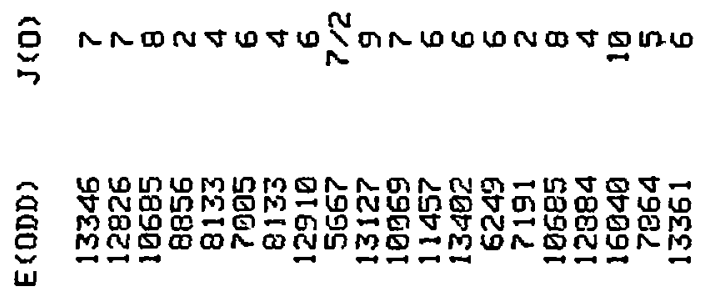

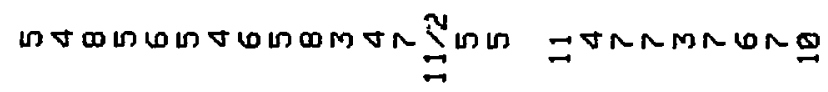

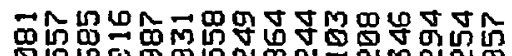

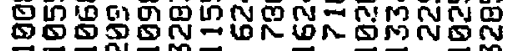

Mำ

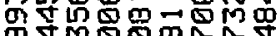

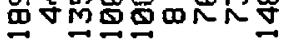

包

峁

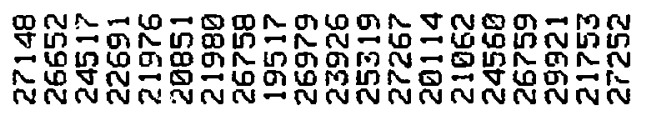

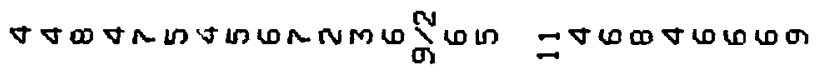

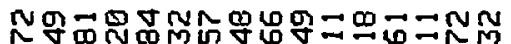
O

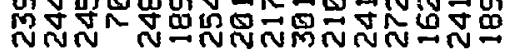

9m9ñ

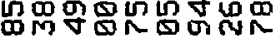

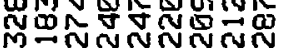

금

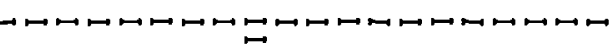

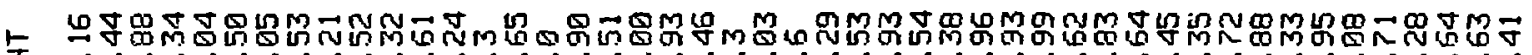

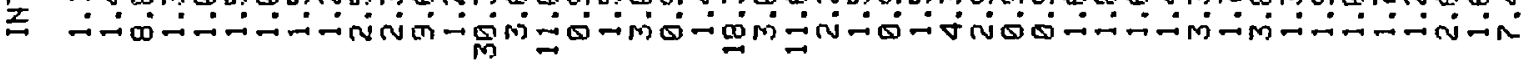

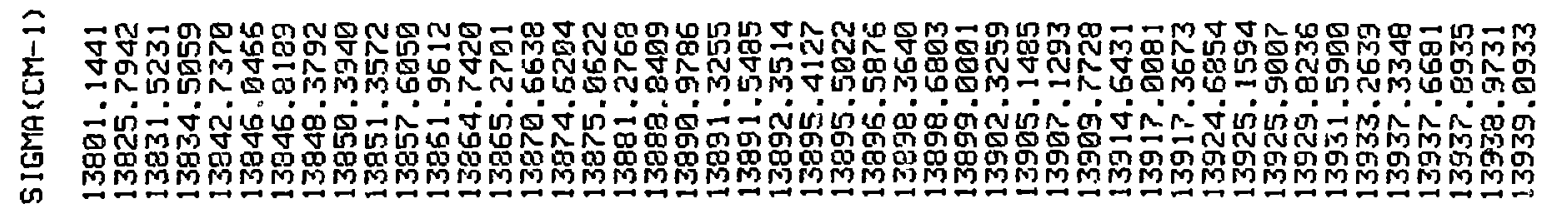

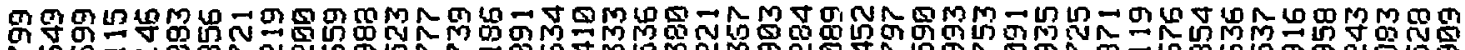

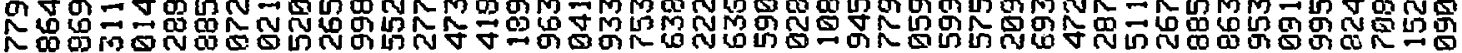

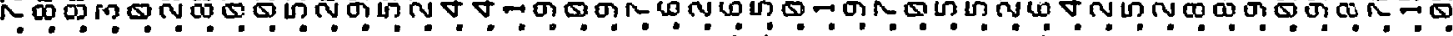

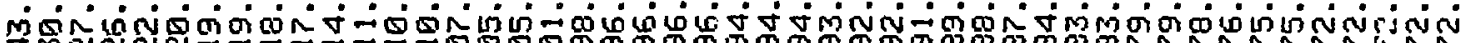

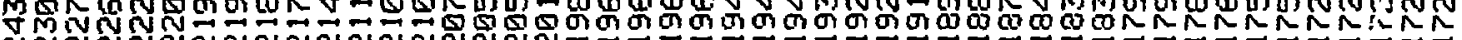

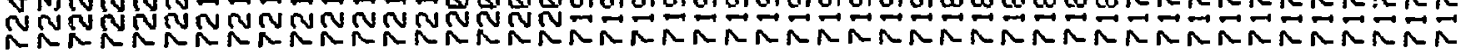




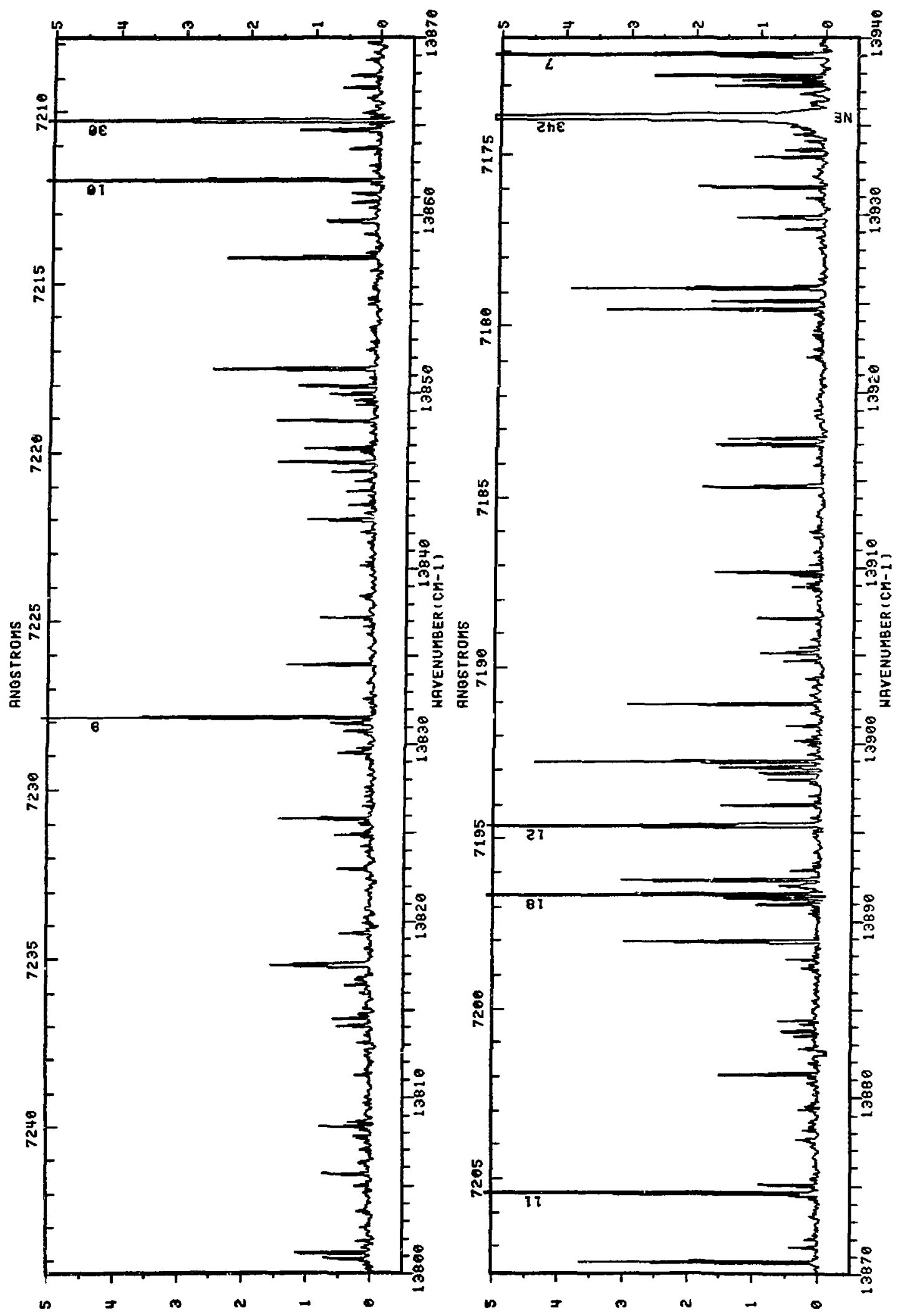




\begin{tabular}{|c|c|c|c|c|}
\hline$\widehat{\varrho}$ & 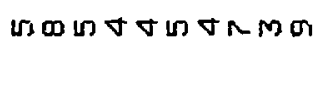 & ヘのスமの & ル & 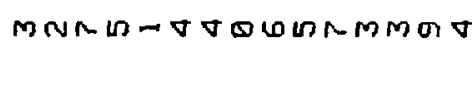 \\
\hline 总 & 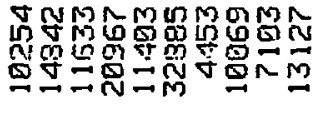 & 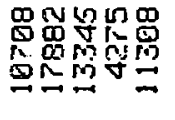 & 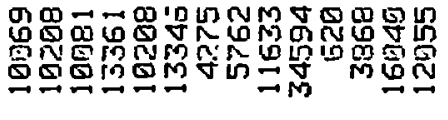 & 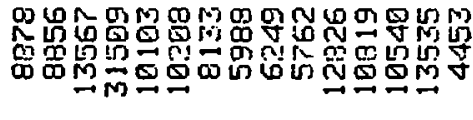 \\
\hline
\end{tabular}

岂

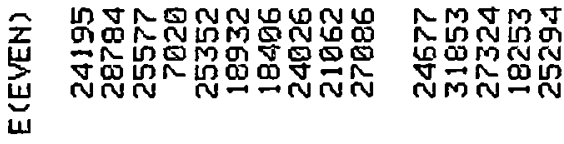

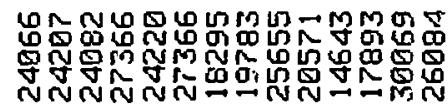

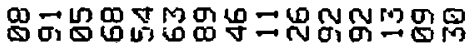

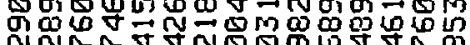

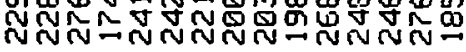

금

ロロロッッッロロッー

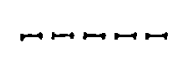

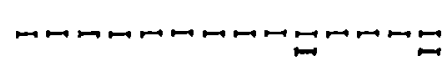

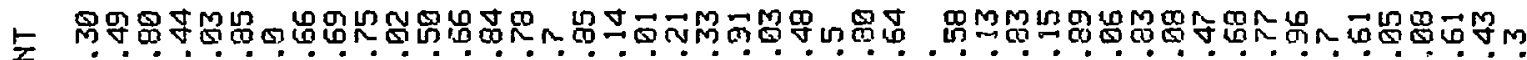

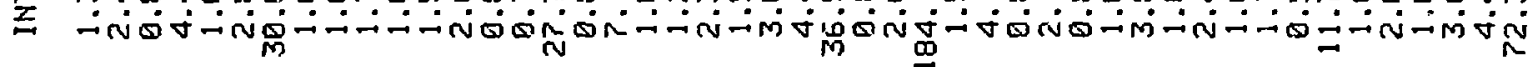

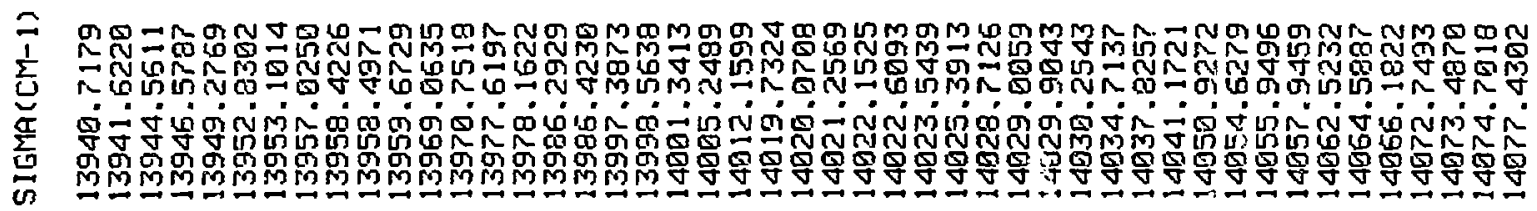

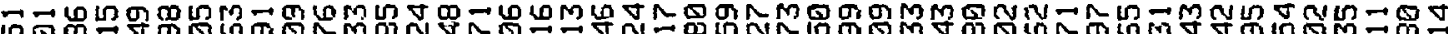

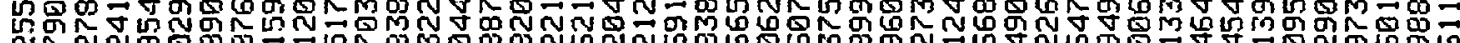

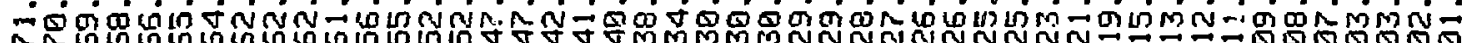

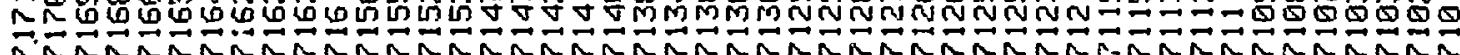




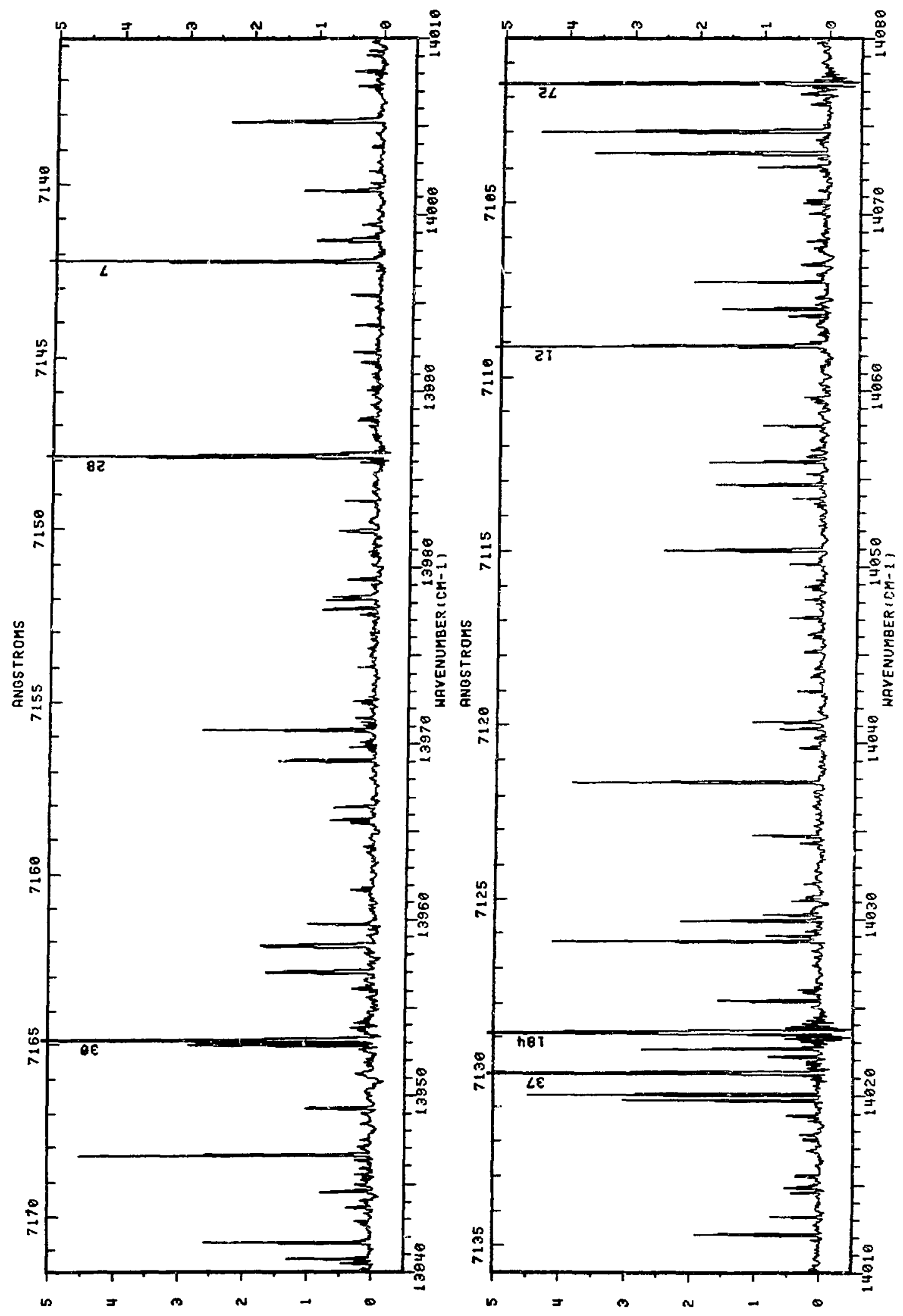




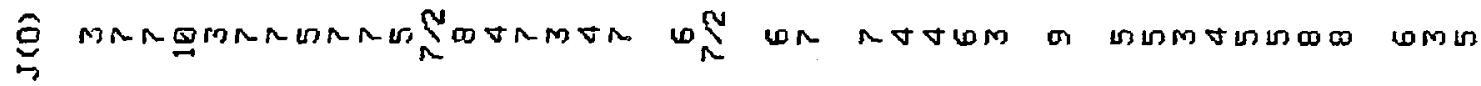

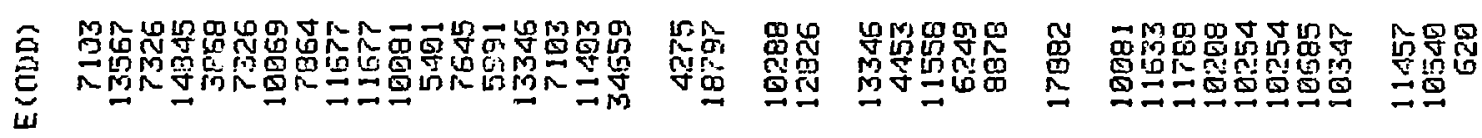

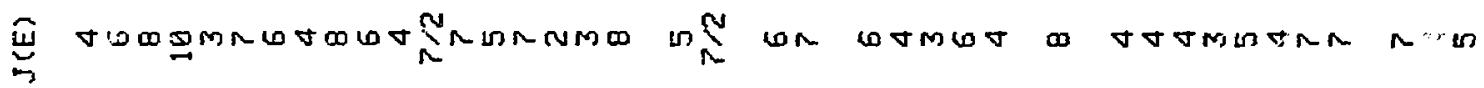

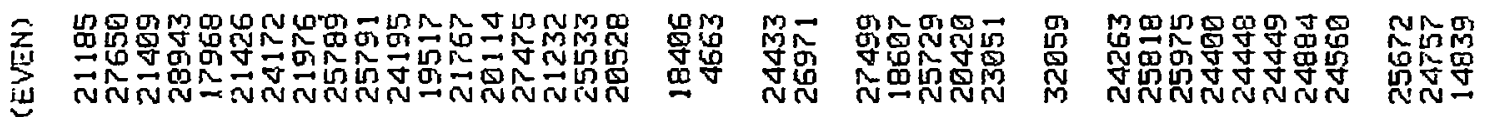

吾
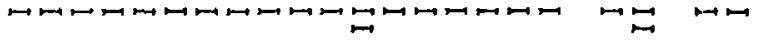

$\rightarrow \rightarrow n \rightarrow m$

a mamamat and

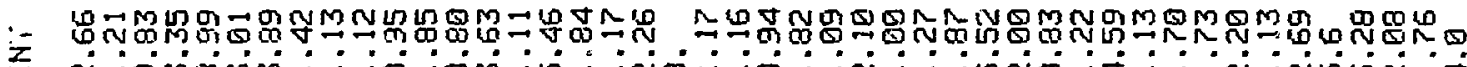
方

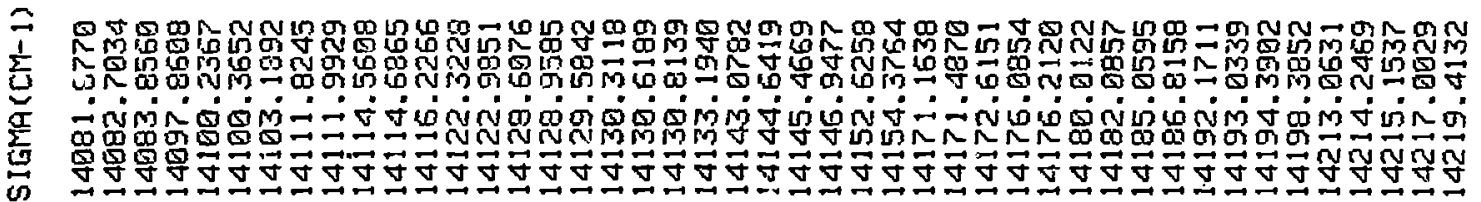

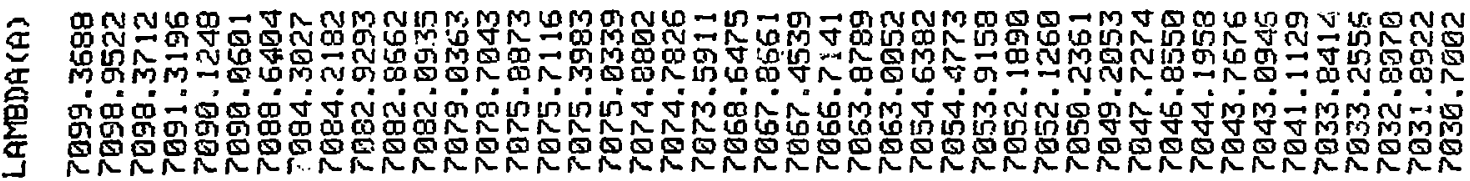





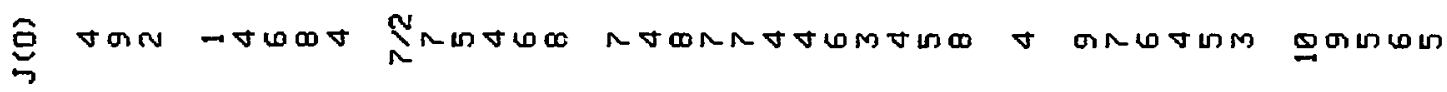

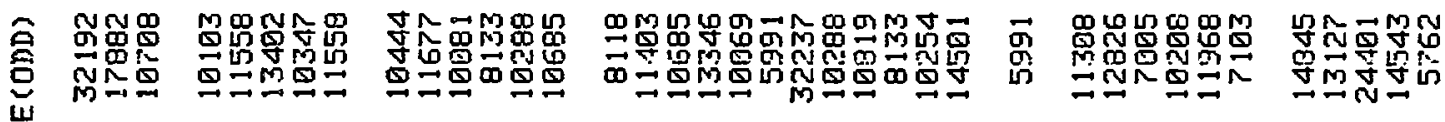

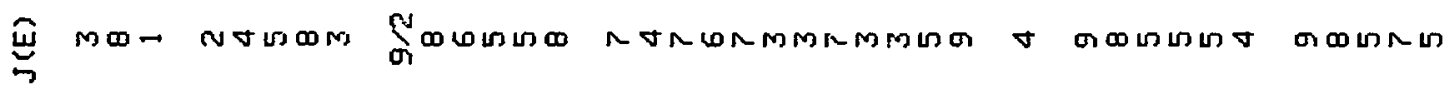
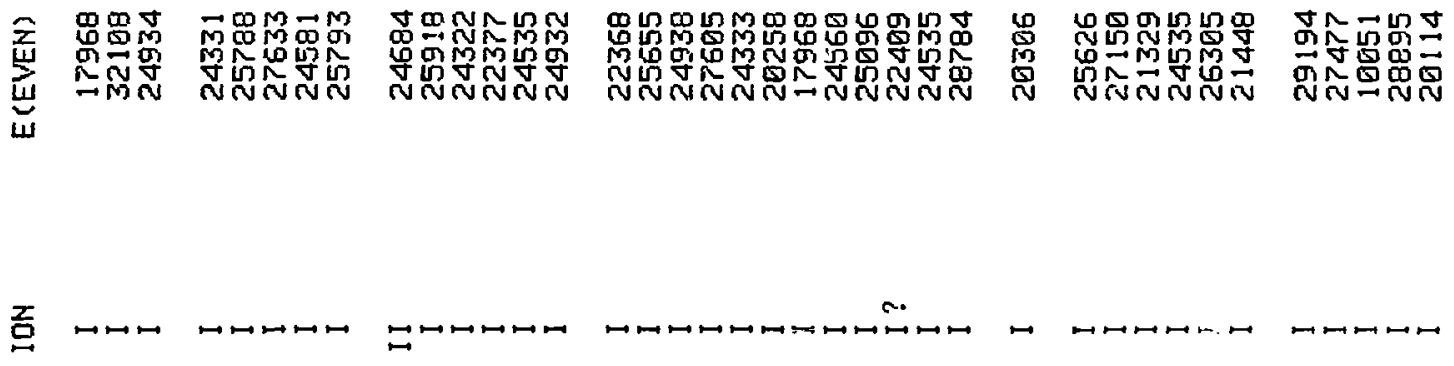

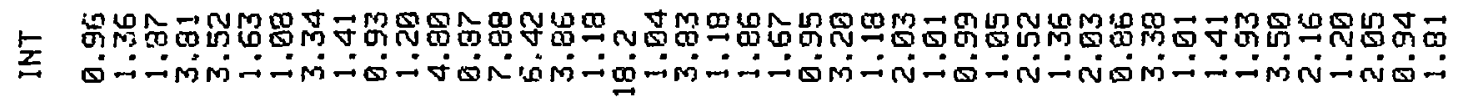
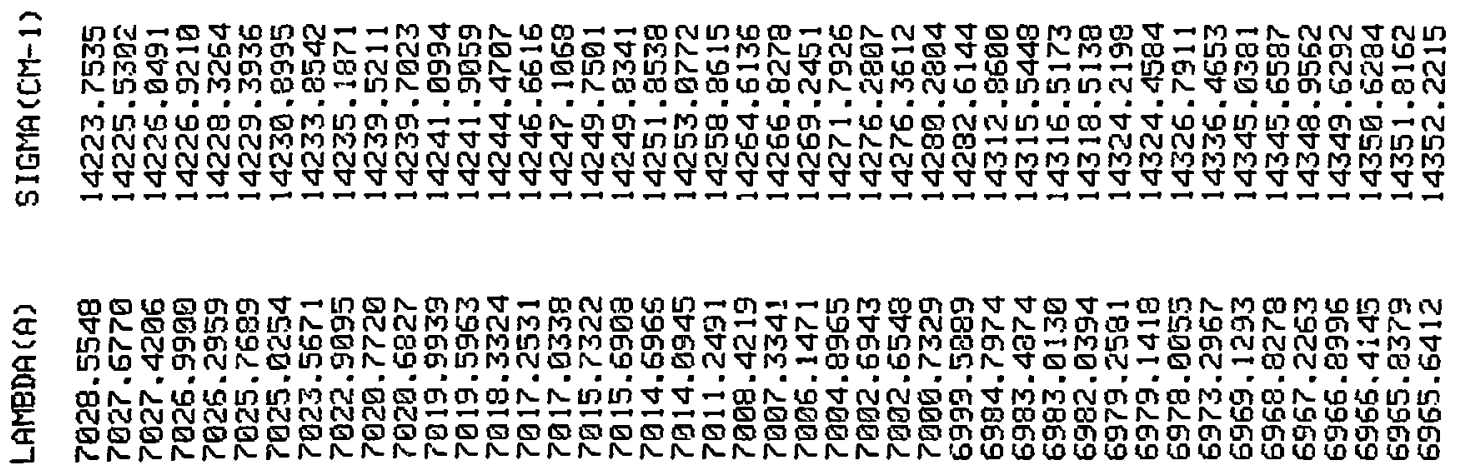

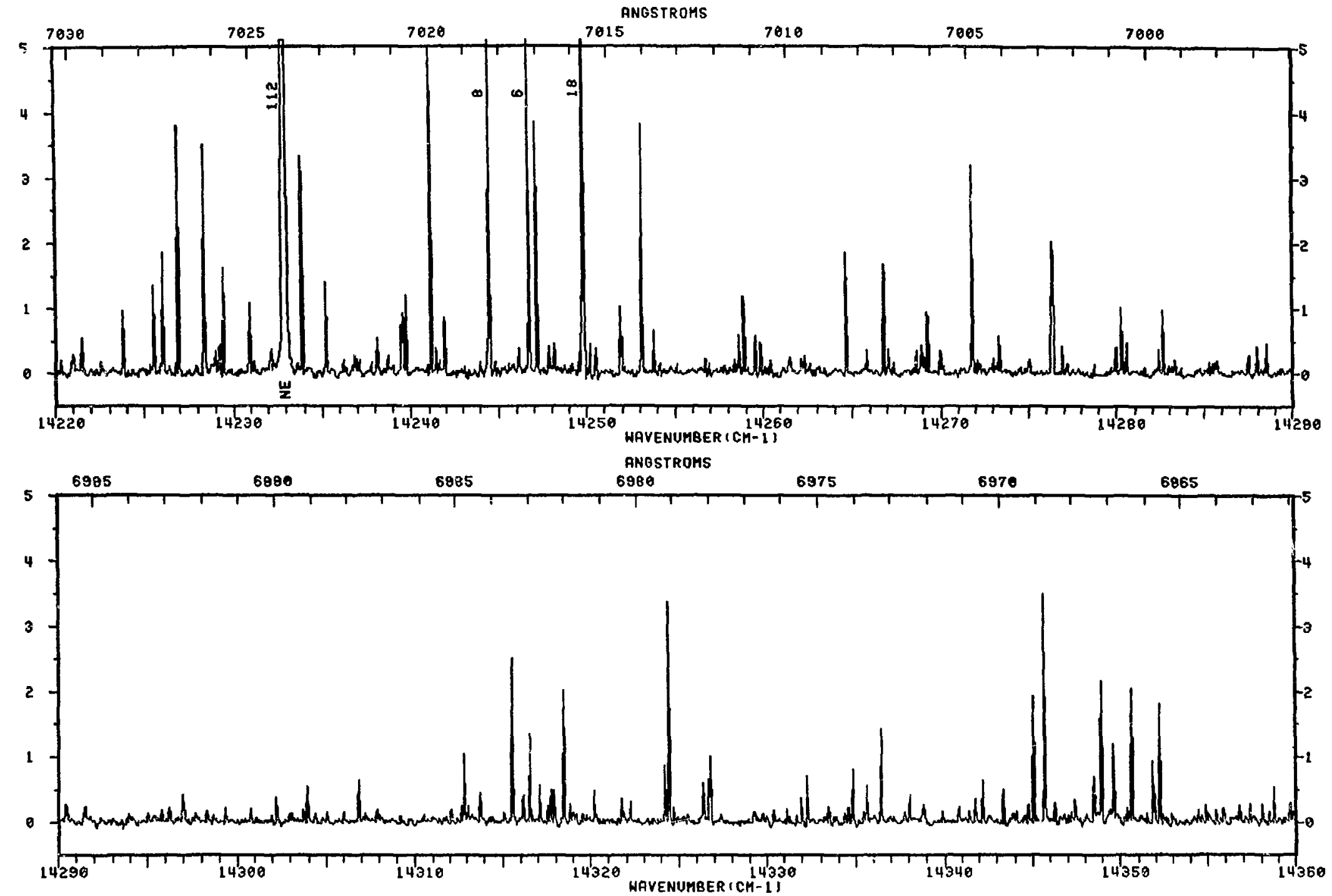
OC $\quad$ v

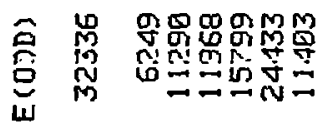

एँ

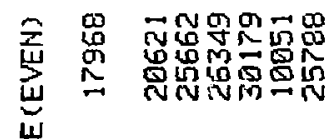

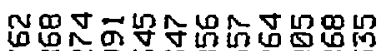

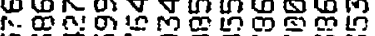

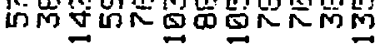

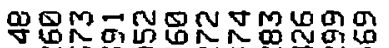

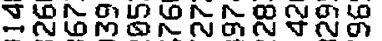

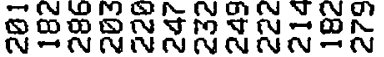

ヘேマーMn

MMOLMLOVRMOMN

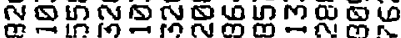

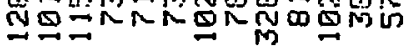

WR TOWRARMCO

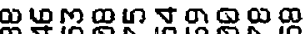

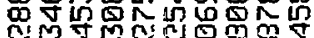

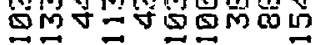

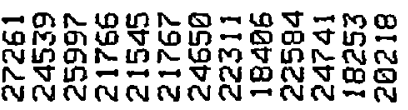

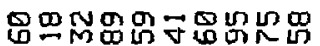

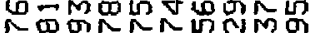

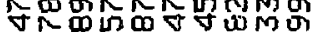

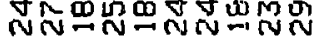

a.

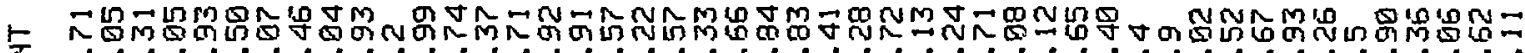

I

7

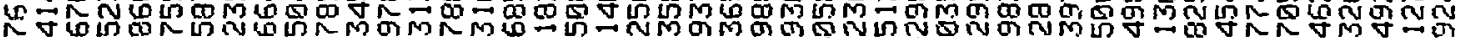

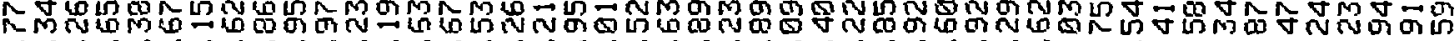
r 40 N

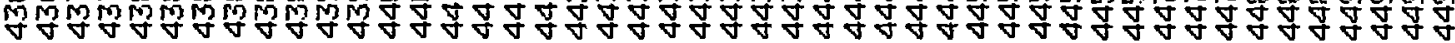

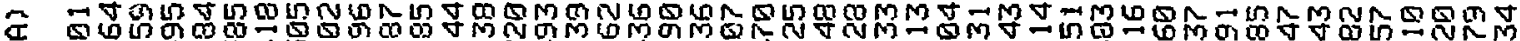

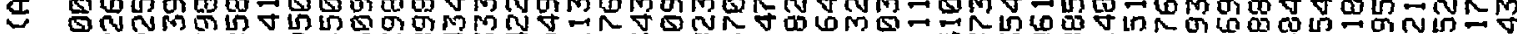

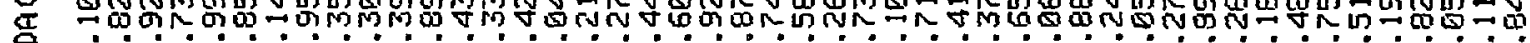

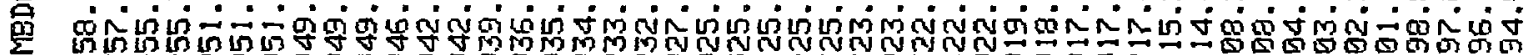

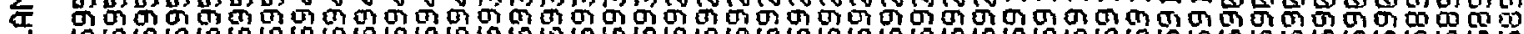
 

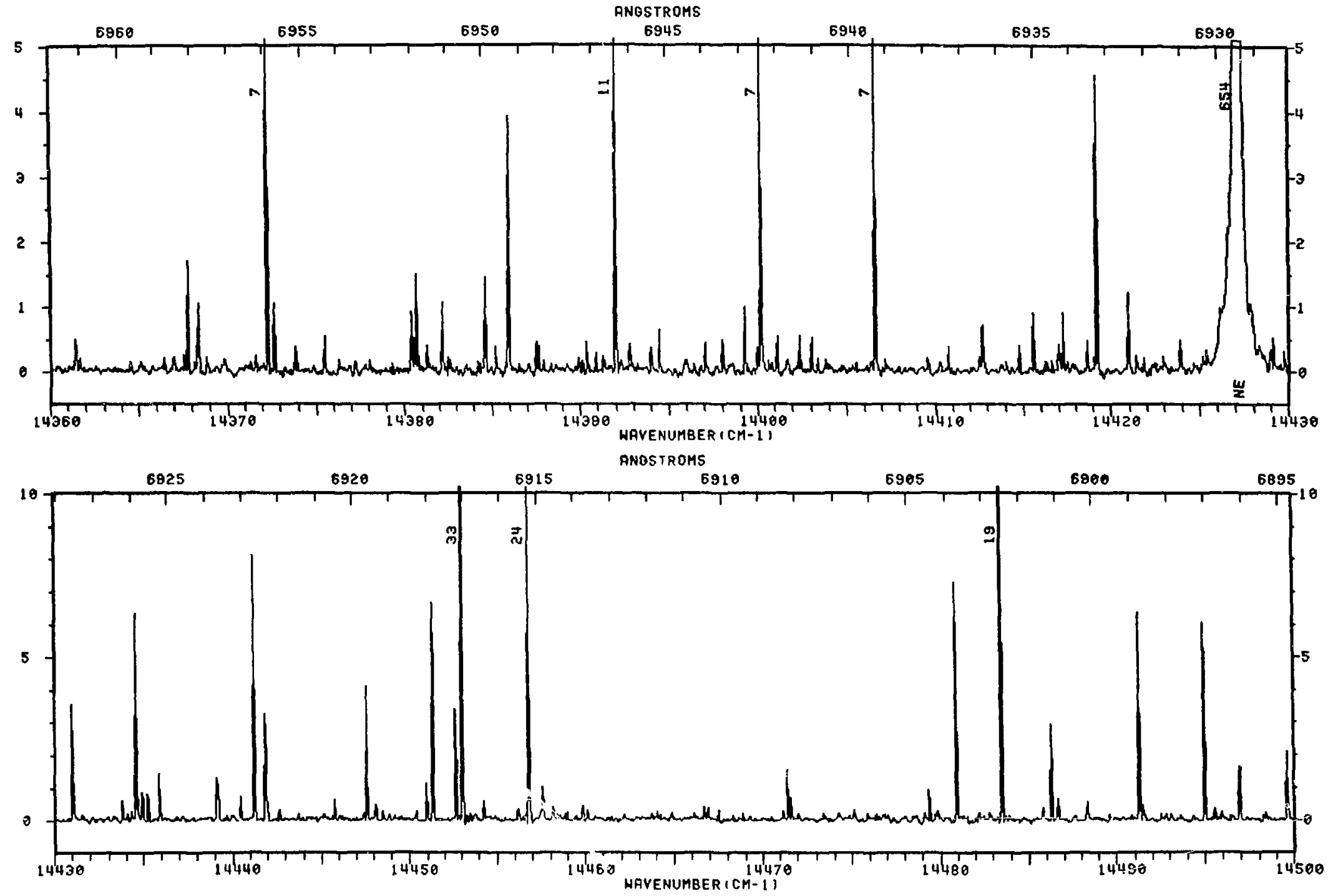


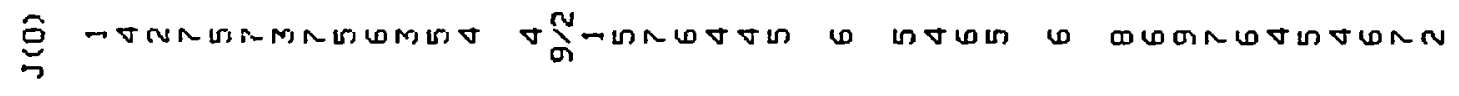

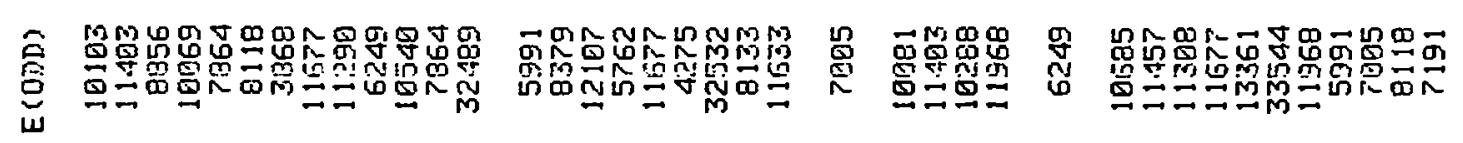

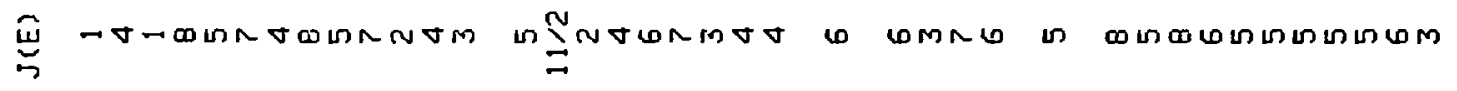

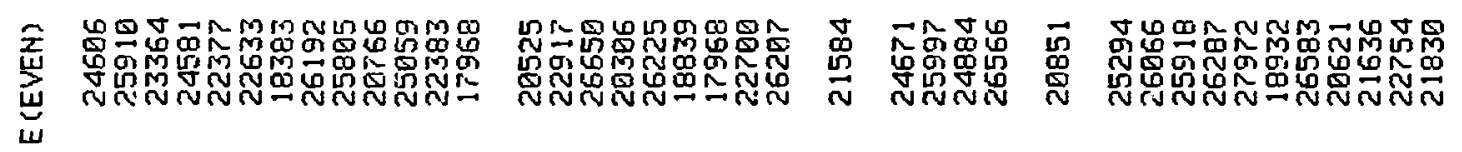

\begin{abstract}
(2)
\end{abstract}

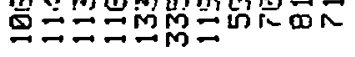

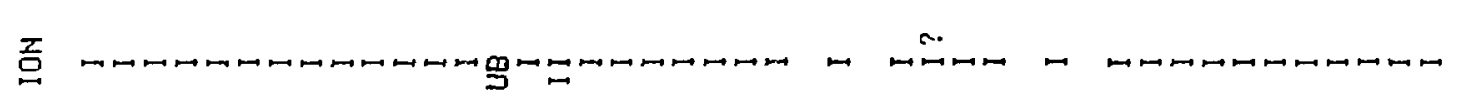

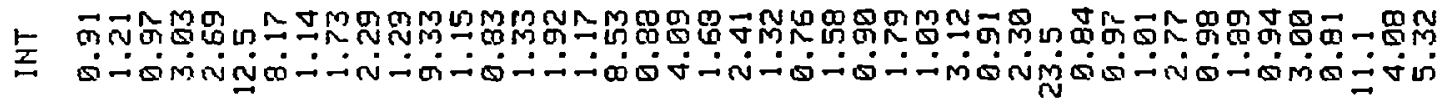

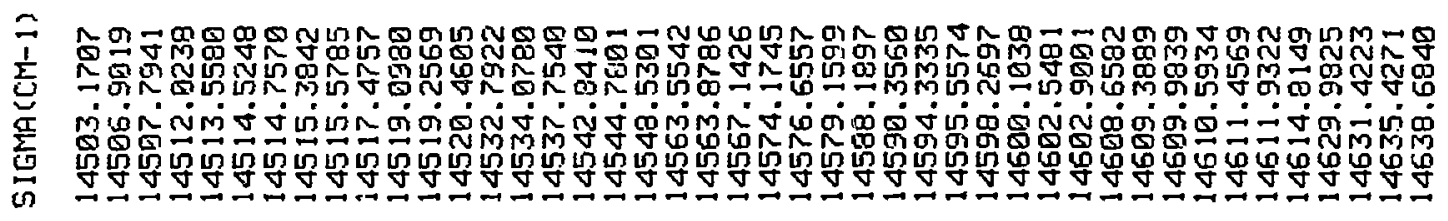

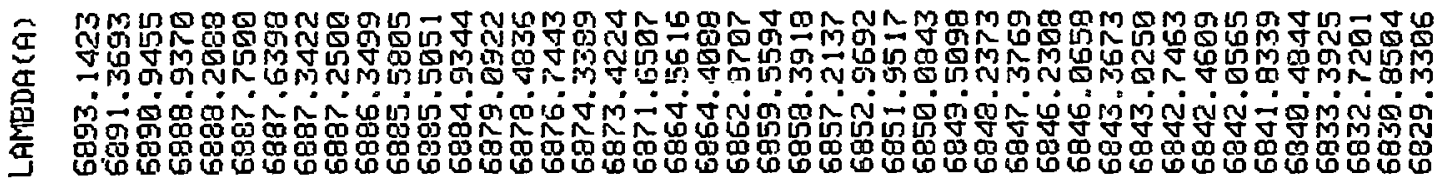



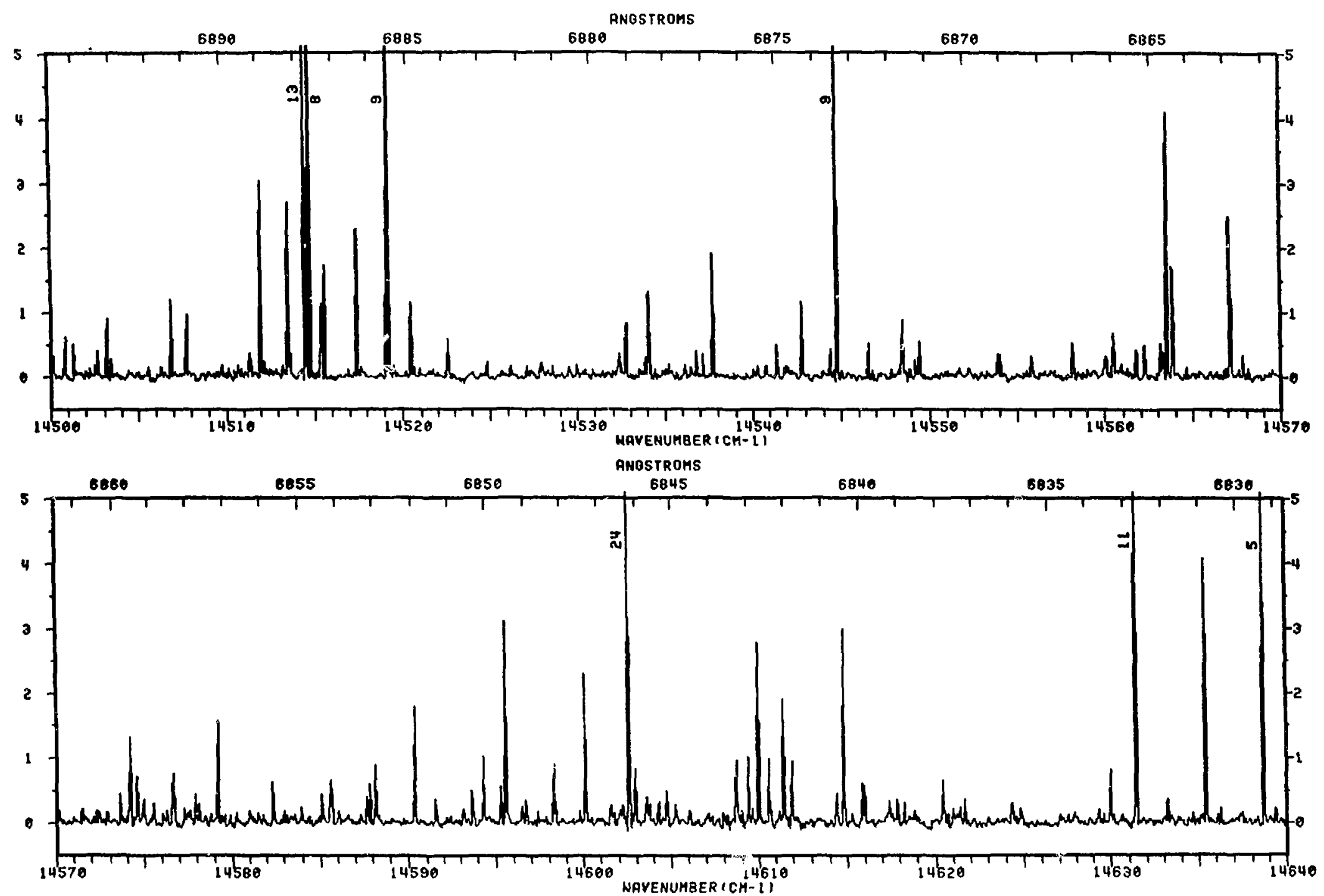
o

合

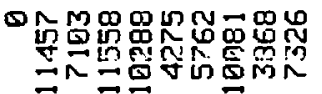

Шิ

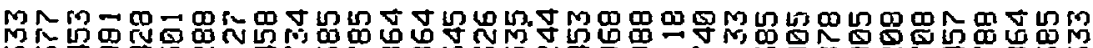

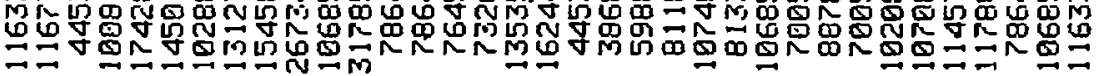

nएव

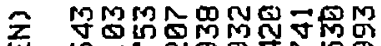

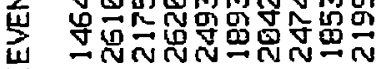

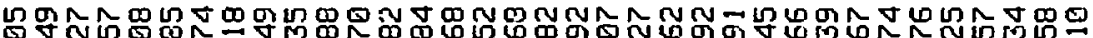

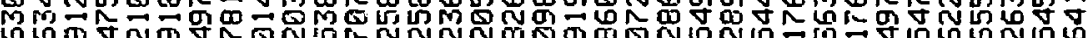

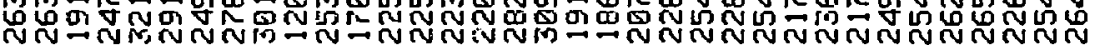

ż口

$\mapsto \mapsto \mapsto \mapsto \mapsto \mapsto \mapsto \mapsto \mapsto$

$-$

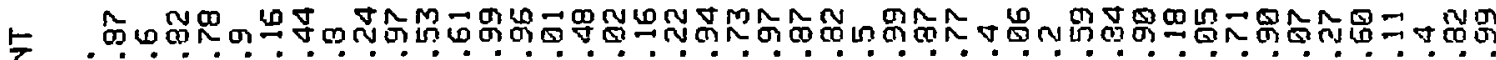

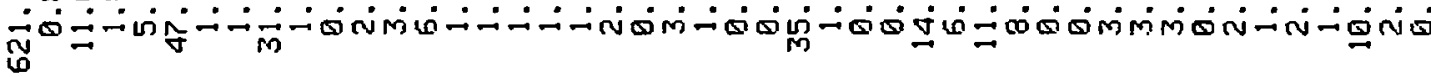

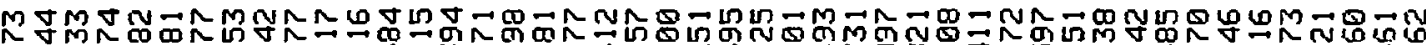

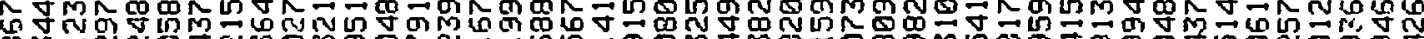

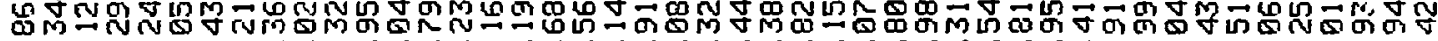
mgign்

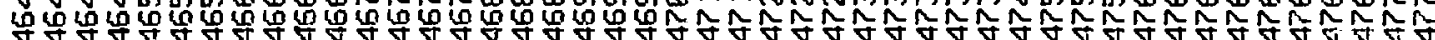

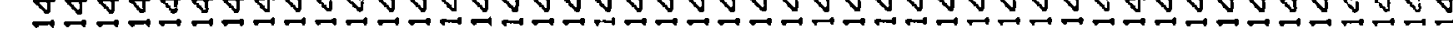

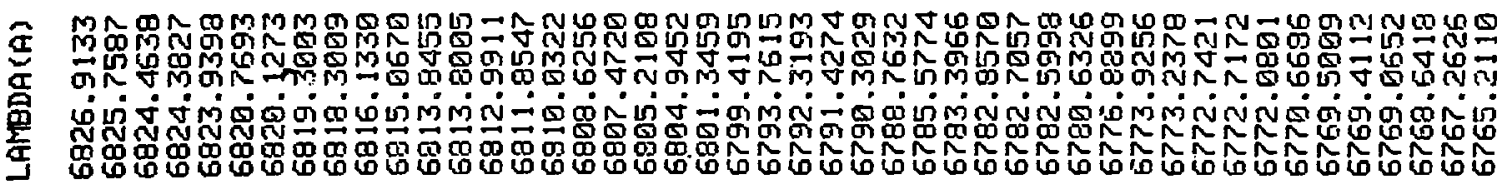



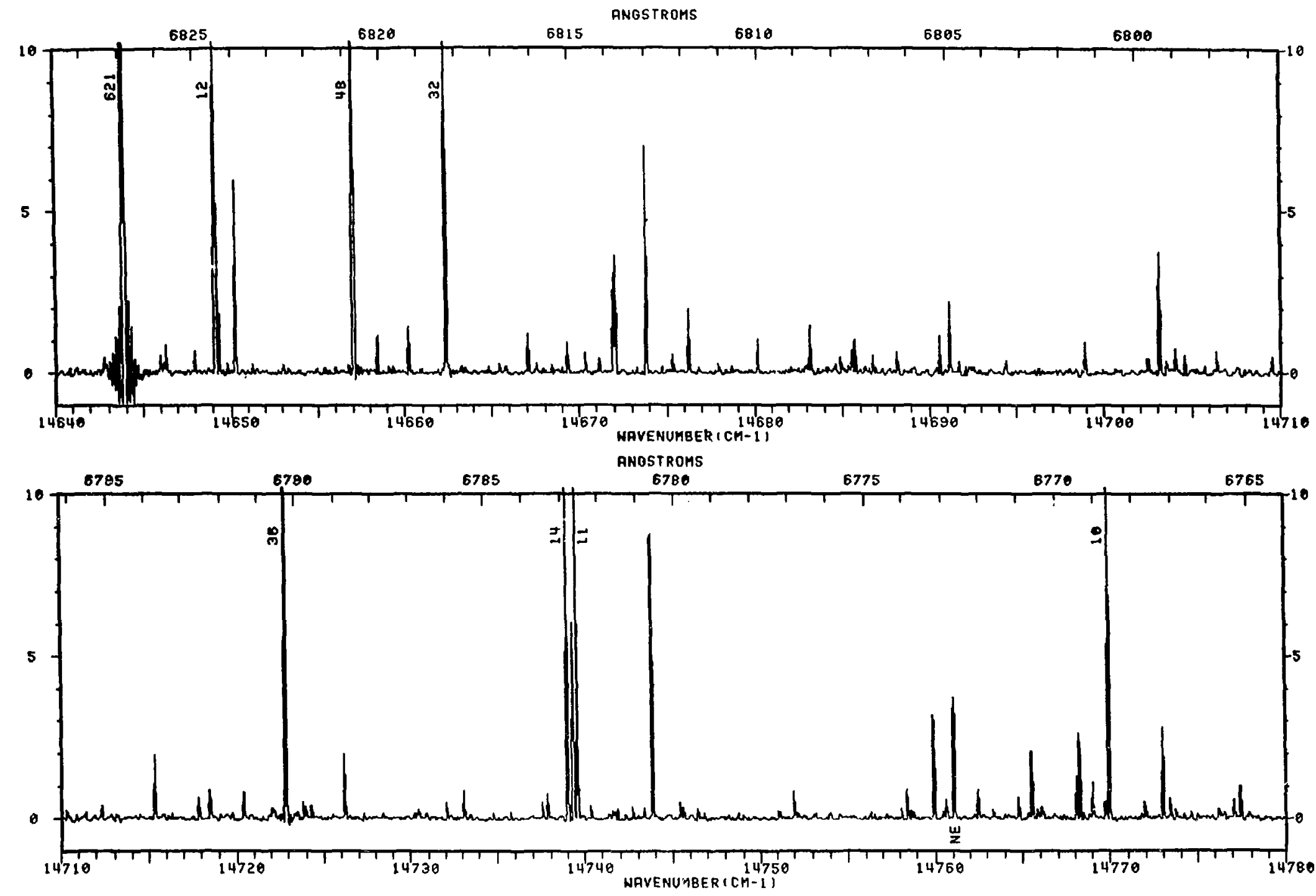


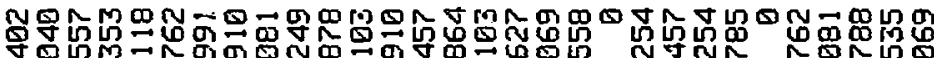

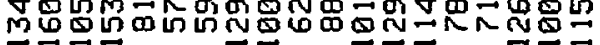

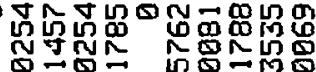

告 N思思思思 怘

员员 M气

(1)

$\rightarrow \rightarrow M \quad 0-0$

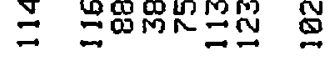

డั

畐

Шुّ
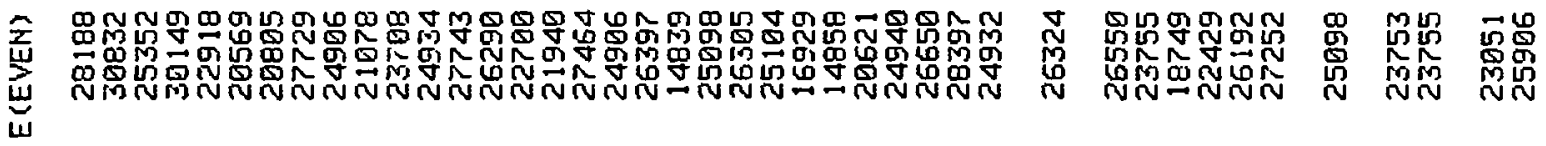

곰

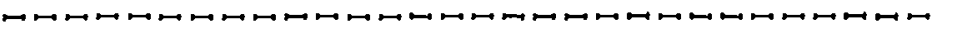

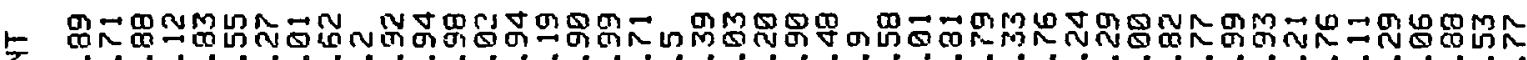

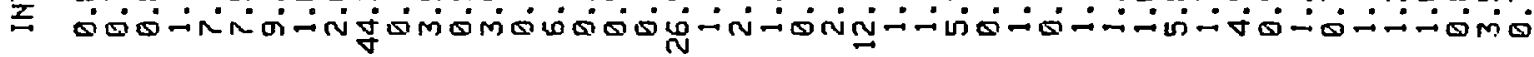

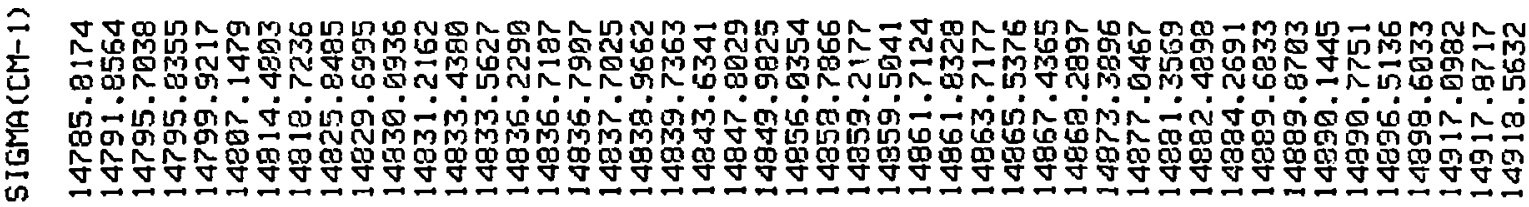

ம=L

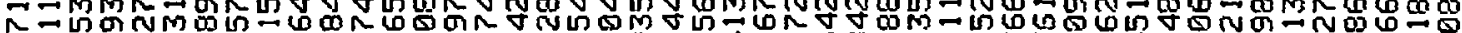
ल

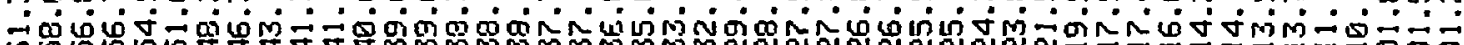

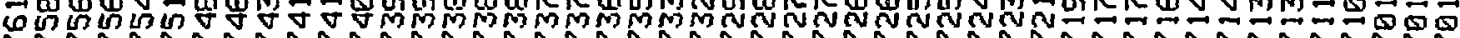
6 

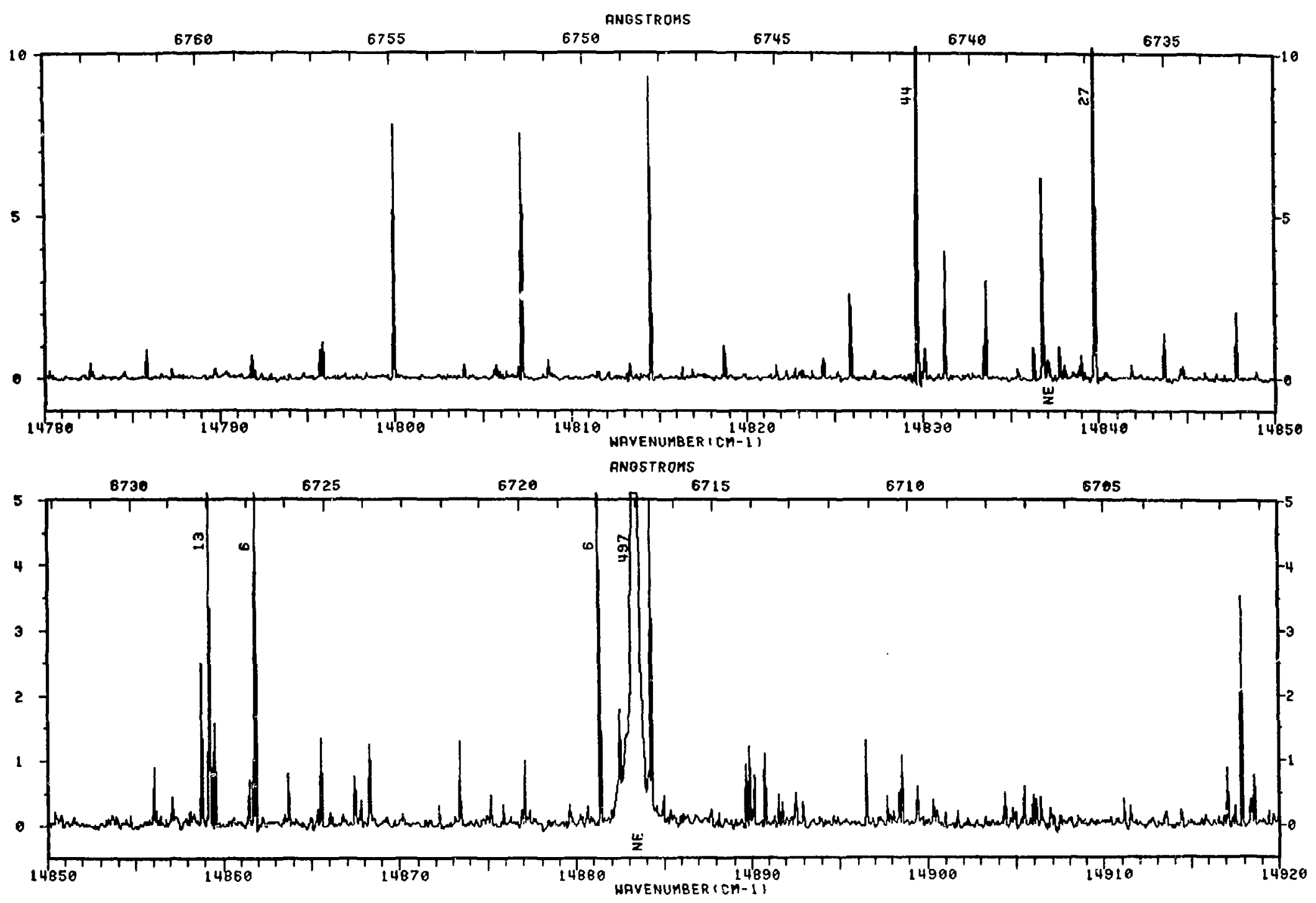
吉

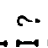

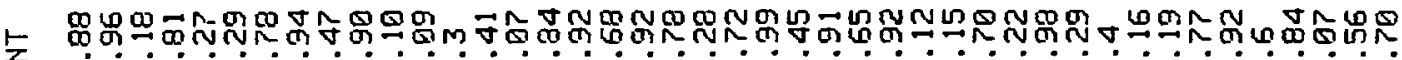

I

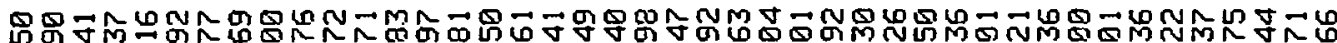

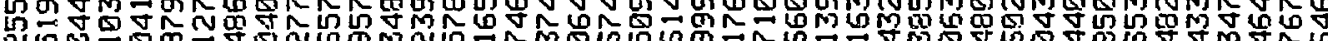

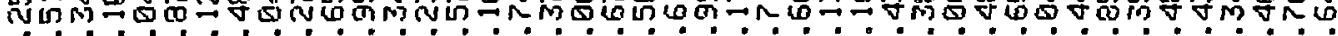
बí

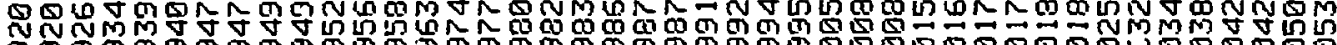

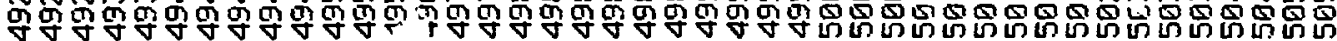
ปั

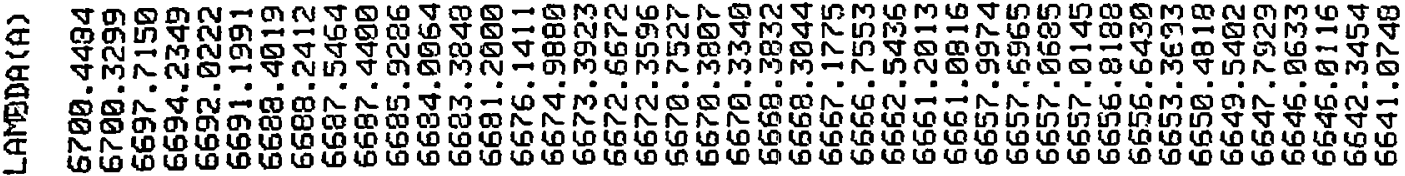



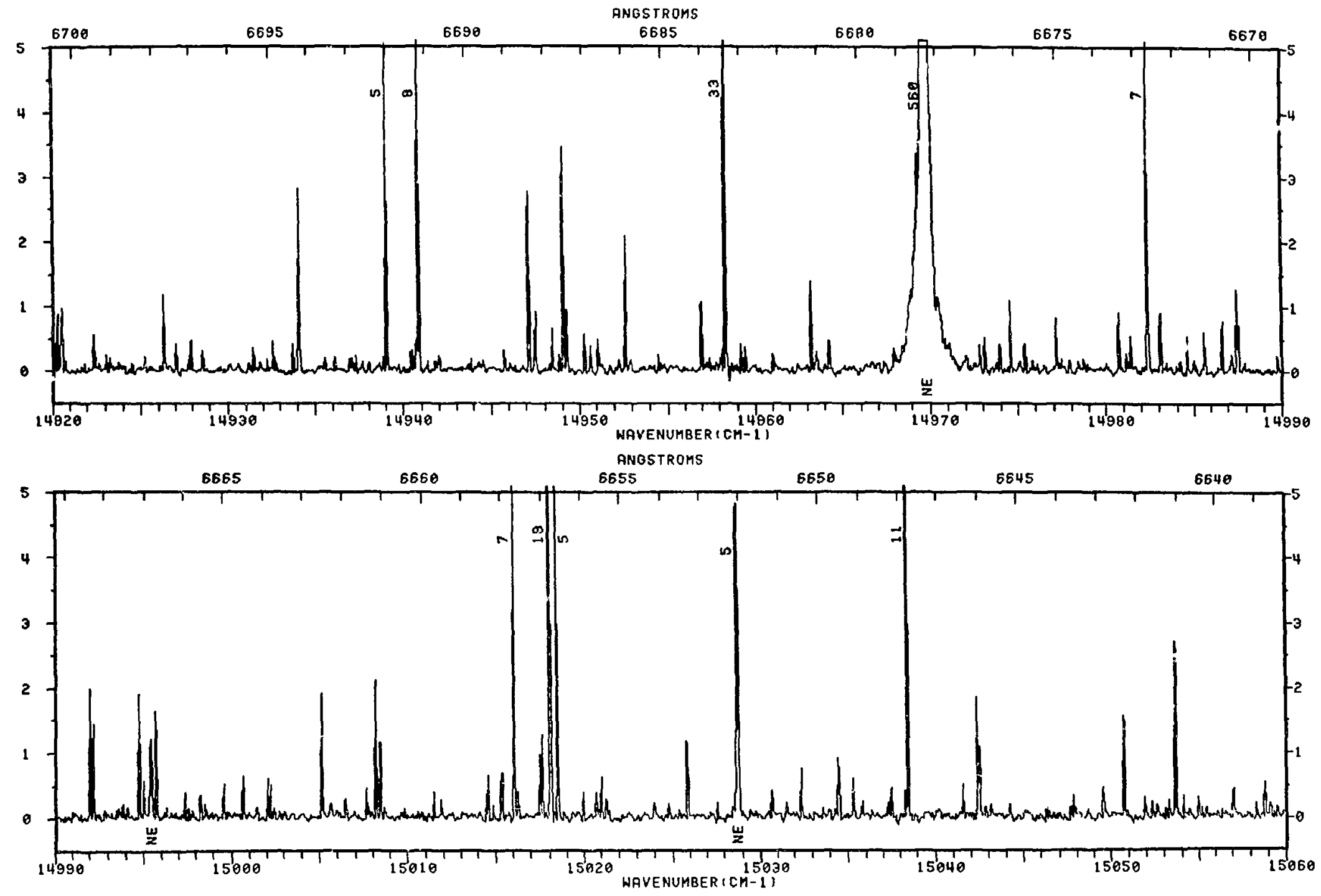


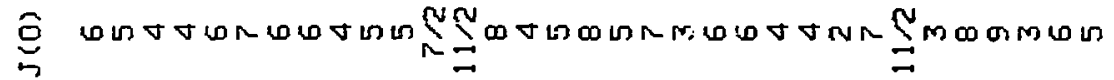

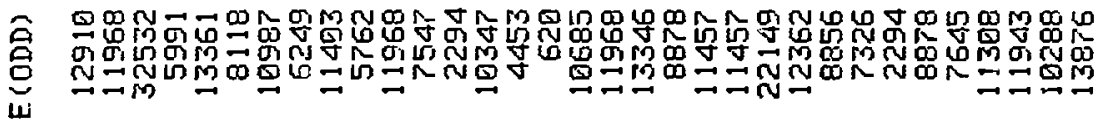

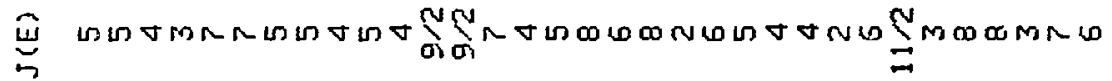

$\frac{2}{3}$

台

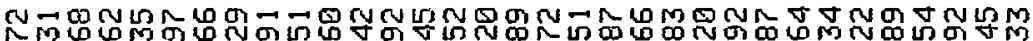

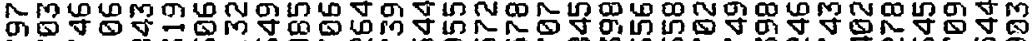
NN N m

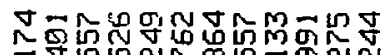

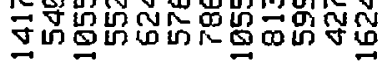

$\sin \sum_{m}^{N} r \sin \theta \sin$

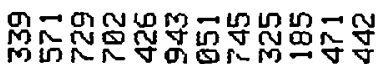

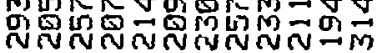

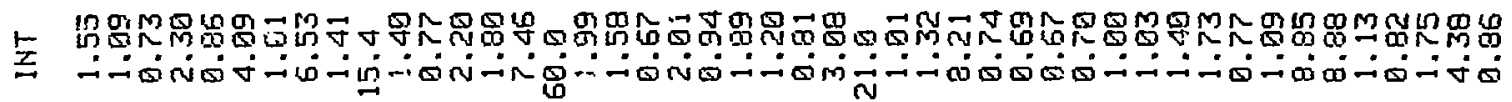

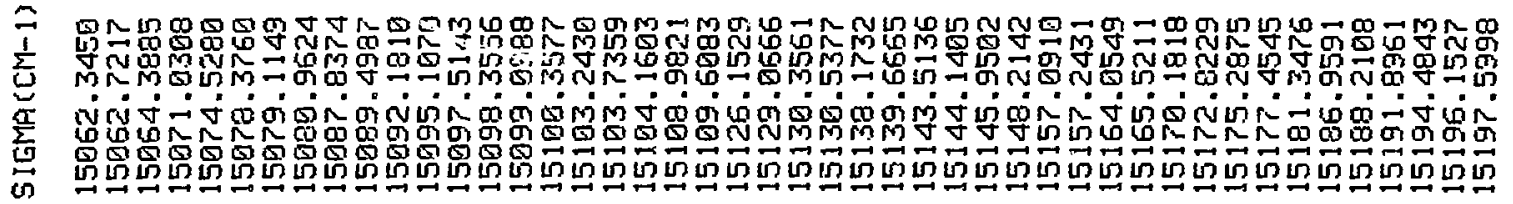

"

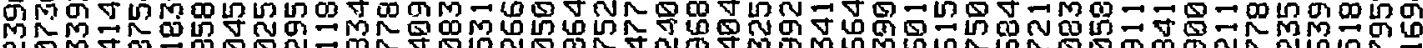

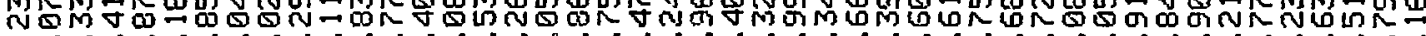
술 -

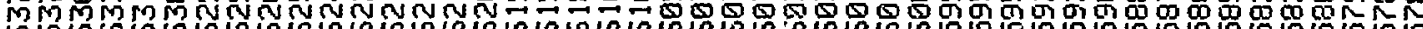

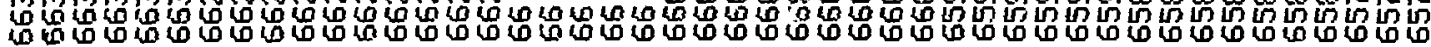



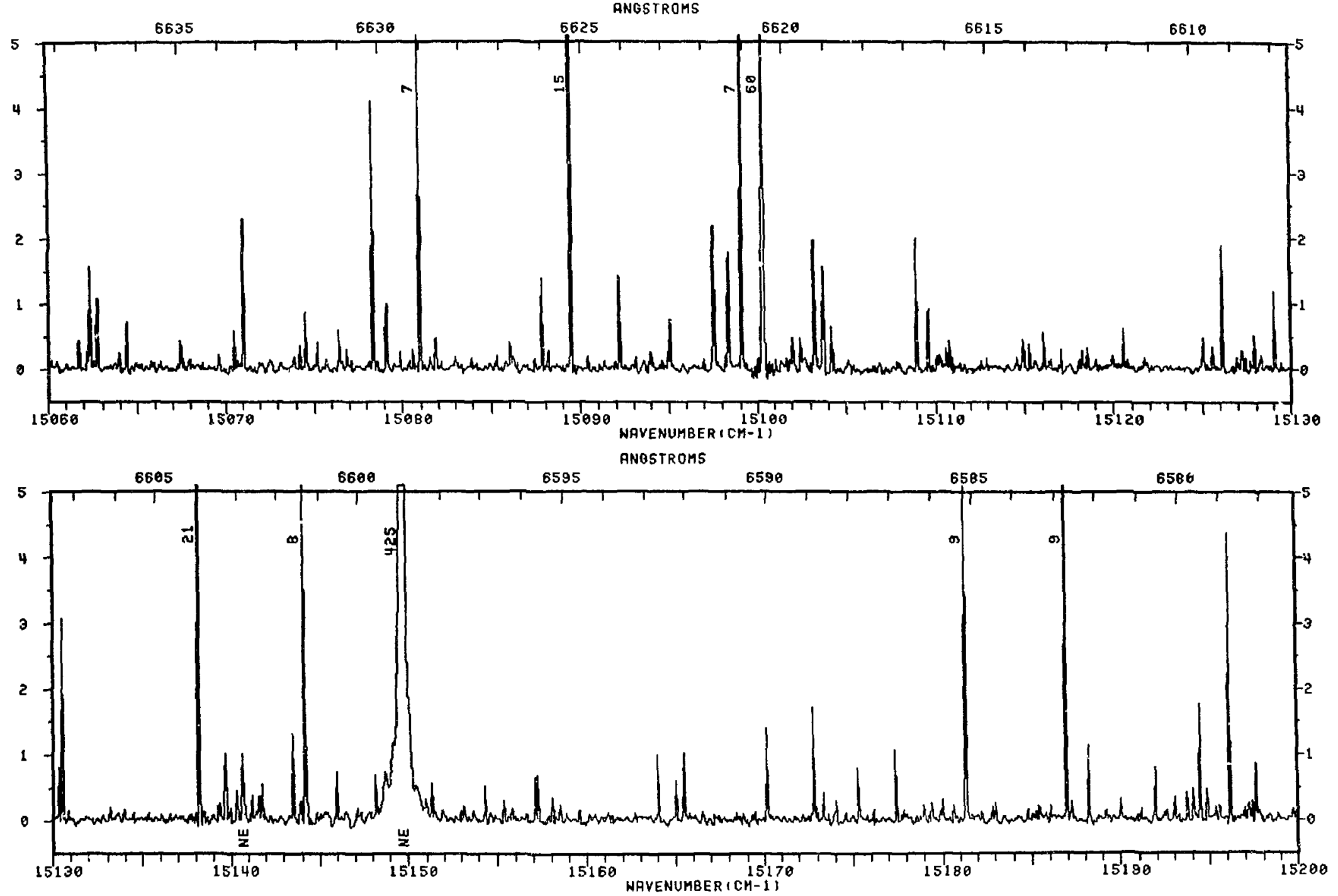


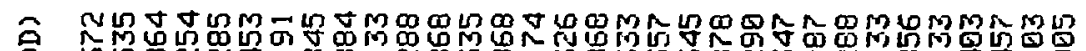

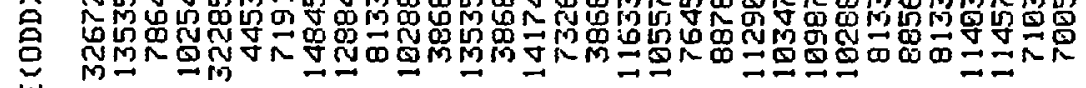

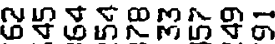

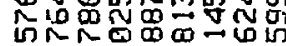
UN

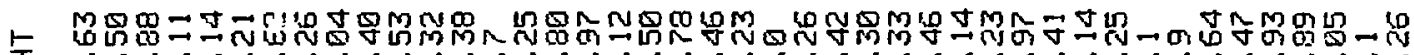

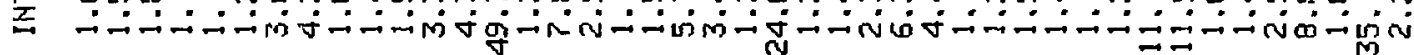

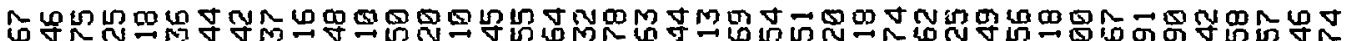

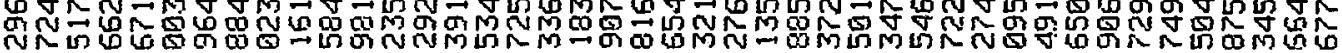
+

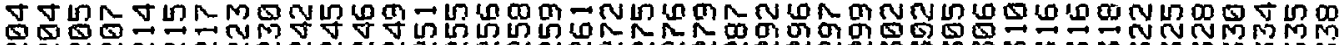

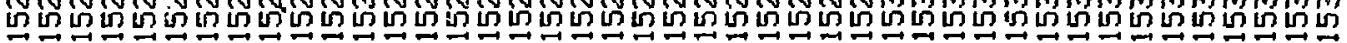

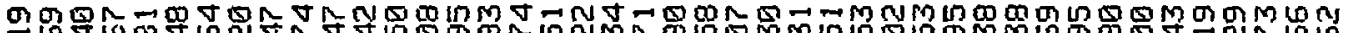

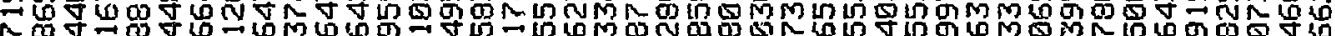
ก ம்

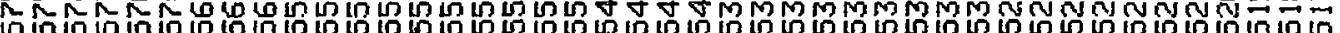
苗 


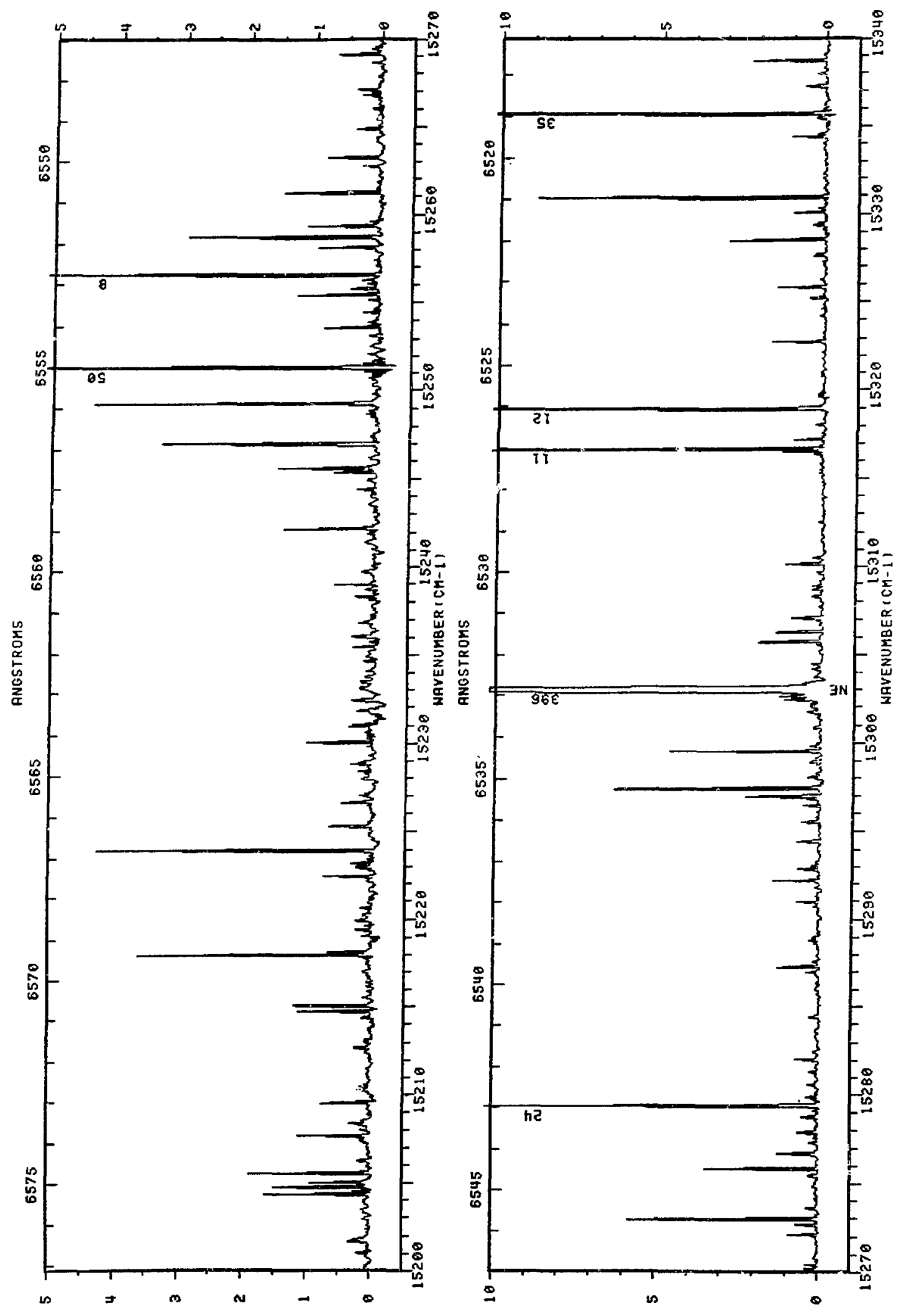




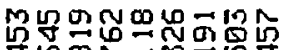

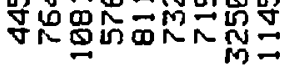

舟守 $\min _{m} \underset{\sim}{m}$

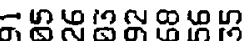

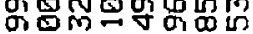

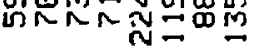

$\infty$ nwonm

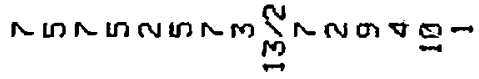

MNMVNWMLOR

ron

anond

立

ก

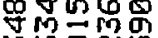
MNNNN

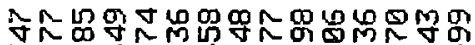

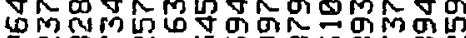

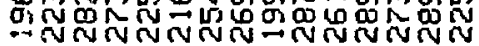

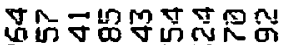

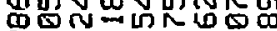
OMm $\rightarrow$ MNN तNญ心 N N

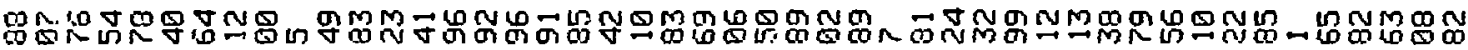

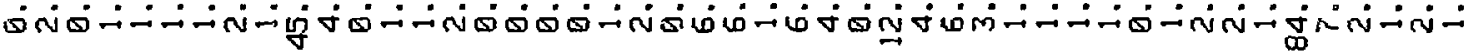

$\frac{1}{3}$

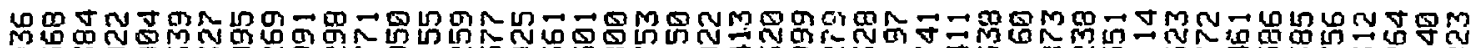

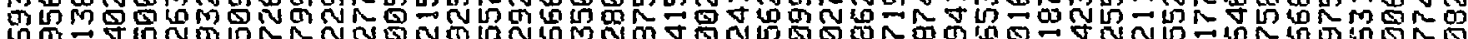

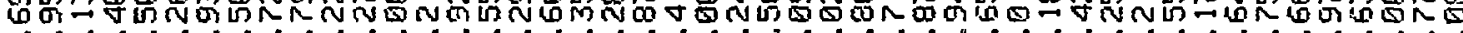
MN 才寸寸

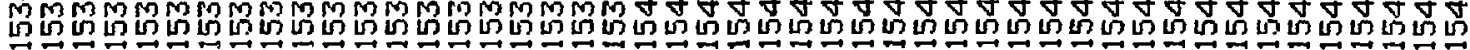

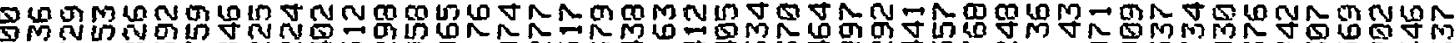

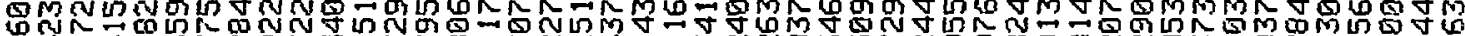

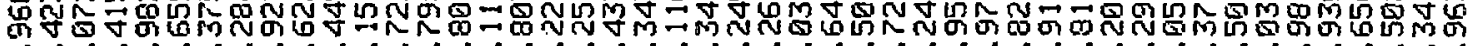
ம்

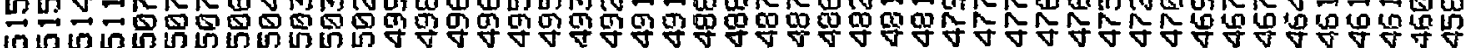

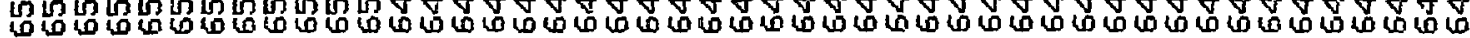



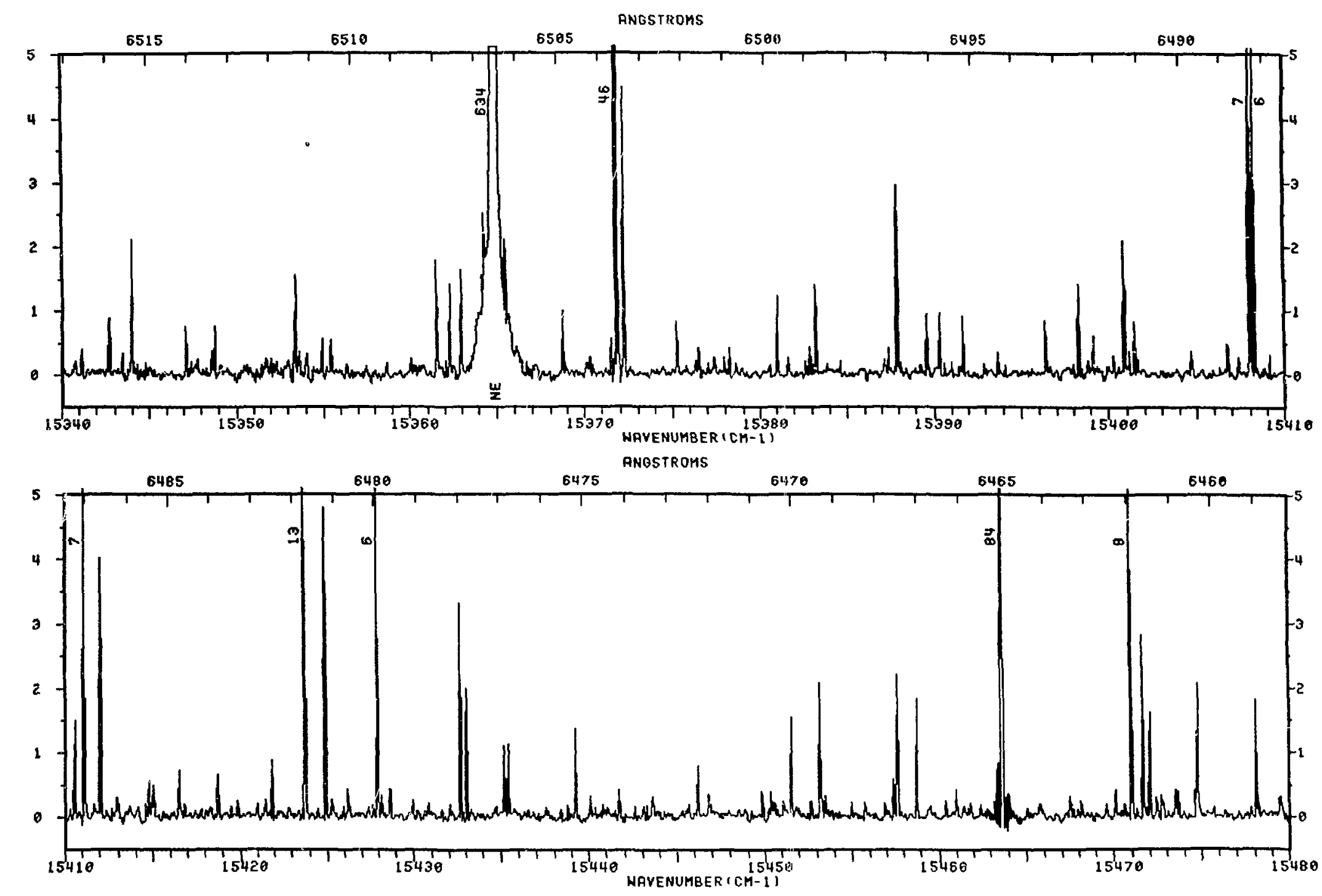


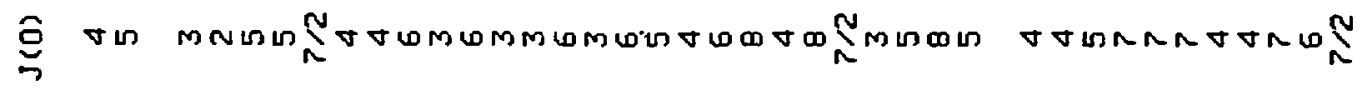

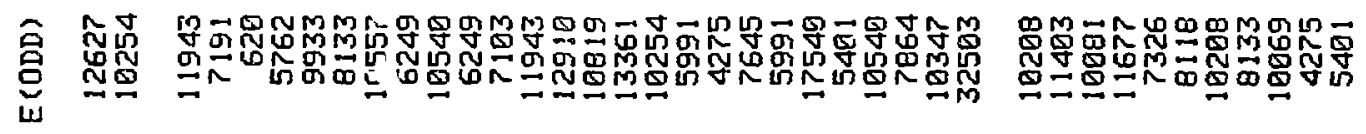

㞾

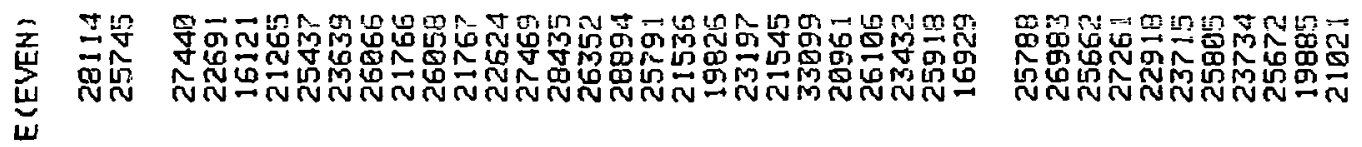

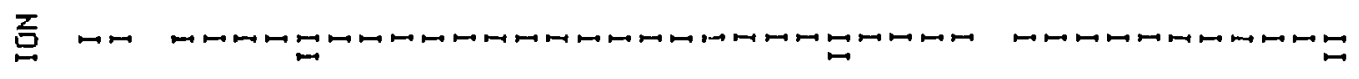

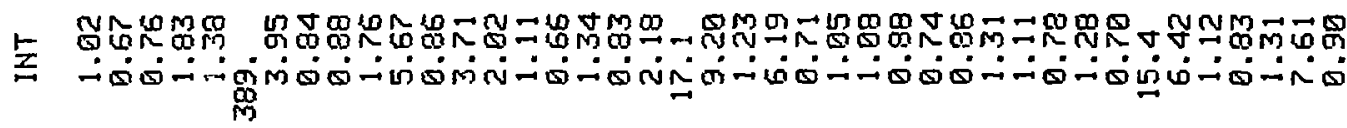

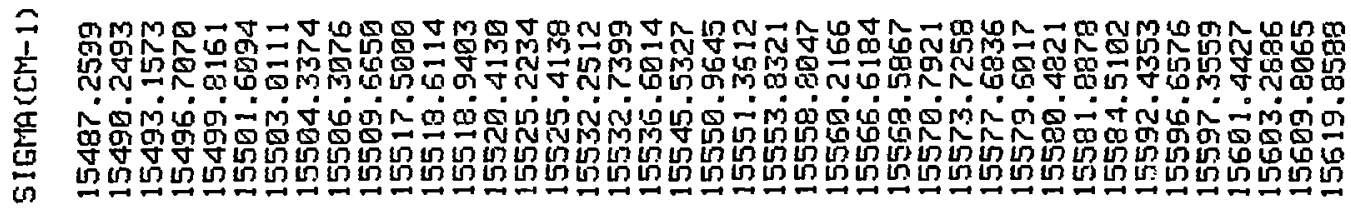

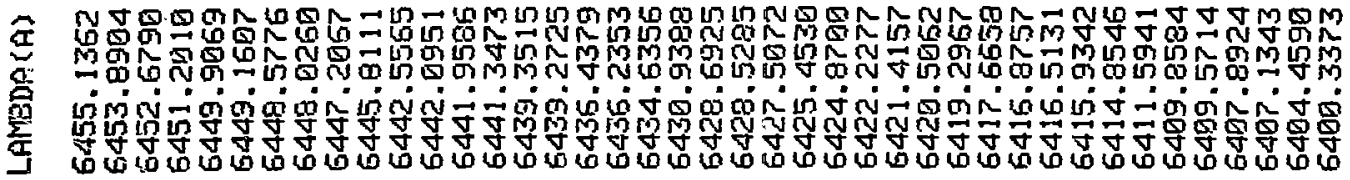


1

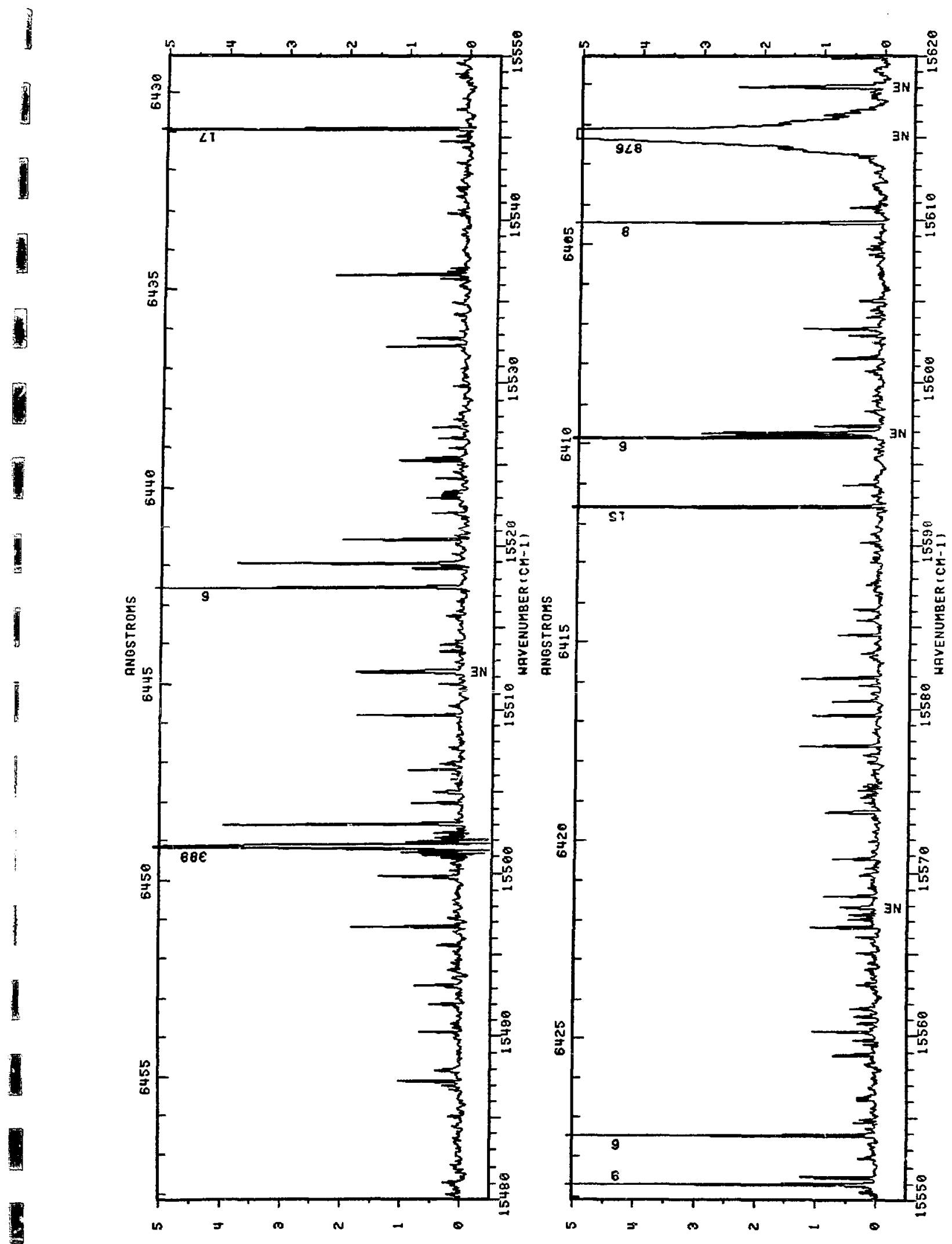




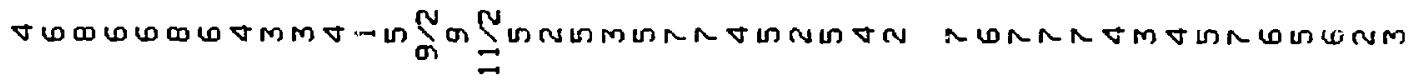
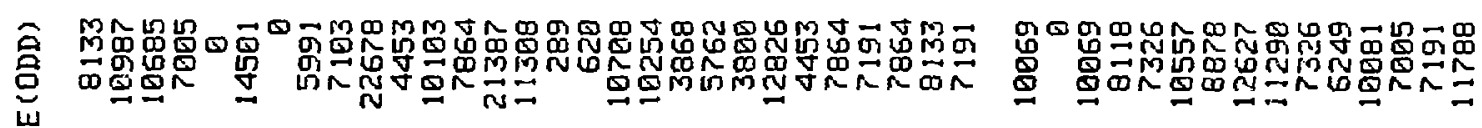

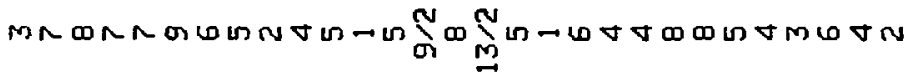

angRM

논

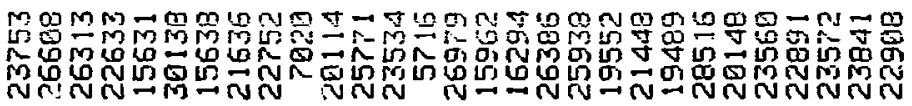

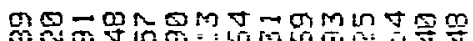

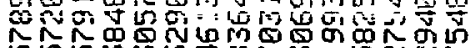

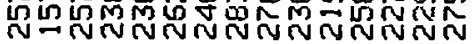

$\underset{0}{\overparen{D}}$

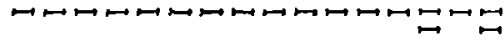

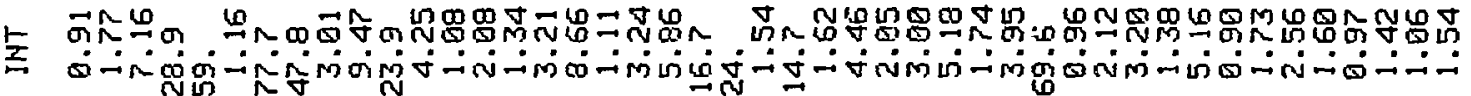
楚

$\underset{\frac{1}{2}}{\frac{1}{2}}$

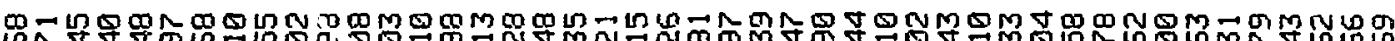

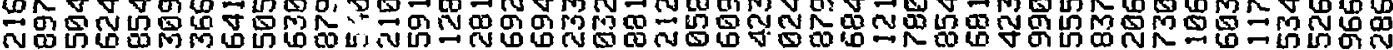

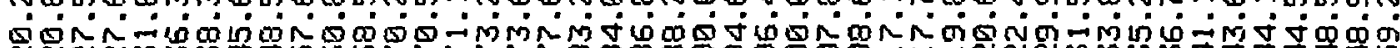

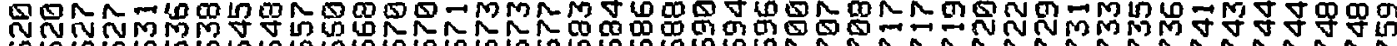

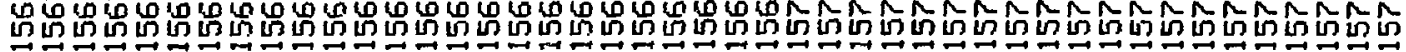

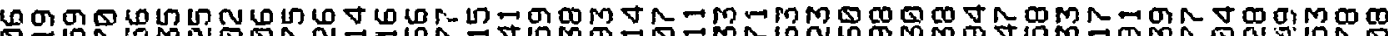
S-

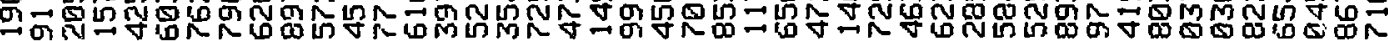

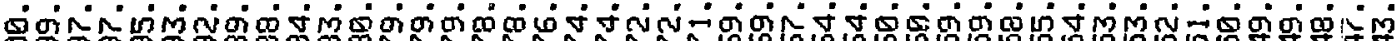

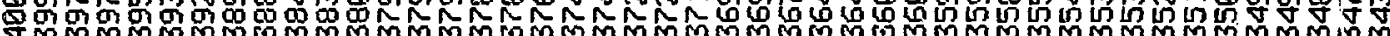
प 

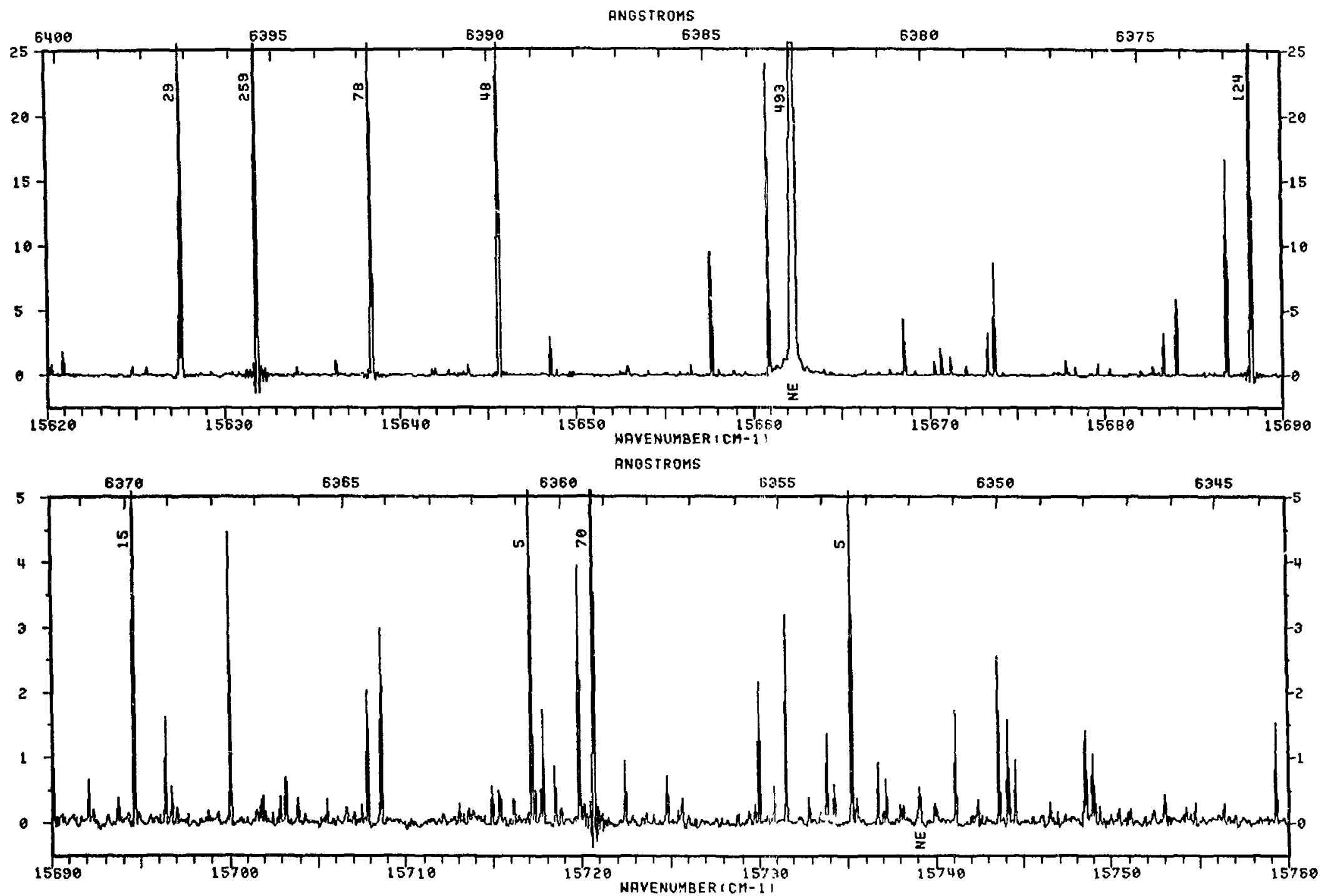
कृ

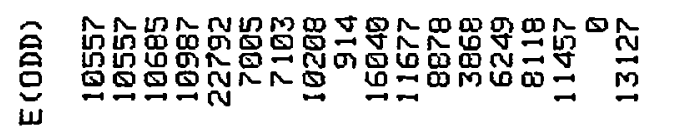

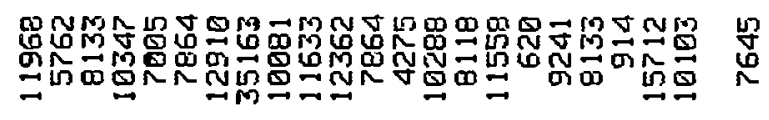

岁 MUDN

जक

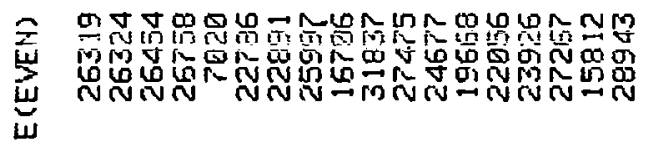

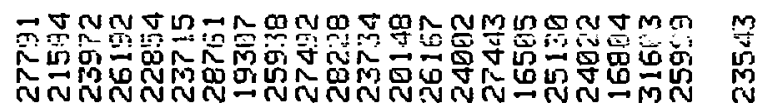

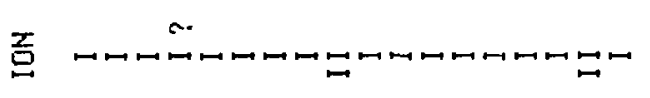

ロロロロロロロロロローロロロロロロコロロロー ロ

与 ำำ

I
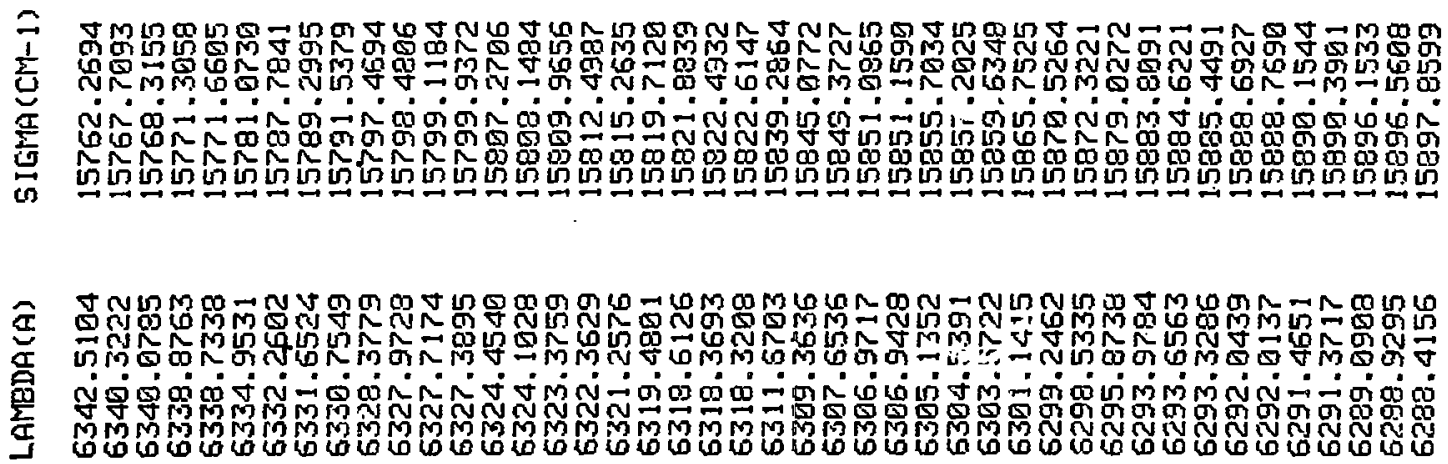

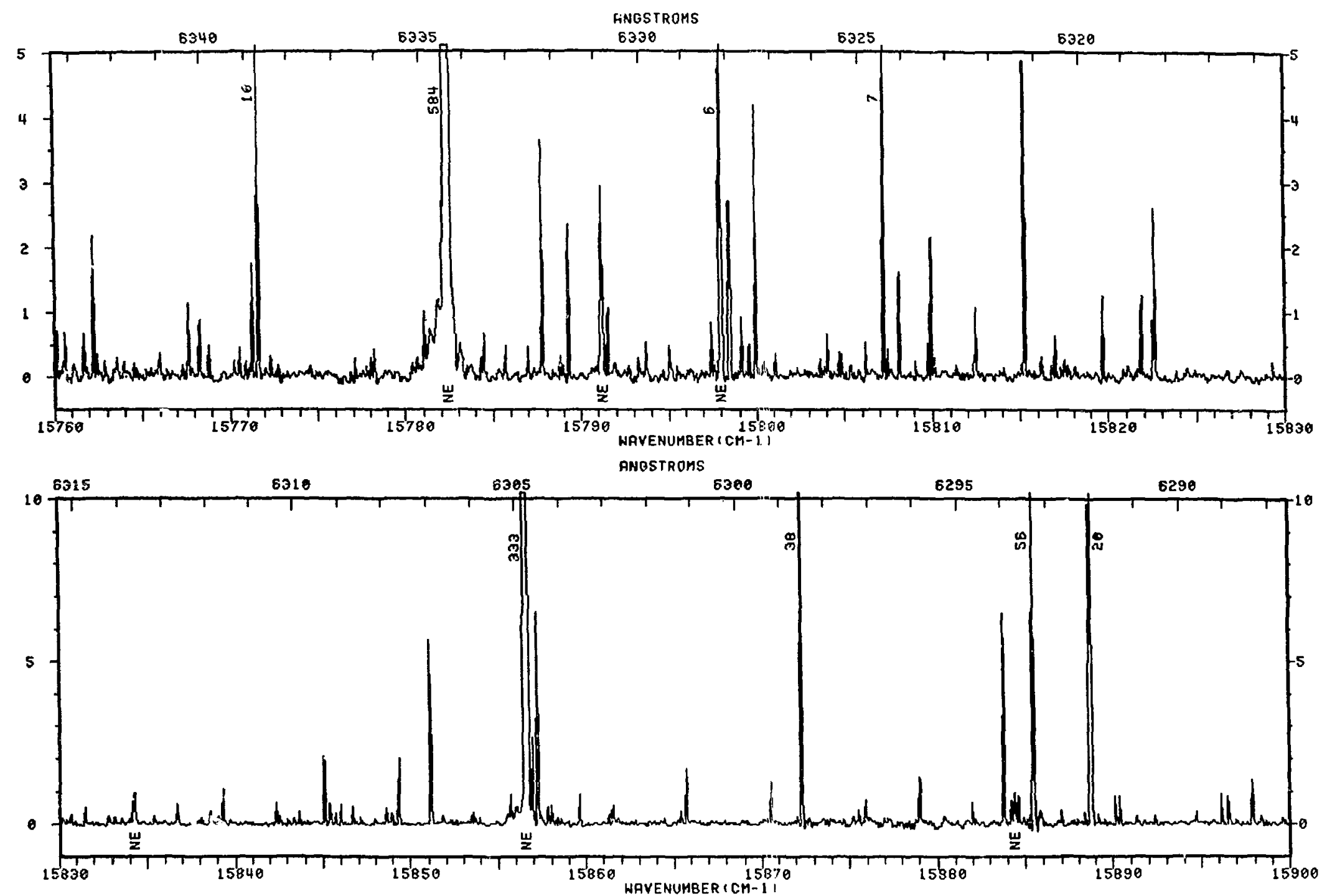


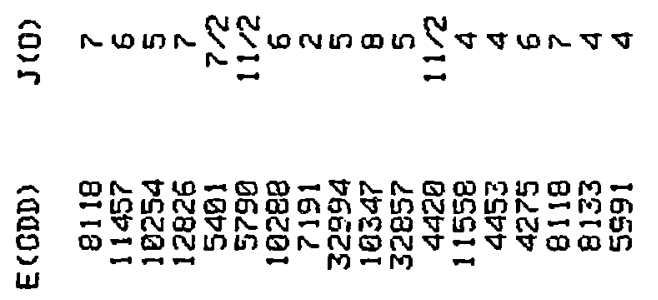

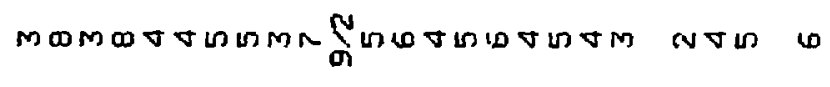

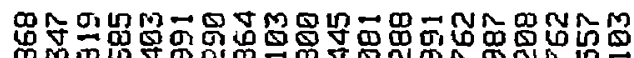

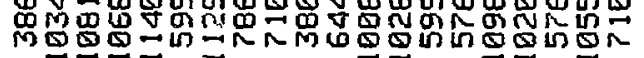

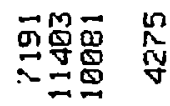

岂

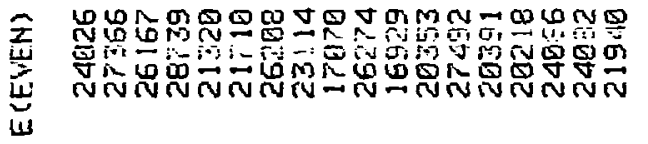

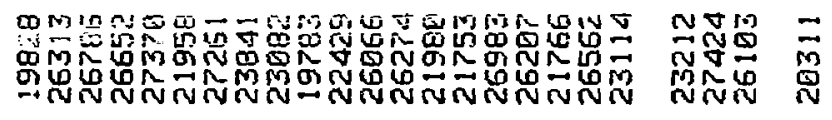

空

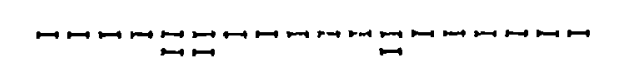

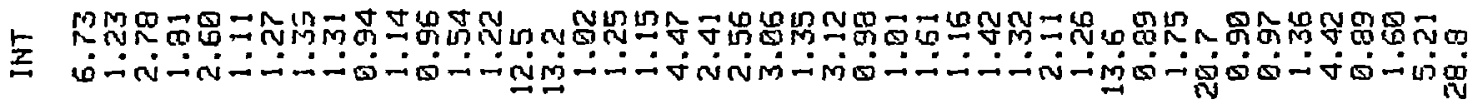
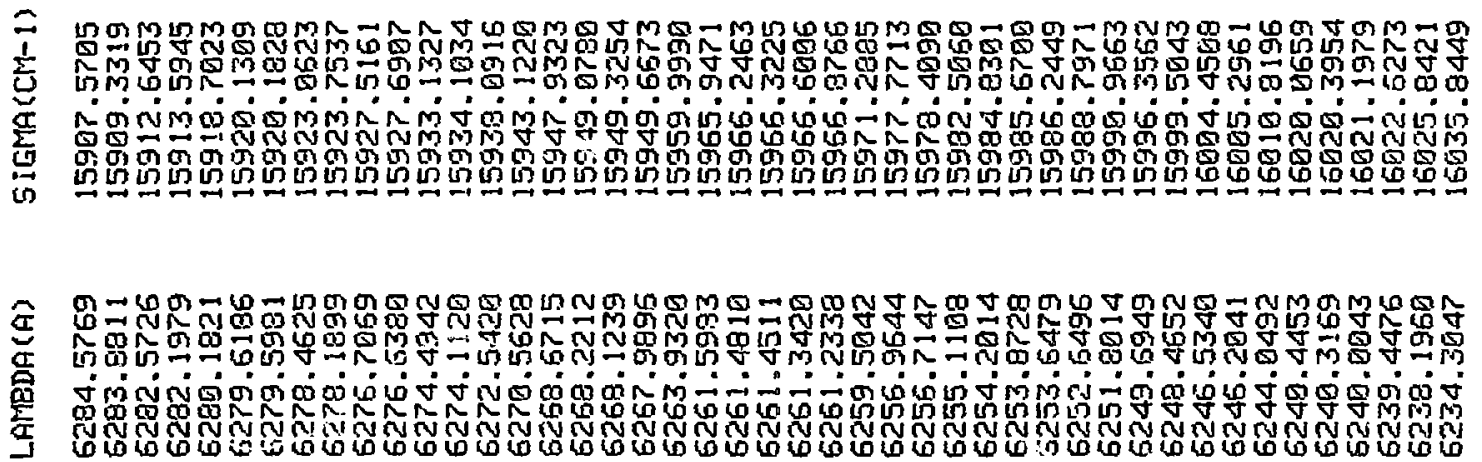
ANGS TROHS
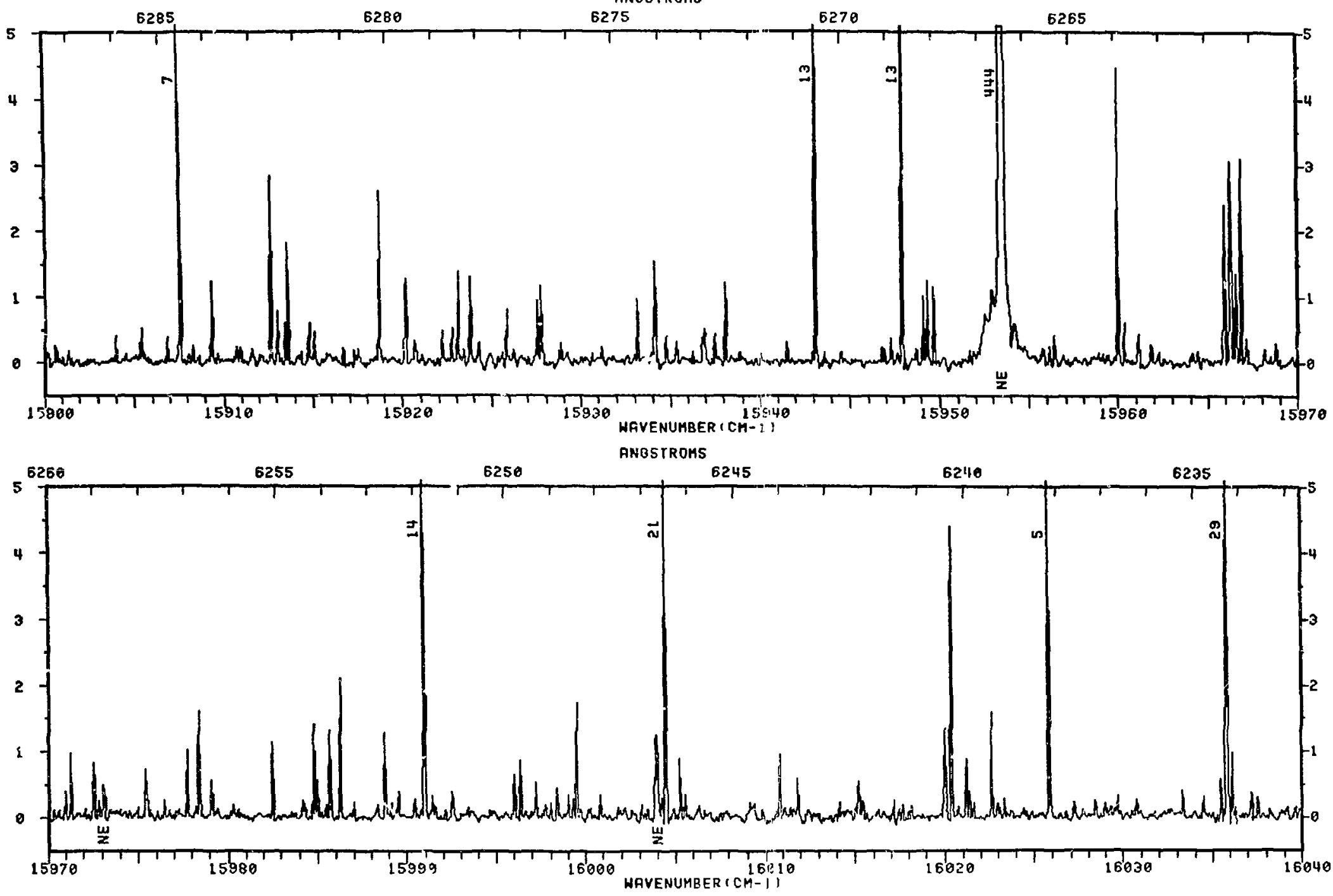


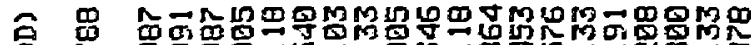

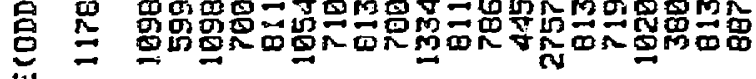

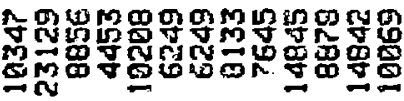

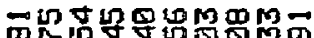

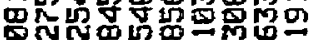

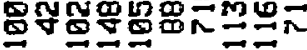

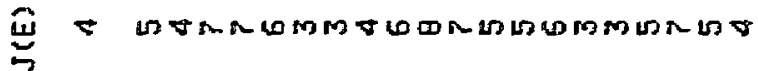

Z M FCH

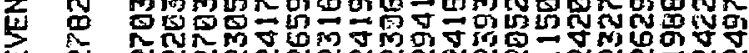

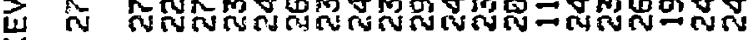

母Q⿻日禸

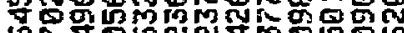

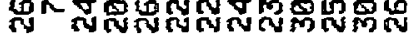

$10000 \cos \theta 0$

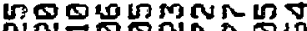
NOSONR \%

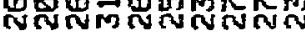
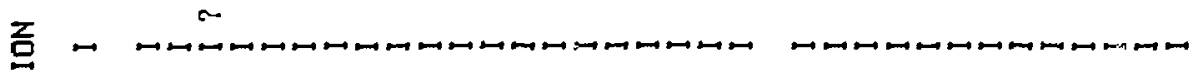

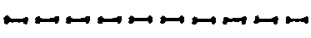

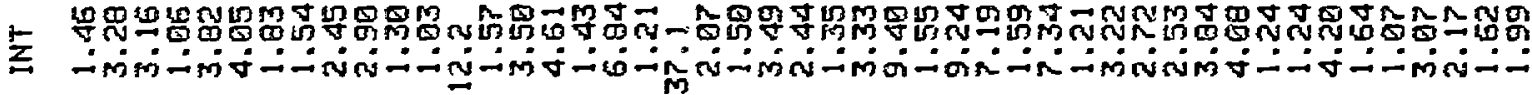

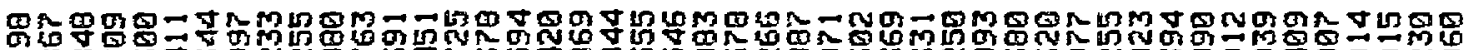

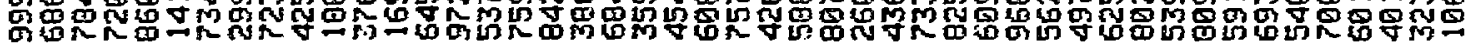
onminci

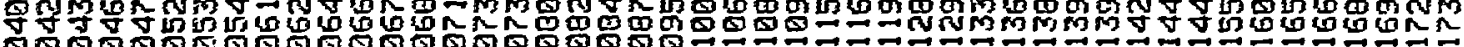

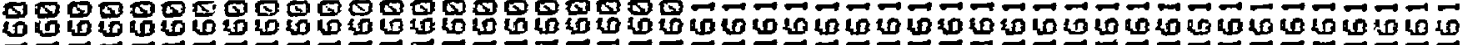

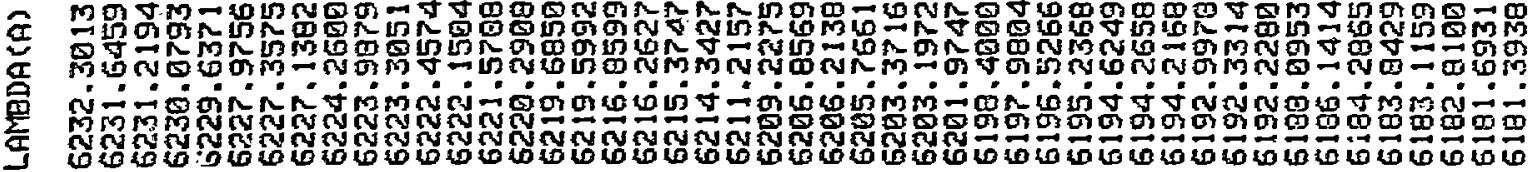



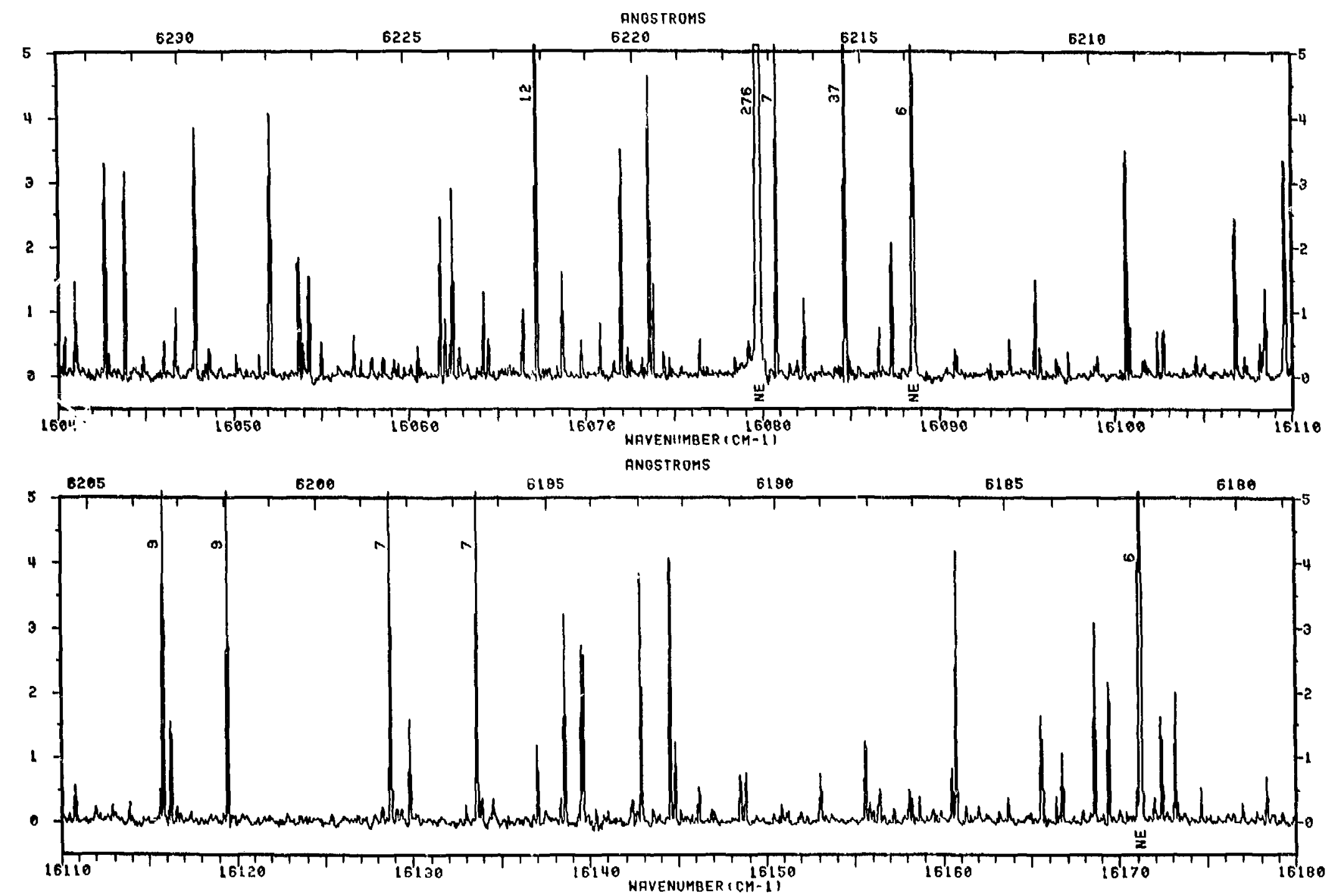
9

莣

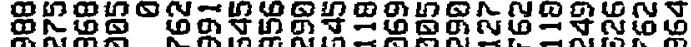

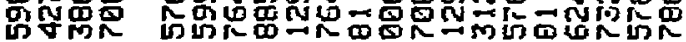
$\rightarrow$

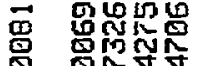

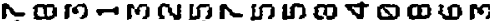

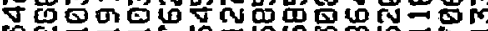

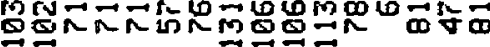

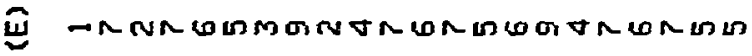

n $\cos 2$

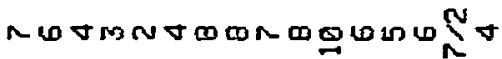

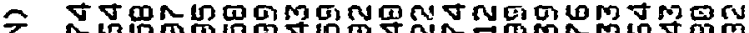

竞

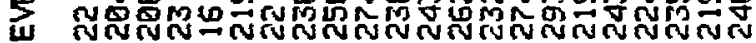

in Noin-

永

只

MG 品

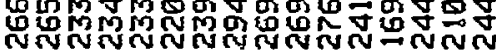

곰

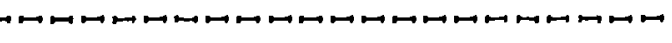

$\rightarrow$
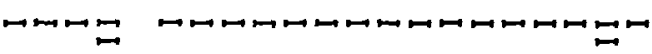

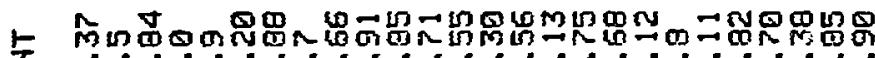

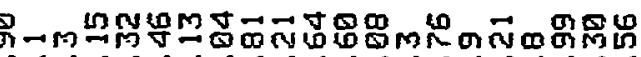

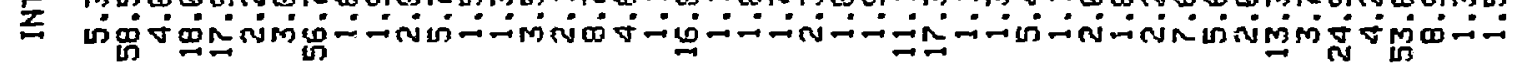

6日勺

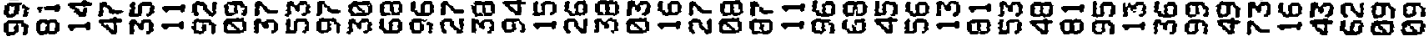

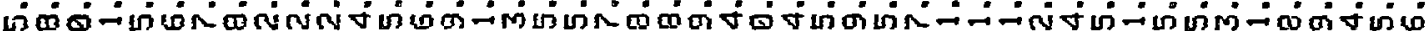

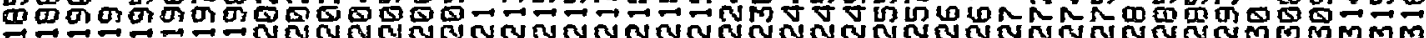

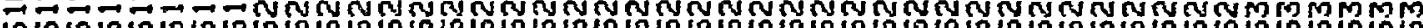

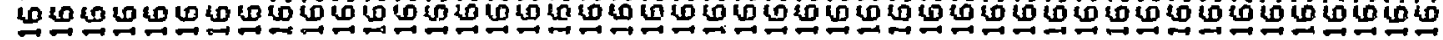

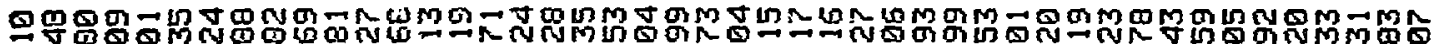

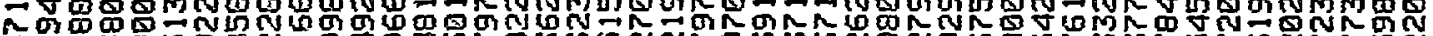

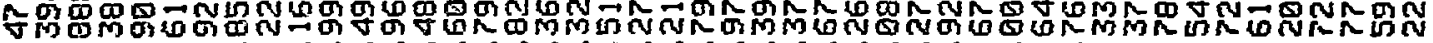

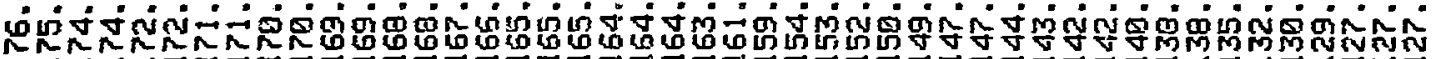

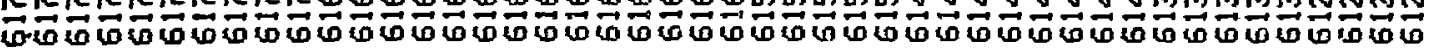



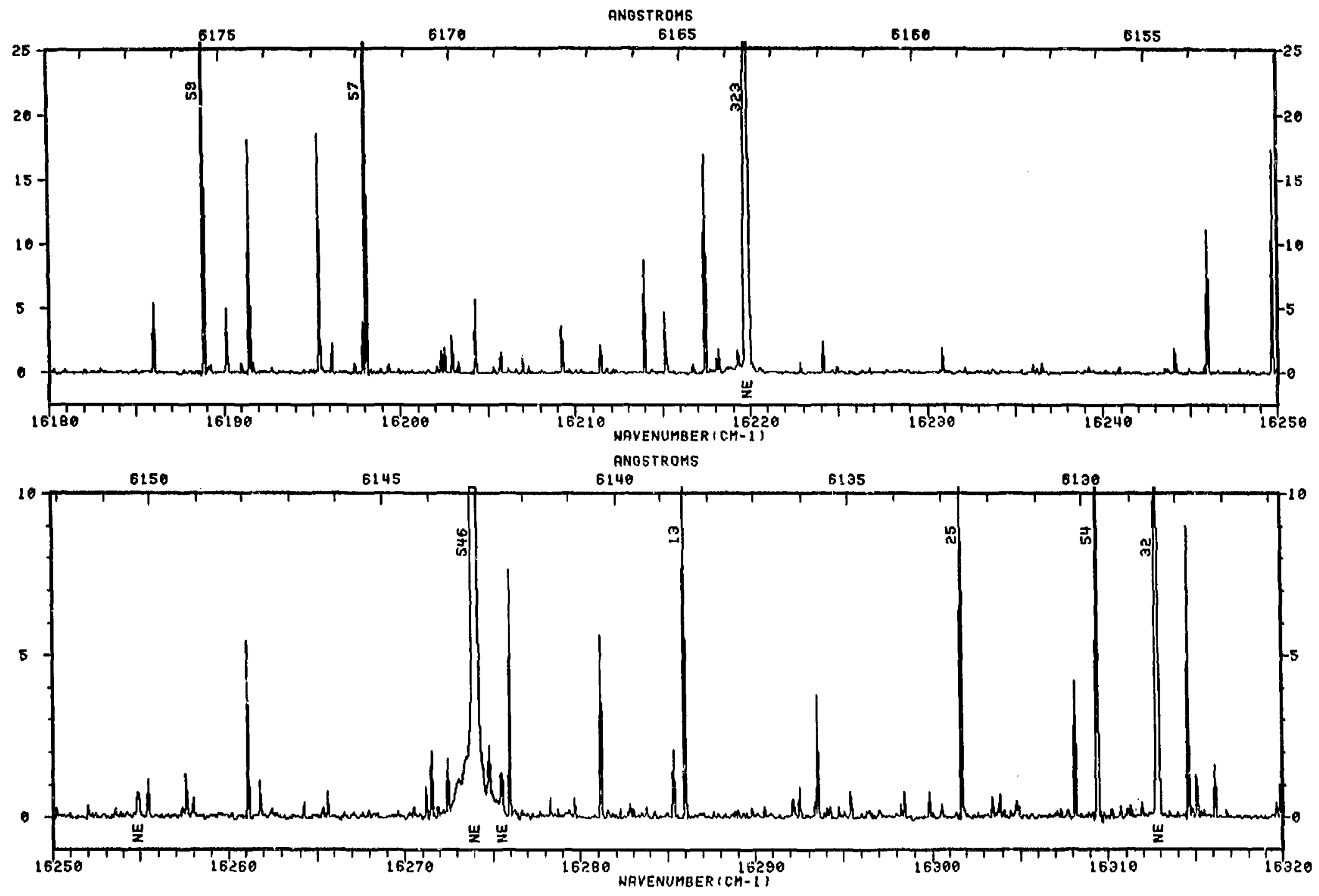


\begin{tabular}{|c|c|c|c|c|c|c|}
\hline$\underline{=}$ & mMnem & $\omega \nabla \nabla \backsim 0 \mathrm{~mm}$ & $\infty$ & $6 M G \sim M \nabla M$ & 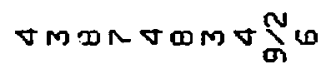 & $\sim \backsim M \cap N \backsim \sim M M$ \\
\hline 5 & 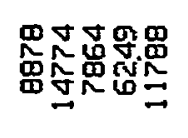 & 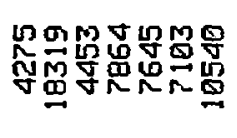 & 営 & 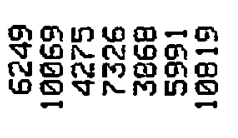 & 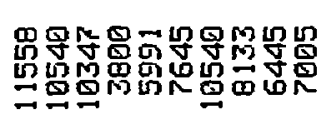 & 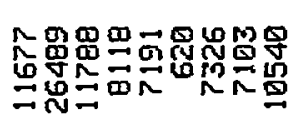 \\
\hline
\end{tabular}

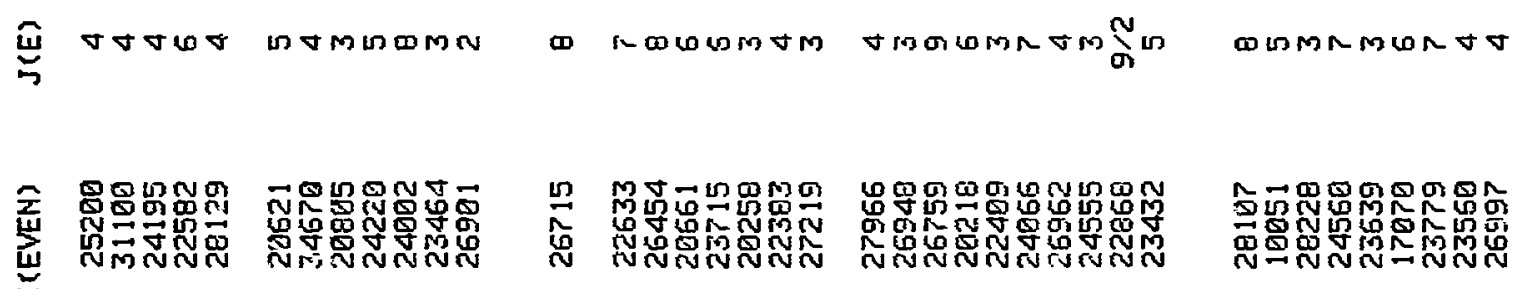

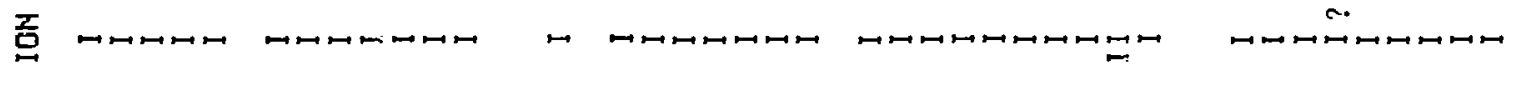

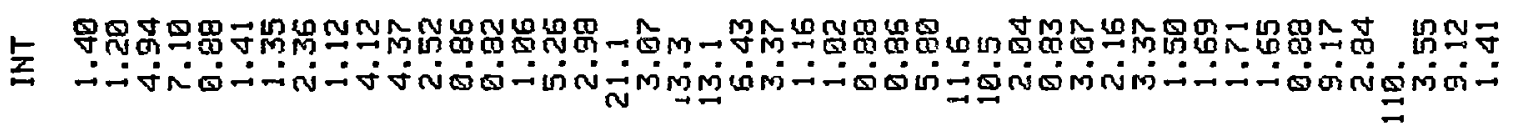

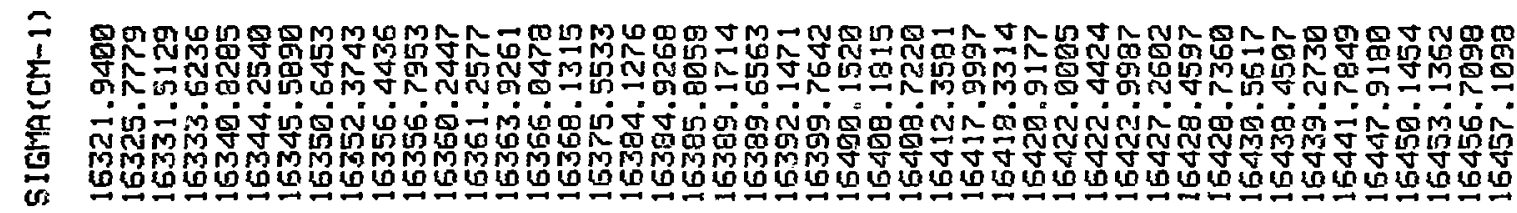

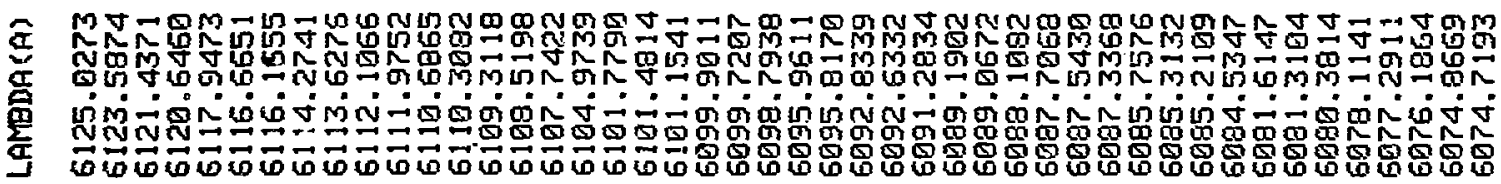





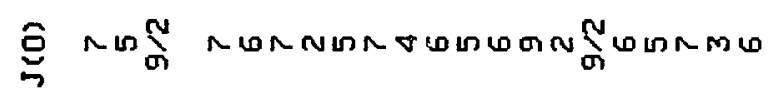

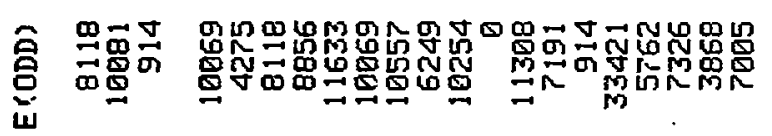

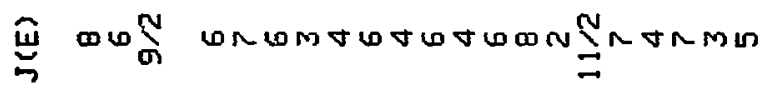

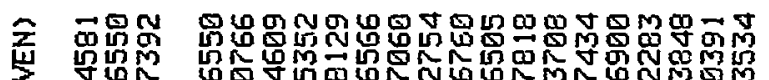

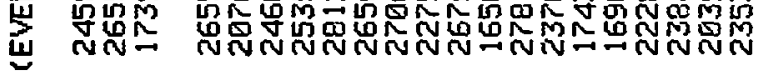

G

ต ตั

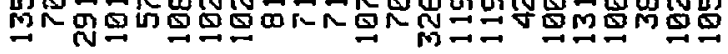

름

$\rightarrow-=$
6冈刂 ON MN.

U1

का M M

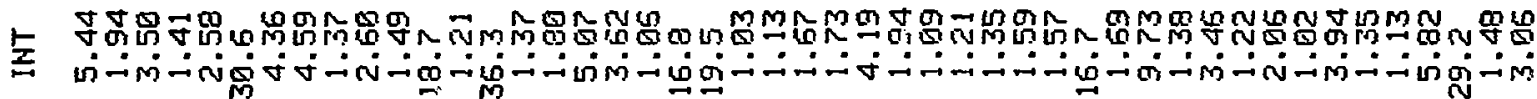

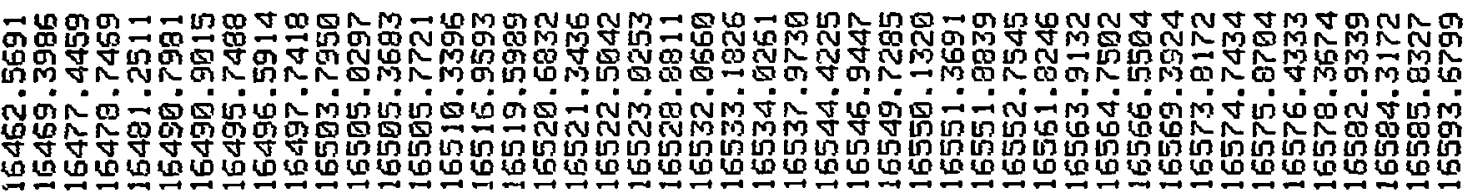

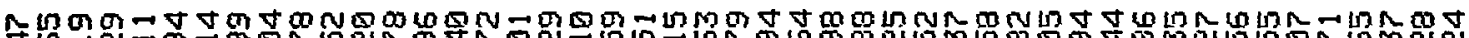

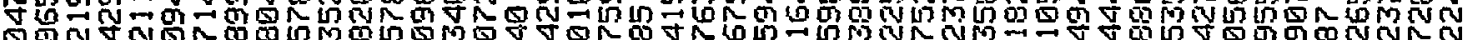

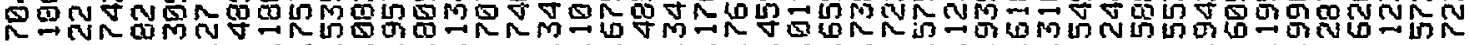

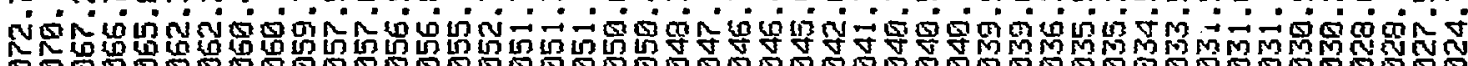

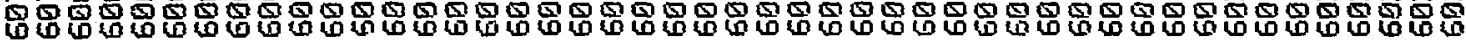



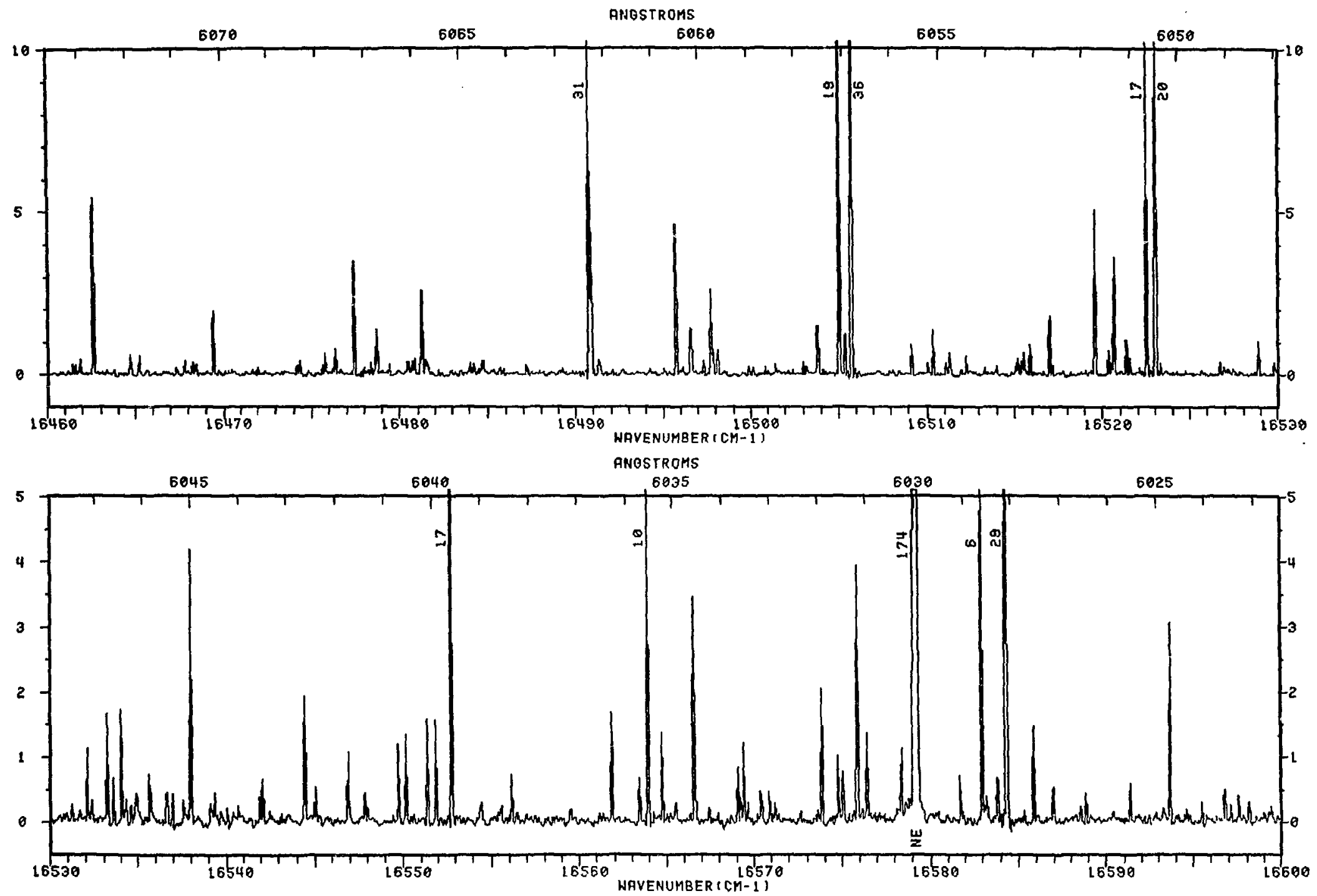

81 
官 MUV

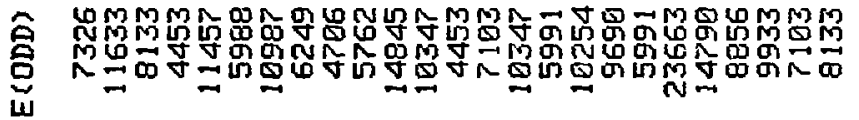

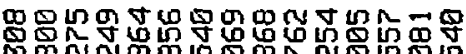

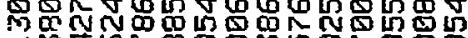

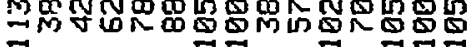

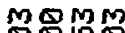

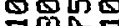
$\sim ⿻ \underset{m}{\sim} \bar{N}$ UI

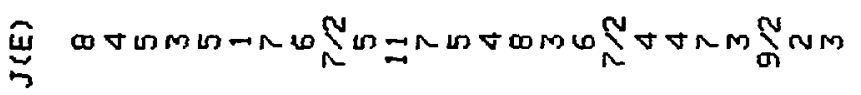

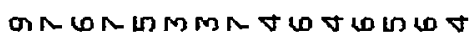
$\nabla \infty \nabla \nabla$

管

MUNAGQNeR

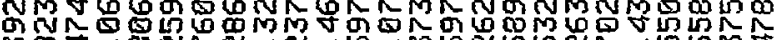

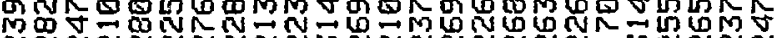

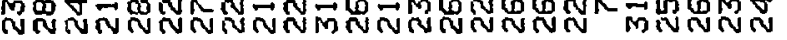

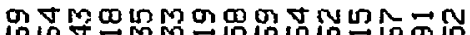

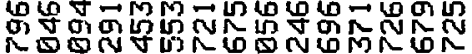

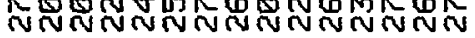

$\ln \infty \vec{\nabla}$ NNE m品畏 NON

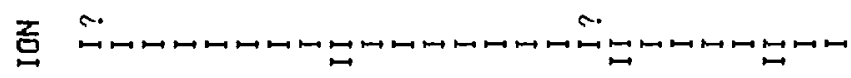

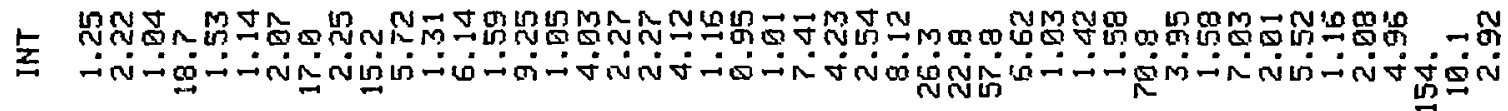

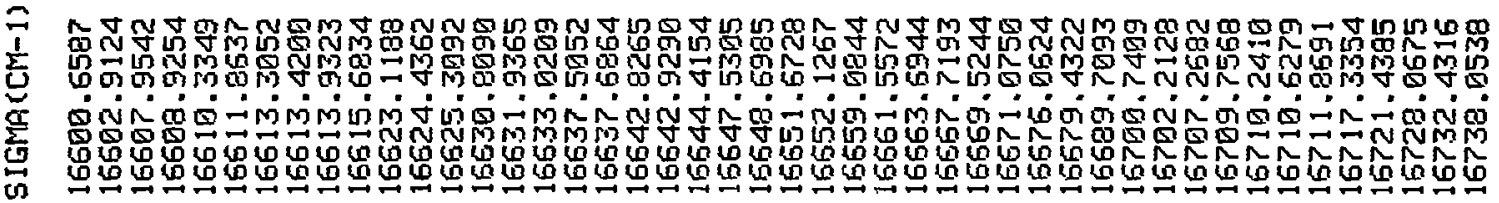

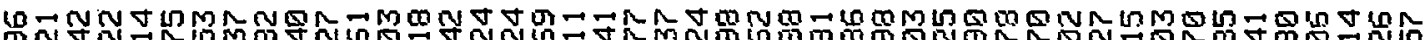
ดN寸

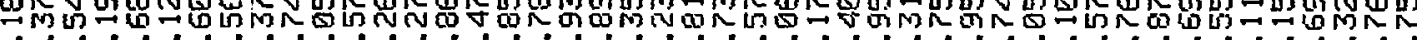
نं

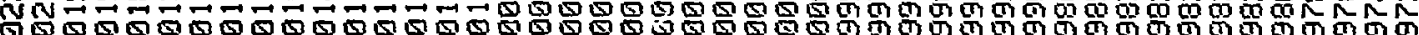

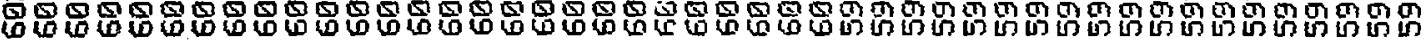



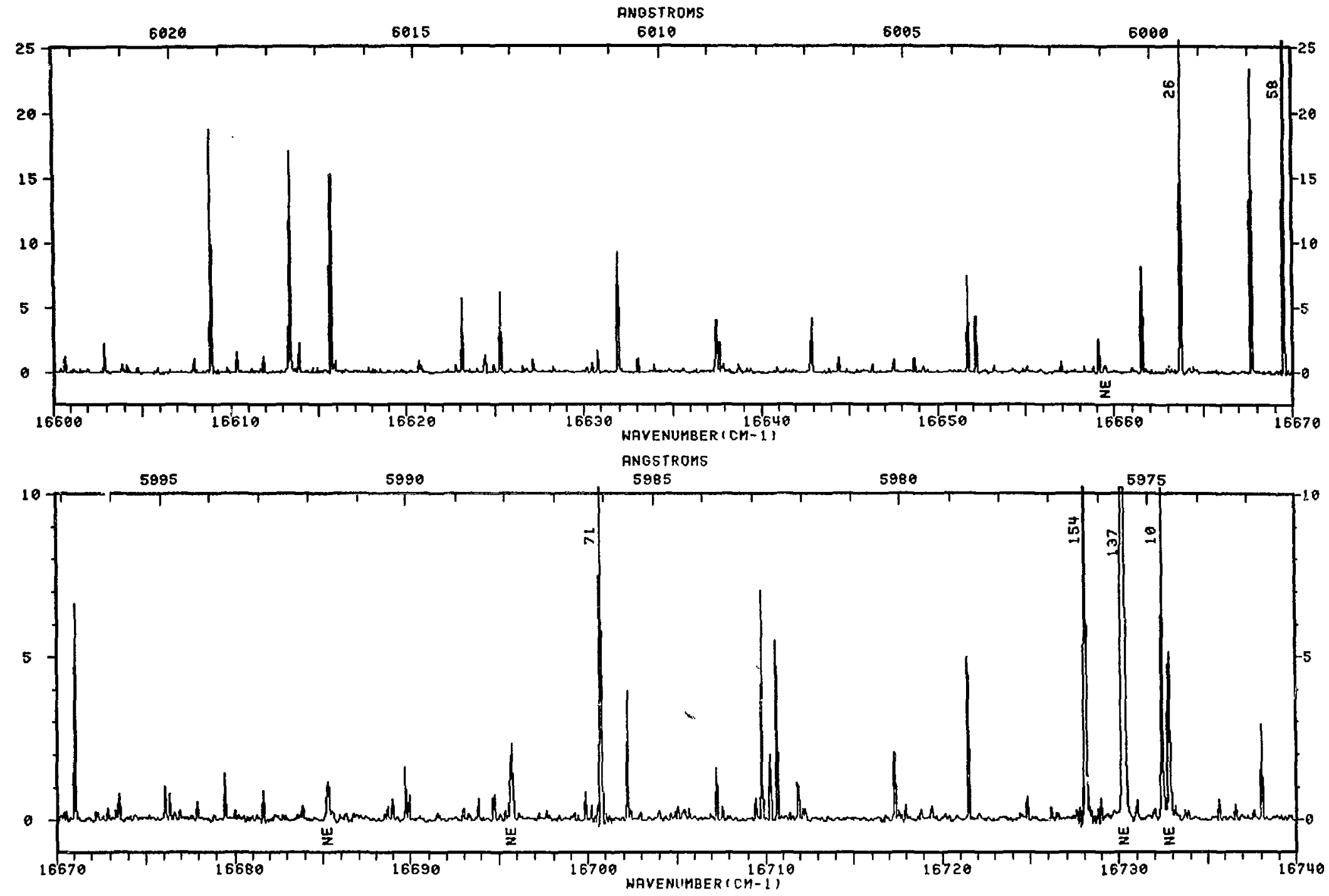


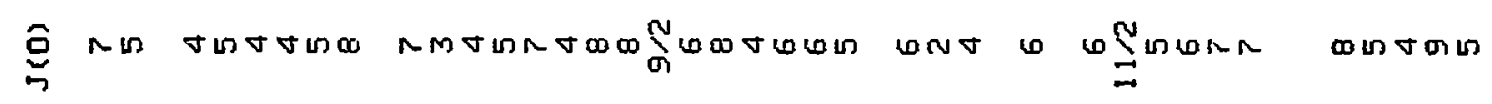

脸

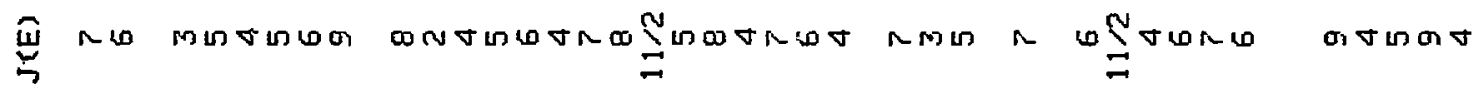

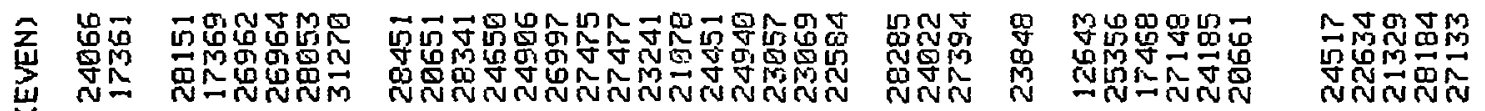

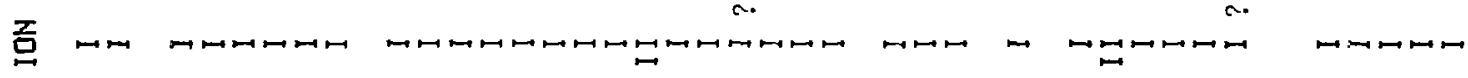

ㄴ I

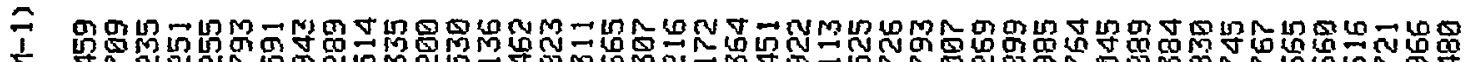

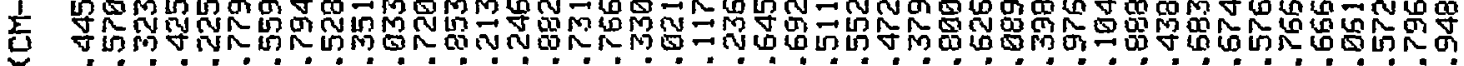
๔

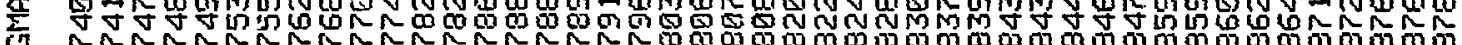

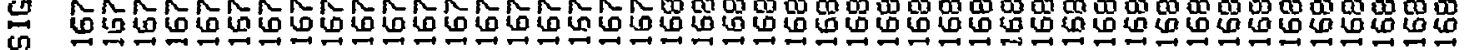

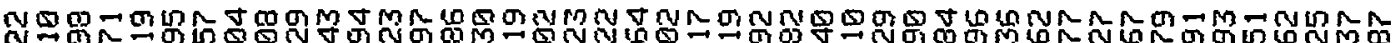

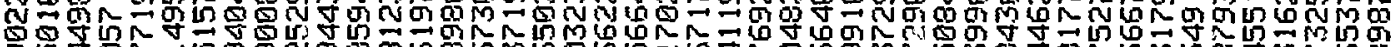

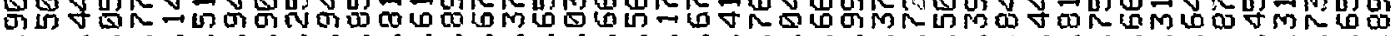

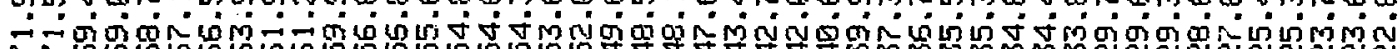
R M

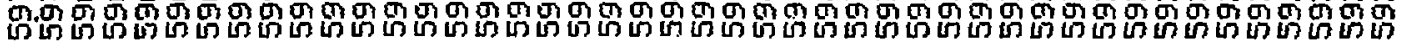



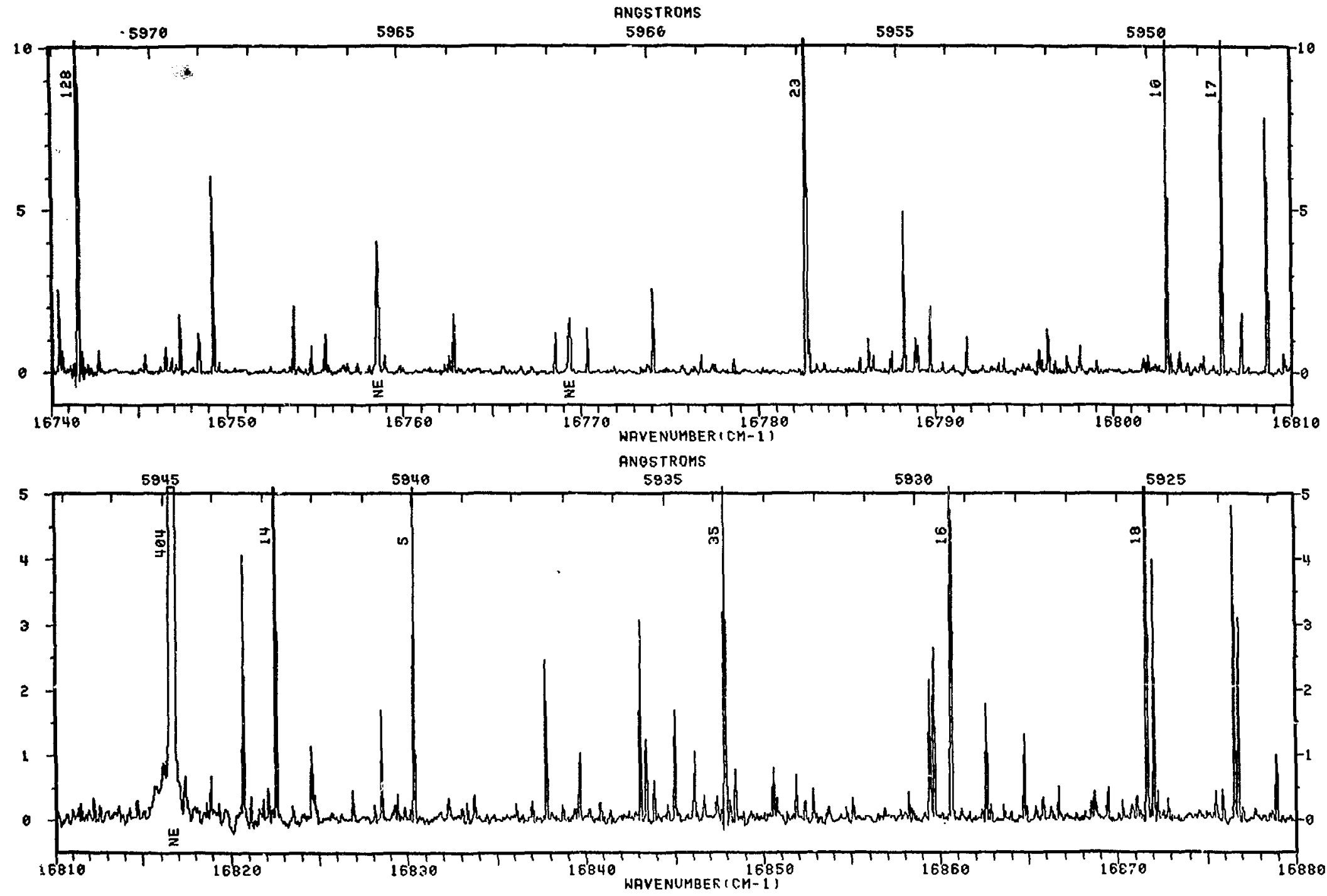


\begin{tabular}{|c|c|c|c|c|c|c|c|c|}
\hline$\hat{\theta}$ & $m n-6$ & $n$ & $n=$ & 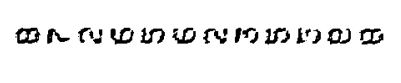 & 075 & $\nabla$ & 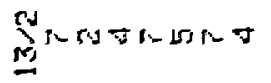 & EUSTMRTh \\
\hline 总 & 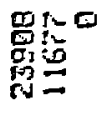 & 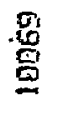 & $\begin{array}{l}19 \\
9 \\
9\end{array}$ & 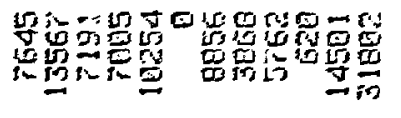 & 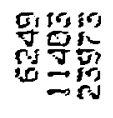 & 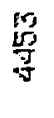 & 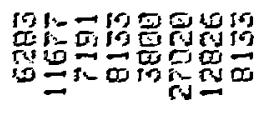 & 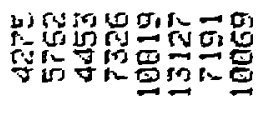 \\
\hline
\end{tabular}

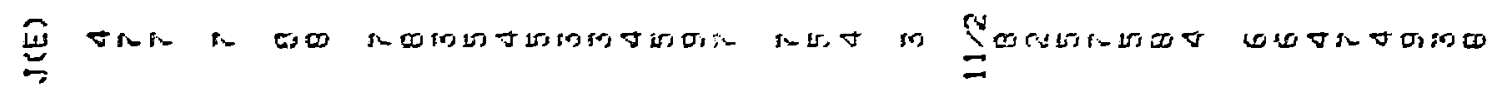
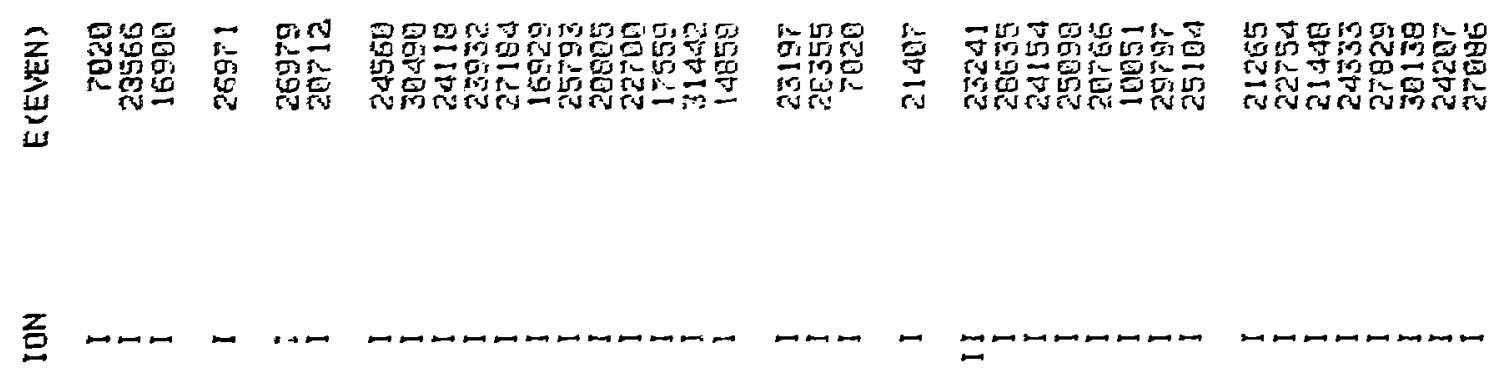

年

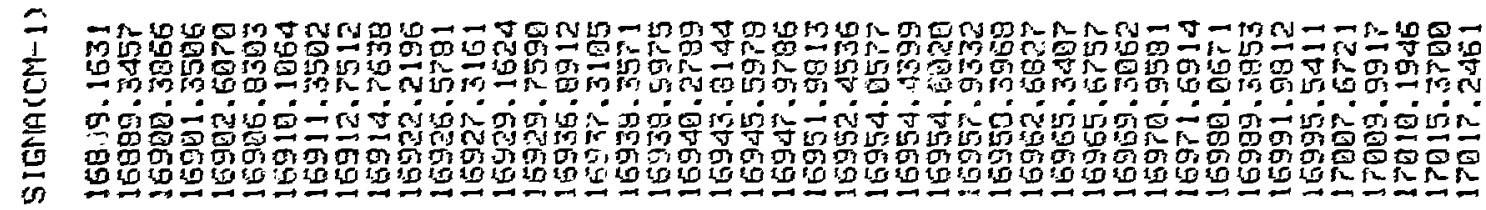

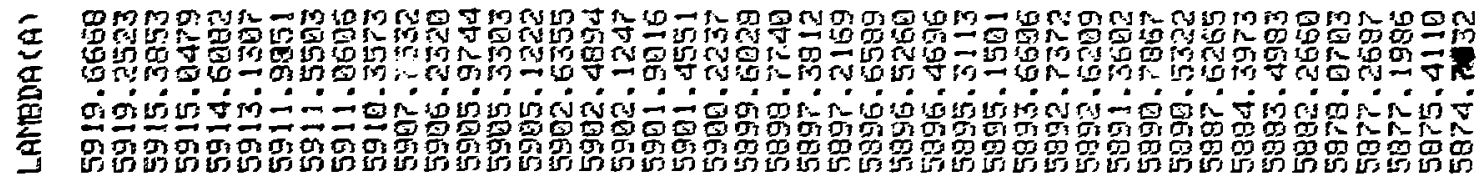



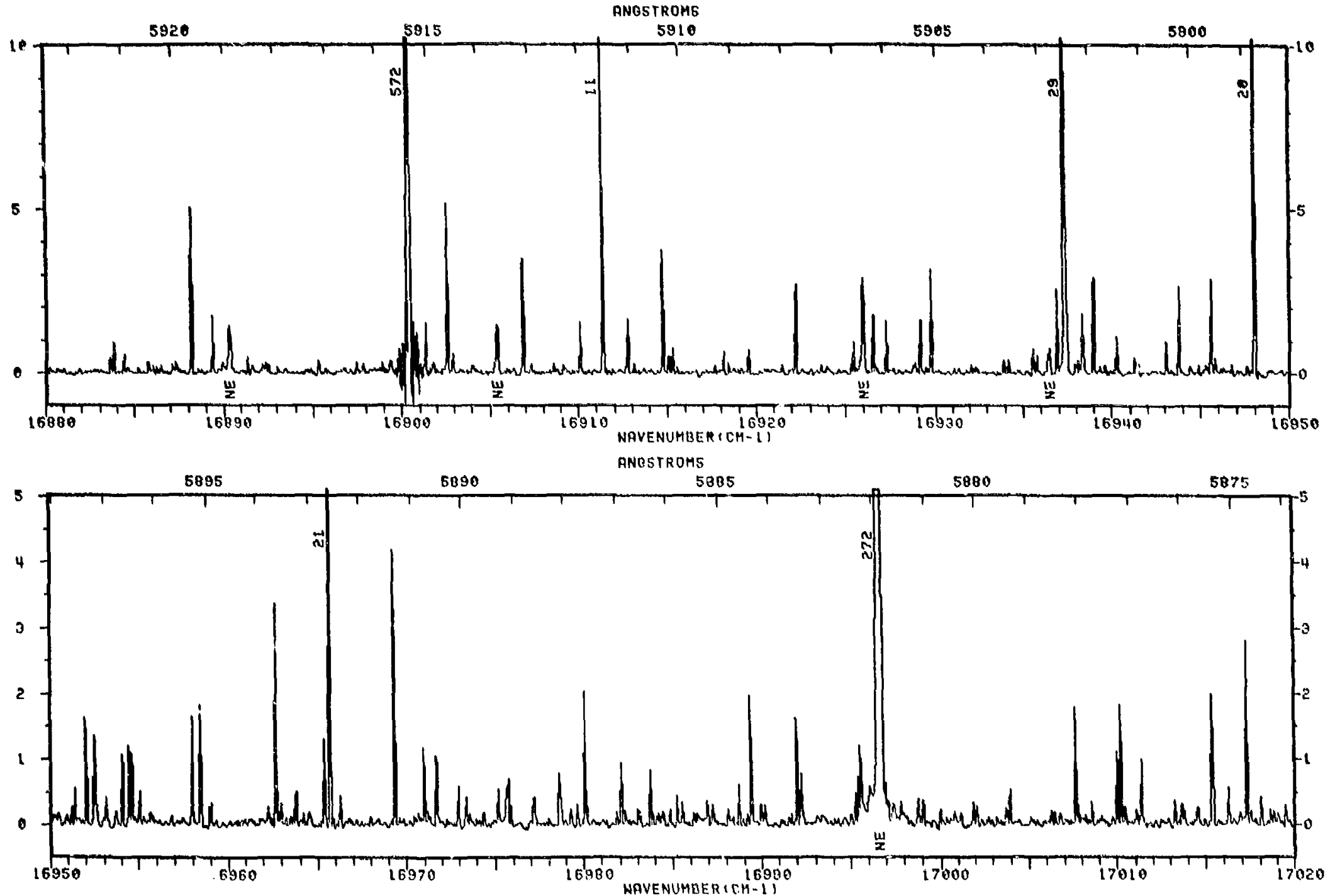

87 


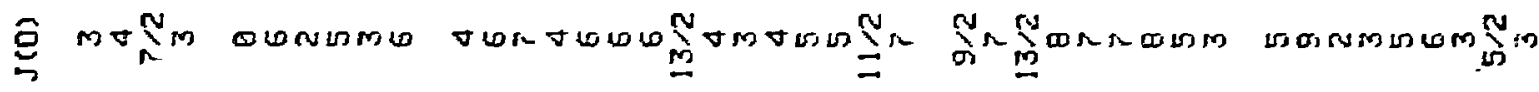

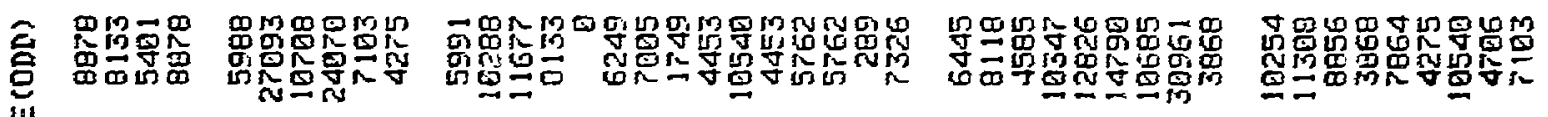

$=m$ m

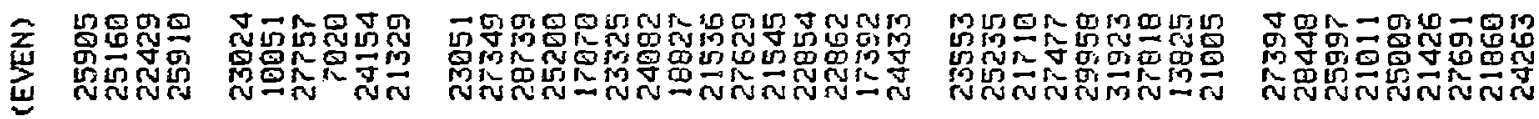

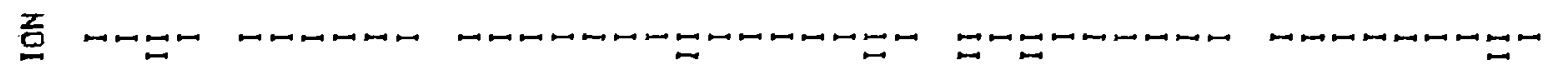

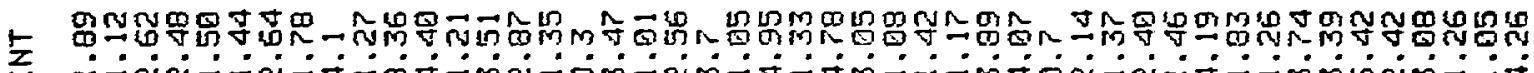

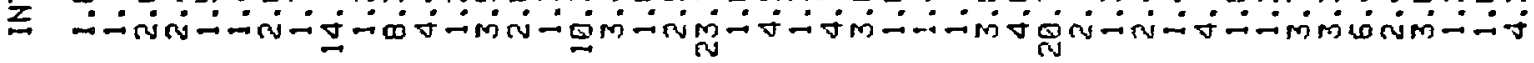

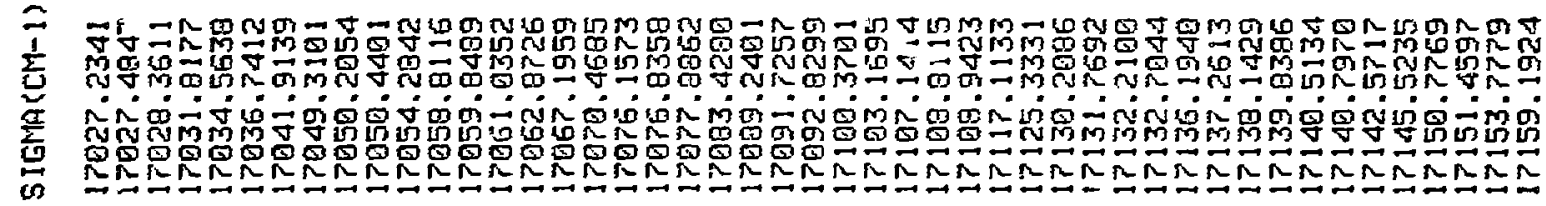

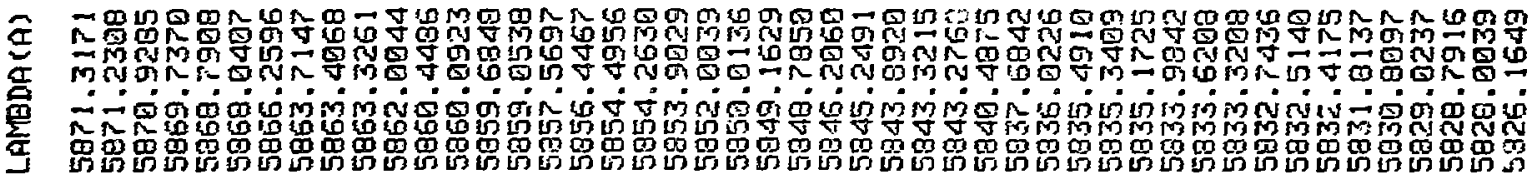



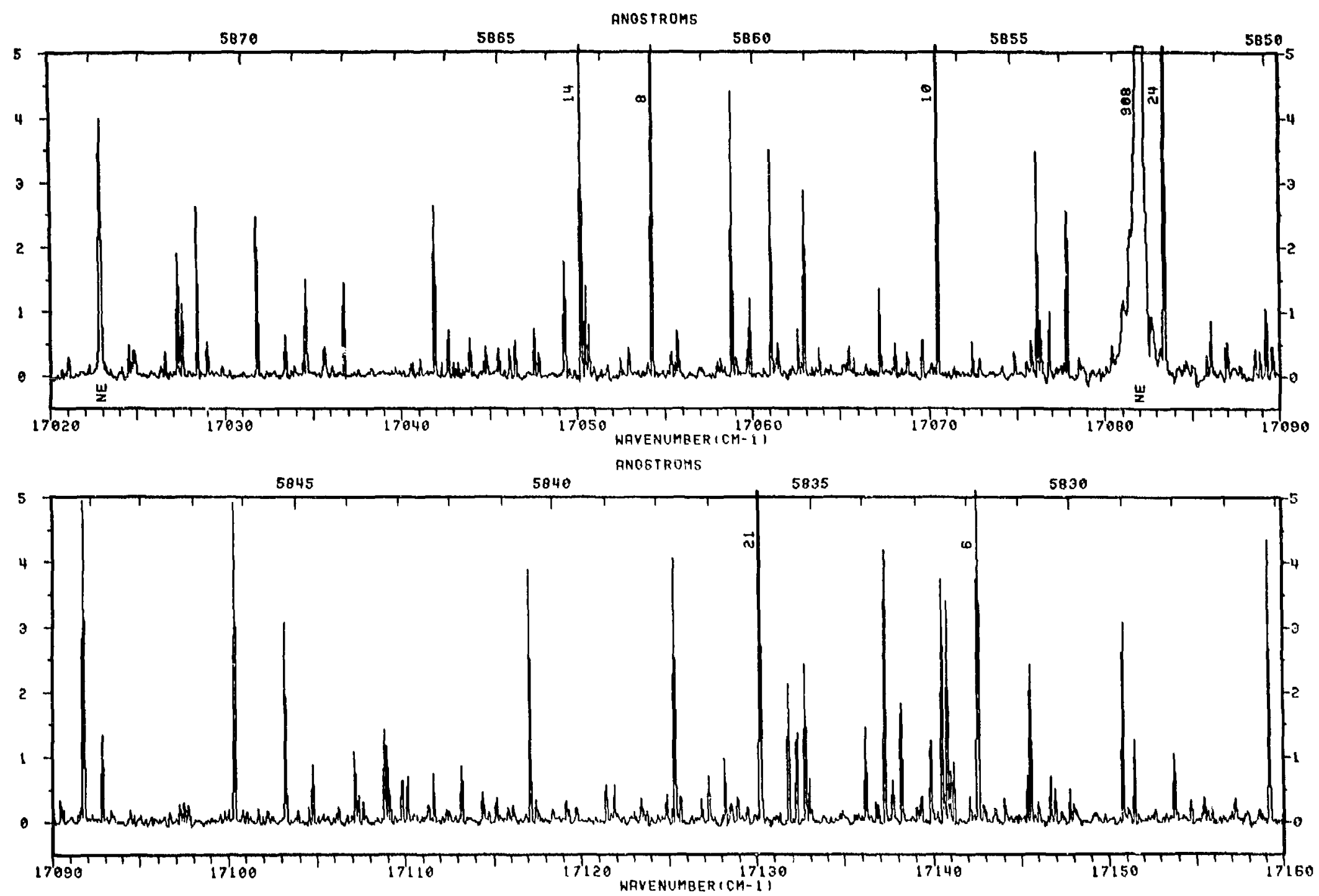


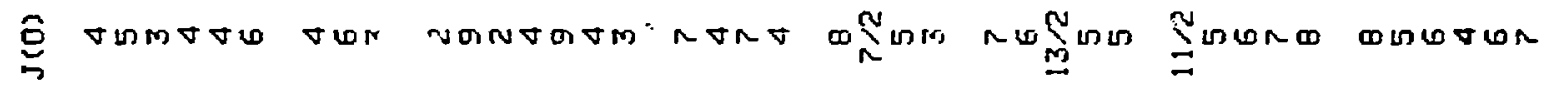

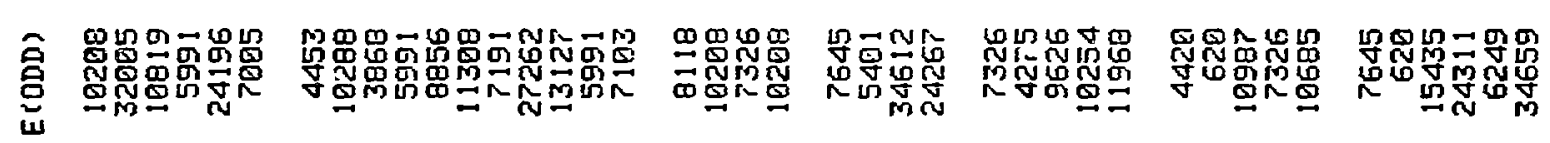

$\stackrel{9}{9}$

จルกM

$\operatorname{con} \backsim \int_{\pi}^{\pi}$

$\cos \infty \sum_{\pi}^{\infty} 0000$

$\cos 2 \pi \cos$

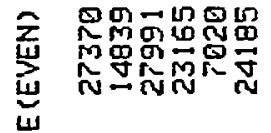

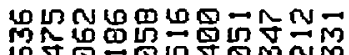

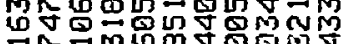

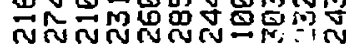

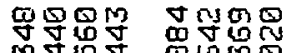

m约需

กิñ

ging

N $N$

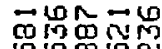

a)

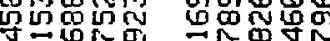

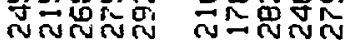

M四罗品

$\operatorname{Tr}$

N I

ż口<smiles>[CH]C=[CH]</smiles>
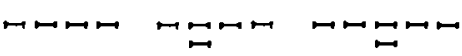

$\varpi$

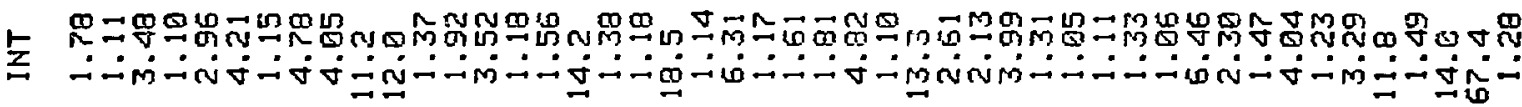

ด

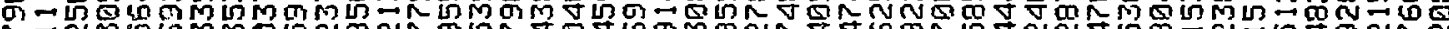

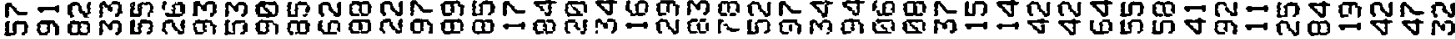

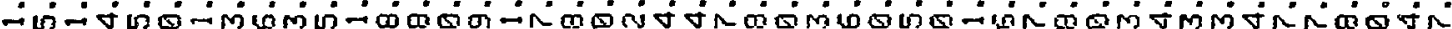
ம

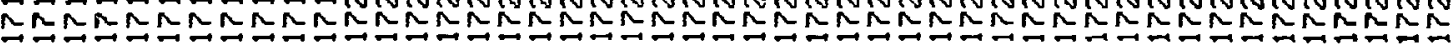

L Ұㅁำ

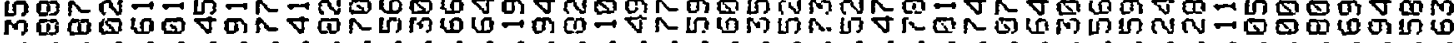

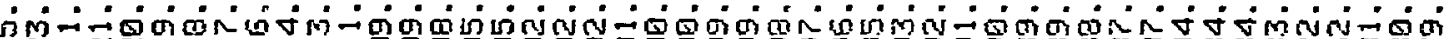

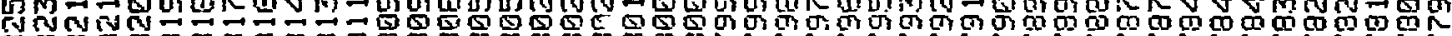

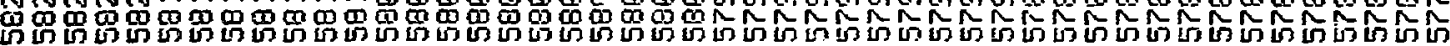



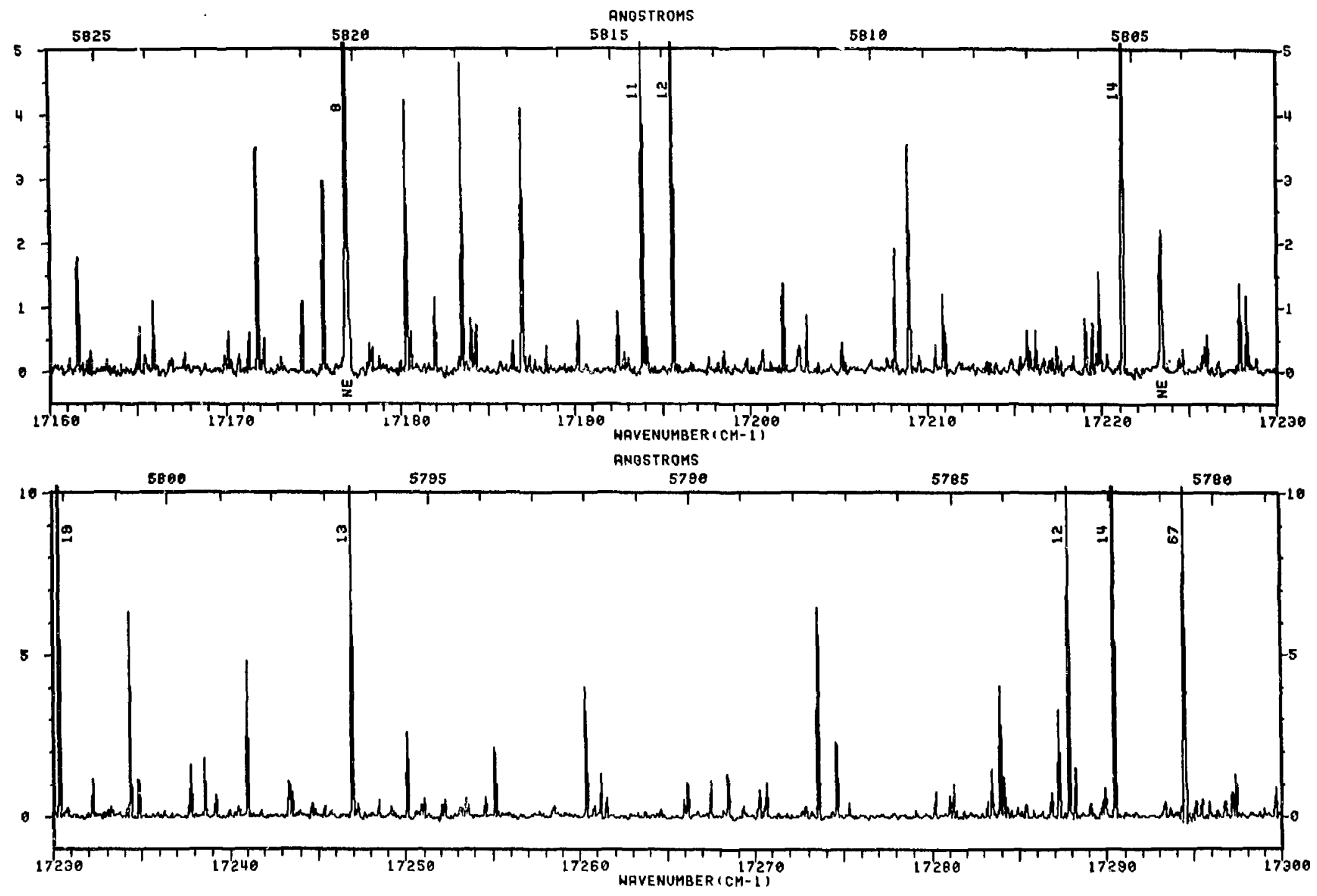


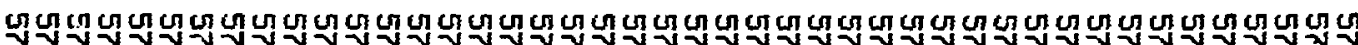
WWWWWWWWWN

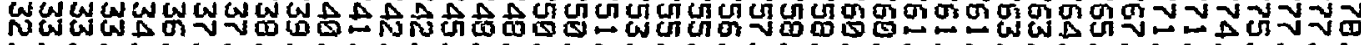

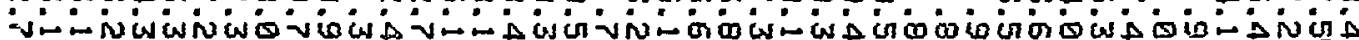
Nم

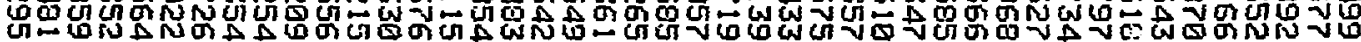

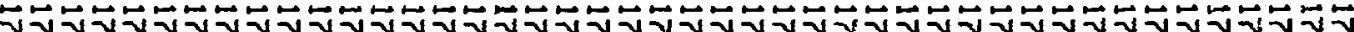
A

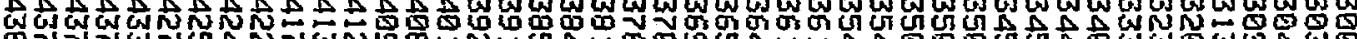

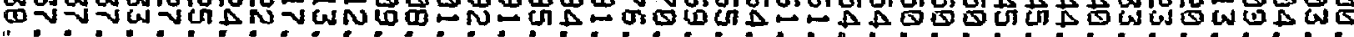
gin

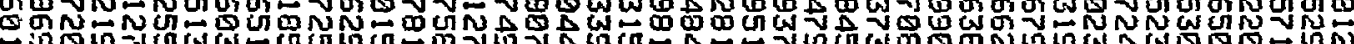

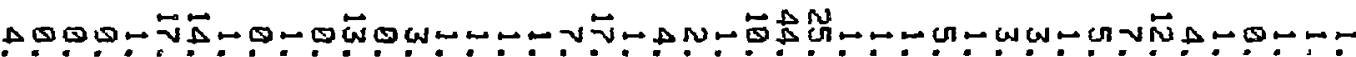

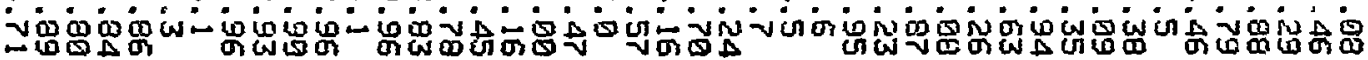
$\ddot{n}$

$\because$

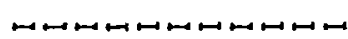

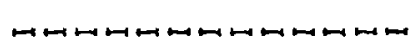

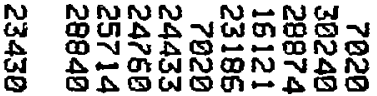

ปู

$N N N \mapsto N N \mapsto N N \Omega$

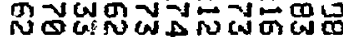

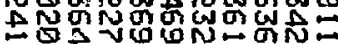

NNNNNNNNNMNMN

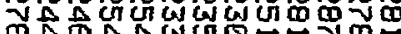

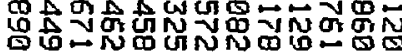

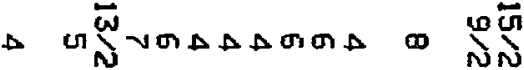

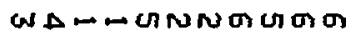

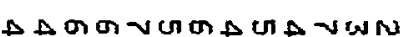

$\sum_{\frac{m}{z}}^{m}$

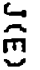

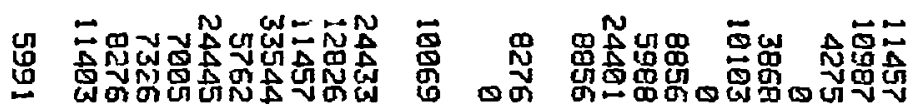

$\Delta \triangle \vec{\omega} v \sin a \cos v$ v

NUONOR WTOMT

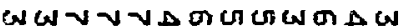

总

品 

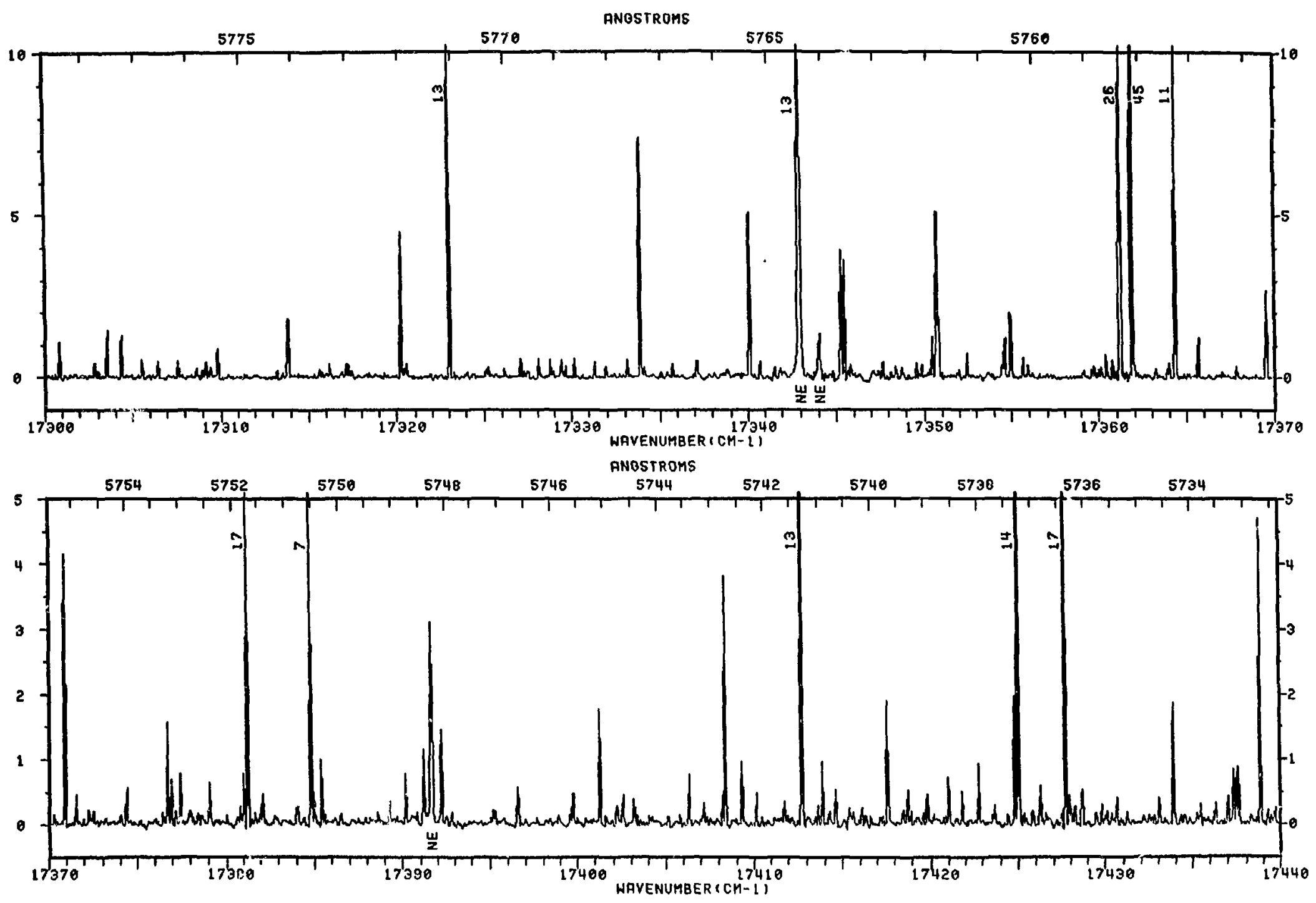

93 


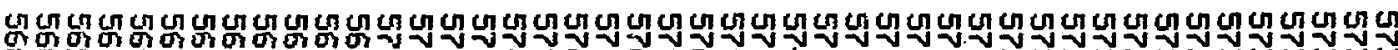

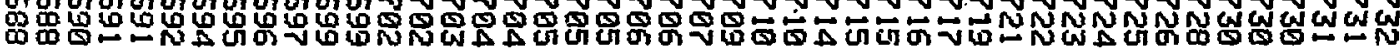
-

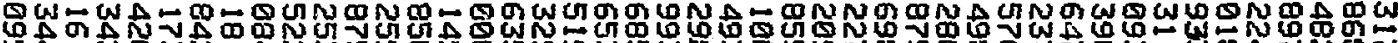

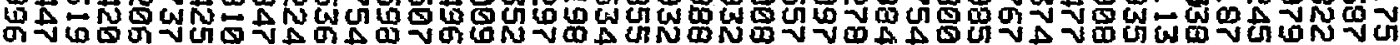

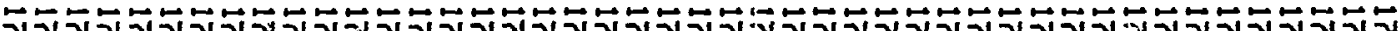

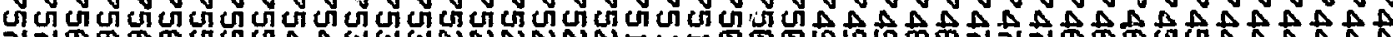

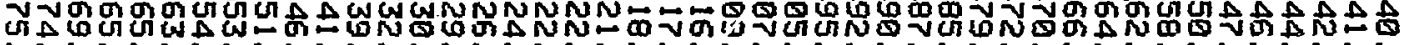

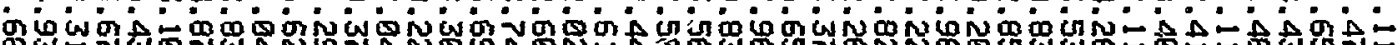

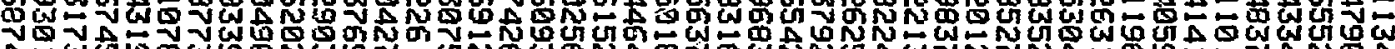
А

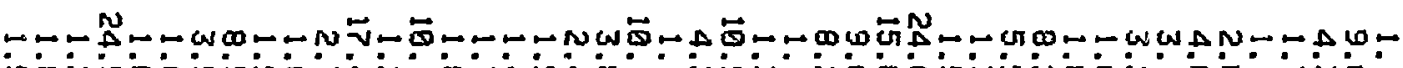

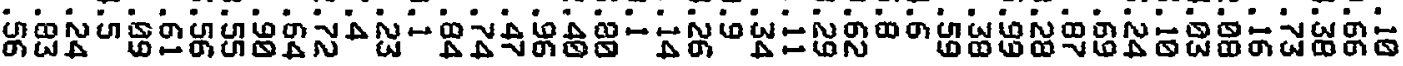

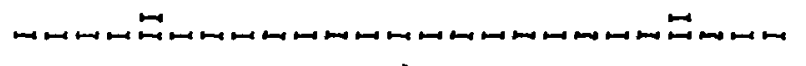

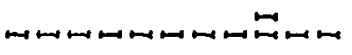

ロேロロロッே

WGNGNANGWN NWNNNNNAN NNGNN

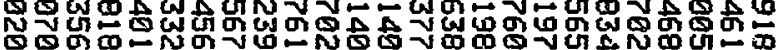

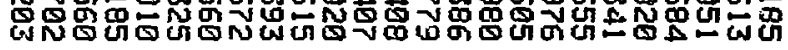

NNNNN NONNM Gた

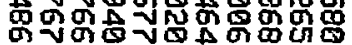

NGM

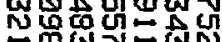

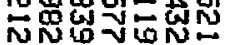



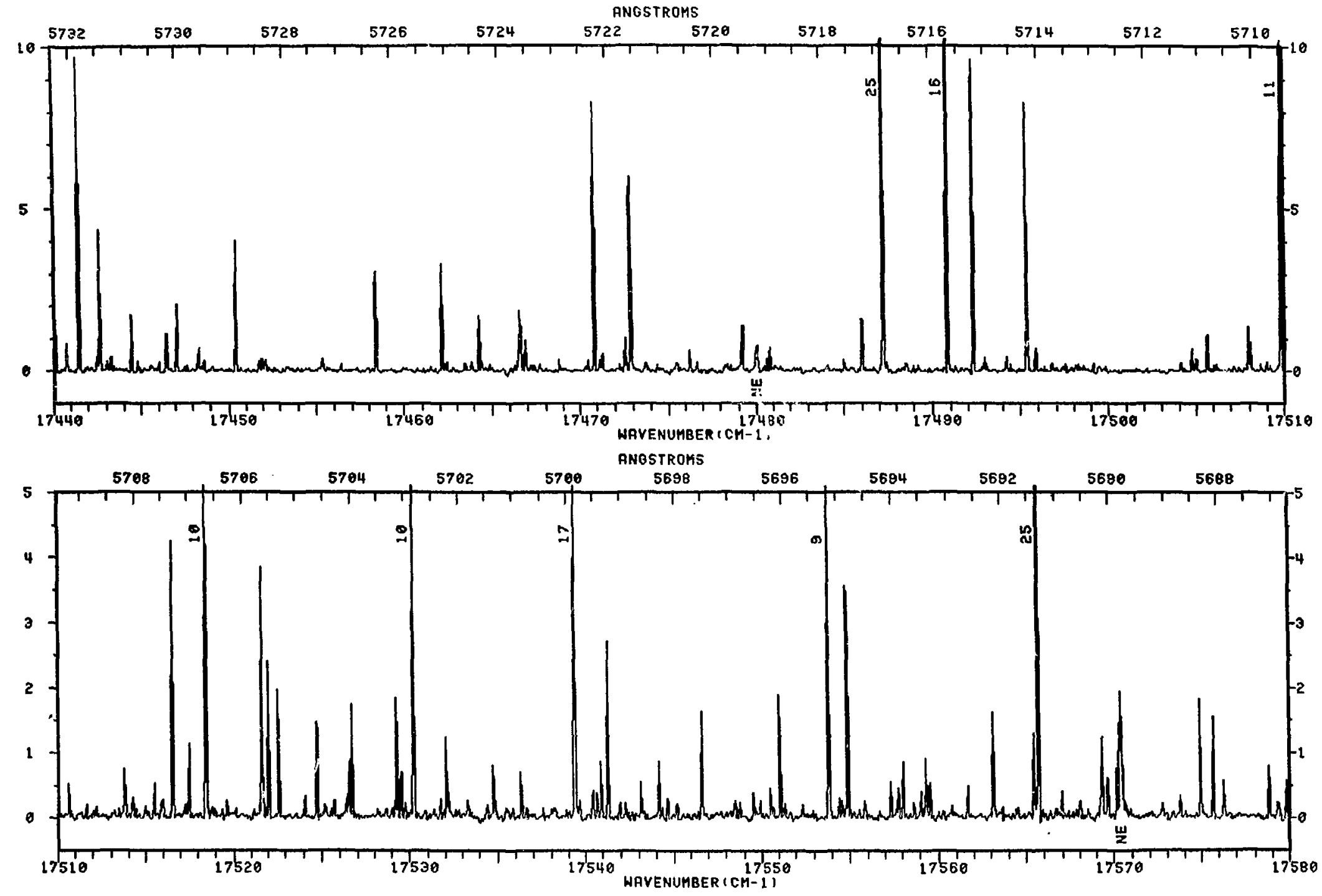


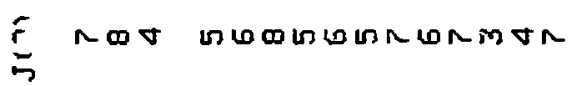

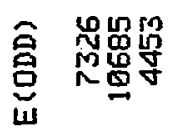

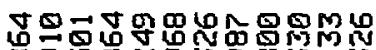

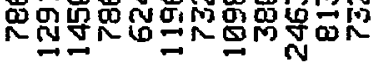

$\infty \boldsymbol{\infty}$

它罢 MM

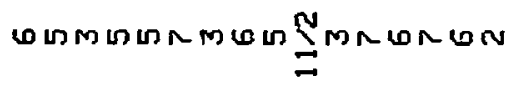

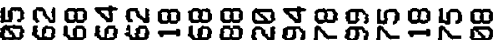

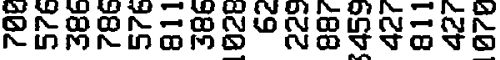

艺

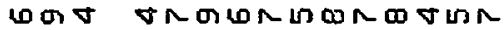

non

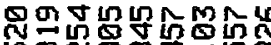

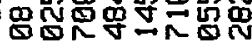

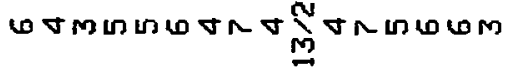

ー

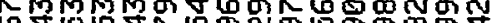

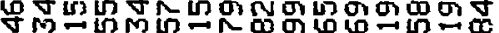

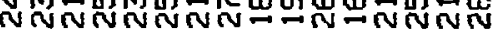

믐

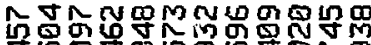

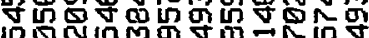

\%

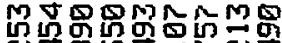

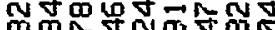

政

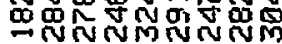
TNO 

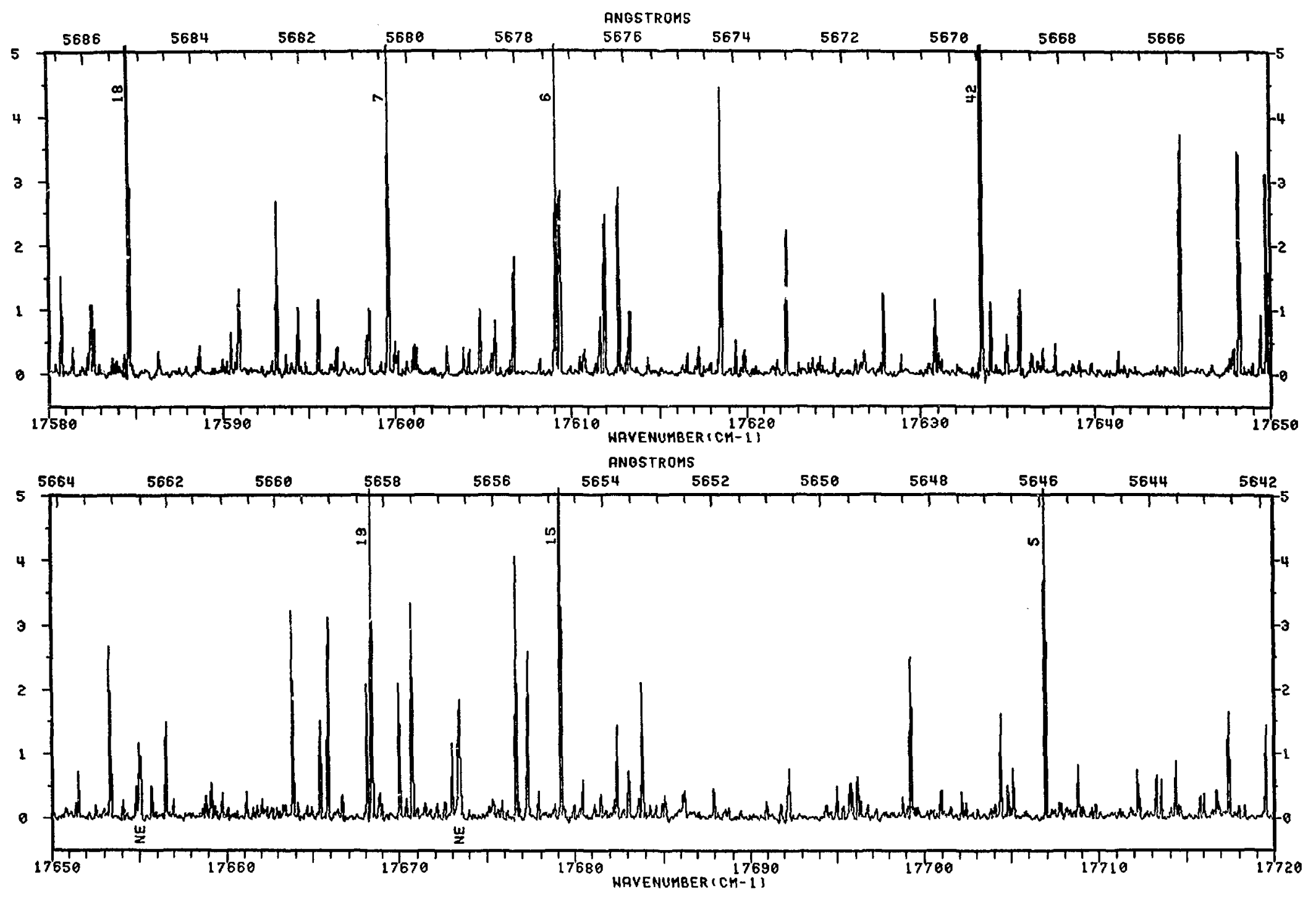

97 
各 ロロロロロロロロ
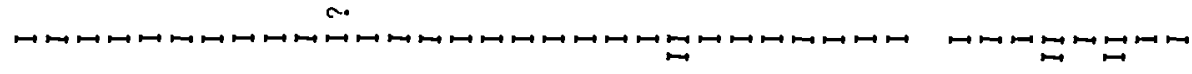

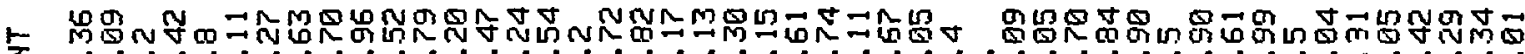

Z Ni⿻日禸

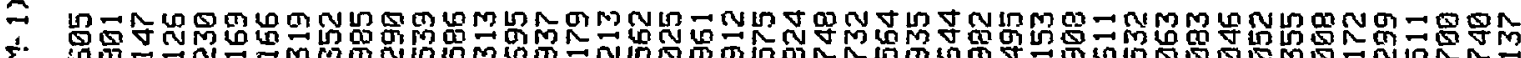

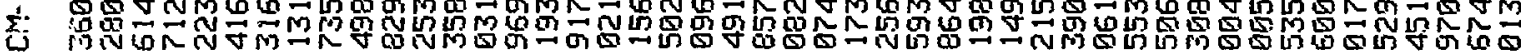

-

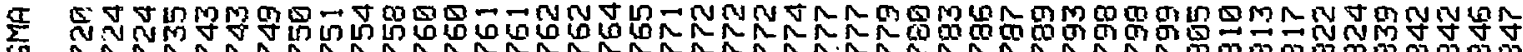

m

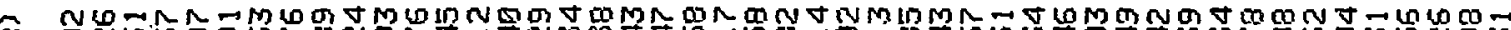

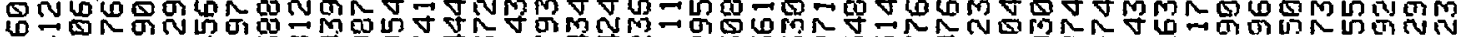

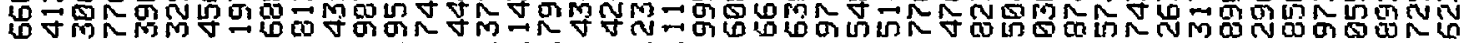
-ه் Ұ

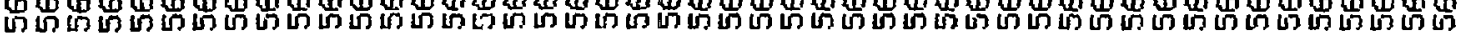



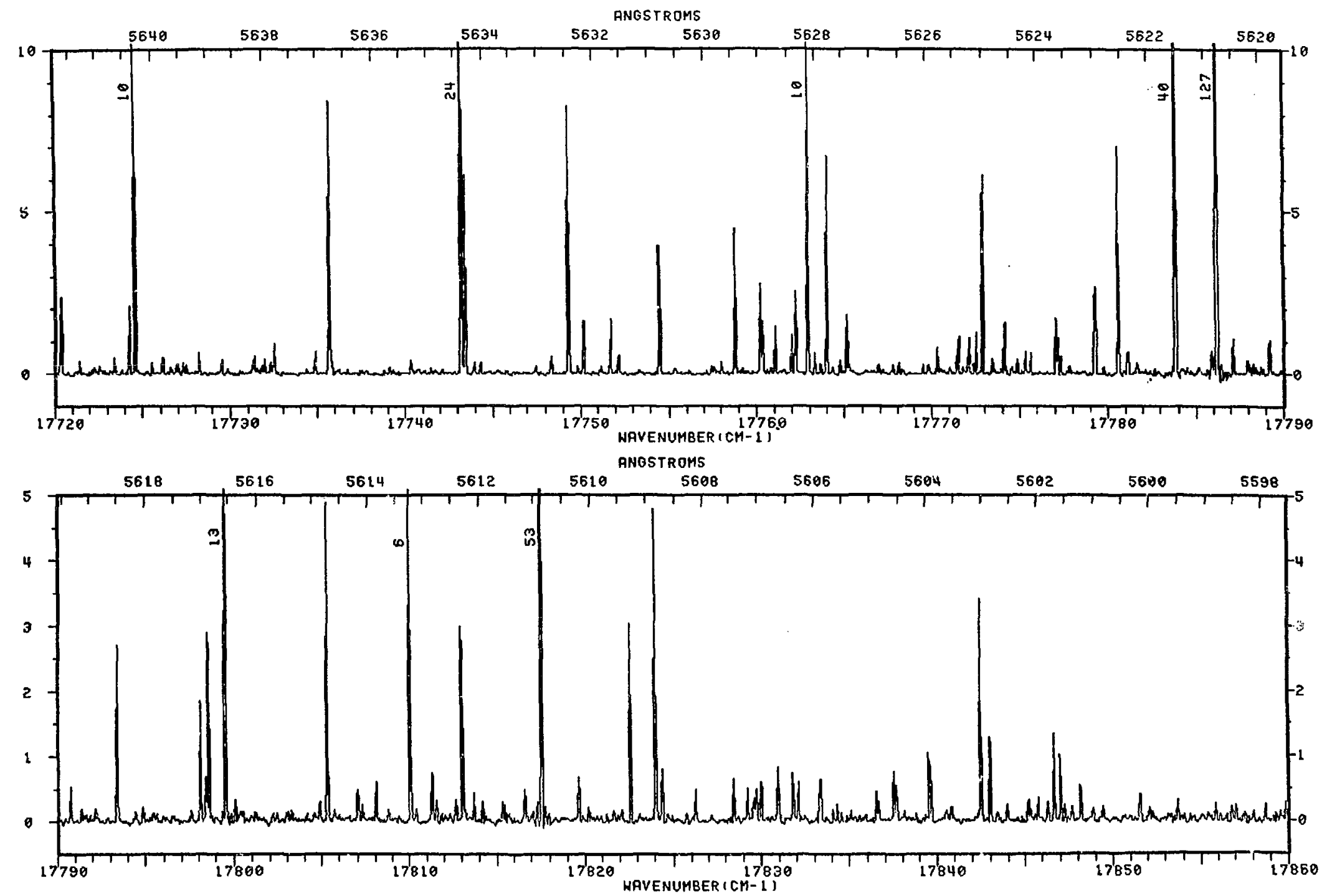


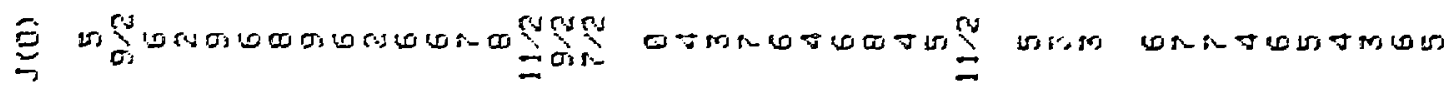

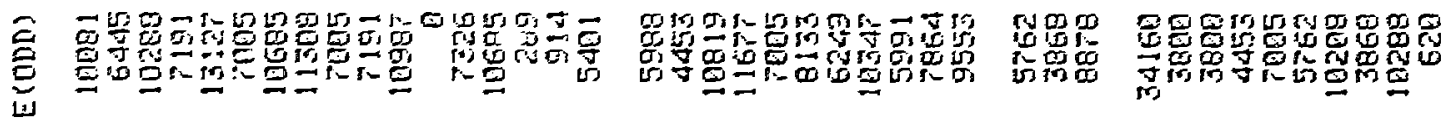

些

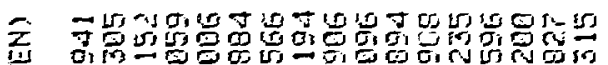

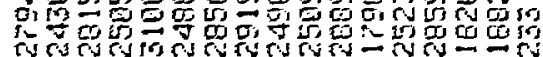

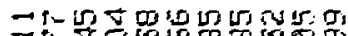

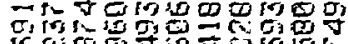

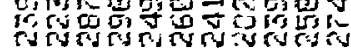

159 ing âd

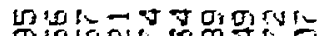
Tis

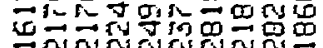
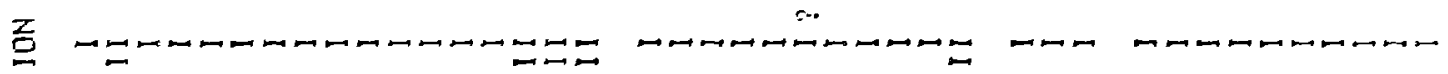

至

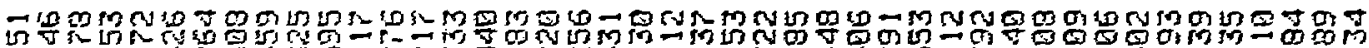

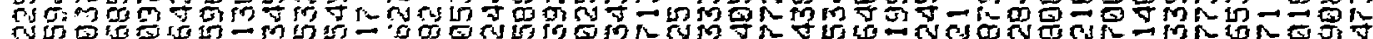
-

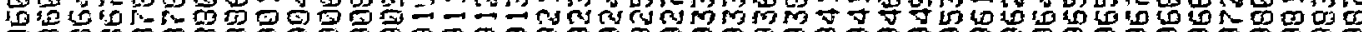

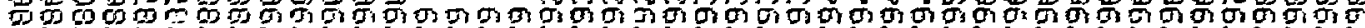

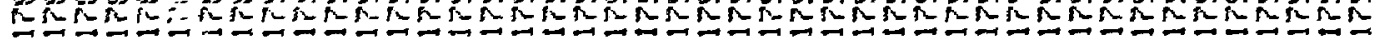

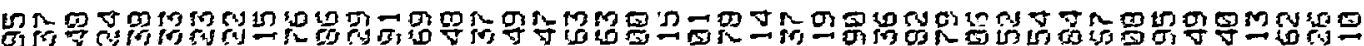

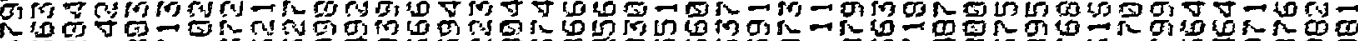

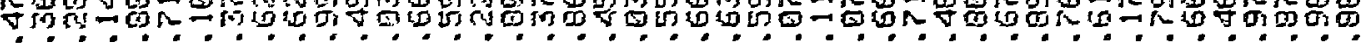

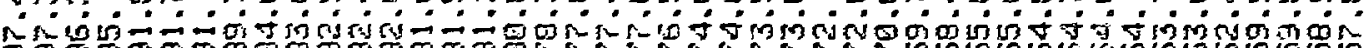

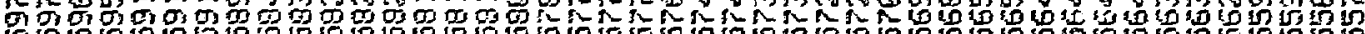

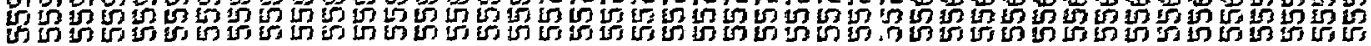



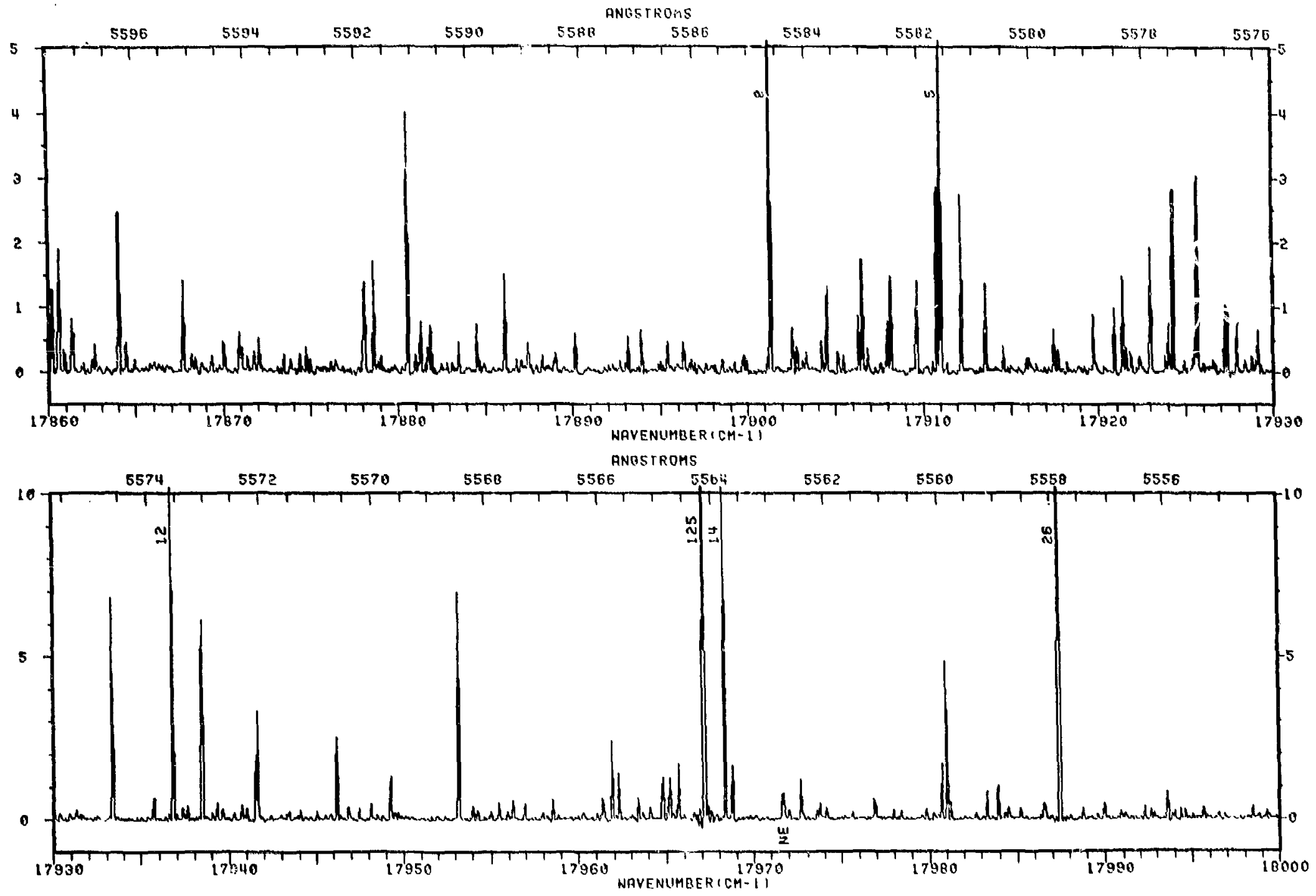
马 m⿻

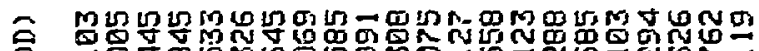

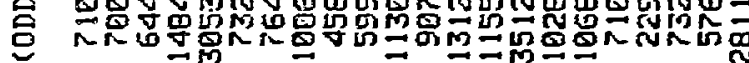

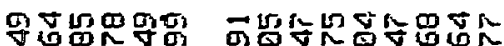

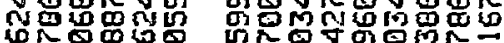

$\sim \sim \tilde{\sigma}_{1} \dot{0}$

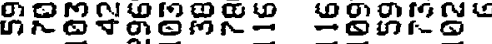

w$$
\text { 穴 }
$$

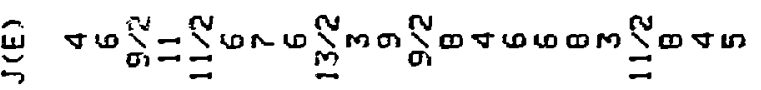

$\operatorname{lon} \nabla \pi \stackrel{2}{2}$

แ⿻

OMN

$\sum_{w}^{\underline{w}}$

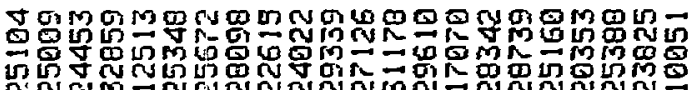

TM $\rightarrow M M$

Gog

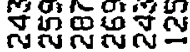

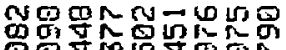

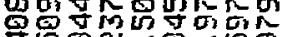

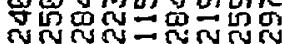

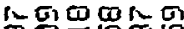

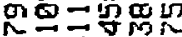

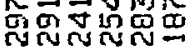

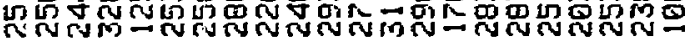

금

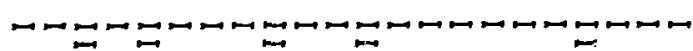

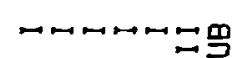

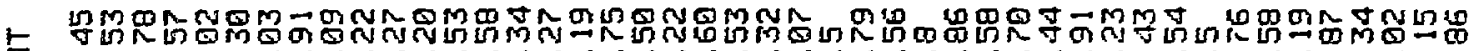

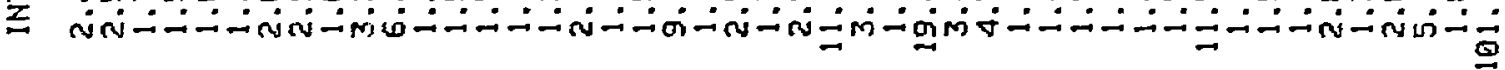

竞

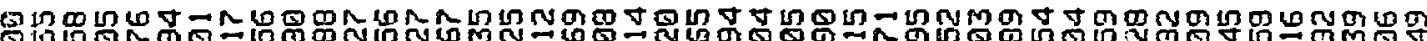

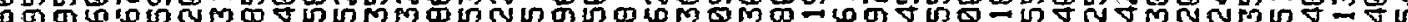

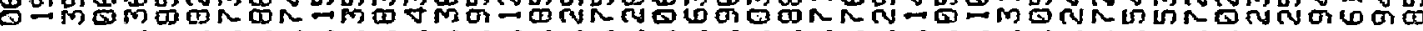

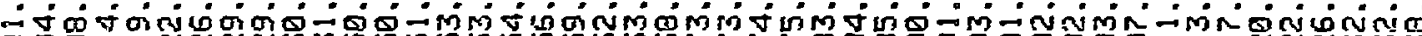

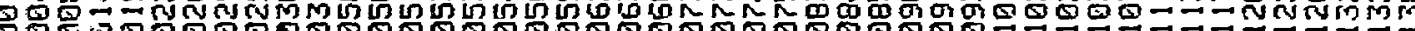

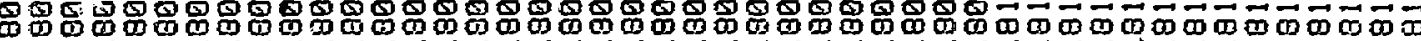



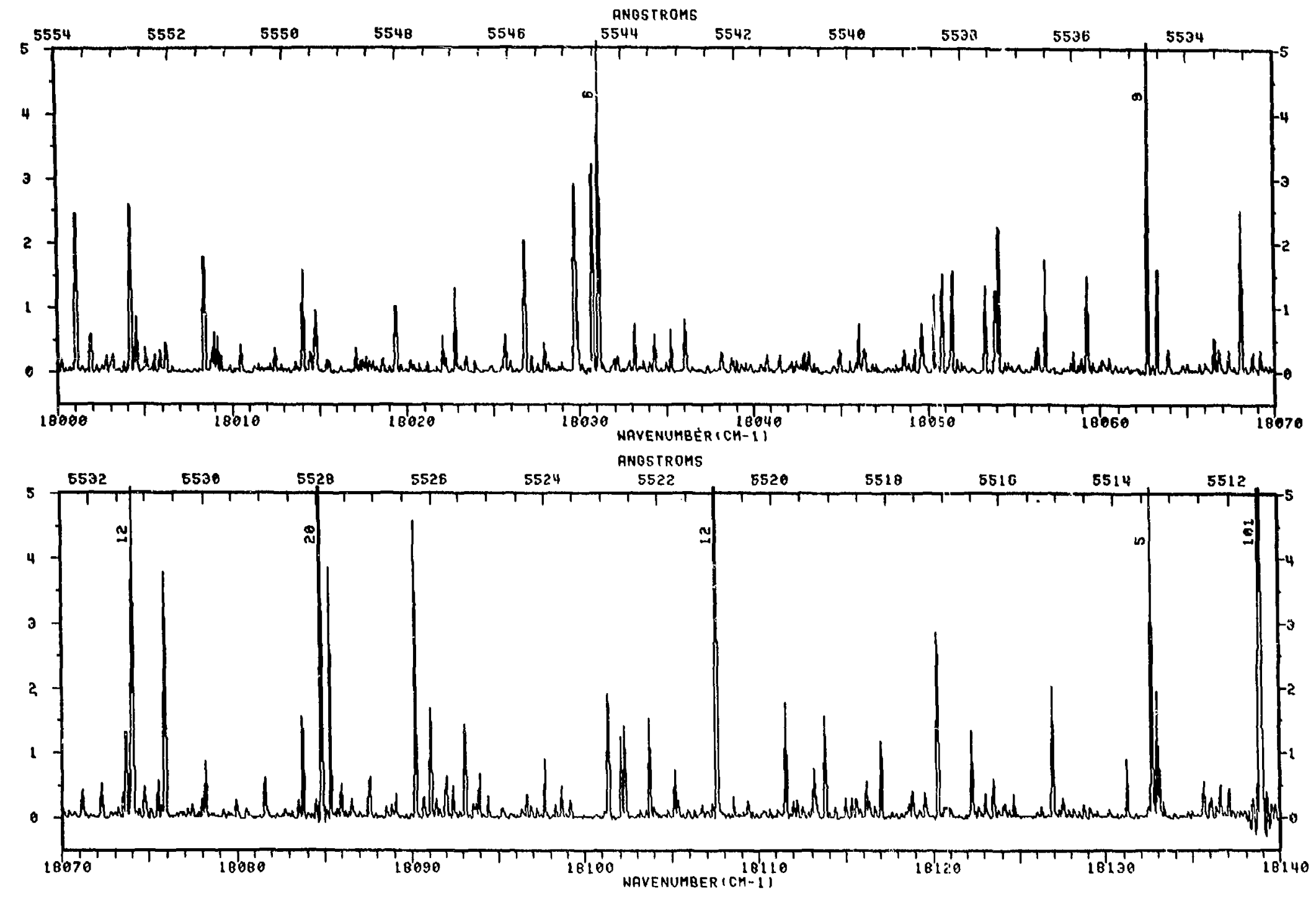
g

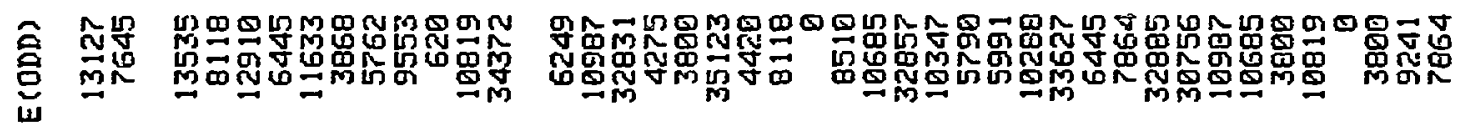

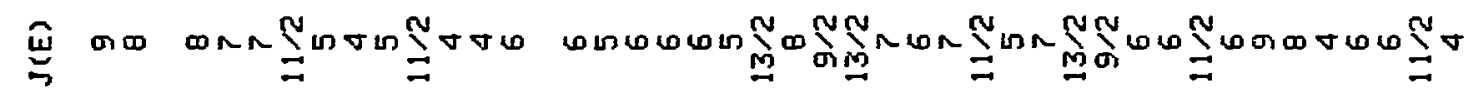

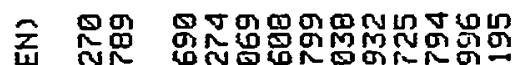

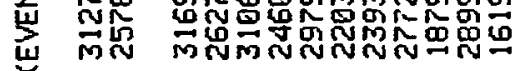

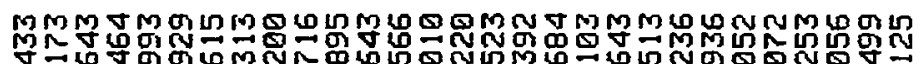

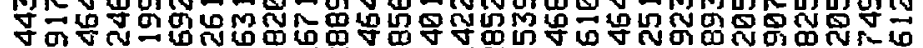
w W

豆

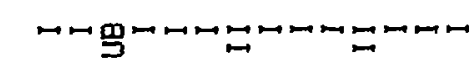

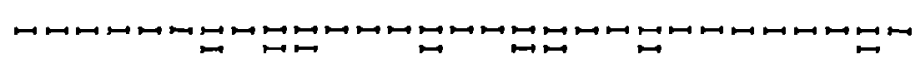

₹

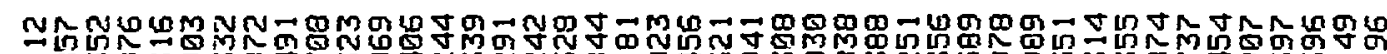

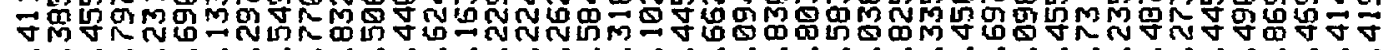

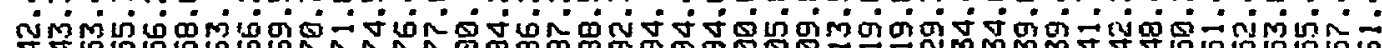

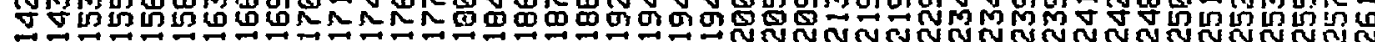

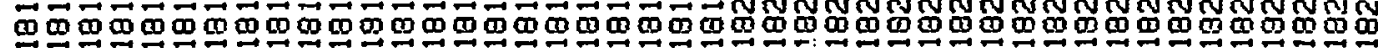

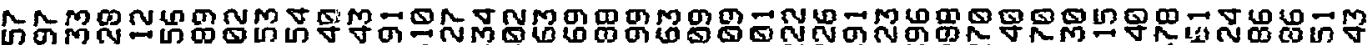

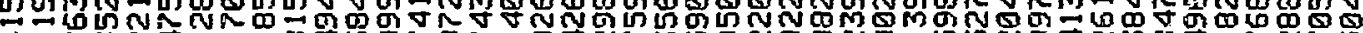

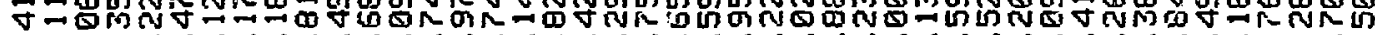

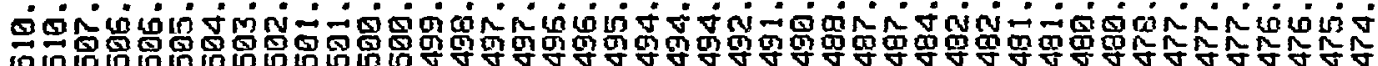

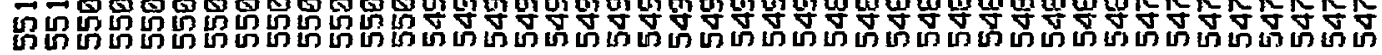



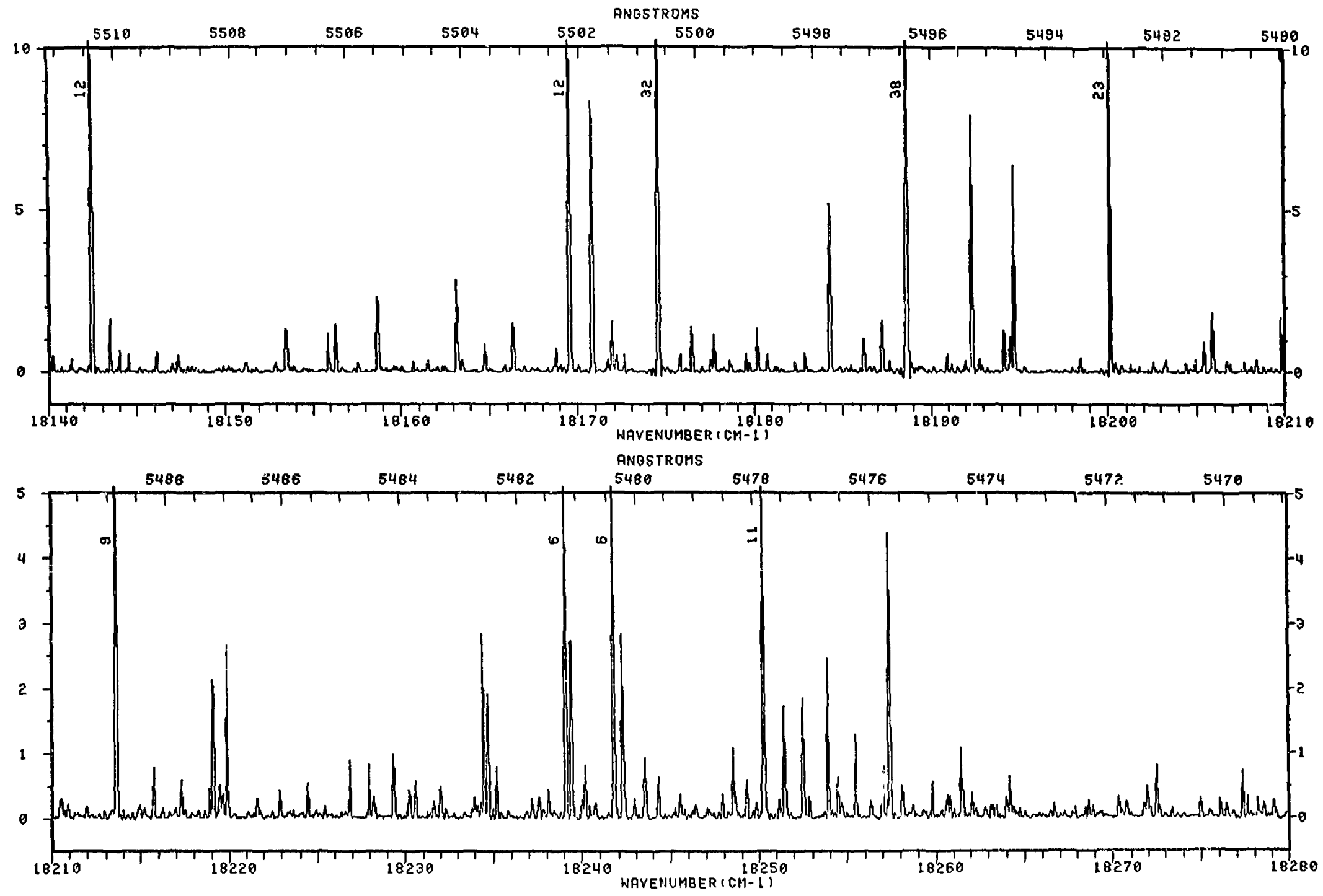


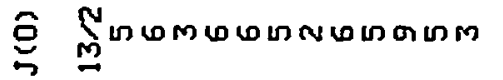

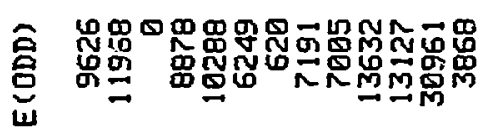

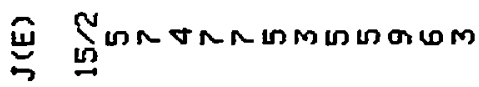

密

Nํำ

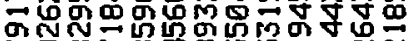

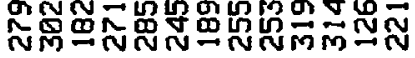

mowormon

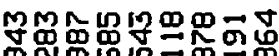

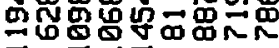

$\checkmark \stackrel{N}{\Xi} 00000 \mathrm{mmv}$

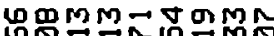

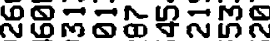

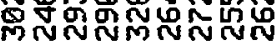

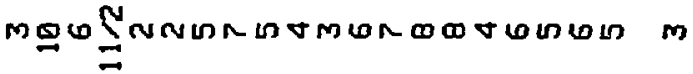

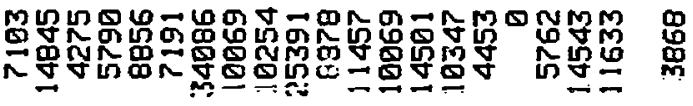

$\nabla$

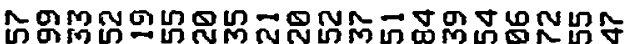

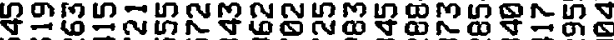
फ़N

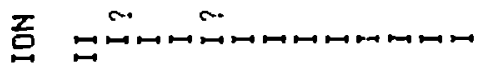

a.

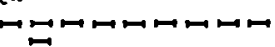

$\mapsto \mapsto \mapsto \mapsto$

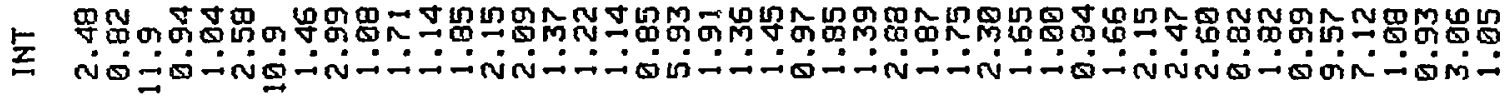

MM

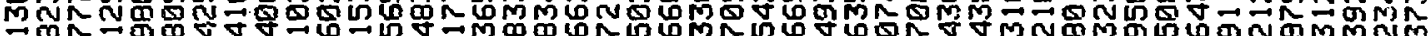

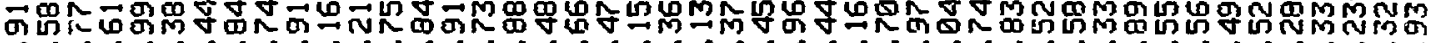

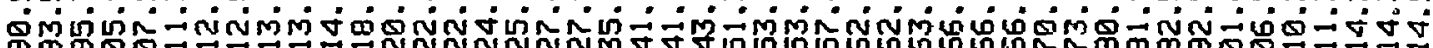

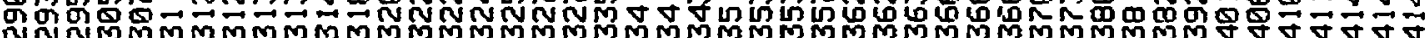

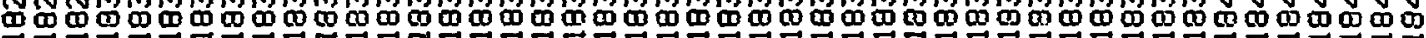

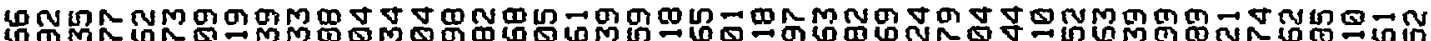
COM D

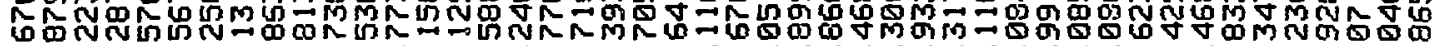
n்ं

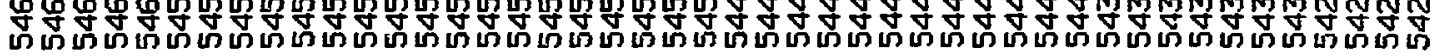




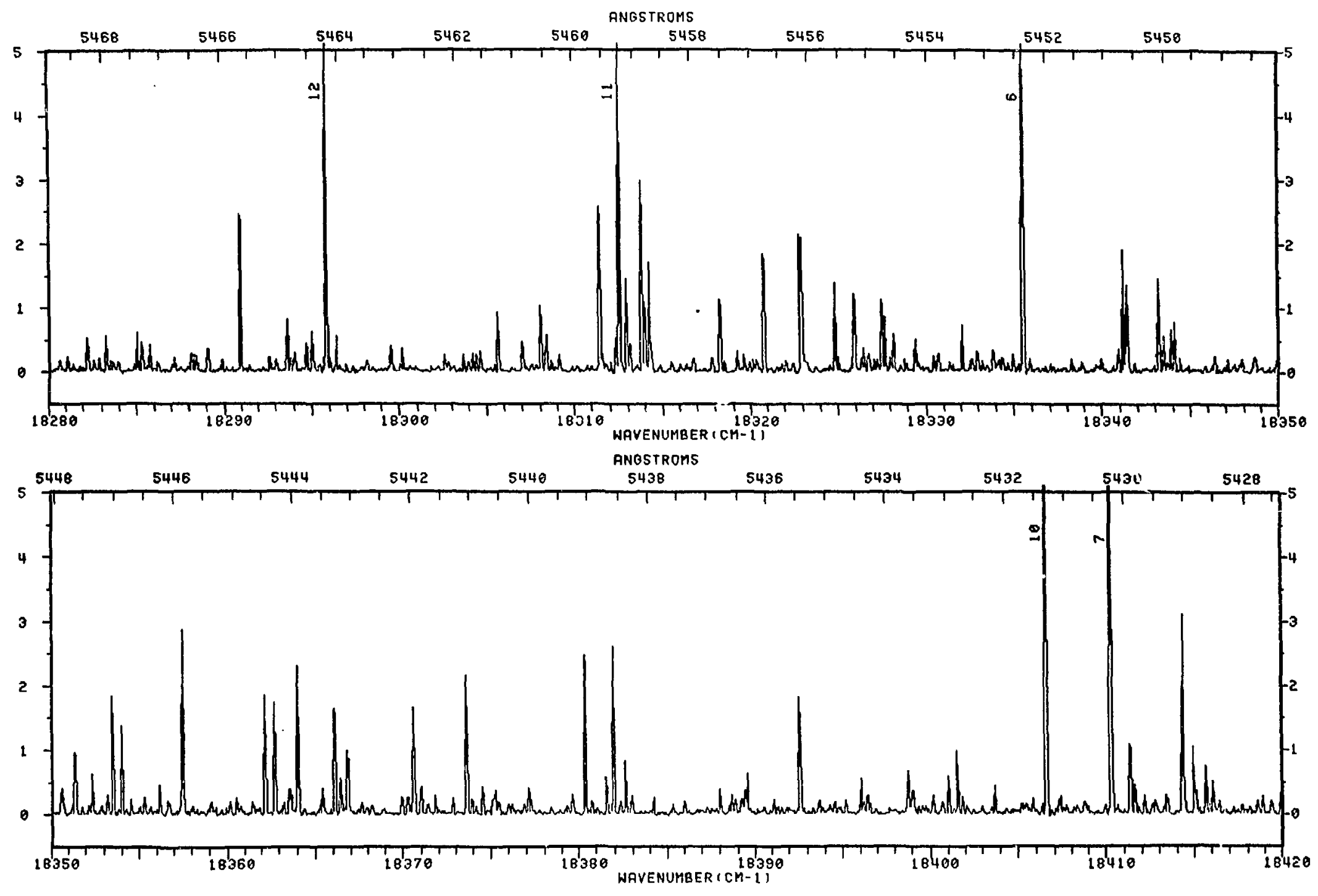


gु

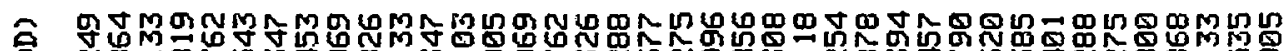

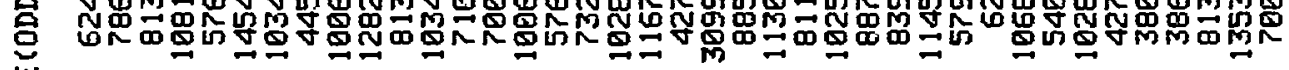

$$
\text { (1) }
$$

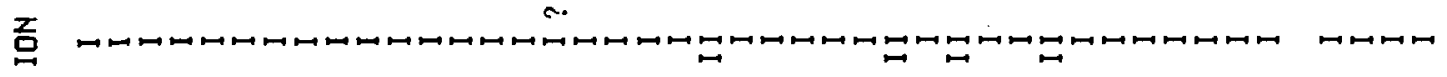

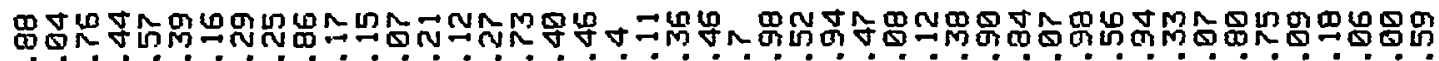

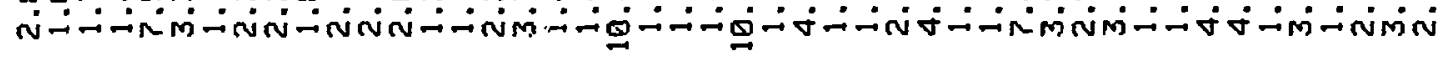

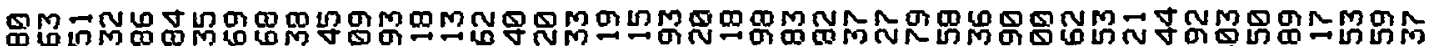

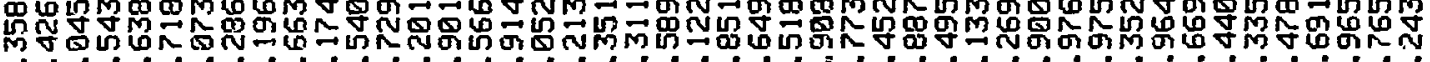

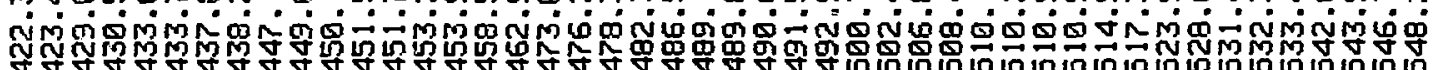
ఏ

M

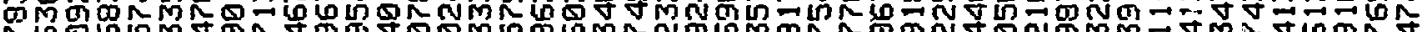

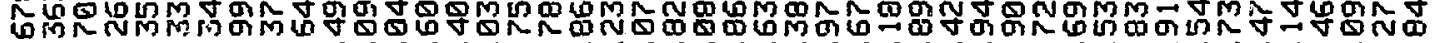

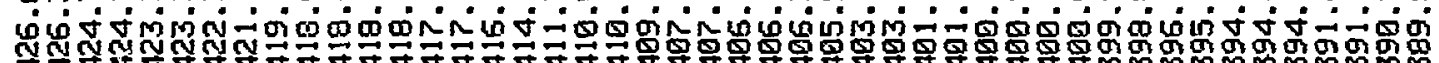
ตัง 

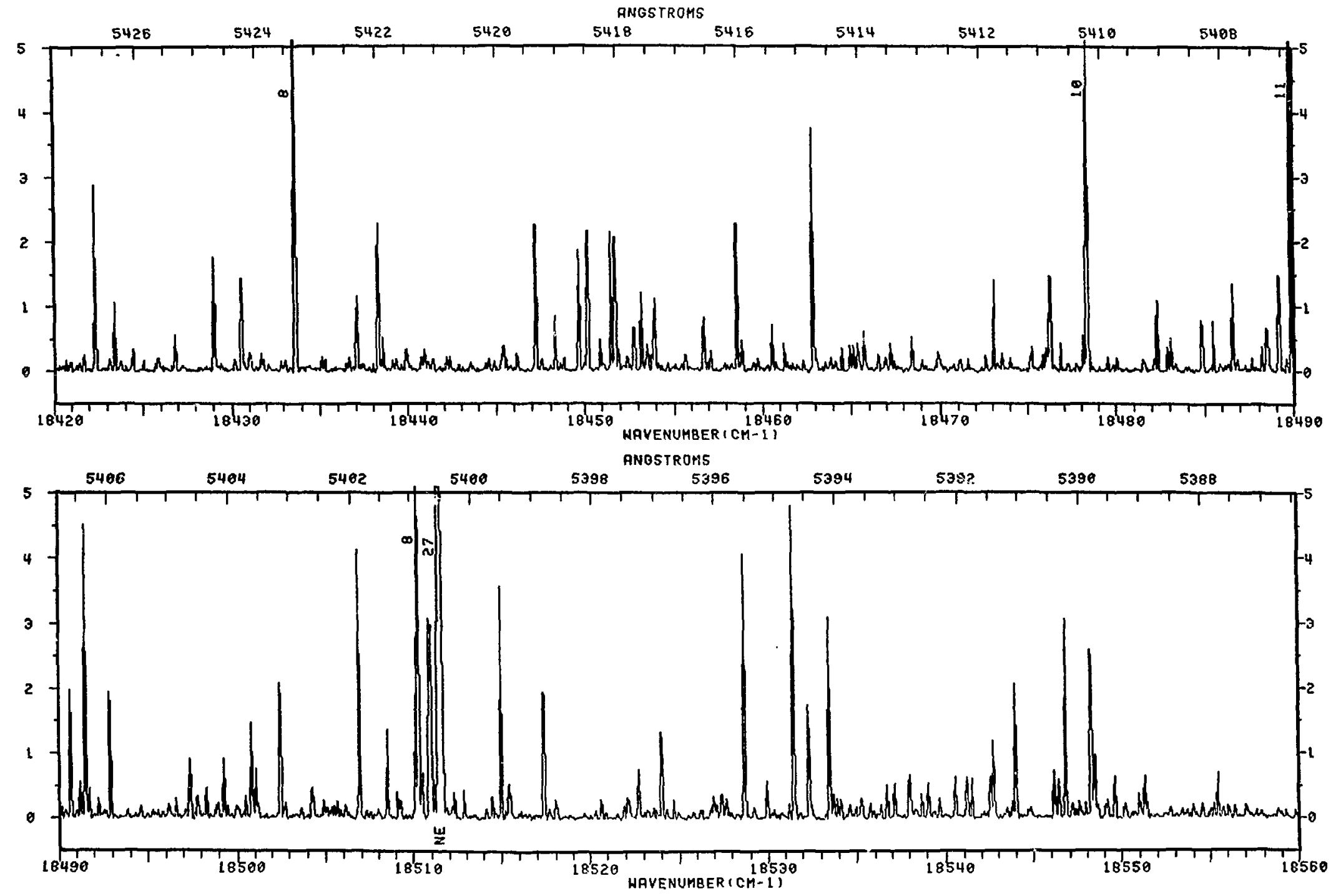


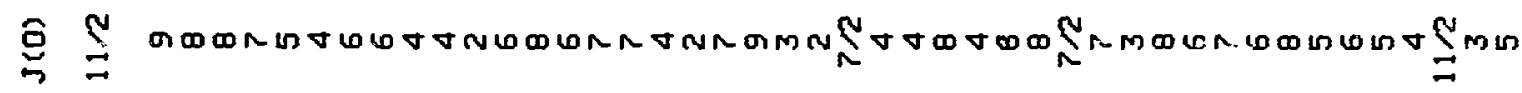

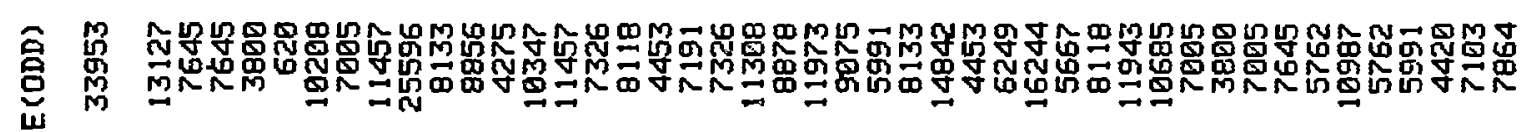

यू.

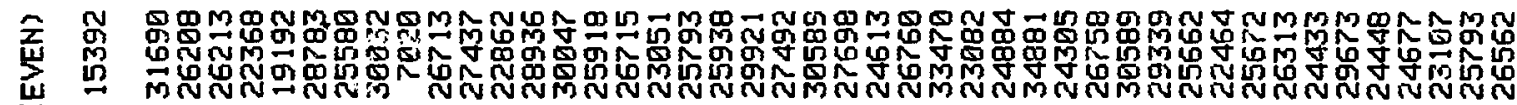

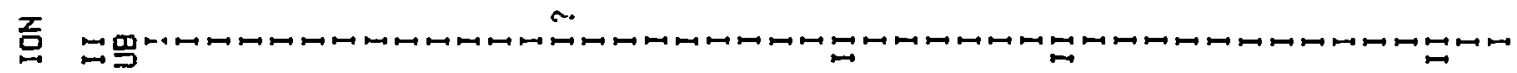

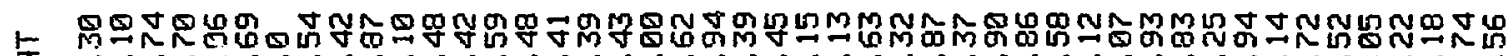

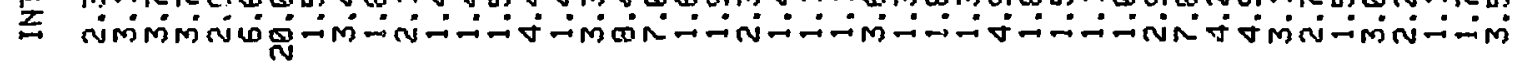

$\frac{7}{1}$
$\frac{1}{0}$
$\frac{1}{2}$
$\frac{1}{5}$
0
0

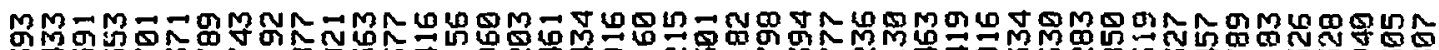
Mำ

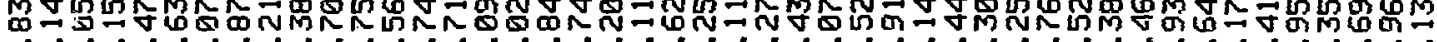
बinmiñ on

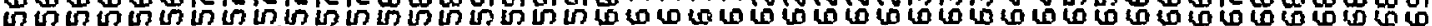

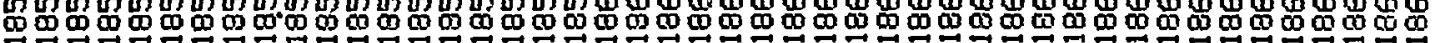

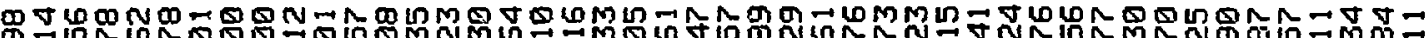

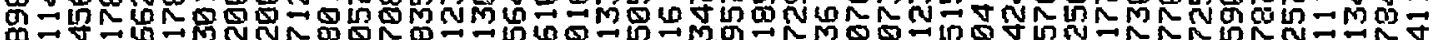

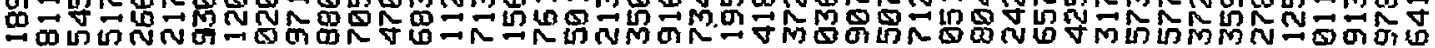
ம்

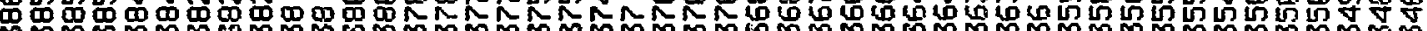

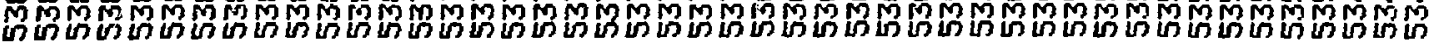




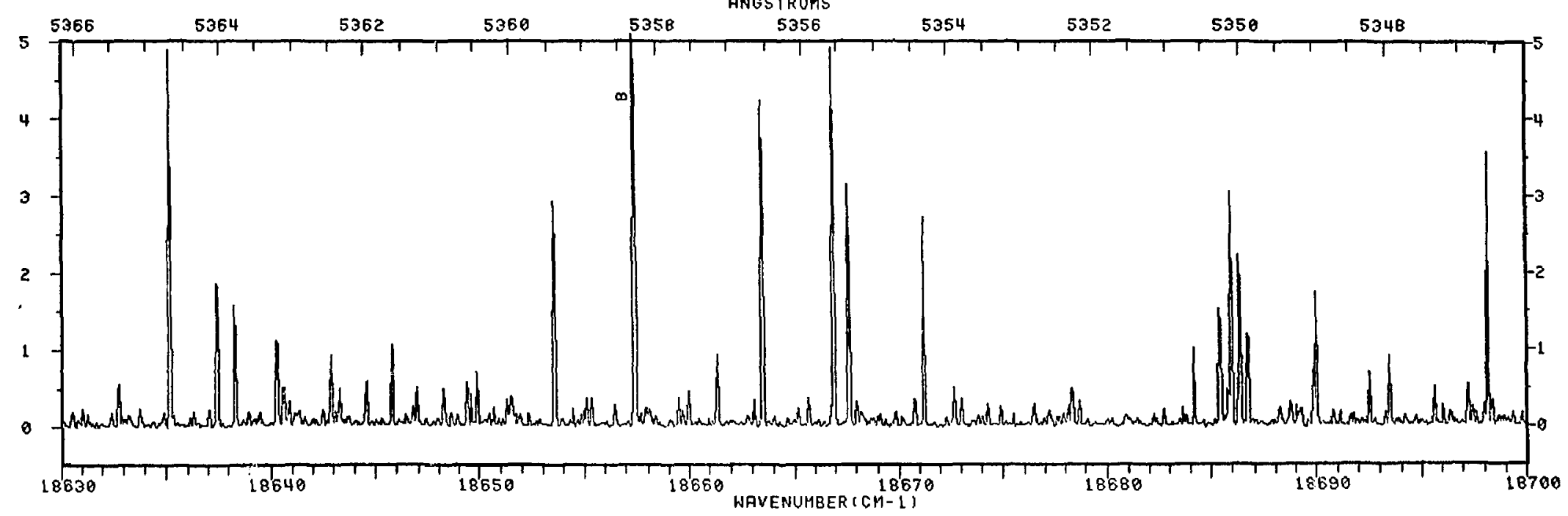




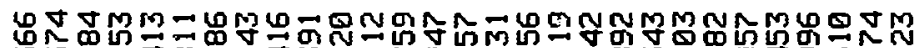

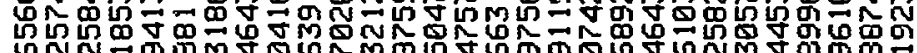

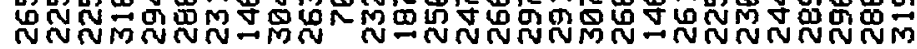

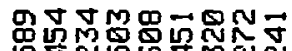

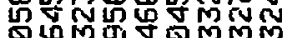
MNMUNW MNM

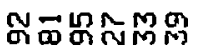

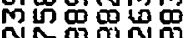

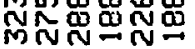

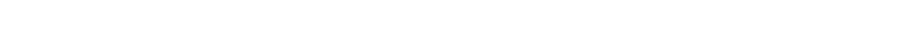

$a$

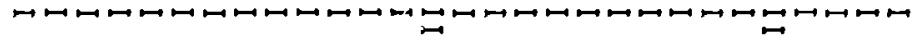

$a$

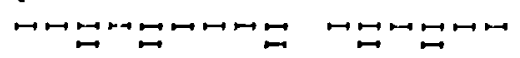

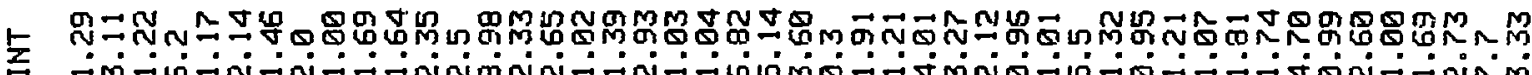

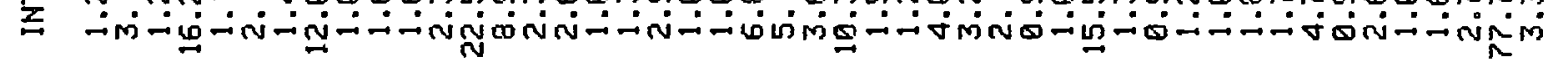

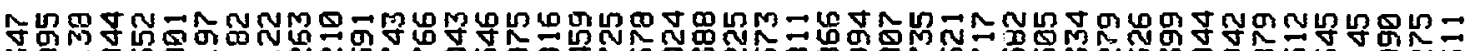

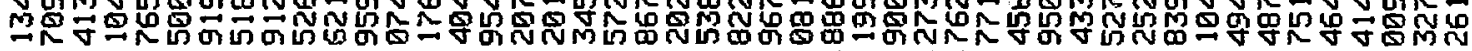

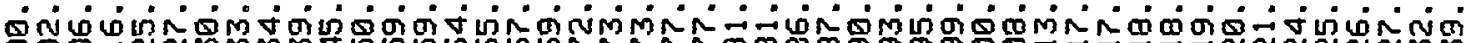

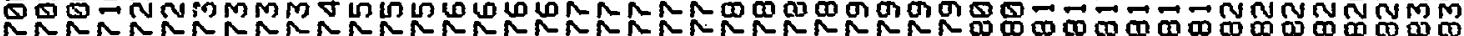

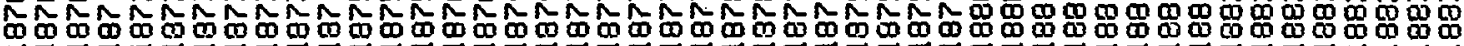

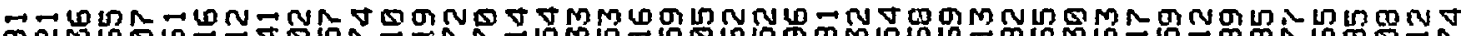

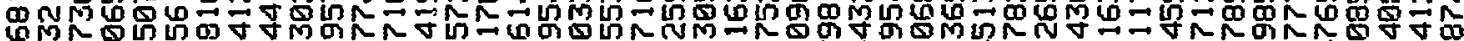

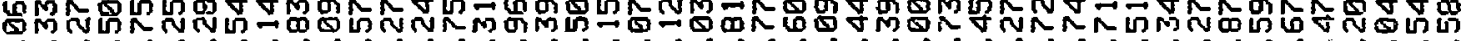
乌ீं м 


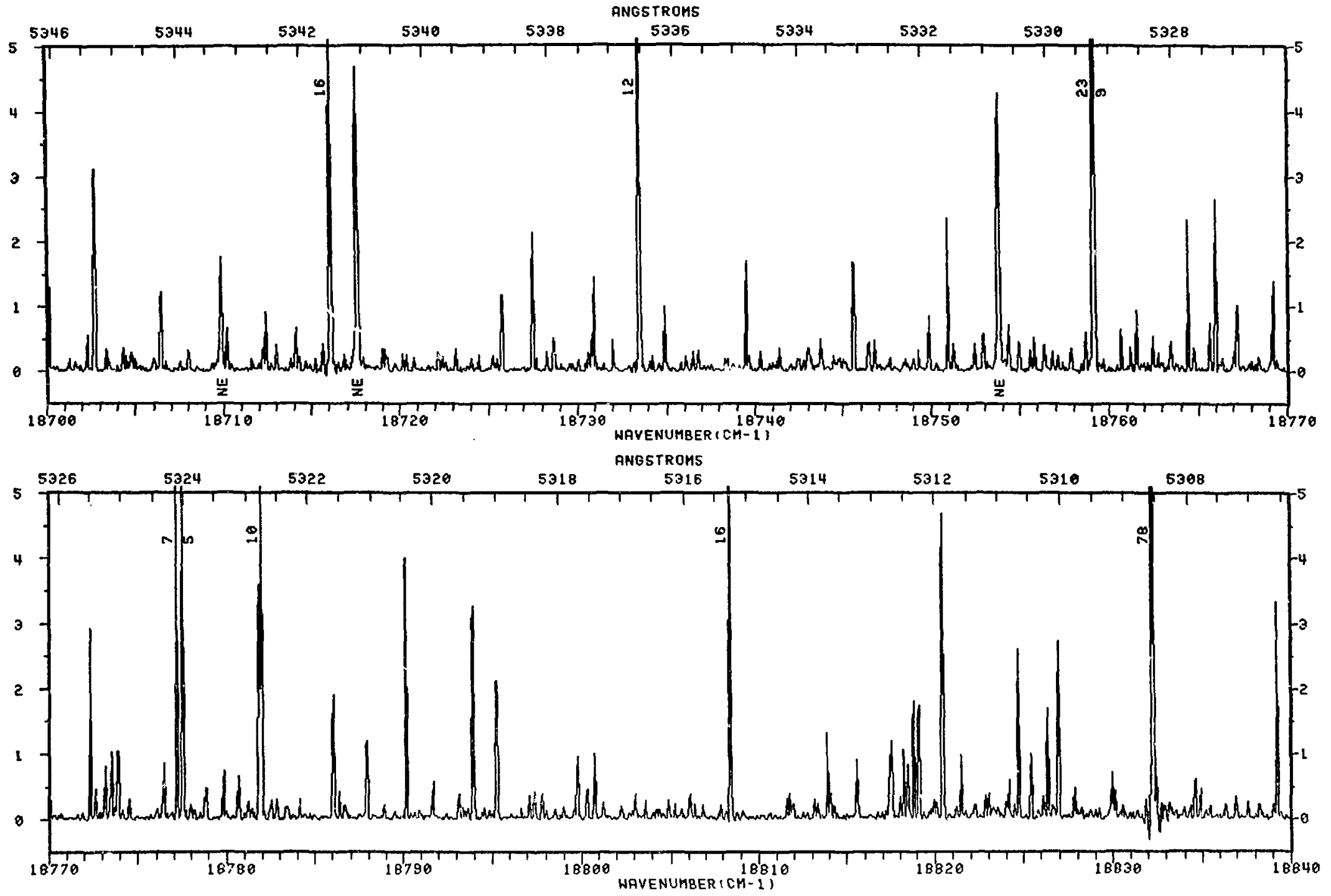

113 


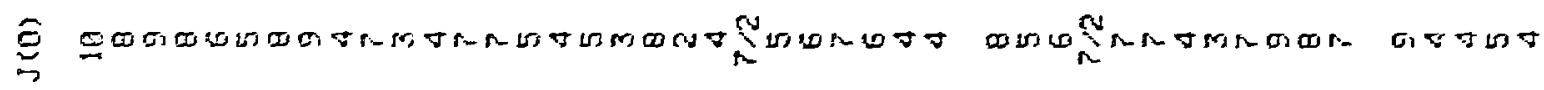

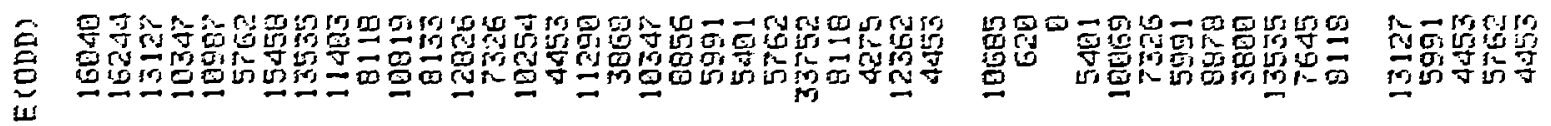

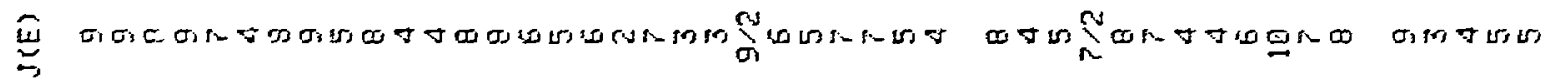

之

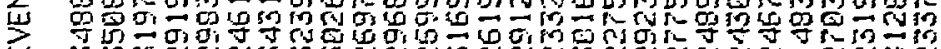

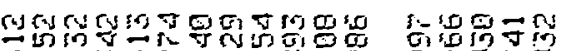

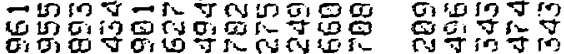

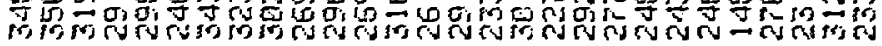

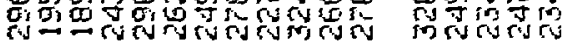

壱

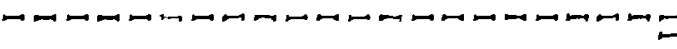

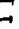

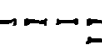

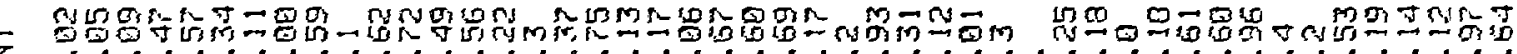

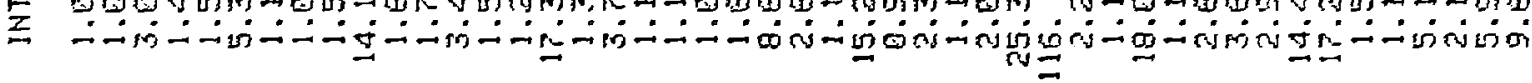

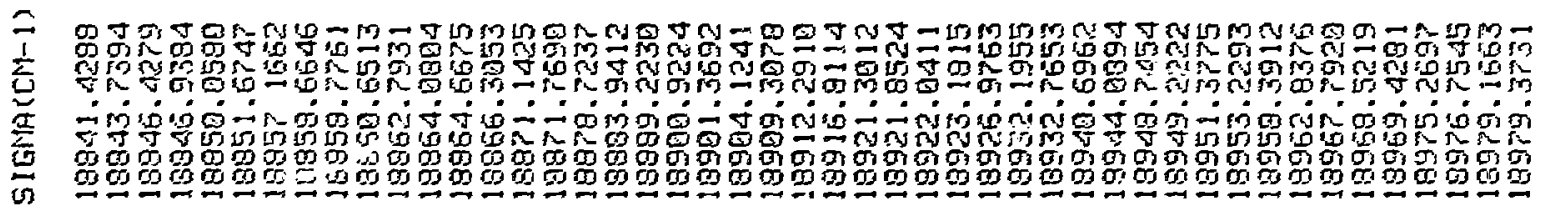

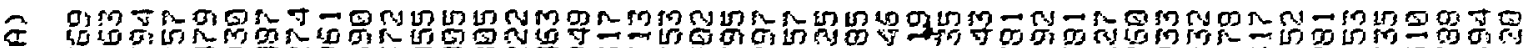

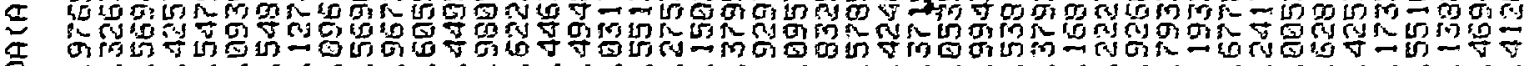
भi்

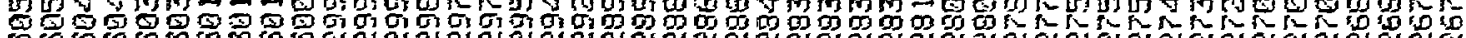

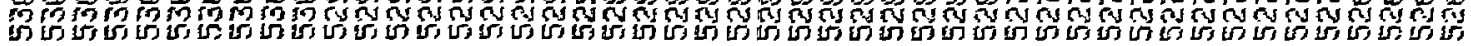



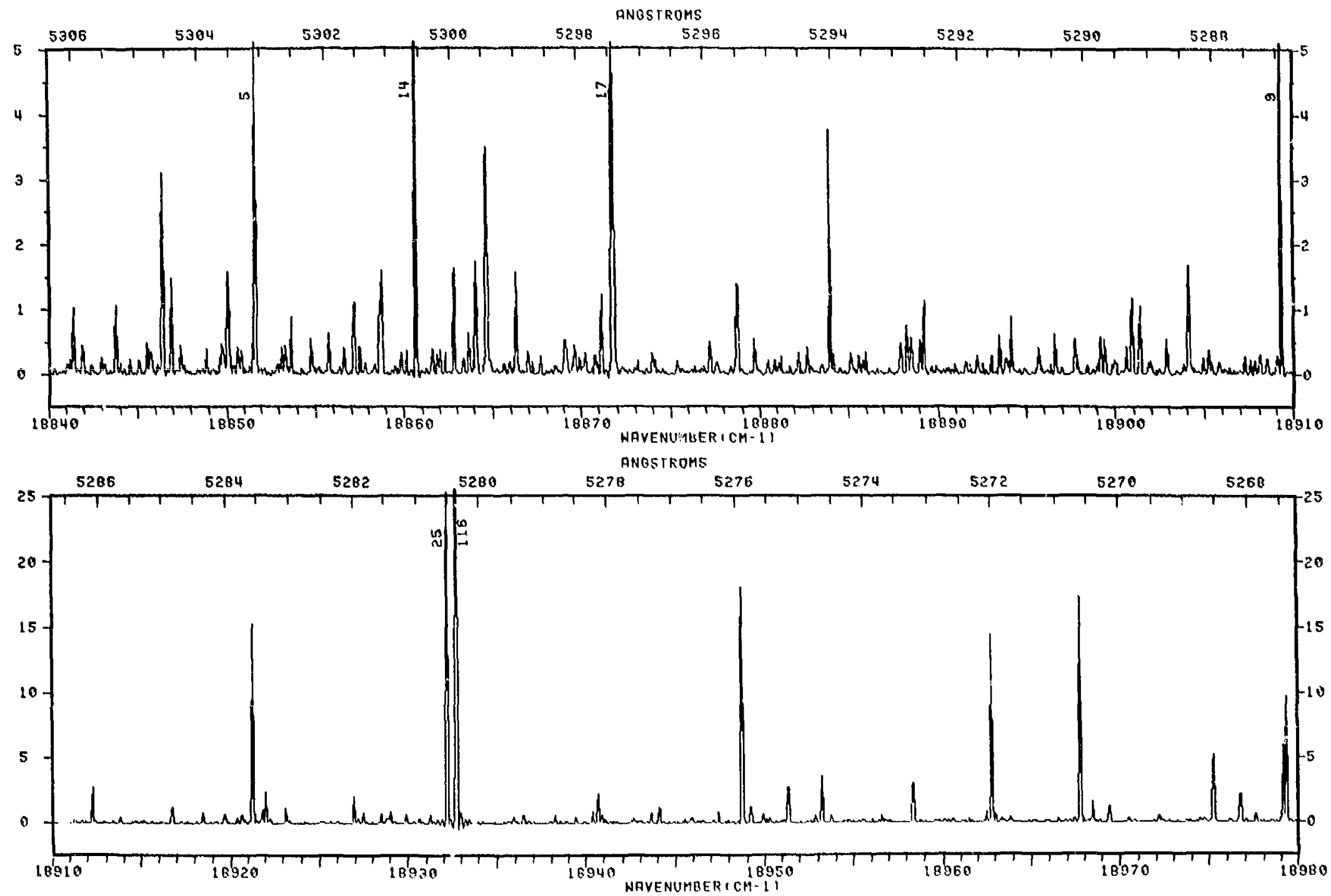
D

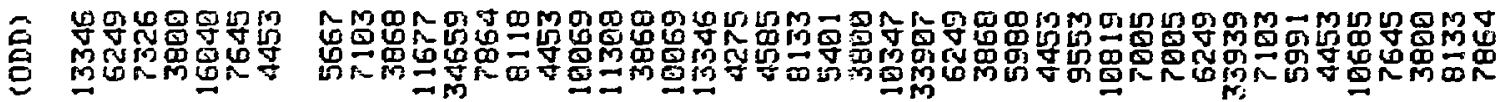

تु

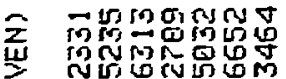
Niving in

Бמ

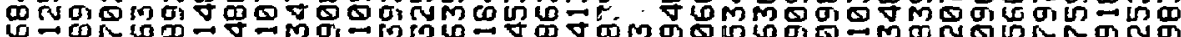

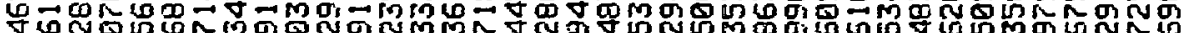

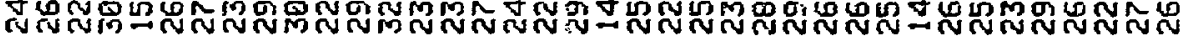

号

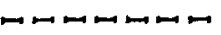

$m$

$\underset{z}{ \pm}$

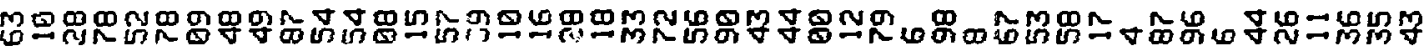

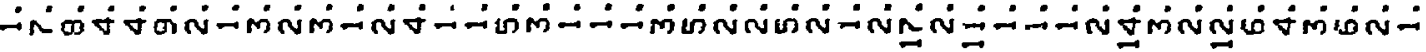

 ڤ

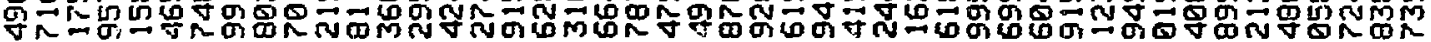

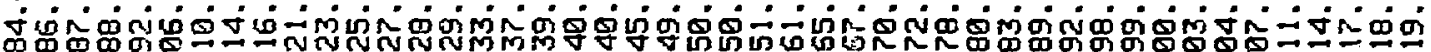

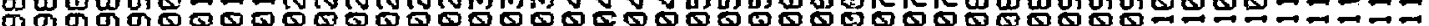

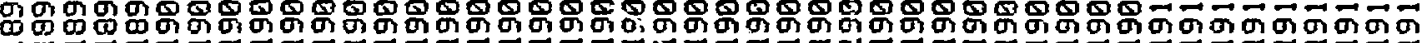

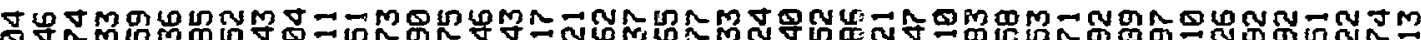

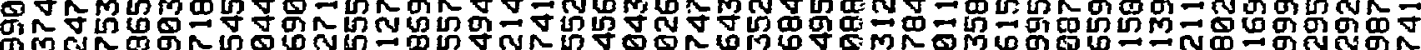

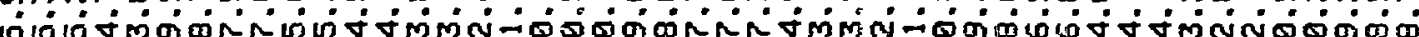
ต NAN N N N 


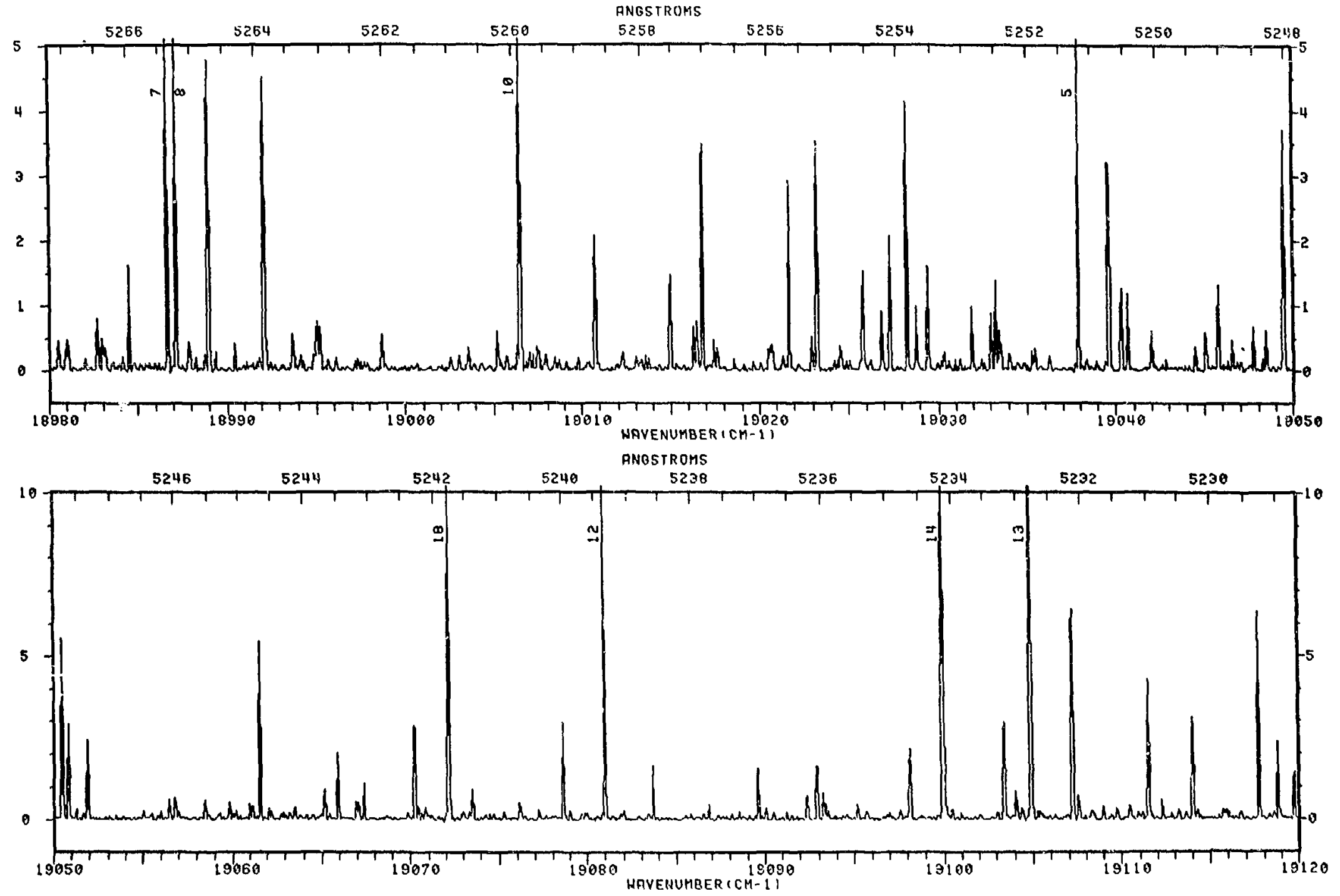




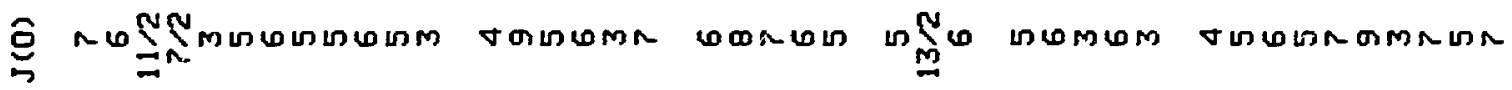

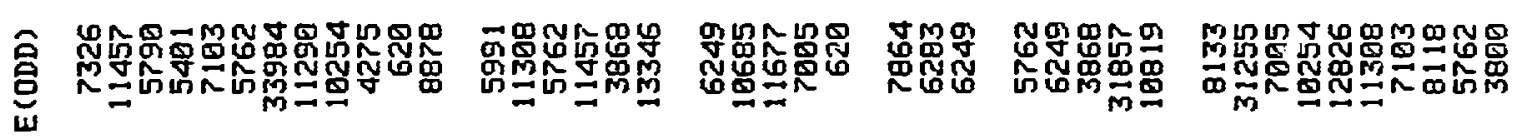

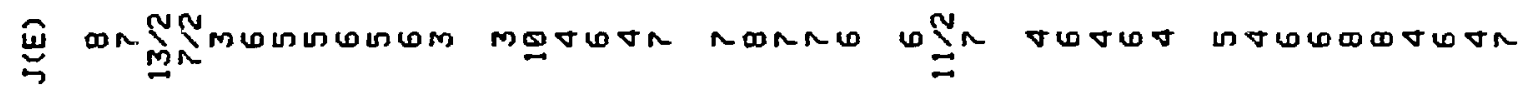

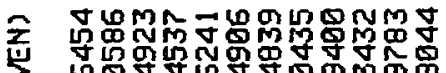

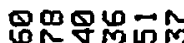

ง

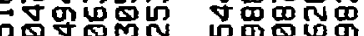

员罢罣思

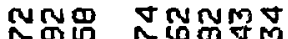

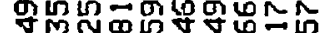

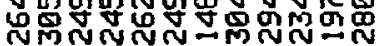

UM

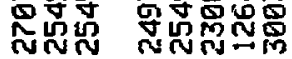

m心

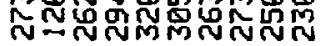

$\boldsymbol{w}$

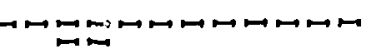

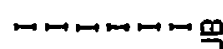

䍐円ロロロロ ロツロ䍐

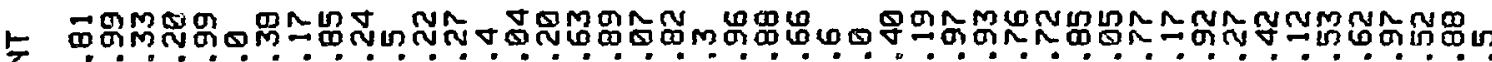

Z Ḿ⿴囗ن்

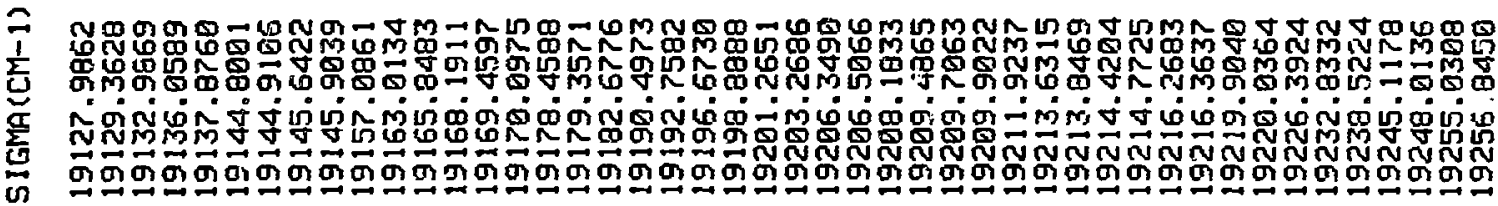

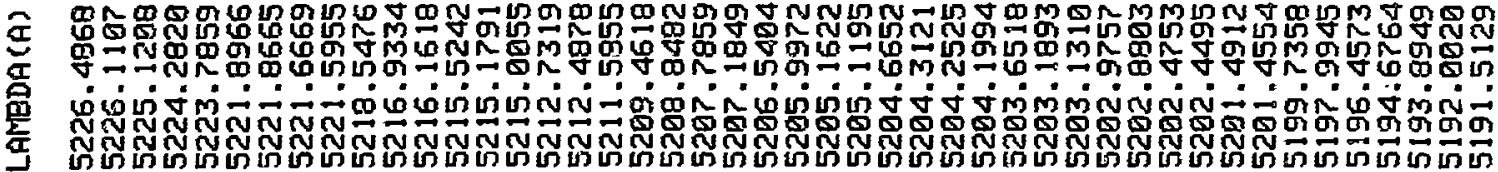




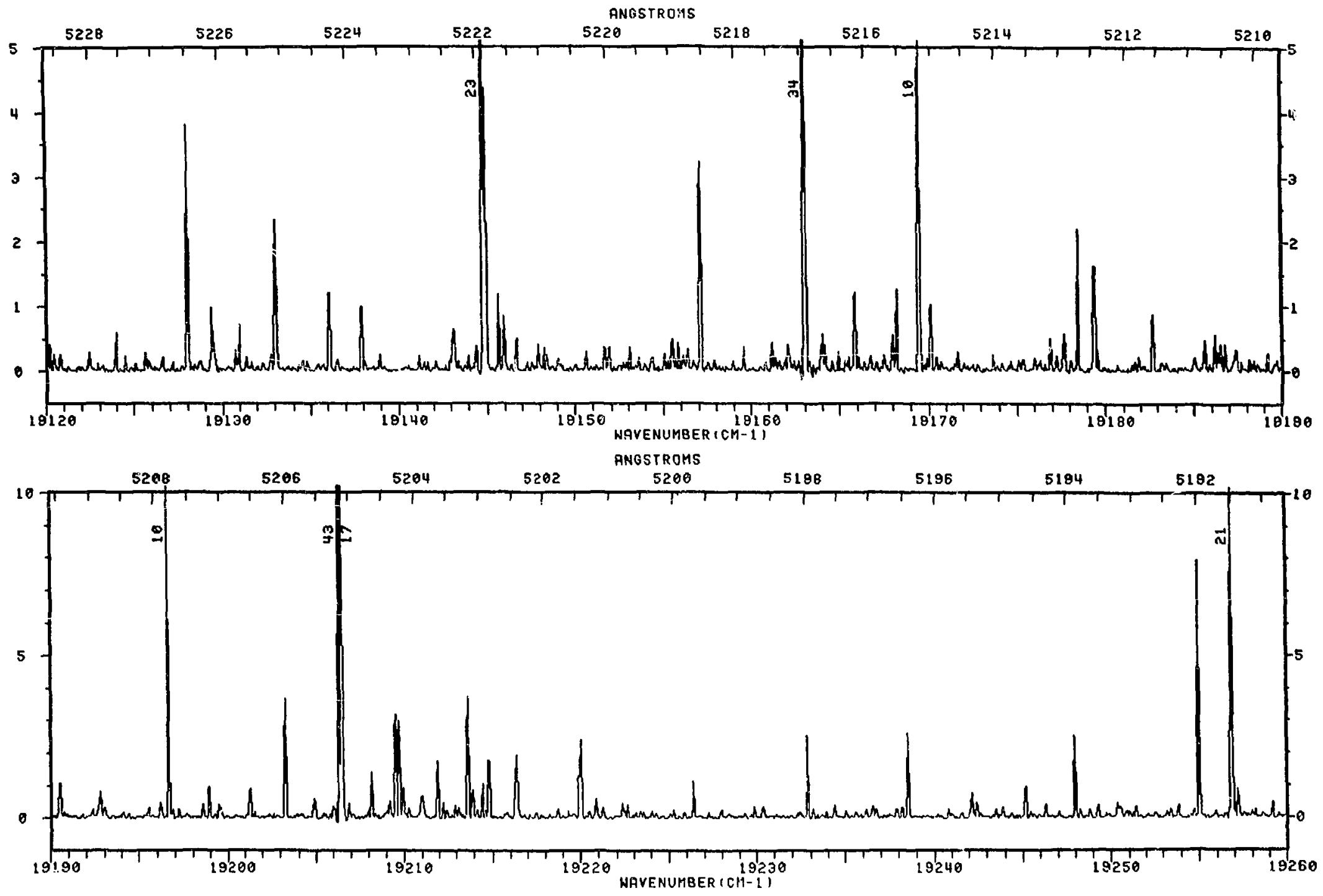

119 


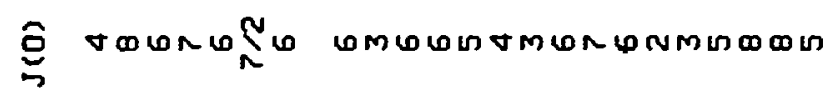

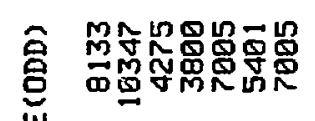

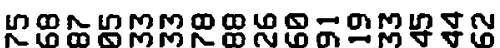

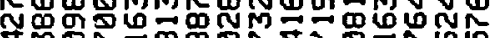

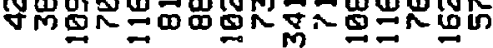

on

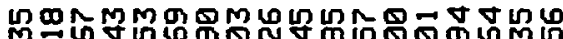

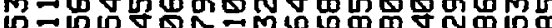

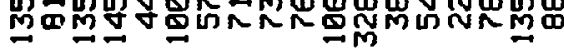

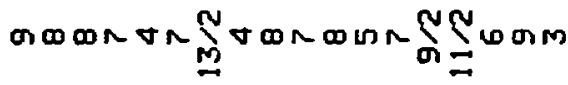

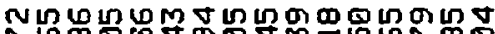

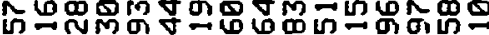

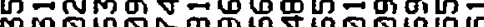

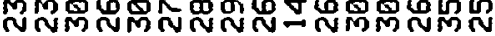

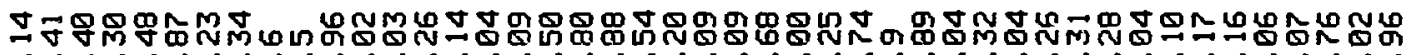
- மா-

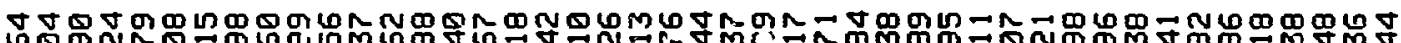

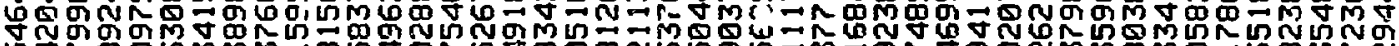

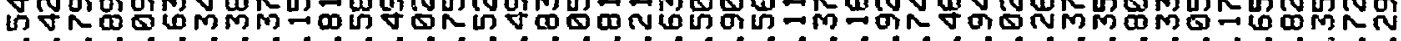
$\therefore$ ம் 它

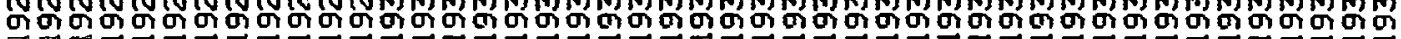

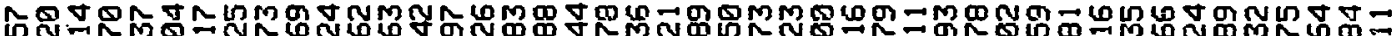

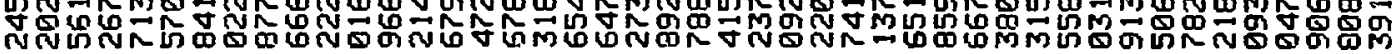

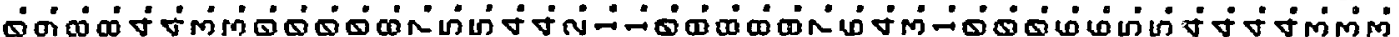
ต

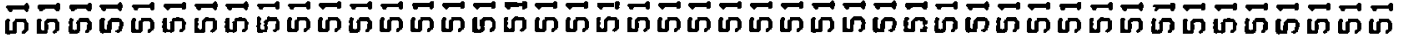



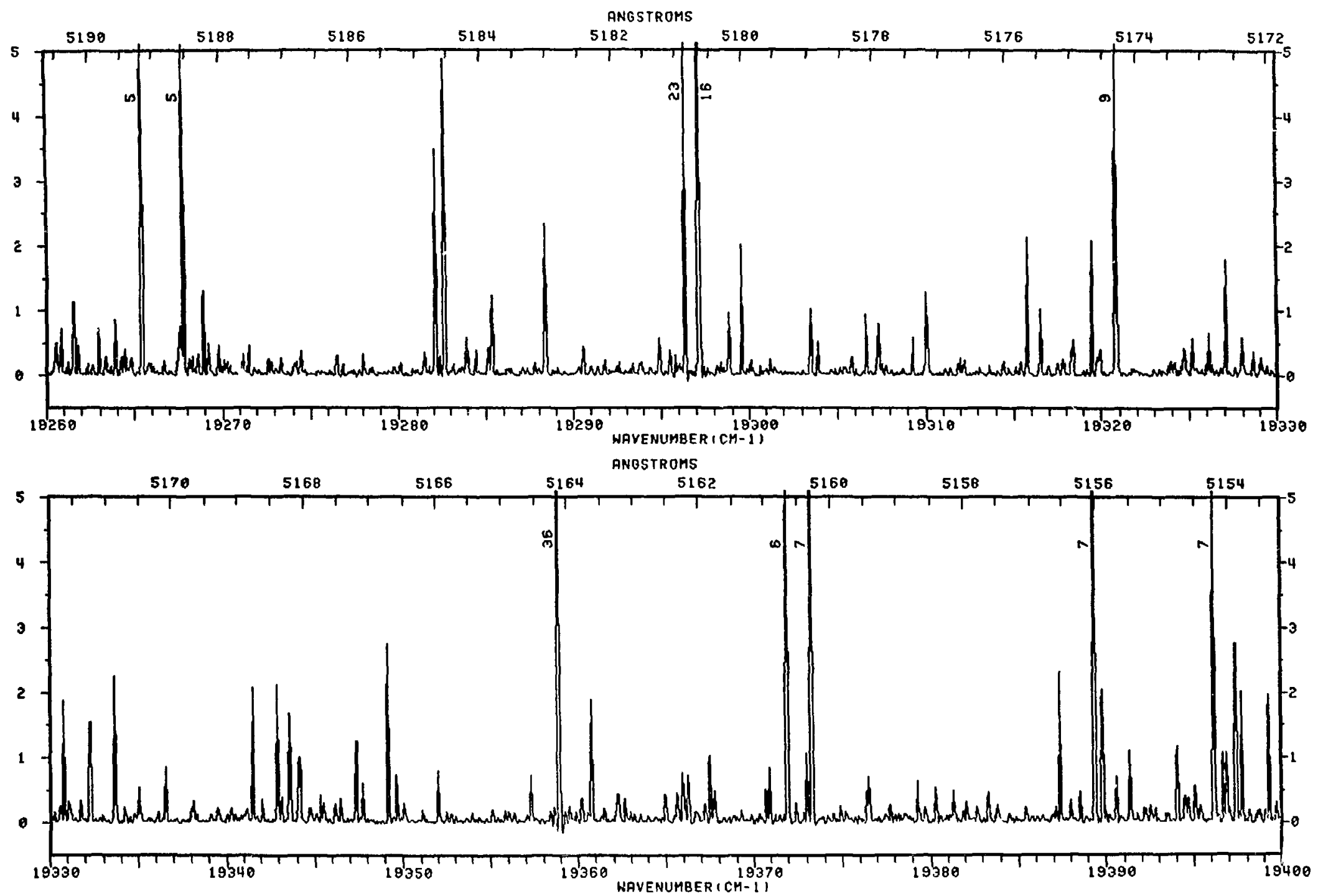
兑 $\nabla$ m m

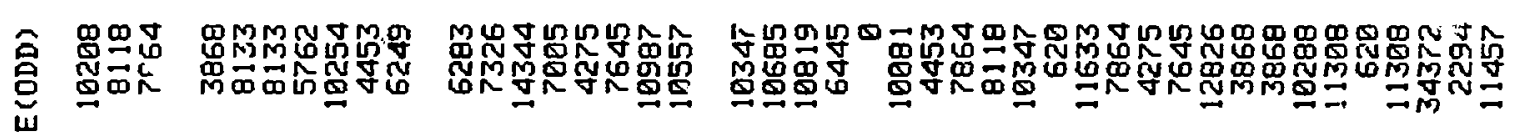

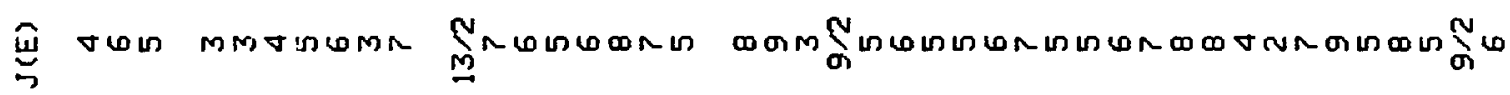

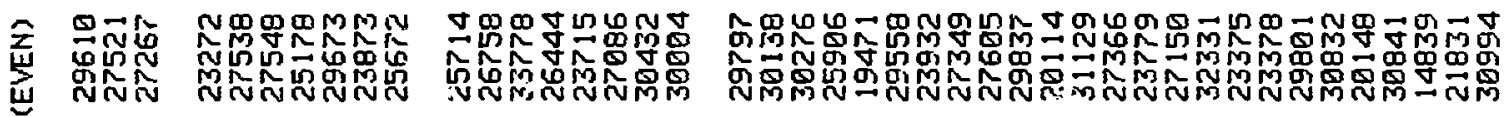

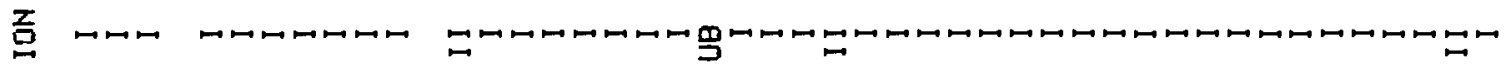

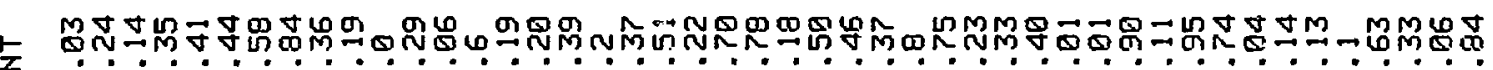

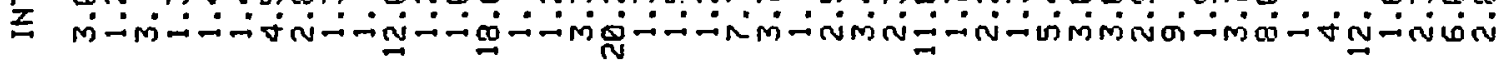

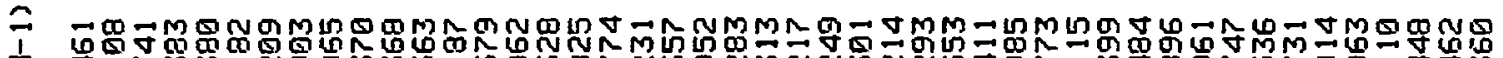

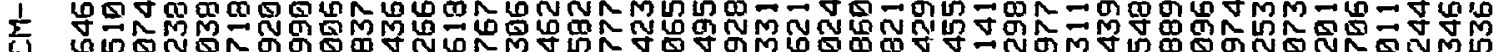

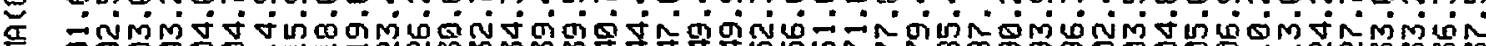

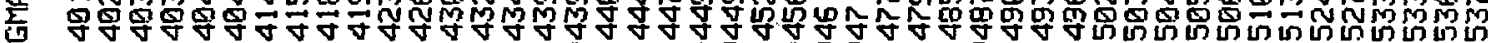

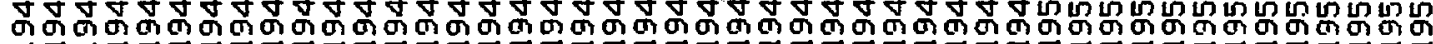

oi

ส

๔

( Niñ

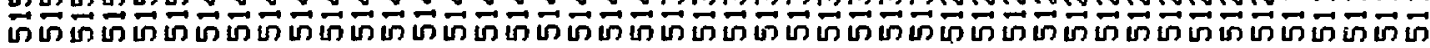



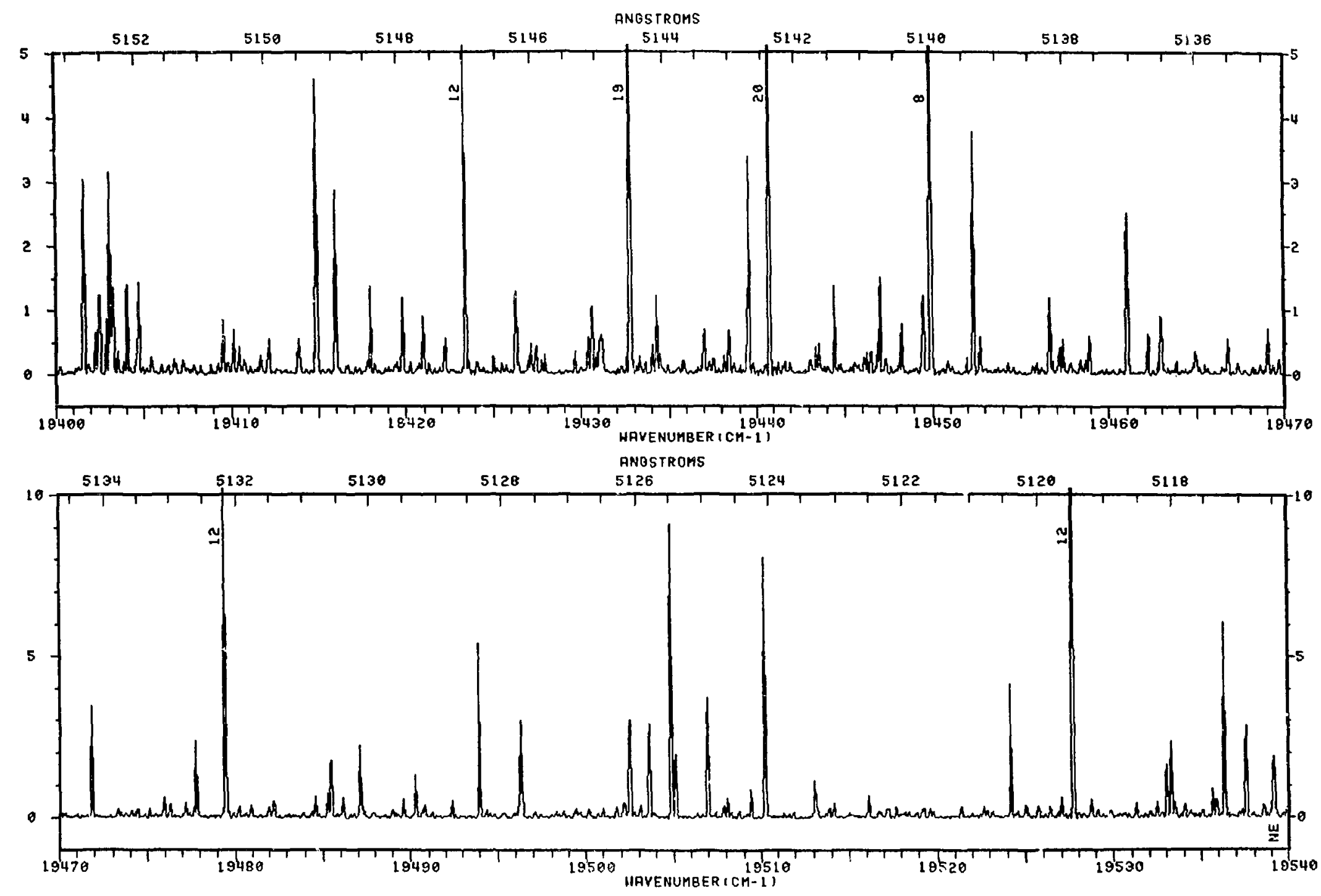


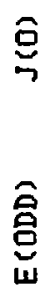

M⿱宀⿻上丨

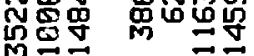

eq

$\omega \boldsymbol{\infty} \boldsymbol{\infty}$

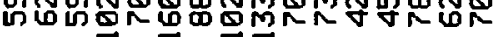

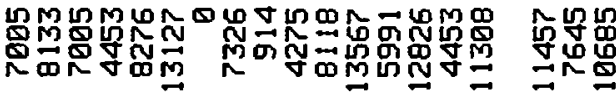

는

mounconerwen

Neon mos

Mmø⿺𠃊八刀

won

交

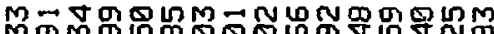

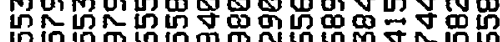

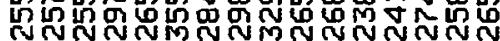

c.<smiles>[B]C</smiles>

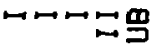

ロேロのほ

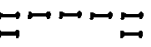

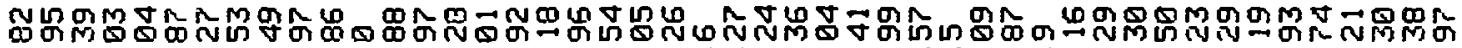

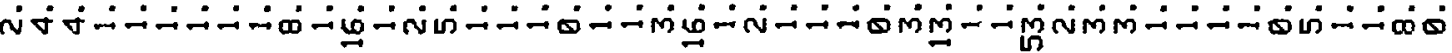

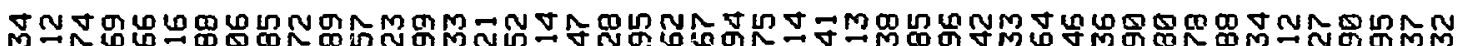

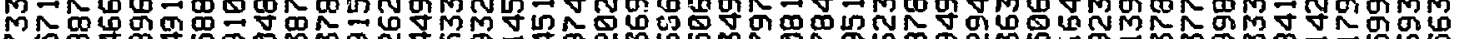

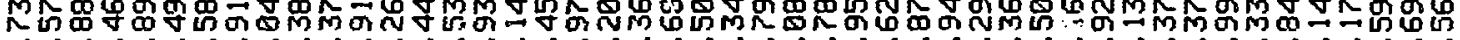
- م் ர் 寸寸寸 寸 寸 in

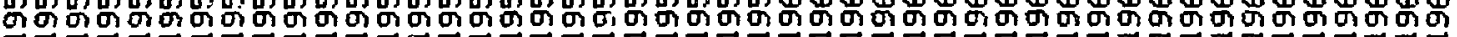

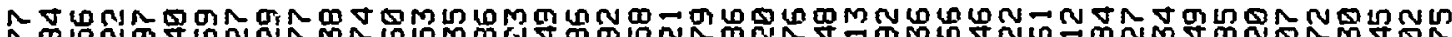

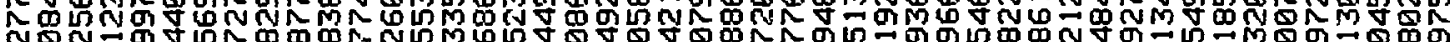

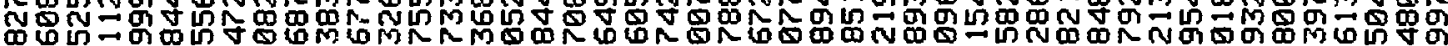

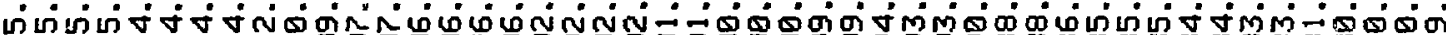

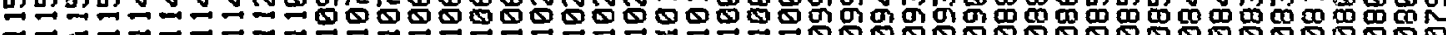
மே 


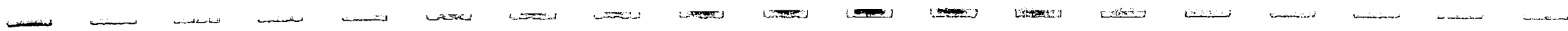
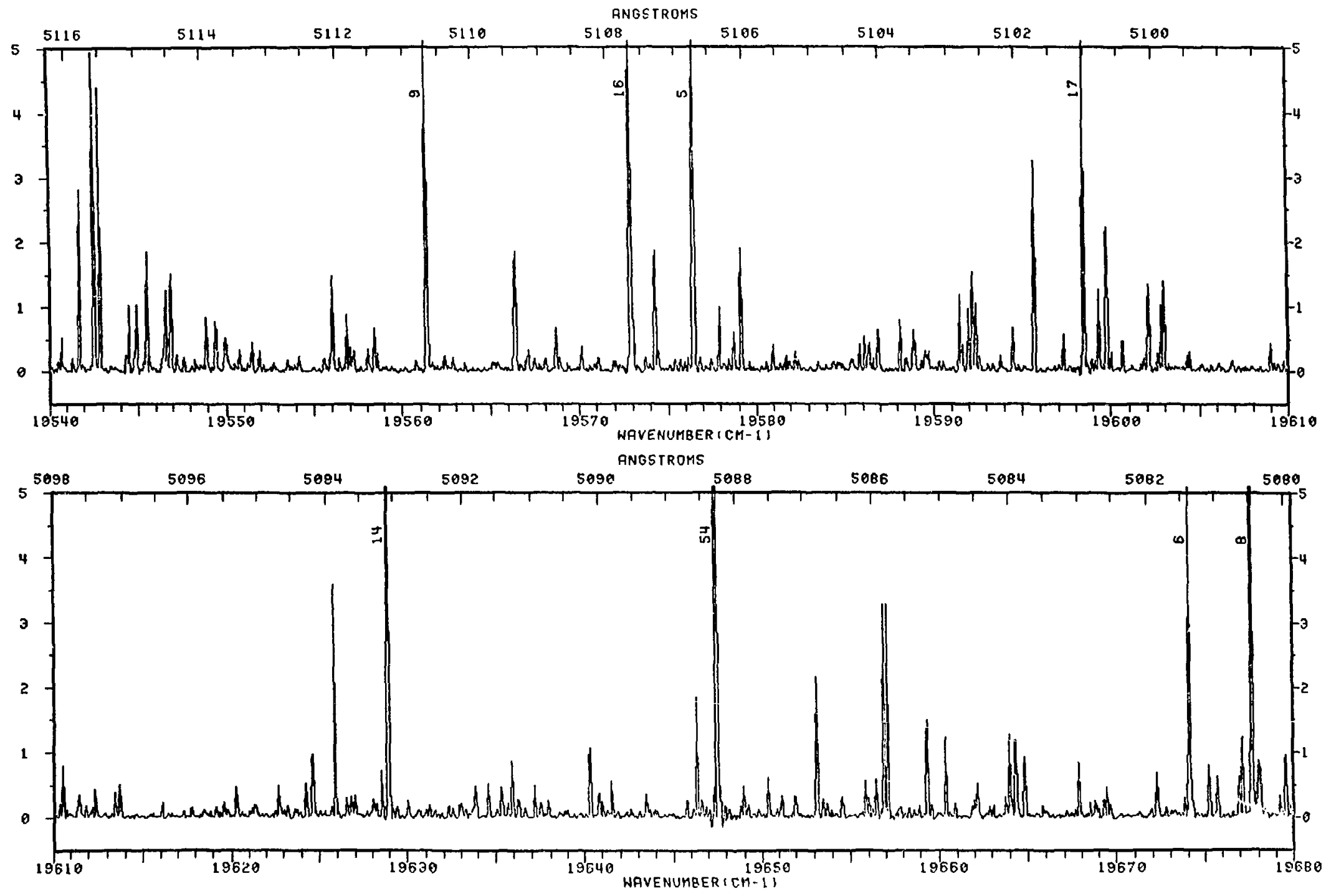

125 
总

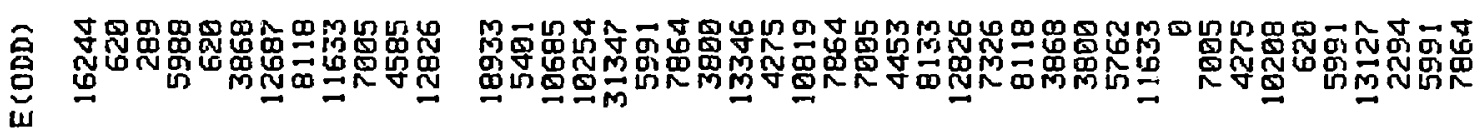

岁

妾

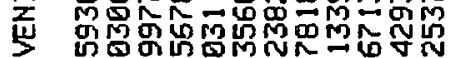

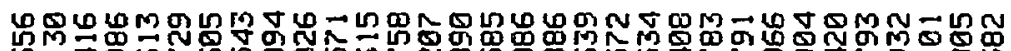

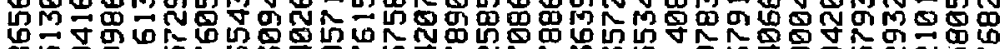

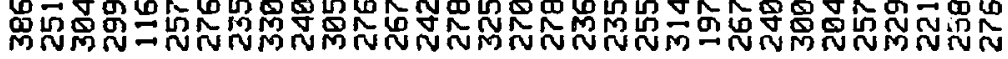

$\underset{\sim}{Z}$
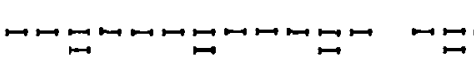

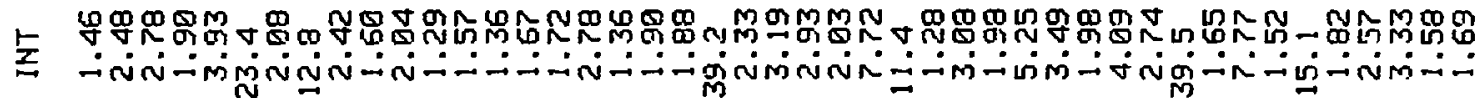
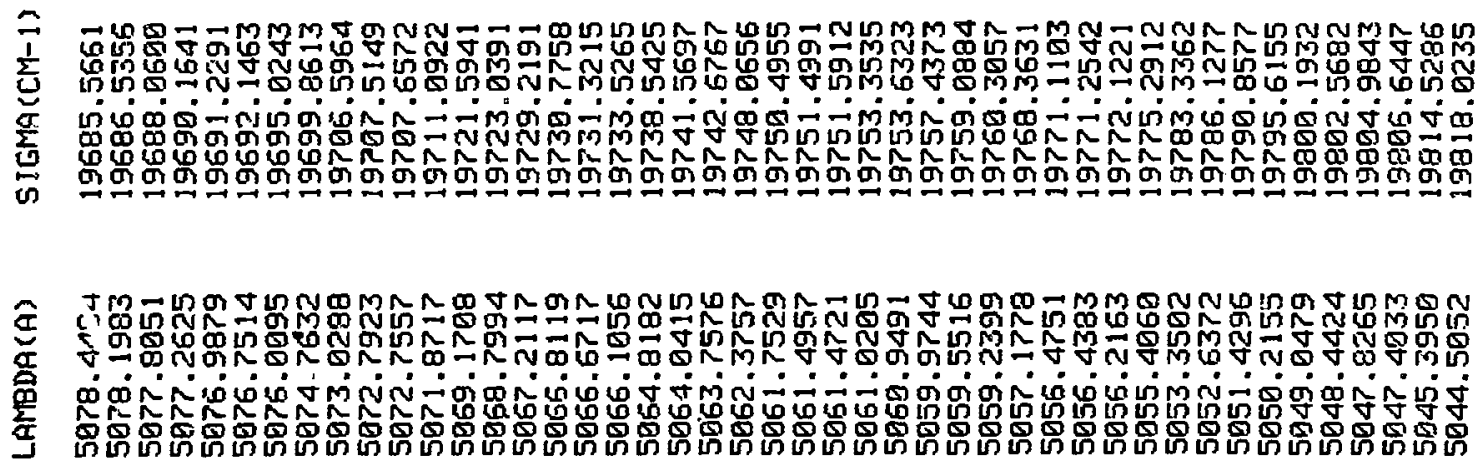

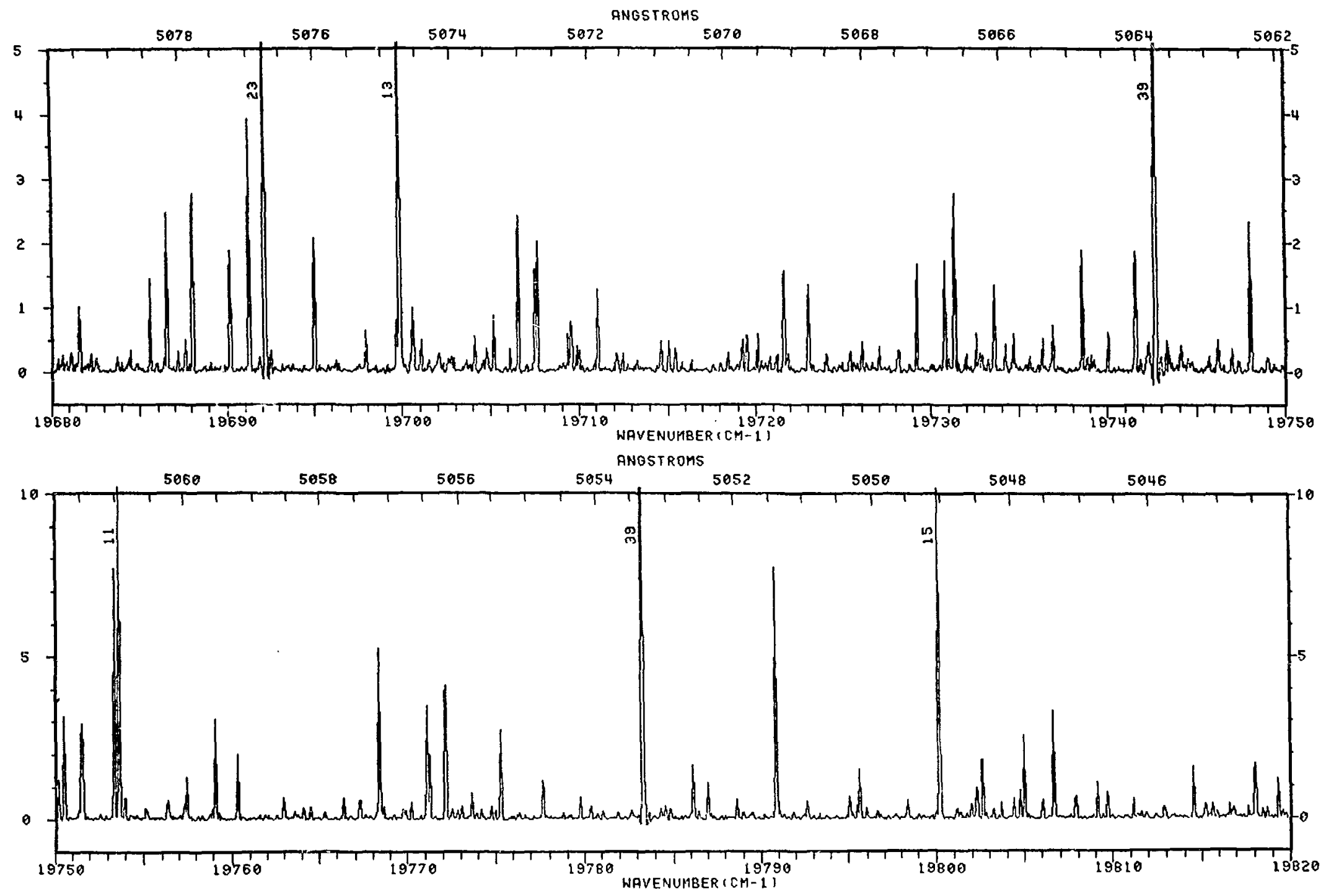
g

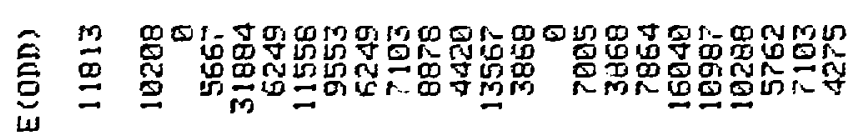

$\cos \pi-1000$

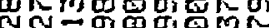

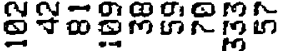

$\cos \theta \operatorname{tin} \sec 6$

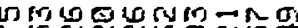

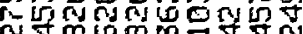

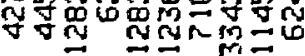

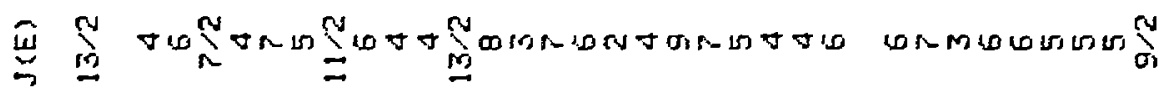

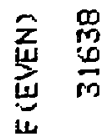

T

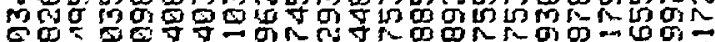

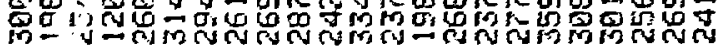

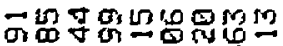

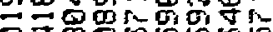

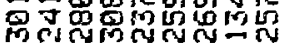

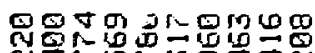

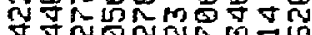

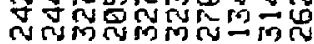

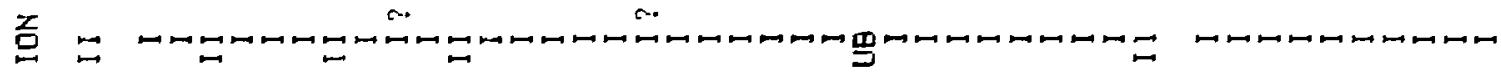

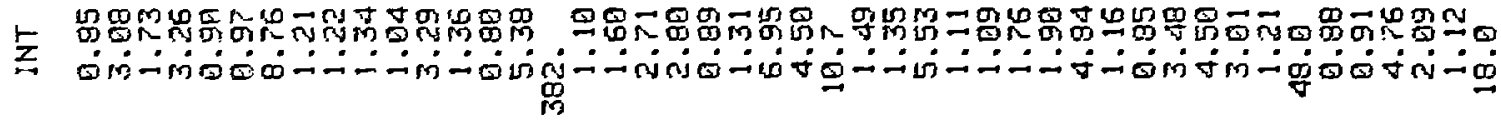

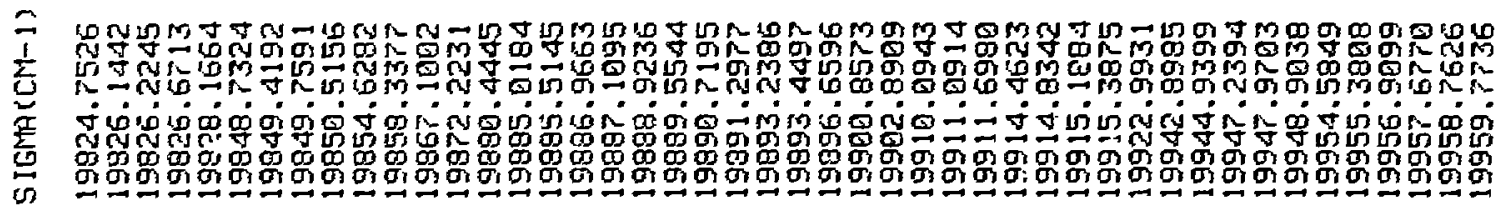

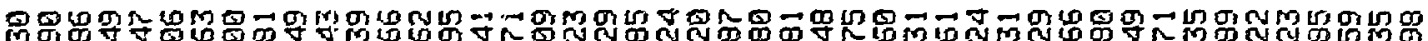

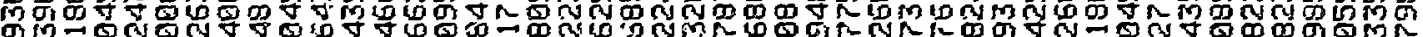

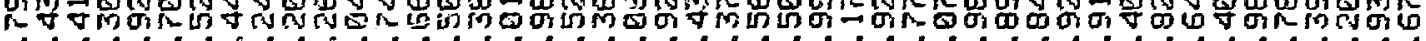

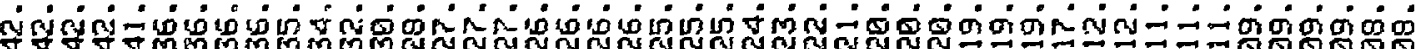
पु

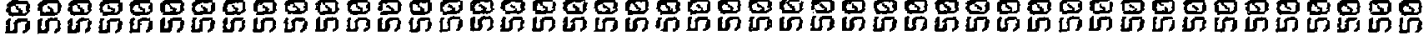




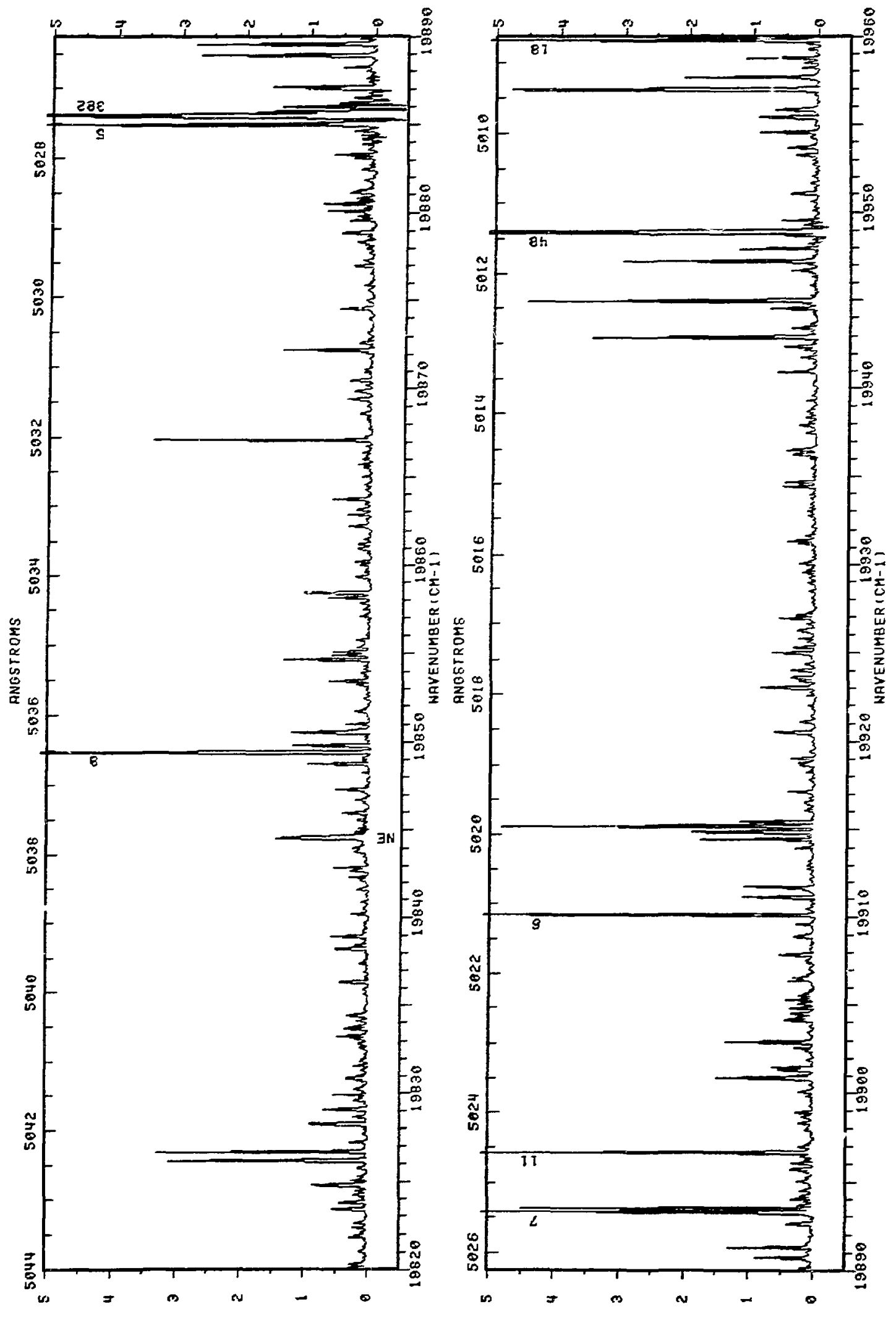


包

\begin{tabular}{|c|c|c|c|c|c|}
\hline 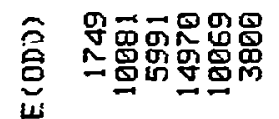 & 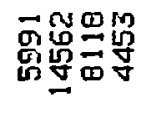 & 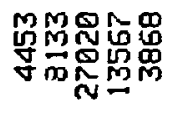 & 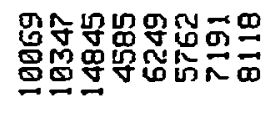 & 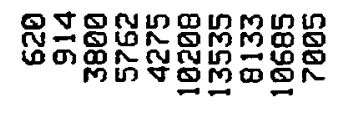 & 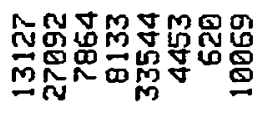 \\
\hline
\end{tabular}

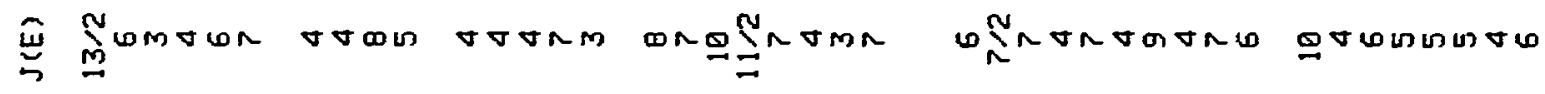

\begin{tabular}{|c|c|c|c|c|}
\hline 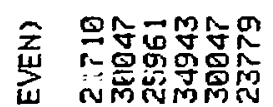 & $\begin{array}{l}\text { 䈍思思导 } \\
\text { 思 }\end{array}$ & 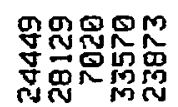 & 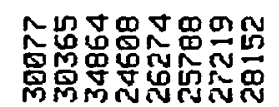 & 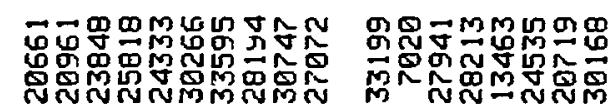 \\
\hline
\end{tabular}

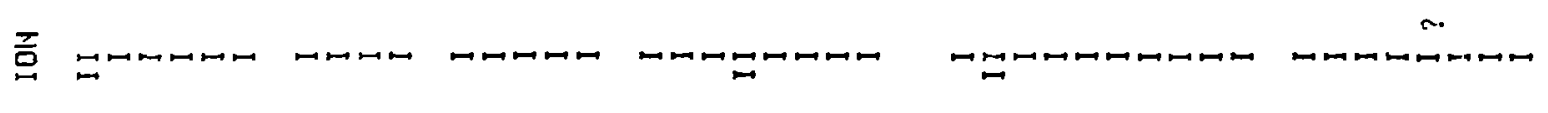

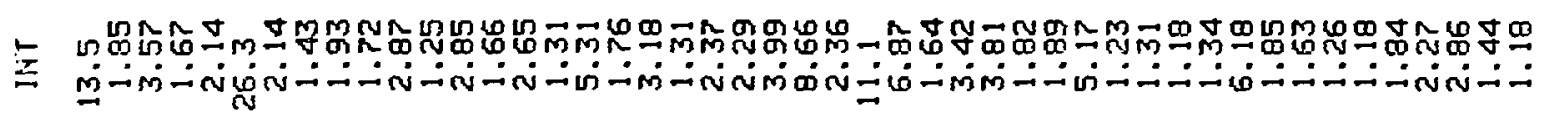

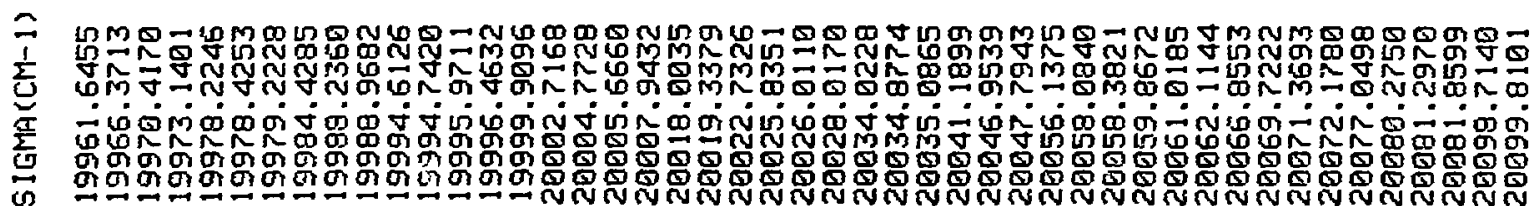

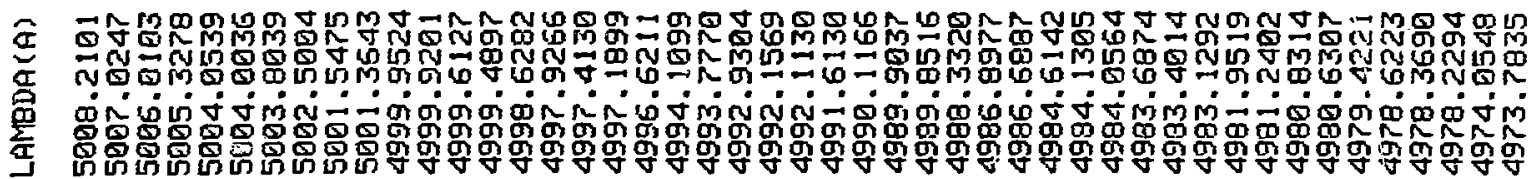



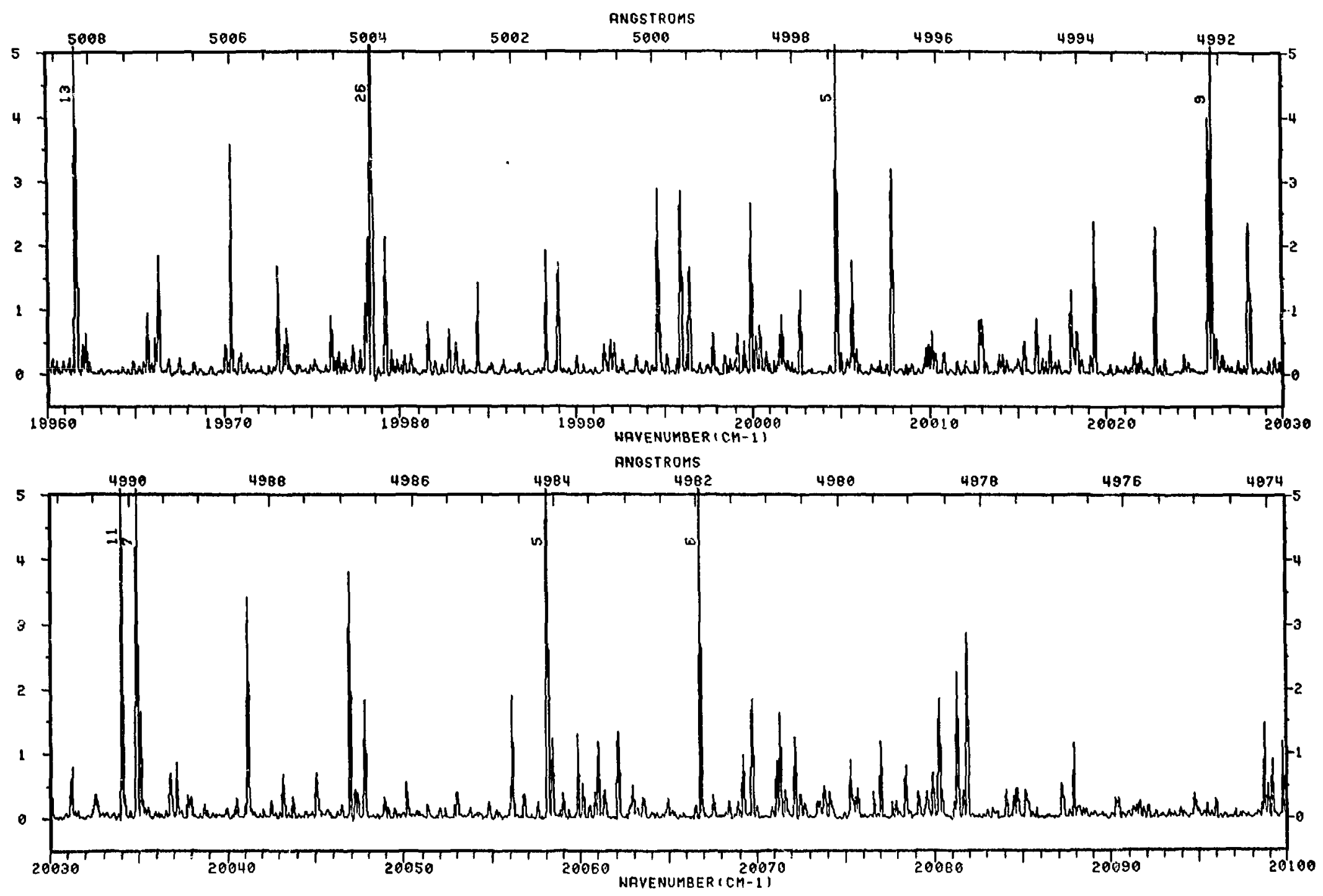


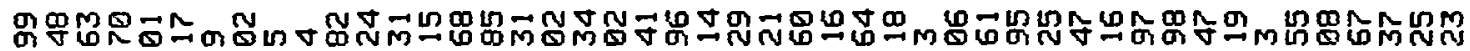

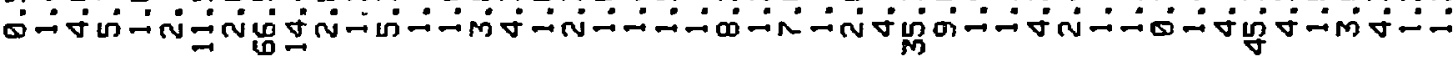

MMN

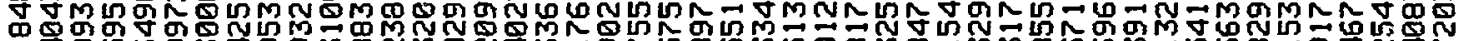

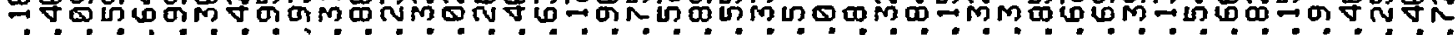

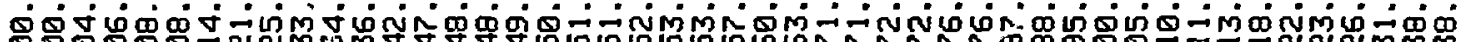

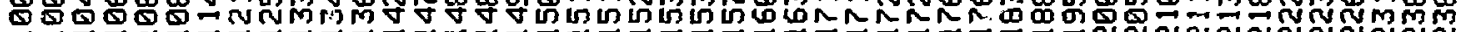

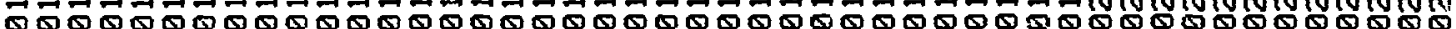

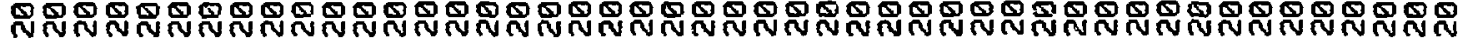

\%

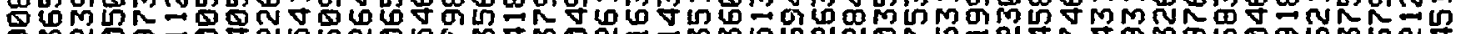

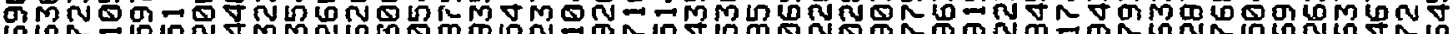
an

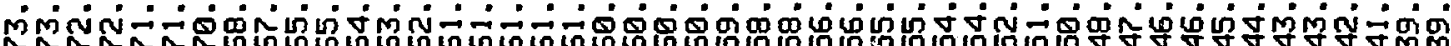

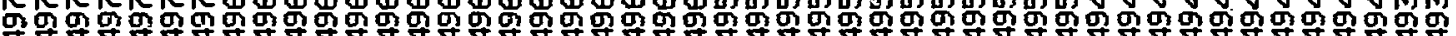



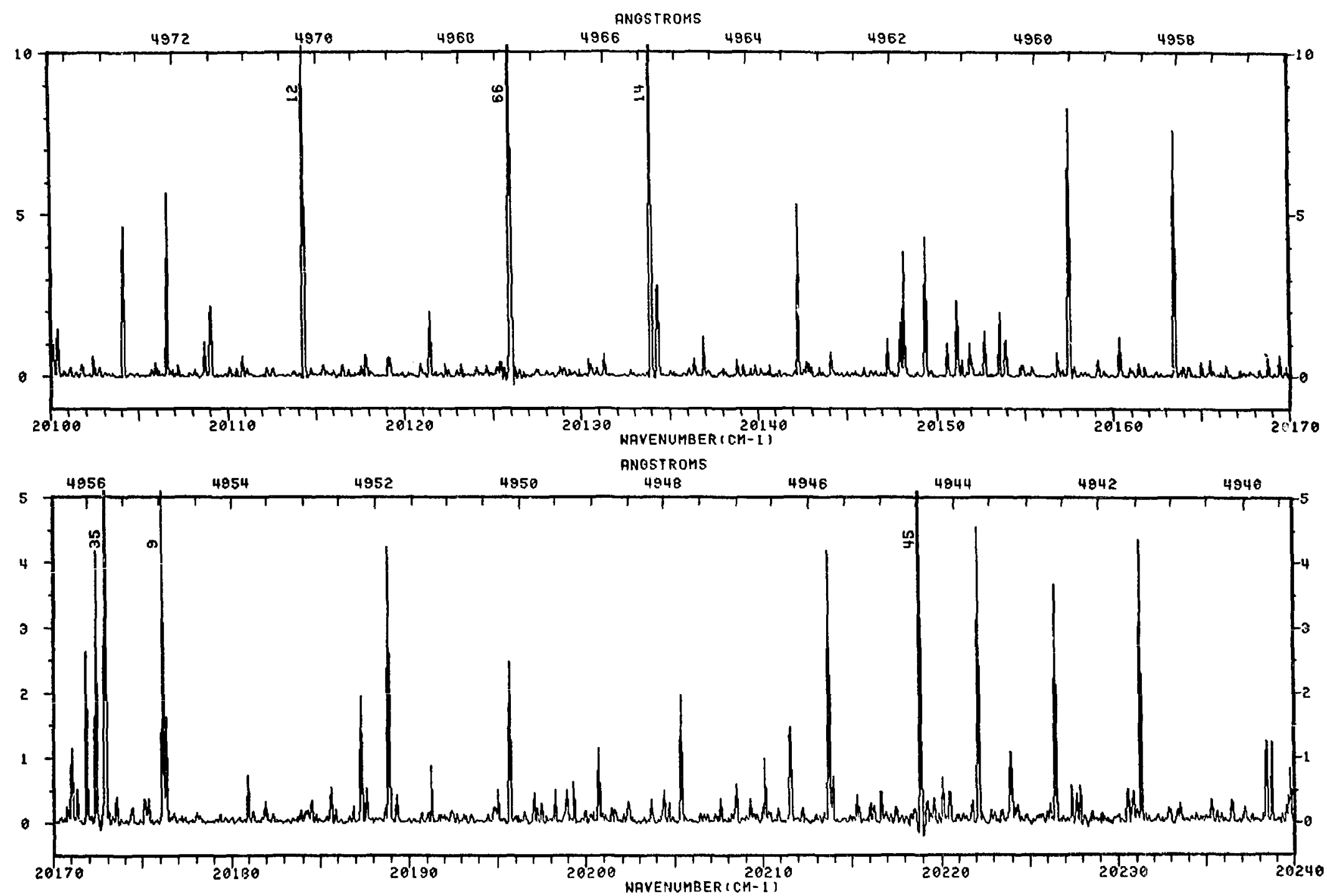
总

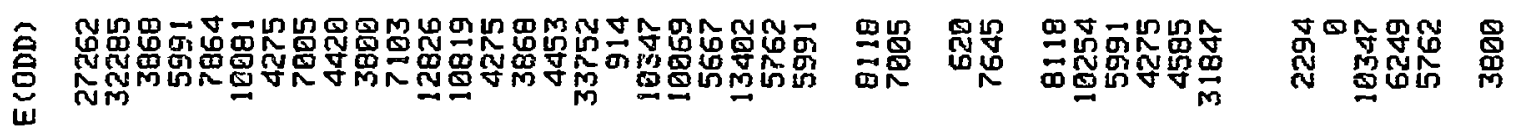

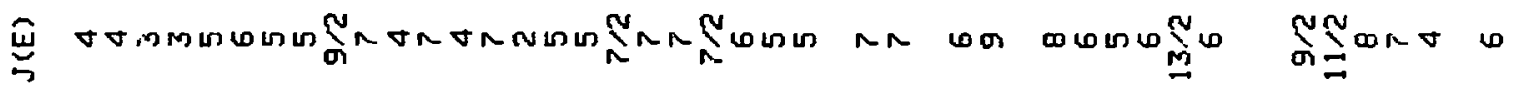
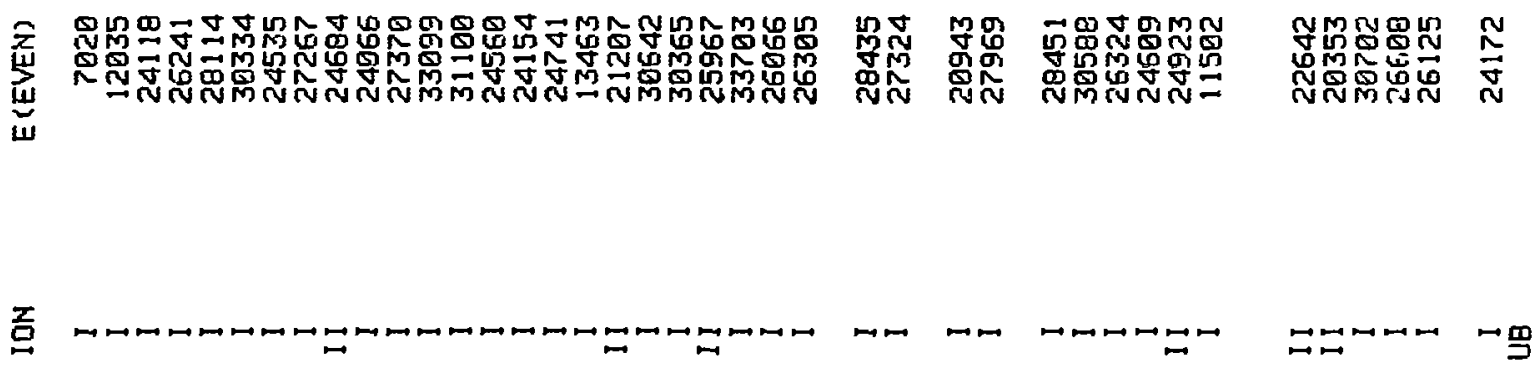

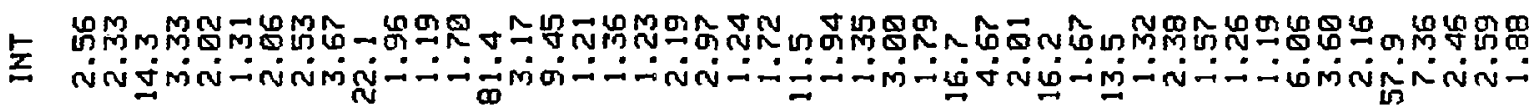

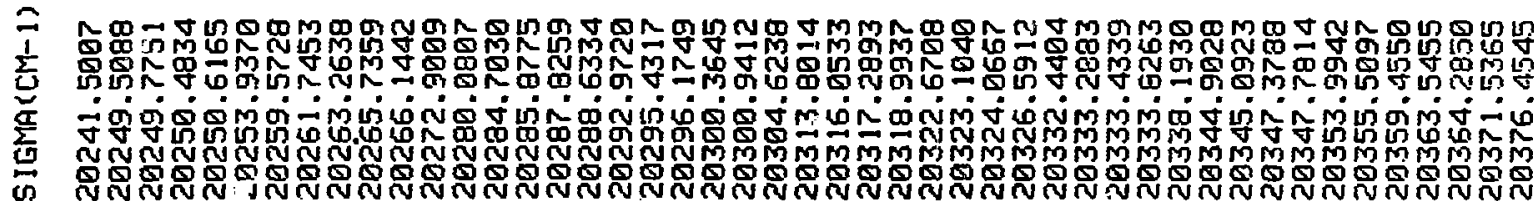

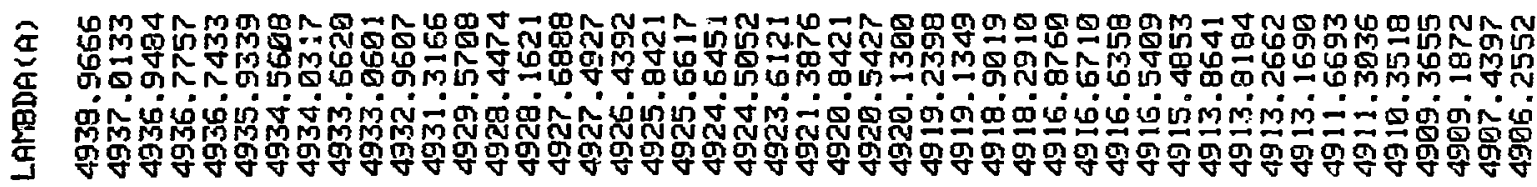



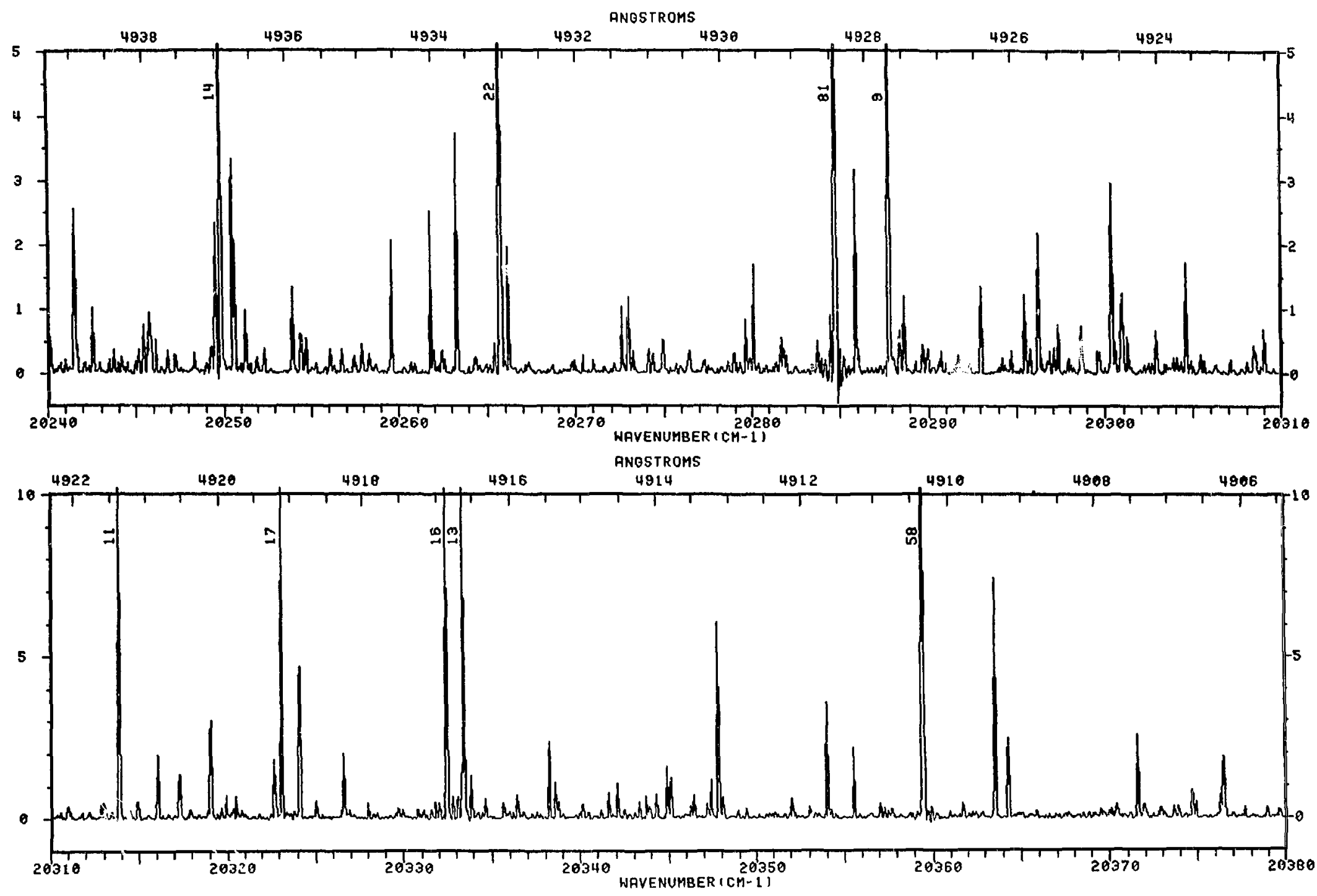


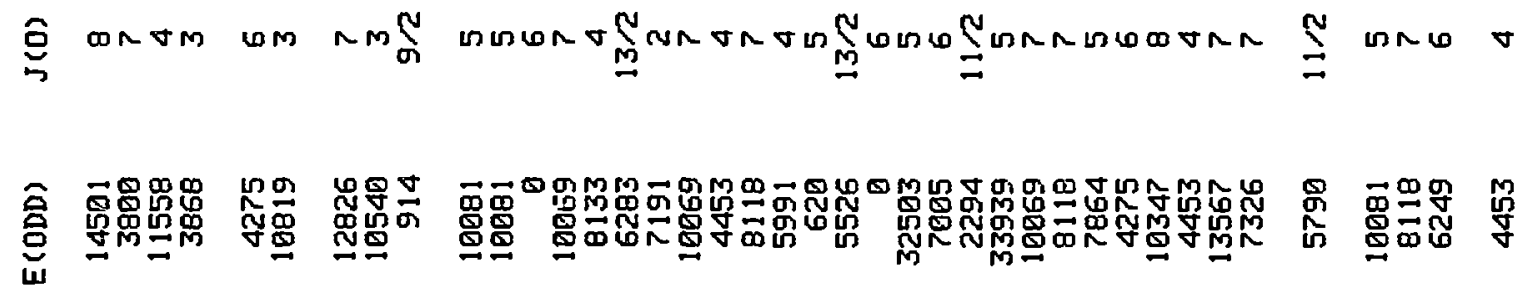

E్

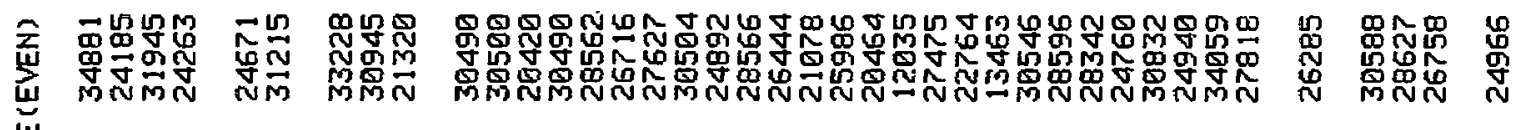

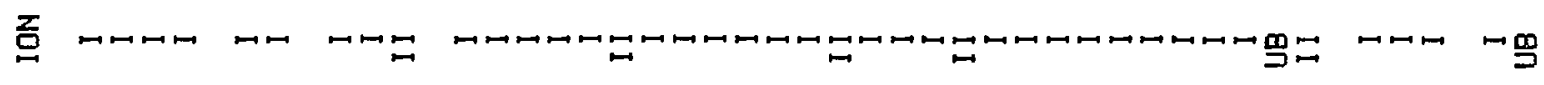

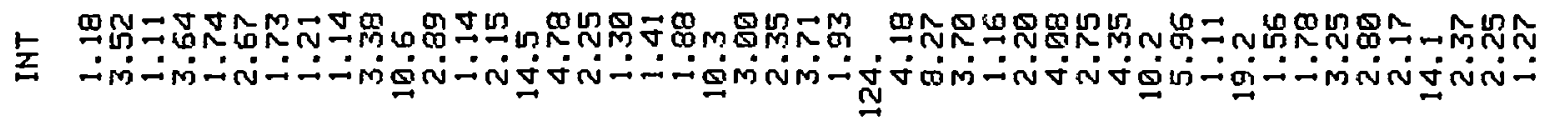

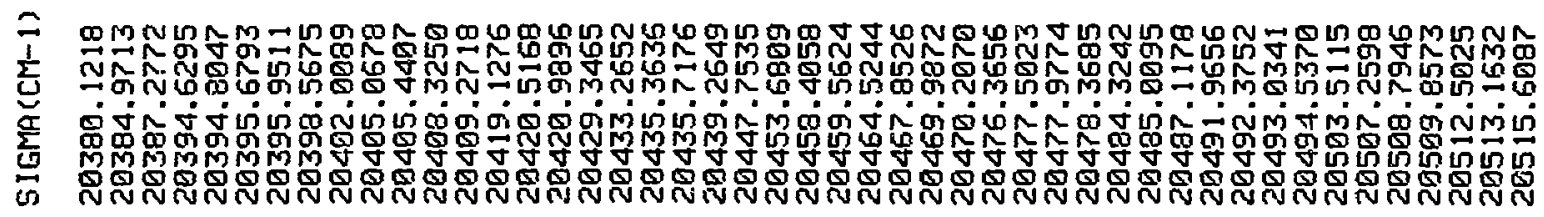

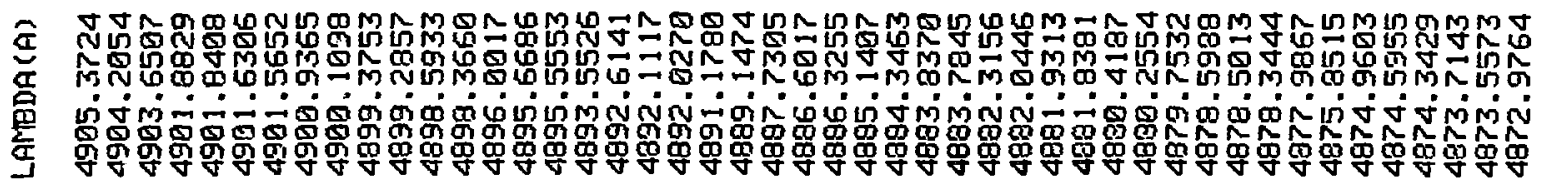



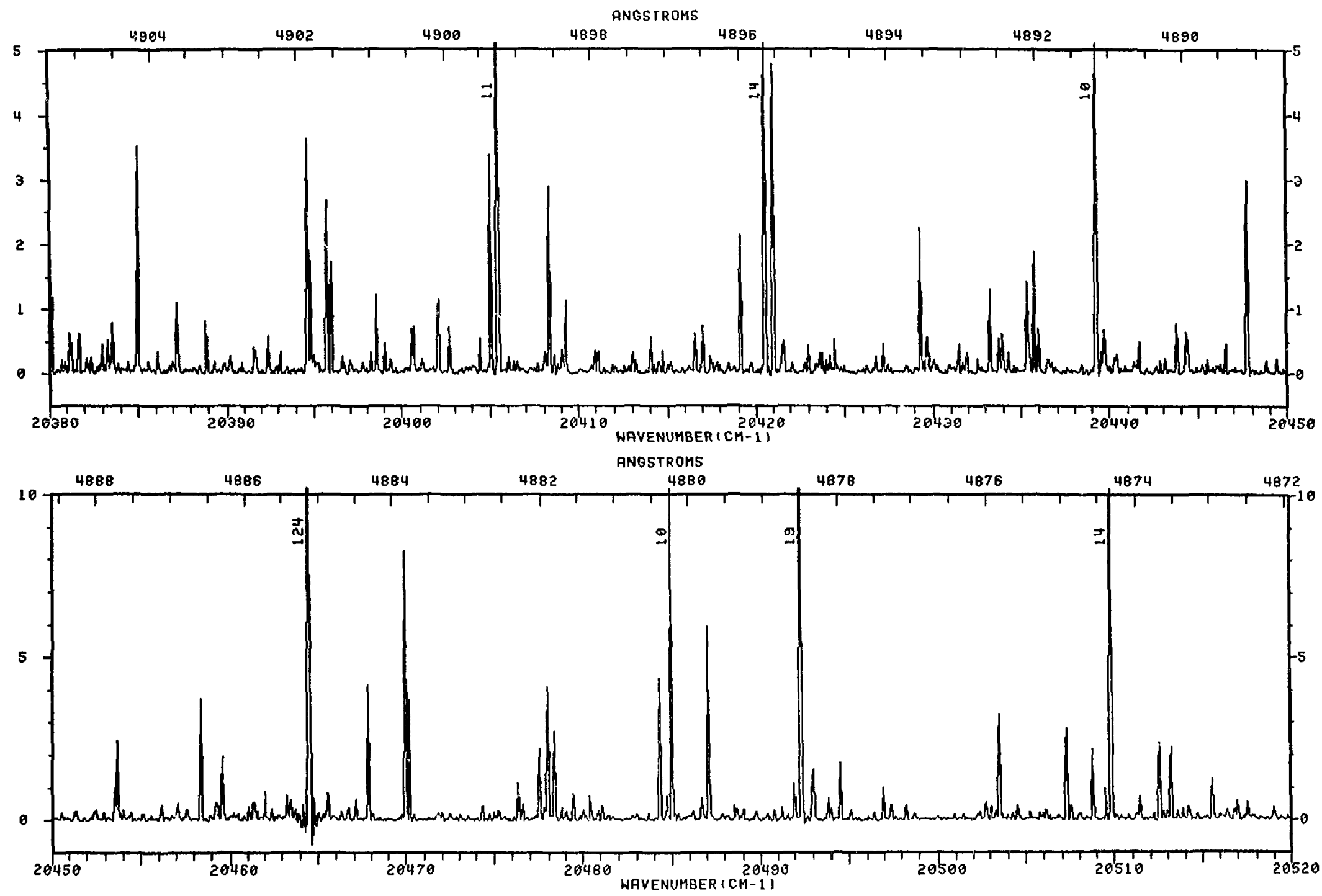


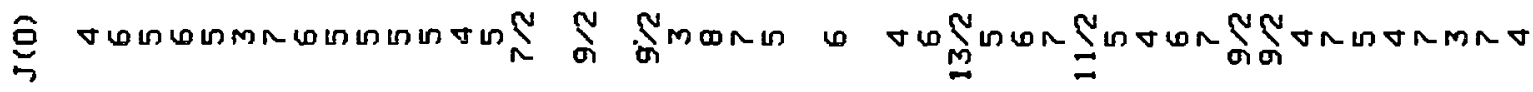

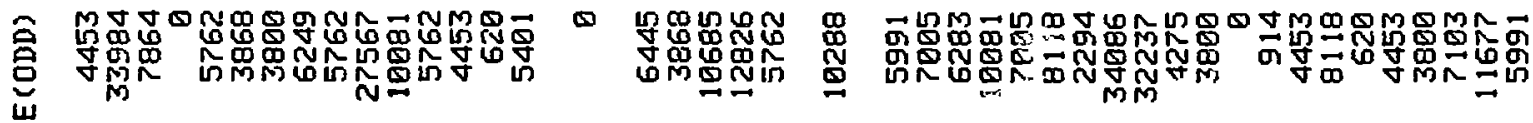

己

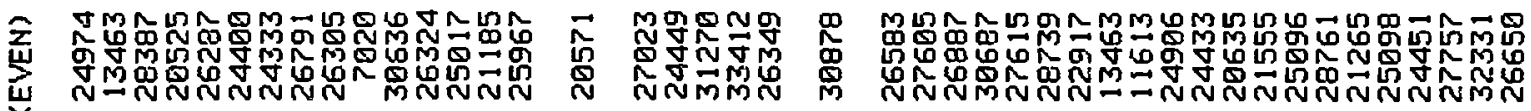

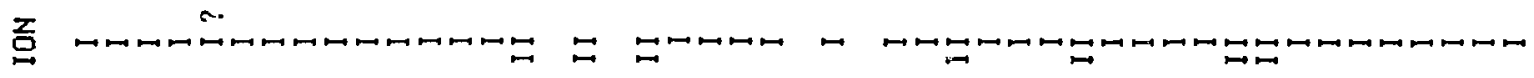

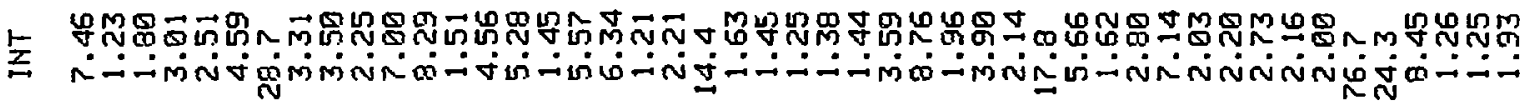

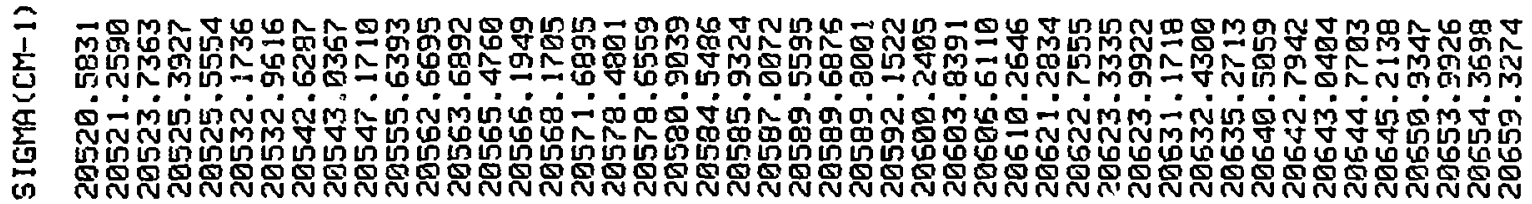

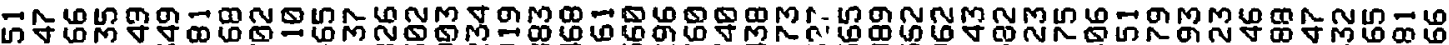

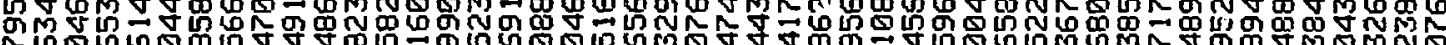
几

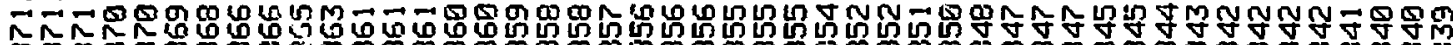

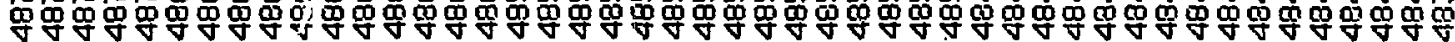



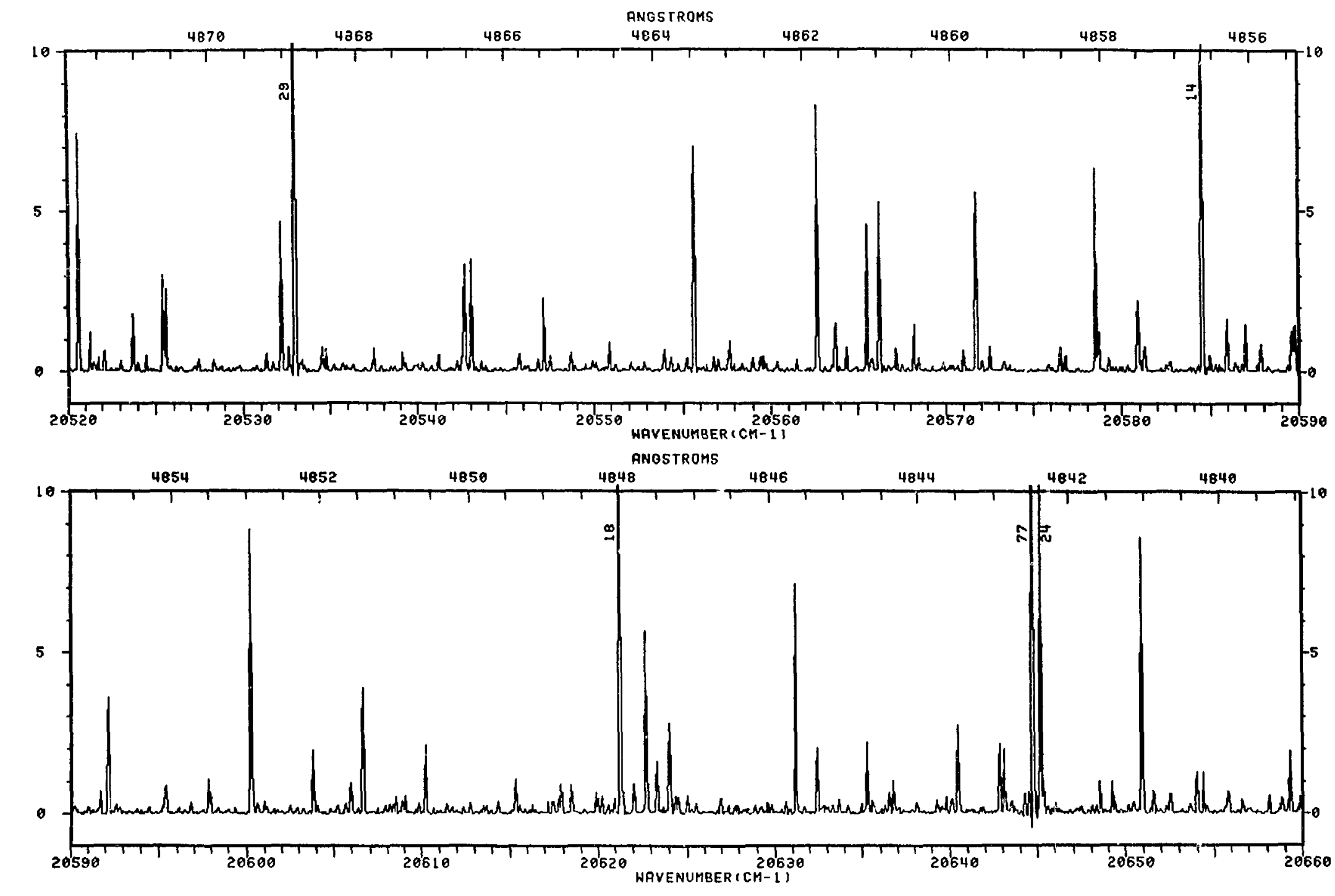
以す山

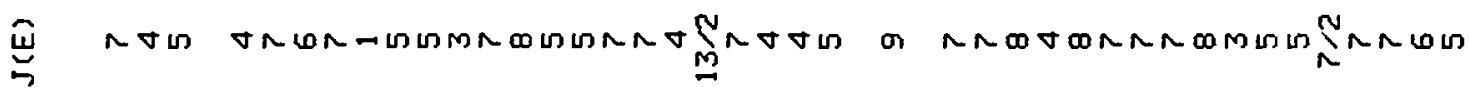

a. r.

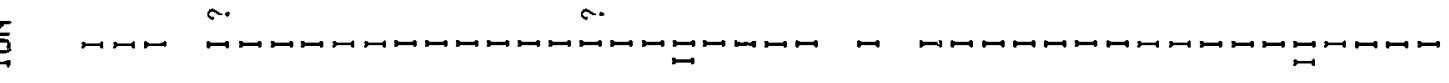

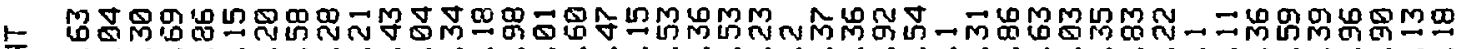

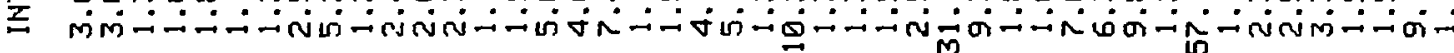

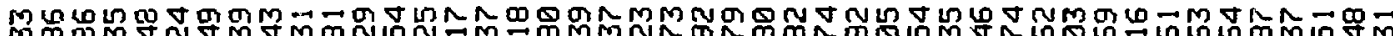

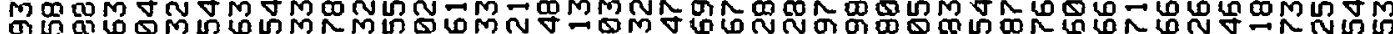

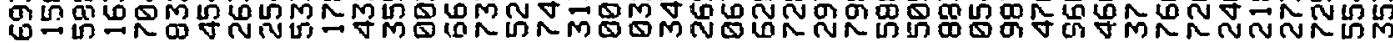

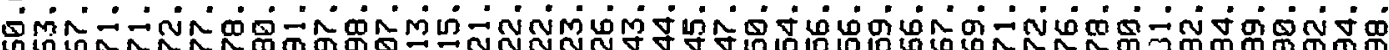

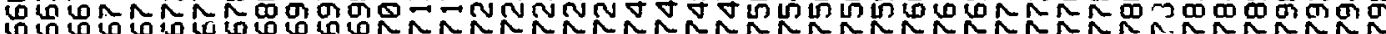

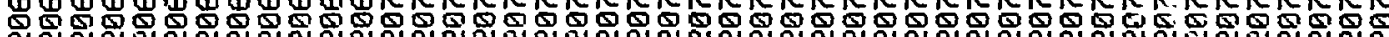
N N

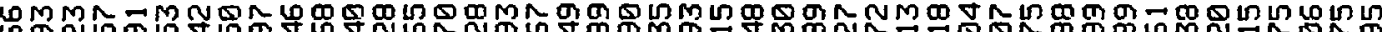

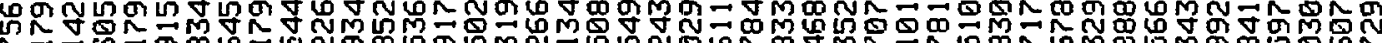
N N giń

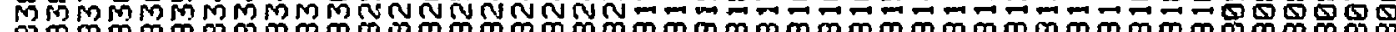

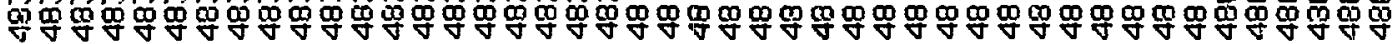



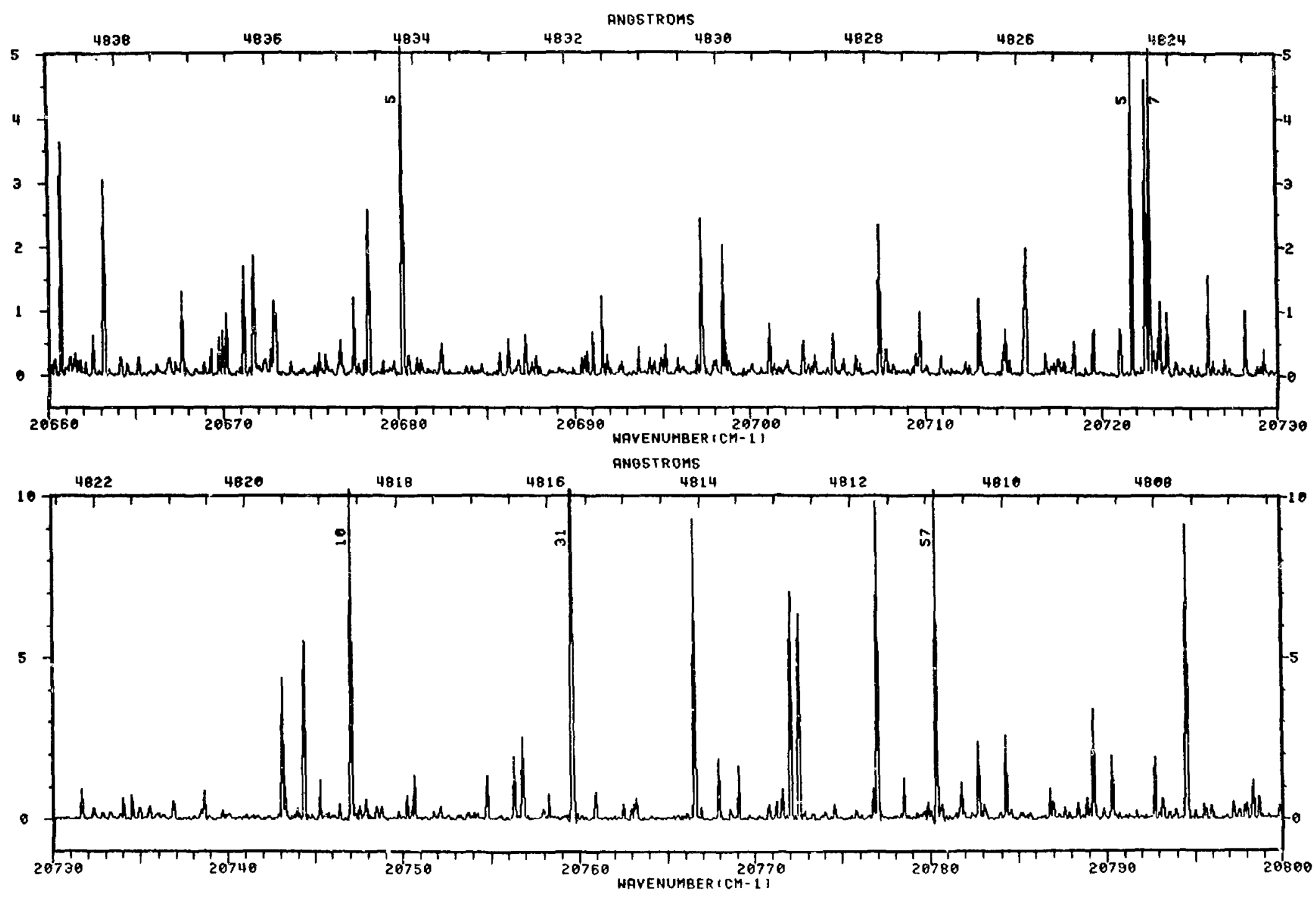


\begin{tabular}{|c|c|c|c|c|c|c|}
\hline$\widetilde{\sigma}(s)$ & 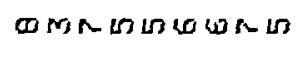 & $\nabla N$ & $\nabla \nabla \nabla \infty \sin$ & $\nabla$ & $6 \nabla m \operatorname{~os~}$ & 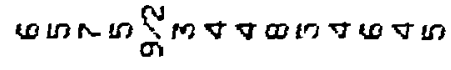 \\
\hline$\underbrace{0}_{0}$ & 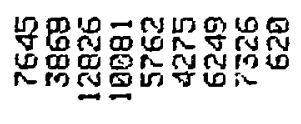 & $\overrightarrow{\Omega_{0}}$ & 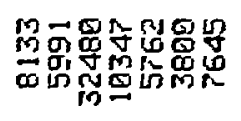 & $\begin{array}{l}\text { 兽 } \\
\text { 怘 }\end{array}$ & 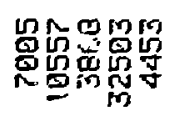 & 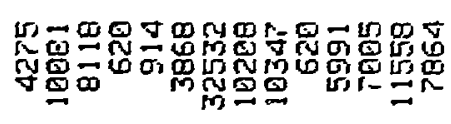 \\
\hline
\end{tabular}

峞
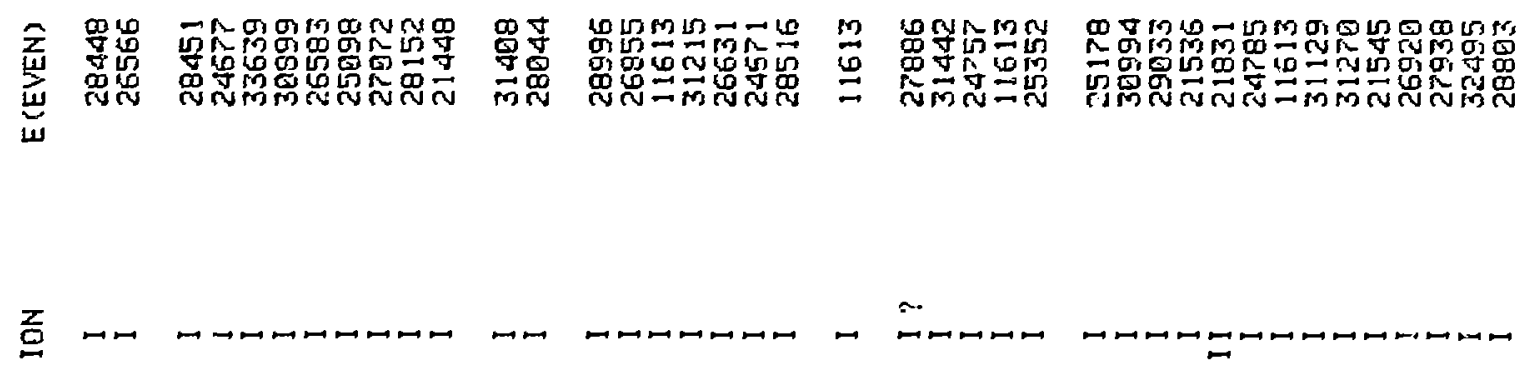

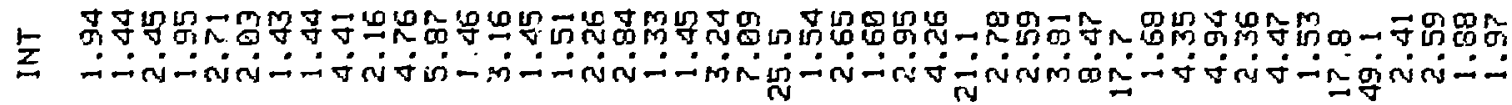

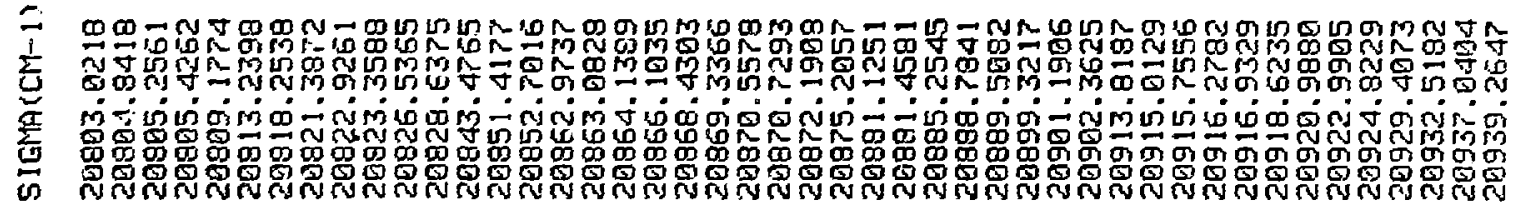

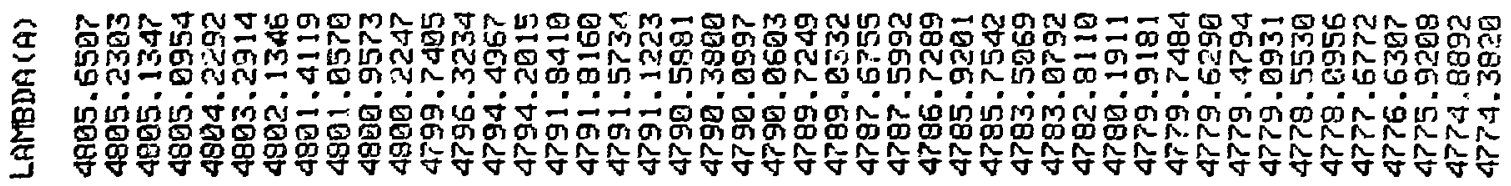




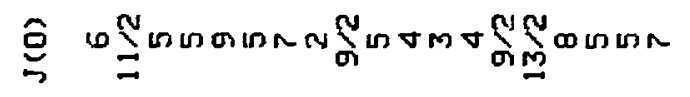
n
NMn o o

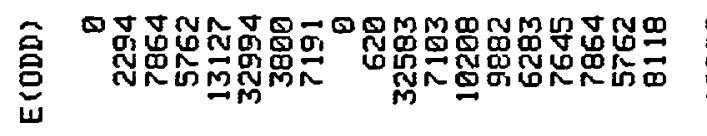

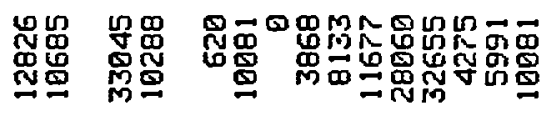

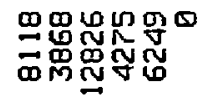

कु

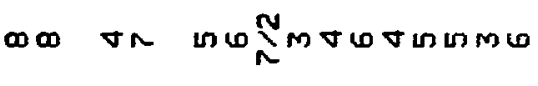

m

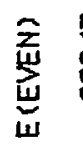

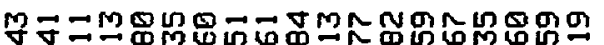

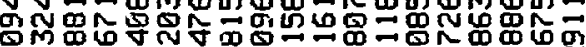

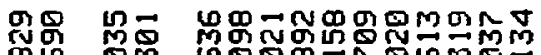

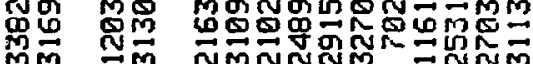

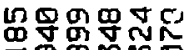

$\vec{\sigma} \operatorname{smm}$

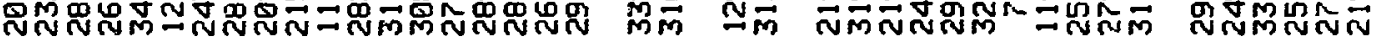

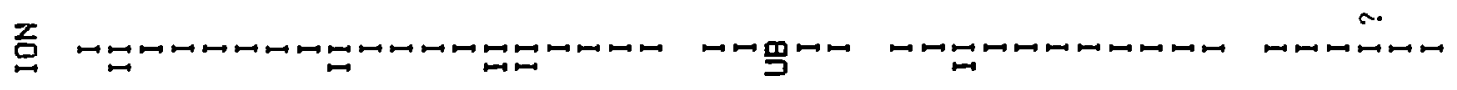

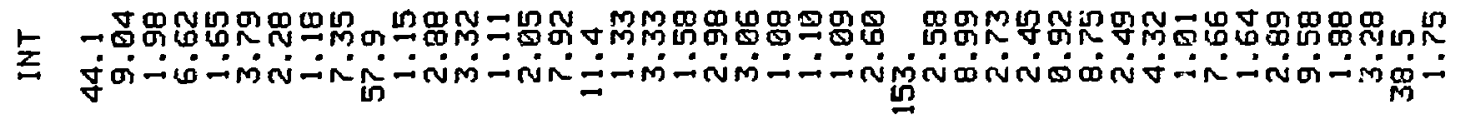

2

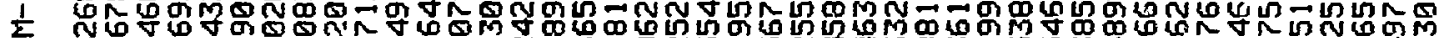

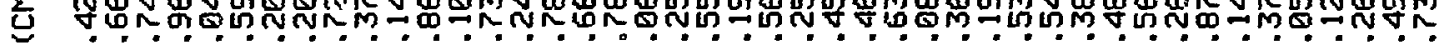

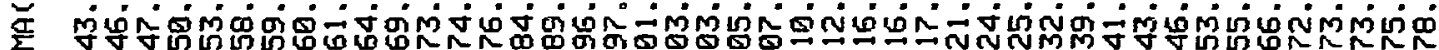

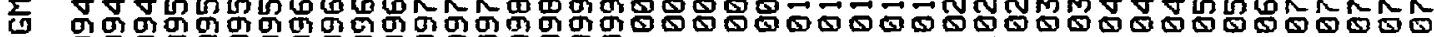

G N

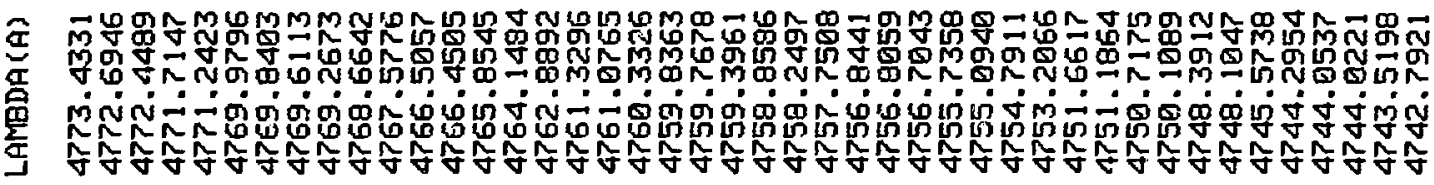




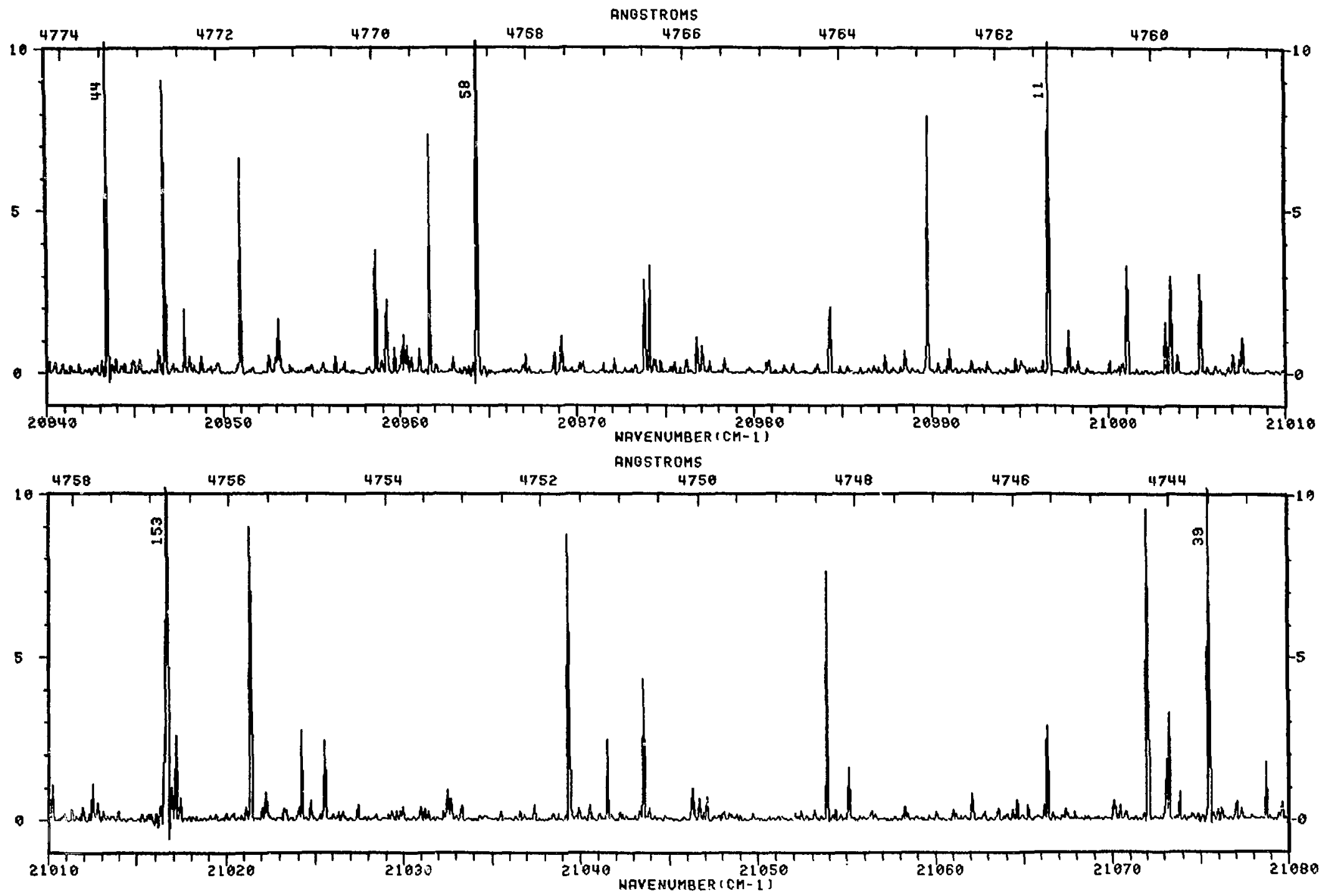


ğ

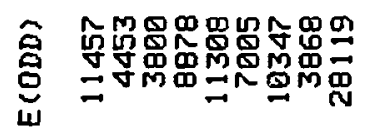

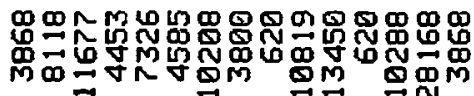

界

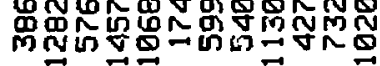

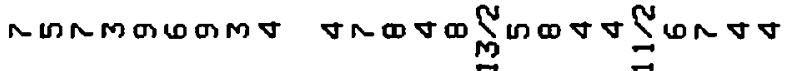

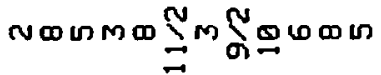

mine in

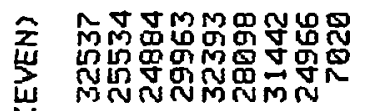

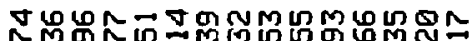

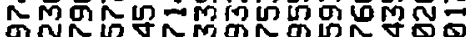

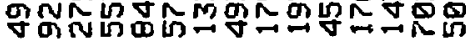

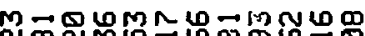

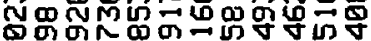

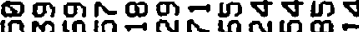

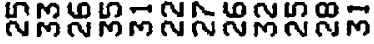

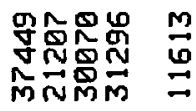
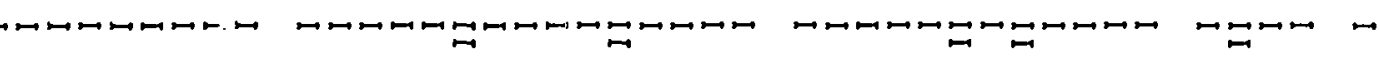

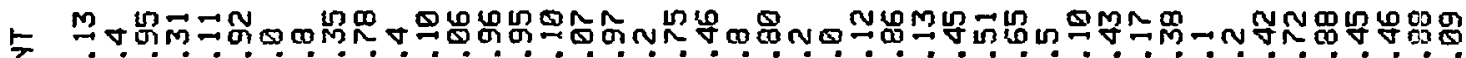

-

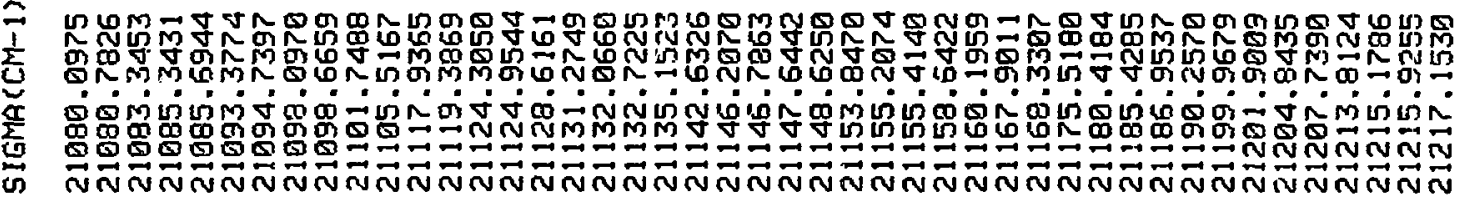

6ตำ भด

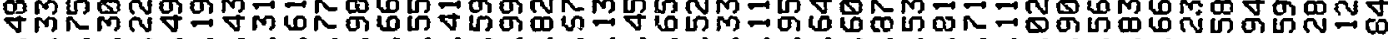

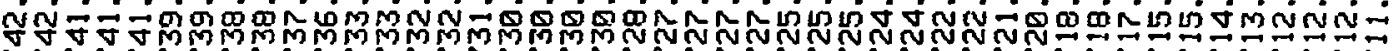

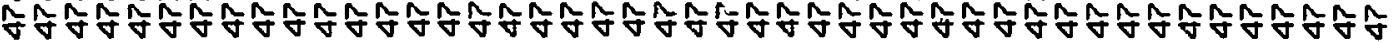



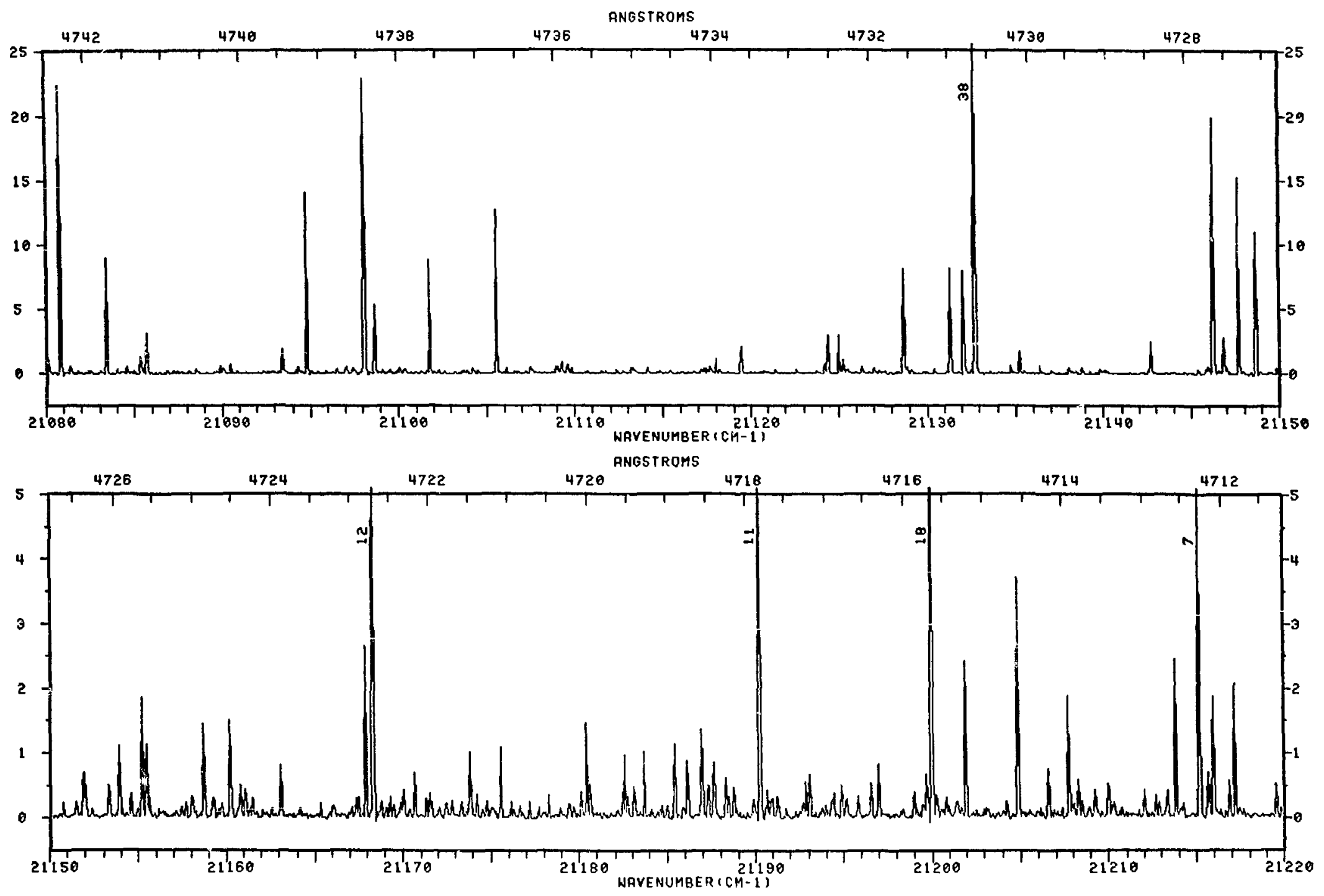

147 
总 novR

ล

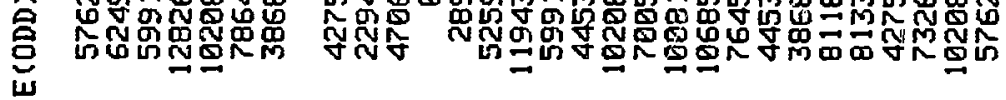

$\Delta \sim M \otimes R Q M \sim g m$

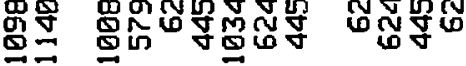

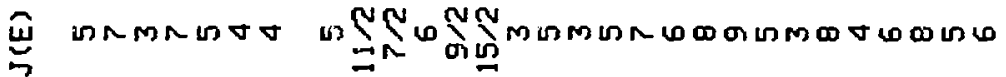

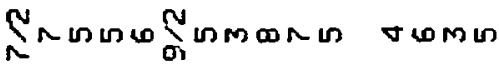

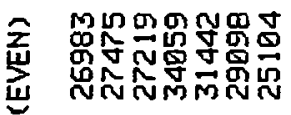

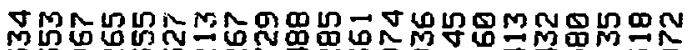
น

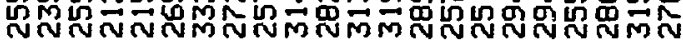

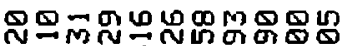
mMNM

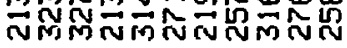

以四岛 \%ั0 त低空

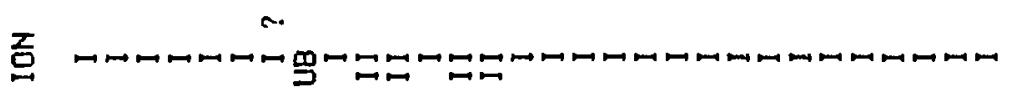

$\varpi$

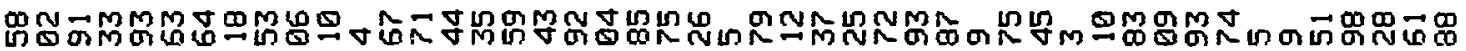

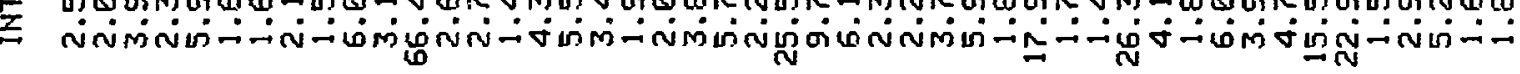

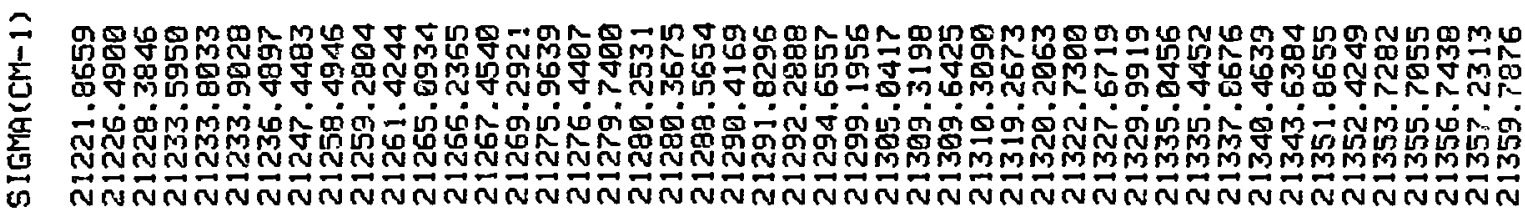

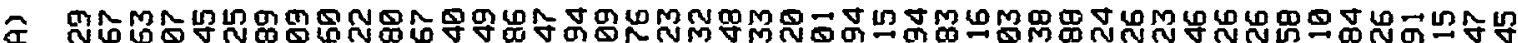

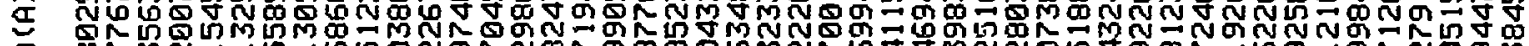

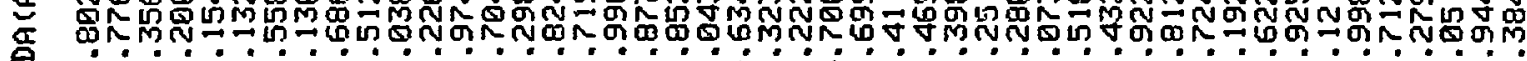

ब

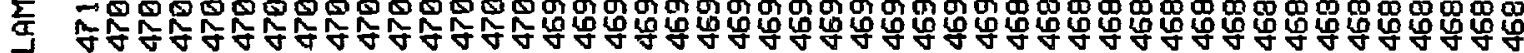



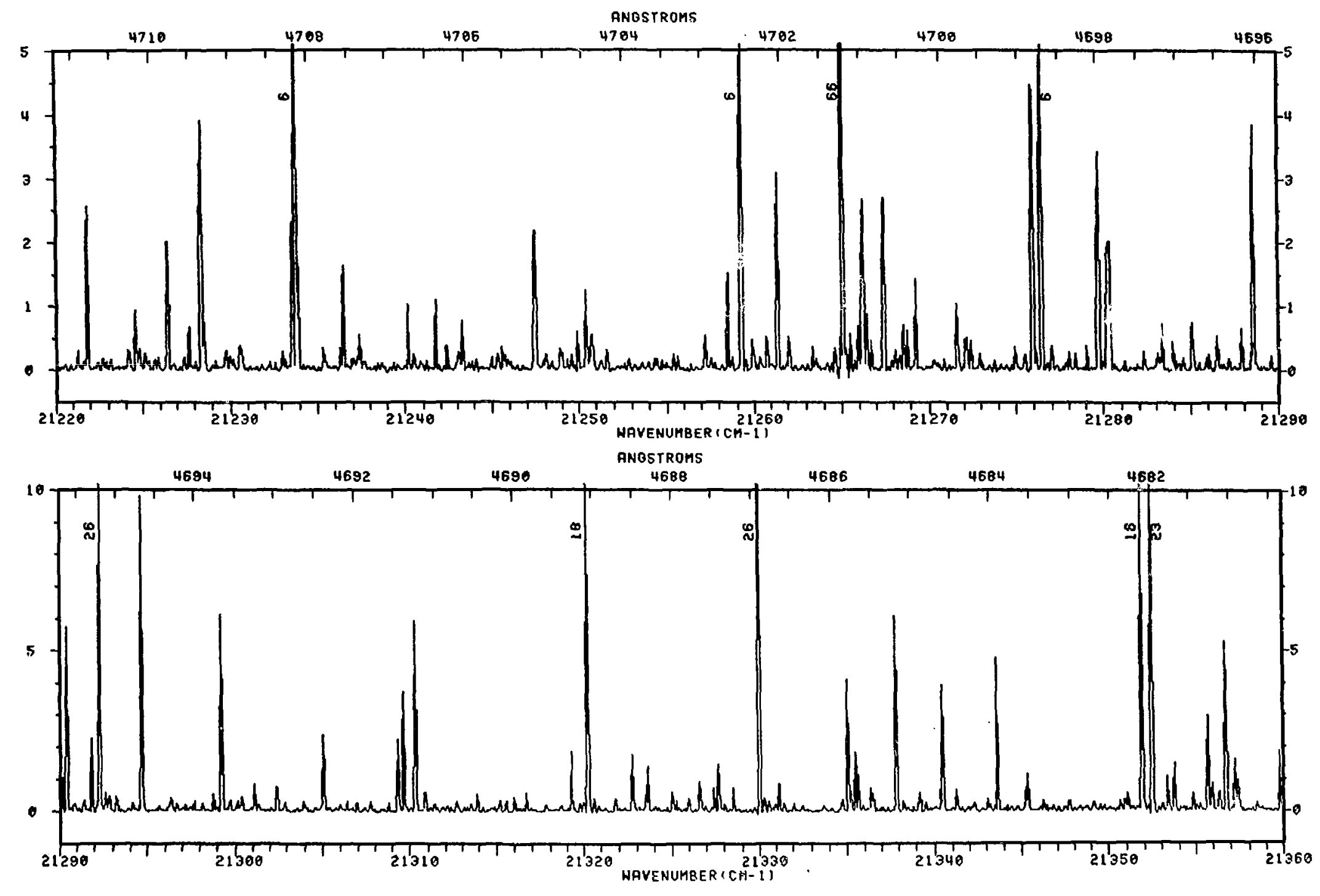


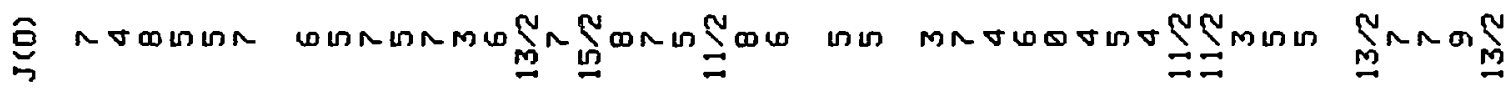

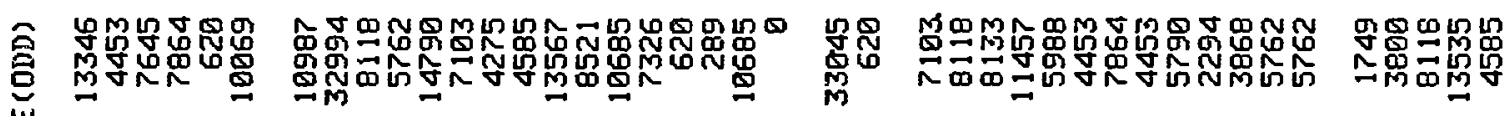

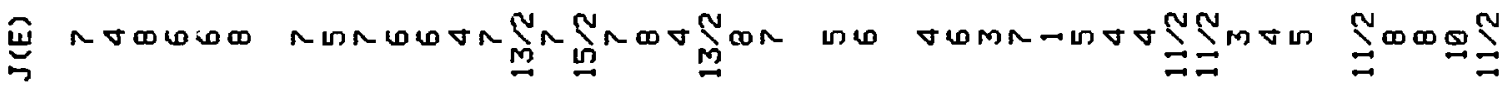

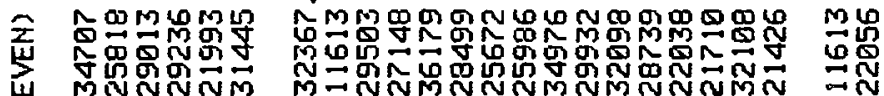

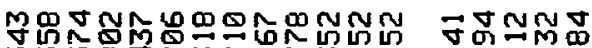

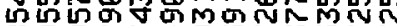

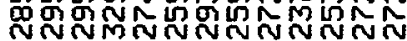

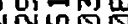

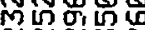
NNMN

흠
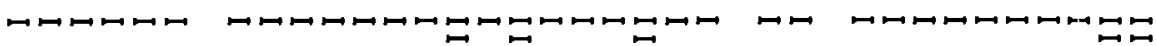

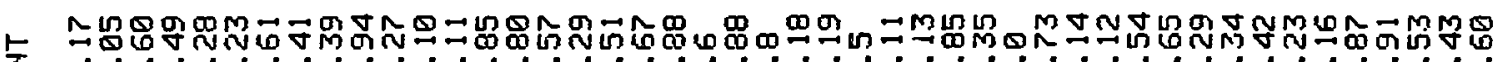

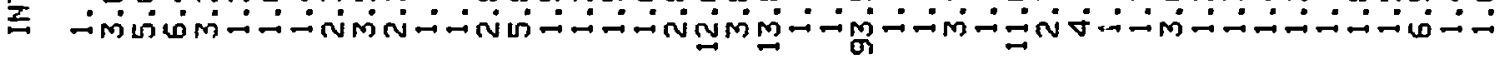

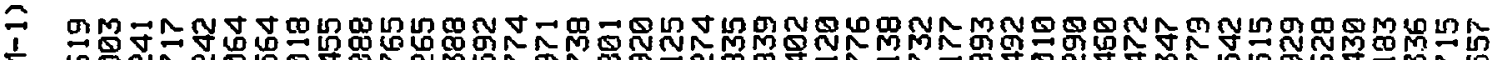

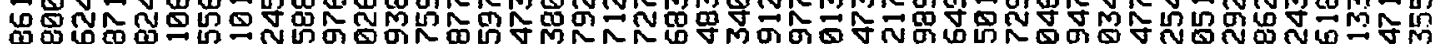

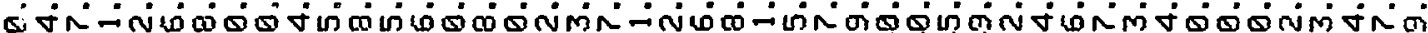
ऊผ MMMMMm

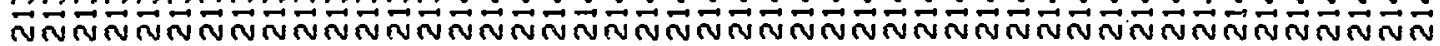

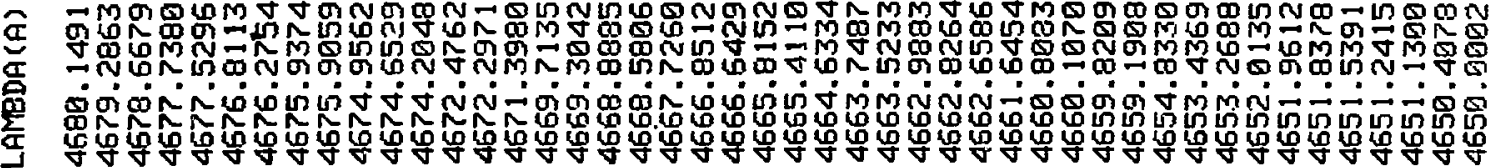




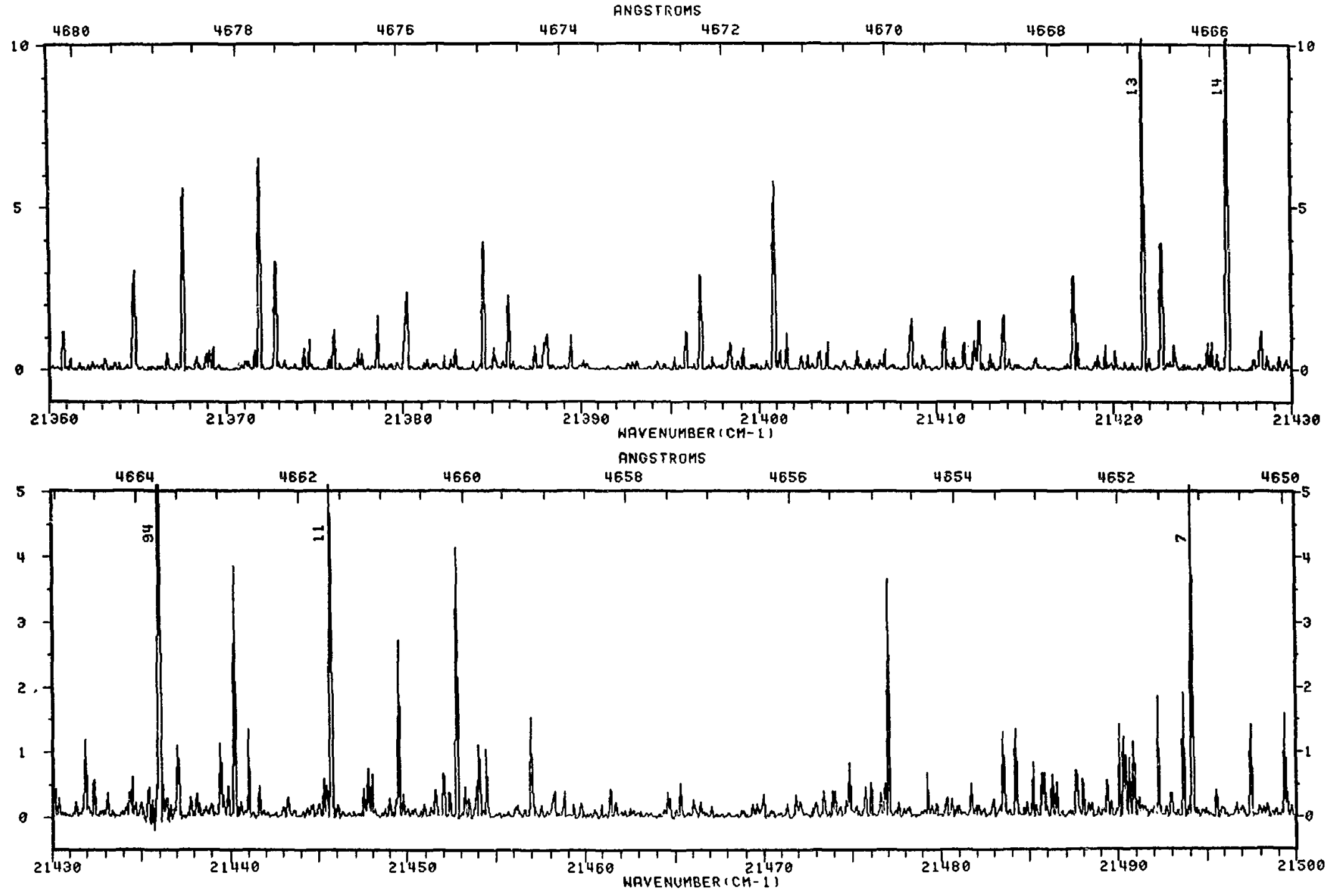

151 


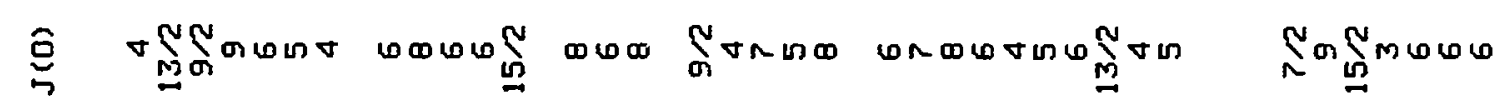

\begin{tabular}{|c|c|c|c|c|c|}
\hline 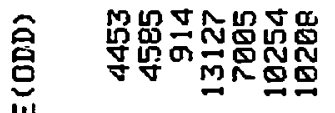 & 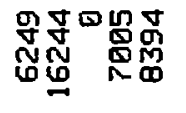 & 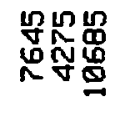 & 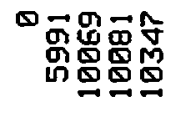 & 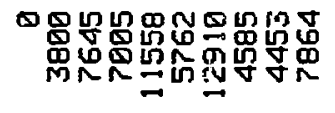 & 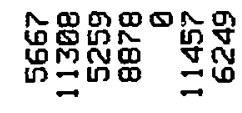 \\
\hline
\end{tabular}

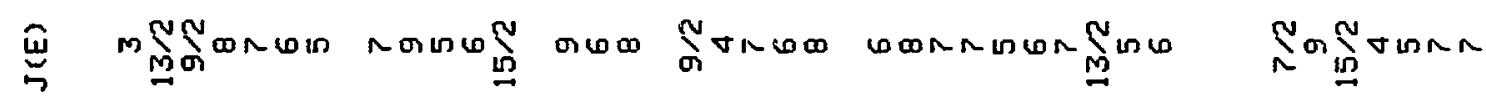

\begin{tabular}{|c|c|c|c|c|}
\hline & 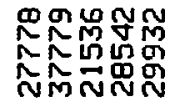 & 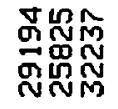 & 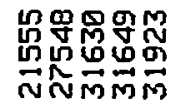 & 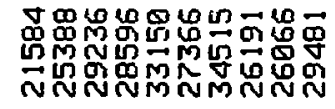 \\
\hline
\end{tabular}

긍
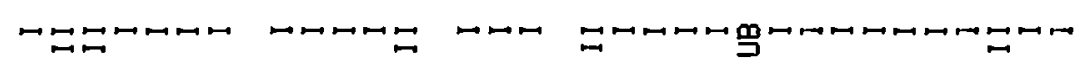

罗コロロロローロ

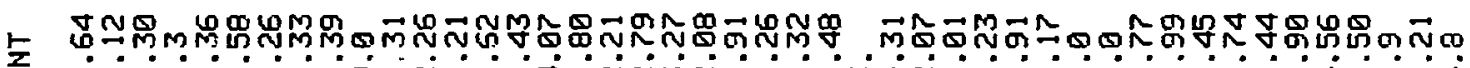

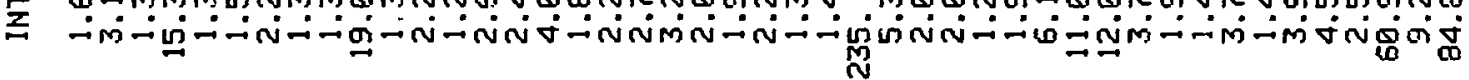

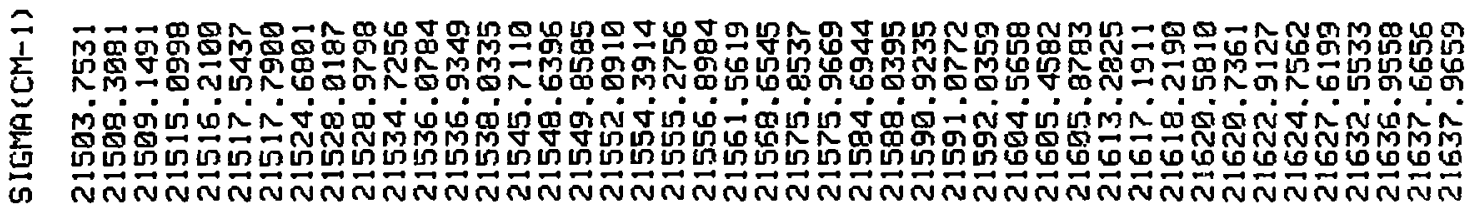

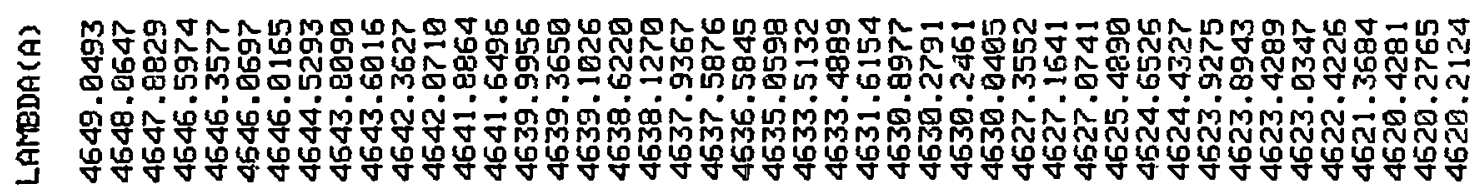



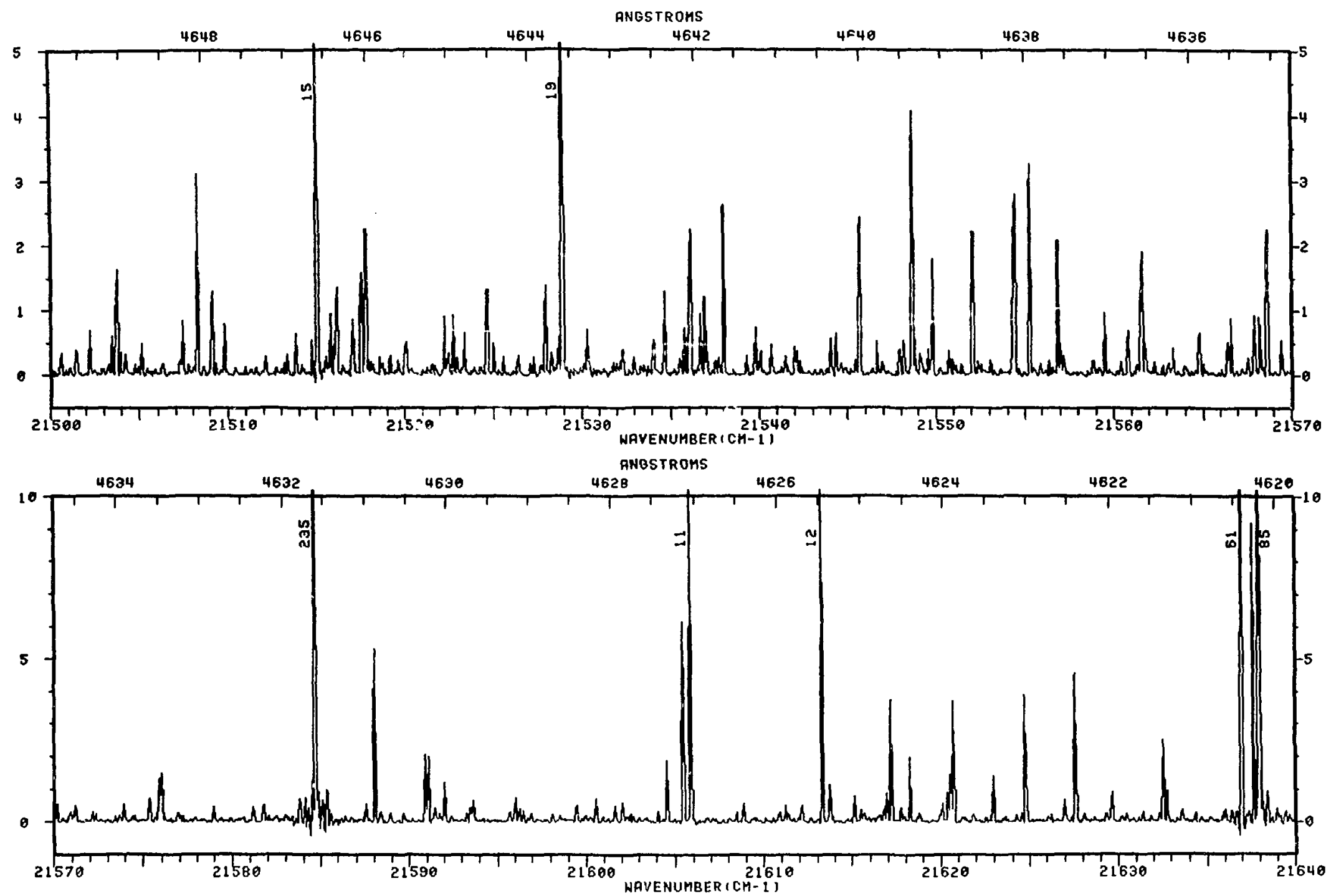


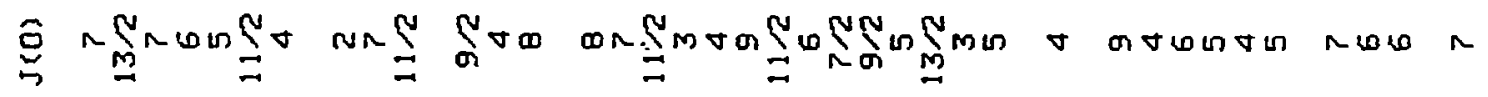

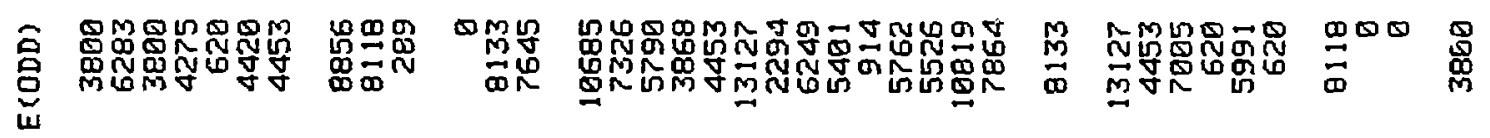

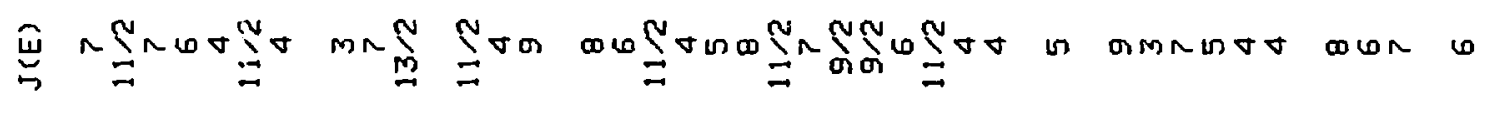

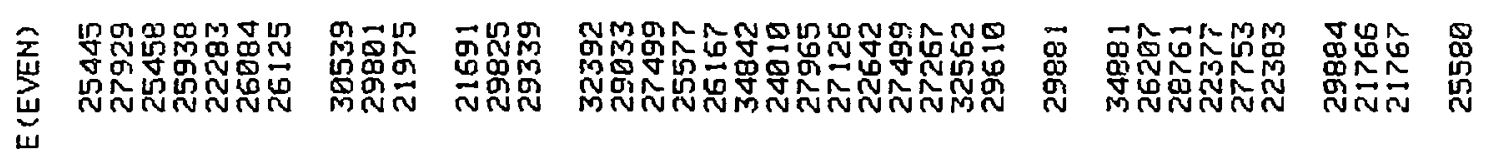

吾

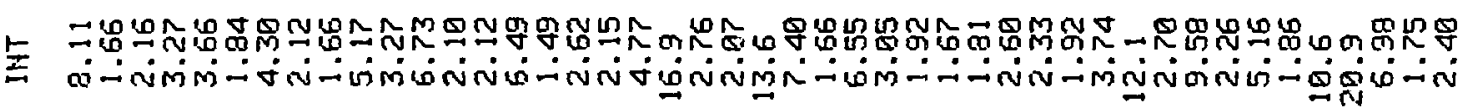

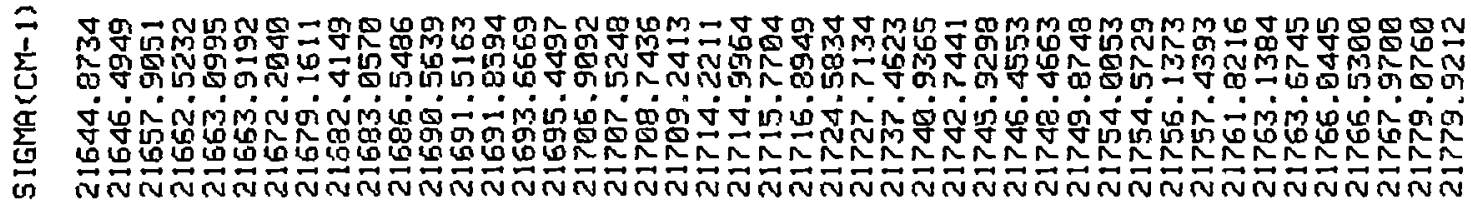

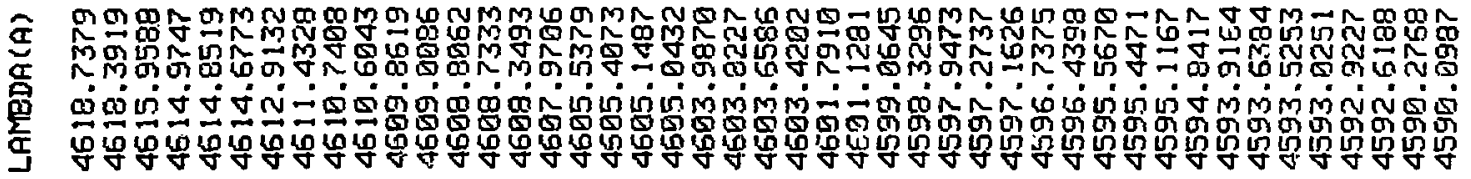




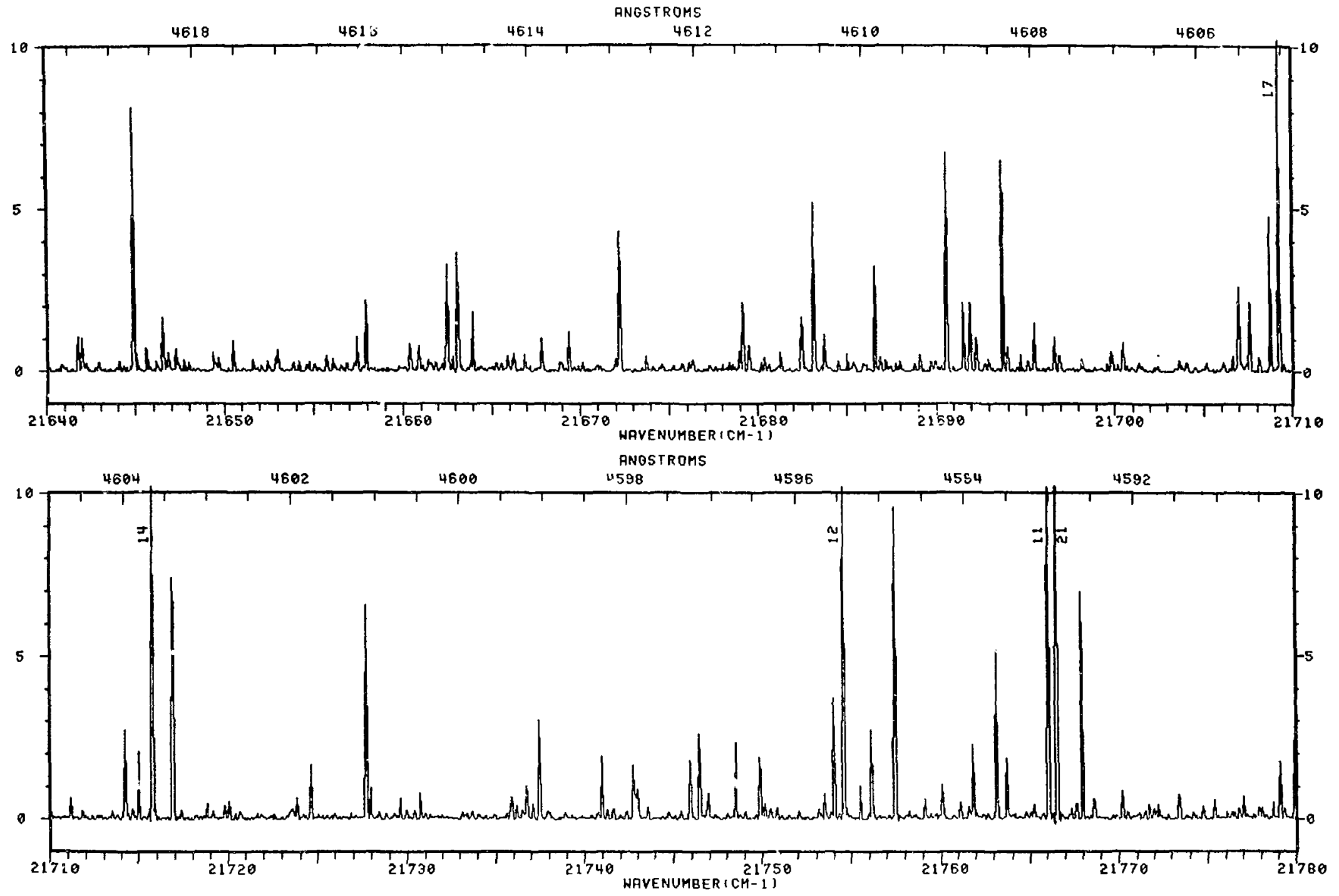

155 


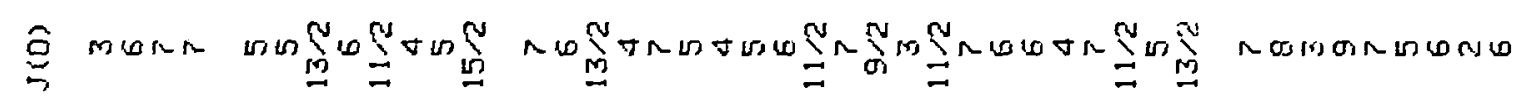

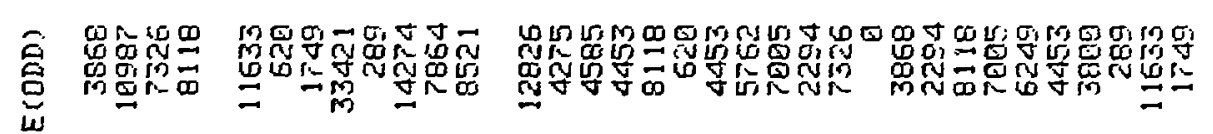

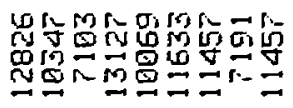

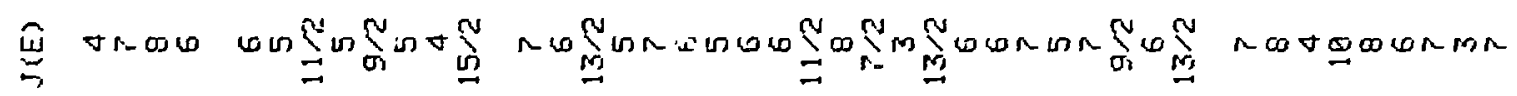

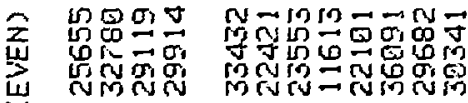

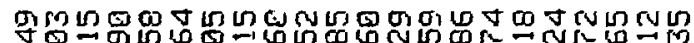

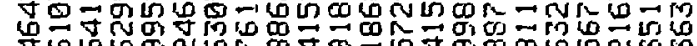

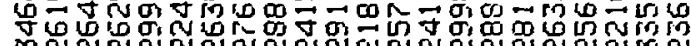

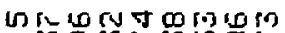

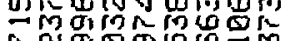

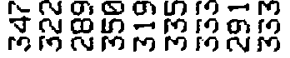

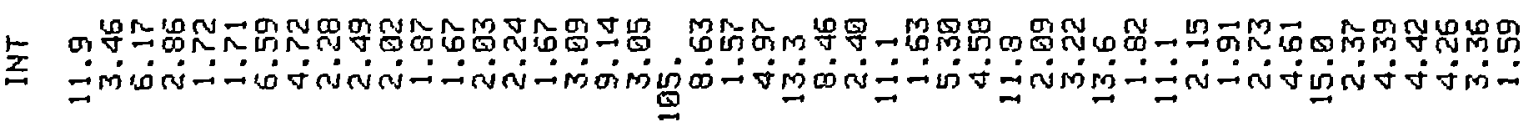

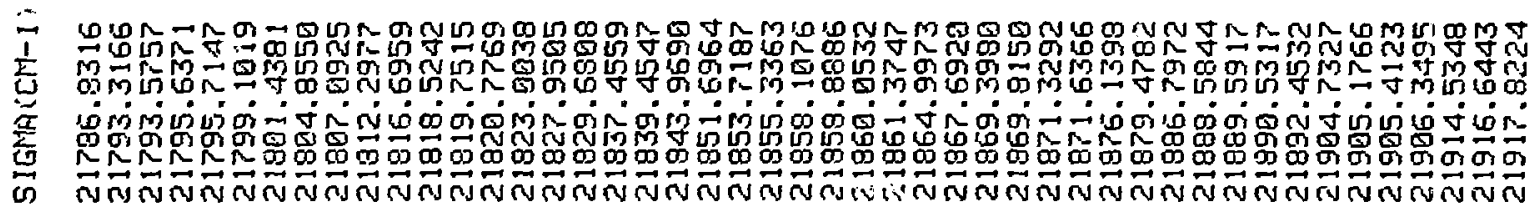

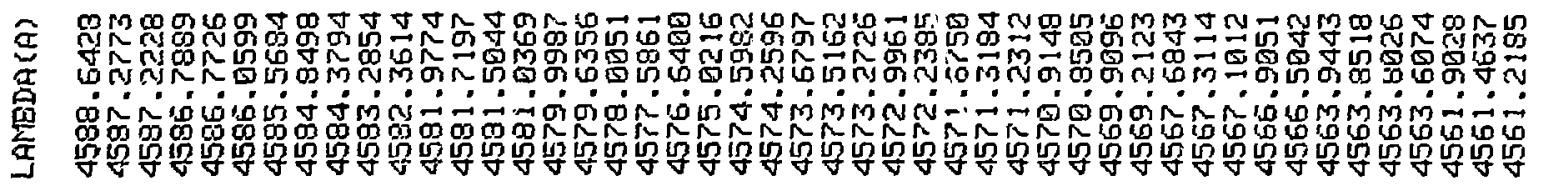




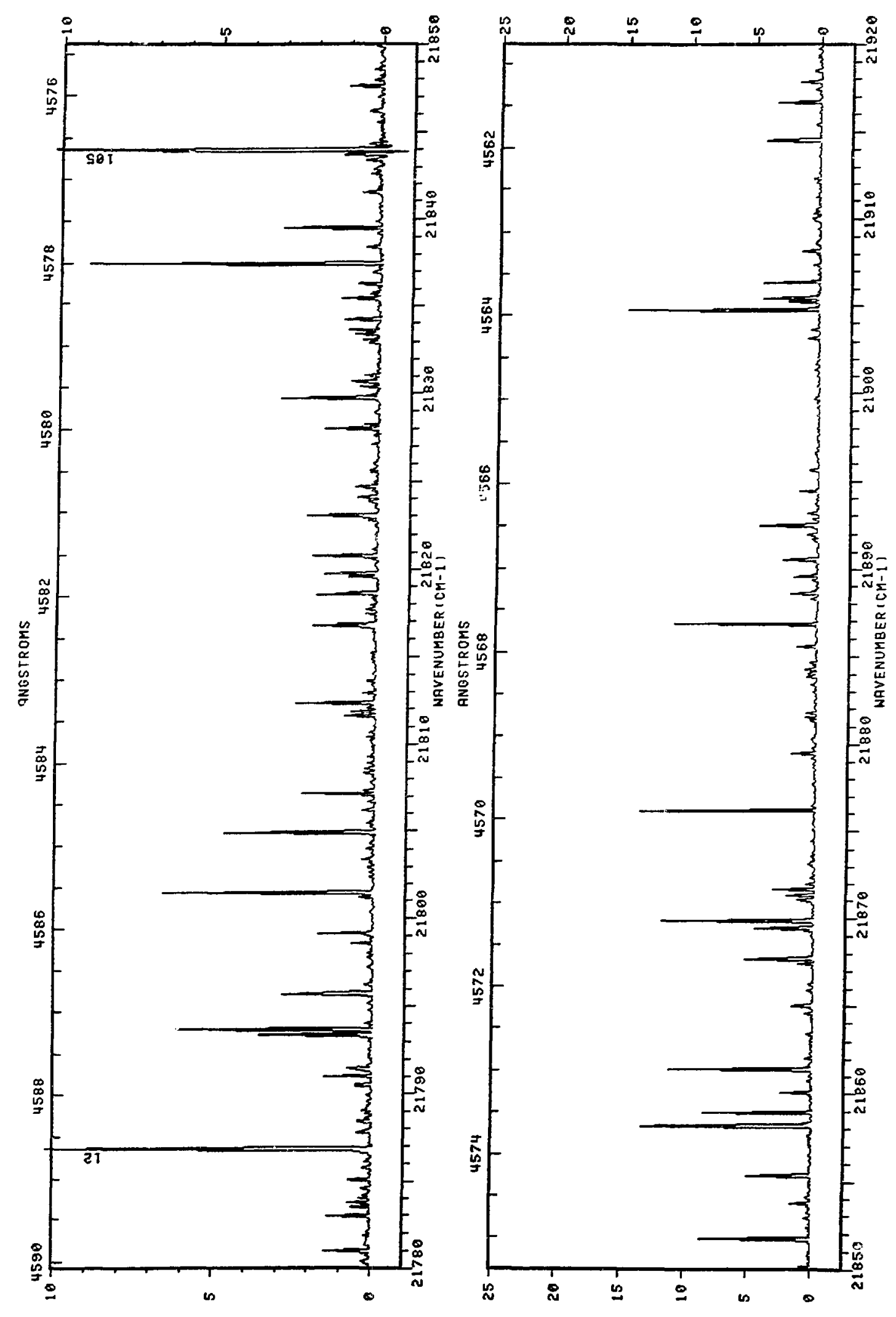

E 


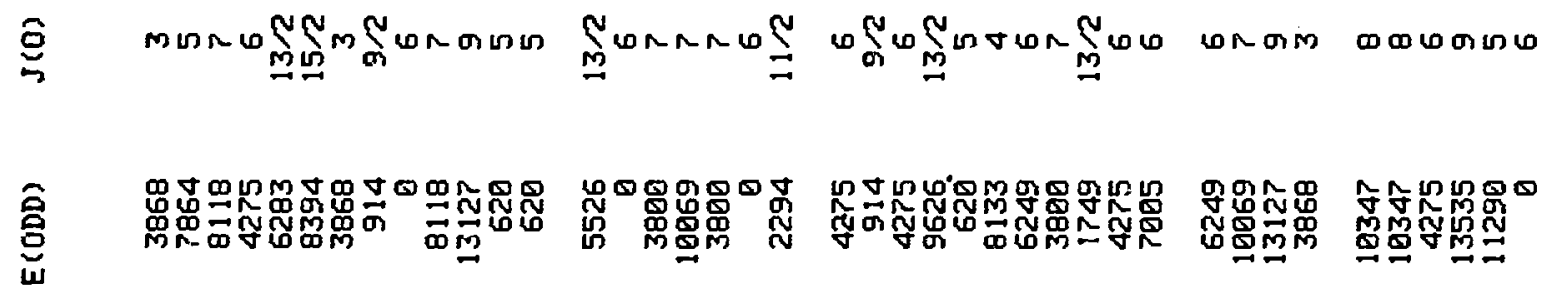

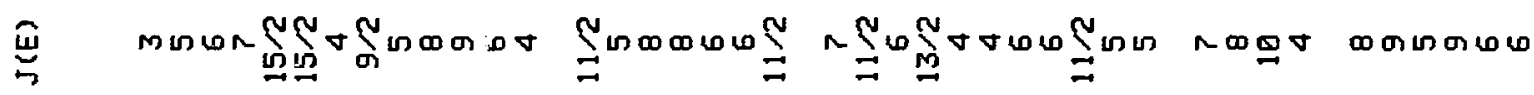

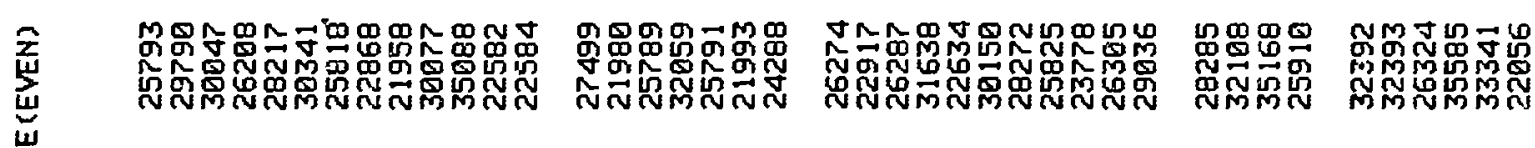

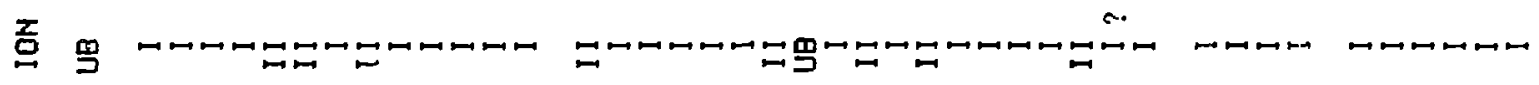

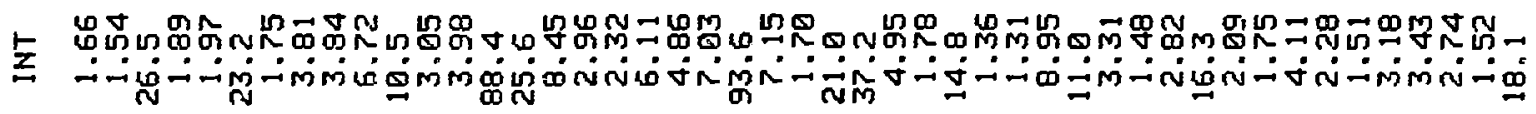
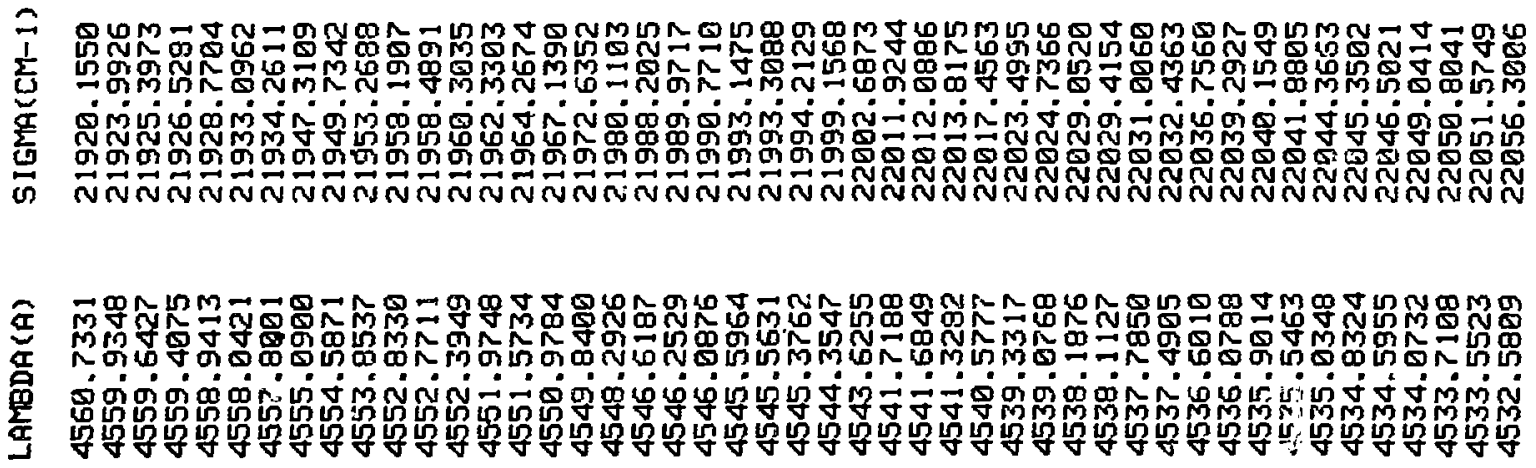



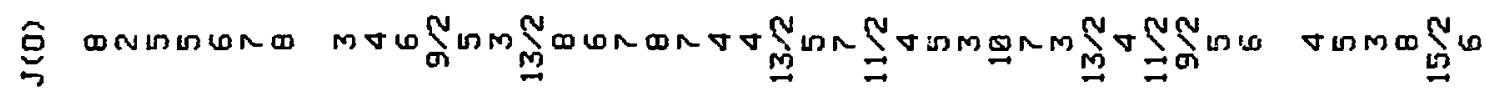

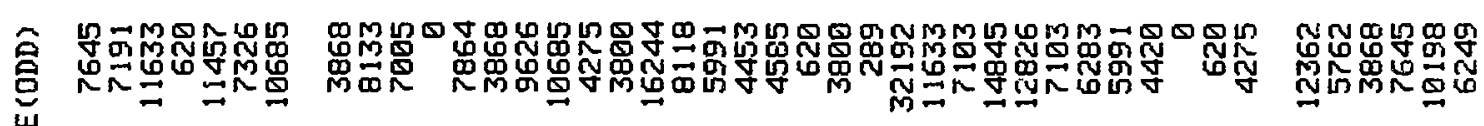

包

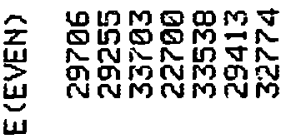

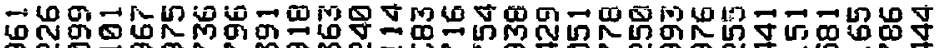

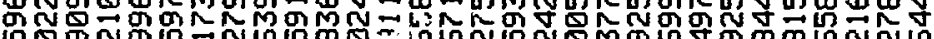

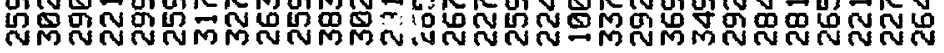

M品自罢

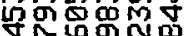
MÑ

$\underset{D}{\mathrm{Z}}$

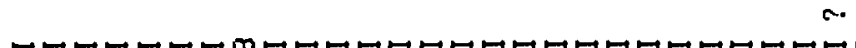
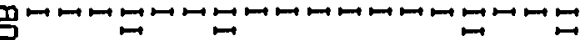

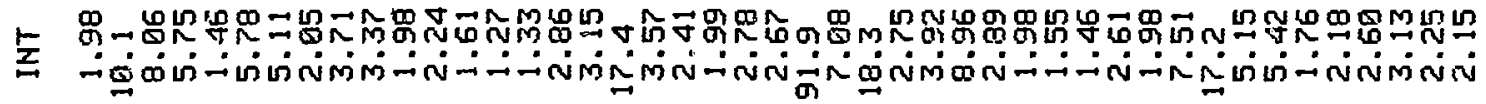

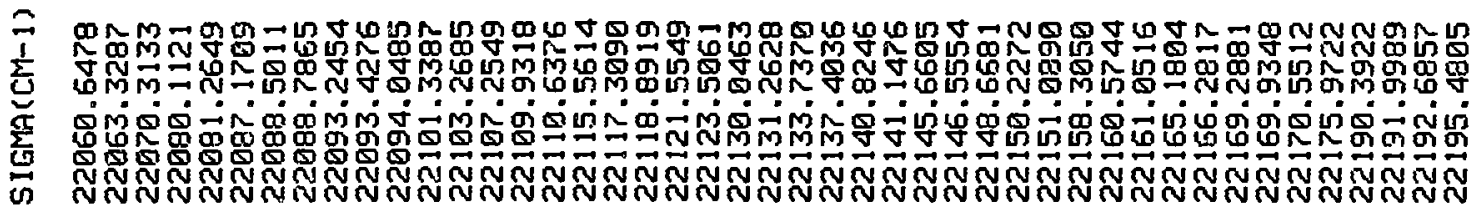

和

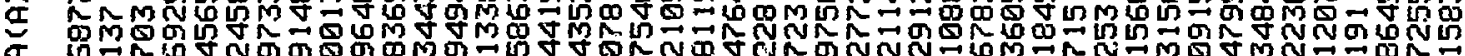

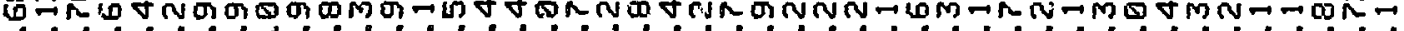

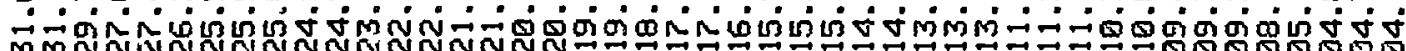

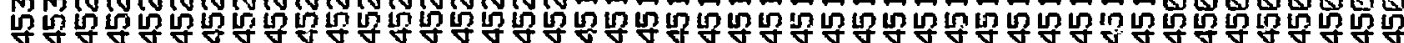





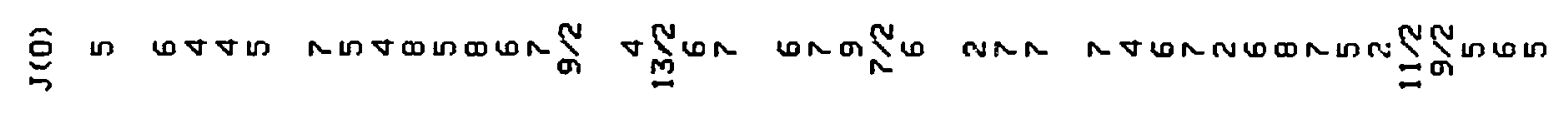

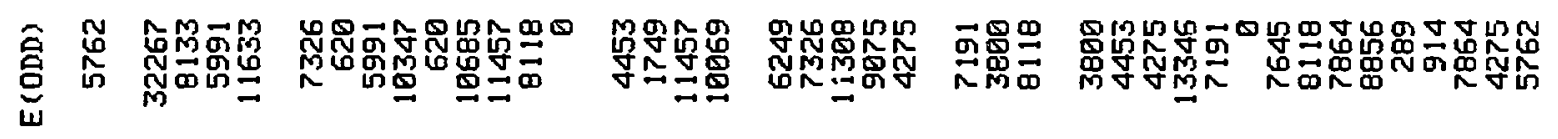

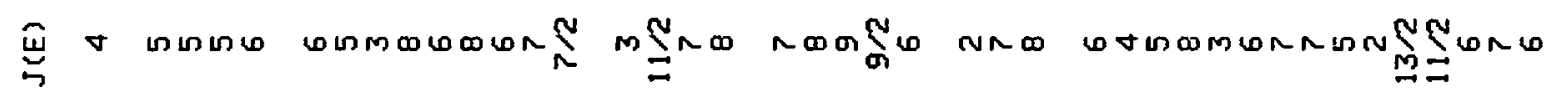

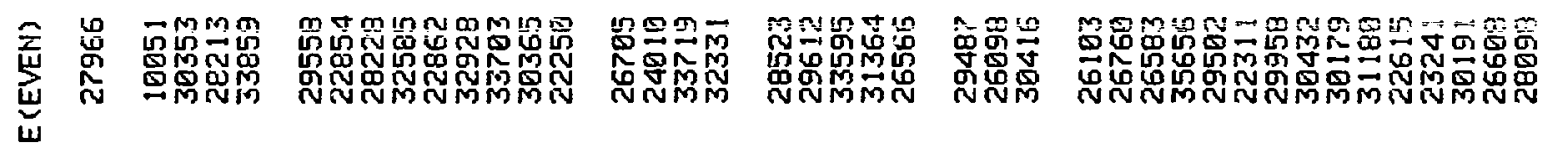

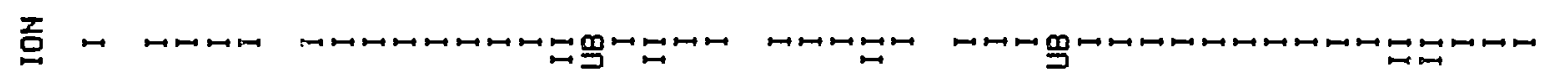

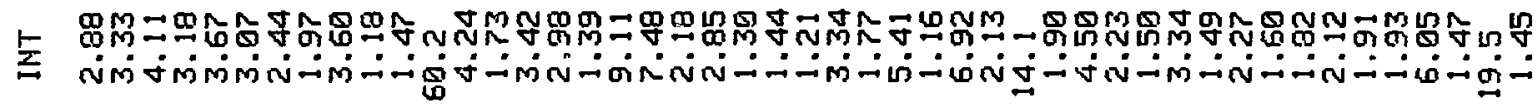

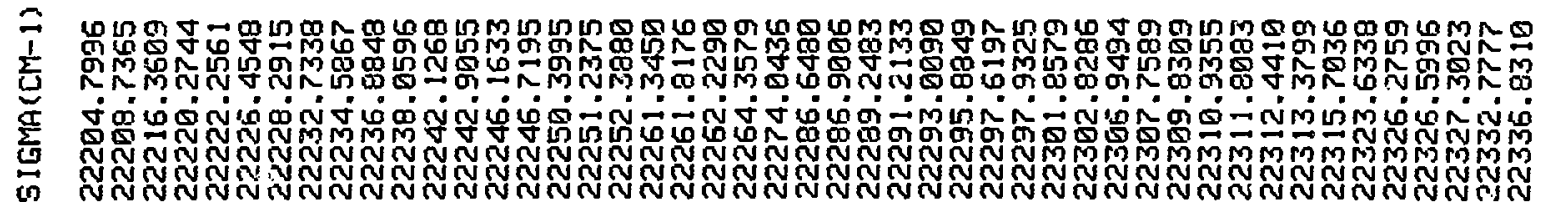

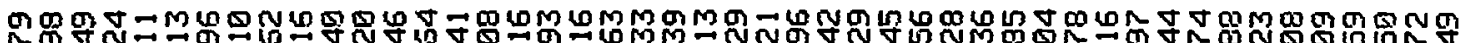
员 -

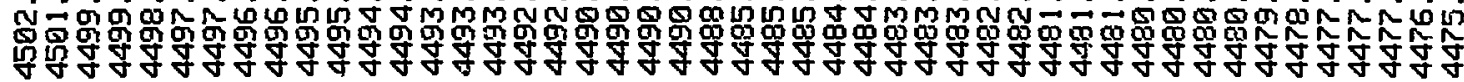




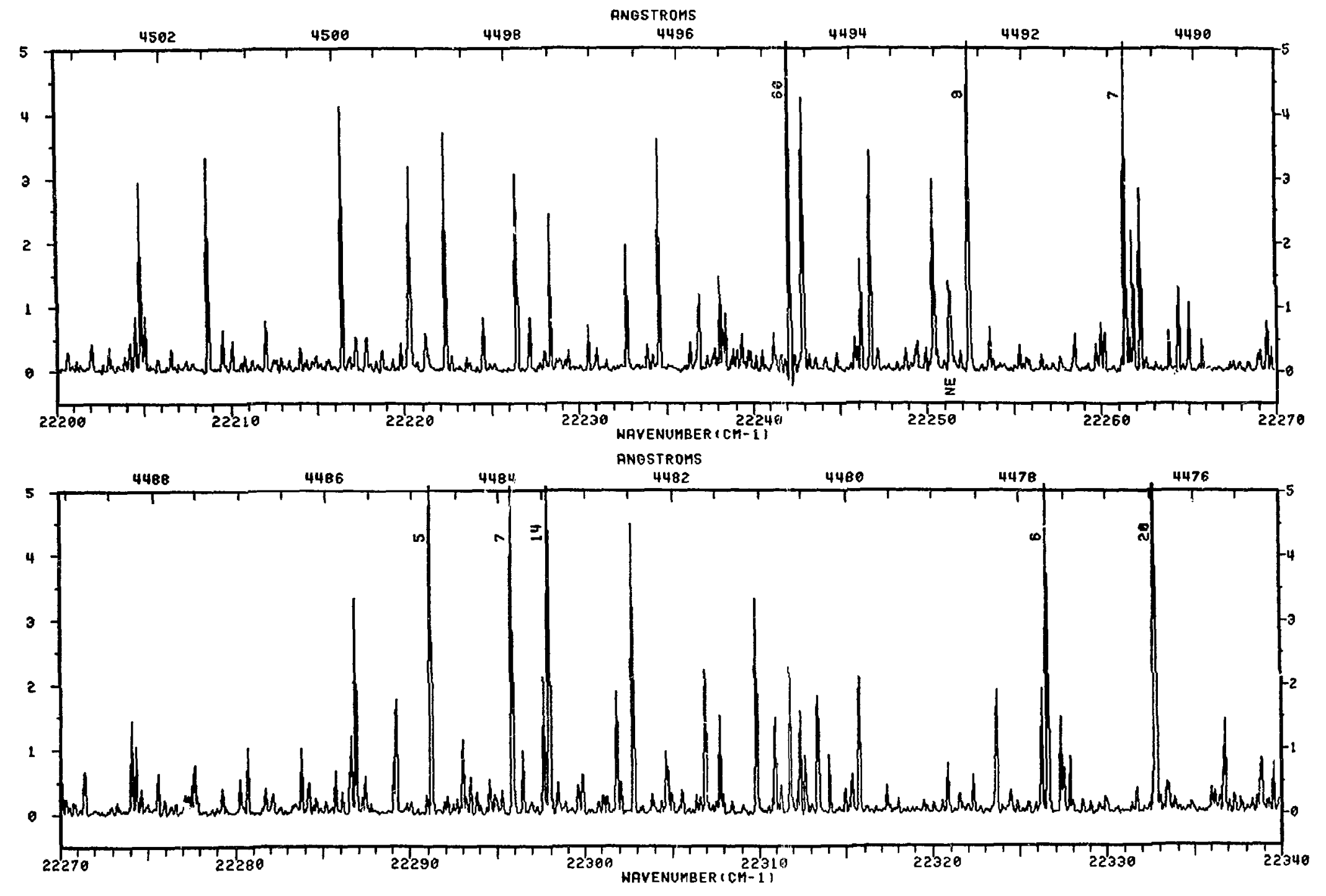




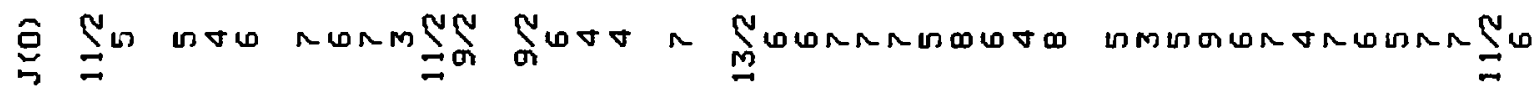

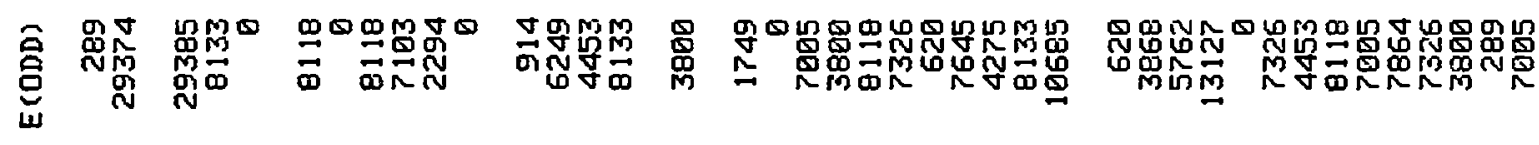

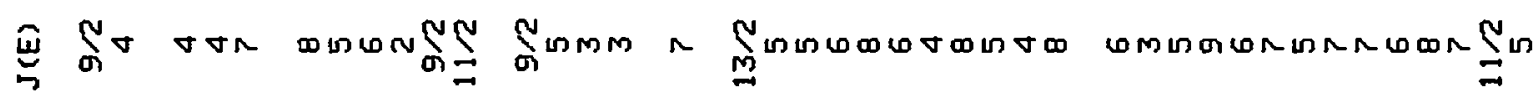

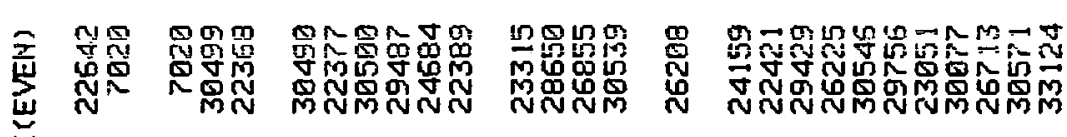

grom 000000407

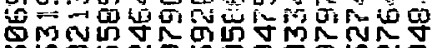
N

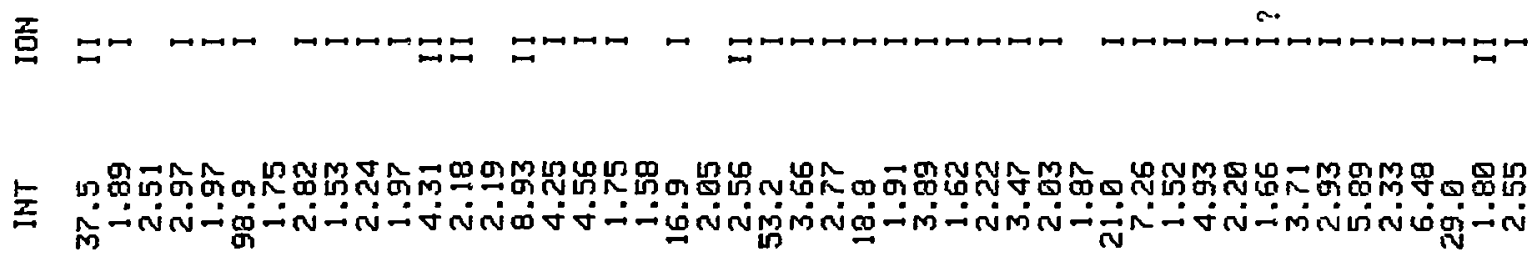

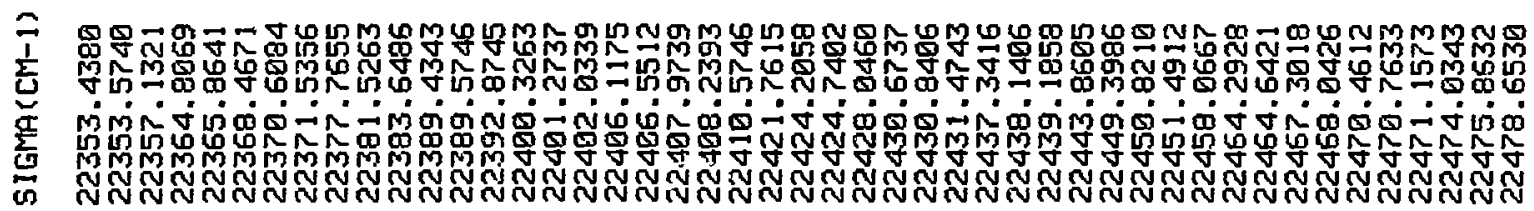

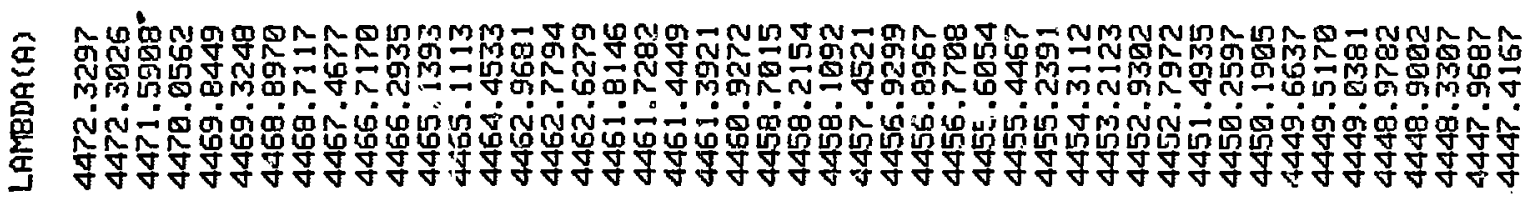



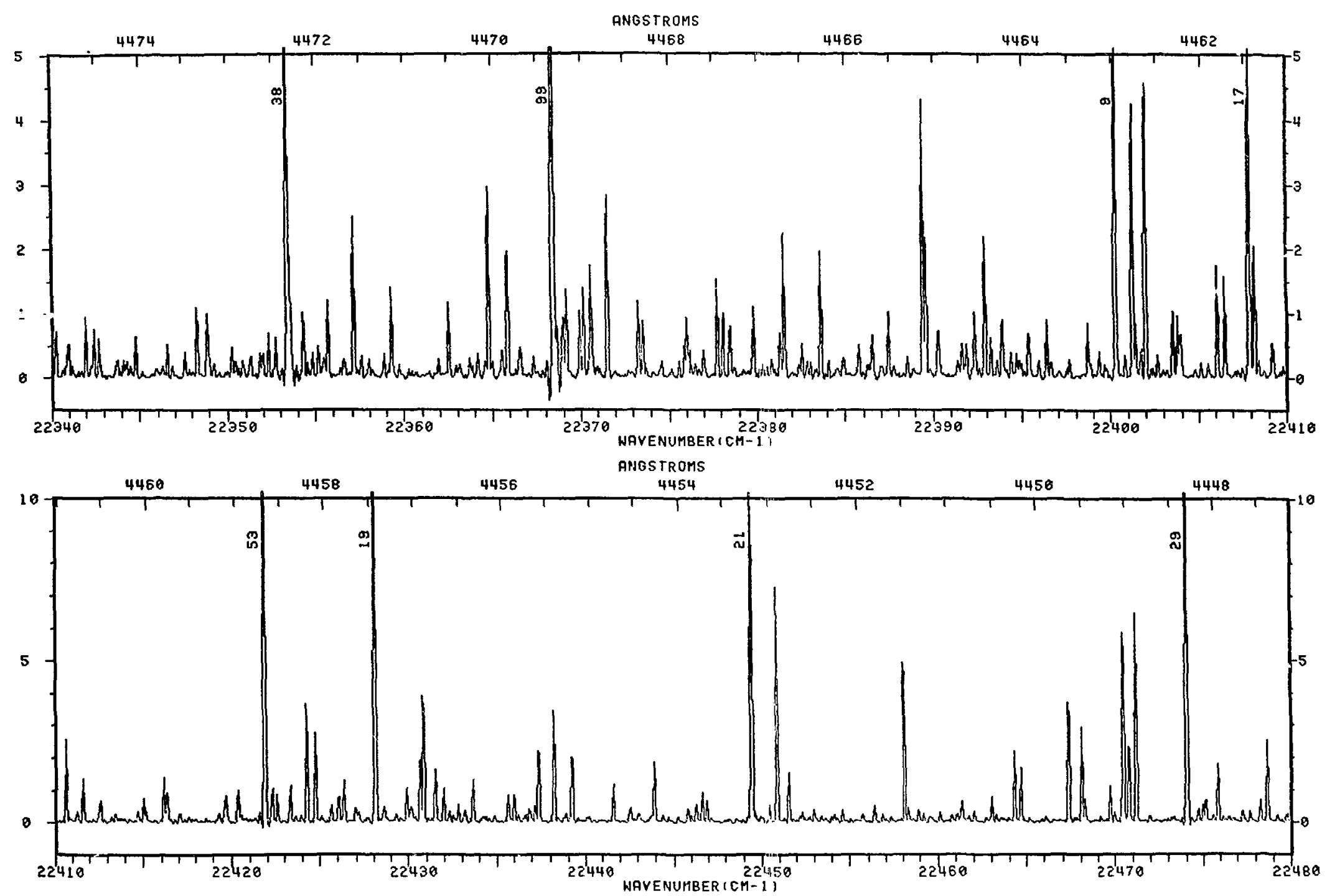


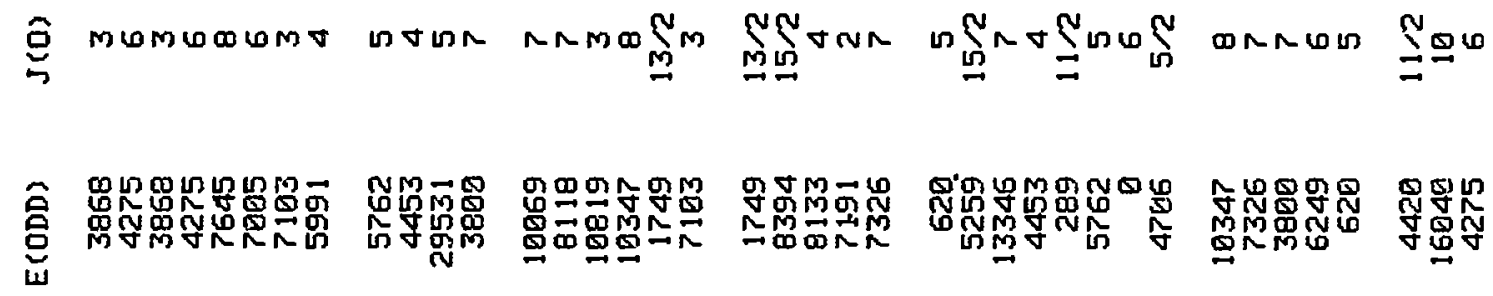

岂 VMNLOR

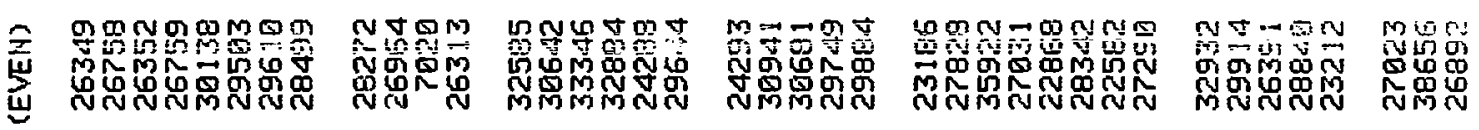

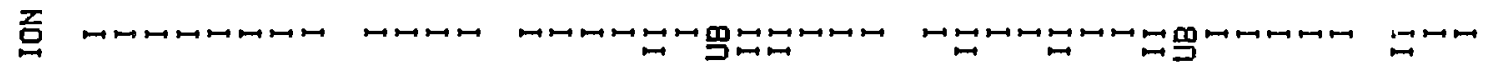

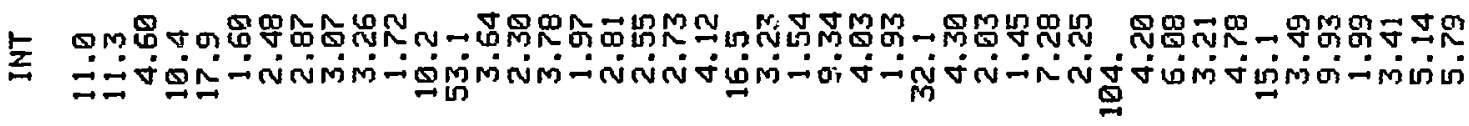

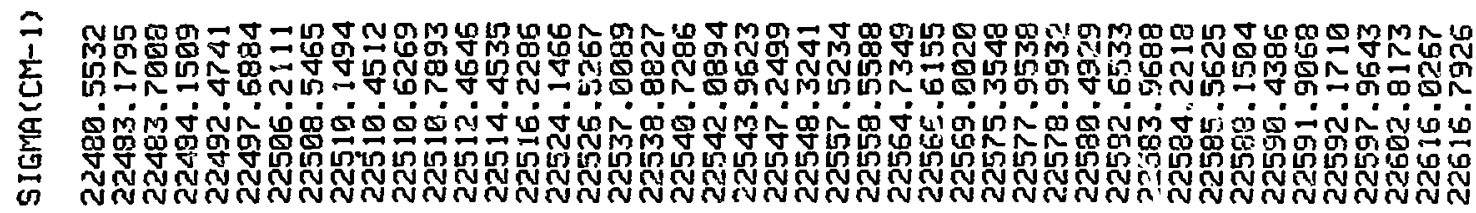

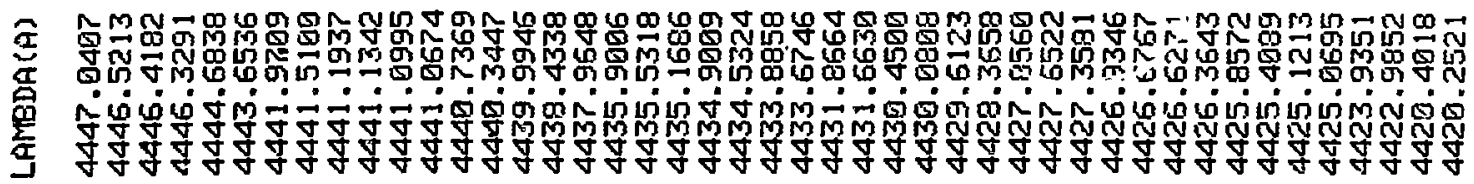



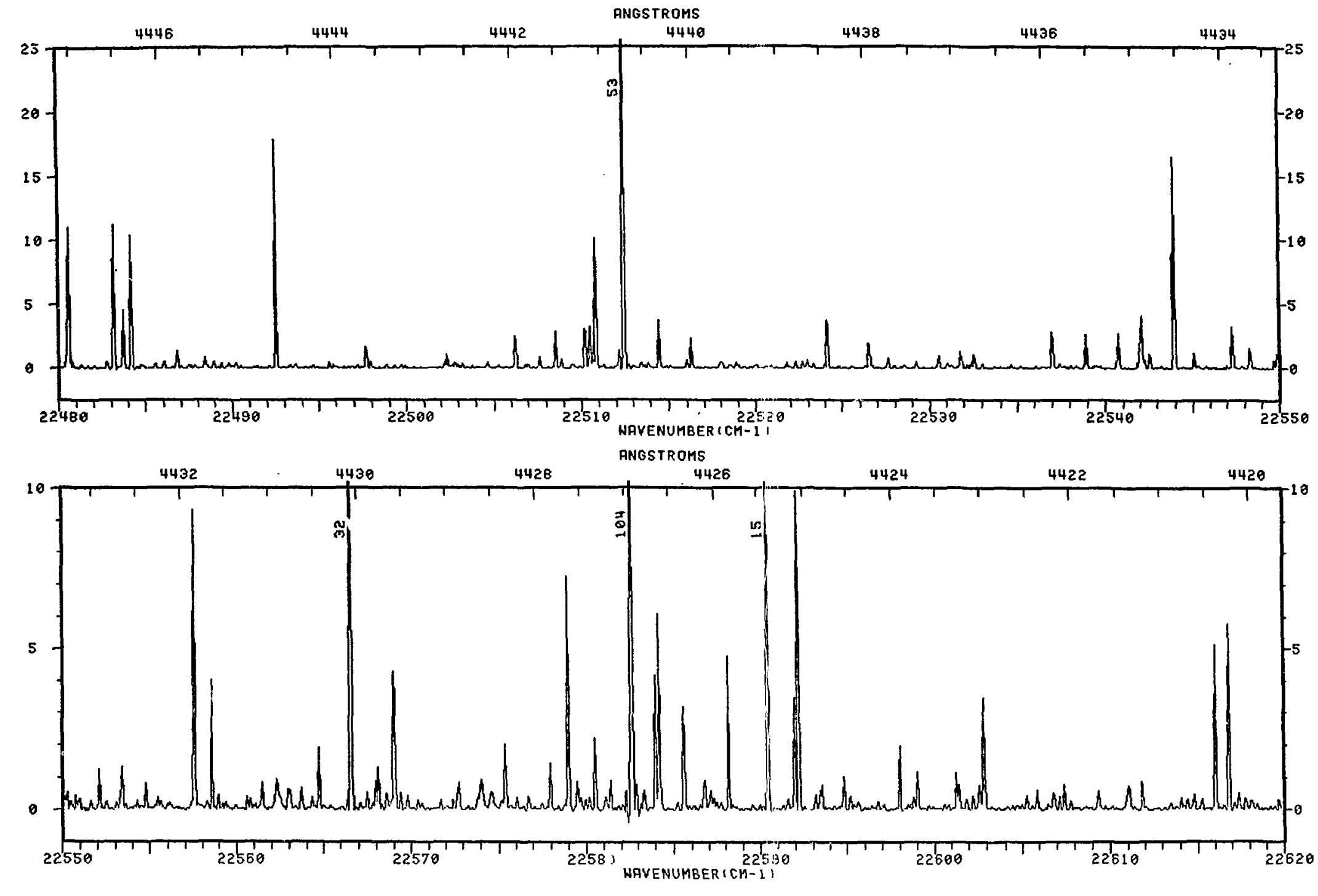


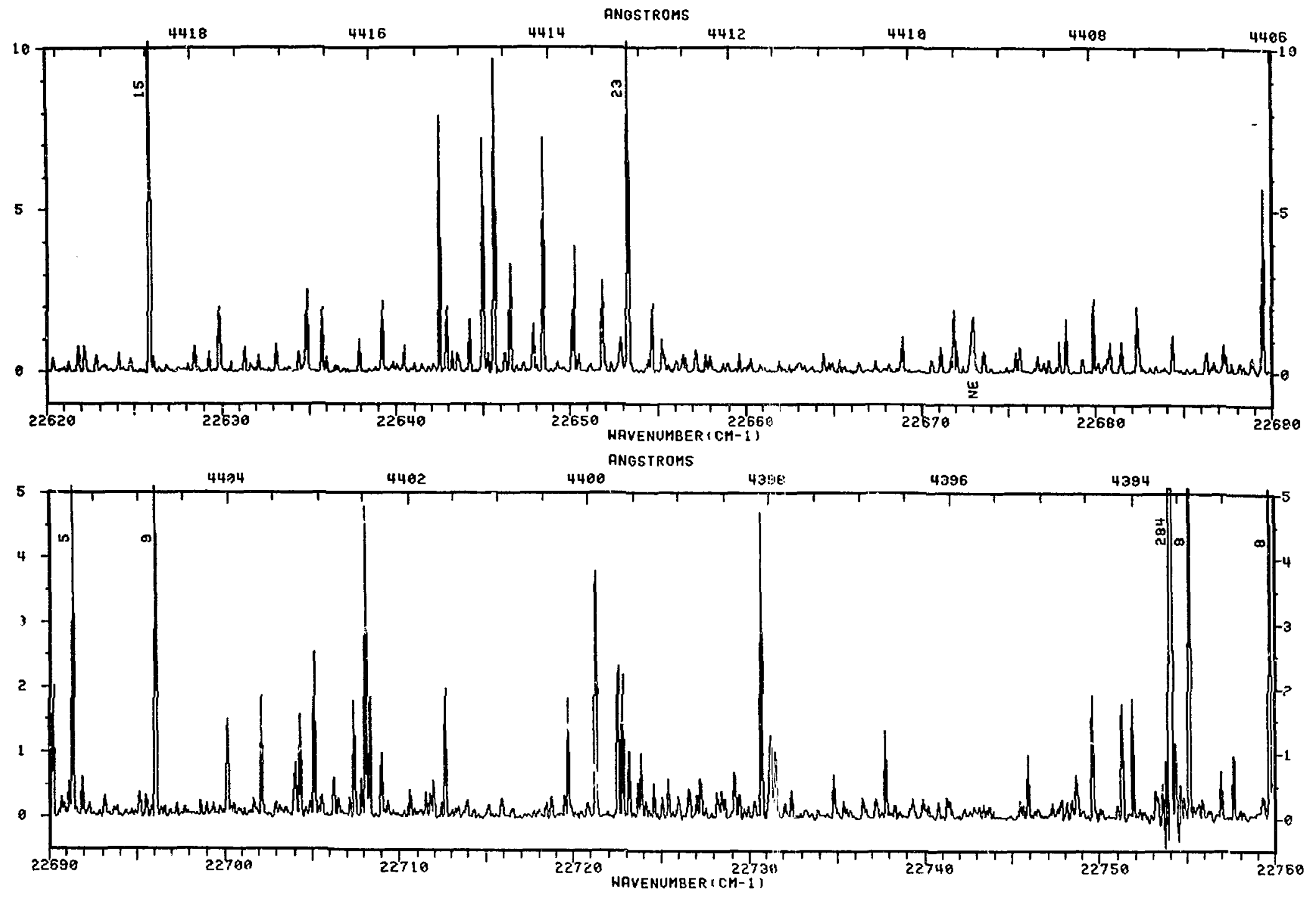

169 


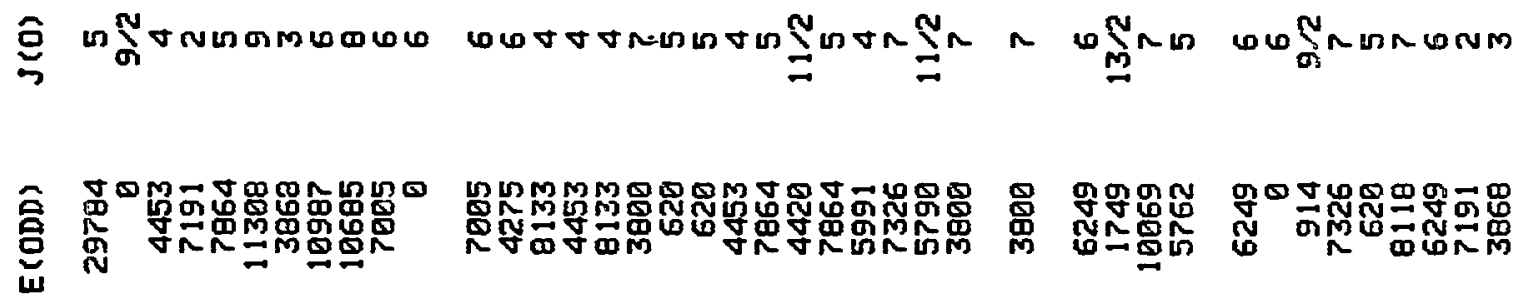

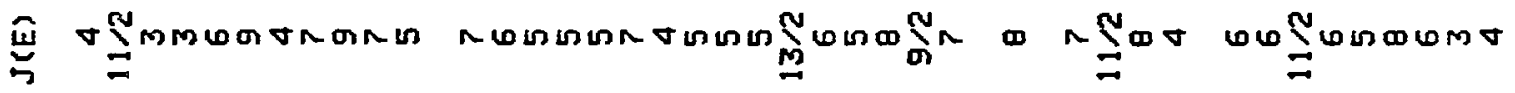

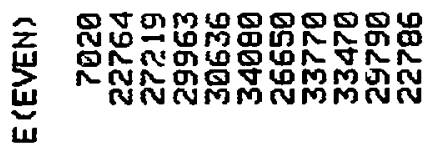

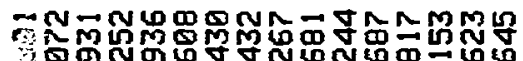

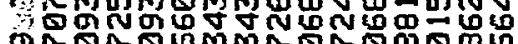

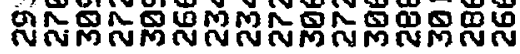

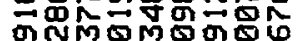

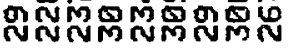

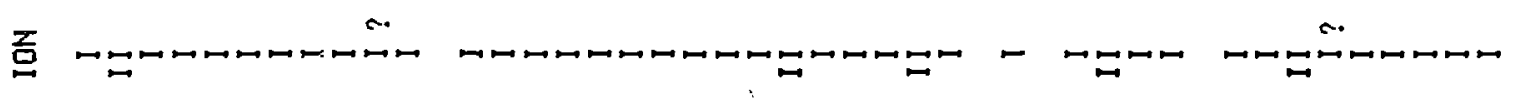

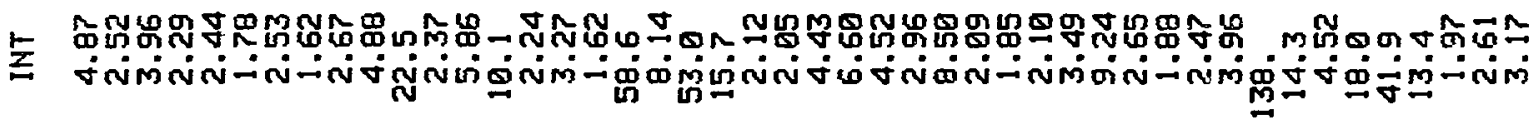

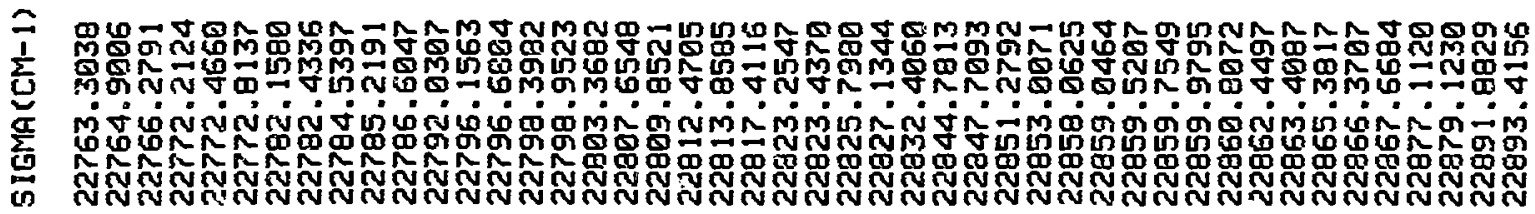

61 N

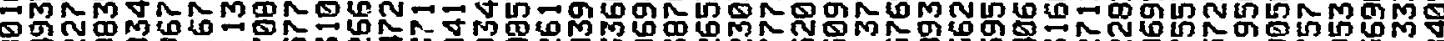

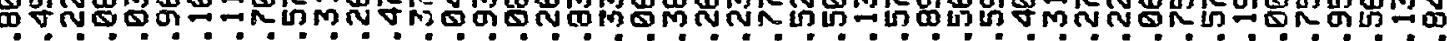

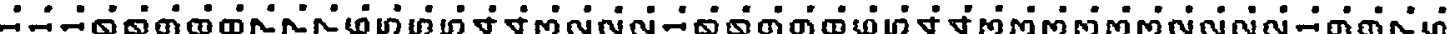

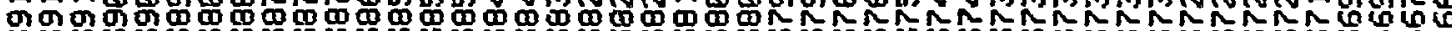

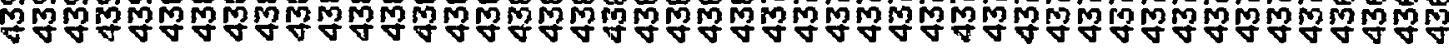




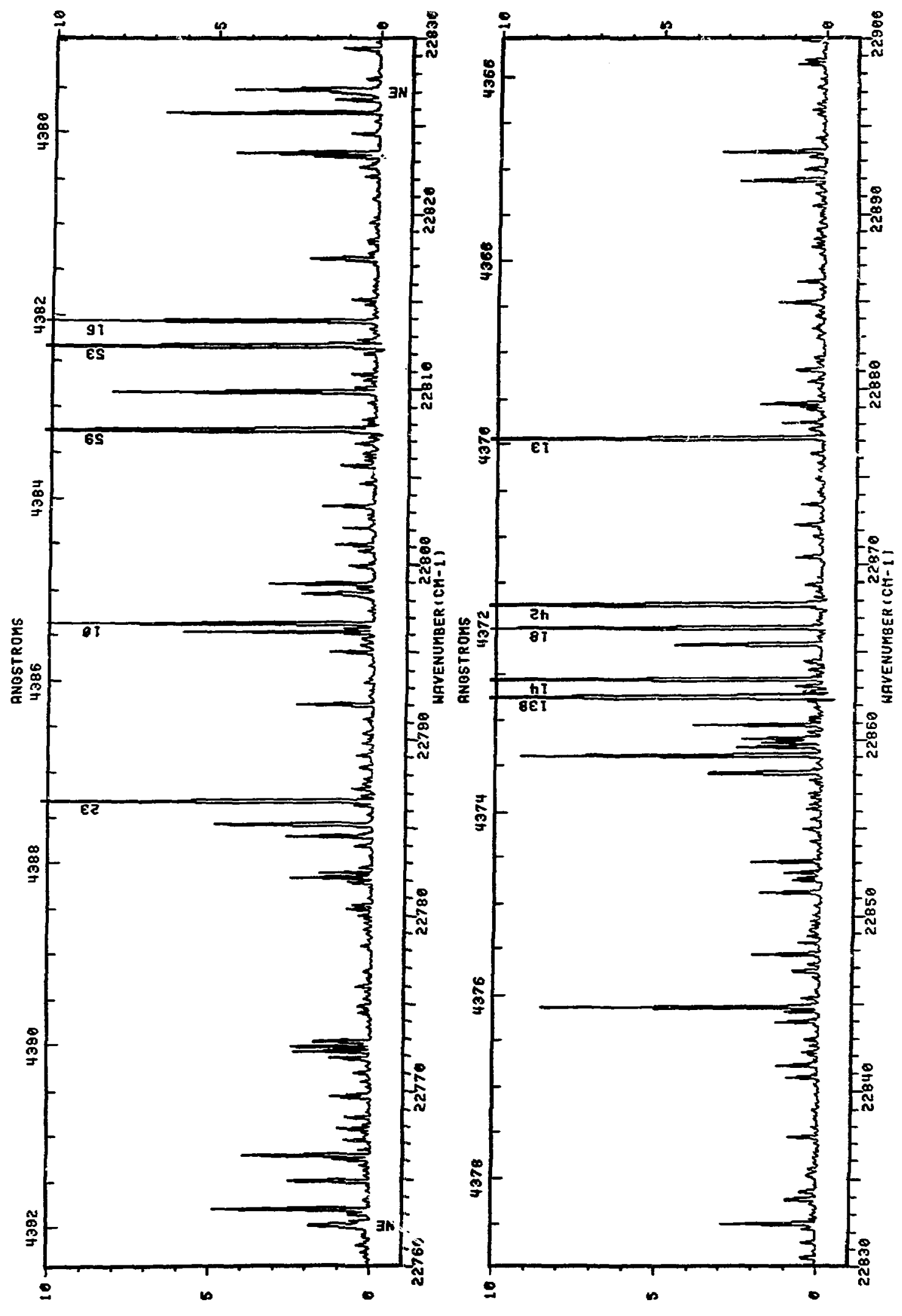




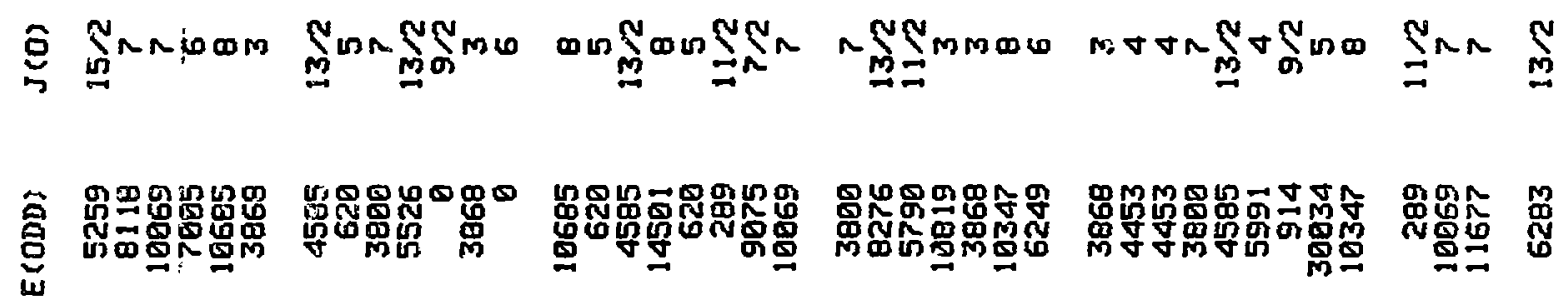

岂

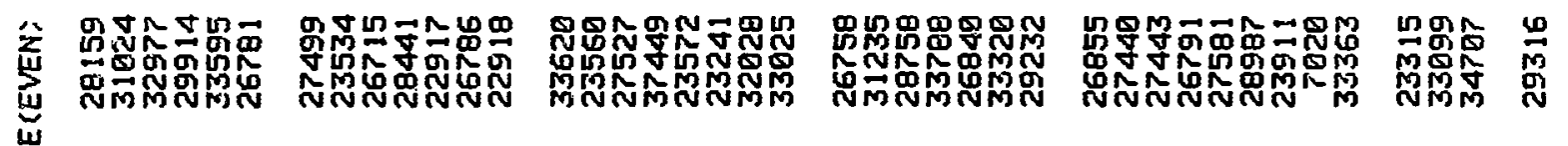

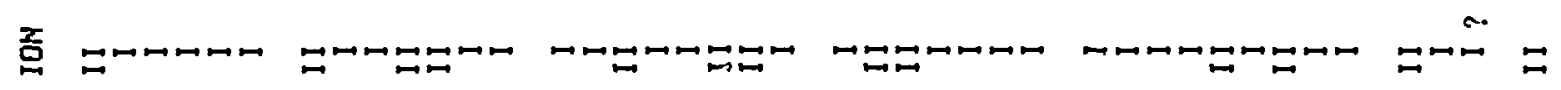

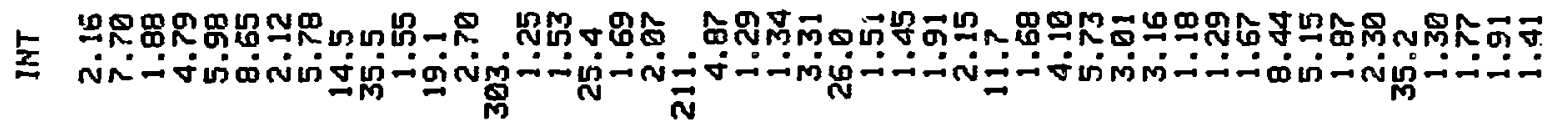

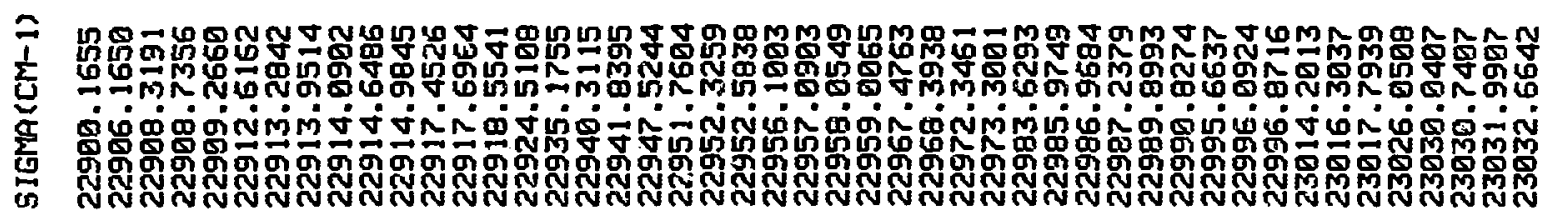

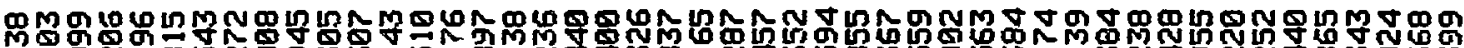

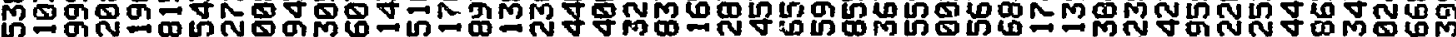

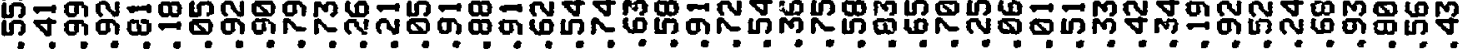

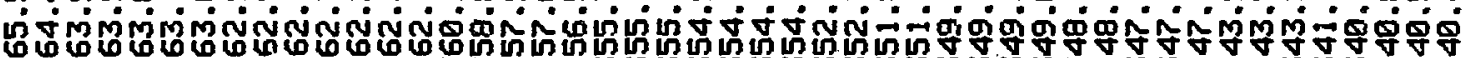

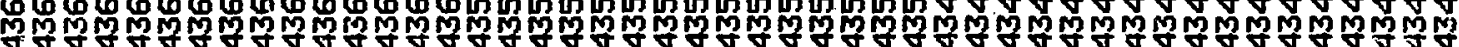




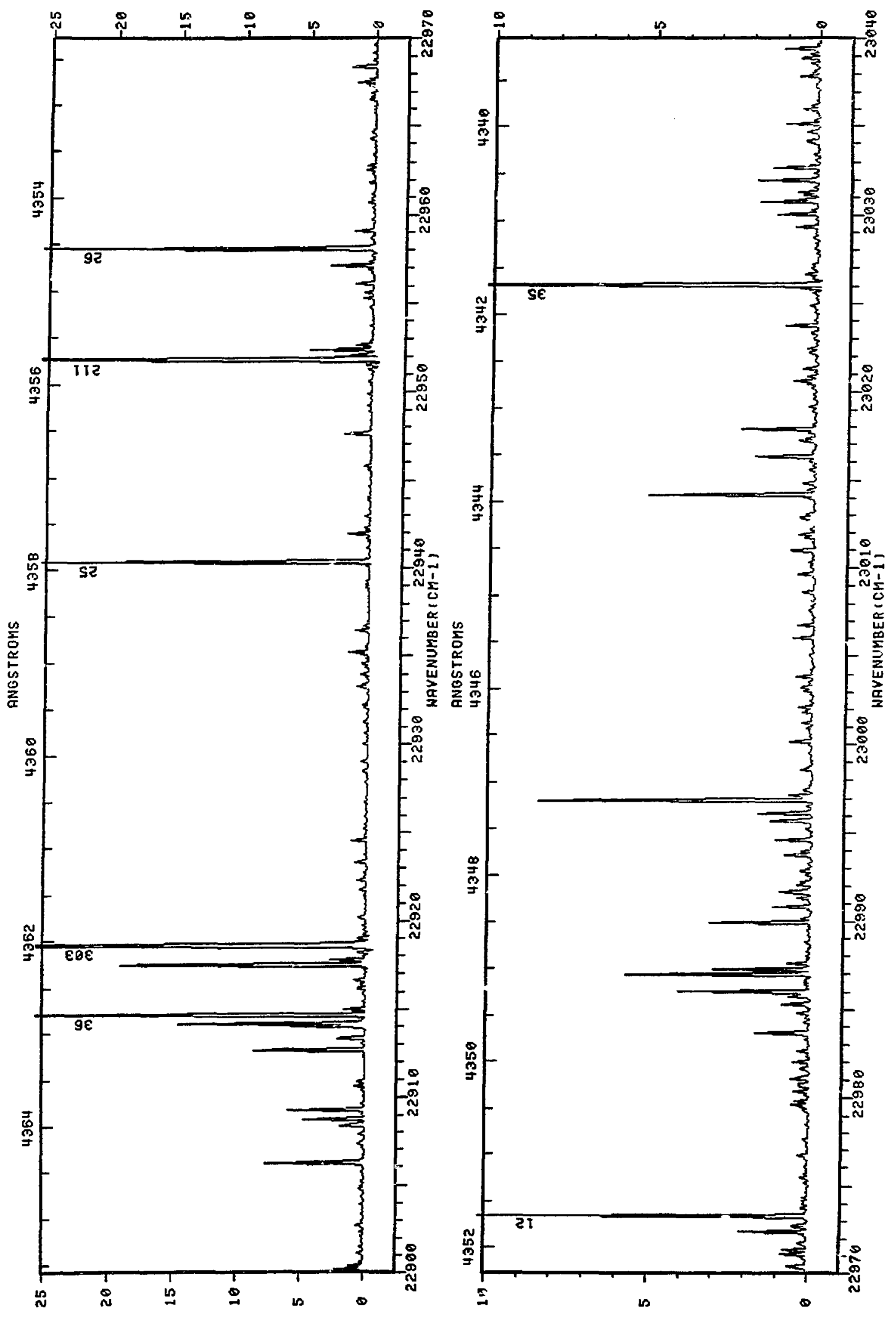




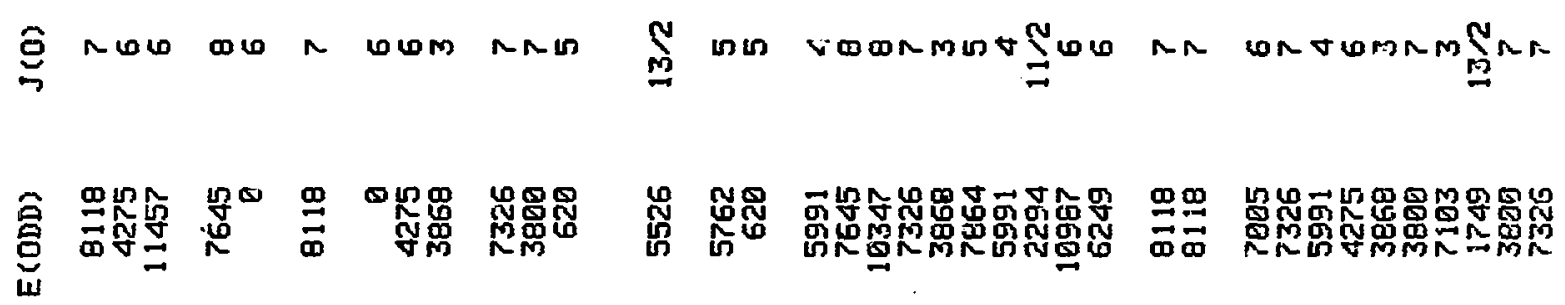

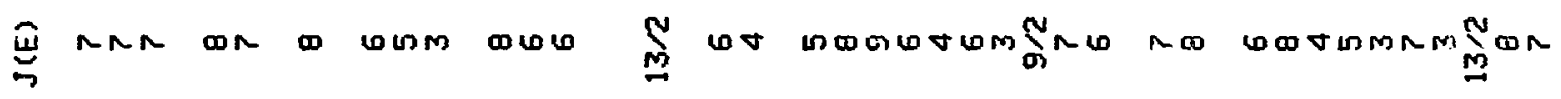

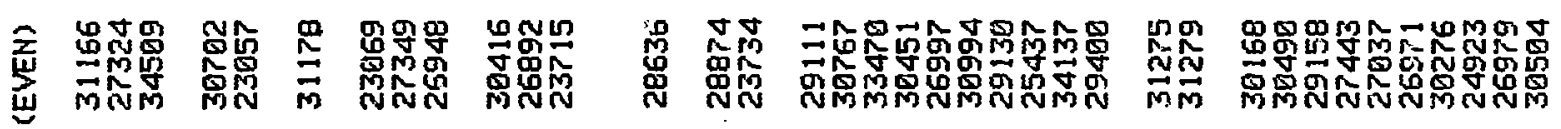

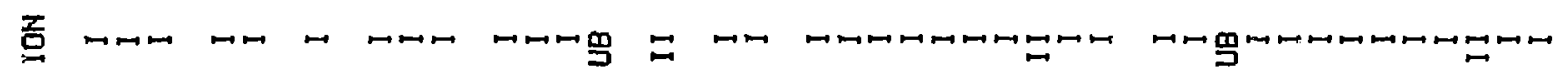

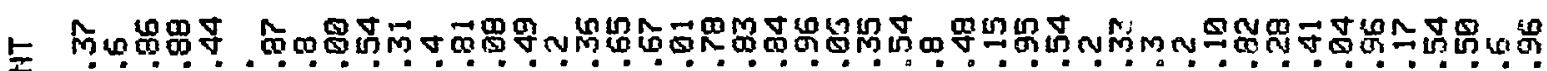
F

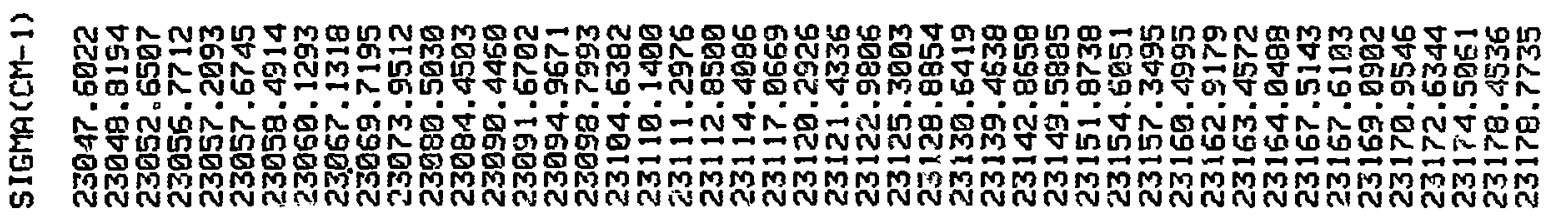

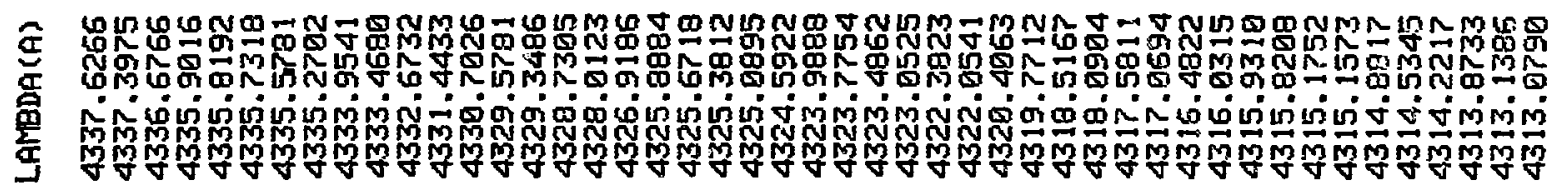




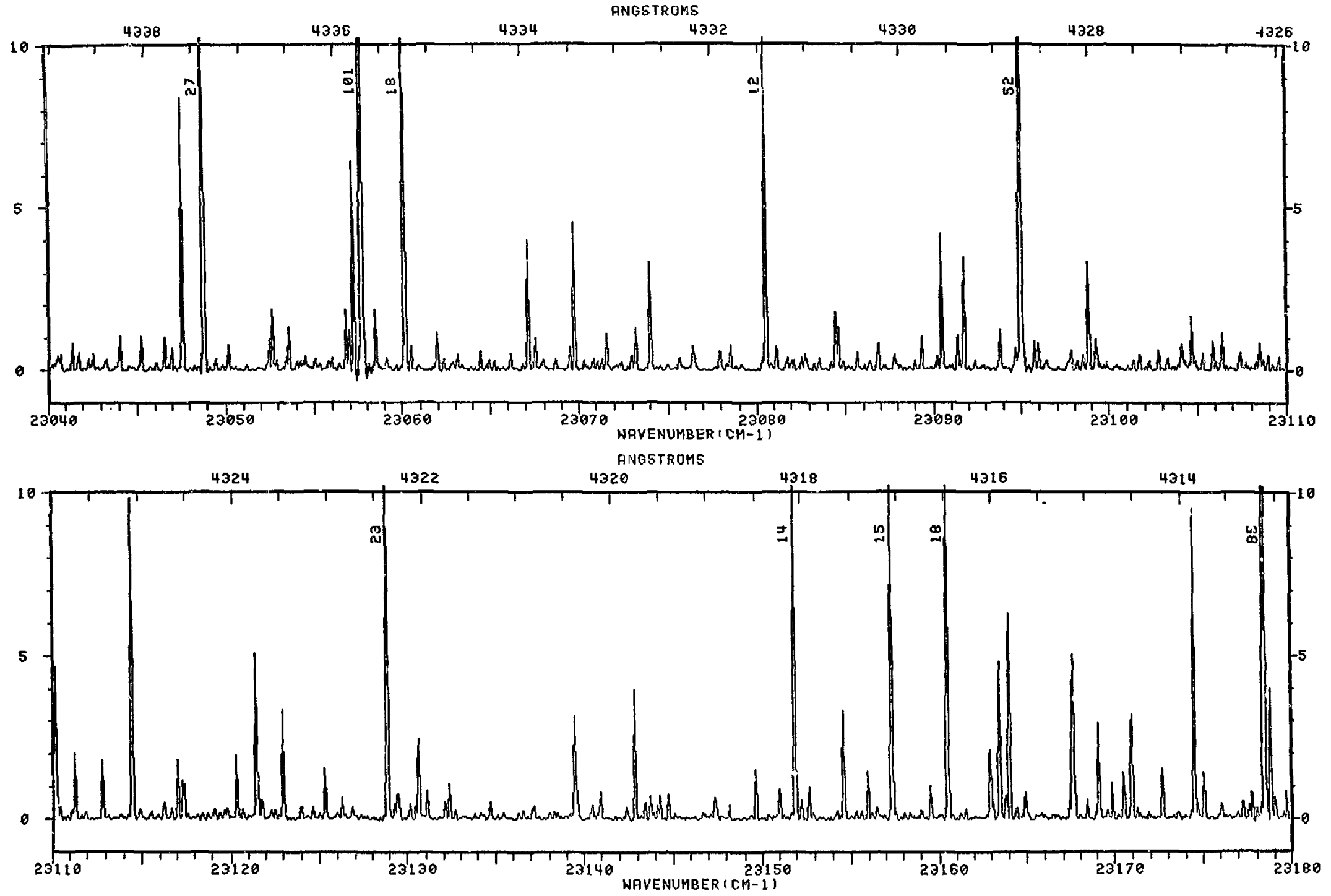

175 


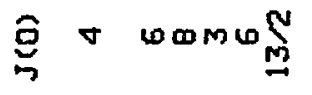
$\cos \cos \theta$
6 comsin
$\operatorname{Non}$
$\cos \Xi$
थषค
$\operatorname{sen} \sin m$

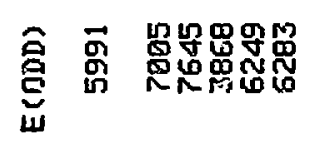

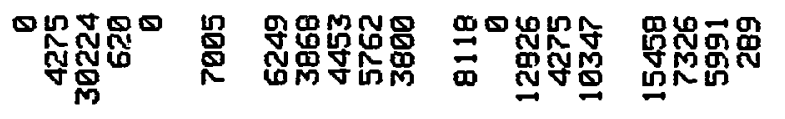

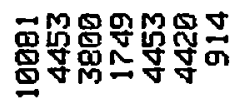

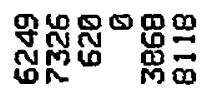

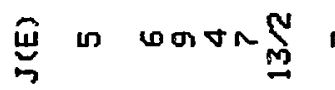

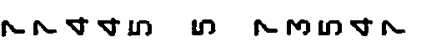

$n \cong 000$ on

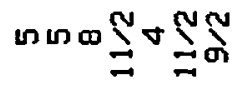

$\omega \cos \frac{N}{\sigma} \sigma \alpha$

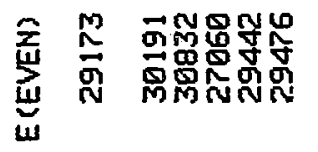

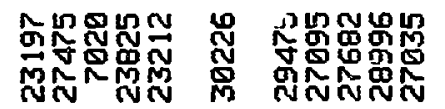

思灾怘塄

MN罢赑

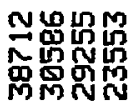

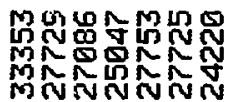

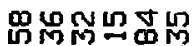

मूलm

思佥

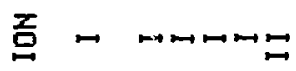
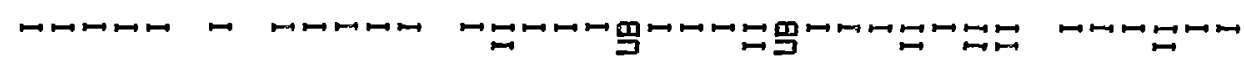

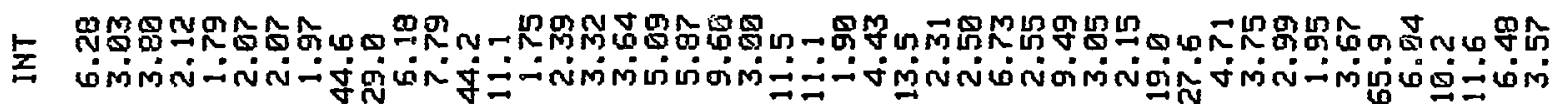

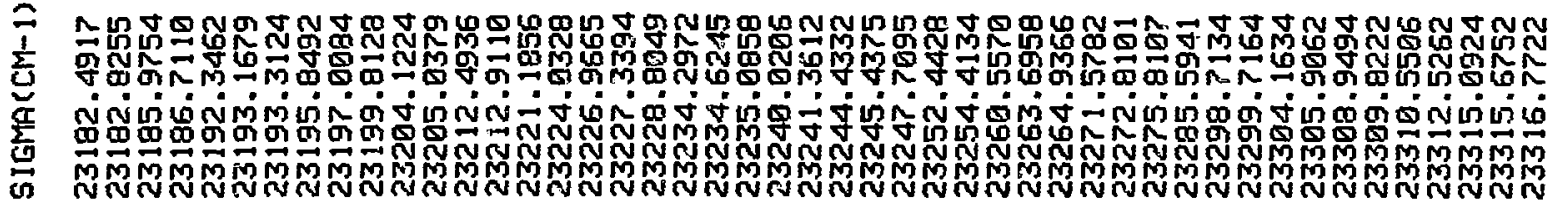

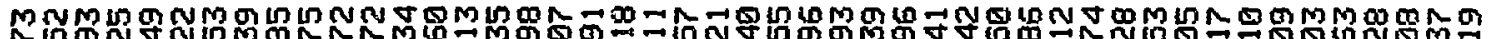
ڤ

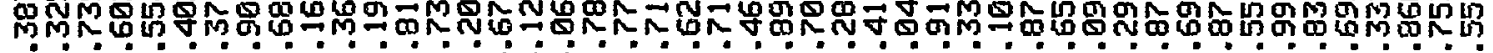

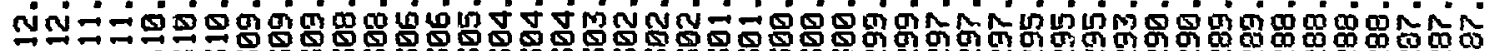

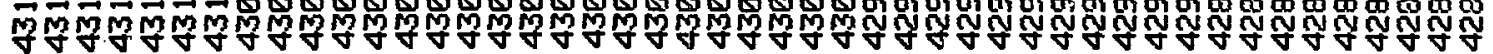



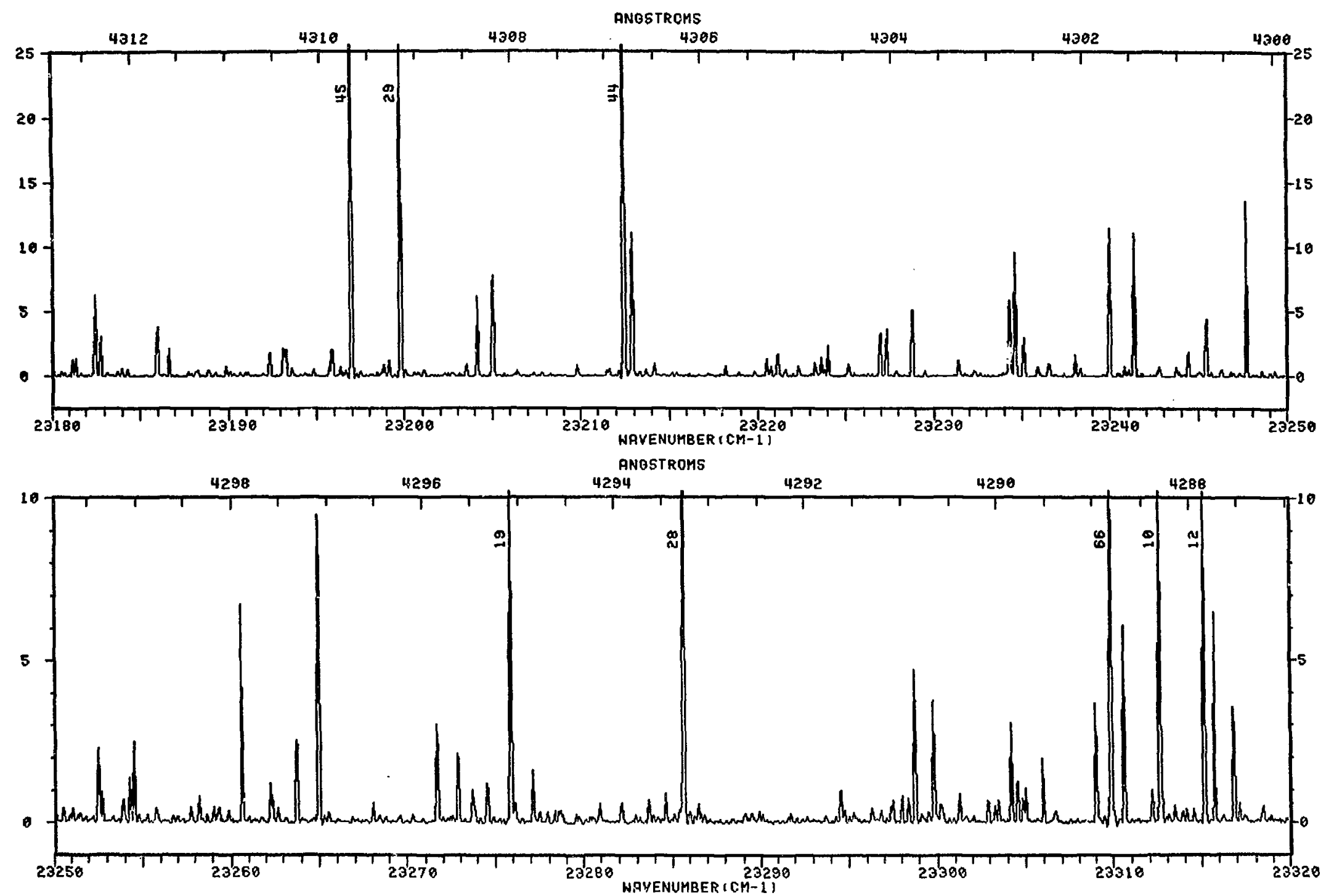


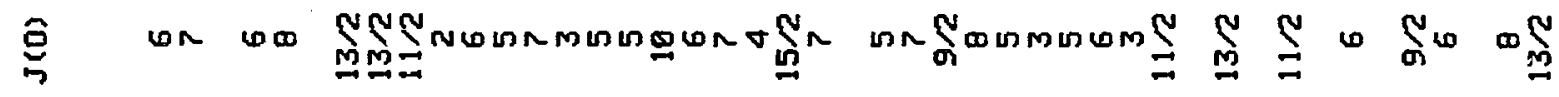

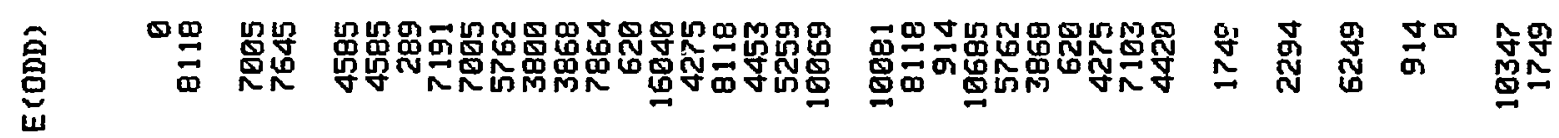

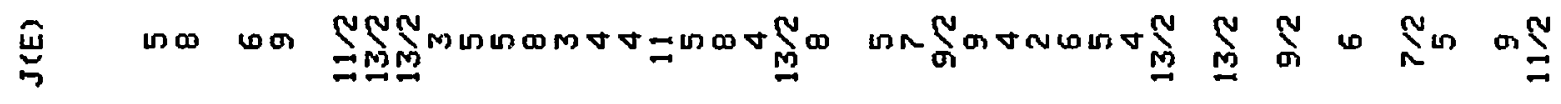
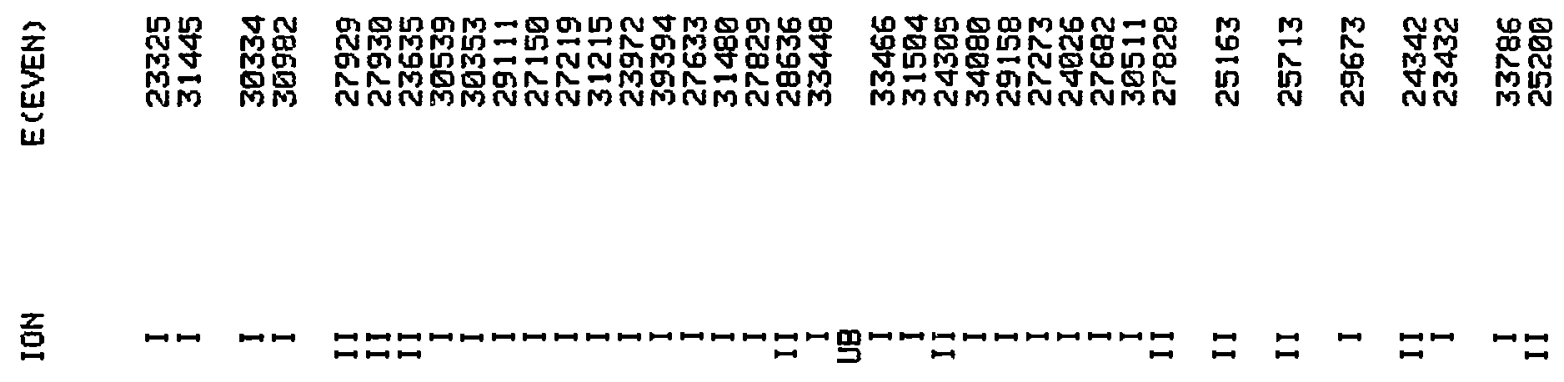

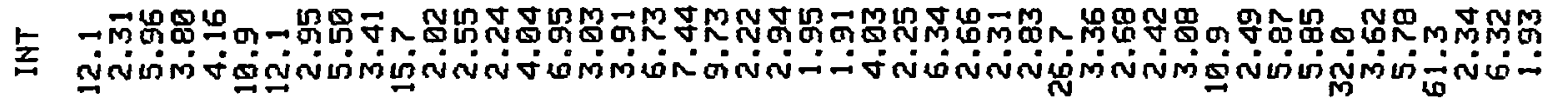

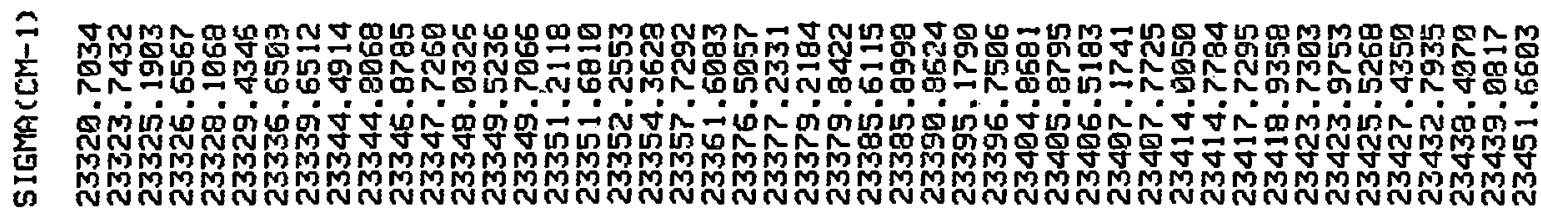

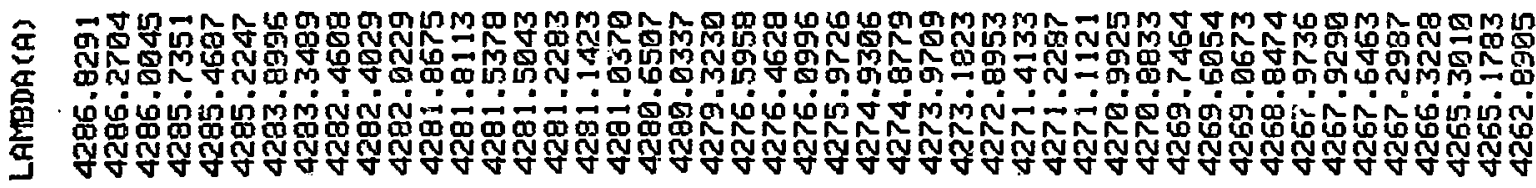



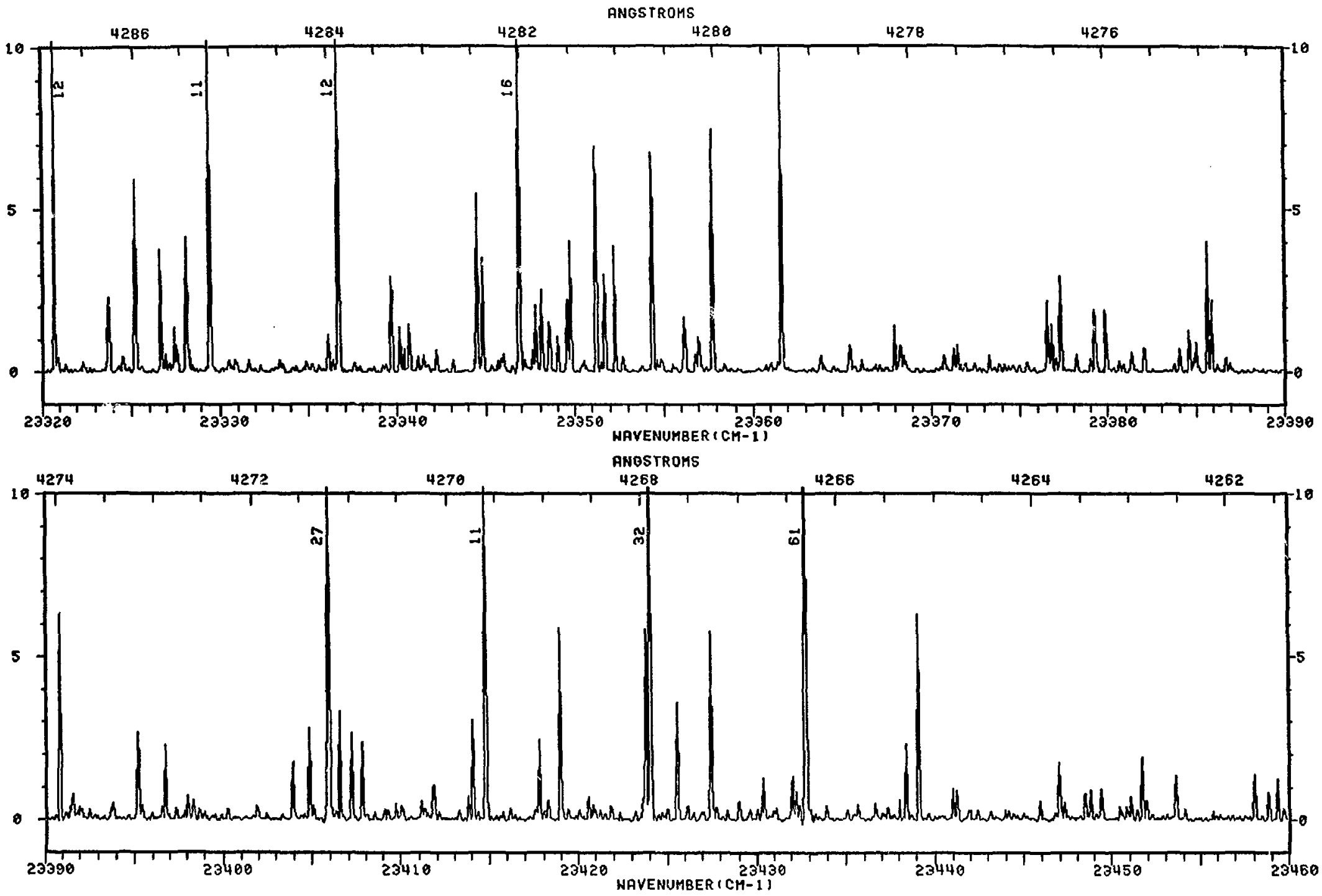
吕 n⿴囗十

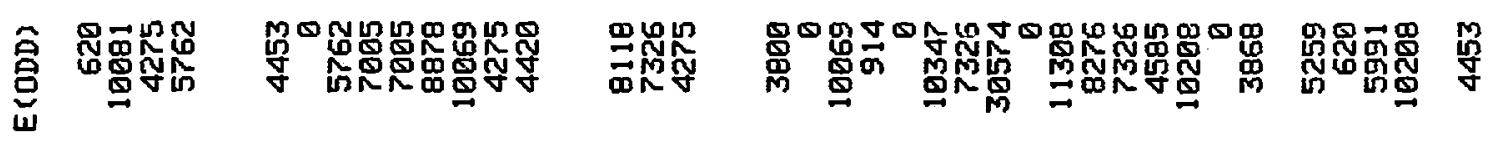

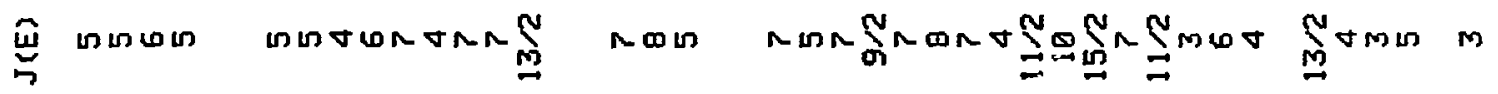

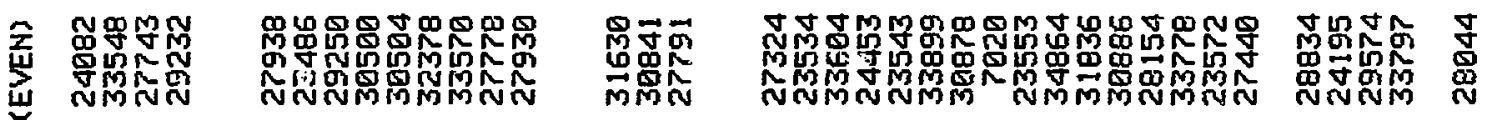

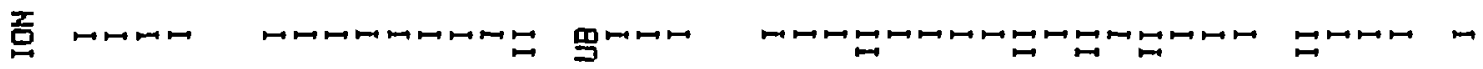

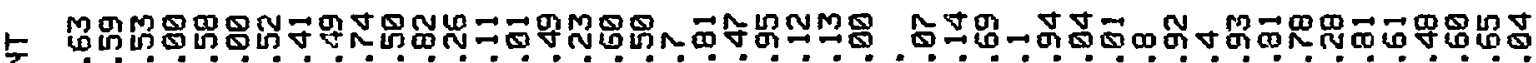

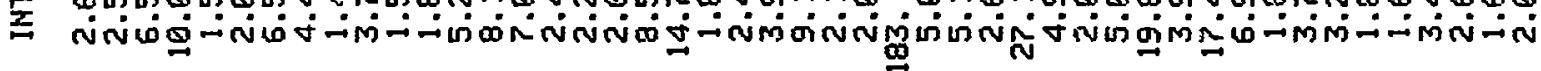

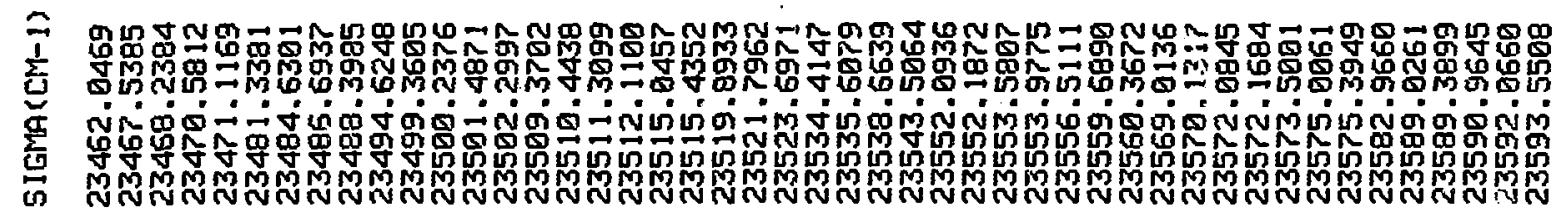

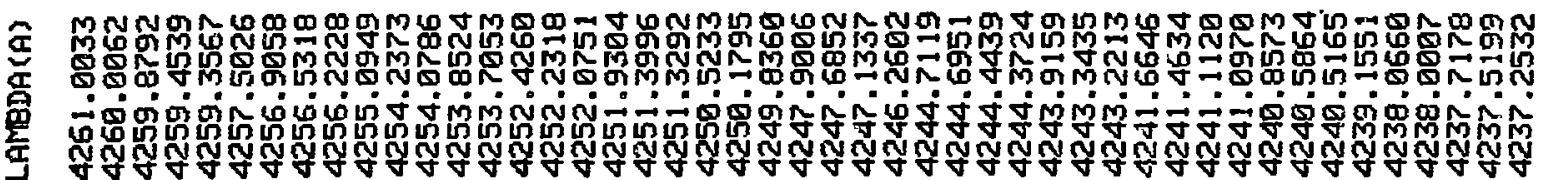



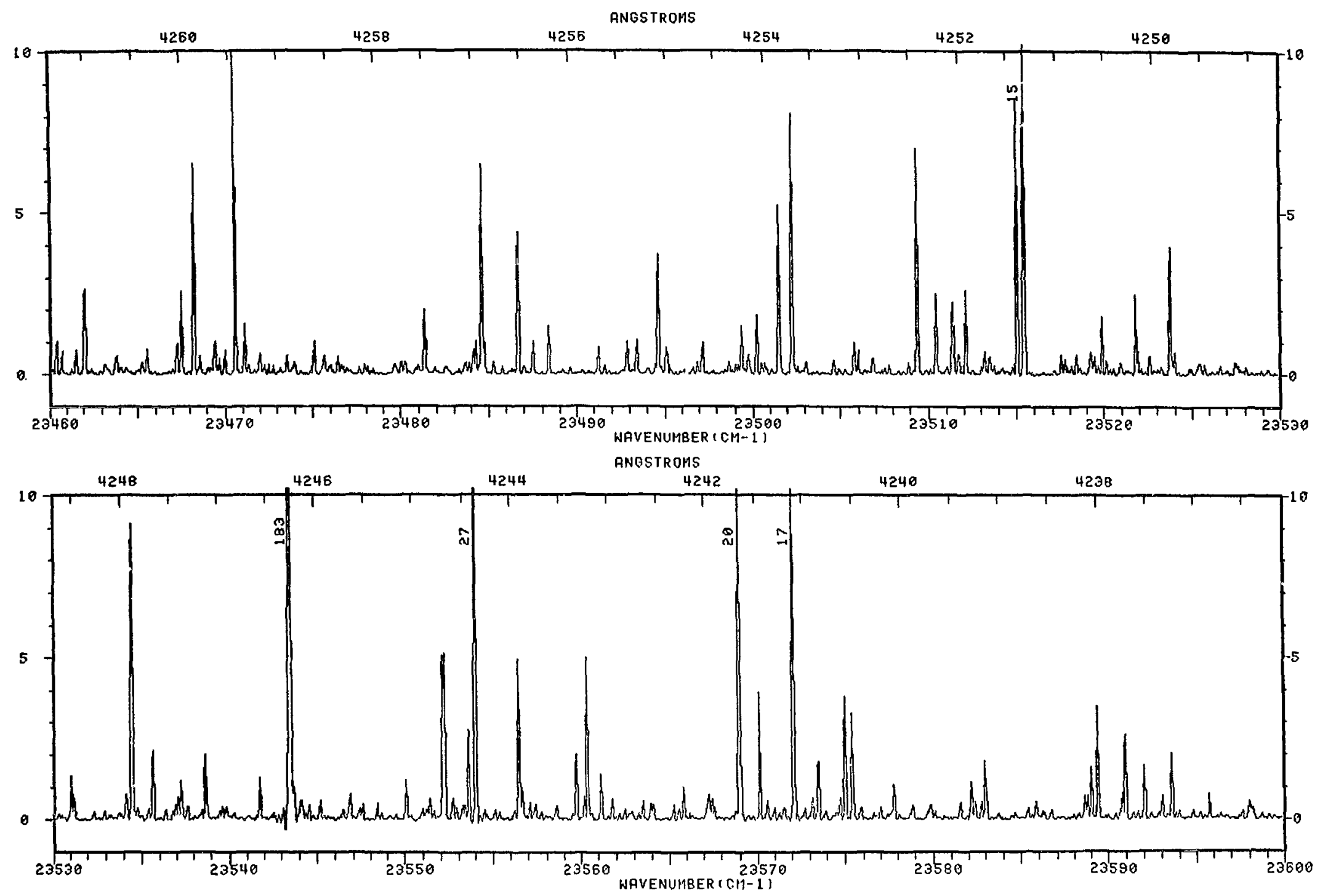

$18 j$ 

ถั

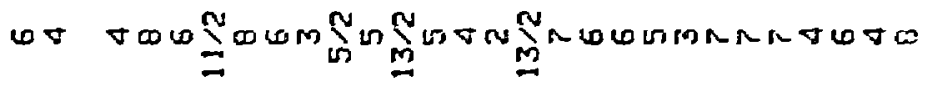

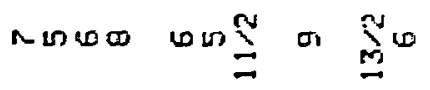

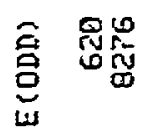

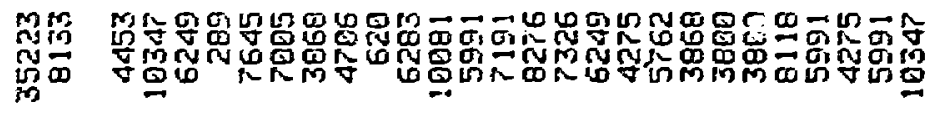

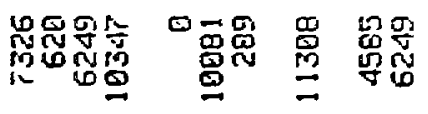

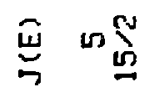

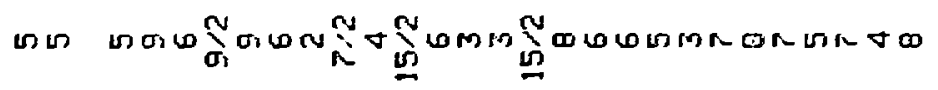

ron

$\sum_{\text {u. }}^{2}$ का

喿柔

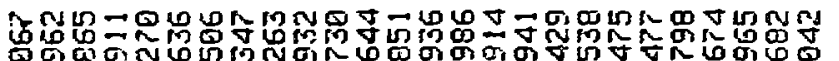

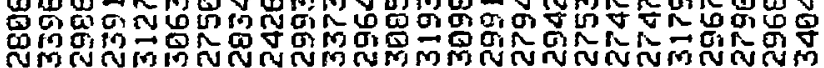

ปัญ恐思

Q日勺

ing

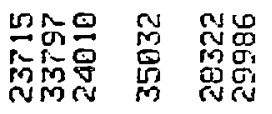

z믐

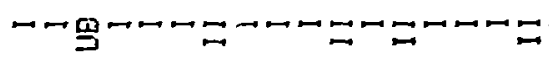

上

z

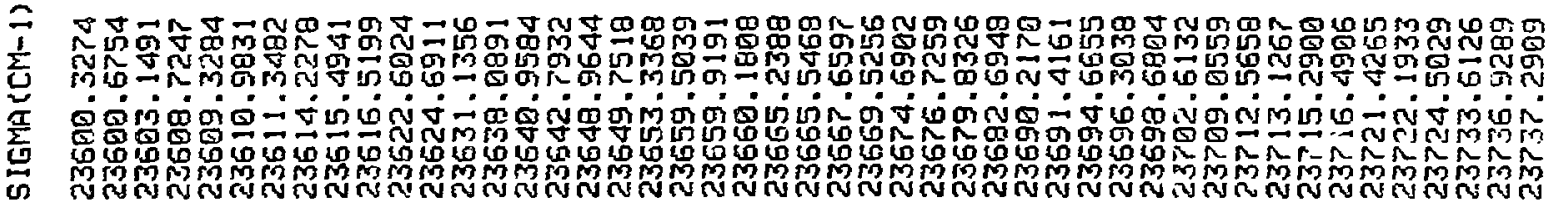

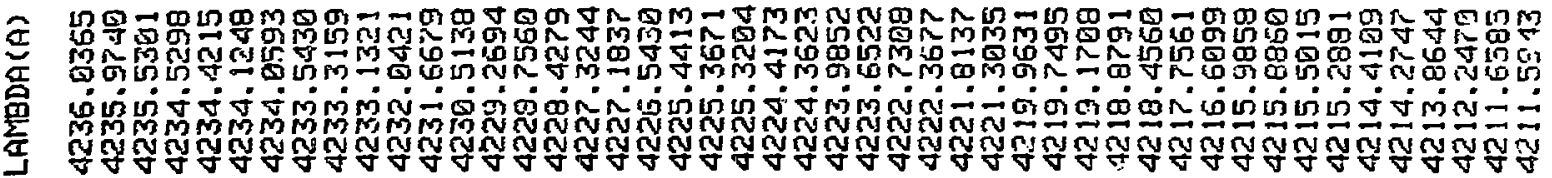



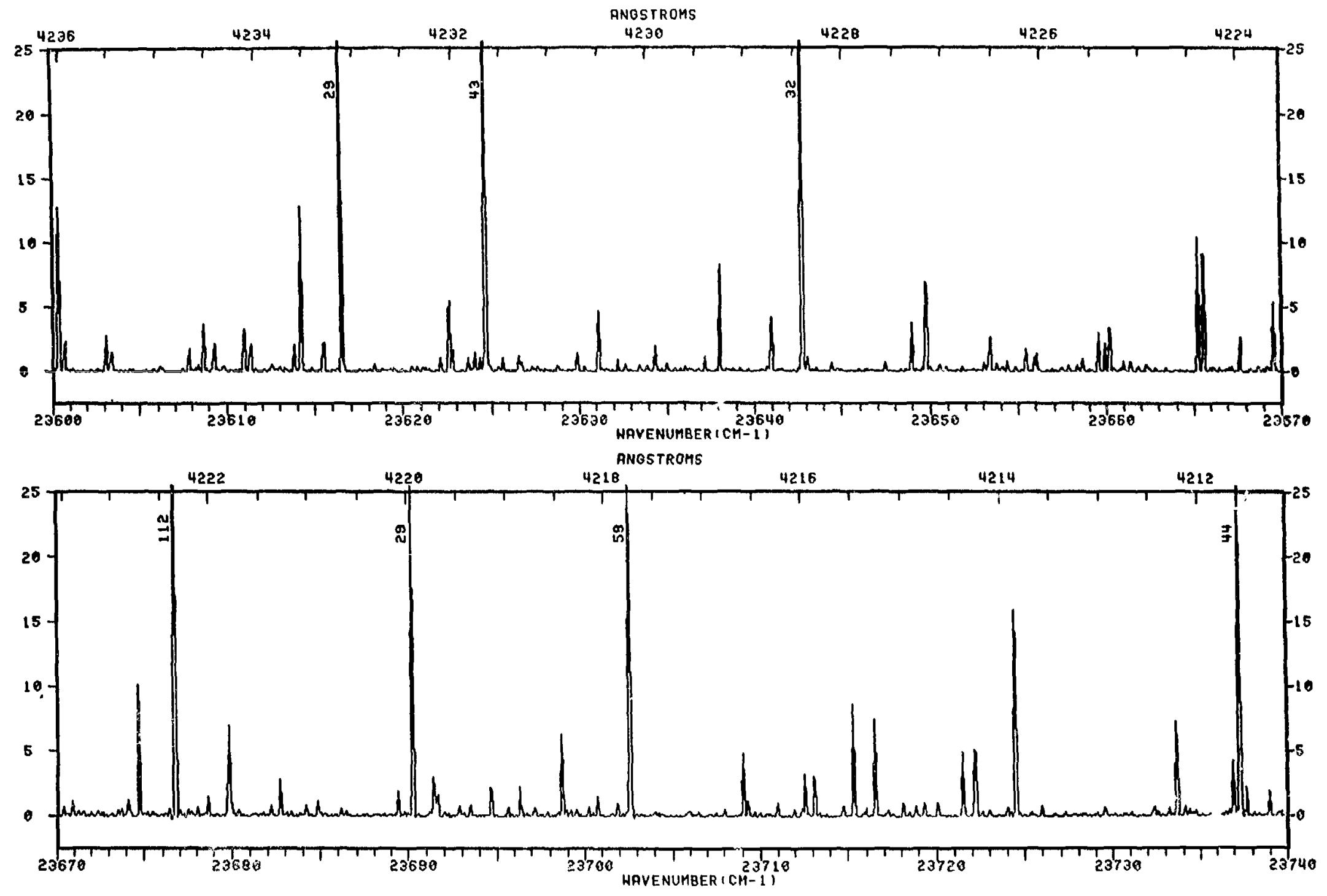


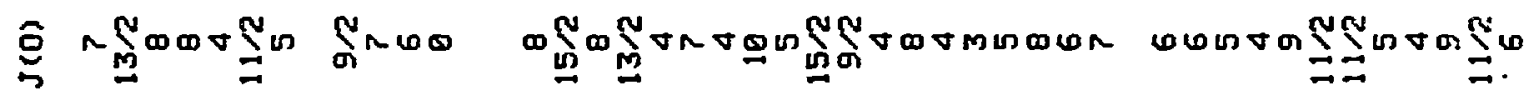

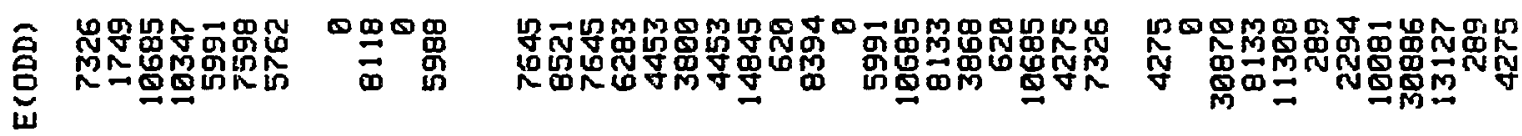

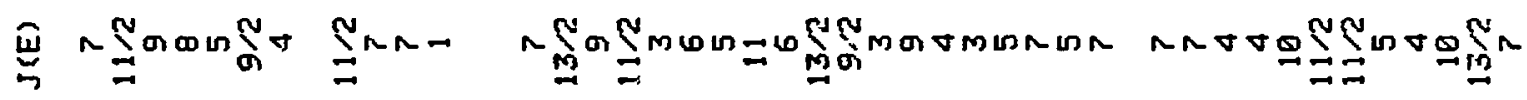

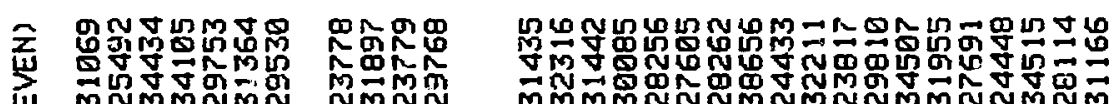

WQ

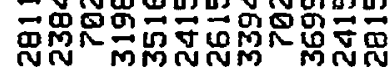
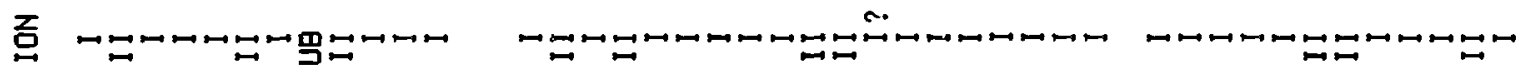

度

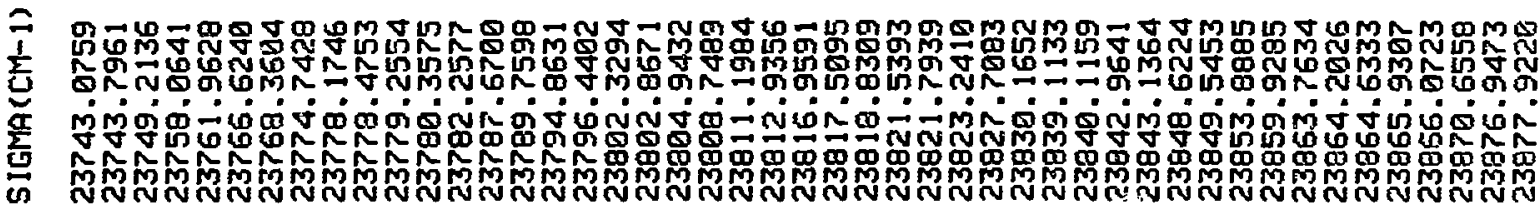

$\overrightarrow{8} 8$ 药 \% 付

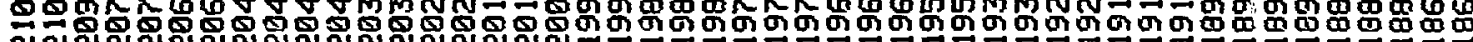

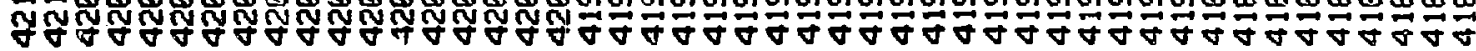



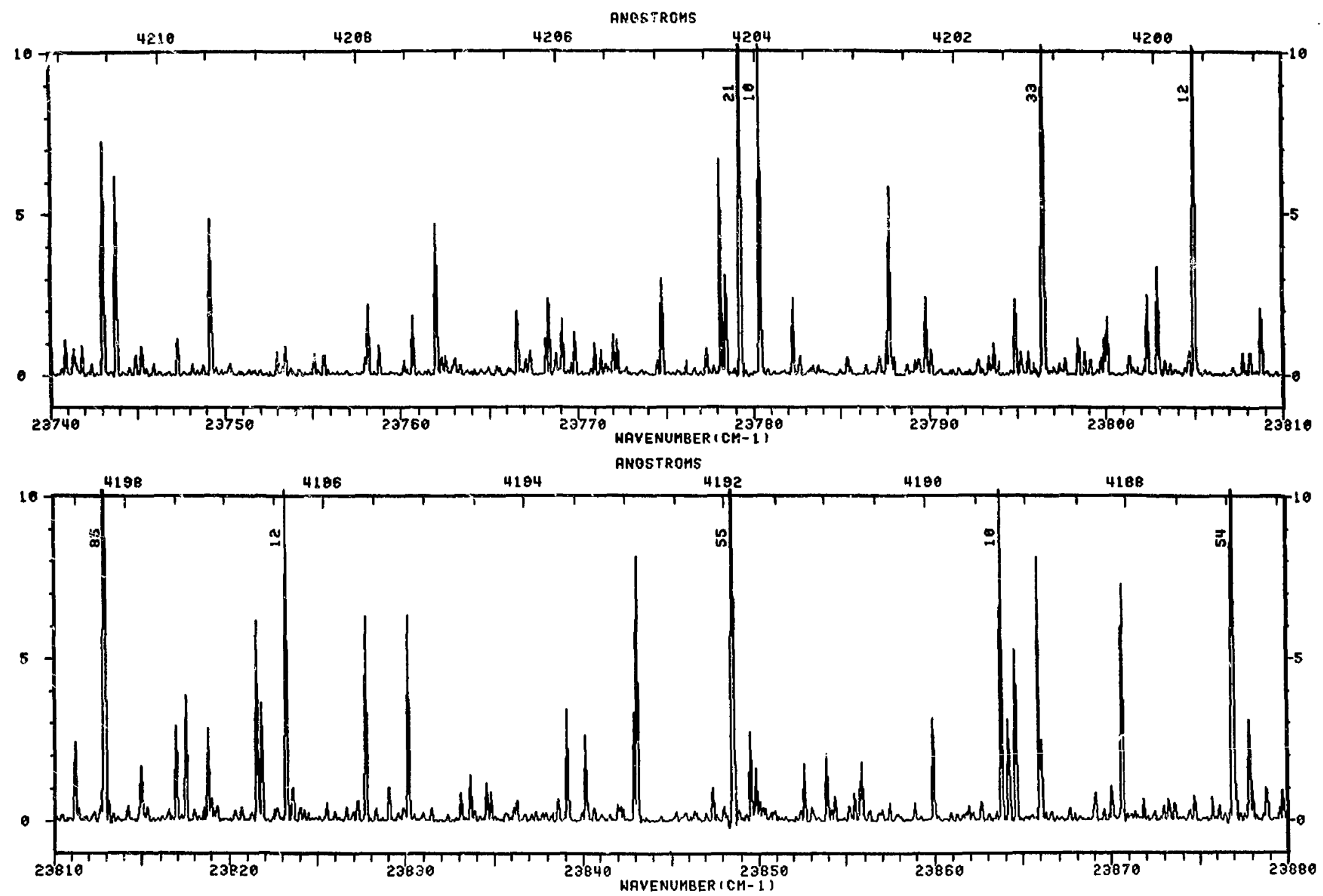


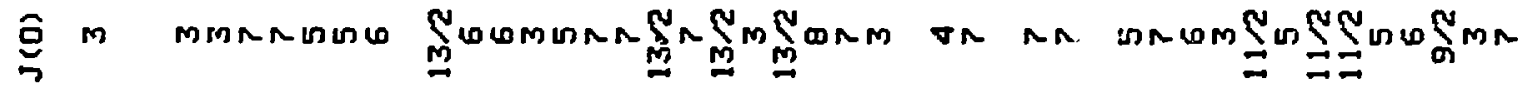

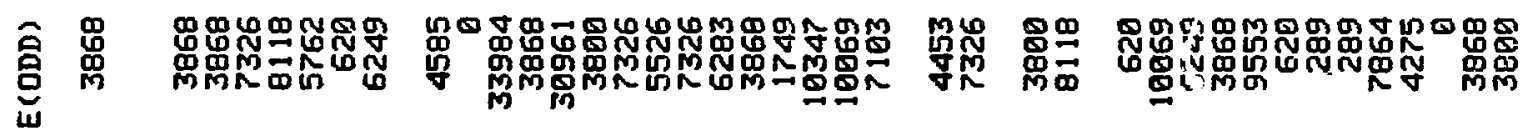

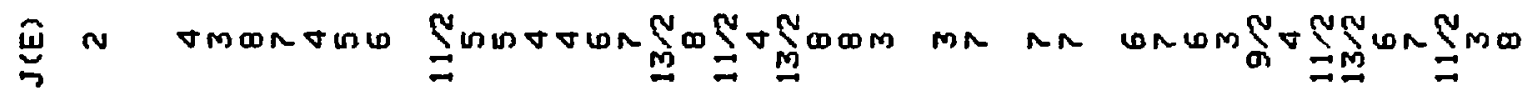

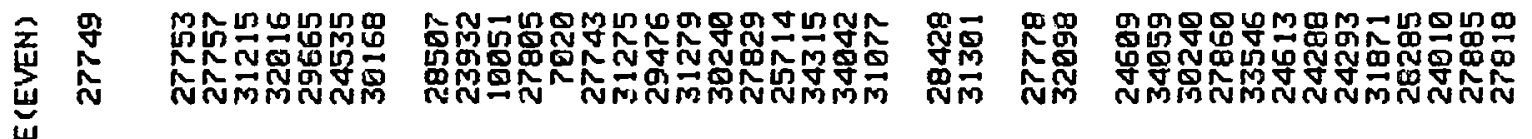

z-

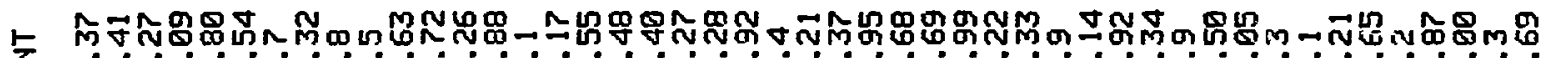

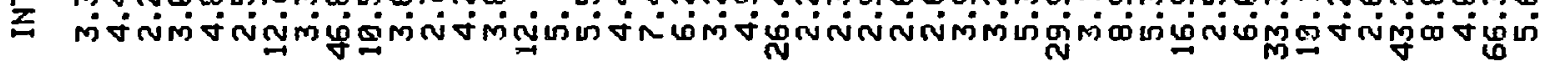

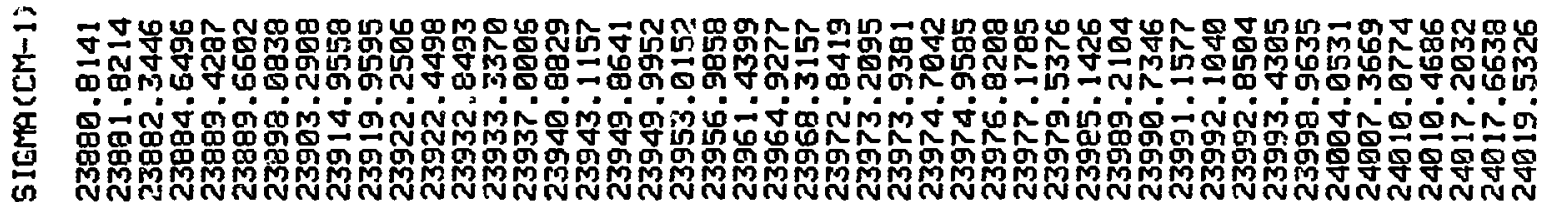

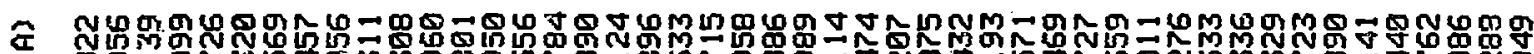

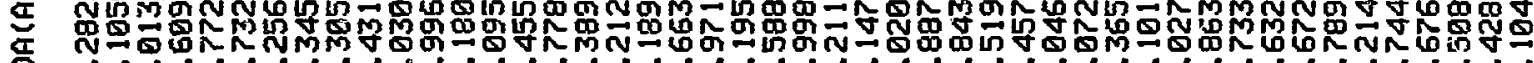
की

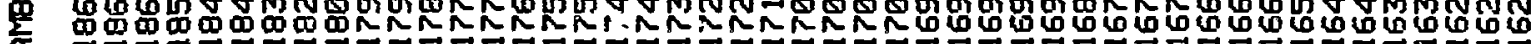

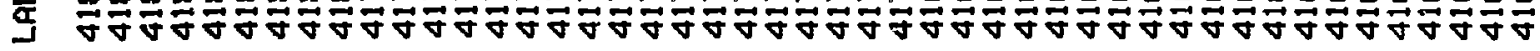



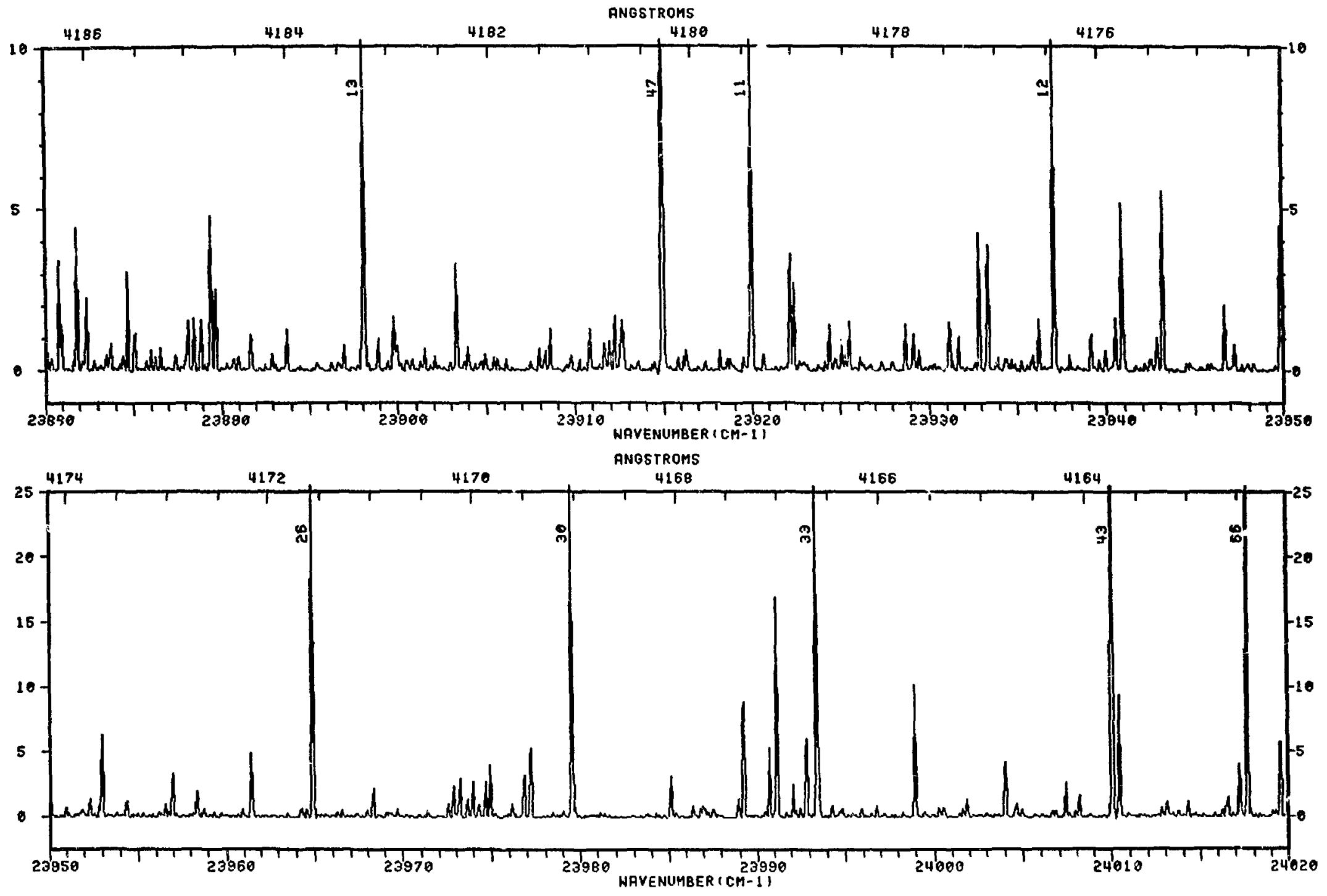

187 


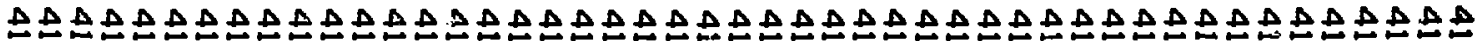

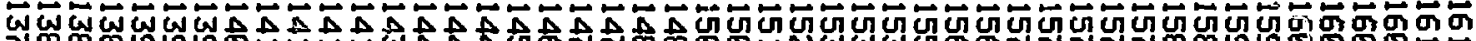

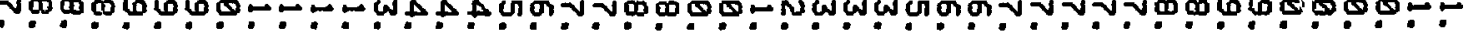

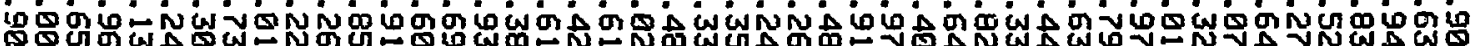

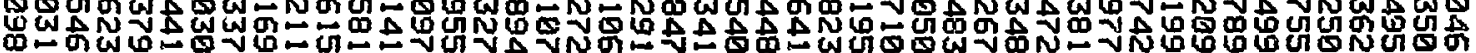

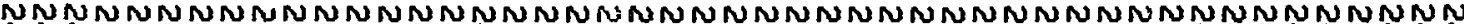

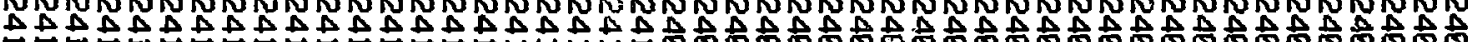

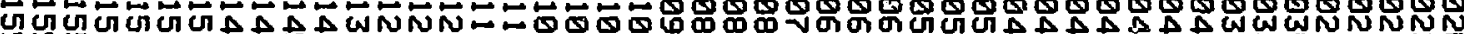

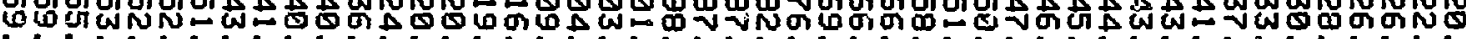

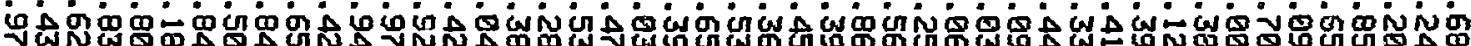

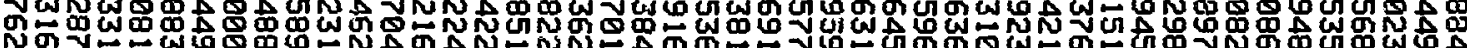

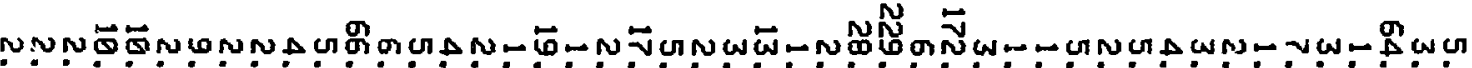

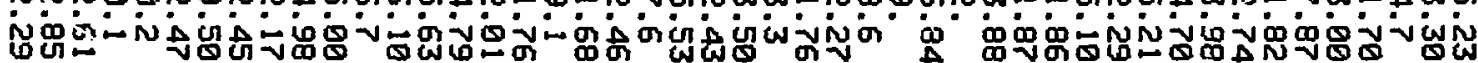

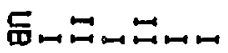
$=$ $-1$

W

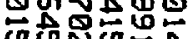

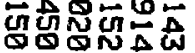
WNNWNNW

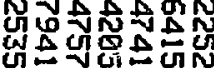

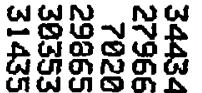
NW NNWNN

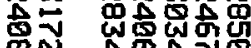

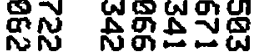
令尔 WN 产 a d N $\doteq$ 贾 心怘䍐

NNG $\rightarrow \underset{1}{0}$

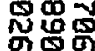

奋

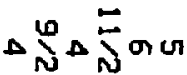

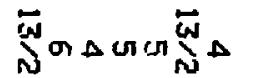

$\operatorname{ven} \theta A \operatorname{sos}$

$\operatorname{vi}$

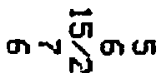
$\Delta \boldsymbol{\infty}$ $A \sigma$ जUตก $\omega \rightarrow \infty$

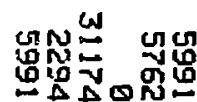

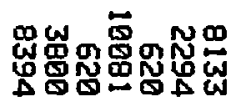

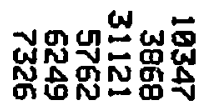

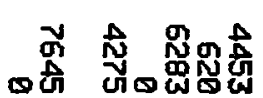
䏝 员罟

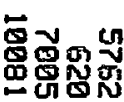
ws 보용 ๑思

$\stackrel{m}{g}$

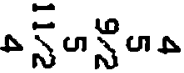

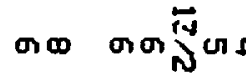

$\Delta \boldsymbol{m}$ 


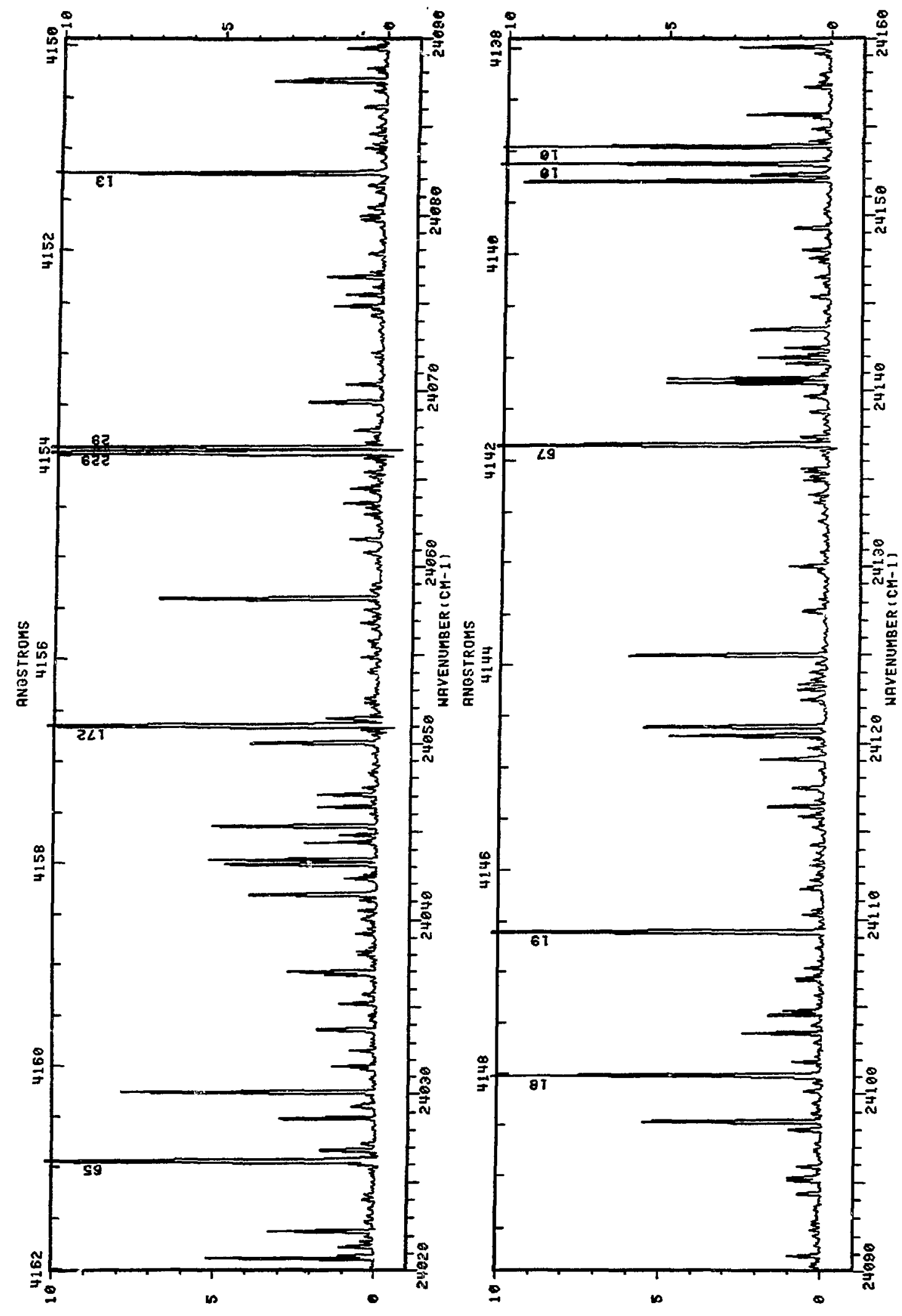




$$
\begin{aligned}
& \text { ఏి }
\end{aligned}
$$

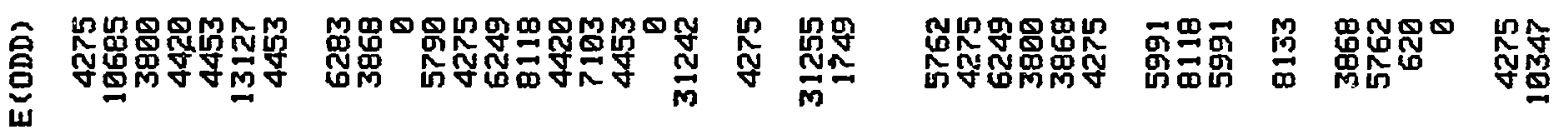$$
\text { פ⿹ }
$$

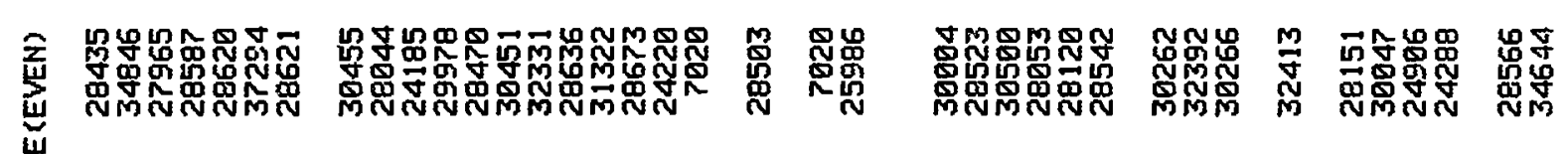

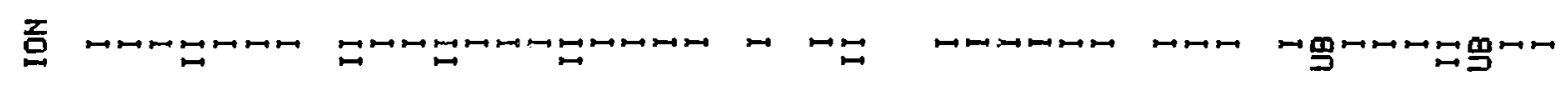

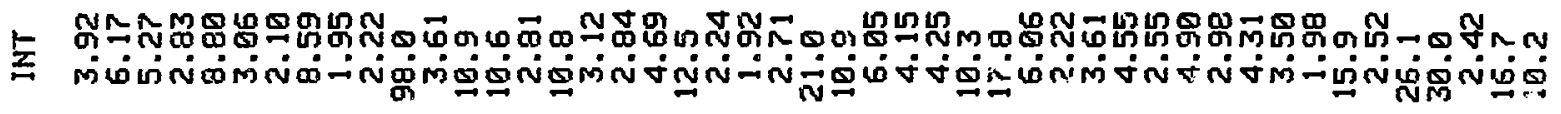

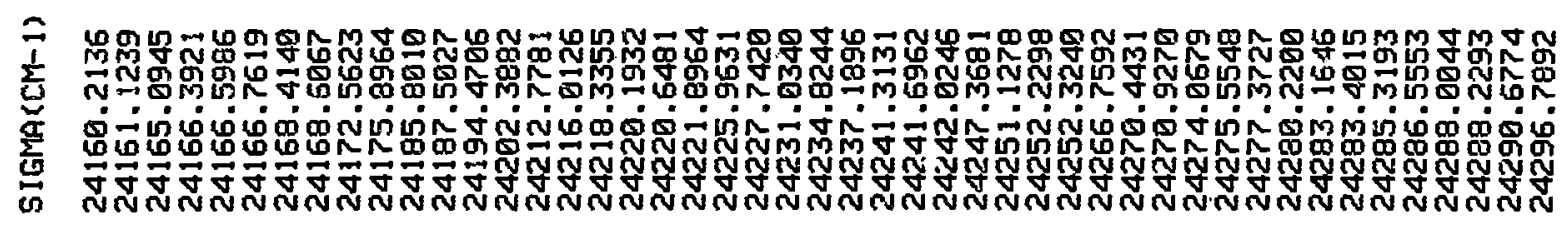

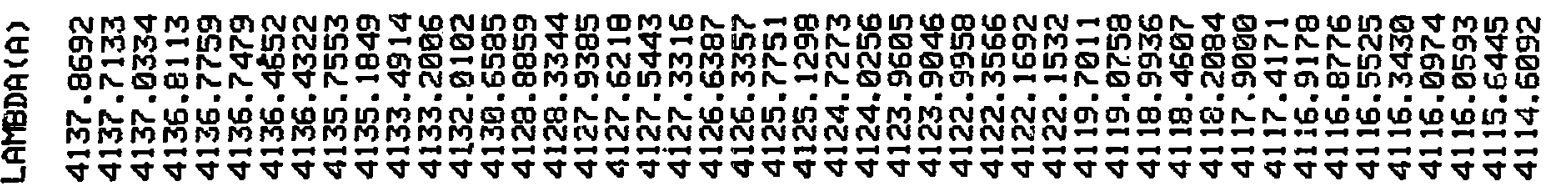



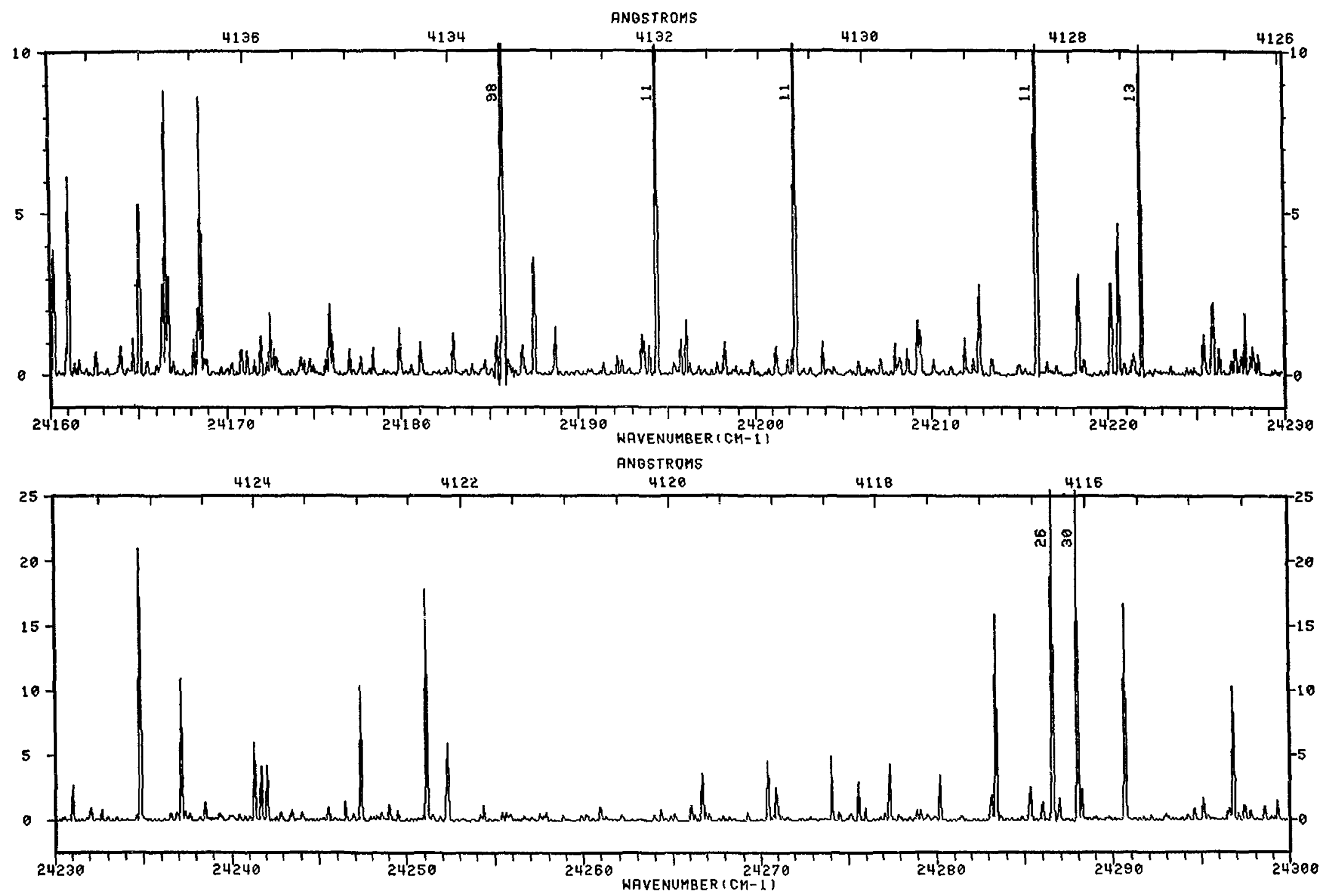


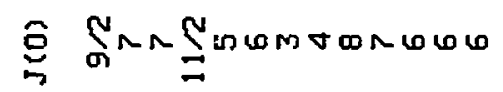

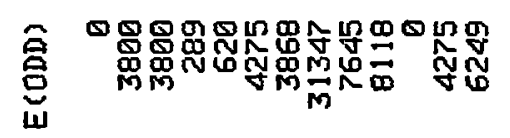

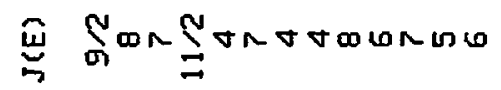

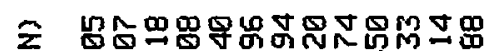

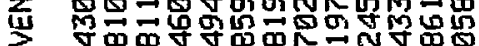

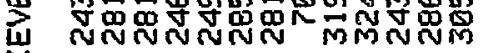

के ल

$\infty$

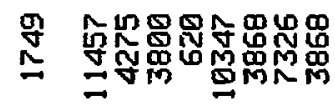

แ

$\min$

§n

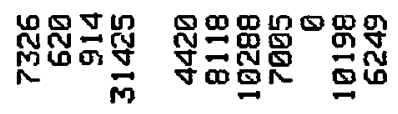

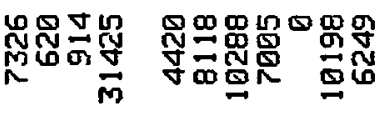

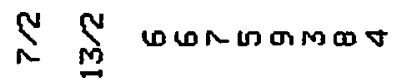

㲎

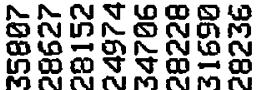

NMg日o

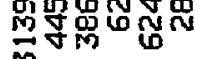

$\sum_{m}^{M} \sim n N_{N}$

果禺等 心ल心

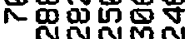

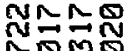
N⿴囗十心 ming

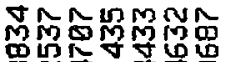

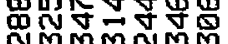

$\underset{0}{Z}$

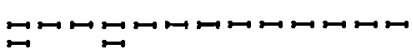

$\cong \cong$

a.

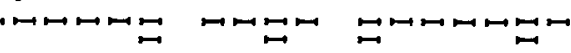

与 㟧于 I

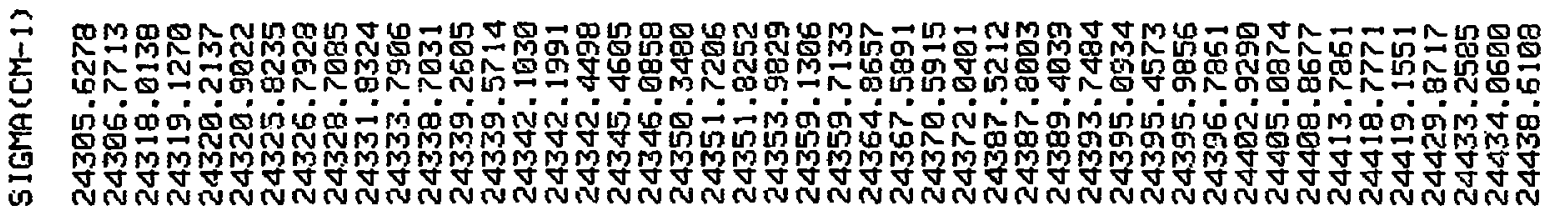

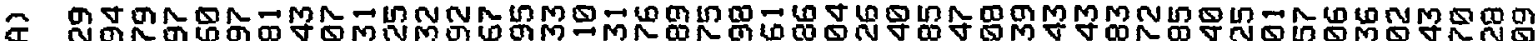

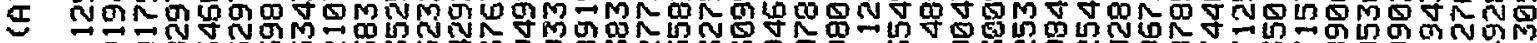

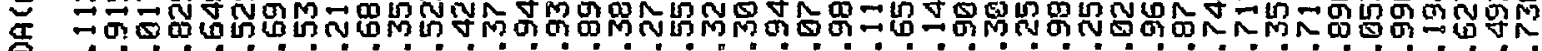

鱼

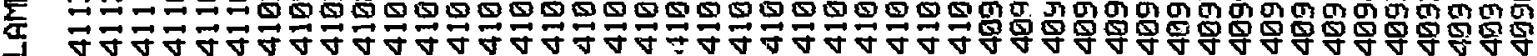



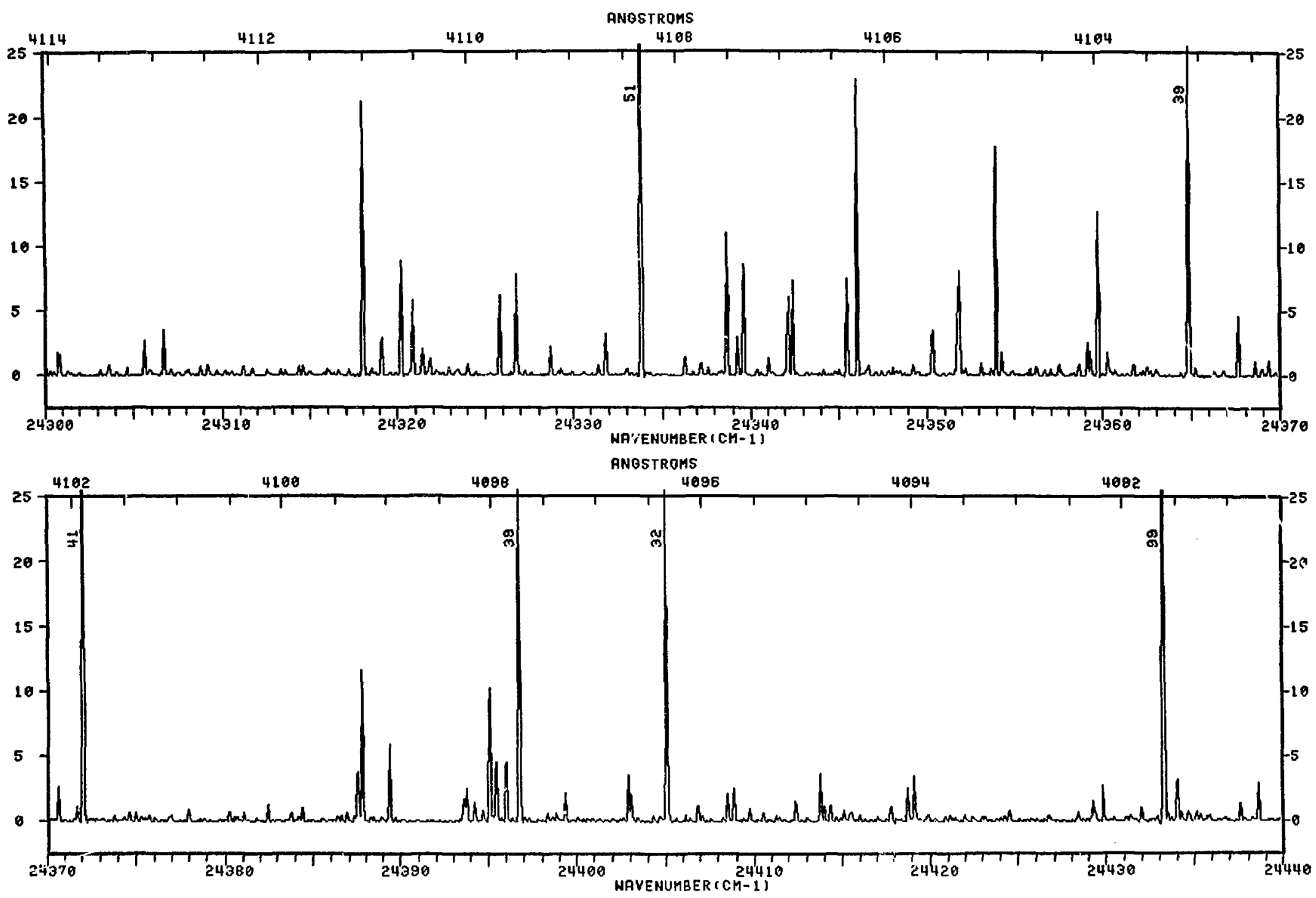


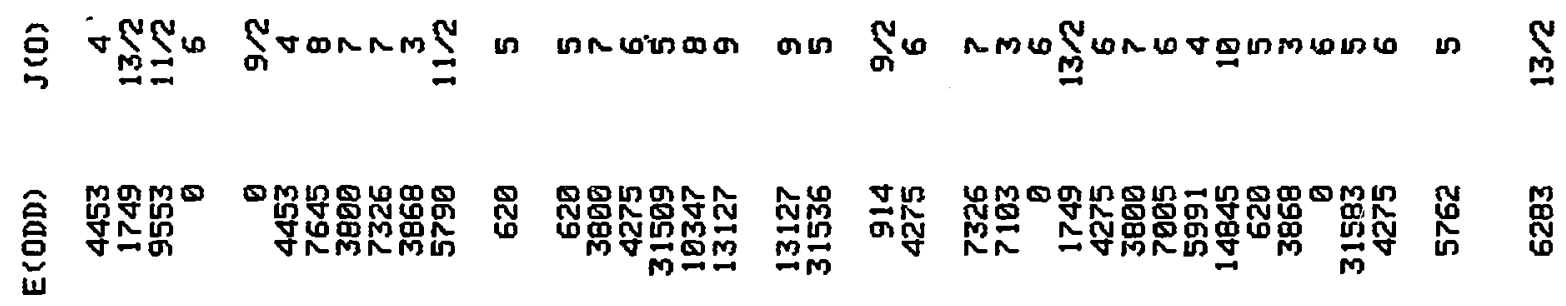

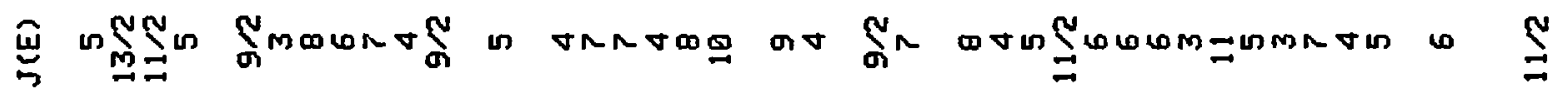

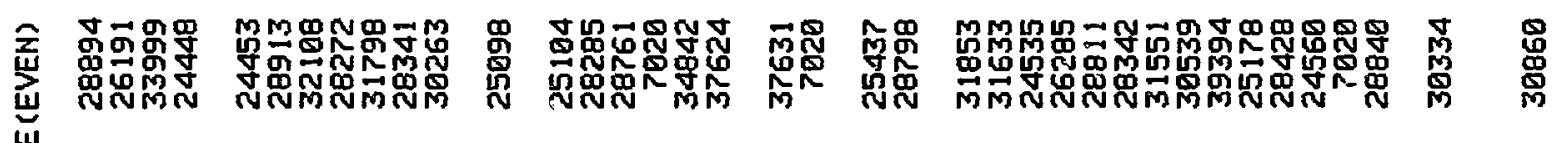

吾

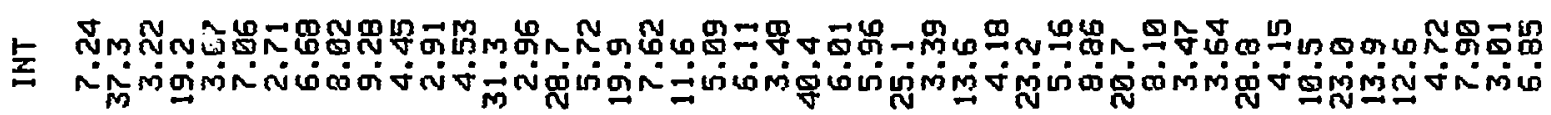

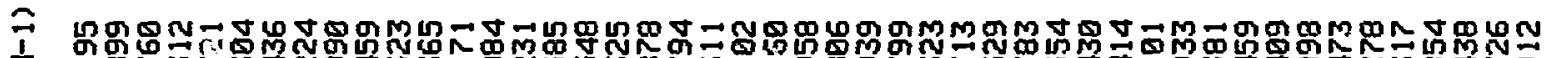

仿

z

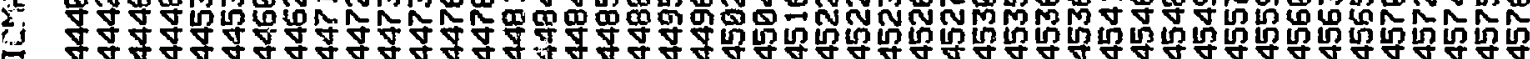

品

क

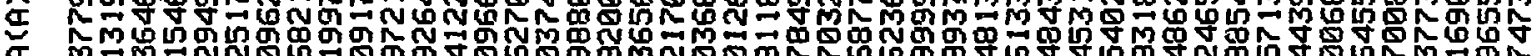

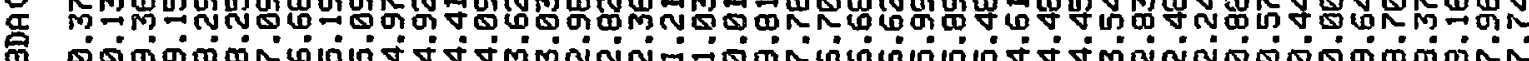

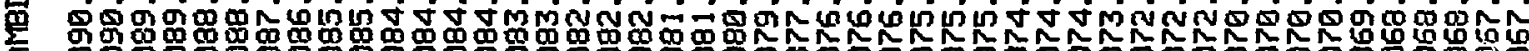

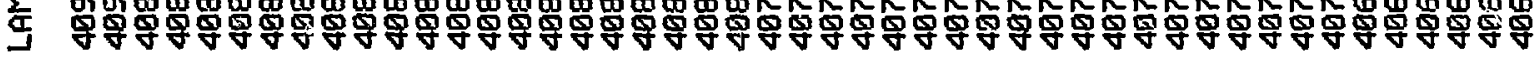




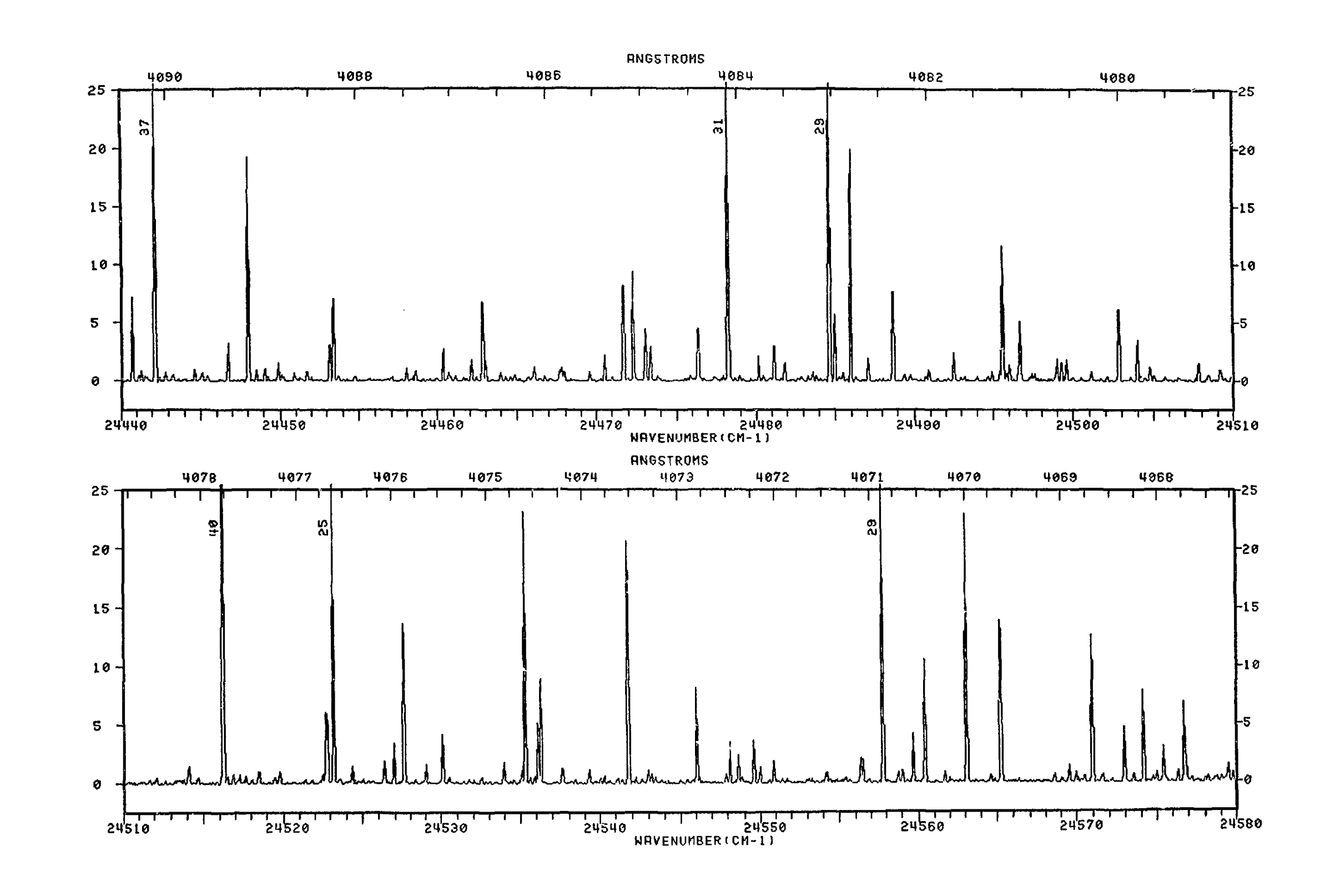




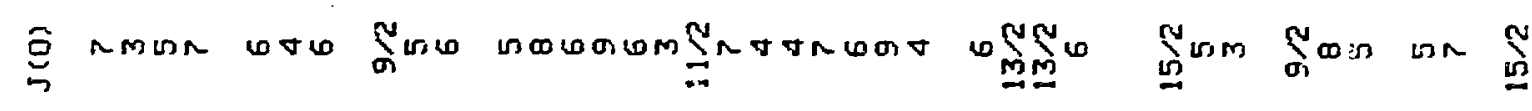

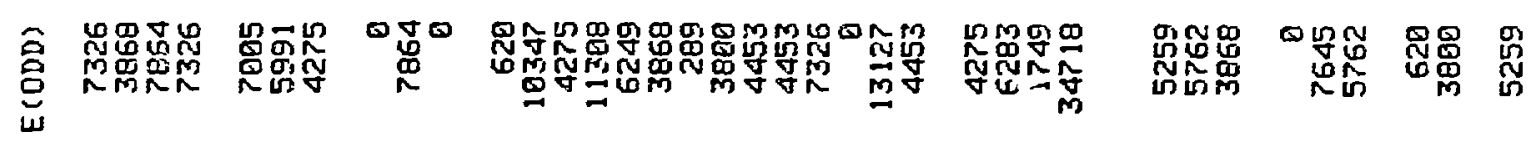

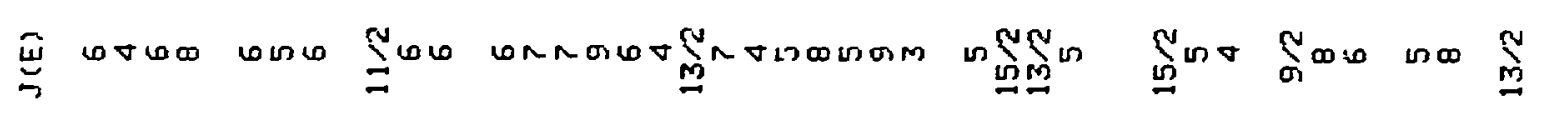

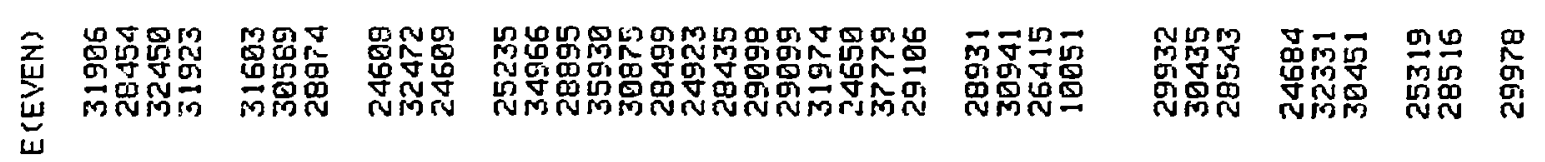

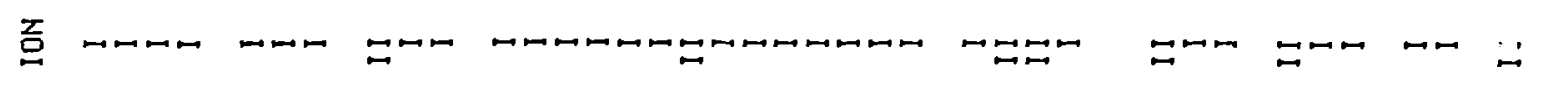

参

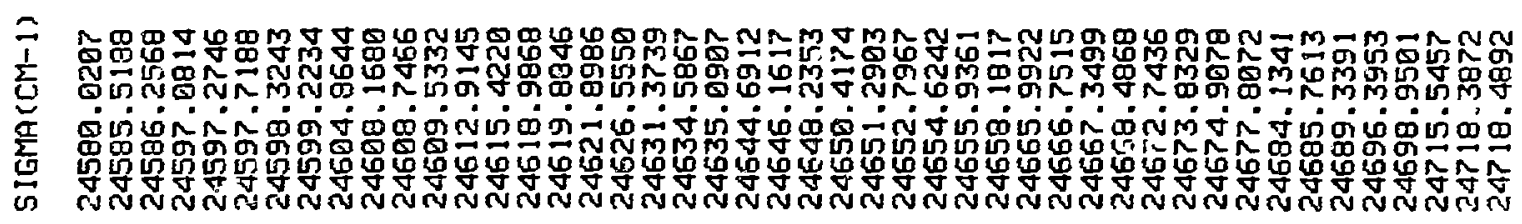

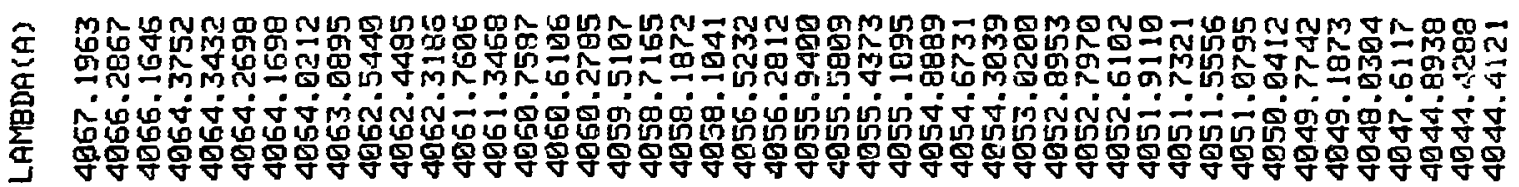



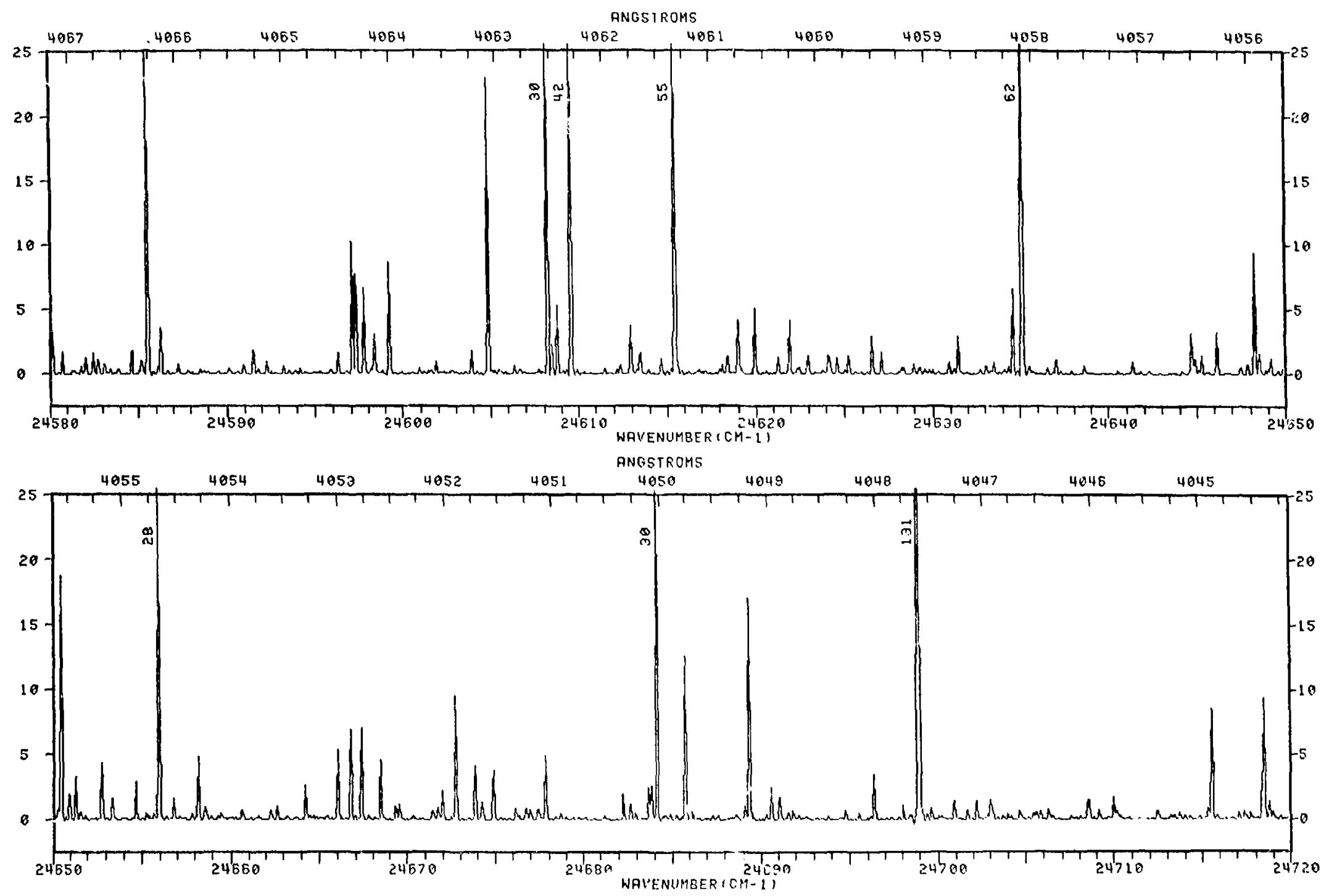

气
$\ln \cos$
$\boldsymbol{\omega} \boldsymbol{\omega} \boldsymbol{\omega}$
$\operatorname{snn} \tan$
$\sim \underset{\boldsymbol{N}}{\boldsymbol{N}}$
m INMment
$\sum_{n} \cos \theta \sin \cos$

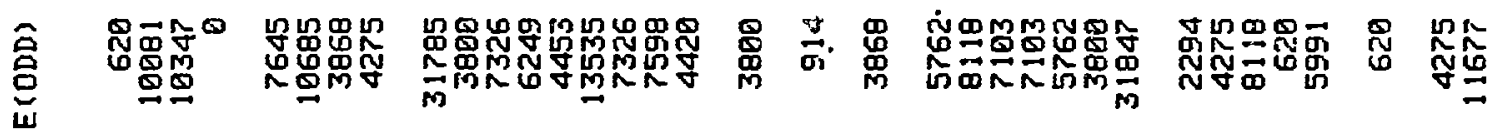

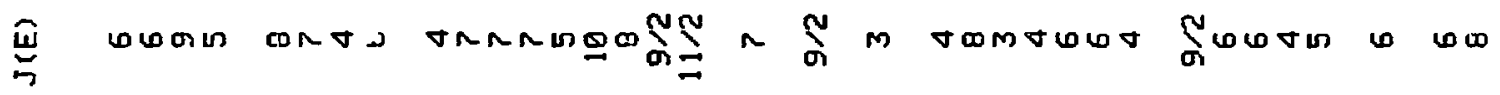

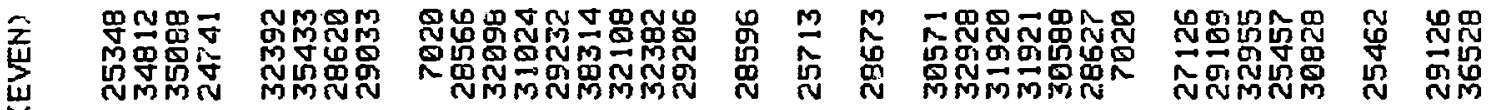

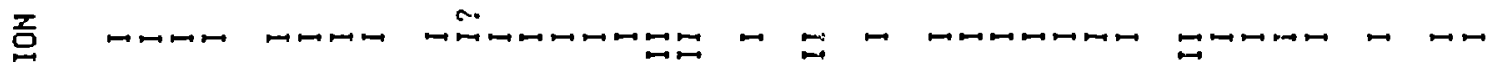

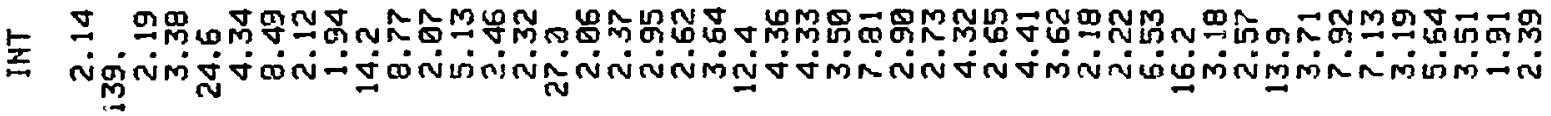

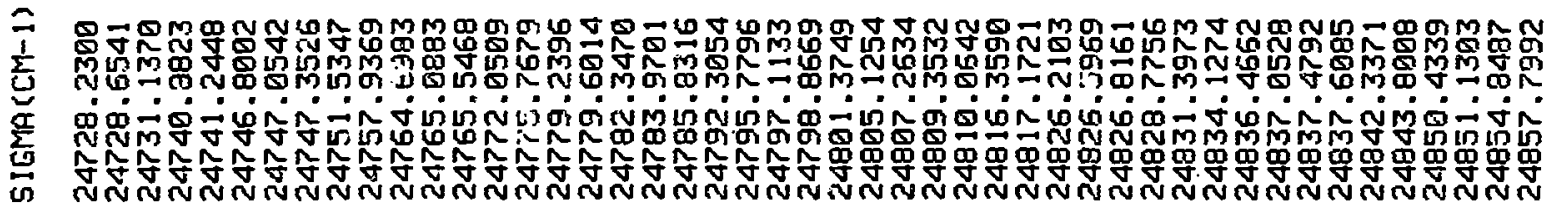

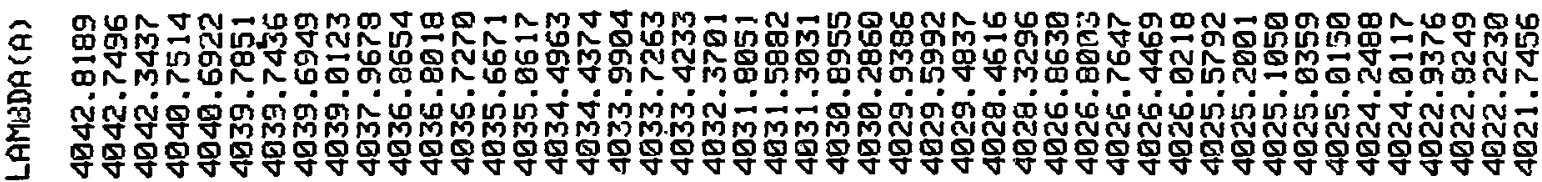



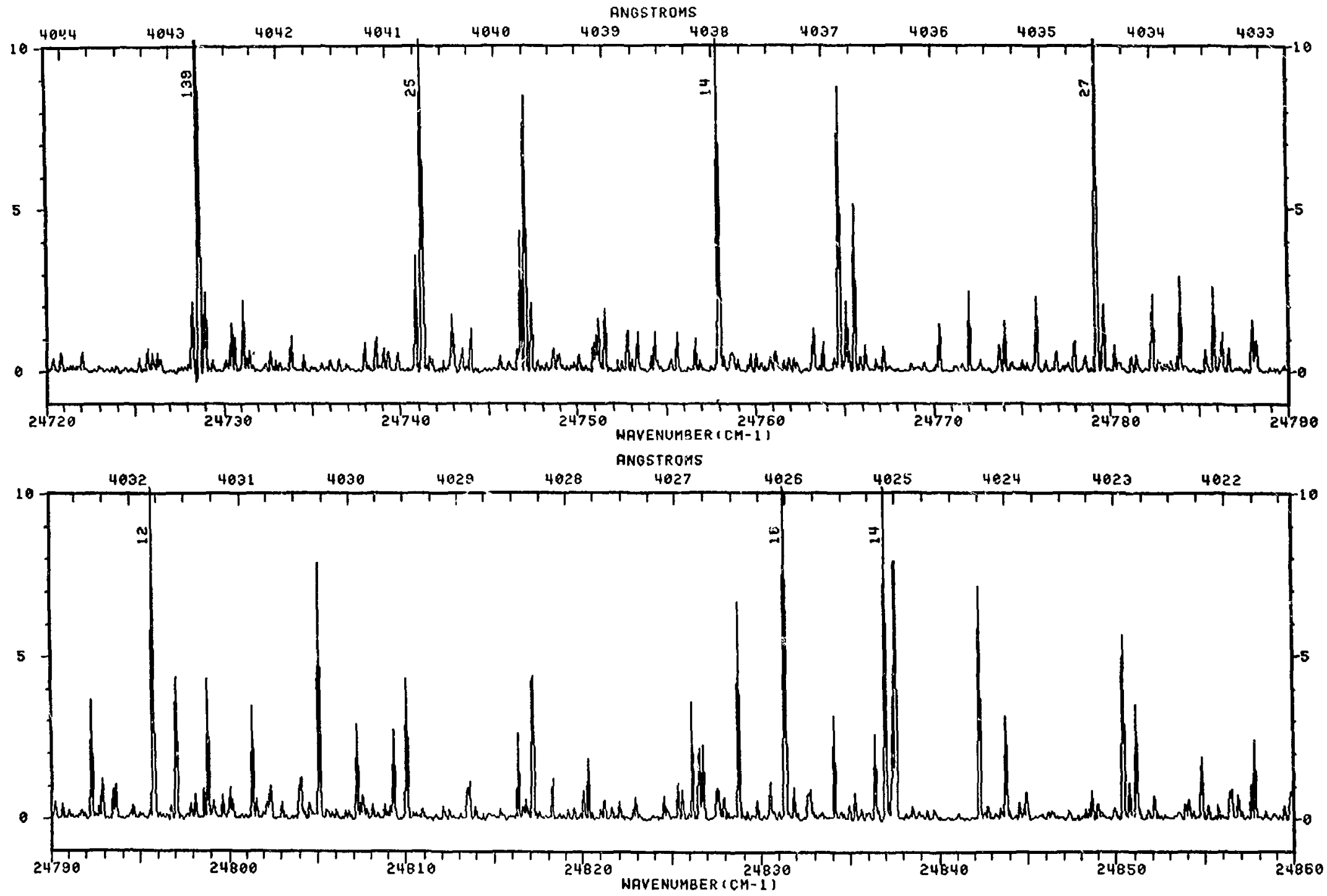
弟

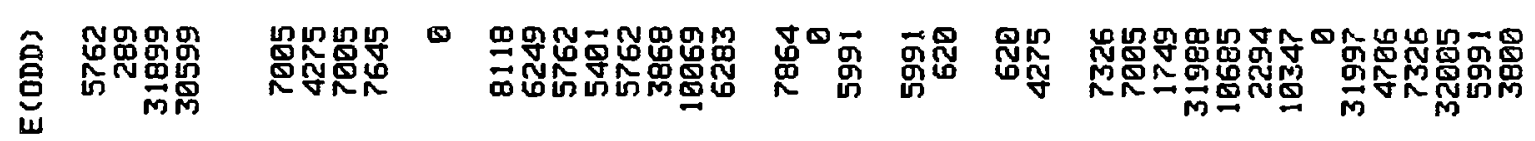

岸

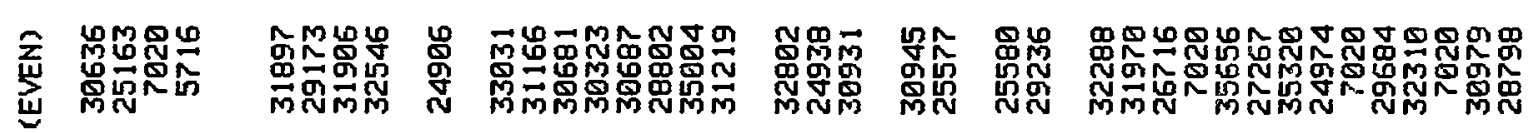

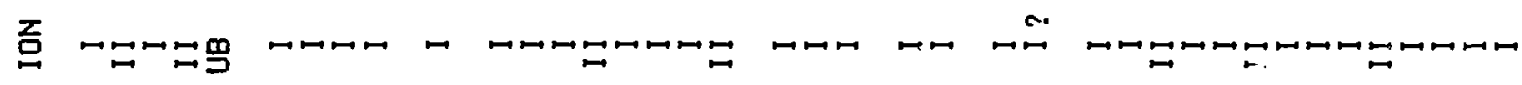

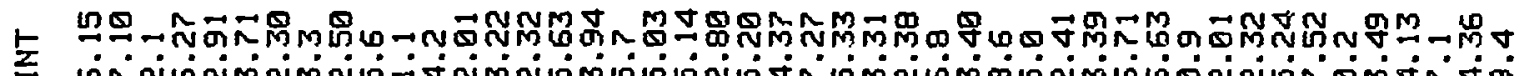

$z_{n}$

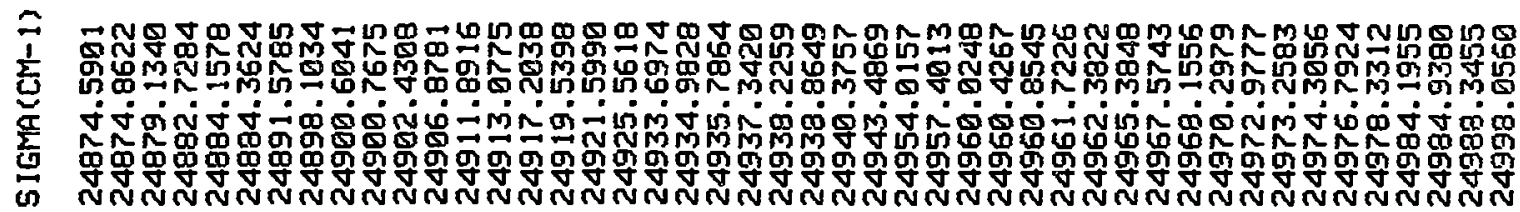

m

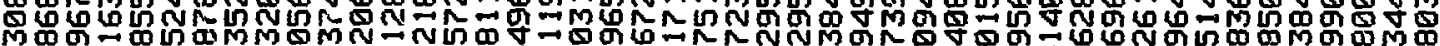

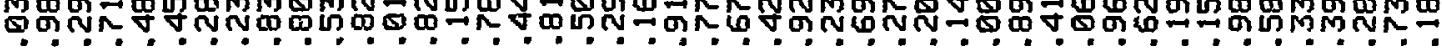

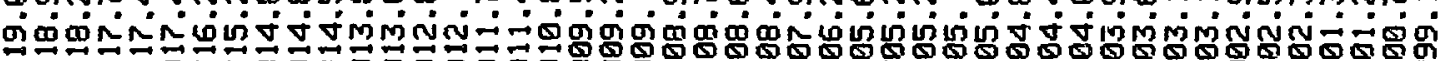

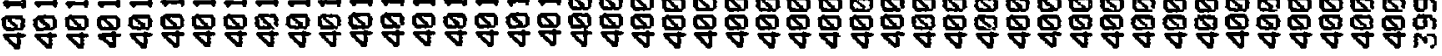



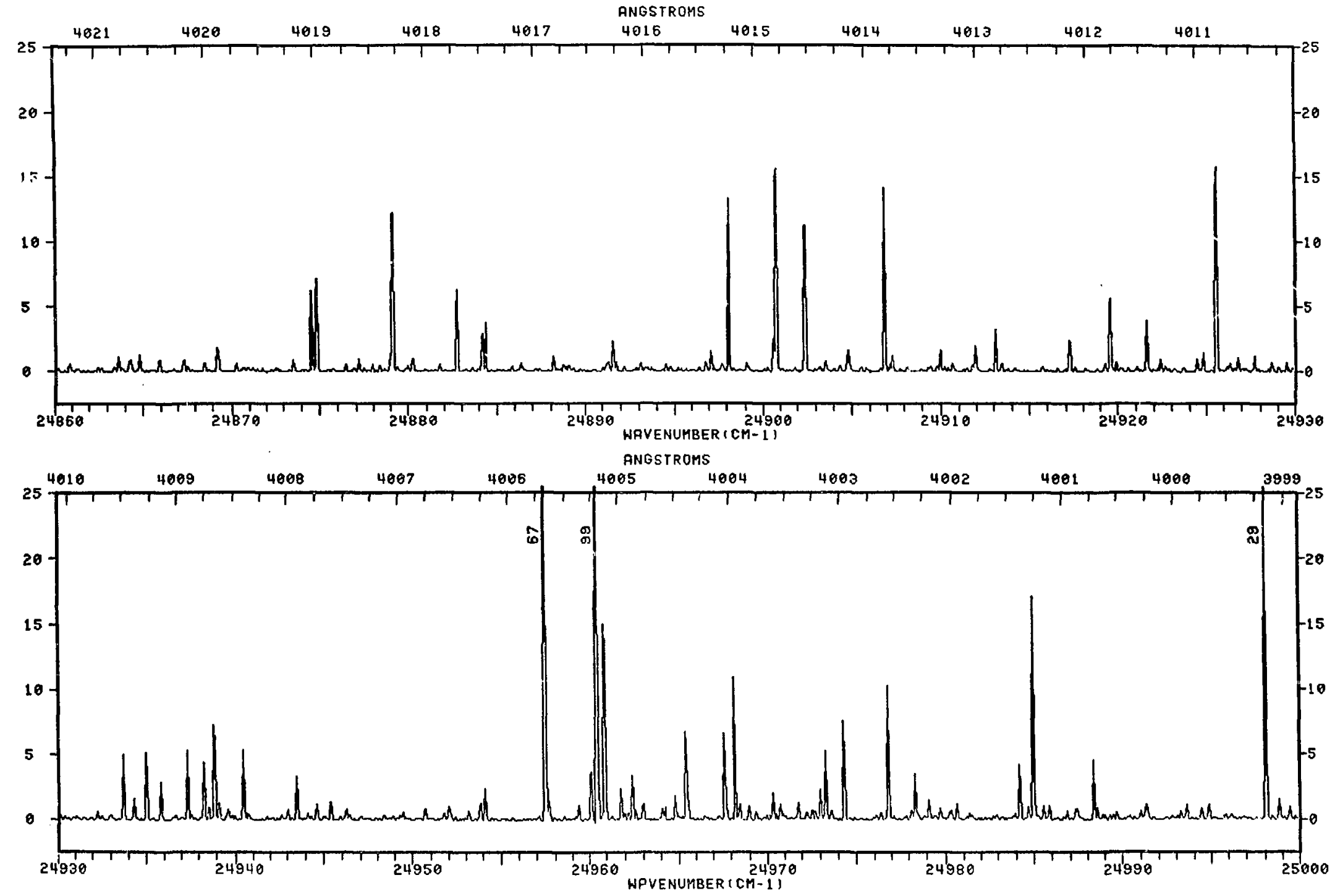


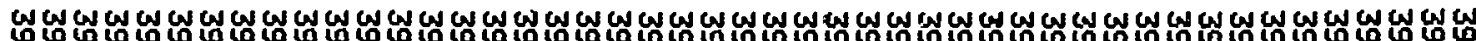

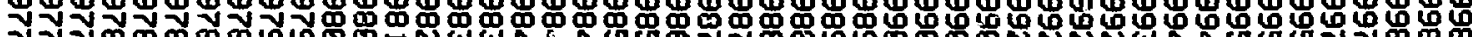
VVM0000600. NWWA

Q

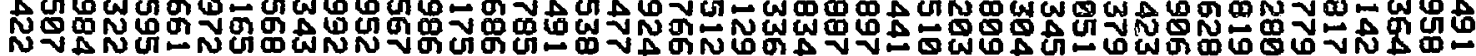

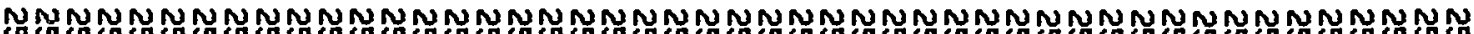

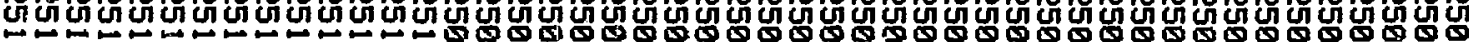

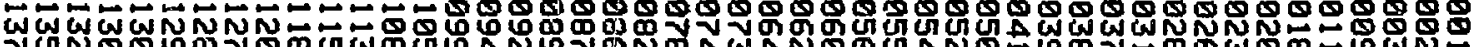
V GN

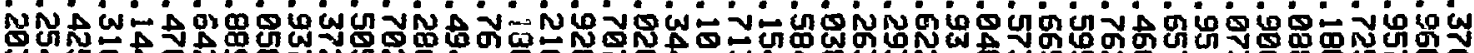

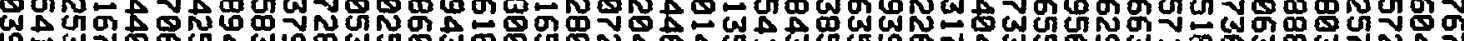

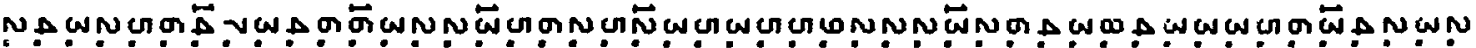

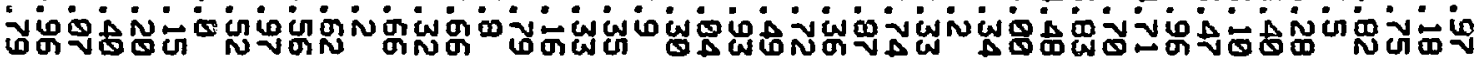
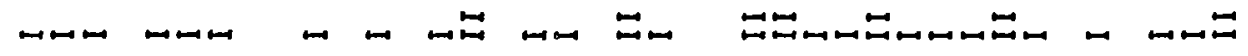

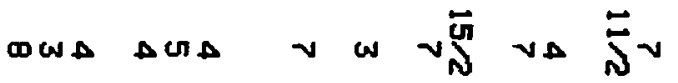

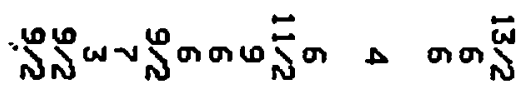




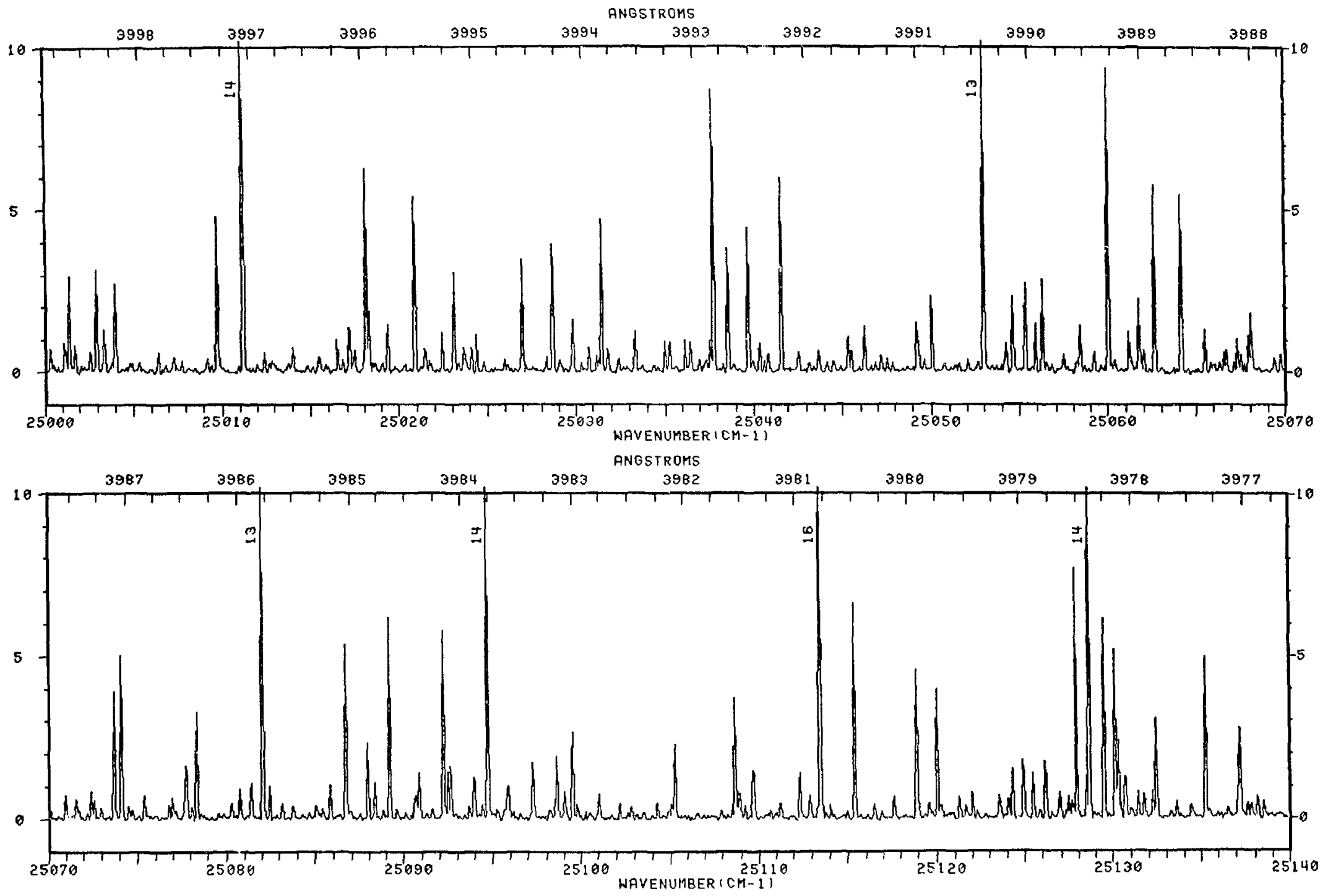




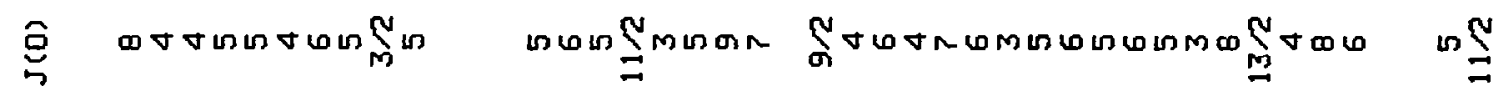

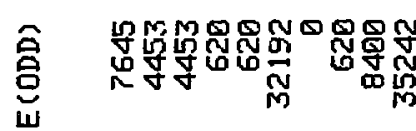

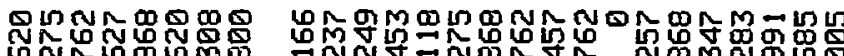

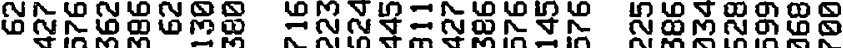

䍐

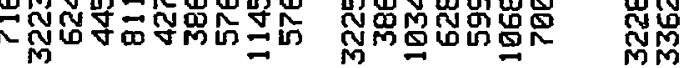

峞 $\cos \theta \operatorname{son} \sin$

$\nabla \sim \omega \int_{0}^{N} \nabla \omega \sigma \infty$

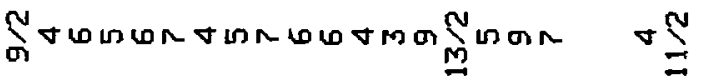

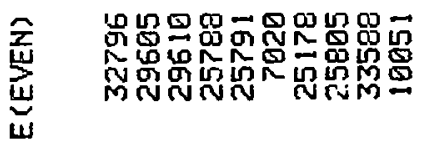

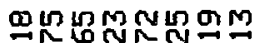

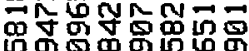

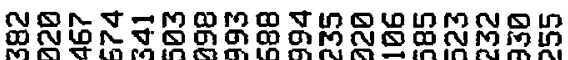
N

品

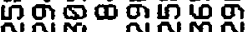
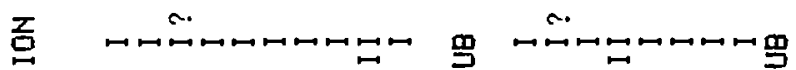

罗ニ

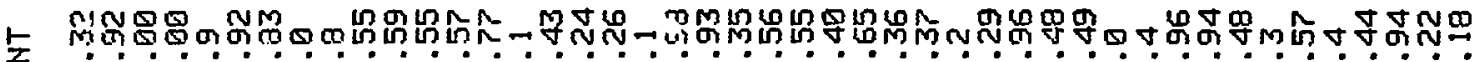
ב

$\frac{2}{2}$

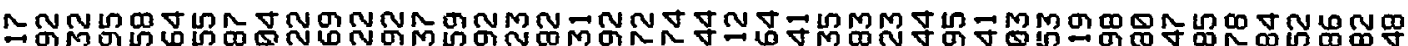

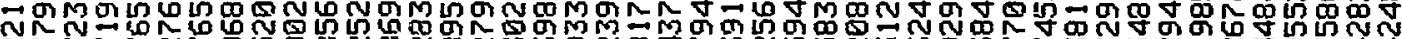

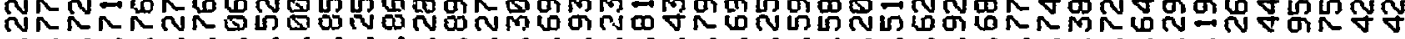

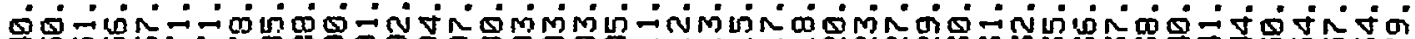
vin

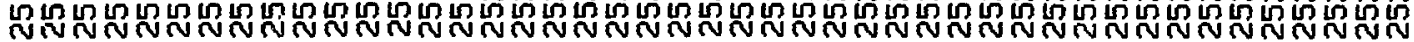

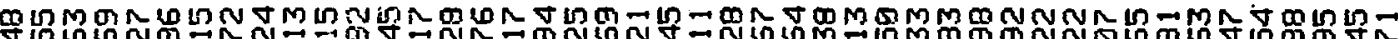
母

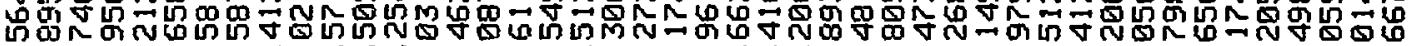

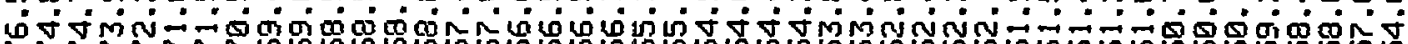

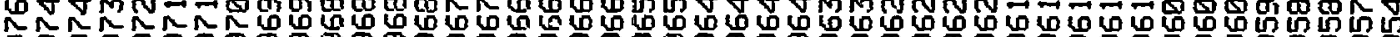

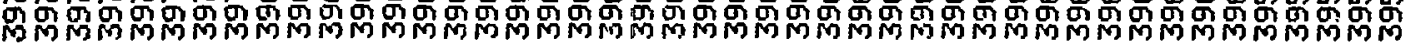



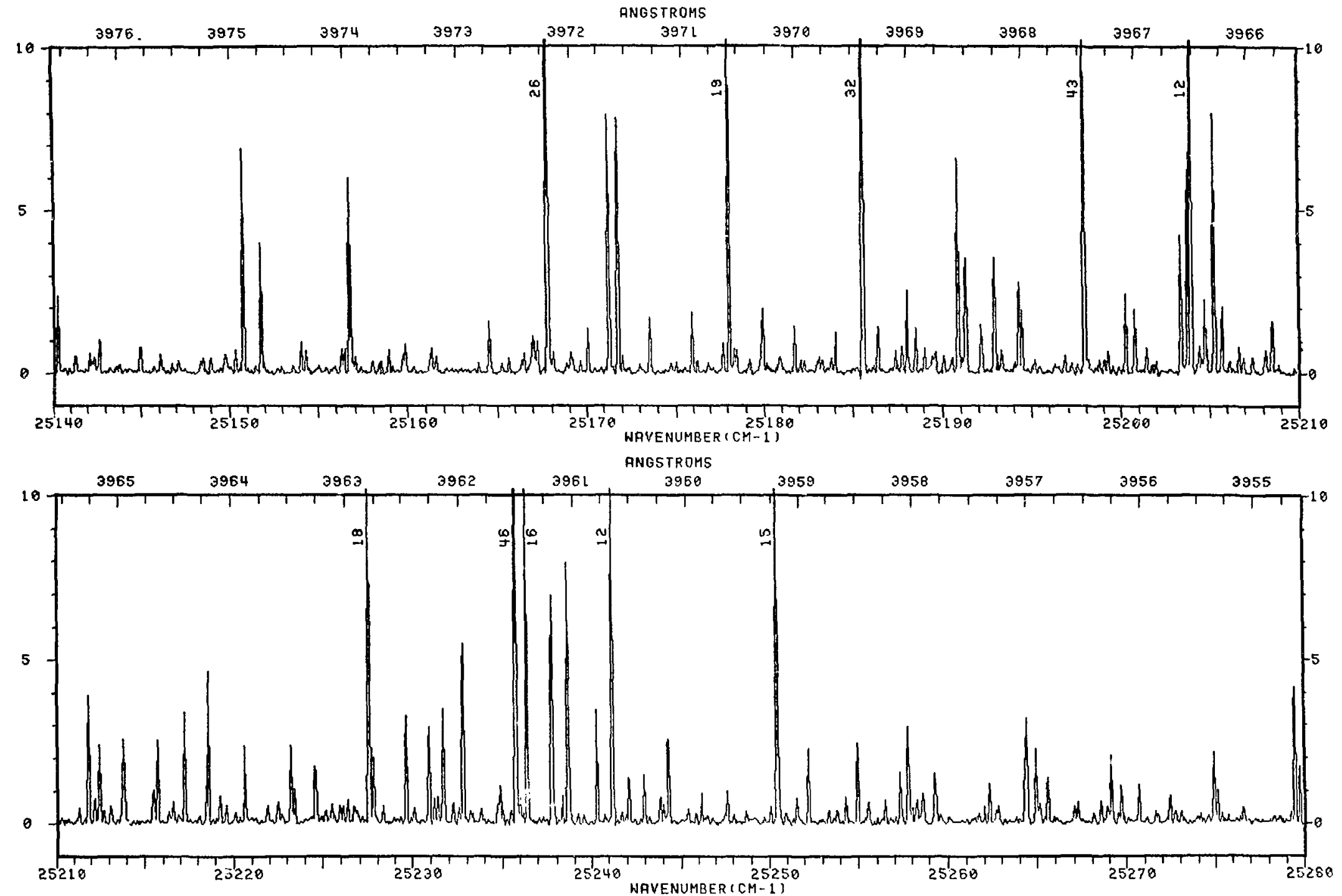
总

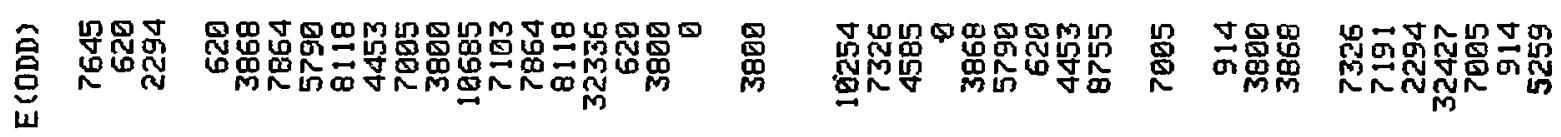

岂

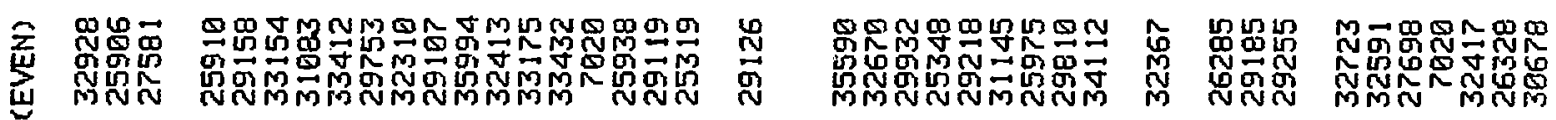

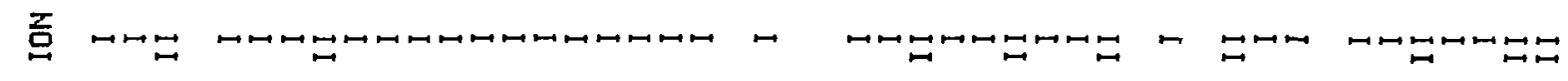

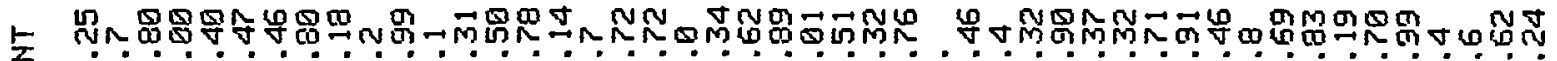

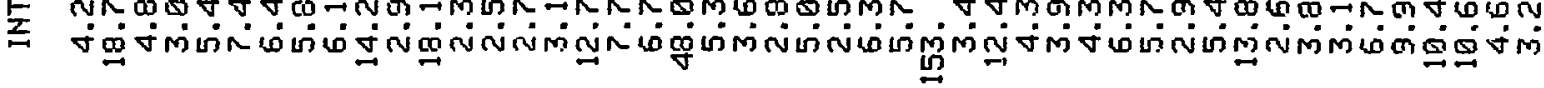

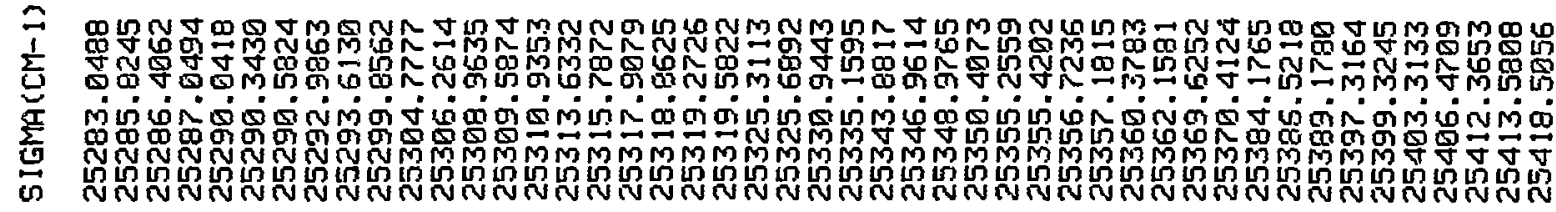

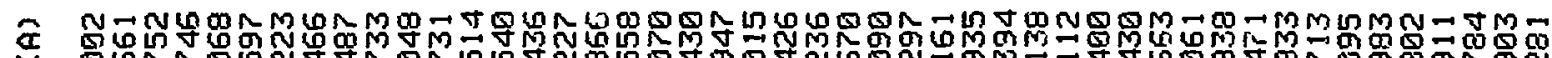

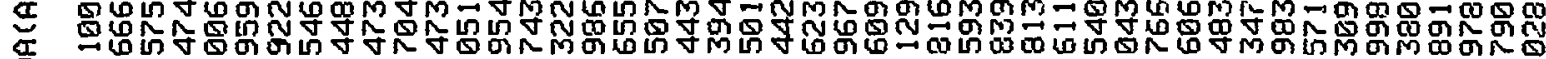

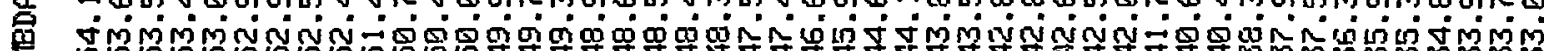

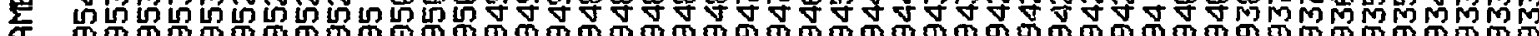

उ 

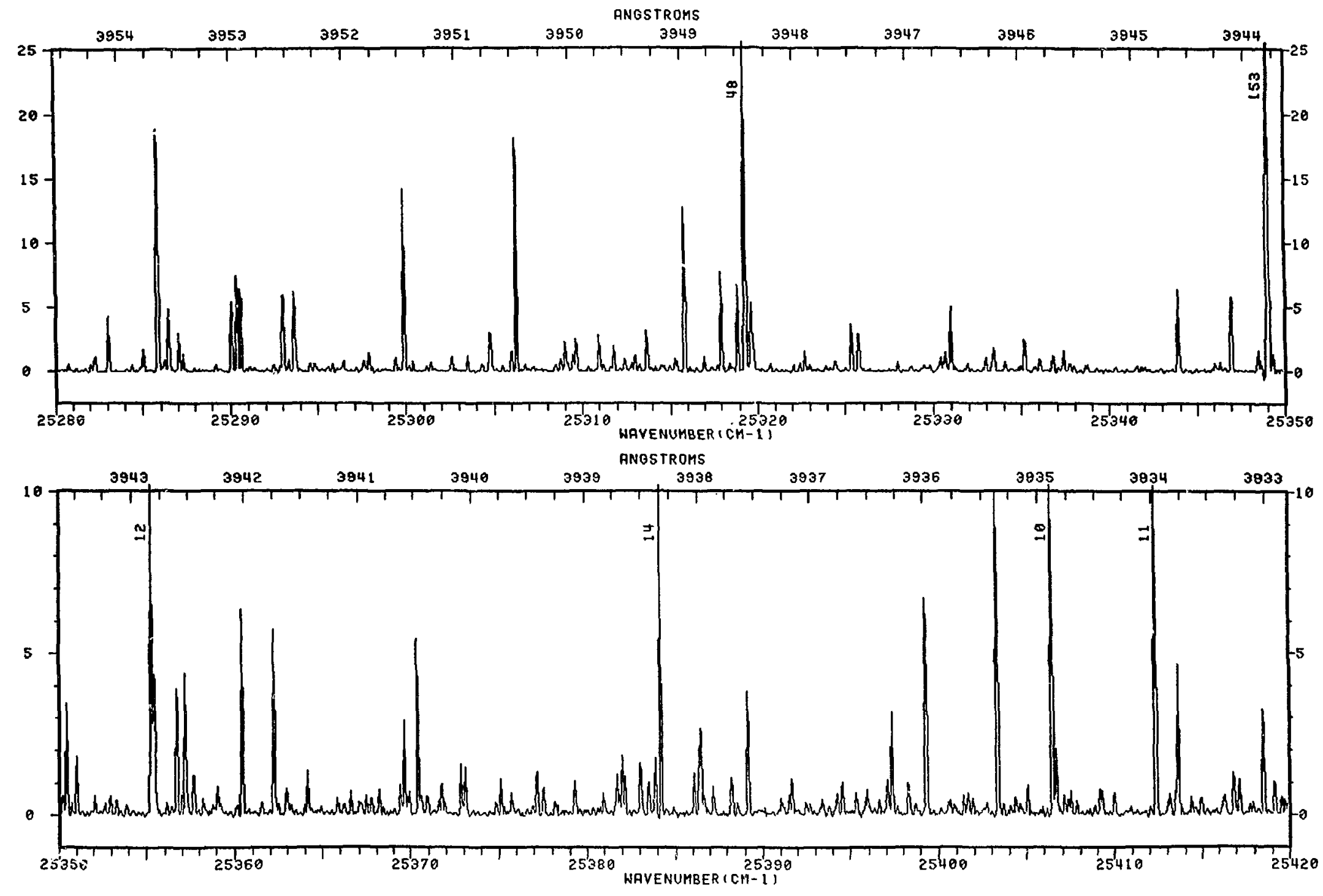


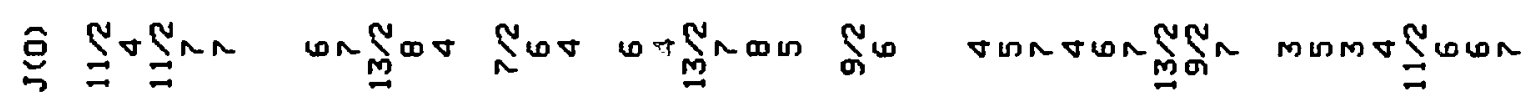

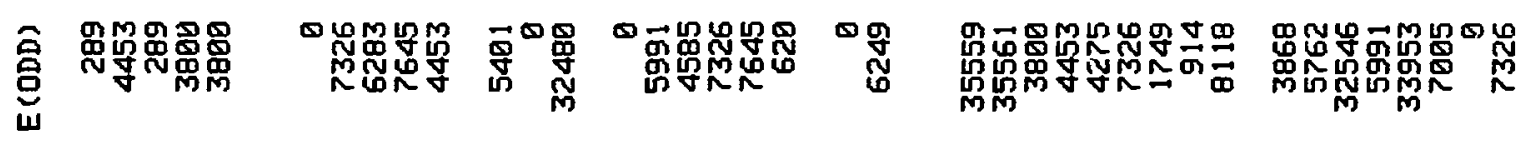

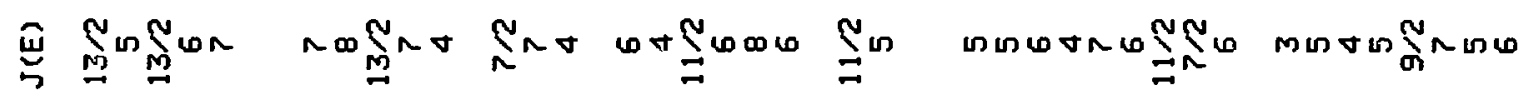

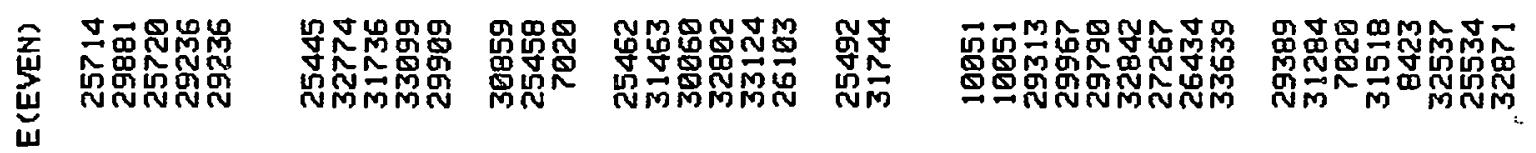

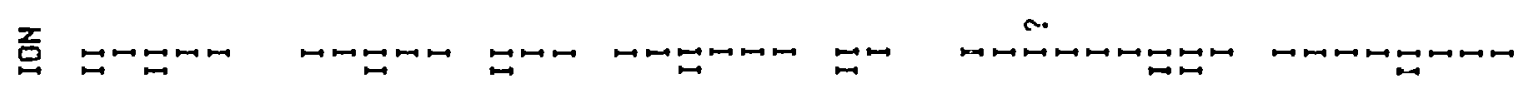

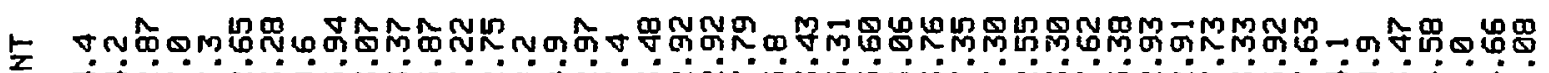

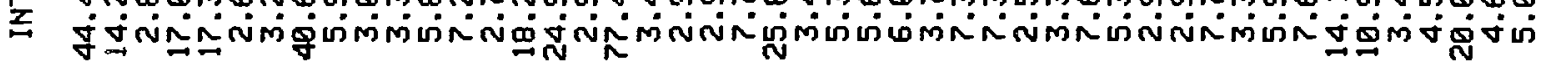

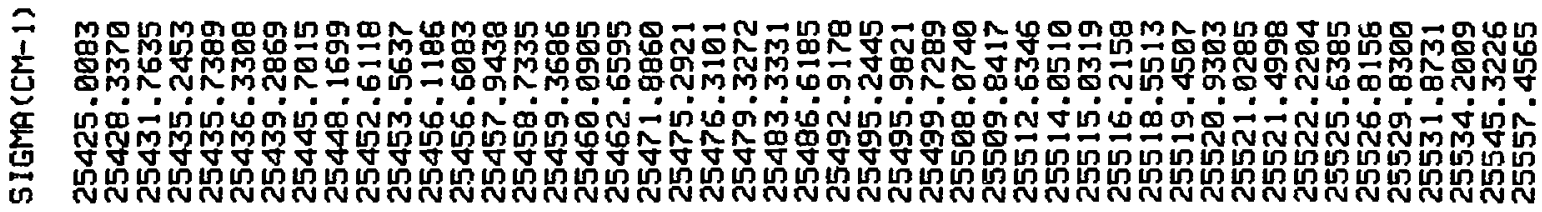

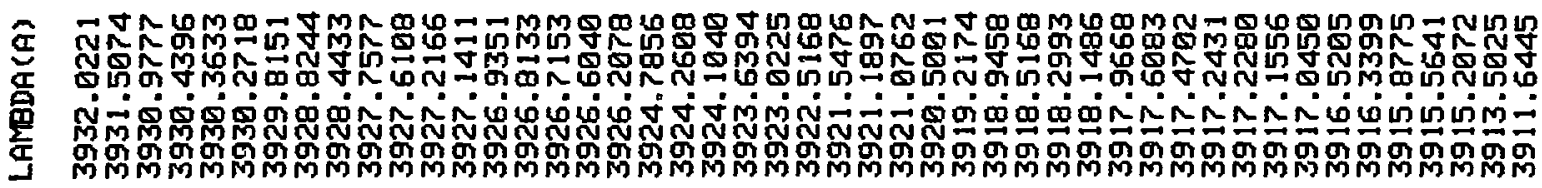



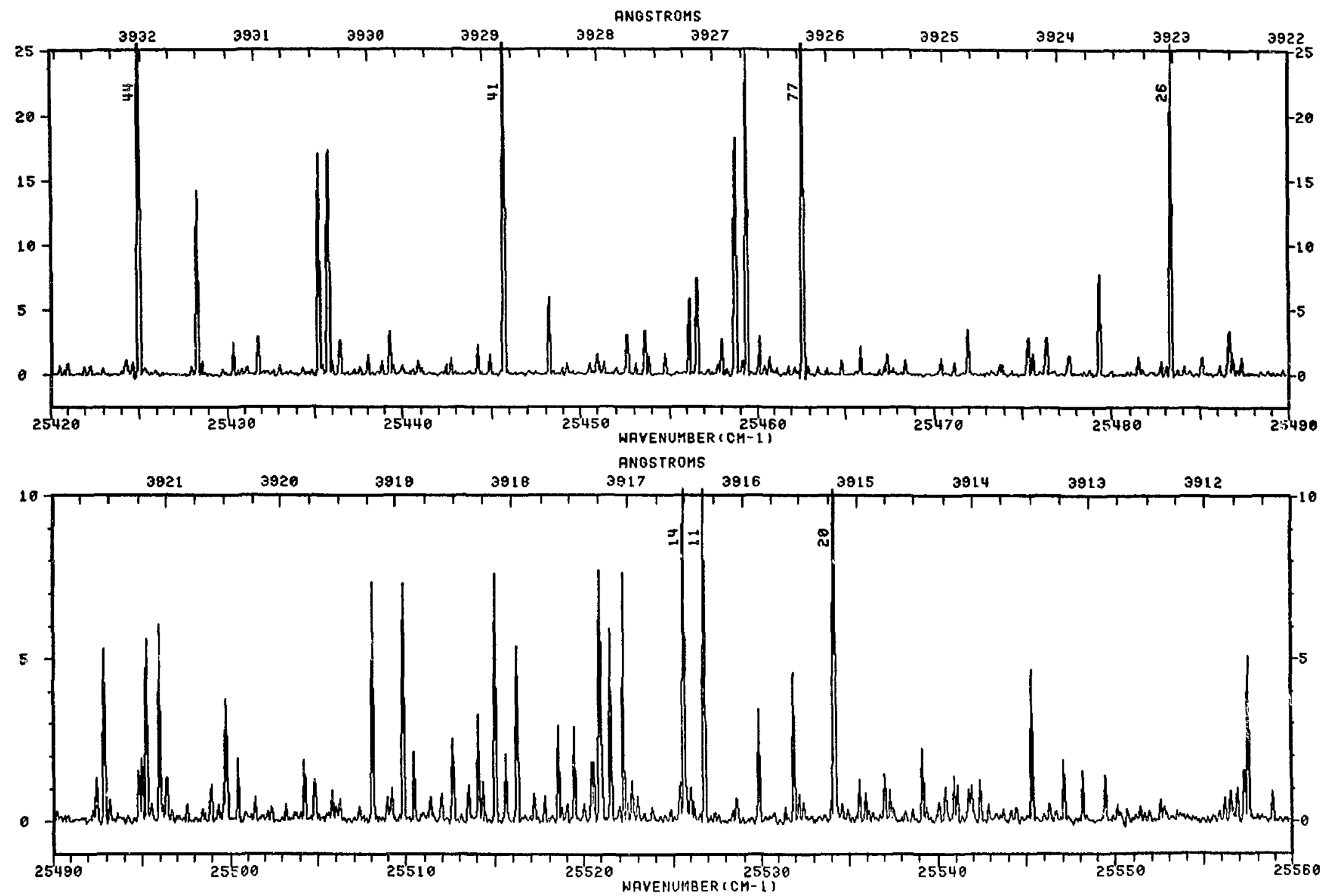
莺

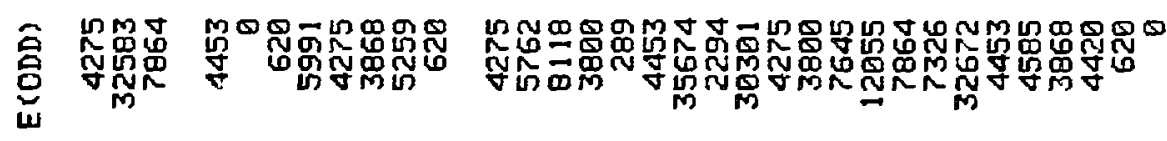

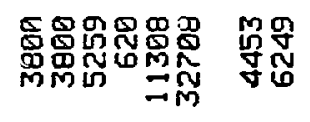

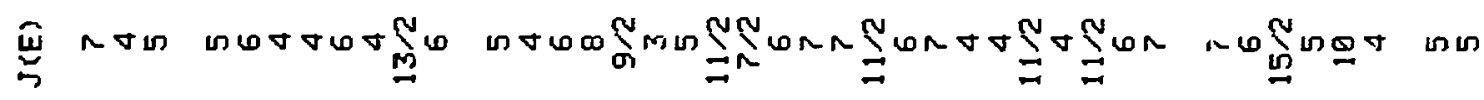

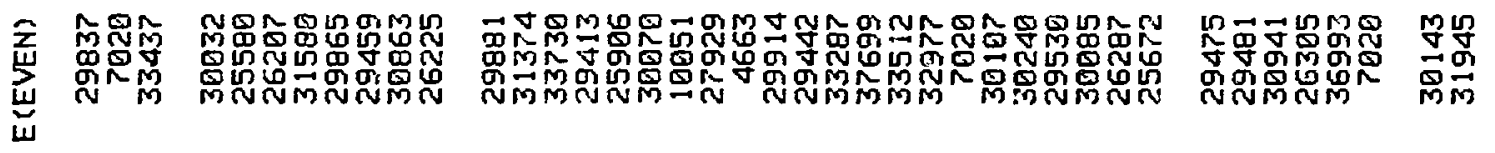

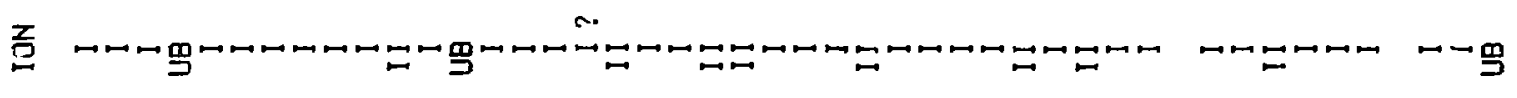

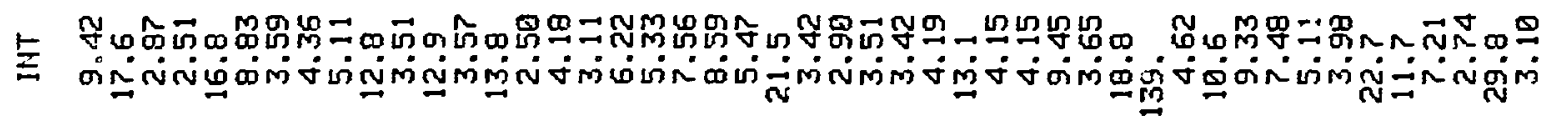

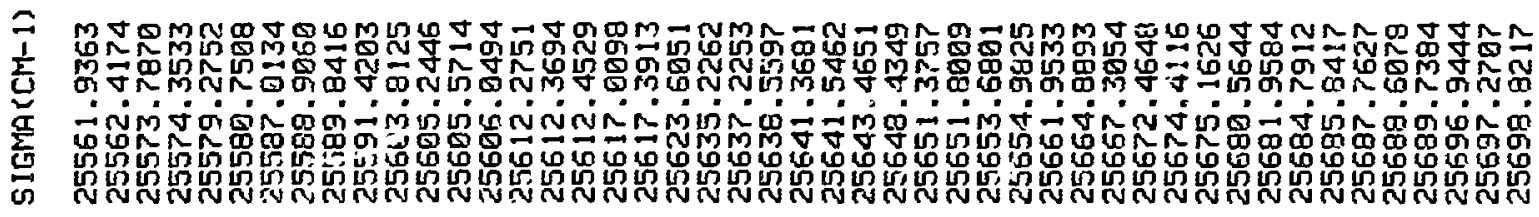

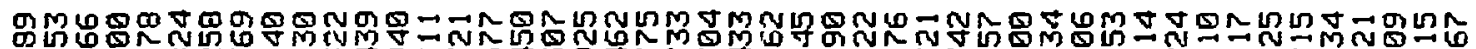

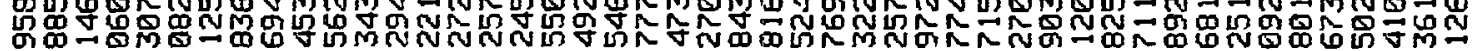
ऽهigín் -79 M 


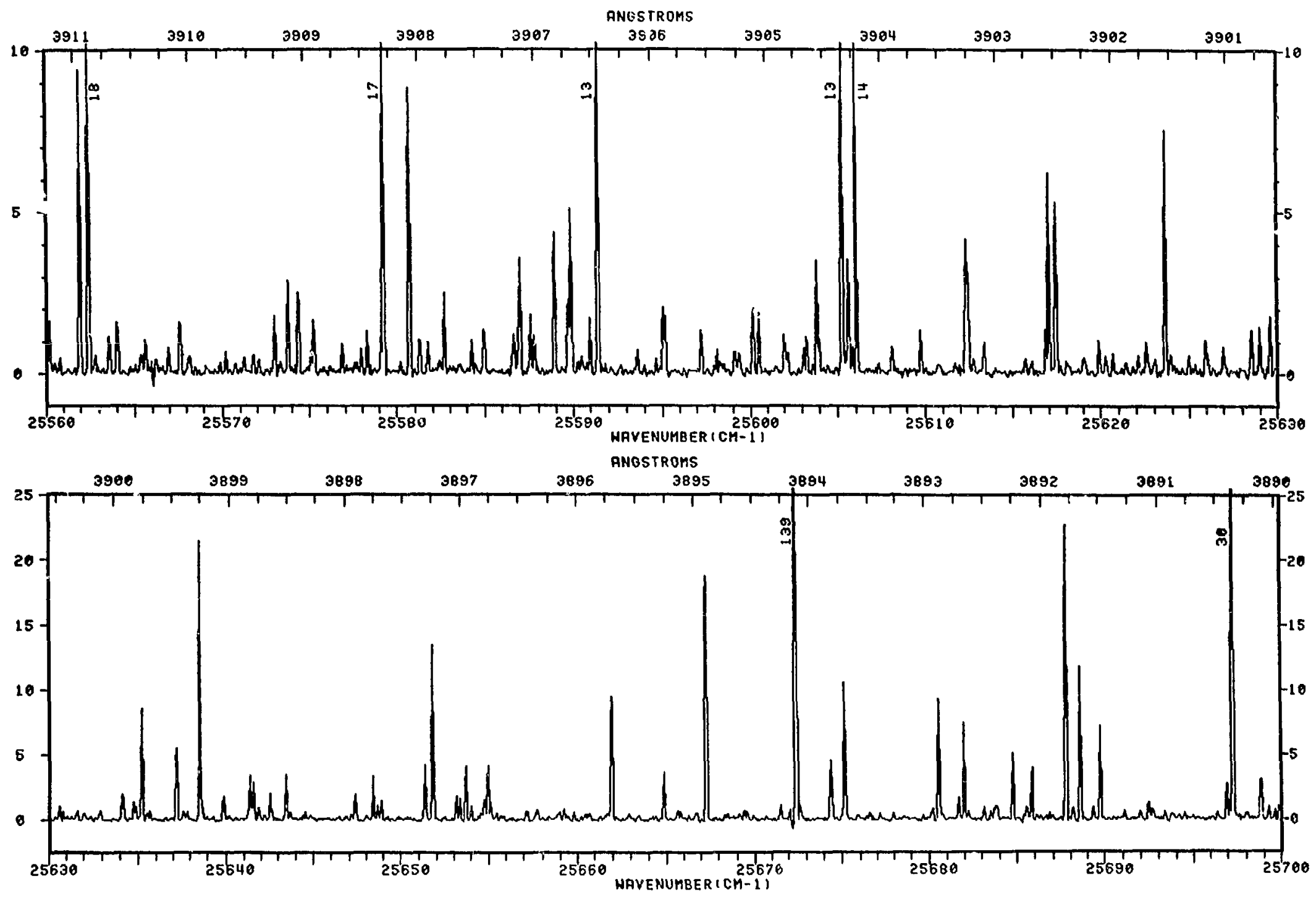




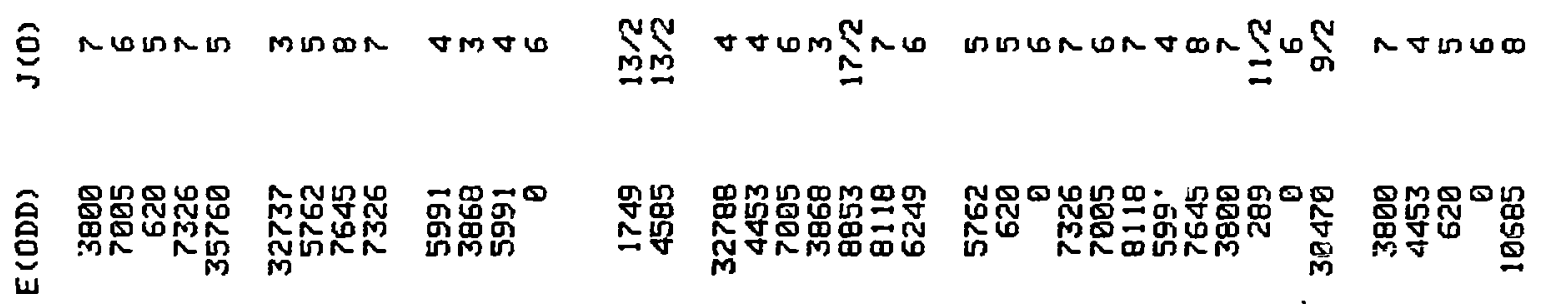

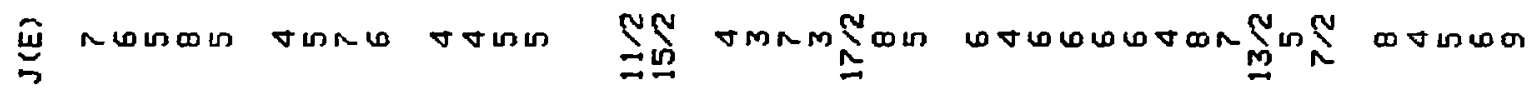

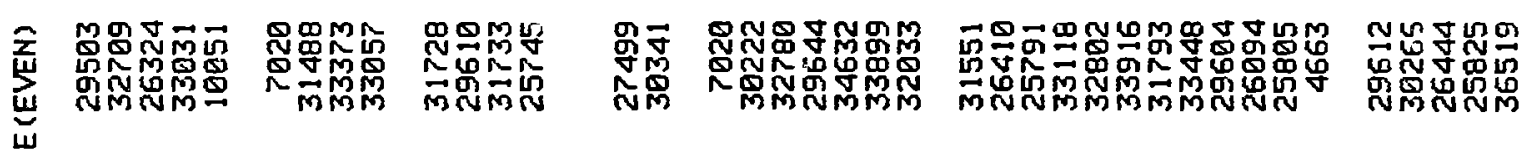

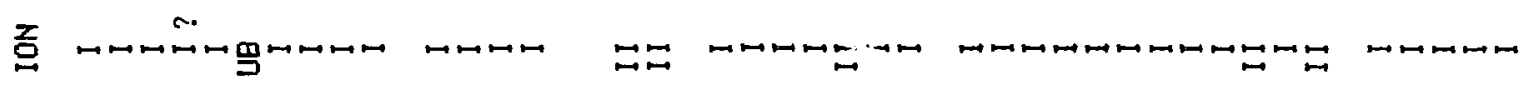

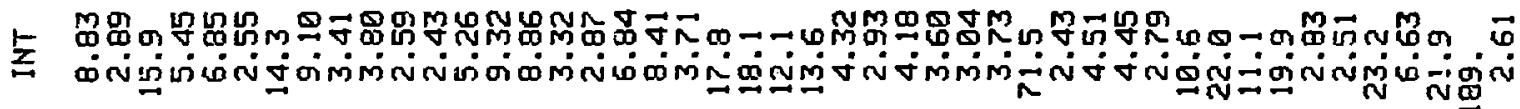

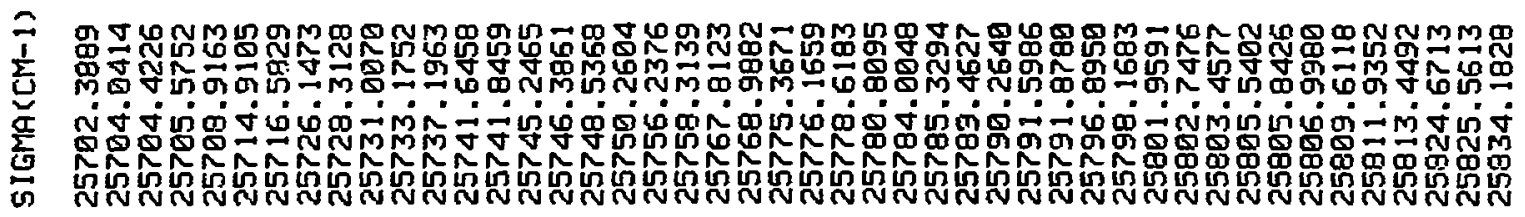

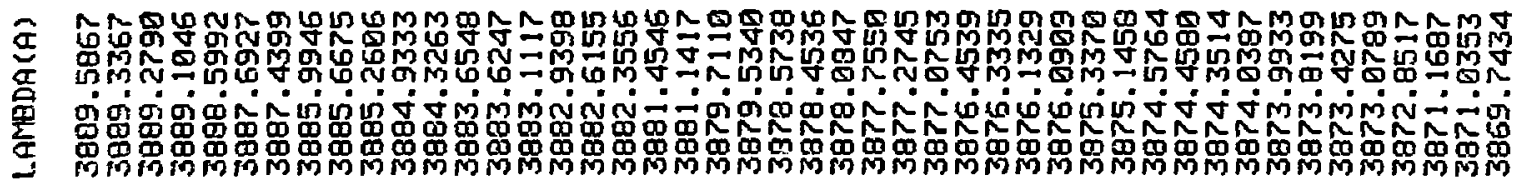



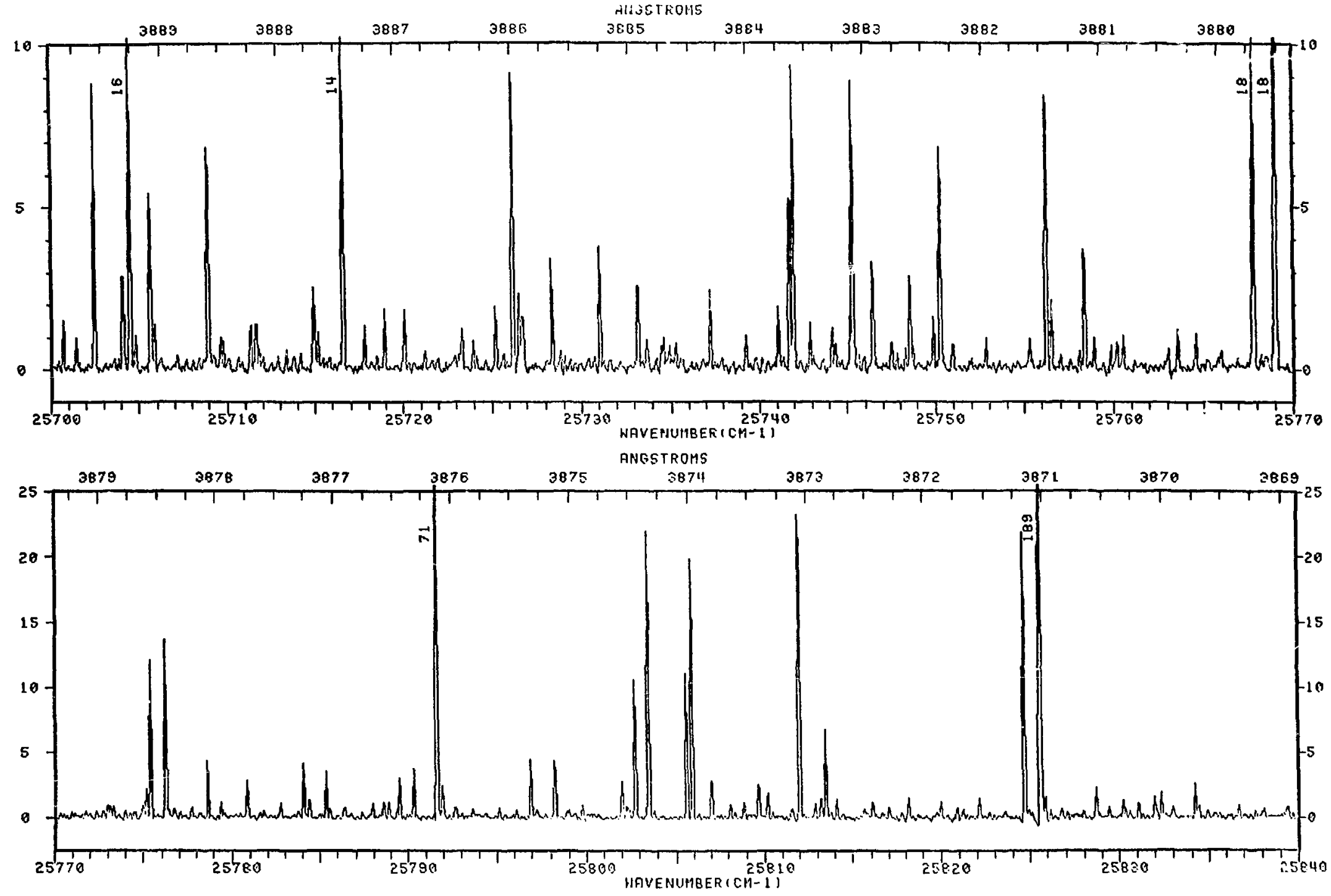


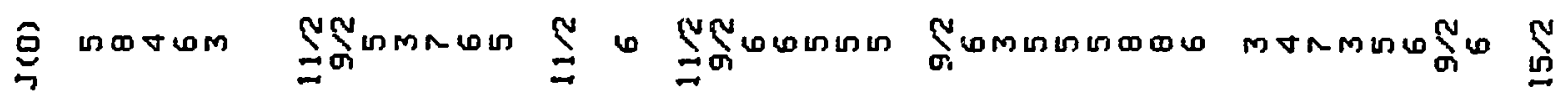

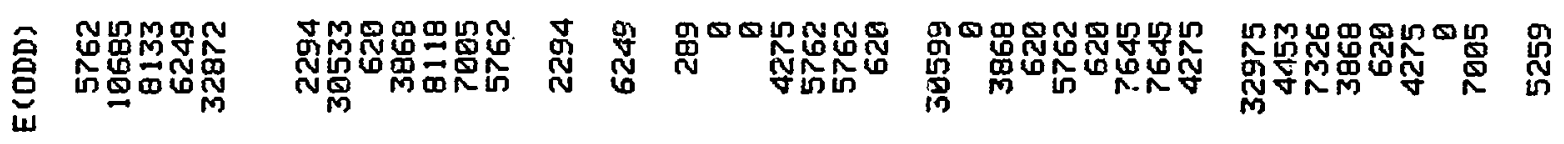

矛

\begin{tabular}{|c|c|c|c|c|c|}
\hline 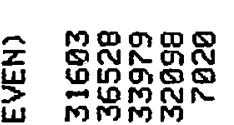 & 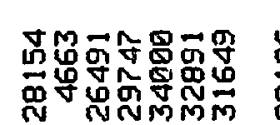 & 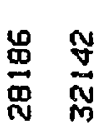 & 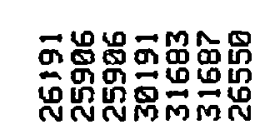 & 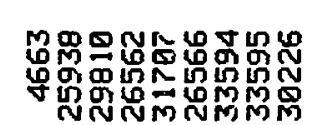 & 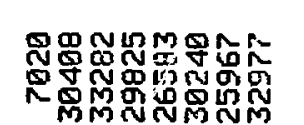 \\
\hline
\end{tabular}

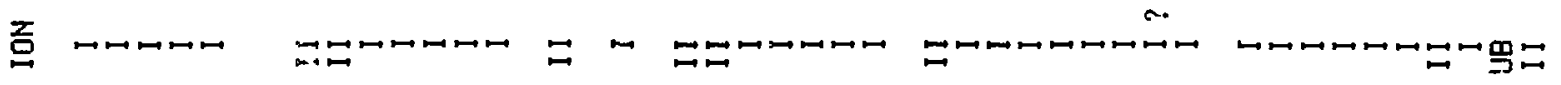

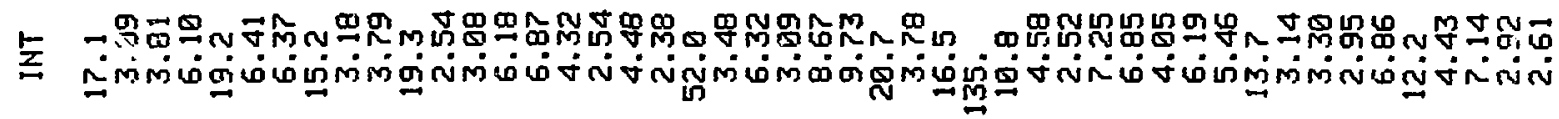

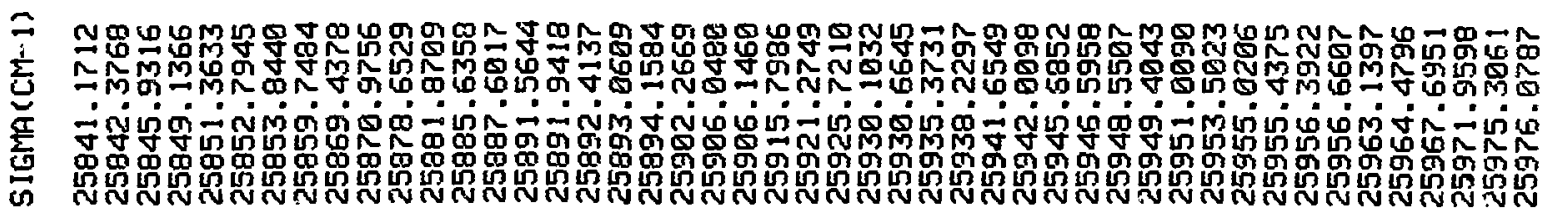

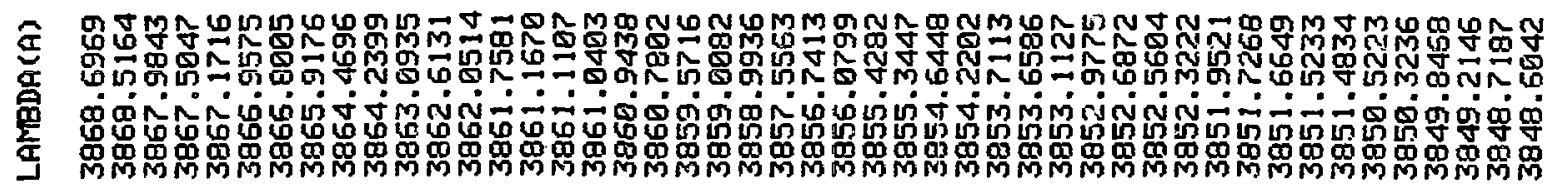




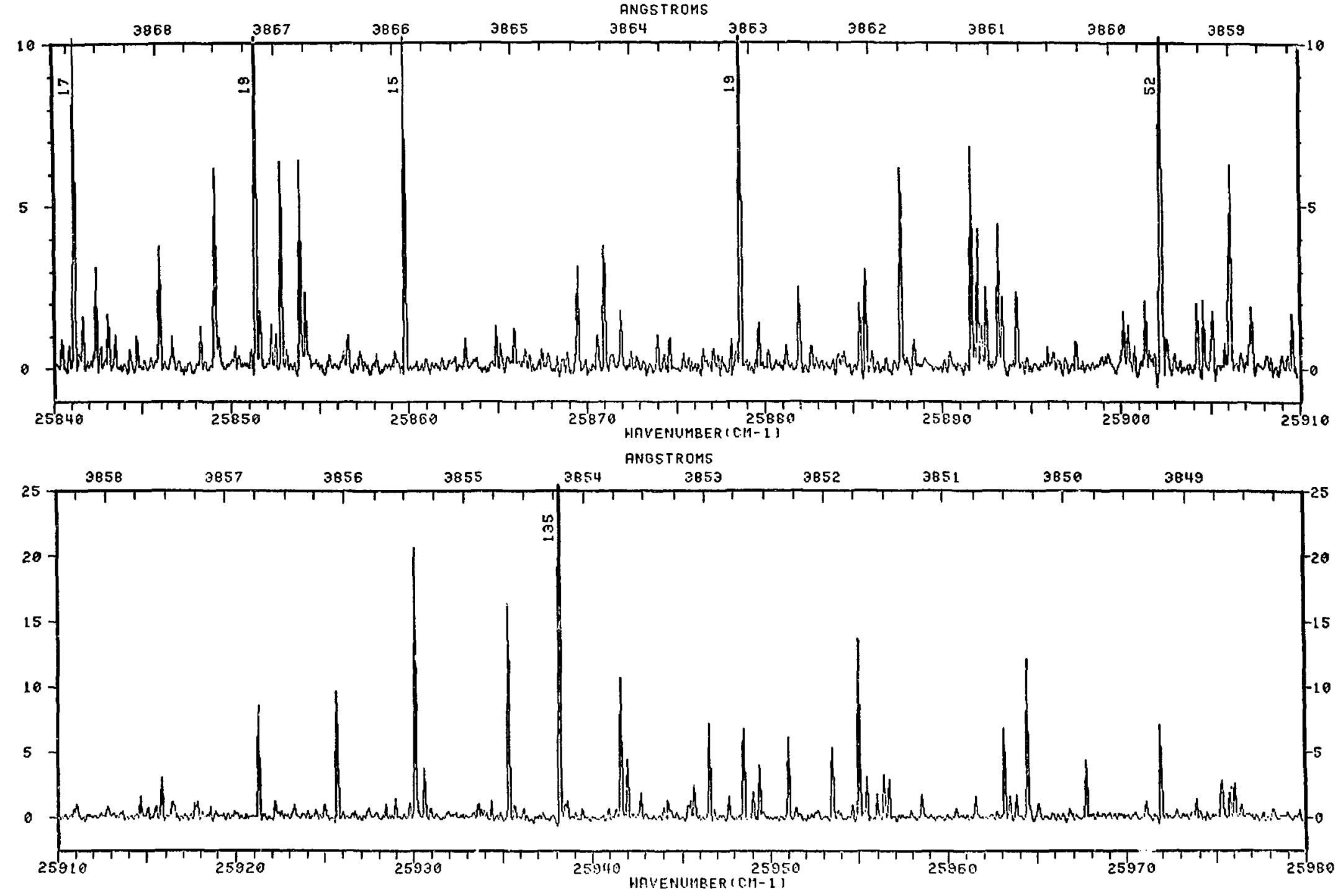


APPENDIX

\section{Atlas for optogalvanic wavelength calibration}

Richard A. Keller, Rolf Engleman, Jr., and

Byron A. Palmer

University of California, Los Alamos Scientific Laboratory, P.O. Box 1663, Los Alamos, New Mexico 87545.

Received 27 September 1979.

0003-6935/80/060836-02\$00.50/0.

(C) 1980 Optical Society of America.

There is consid srable interest in the use of the optogalvanic effect for laser wavelength calibration. ${ }^{2}$ For this use a portion of the laser beam is deflected into a commercial hollow cathode discharge tube. When the laser wavelength corresponds to an optical transition of a species present in the discharge, the voltage across the discharge changes. ${ }^{2,3}$ These voltage changes are easily measured and form the basis for a calibration process. Commercial hollow cathode discharge tubes are available for all the well-known calibration sources-iron, thorium, rare gases, etc.

As an aid to this calibration it is useful to know how the relative magnitude of the induced voltage change compares to the relative intensity of the corresponding emission line. It was demonstrated in an uranium hollow cathode discharge at constant current that the ratio of the voltage change to the laser intensity for $\mathrm{cw}$ excitation is given by ${ }^{4}$

$$
\frac{\Delta V_{i j}}{I} \propto \lambda_{i j} f_{i j} g_{i} \exp \left(-E_{i} / k T\right)
$$

$\Delta V_{i j}$ is the observed voltage change when the laser wavelength $\left(\lambda_{i j}\right)$ corresponds to the $E_{i} \rightarrow E_{j}$ transition, $f_{i j}$ is the oscillator strength of this transition, $g_{i}$ is the degeneracy of the initial ( $i$ th) state, $E_{i}$ is the energy of this state, and $l$ is the laser intensity. Care must be taken to avoid laser powers in excess of $100 \mathrm{~mW}$ because of saturation effects. This can be a serious problem when using pulsed excitation. The intensity of an emission line is given by

$$
F_{j i} \propto \frac{g_{i}}{g_{j}} \cdot \frac{f_{i j}}{\left(\lambda_{i j}\right)^{3}} \cdot g_{j} \exp \left(-E_{j} / k T\right) .
$$

In this case the initial state is the jth state. In hoth (1) and (2) we are interested in calibration over a small wavelength region $(<50 \AA)$ so $\lambda_{i j}$ does not change appreciably. $E_{j}=E_{i}$ $+h v_{i j}$. Thus, (1) and (2) can be rewritten for comparison over small wavelength regions as

$$
\begin{array}{r}
\frac{\Delta V_{i j}}{I} \propto f_{i j} \cdot g_{i} \cdot \exp \left(-E_{i} / k T\right), \\
\quad F_{j i} \propto f_{i j} \cdot g_{i} \cdot \exp \left(-E_{i} / k T\right),
\end{array}
$$



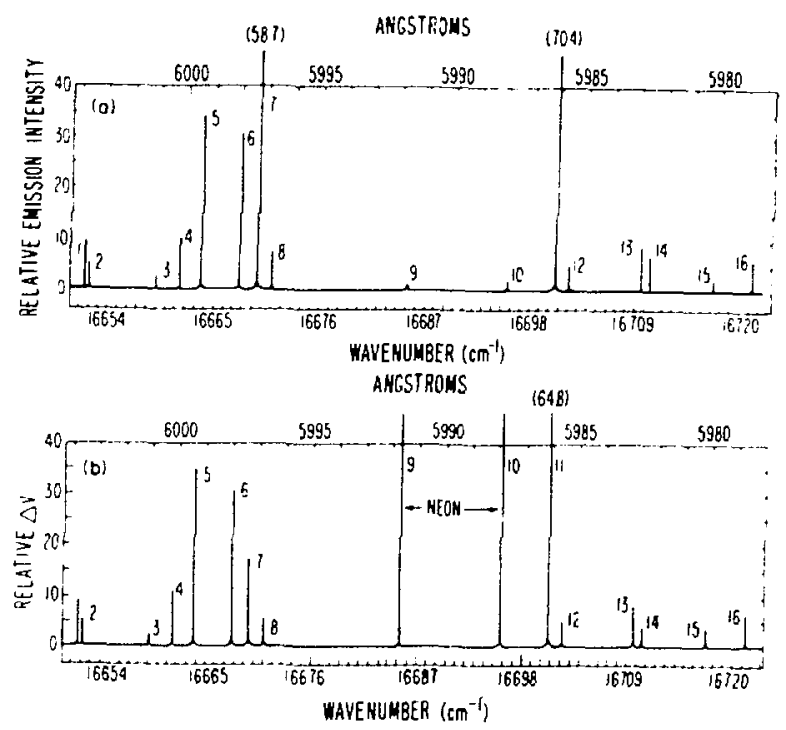

Fig. 1. Comparison of the optical emission spectrum (a) with the (op)tugalvanic spectrum (b). Discharge tube current: (a) $75 \mathrm{~mA}$; (b) $25 \mathrm{~mA}$. Laser puwer: $50150 \mathrm{~mW}$. Lat er linewidth: three modes with an intermode spacing rof 1.2 GHz. Source: neon-filled uranium hollow cathode discharge tube manufactured by Westinghouse corporation.

where the factors $\lambda^{n}$ and $\exp (-h w / h T)$ have been incorporated into the proportionality constant. Inspection of (3) and (4) indicates that there should be a correspondence between the laser-induced voltage change and the intensity of the emission line. This is usually the case as can be seen in Fig. 1. In this figure both the emission spectrum (a) and the optogalvanic spectrum (b) are displayed. With the exception of line 7 the agreement of intensities in the two spectra is within a factor of 2. Measurements over a more extensive range show that this agreement is good $10 \pm 30^{\circ} \mathrm{c}$. Care must be taken on lines that appear $t o$ be single but are really blends of more than one line. Occasionally a line such as 7 appears that has anomalous intensity in optogalvanic or emission spectrum (or both). Note that the optogalvanic signals from the neon carrier gas are much larger than those from the sputtered atomic species, even when the neon emission is barely visible in the spectrum. Note also that the discharge currents in the two spectra are different $[75 \mathrm{~mA}$ in (a) and 25 $\mathrm{mA}$ in (b)]. Although the absolute intensities of the emission lines and the optogalvanic signals increase with increasing discharge current, the relative intensit: s within a spectrum are essentially unchanged over this current region. An operating current of $25 \mathrm{~mA}$ was used, as suggested by the tube manufacturer, for the optogalvanic measurements.

The correspondence between laser-induced voltage changes and emission intensities means that an at las of emission lines is useful for optogalvanic wavelength calibration. Such an atlas exists for the uranium transitions in the $3848-9085-\AA$ wavelength region. Current plans include the preparation of a similar atlas for thorium and extension of both atlases into the UV and IR regions. 
The uranium emission intensities are displayed as recorded on the Fourier transform spectrometer (FTS) at Kitt Peak National Observatory. The presentation in Fig. 1 is similar to that in the atlas. The wavelength can be read from these tracings to an accuracy of better than $0.1 \AA$. The wave numbers of the stronger transitions are iabulated with an estimated accuracy of $0.005 \mathrm{~cm}^{-1}$ or better. Both uranium and thorium make excellent wavelength standards because they are heavy, essentially monoisotopic, and have no hyperfine structure. Doppler widths in the commercial hollow cathode discharges were measured to be $\sim 0.03 \mathrm{~cm}^{-1} .{ }^{7}$ Uranium has the advantage of having a denser spectrum over the visible and $\mathrm{IR}$ region. For additional reference, $a$ list of intensities and wave numbers of over 100,000 uranium transitions is available. ${ }^{8}$

Transitions associated with the rare gas carrier are often used for optogalvanic wavelength calibration because of their high intensity. For this rcason, the neon lines are also included in the atlas. They have larger Doppler widths and tend to saturate easier. The optogalvanic signal intensity has no direct correlation to the emission intensity for the neon lines in the atlas.

When the optogalvanic effect is used for wavelength calibration in the UV spectral region, often photoelectric emission from the cathode masks the optogalvanic signal. This is especially true for pulsed excitation where it can even be a problem in the visible region. Construction of a uranium hollow cathode tube with a clear optical path through the cathode would be very useful for optogalvanic wavelength calibration at shorter wavelengths. When working in the UV region with frequency doubled lasers, it is usually better to do the wavelength calibration in the visible region on the fundamental laser beam.

Richard Zare, Stanford University, pointed out the usefulness of an atlas for optogalvanic wavelength calibeation, and John Travis, National Bureau of Standards, suggested inclusion of the paragraph on problems associated with photoelectric emission. We appreciate the help of Jim Brault, Kitt Peak National Observatcry, in obtaining the Fourier transform spectra used in this work.

\section{References}

1. D. S. King, P. K. Schenck, K. C. Smyth, and J. C. Travis, Appl. Opt. 16, 2617 (1977).

2. R. B. Green, R. A. Keller, G. G. Luther, P. K. Schenck, and J. C. Travis, Appl. Phys. Lett. 29, 727 (1976).

3. E. F. Zalewski, R. A. Keller, and R. Engleman, Jr., J. Chem. Phys. 70, 1015 (1979).

4. R. A. Keller, R. Engleman, Jr., and E. F. Zalewski, J. Opt. Soc. Am. 69, 733 (1979).

5. A. P. Thorne, Spectrophysics (Chapman and Hall, London, 1974), Chap. 9.

6. B. A. Palmer, R. Engleman, Jr., and R. A. Keller, "An Atlas of Uranium Emission Intensities in a Hollow Cathode Discharge," LASL report, LA-8251-MS.

7. Measured by optogalvanic spectroscopy with a single frequency dye laser on the Gaussian-shaped $5915-\AA$ uranium emission from a commercial hollow cathode discharge operating at $20 \mathrm{~mA}$.

8. D. W. Steinhaus, L. J. Radziemski, Jr., R. D. Cowan, J. Blaise. G. Guelachvili, Z. B. Osman, and J. Vergés, "Present Status of the Analysis of the First and Second Spectra of Uranium (U I and U II) as Derived from Measurements of Optical Spectra," Los Alamos Scientific Laboratory Report LA-4501 (Oct. 1971). A new report containing many more transitions is in preparation. 